\title{
APLICACIÓN DE NUEVAS TECNOLOGÍAS MONOLÍTICAS Y FOTÓNICAS A REDES CONFORMADORAS DE HACES EN AGRUPACIONES DE ANTENAS PARA BANDAS DE MICROONDAS Y ONDAS MILIMÉTRICAS
}

\author{
Juan Luis Corral González \\ Director: Dr. Javier Martí Sendra
}

Tesis Doctoral presentada en la Universidad Politécnica de Valencia para la obtención del título de Doctor Ingeniero de Telecomunicación

Gandia, Mayo 1998 

A Simone, y a mi madre $y$ hermanos 



\section{Agradecimientos}

Esta Tesis Doctoral no habría existido, tal como es, sin los consejos y ayudas de Francesc Coromina, quien durante los dos años de mi estancia en la Agencia Espacial Europea me contagió su entusiasmo sobre las redes conformadoras de haces. También me gustaría agradecer a mi director de tesis, Javier Martí, su ayuda en esos momentos en los que un par de comentarios, una referencia o un consejo permiten superar barreras que se antojaban insalvables.

En estos momentos, no puedo olvidar a Santiago Flores ni a Jordi Bataller, con quienes he compartido los últimos meses de redacción de nuestras respectivas Tesis Doctorales y gracias a los cuales se ha hecho un poco menos solitaria esta carrera de fondo en que consiste una tesis. 



\section{Índice General}

1 Introducción 1

1.1 Marco de la Tesis . . . . . . . . . . . . . . . . . 1

1.2 Breve descripción del campo de investigación . . . . . . . . . . 2

1.3 Objetivos de la Tesis . . . . . . . . . . . . . . . . . 4

1.4 Estructura de la Tesis $\ldots \ldots \ldots \ldots \ldots$

$\begin{array}{lll}2 & \text { Introducción a las redes conformadoras de haces } & 7\end{array}$

2.1 Introducción . . . . . . . . . . . . . . . . . . 7

2.2 Agrupación de antenas . . . . . . . . . . . . . . . . 7

2.2.1 Apuntamiento de la agrupación . . . . . . . . . . . . 9 9

2.2 .2 Propiedades de radiación . . . . . . . . . . . . . . . 11

2.2.3 Distribución de amplitudes . . . . . . . . . . . . . . . 16

2.2.4 Agrupaciones desfasables de antenas . . . . . . . . . 17

2.2.5 Agrupación retardable de antenas frente a agrupación desfasable de antenas. Subagrupaciones . . . . . . . . . . 19

2.3 Antenas multihaz . . . . . . . . . . . . . . . . . . 23

2.3.1 Haces ortogonales. Límite de Stein . . . . . . . . . . . 24

2.3.2 Antenas multihaz en agrupaciones lineales . . . . . . . 27

2.3.3 Antenas multihaz en agrupaciones planas . . . . . . . . . 32

2.4 Redes conformadoras de haces eléctricas $\ldots \ldots \ldots \ldots$

2.4 .1 Redes de distribución . . . . . . . . . . . . . . . . . . 33

2.4.2 Redes conformadoras para antenas multihaz . . . . . . . . 34

2.4 .3 Impacto de la tecnología MMIC . . . . . . . . . . . 36 
2.5 Redes ópticas conformadoras de haces . . . . . . . . . . . 37

2.6 Efecto sobre el diagrama de radiación de los errores en amplitud y fase en la alimentación de la agrupación . . . . . . . . . . . . . 41

2.6.1 Errores Aleatorios ................. 41

2.6.2 Efecto de la cuantificación de la alimentación de la agrupación 46

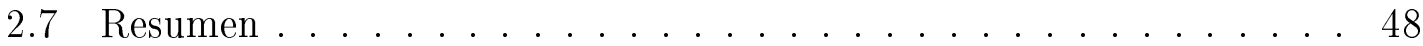

\section{Aplicación de tecnología monolítica a agrupaciones desfasables de antenas}

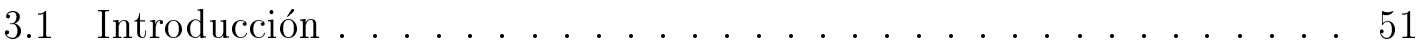

3.2 Efecto combinado de los errores de fase aleatorios y de cuantificación.

Calibración .................... 52

3.2 .1 Sin calibración .................. 53

3.2.2 Calibración simple . . . . . . . . . . . . 59

3.2.3 Calibración según desfasador promedio . . . . . . . . 62

3.2.4 Calibración completa de cada desfasador . . . . . . . . . 62

3.2.5 Resultados combinados . . . . . . . . . . . 63

3.2.6 Redundancia. Desfasadores no binarios . . . . . . . . . . . 71

3.3 Diseño de un desfasador variable a $24 \mathrm{GHz}$ con tecnología MMIC . . 77

3.3 .1 Introducción . . . . . . . . . . . . . 77

3.3.2 Datos generales .................... 77

3.3.3 Primeros enfoques . . . . . . . . . . . . 78

3.3.4 Tres dígitos más significativos $\left(180^{\circ}, 101^{\circ}\right.$ y $\left.57^{\circ}\right) \ldots 82$

3.3.5 Tres dígitos menos significativos $\left(32^{\circ}, 18^{\circ}\right.$ y $\left.10^{\circ}\right) \ldots . . . .93$

3.3.6 Diseño completo . . . . . . . . . . . . . 101

3.3.7 Resultados de simulación para las medidas sobre la oblea del desfasador variable . . . . . . . . . . . . 108

3.4 Medidas RFOW de un desfasador variable a $24 \mathrm{GHz}$. . . . . . . . . 112

3.4.1 Introducción . . . . . . . . . . . . . . 112

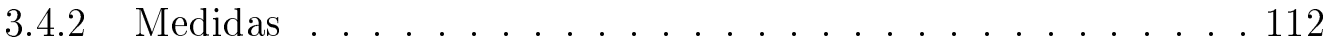

3.5 Conclusiones . . . . . . . . . . . . . . . . . 119 


\section{Aplicación de tecnologías monolíticas a redes conformadoras fijas} para agrupaciones de antenas multihaz

4.1 Introducción . . . . . . . . . . . . . . . . . . . . . 121

4.2 Arquitectura eficiente de red conformadora de haces de radiofrecuencia para agrupaciones hexagonales de antenas . . . . . . . . 122

4.2 .1 Introducción . . . . . . . . . . . . . . . . . . . 122

4.2.2 Análisis de la matriz de Butler generalizada para muestreo hexagonal . . . . . . . . . . . . . . . . . . . 124

4.2.3 Características de las celdas básicas del conformador de haces de radiofrecuencia con peso reducido . . . . . . . . . . . 140

4.3 Diseño de la celda básica $3 \times 3$ con tecnología MMIC . . . . . . . 144

4.3 .1 Datos generales . . . . . . . . . . . . . . . . . 146

4.3.2 Matriz $3 \times 3$ fija . . . . . . . . . . . . . . 147

4.3.3 Matriz $3 \times 3$ sintonizable . . . . . . . . . . . . . . 157

4.3.4 Simulaciones de la red conformadora de haces . . . . . . 168

4.4 Medidas de las matrices $3 \times 3 \ldots \ldots \ldots \ldots$. . . . . . . . . 178

4.4 .1 Matriz $3 \times 3$ fija $\ldots \ldots \ldots \ldots \ldots \ldots$

4.4.2 Matriz $3 \times 3$ sintonizable . . . . . . . . . . . . . 179

4.4.3 Comentarios a los resultados de las medidas de las celdas básicas 183

4.5 La matriz $3 \times 3$ como celda básica. Conformadores de haces $9 \times 9 \mathrm{y}$

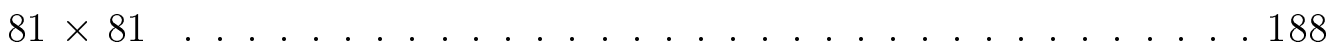

4.5.1 Medidas y simulaciones del conformador $9 \times 9 \ldots \ldots \ldots$

4.5.2 Simulaciones del conformador de haces $81 \times 81 \ldots 195$

4.5.3 Comentarios a las medidas del conformador $9 \times 9 \ldots 201$

4.6 Conclusiones . . . . . . . . . . . . . . . . . . . . . . . . . . . 202

5 Diseño, implementación y medida de una red conformadora de haces controlada ópticamente mediante el uso de redes de difracción en fibra

5.1 Introducción . . . . . . . . . . . . . . . . . 205

5.2 Redes de difracción en fibra . . . . . . . . . . . . . . 206

5.2.1 Redes de difracción con periodo constante . . . . . . . . . 209 
5.2.2 Redes de difracción con periodo variable . . . . . . . . . 213

5.3 Líneas de retardo basadas en redes de difracción . . . . . . . . . . 217

5.3.1 Líneas de retardo programable basadas en redes de difracción con periodo constante . . . . . . . . . . . . . . 217

5.3.2 Líneas de retardo continuamente variables basadas en redes de difracción con periodo variable . . . . . . . . . . 219

5.3.3 Limitaciones en el uso de una red de difracción con periodo lineal como elemento de retardo verdadero en sistemas IM/DD 220

5.3.4 Comparación entre el uso de varias redes de difracción discretas y el uso de una única red de difracción con periodo variable 262

5.3.5 Otros retardadores variables en fibra basados en dispositivos altamente dispersivos .................. 263

5.4 Arquitectura de red conformadora de haces controlada ópticamente . 263 5.4.1 Prestaciones de la red óptica conformadora de haces . . . . . . 266

5.5 Medidas del conformador óptico . . . . . . . . . . . 278

5.5.1 Atenuación dispersiva . . . . . . . . . . . . 278

5.5.2 Caracterización de la red conformadora. Retardos . . . . . . 279

5.5.3 Propiedades de conformación ............... 285

5.6 Conclusiones . . . . . . . . . . . . . . 286

6 Conclusiones y líneas abiertas $\quad 289$

6.1 Conclusiones . . . . . . . . . . . . . . . . 289

6.2 Contribuciones de la Tesis . . . . . . . . . . . . . . 291

6.3 Líneas abiertas y trabajos de continuación . . . . . . . . . . . 291

A Análisis estadístico del efecto de los errores de iluminación de una

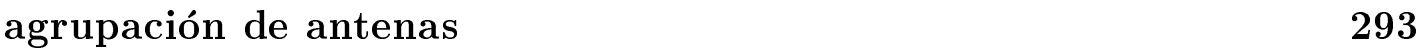

A.1 Caso general . . . . . . . . . . . . . . . 293

A.2 Expresiones particulares . . . . . . . . . . . . . 297

A.3 Errores de fase . . . . . . . . . . . . . . 298

B Características del proceso D02AH 301 
C Elementos de conmutación a $24 \mathrm{GHz}$ en el proceso D02AH

303

C.1 Introducción . . . . . . . . . . . . . . . . . . . . . . 303

C.2 Conmutador serie . . . . . . . . . . . . . . . . . 303

C.3 Conmutador paralelo . . . . . . . . . . . . . . . 305

C.4 Conmutador serie/paralelo . . . . . . . . . . . . . . . . 305

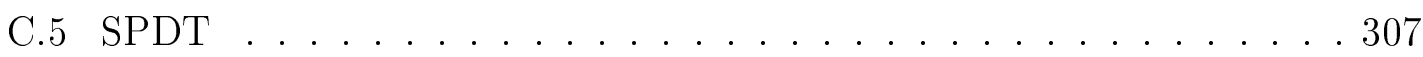

$\begin{array}{ll}\text { BIBLIOGRAFÍA } & 309\end{array}$ 



\section{Índice de Figuras}

2.1 Geometría de una agrupación de antenas genérica. . . . . . . . . 8

2.2 Ejemplo de proyección de contornos de haces sobre el plano $(u, v) \ldots \quad$. 12

2.3 Agrupaciones planas con distribución regular de los elementos. a) Rejilla rectangular. b) Rejilla triangular/hexagonal. . . . . . . . . . 13

2.4 Posiciones de los máximos de radiación en el espacio $(u, v)$ sin apuntamiento $\left(u_{o}=v_{o}=0\right)$ y con apuntamiento. a) Distribución rectangular de los elementos. b) Distribución hexagonal de los elementos. 14

2.5 Agrupaciones desfasables en modo transmisión. a) Agrupación pasiva. b) Agrupación activa. . . . . . . . . . . . . . . . . 18

2.6 Aplicación de retardadores temporales a agrupaciones de antenas. a) Retardadores limitados en cada elemento. b) Retardadores completos a nivel de subagrupaciones. . . . . . . . . . . . . . . . . . 22

2.7 Ejemplo de red conformadora de haces para antena multihaz. . . . . . 23

2.8 Coeficiente de acoplo $\left(C_{p q}\right)$ entre dos haces separados $\Delta u$ para una agrupación lineal de $N=10$ elementos. . . . . . . . . . . 28

2.9 Factor de agrupación en plano $u$ y en espacio real para los $N$ haces ortogonales de una MBA lineal. a) $N=8$. b) $N=9 . \ldots \ldots \ldots .29$

2.10 Nivel de cruce de los haces en agrupaciones lineales (trazo contínuo), planas rectangulares (trazo discontínuo) y hexagonales (trazo punteado) y pérdidas (circunferencias) en los accesos terminados para conformadores de haces sobredimensionados en un factor $M / N$. . . 31

2.11 a) Distribución rectangular de haces. b) Distribución hexagonal de haces. . . . . . . . . . . . . . . . . . 33

2.12 Ejemplo de matriz de Butler para $N=8$ (los desfasadores están indicados en múltiplos de $\left.22.5^{\circ}\right) \ldots \ldots \ldots \ldots \ldots \ldots$

2.13 Ejemplo de matriz de Blass $\ldots \ldots \ldots \ldots \ldots \ldots$

2.14 Diferentes posibilidades de aplicación de tecnología óptica a redes conformadoras de haces. . . . . . . . . . . . . 38 
2.15 Módulo T/R MMIC controlado ópticamente. a) Detección directa y amplificación de la señal de referencia. b) Oscilador local enganchado por inyección. . . . . . . . . . . . . . . . . 39

2.16 Ejemplo de conformador de haces mediante procesado óptico de Fourier en espacio libre. . . . . . . . . . . . . . . . . 40

2.17 Nivel residual normalizado de los lóbulos secundarios de fondo. . . . . 43

2.18 Nivel residual normalizado de lóbulos secundarios respecto a la relación entre nivel de pico de los lóbulos y nivel de diseño parametrizado respecto a la probabilidad de quedar por debajo del nivel de pico. . . 45

3.1 Esquema de un desfasador digital variable de $p$ dígitos y factor $\beta$. . . 54

3.2 Comparativa de los casos A y D en función del error de cada dígito cuando los errores son iguales y con distribución uniforme. a) Error

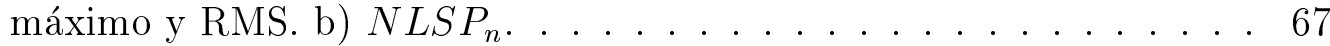

3.3 Comparativa de los casos A y D en función del error de cada dígito cuando los errores son iguales y con distribución gausiana. a) Error

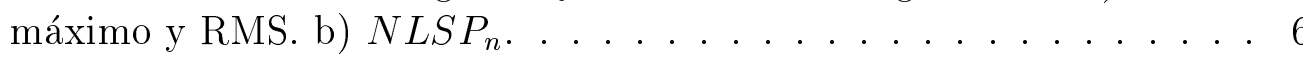

3.4 Comparativa de los casos A y D en función del error de cada dígito cuando los errores son proporcionales y con distribución uniforme. a)

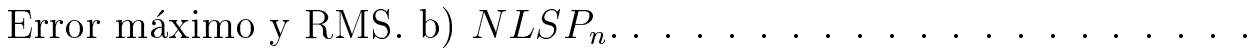

3.5 Comparativa de los casos A y D en función del error de cada dígito cuando los errores son proporcionales y con distribución gausiana. a) Error máximo y RMS. b) $N L S P_{n} \ldots$. . . . . . . . . . . . . 70

3.6 Comparativa en función del error de cada dígito cuando los errores son proporcionales y con distribución uniforme para desfasadores digitales: binario de cinco dígitos (5), binario de seis dígitos (6), binario de cinco dígitos con LSB repetido $(5+\mathrm{LSB})$, no binario de seis dígitos con cinco equivalentes $(5+1)$ y no binario de siete dígitos con cinco equivalentes (5+2). a) Error máximo y RMS. b) $N L S P_{n} \ldots$. . . . . 75

3.7 Diversos tipos de desfasadores variables de microondas. . . . . . . . 81

3.8 Desfasador variable de un dígito con configuración líneas-conmutadas. 82

3.9 Modelo del conmutador P-HEMT del proceso D02AH. . . . . . . . . 84

3.10 Diversas configuraciones SPDT a) Conmutadores en serie. b) Conmutadores en paralelo. c) Conmutadores en serie y paralelo. . . . . . 85

3.11 Configuración final del SPDT. . . . . . . . . . . 87

3.12 Distribución espacial del dígito de $180^{\circ}$ con configuración líneas-conmutadas. . . . . . . . . . . . . . . 8 
3.13 Distribución espacial del dígito de $101^{\circ}$ con configuración líneas-conmutadas. . . . . . . . . . . . . . . . . . . . 9 91

3.14 Distribución espacial del dígito de $57^{\circ}$ con configuración líneas-conmutadas. . . . . . . . . . . . . . . . . . . 992

3.15 Desfasador variable con configuración filtros-conmutados reconfigurable. a) Circuito. b) Red equivalente en estado paso-alto. c) Red equivalente en estado paso-bajo. . . . . . . . . . . . . . . . 94

3.16 Distribución espacial del dígito de $32^{\circ}$ con configuración filtros-conmutados. . . . . . . . . . . . . . . . . . 97

3.17 Distribución espacial del dígito de $18^{\circ}$ con configuración filtros-conmutados. . . . . . . . . . . . . . . . . . . . . 99

3.18 Distribución espacial del dígito de $10^{\circ}$ con configuración filtros-conmutados. . . . . . . . . . . . . . . . . . . . . 100

3.19 Adaptación del desfasador variable completo, incluyendo el efecto de los hilos de conexión (64 estados). a) Adaptación a la entrada. b) Adaptación a la salida. . . . . . . . . . . . . . . . . . . . . . . . 104

3.20 Resultados de simulacion del desfasador variable completo incluyendo el efecto de los hilos de conexión (64 estados). a) Pérdidas de inserción. b) Error de fase respecto al desfase nominal para la frecuencia central. . . . . . . . . . . . . . . . . . . 105

3.21 Distribución espacial del desfasador variable a $24 \mathrm{GHz}$. . . . . . . . . 106

3.22 Simulación RFOW del desfasador variable para catorce estados. a) Adaptación a la entrada. b) Adaptación a la salida. . . . . . . . . . . 110

3.23 Simulación RFOW del desfasador variable para catorce estados. a) Pérdidas de inserción. b) Desfase respecto al estado $0 . \ldots$. . . . . 111

3.24 Medidas RFOW del desfasador variable para catorce estados. a) Adaptación a la entrada. b) Adaptación a la salida. . . . . . . . . . 116

3.25 Medidas RFOW del desfasador variable para catorce estados. a) Pérdidas de inserción. b) Desfase. . . . . . . . . . . . . . . . . . 117

4.1 Ejemplo de matriz de Butler aplicada a una agrupación lineal de 10 elementos, mostrando claramente las relaciones entre los dominios espacial discreto (elementos de la agrupación), frecuencial contínuo (factor de agrupación) y frecuencial discreto (accesos haces) . . . . . 125

4.2 Esquema de DFT de 2 puntos $(\phi=0)$ e implementación analógica de la misma. . . . . . . . . . . . . . . . . . . 127

4.3 Esquema general de una celda básica $N \times N \ldots \ldots \ldots \ldots \ldots$ 
4.4 Matriz de Butler para agrupaciones planas rectangulares de $N \times M$ elementos. . . . . . . . . . . . . . . 130

4.5 Rejilla hexagonal $L_{V}$ generada por la matriz de muestreo $\mathbf{V}=\left[\mathbf{v}_{1}, \mathbf{v}_{2}\right] .131$

4.6 Ejemplo de secuencia finita con doce elementos (trama más oscura) y que cumple la propiedad de cobertura con la matriz de repetición $\mathbf{N}=\left[\mathbf{n}_{\mathbf{1}}, \mathbf{n}_{\mathbf{2}}\right]$ en la rejilla hexagonal $L_{V}, \mathbf{V}=\left[\mathbf{v}_{\mathbf{1}}, \mathbf{v}_{\mathbf{2}}\right] \ldots 133$

4.7 Resumen del cálculo de la arquitectura óptima de red conformadora para agrupaciones con rejilla hexagonal. . . . . . . . . . . . 138

4.8 Ejemplo de secuencia bidimensional sobre rejilla hexagonal, que cumple la propiedad de cobertura. . . . . . . . . . . . . 139

4.9 Relleno de la secuencia correspondiente a una agrupación de $E=19$ elementos, con contorno hexagonal (trama más oscura) cumpliendo cobertura y distribución hexagonal de los haces. a) Secuencia hexagonal no regular con $H=27$ elementos. b) Secuencia rectangular con $H=36$ elementos. . . . . . . . . . . . . . . . 141

4.10 Arquitectura funcional del conformador de haces de Butler $27 \times 27$ para agrupaciones planas hexagonales. . . . . . . . . . . . 142

4.11 Arquitectura funcional del conformador de haces de Butler $81 \times 81$ para agrupaciones planas hexagonales. . . . . . . . . . 143

4.12 Diagrama de bloques funcional de la DFT unidimensional $3 \times 3$. . 145

4.13 Numeración de los 27 elementos de la secuencia hexagonal no regular y de los 19 elementos de la agrupación hexagonal regular. . . . . . . . 145

4.14 Arquitectura de la matriz fija $3 \times 3$ (Topología 1) . . . . . . . . 147

4.15 Implementación real de la topología 1. a) Opción 1. b) Opción 2. . 148

4.16 Circuitos equivalentes concentrados de acopladores direccionales con ramas laterales para una frecuencia de $2.3 \mathrm{GHz}$. a) Acoplamiento $-3 \mathrm{~dB}$ y desfase entre salidas $90^{\circ}$. b) Acoplamiento $-1.76 \mathrm{~dB}$ y desfase entre salidas $90^{\circ}$. . . . . . . . . . . . . . . 149

4.17 Arquitectura de la matriz fija $3 \times 3$ (topología 2) . . . . . . . 150

4.18 Resultados de simulación de la matriz fija $3 \times 3$. a) Adaptación. b) Aislamiento. . . . . . . . . . . . . . . 154

4.19 Resultados de simulación para las pérdidas de inserción de la matriz fija $3 \times 3 \ldots \ldots \ldots \ldots . \ldots \ldots \ldots$

4.20 Distribución espacial del MMIC de la matriz fija $3 \times 3 \ldots . \ldots . . .156$

4.21 Capacidad serie (medidas y simulación) del diodo BE $4 \times 30$ del proceso D02AH. . . . . . . . . . . . . 159 
4.22 Red de polarización de la matriz sintonizable dentro del circuito integrado. . . . . . . . . . . . . . . . 160

4.23 Resultados de simulación de la matriz sintonizable $3 \times 3$. a) Adaptación. b) Aislamiento. . . . . . . . . . . . . . . . . . . . . 162

4.24 Resultados de simulación para las pérdidas de inserción de la matriz

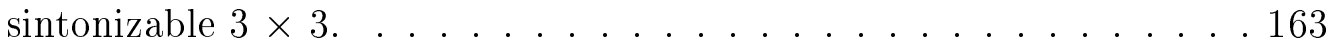

4.25 Distribución espacial del MMIC de la matriz sintonizable $3 \times 3 \ldots 167$

4.26 Conformador de haces $27 \times 27$ como subredes en cascada. . . . . . 168

4.27 Red $2 \times 2$ generalizada para una red con $2 N$ accesos. . . . . . . . 169

4.28 Contornos del factor de agrupación en espacio real $(\theta, \phi)$ para los 27 haces (lóbulos de difracción incluidos) al nivel de cruce (-3.8 dB), para una agrupación de 19 elementos, separados entre sí, una longitud de onda sobre una rejilla hexagonal y utilizando el conformador de haces de tamaño $27 \times 27$, construido con matrices $3 \times 3$ sintonizables y con la numeración de haces y de elementos indicada en la figura 4.10. . . 174

4.29 Contornos Iso-PFD para la agrupación de 37 elementos con rejilla hexagonal de la misión ARCHIMEDES. a) Antena alimentada con el conformador de haces $81 \times 81$ ideal. b) Antena alimentada con el conformador de haces $81 \times 81$ construido con matrices sintonizables

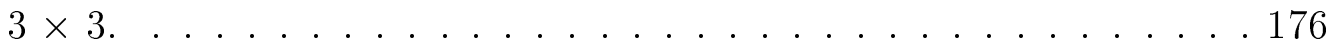

4.30 Ejemplo de área de cobertura para la misión ARCHIMEDES de Difusión de Audio Digital (DAB). . . . . . . . . . . . . . 177

4.31 Esquemático del soporte de prueba usado en las medidas de las matrices $3 \times 3$ fija y sintonizable. . . . . . . . . . . . 178

4.32 Medidas de la matriz $3 \times 3$ fija. a) Respuesta en fase, relativa al camino 2 a 4, para los caminos 2 a 4, 2 a 5 y 2 a 6 . b) Aislamiento entre los accesos 5 y $6 \ldots \ldots \ldots \ldots$

4.33 Medidas de la matriz $3 \times 3$ fija. a) Pérdidas de inserción, relativas al camino 2 a 5 , para los caminos 2 a 4,2 a 5 y 2 a 6 . b) Pérdidas de inserción para el camino 2 y 4 . . . . . . . . . . . . . . 181

4.34 Medidas de la matriz $3 \times 3$ sintonizable en función de la tensión de polarización. a) Desfase relativo entre las salidas 4 y 5 y las salidas 6 y 5 . b) Pérdidas de inserción para las salidas 4,5 y 6 cuando la

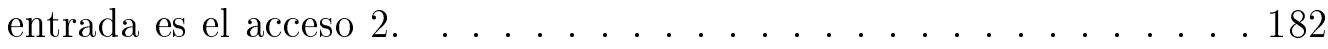

4.35 Esquemático de la matriz $3 \times 3$ mostrando los cinco diferentes valores de inductancia. . . . . . . . . . . . . . . . . . 184 
4.36 Inserción de fase diferencial para la matriz sintonizable $3 \times 3$, en función de la tensión de polarización fuera del circuito integrado. a) Diferencia de desfases entre las salidas 4 y 5 , cuando la entrada es el acceso $2\left(\phi_{24}-\phi_{25}\right)$. b) Diferencia de desfases entre las salidas 6 y 5 , cuando la entrada es el acceso $2\left(\phi_{26}-\phi_{25}\right) \ldots \ldots . \ldots 186$

4.37 Diferencia de pérdidas de inserción para la matriz sintonizable $3 \times 3$, en función de la tensión de polarización fuera del circuito integrado. a) Entre las salidas 4 y 5 , cuando la entrada es el acceso $2\left(P I_{24}-P I_{25}\right)$.

a) Entre las salidas 6 y 5 , cuando la entrada es el acceso $2\left(P I_{26}-P I_{25}\right) .187$

4.38 Esquemático de la red conformadora de haces $9 \times 9$, usando las matrices $3 \times 3$ MMIC como celdas básicas. . . . . . . . . . . . 188

4.39 Sección transversal de la estructura multicapa diseñada por Dassault Electronique. ....................... 189

4.40 Nivel de haz en función del gradiente de fase de la onda incidente, para el conformador $9 \times 9$ alimentando una agrupación lineal de nueve elementos. a) Accesos 1, 2 y 3. b) Accesos 4, 5 y 6. c) Accesos 7, 8 y 9.196

4.41 Contornos del factor de la agrupación para el haz 22 para la antena ARCHIMEDES alimentada por un conformador $81 \times 81$. a) Simulación a partir de medidas del conformador $9 \times 9$. b) Según conformador $81 \times 81$ ideal. . . . . . . . . . . . . 198

4.42 Contornos del factor de la agrupación para el haz 77 para la antena ARCHIMEDES alimentada por un conformador $81 \times 81$. a) Simulación a partir de medidas del conformador $9 \times 9$. b) Según conformador $81 \times 81$ ideal. . . . . . . . . . . . . 199

4.43 Ejemplo de iluminación no uniforme en amplitud para la agrupación hexagonal de ARCHIMEDES. . . . . . . . . . . . . 200

4.44 Fotografía del conformador $9 \times 9$ montado en un soporte de prueba junto a otra copia de la estructura triplaca multicapa con los circuitos integrados montados. . . . . . . . . . . . . . . 203

5.1 Ejemplo de red de difracción. . . . . . . . . . . . . 206

5.2 Configuraciones y respuesta típica de una red de difracción en reflexión.207

5.3 Ejemplo de red de difracción con periodo espacial variable mostrando como el retardo introducido es proporcional a la distancia. . . . . . . 214

5.4 Ejemplo de respuesta en frecuencia de una red de difracción de longitud $L_{\text {red }}=25 \mathrm{~cm}$, con acoplo constante $K_{o} L=5$ y función de fase lineal con factor $F=50$. a) Magnitud de la función de transferencia en reflexión. b) Retardo de grupo de la función de transferencia en reflexión. . . . . . . . . . . . . . . . . 215 
5.5 Ejemplo de línea de retardo basada en el uso de redes de difracción discretas. . . . . . . . . . . . . . . . . . . 218

5.6 Ejemplo de línea de retardo basada en el uso de redes de difracción con periodo variable. . . . . . . . . . . . . . . . . . . . 219

5.7 Esquema de modulador interferométrico Mach-Zehnder con alimentación simétrica. . . . . . . . . . . . . . . . . . . . 221

5.8 Potencia óptica de salida de un modulador Mach-Zehnder en función de la diferencia de tensión entre ambos electrodos. . . . . . . . . . . 222

5.9 Atenuación dispersiva de una señal de radiofrecuencia en la transmisión a través de un enlace IM/DD que implementa una línea de retardo con una red de difracción con periodo variable lineal $(\delta=850 \mathrm{ps} / \mathrm{nm}$,

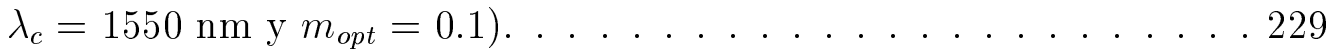

5.10 Potencia relativa de los primeros armónicos de la señal moduladora alrededor de la portadora óptica a la salida de un EOM simétrico. a) Polarización en MIP. b) Polarización en LIN. c) Polarización en MAP. 231

5.11 Potencias relativas de los armónicos de la señal moduladora a la salida del fotodetector de una línea de retardo variable con una red de difracción con dispersión $\delta=100 \mathrm{ps} / \mathrm{nm}$, parametrizadas respecto al índice de modulación del EOM $\left(f_{R F}=1 \mathrm{GHz}\right)$. a) Polarización en MIP. b) Polarización en LIN. c) Polarización en MAP. . . . . . . . . . 235

5.12 Potencias relativas de los armónicos de la señal moduladora a la salida del fotodetector de una línea de retardo variable con modulación externa $\left(m_{o}=0.1\right)$, parametrizadas respecto a la dispersión de la red de difracción $\left(f_{R F}=5 \mathrm{GHz}\right)$. a) Polarización en MIP. b) Polarización en LIN. c) Polarización en MAP. . . . . . . . . . . . . . . . . . 236

5.13 Potencias relativas de contínua y de los cinco primeros armónicos de la moduladora a la salida del fotodetector, en función del producto de dispersión, $\phi_{\text {disp }}$, para un modulador trabajando en la zona lineal. a) $m_{o}=0.1$ b) $m_{o}=0.5 \ldots \ldots \ldots \ldots \ldots \ldots \ldots$

5.14 Potencias relativas de contínua y de los cinco primeros armónicos de la moduladora a la salida del fotodetector, en función del índice de modulación, $m_{o}$, para un modulador trabajando en la zona lineal. a) $\phi_{d i s p}=0 \mathrm{rad} \mathrm{y} \mathrm{b)} \phi_{\text {disp }}=0.1 \mathrm{rad} \ldots \ldots \ldots 239$

5.15 Potencias relativas de contínua y de los cinco primeros armónicos de la moduladora a la salida del fotodetector, en función del índice de modulación, $m_{o}$, para un modulador trabajando en la zona lineal. a) $\phi_{\text {disp }}=1 \mathrm{rad}$. b) $\phi_{\text {disp }}=2 \mathrm{rad} \ldots \ldots \ldots \ldots . \ldots . \ldots 240$ 
5.16 Potencia relativa de los armónicos y de los productos de intermodulación a la salida del fotodetector para una línea de retardo con pendiente $\delta(\mathrm{ps} / \mathrm{nm})$ y un modulador interferométrico polarizado en la zona lineal y modulado por dos tonos de $5 \mathrm{GHz}$ y $6 \mathrm{GHz}$ con índices de modulación $\left(m_{o}=m_{o 1}=m_{o 2}\right)$. a) Resultados para $m_{o}=0.1,0.2$, $0.3,0.4$ y 0.5, y $\delta=100 \mathrm{ps} / \mathrm{nm}$. b) Resultados para $\delta=0,250,500$, 750 y $1000 \mathrm{ps} / \mathrm{nm}, \mathrm{y} m_{o}=0.1$. . . . . . . . . . . . 245

5.17 Potencia relativa de las frecuencias fundamentales y de los productos de intermodulación de tercer orden diferencia a la salida del fotodetector en función del producto de dispersión de la línea de retardo para $\omega_{R F 1}=n \omega_{u}, \phi_{\text {disp }}^{R F 1}$; tomando $m_{o 1}=m_{o 2}=0.1$. a) $n=4, m=5$. b) $n=7, m=8$. c) $n=10, m=11 \ldots . . \ldots 247$

5.18 Potencia relativa de las frecuencias fundamentales y de los productos de intermodulación de tercer orden diferencia a la salida del fotodetector en función del índice de modulación óptico $\left(m_{o}=m_{o 1}=m_{o 2}\right)$ para una dispersión de $\phi_{\text {disp }}^{R F 1}=0.1 \mathrm{rad}$. a) $n=4, m=5$. b) $n=7$,

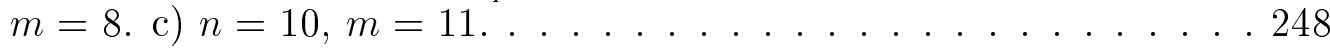

5.19 Potencia relativa de las frecuencias fundamentales y de los productos de intermodulación de tercer orden diferencia a la salida del fotodetector en función del índice de modulación óptico $\left(m_{o}=m_{o 1}=m_{o 2}\right)$ para una dispersión de $\phi_{\text {disp }}^{R F 1}=1 \mathrm{rad}$. a) $n=4, m=5$. b) $n=7$, $m=8$. c) $n=10, m=11 \ldots \ldots . \ldots . \ldots . \ldots . . . \ldots 249$

5.20 Modulador electro-óptico dual aplicado como modulador en banda

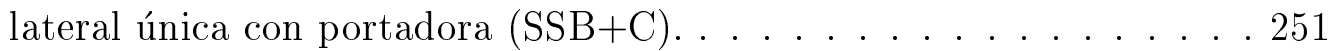

5.21 Generación de señal óptica autoheterodina con una única fuente óptica

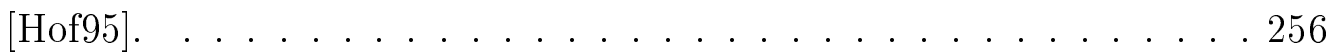

5.22 Modulador óptico pseudo-autoheterodino. . . . . . . . . . . . 257

5.23 Atenuación dispersiva del término de la señal detectada $\omega_{R F}=2 \omega_{O L}+$ $\omega_{I F}$ en la transmisión a través de un enlace IM/DD con modulación pseudoheterodina que implementa una línea de retardo con una red de difracción con periodo variable lineal $\left(\delta=850 \mathrm{ps} / \mathrm{nm}, \lambda_{c}=1550 \mathrm{~nm}\right.$, $\left.m_{o p t}=0.1, \omega_{I F}=1 \mathrm{GHz}\right)$. En traza discontínua, la atenuación si se utilizara modulación AM convencional para la misma frecuencia

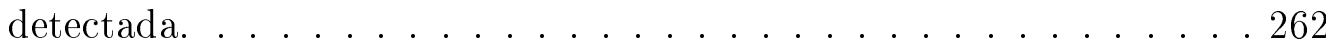

5.24 Diagrama de bloques en transmisión de la red conformadora de haces controlada ópticamente basada en una red de difracción como elemento de retardo verdadero variable. . . . . . . . . . . . 264

5.25 Respuesta ideal de una red de difracción con variación lineal del periodo y posición nominal de las $N$ longitudes de onda de la red conformadora, mostrando el margen de sintonización disponible. . . . . . 265 
5.26 Retardo máximo $\left( \pm \tau_{\max }\right)$ en función del ángulo de barrido $\left( \pm \theta_{\max }\right)$ respecto al ángulo de reposo para diferentes valores del producto $N \cdot d .265$

5.27 Diagrama de bloques en recepción de la red conformadora de haces controlada ópticamente basada en una red de difracción como elemento de retardo verdadero variable. . . . . . . . . . . 267

5.28 Efecto de la dispersión de una rama del conformador sobre el punto de compresión a $1 \mathrm{~dB}$, en función del producto de dispersión, $\phi_{\text {disp }}$. a) Índice de modulación a la entrada del EOM, correspondiente al punto de compresión a $1 \mathrm{~dB}, m_{o}^{1 d B c}$. b) Nivel de potencia relativa de la señal detectada a la salida del fotodetector en el punto de compresión a 1 dB.271

5.29 Efecto de la dispersión de una rama del conformador sobre el punto de intercepción de tercer orden, en función del producto de dispersión, $\phi_{\text {disp. }}$ a) Índice de modulación a la entrada del EOM, correspondiente al punto de intercepción de tercer orden, $m_{o}^{3 I P}$. b) Nivel de potencia relativa de la señal detectada a la salida del fotodetector en el punto de intercepción de tercer orden. . . . . . . . . . . . . . 272

5.30 Obtención de los márgenes dinámicos del conformador óptico basado en redes de difracción con variación lineal del periodo. a) Margen dinámico de compresión. b) Margen dinámico libre de espúreos, SFDR.274

5.31 Efecto del filtrado no ideal en la separación de las portadoras ópticas, sobre una agrupación de diez elementos con separación entre elementos $d=\lambda / 2$, en función del ángulo de apuntamiento y de la caída del filtro entre portadoras $(\mathrm{dBo} / \Delta \lambda)$. a) Valores RMS del error de fase en la alimentación. b) Valores RMS del error de amplitud en la alimentación. c) Error de apuntamiento del haz principal. . . . . . . 277

5.32 Configuración del experimento para la caracterización de los retardos relativos de una única rama de la red óptica conformadora. . . . . . . 279

5.33 Respuesta en reflexión de la red de difracción utilizada en los experimentos. a) Respuesta en amplitud. b) Retardo de grupo. . . . . . . . 280

5.34 Medidas y simulaciones de la atenuación dispersiva de una señal de radiofrecuencia en la transmisión en un sistema IM/DD (modulaciones $\mathrm{AM}$ y SSB+C) que incluye una red de difracción $(\delta=850 \mathrm{ps} / \mathrm{nm})$. (i) Traza contínua: medidas AM IM/DD. Traza discontínua: simulación AM IM/DD. (ii) Traza contínua: medidas SSB+C IM/DD. Traza discontínua: simulación SSB+C IM/DD. $\left(\lambda_{c}=1547.5 \mathrm{~nm}\right.$ y $\left.m_{o}=0.1\right) .281$ 
5.35 Medidas (traza contínua) y simulación (circunferencias) de la atenuación dispersiva del término de la señal detectada $\omega_{R F}=2 \omega_{O L}+\omega_{I F}$ en la transmisión a través de un enlace IM/DD con modulación pseudoheterodina que implementa una línea de retardo con una red de difracción con periodo variable lineal $\left(\delta=850 \mathrm{ps} / \mathrm{nm}, \lambda_{c}=1547.5 \mathrm{~nm}\right.$, $\left.m_{\text {opt }}=0.1, \omega_{I F}=0.5 \mathrm{GHz}\right)$. En traza discontínua, simulación de la atenuación si se utilizara modulación AM convencional para la misma frecuencia detectada. . . . . . . . . . . . . . . . 282

5.36 Medida de los retardos correspondiente a cada uno de los cuatro elementos de la agupación para dos frecuencias de microondas diferentes (2 y $5 \mathrm{GHz}$ ) en función de la desviación respecto a la longitud de onda central $\left(\Delta \lambda_{i}\right)$. Cada figura corresponde a una longitud de onda diferente. . . . . . . . . . . . . . . . 284

5.37 Medidas (traza discontínua) de retardo en cada elemento en función del ángulo de apuntamiento cuando $f_{R F}=5 \mathrm{GHz}$. Se muestran también los resultados teóricos con traza contínua. . . . . . . . . . 285

5.38 Diagrama de radiación ideal (traza discontínua) y simulación del diagrama de radiación a partir de las medidas obtenidas de una rama del conformador (traza contínua) para una agrupación lineal de cuatro elementos isotrópicos con un apuntamiento de $45^{\circ}$. . . . . . . . 286 


\section{Índice de Tablas}

2.1 Características de diversas funciones de iluminación para aperturas contínuas lineales y planas circulares $[$ Sko90a] . . . . . . . . 17

3.1 Comparativa de los estadísticos del error total para desfasadores digitales de cinco y seis dígitos para diferentes estadísticas de error en los dígitos individuales (UI=uniforme,iguales; $\mathrm{GI}=$ gausiana,iguales; $\mathrm{UP}=$ uniforme,proporcionales; $\mathrm{GP}=$ gausiana,proporcionales) y para los casos A (sin calibración) y B (calibración simple). . . . . . . . 63

3.2 Efecto combinado de los errores de cuantificación y errores aleatorios en cada dígito iguales y con estadística uniforme. . . . . . . . . 64

3.3 Efecto combinado de los errores de cuantificación y errores aleatorios en cada dígito iguales y con estadística gausiana (el error en cada dígito corresponde a la desviación estándar del desfase de cada estado). 65

3.4 Efecto combinado de los errores de cuantificación y errores aleatorios en cada dígito proporcionales iguales y con estadística uniforme. . . . 66

3.5 Efecto combinado de los errores de cuantificación y errores aleatorios en cada dígito proporcionales y con estadística gausiana (el error en cada dígito corresponde a la desviación estándar del desfase de cada estado). . . . . . . . . . . . . . . 66 66

3.6 Porcentaje de error tolerable $(\partial)$ en función del número de dígitos reales $(p)$ para cinco dígitos equivalentes $(N=5) \ldots \ldots \ldots 74$

3.7 Número de dígitos equivalentes $(N)$ en función del porcentaje de error tolerable $(\partial)$ para seis dígitos reales $(p=6) \ldots \ldots \ldots \ldots$

3.8 Comparación de resultados estadísticos para diversas configuraciones de desfasadores variables digitales. . . . . . . . . . . . . 79

3.9 Pesos para un desfasador digital variable no binario de seis dígitos con una resolución de cinco dígitos binarios equivalentes. . . . . . 8 80

3.10 Resultados de simulación del dígito de $180^{\circ}$ tipo líneas-conmutadas. . 90

3.11 Resultados de simulación del dígito de $101^{\circ}$ tipo líneas-conmutadas 90 
3.12 Resultados de simulación del dígito de $57^{\circ}$ tipo líneas-conmutadas. $\quad$ - 90

3.13 Resultados de simulación del dígito de $32^{\circ}$ tipo filtros-conmutados. $\quad$ - 98

3.14 Resultados de simulación del dígito de $18^{\circ}$ tipo filtros-conmutados. $\quad 100$

3.15 Resultados de simulación del dígito de $10^{\circ}$ tipo filtros-conmutados. . 101

3.16 Comparativa de los resultados medios del desfasador variable completo, para la mejor y la peor ordenación de los seis dígitos individuales. 102

3.17 Selección de catorce estados del desfasador variable para su medida sobre la oblea. . . . . . . . . . . . . . . . . 108

3.18 Resultados de simulación (adaptación a la entrada y a la salida, pérdidas de inserción e inserción de fase), para catorce estados del desfasador variable completo, correspondientes a medidas RFOW sin hilos de conexión para una frecuencia de $24.15 \mathrm{GHz}$. . . . . . . . . . 109

3.19 Medidas RFOW de desfase para catorce estados del desfasador variable completo correspondientes a tres circuitos integrados distintos y comparadas con los resultados ideales esperados. . . . . . . . . . 113

3.20 Interacciones entre dígitos al conmutar estados. . . . . . . . . . 113

3.21 Medidas RFOW de pérdidas de inserción y de adaptación para catorce estados del desfasador variable completo $\mathrm{CCT}_{3}$. . . . . . . . . . 114

3.22 Comparación entre las medidas del desfasador $\mathrm{CCT}_{3}$ con un desfasador digital binario de cinco dígitos y con un desfasador digital, no binario, de seis dígitos y cinco equivalentes $(6 / 5)$. . . . . . . . 118

3.23 Valor máximo y RMS del error total, nivel normalizado de los lóbulos secundarios respecto al lóbulo principal y variación de la ganancia del haz principal, para cada una de las tres iteraciones del desfasador variable MMIC a $24 \mathrm{GHz}$; resultados obtenidos a partir de las medidas de los desfases de cada uno de los seis dígitos. . . . . . . . . . . . 119

4.1 Resultados de simulación de inserción de fase para la matriz fija MMIC $3 \times 3 \ldots \ldots \ldots \ldots . \ldots \ldots \ldots$

4.2 Resultados de simulación de pérdidas de inserción para la matriz fija

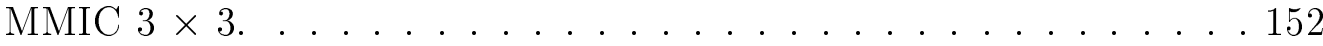

4.3 Resultados de simulación de adaptación y aislamiento para la matriz

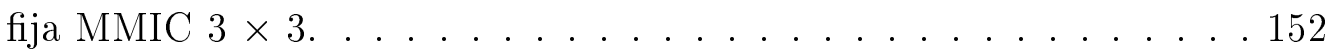


4.4 Efecto de las variaciones de la capacidad del proceso D02AH sobre las prestaciones del conformador $27 \times 27$, basado en la matriz $3 \times 3$ fija (para los 27 haces): error de fase RMS medio $\left(\overline{\delta^{2}}\right)$, error de amplitud RMS medio $\left(\overline{\Delta^{2}}\right)$, nivel de fondo de los lóbulos secundarios respecto al principal $\left(N L S P_{\text {fondo }}\right)$ y variación en la ganancia $(\Delta G)$. . . . 157

4.5 Resultados de simulación de inserción de fase, para la matriz sintonizable MMIC $3 \times 3$. Tensión de polarización de los diodos: $-0.4 \mathrm{~V}$. . 160

4.6 Resultados de simulación de pérdidas de inserción, para la matriz sintonizable MMIC $3 \times 3$. Tensión de polarización de los diodos: -0.4 V. . . . . . . . . . . . . . . . . . 161

4.7 Resultados de simulación de adaptación y aislamiento, para la matriz sintonizable MMIC $3 \times 3$. Tensión de polarización de los diodos: -0.4 V.161

4.8 Resultados de simulación de inserción de fase, para la matriz sintonizable MMIC $3 \times 3$. Tensión de polarización de los diodos: $-0.8 \mathrm{~V}$. Variación de capacidad corregida: $12.8 \%$. . . . . . . . . . . . 164

4.9 Resultados de simulación de pérdidas de inserción, para la matriz sintonizable MMIC $3 \times 3$. Tensión de polarización de los diodos: -0.8 V. Variación de capacidad corregida: $12.8 \%$. . . . . . . . . . 164

4.10 Resultados de simulación de adaptación y aislamiento, para la matriz sintonizable MMIC $3 \times 3$. Tensión de polarización de los diodos: -0.8 V. Variación de capacidad corregida: $12.8 \%$. . . . . . . . . . . 164

4.11 Resultados de simulación de inserción de fase, para la matriz sintonizable MMIC $3 \times 3$. Tensión de polarización de los diodos: $0 \mathrm{~V}$. Variación de capacidad corregida: $-15.4 \%$. . . . . . . . . 165

4.12 Resultados de simulación de pérdidas de inserción, para la matriz sintonizable MMIC $3 \times 3$. Tensión de polarización de los diodos: 0 V. Variación de capacidad corregida: $-15.4 \%$. . . . . . . . 165

4.13 Resultados de simulación de adaptación y aislamiento, para la matriz sintonizable MMIC $3 \times 3$. Tensión de polarización de los diodos: $0 \mathrm{~V}$. Variación de capacidad corregida: $-15.4 \%$. . . . . . . . . 165

4.14 Error de fase medio, y error de amplitud medio para la red conformadora de tamaño $27 \times 27$, basada en la matriz $3 \times 3$ sintonizable. . 172

4.15 Resultados de simulación de una agrupación hexagonal de 27 elementos alimentada por una red conformadora de tamaño $27 \times 27$ fabricada a partir de la matriz $3 \times 3$ fija. . . . . . . . . . 173

4.16 Resultados de simulación de una agrupación hexagonal de 27 elementos alimentada por una red conformadora de tamaño $27 \times 27$ fabricada a partir de la matriz $3 \times 3$ sintonizable. . . . . . . . 173 
4.17 Mejor haz y niveles de densidad de potencia mínimo y máximo para el área de cobertura de la figura 4.30, para doce situaciones distintas, correspondientes a tres posiciones orbitales del satélite y cuatro periodos del año. . . . . . . . . . . . . . . . . 178

4.18 Medidas de la matriz $3 \times 3$ fija. . . . . . . . . . . . . 179

4.19 Medidas de la matriz $3 \times 3$ sintonizable. . . . . . . . . . . 182

4.20 Resultados de simulación de la matriz $3 \times 3$ sintonizable. . . . . . . 185

4.21 Resultados de simulación para la matriz sintonizable $3 \times 3$ (inductancias corregidas). .................. 185

4.22 Errores de fase relativos a la fase de la salida $S_{1}$ (Tensión de sintonización: 0.7 Voltios). . . . . . . . . . . . . . . . 190

4.23 Errores de fase relativos al error de fase medio de cada haz (Tensión de sintonización: 0.7 Voltios). . . . . . . . . . . . . 191

4.24 Valor RMS de los errores de fase, en todas las salidas, para cada una de las nueve entradas de haz antes $\left(\sigma_{\delta}\right)$ y después $\left(\sigma_{\delta}^{c}\right)$ de la inserción de las líneas de corrección en cada salida (Tensión de sintonización: 0.7 Voltios). . . . . . . . . . . . . . . . 191

4.25 Errores de fase, relativos al error de fase medio de cada haz, después de la inserción de las líneas de corrección en cada salida de antena (Tensión de sintonización: 0.7 Voltios). . . . . . . . . . . 192

4.26 Pérdidas de inserción para el conformador $9 \times 9$ (Tensión de sintonización: 0.7 Voltios). . . . . . . . . . . . . . . . 192

4.27 Desviaciones de pico $\left(\Delta_{\max }\right)$ y valor RMS $\left(\sigma_{\Delta}\right)$ de las pérdidas de inserción sobre el valor medio para cada haz (Tensión de sintonización: 0.7 Voltios). . . . . . . . . . . . . . . . . . . . 192

4.28 Resumen de las medidas de pérdidas de inserción del conformador $9 \times 9$ (Tensión de sintonización: 0.7 Voltios) . . . . . . . . . 192

4.29 Desviaciones de pico $\left(\Delta_{\max }\right)$ y valor $\operatorname{RMS}\left(\sigma_{\Delta}\right)$ de las pérdidas de inserción sobre el valor medio para cada haz. a) Tensión de sintonización: 0 Voltios. b) Tensión de sintonización: 0.5 Voltios. . . . . . 193

4.30 Resumen de las medidas de pérdidas de inserción del conformador $9 \times 9$ para las tensiones de sintonización $V_{\text {ext }}=0$ Voltios y $V_{\text {ext }}=0.5$ Vol-

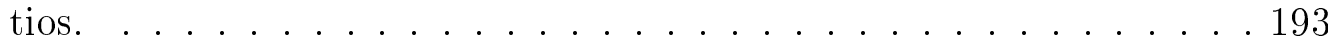

4.31 Valor RMS de los errores de fase en todas las salidas para cada una de las nueve entradas de haz para las tensiones de sintonización $V_{\text {ext }}=0$ Voltios y $V_{\text {ext }}=0.5$ Voltios. . . . . . . . . . . 193 
4.32 Desviaciones de pico $\left(\Delta_{\max }\right)$ y valor $\operatorname{RMS}\left(\sigma_{\Delta}\right)$ de las pérdidas de inserción sobre el valor medio para cada haz (Tensión de sintonización:

0.7 Voltios). Soporte de prueba diseñado por Dassault Electronique. . 194

4.33 Resumen de las medidas de pérdidas de inserción del conformador $9 \times 9$ (Tensión de sintonización: 0.7 Voltios). Soporte de prueba diseñado por Dassault Electronique. . . . . . . . . . . . . . . . . 194

4.34 Valor RMS de los errores de fase en todas las salidas para cada una de las nueve entradas de haz (Tensión de sintonización: 0.7 Voltios). Soporte de prueba diseñado por Dassault Electronique. . . . . . . . . 194

4.35 Efecto sobre el diagrama de radiación de los errores de amplitud y fase de los conformadores $9 \times 9$ de la Agencia Espacial Europea (ESA) y de Dassault Electronique (DE) para diferentes valores de tensión de sintonización. . . . . . . . . . . . . . . 195

4.36 Prestaciones del conformador $81 \times 81$ simulado a partir de las medidas del conformador $9 \times 9$. NLPS $(\mathrm{dB})$ (Valores ideales: $15.8 \mathrm{~dB}$ (iluminación uniforme) $21.1 \mathrm{~dB}$ (iluminación -6/-3/0 dB). . . . . . . 200

4.37 Prestaciones del conformador $81 \times 81$ simulado a partir de las medidas del conformador $9 \times 9$. Ganancia de los haces. . . . . . . . . 200

4.38 Prestaciones del conformador $81 \times 81$ simulado a partir de las medidas del conformador $9 \times 9$. Error en el apuntamiento del centro de los haces. La rejilla usada para las simulaciones es de $0.09^{\circ}$. . . . . . 201

4.39 Prestaciones del conformador $81 \times 81$ simulado a partir de las medidas del conformador $9 \times 9$. Anchos de haz. La rejilla usada en las simulaciones es de $0.09^{\circ}$, siendo el ancho de haz ideal de $4.18^{\circ}$. . . . . 201

5.1 Resultados de simulación de redes de difracción con acoplo y periodo constante en función de la reflectividad máxima a la longitud de onda de Bragg (Anchos de Banda). . . . . . . . . . . . . 211

5.2 Resultados de simulación de la posición y nivel de los tres primeros lóbulos secundarios de la reflectividad para redes de difracción con acoplo y periodo constante en función de la reflectividad máxima a la longitud de onda de Bragg. . . . . . . . . . . . . . . . . 212

5.3 Retardo medio y pendiente para cada una de las cuatro longitudes de onda centrales para 2 y $5 \mathrm{GHz}$, considerando $\Delta \lambda=0.5 \mathrm{~nm}$. . . . . 283

5.4 Desviación estándar del retardo de grupo respecto a la pendiente $\delta_{i}$. . 283

5.5 Valor RMS del error total en el retardo de una rama del conformador óptico. . . . . . . . . . . . . . . . . . . . . . . 284 
C.1 Resultados para diferentes tamaños de conmutador en serie. . . . . . 304

C.2 Resultados para diferentes tamaños de conmutador paralelo. . . . . . 305

C.3 Resultados para diferentes tamaños de conmutador serie/paralelo. . 306

C.4 Resumen de resultados de simulación para diversas configuraciones de conmutadores SPDT. . . . . . . . . . . . 307 


\section{Capítulo 1}

\section{Introducción}

\subsection{Marco de la Tesis}

Esta Tesis Doctoral resume cuatro años de investigación en el campo de las redes conformadoras de haces ${ }^{\mathrm{i}}$ para agrupaciones de antenas ${ }^{\mathrm{ii}}$. Al comienzo del citado periodo y gracias a una beca del Ministerio de Educación y Ciencia, el autor ha trabajado durante dos años en la sección de Tecnología y Equipamiento de Microondas (XRM Section) del Departamento de Sistemas Eléctricos (X Departament) de la Agencia Espacial Europea (ESA, European Space Agency) en su centro de investigación y desarrollo tecnológico ESTEC (ESTEC, European Space Research and Technology Centre) sito en Noordwijk (Países Bajos).

Cada año desde 1987, la sección XRM selecciona uno de los procesos, de una de las diferentes fundiciones de arseniuro de galio (GaAs) y coordina a un grupo de diseñadores para trabajar en el diseño de diferentes circuitos integrados de microondas con tecnología monolítica (MMIC ${ }^{\mathrm{iii}}$ ), con el fin de evaluar directamente las prestaciones de sus procesos, y como medio para adquirir experiencia en las labores de diseño, prueba y medida, de dispositivos MMIC. Durante el año 1994, se escogió el proceso D02AH de la compañia Philips Microwave Limeil (PML); se trata de un proceso Pseudomorphic High Electron Mobility Transistor (P-HEMT) con una longitud de puerta de $0.2 \mu \mathrm{m}$, especialmente adecuado para aplicaciones en la banda de ondas milimétricas hasta $70 \mathrm{GHz}$. Aprovechando esta situación, el autor ha analizado y estudiado la aplicación de circuitos monolíticos a antenas desfasables y antenas multihaz, dos campos de gran interés para el mundo aeroespacial europeo.

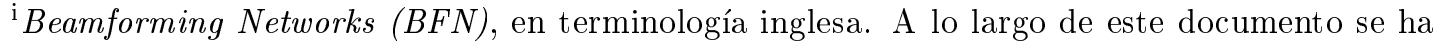
intentado utilizar únicamente nomenclatura en castellano, aún cuando determinados términos sean más conocidos en la comunidad científica y técnica por su denominación en inglés. Solamente se han mantenido los acrónimos en inglés cuando no existe un término equivalente en la literatura en castellano.

ii Antenna Array, en terminología inglesa.

iii Microwave Monolithic Integrated Circuits.
} 
Posteriormente, el autor, reincorporado al Departamento de Comunicaciones de la Universidad Politécnica de Valencia ha continuado sus labores de investigación en el Laboratorio de Comunicaciones Ópticas (LCO) del citado departamento, dentro de grupo de investigación Radio-Fibra, campo realmente prometedor para las aplicaciones de agrupaciones de antenas.

Esta Tesis conjuga por un lado la demostración de nuevos componentes o tecnologías aplicados a arquitecturas ya conocidas, y por lo tanto susceptibles de aplicación inmediata en agrupaciones de antenas actuales, y por otro lado la introducción de arquitecturas totalmente novedosas todavía alejadas de su aplicación comercial.

\subsection{Breve descripción del campo de investigación}

La variedad de nuevos sistemas de comunicaciones y aplicaciones que surgen periódicamente, requieren que las infraestructuras de comunicación sean reconfigurables y flexibles, de forma que se optimicen al máximo los recursos. Para ello es necesario que las antenas sean capaces de modificar sus propiedades de radiación, es decir, la posición de los haces, zonas de cobertura o la frecuencia de trabajo.

La teoría sobre las agrupaciones de antenas es conocida desde hace décadas, pero a la hora de su implementación práctica, ésta ha estado limitada a los casos más simples, dejando los sistemas de mayores prestaciones a aplicaciones militares o espaciales, donde el presupuesto no es siempre la principal limitación. Incluso en esas aplicaciones, es díficil encontrar agrupaciones desfasables electrónicamente, o agrupaciones multihaz, actualmente en funcionamiento.

En 1994, más del 80\% de los sistemas RADAR desplegados disponían de antenas con apuntamiento mecánico, y de aquellos sistemas que utilizaban agrupaciones con apuntamiento electrónico, todos excepto unos pocos eran del tipo pasivo, es decir con un única fuente, generalmente un tubo de onda progresiva. La principal razón para el poco éxito de las agrupaciones desfasables electrónicamente es el coste de adquisición. Durante la última década y gracias al desarrollo de la tecnología monolítica [Mot88, Ima92, Gup91], están disponibles módulos T/R (transmisor/receptor) a precios competitivos y prestaciones más que aceptables, pero todavía queda mucho por hacer.

Actualmente, la tecnología MMIC está lo suficiente avanzada, como para ofrecer módulos $\mathrm{T} / \mathrm{R}$ económicos y con las prestaciones adecuadas; aún así, el desarrollo de agrupaciones de antenas con apuntamiento electrónico y con un gran número de elementos presenta otros inconvenientes como son, el gran volumen, la complejidad de la interconexión de los elementos y de la distribución de las señales de control e información a cada elemento de la agrupación. Si a estas desventajas le añadimos la limitación en cuanto a ancho de banda, justamente se están enunciando las ventajas de los sistemas de distribución ópticos.

La aplicación de tecnologías monolíticas y fotónicas se configuran como las apues- 
tas con mejores perspectivas de cara a futuras aplicaciones prácticas en redes conformadoras de haces, pues las arquitecturas clásicas de distribución de radiofrecuencia pasan a no ser viables cuando se dispone de un alto número de elementos radiantes, debido principalmente a consideraciones de masa, volumen y consumo de potencia. La red conformadora de haces deseada debe ser, a la vez, compacta y ligera. Además se requiere una alta reconfigurabilidad y flexibilidad del sistema completo, así como un nivel de pérdidas reducido, una alta inmunidad frente a interferencias electromagnéticas externas y un gran ancho de banda. Un elemento clave en la integración de las tecnologías monolíticas y fotónicas es el desarrollo de circuitos integrados optoelectrónicos $\left(\mathrm{OEIC}^{\mathrm{i}}\right)$ que facilitan la interconexión de ambos dominios con un tamaño reducido.

Algunas áreas potenciales de aplicaciones comerciales para las antenas de agrupación con apuntamiento electrónico son [Coh94]:

- Uso en vehículos terrestres para, evitar colisiones, comunicaciones, envío de datos para el control del tráfico, y seguimiento y monitorización del vehículo.

- Sistemas aerotransportados y en satélites para predicción metereológica y control medioambiental.

- Comunicaciones móviles, tanto vía satélite como terrestres, con múltiples haces simultáneos con el fin de sectorizar las áreas de cobertura. La necesidad de utilizar haces apuntables viene, para el caso de sistemas vía satélite, de la imposibilidad de trabajar en órbitas geoestacionarias por el retardo que ello implicaría.

- Satélites de difusión directa, optimizando las áreas de cobertura y con asignación dinámica de las mismas.

Y dentro de las aplicaciones militares se pueden citar:

- Sistemas RADAR con blancos múltiples.

- Sistemas de seguimiento y guiado de misiles.

- Sistemas de comunicación de banda ancha.

- Sistemas de guerra electrónica con generación adaptativa de nulos en las direcciones de llegada de interferencias, o inversamente, generación de interferencias en direcciones deseadas.

Verdaderamente, se puede decir que las tecnologías monolíticas y fotónicas están dando nuevas fuerzas al campo de investigación y desarrollo de las agrupaciones de antenas, pues potencialmente permiten obtener las tan ansiadas prestaciones de gran ancho de banda, bajo coste, reconfigurabilidad, peso y volumen reducidos y elevado número de elementos.

\footnotetext{
${ }^{\mathrm{i}}$ Optoelectronic Integrated Circuit.
} 


\subsection{Objetivos de la Tesis}

Cualquier agrupación de antenas desfasable electrónicamente incorpora desfasadores variables, generalmente digitales, ya sean basados en diodos, o en ferritas; la sustitución de estos elementos por sus equivalentes circuitos integrados monolíticos, ha sido el primer paso en la utilización de tecnología MMIC en agrupaciones. Los desfasadores MMIC están sujetos a las tolerancias de los procesos de fabricación, cuya importancia aumenta a medida que se trabaja con frecuencias más altas y se requieren mejores resoluciones. En esta Tesis se pretende analizar el efecto de esas tolerancias sobre el comportamiento de cada desfasador y sobre las prestaciones de la agrupación en su conjunto, buscando nuevas configuraciones que reduzcan su efecto.

Otro campo de aplicación de la tecnología monolítica consiste en las redes conformadoras para antenas multihaz, las cuales son una alternativa a las agrupaciones desfasables, siempre y cuando ofrezcan un número suficientemente elevado de haces cercanos entre sí. Estos conformadores para antenas multihaz, están actualmente limitados en tamaño, por la complejidad de su fabricación y la imprecisión resultante de su ensamblaje. Un aspecto a analizar en esta tesis será la aplicación de nuevas tecnologías electrónicas híbridas que combinen un uso repetitivo de circuitos monolíticos sencillos, interconectados mediante tecnologías de alta integración como pueda ser triplaca multicapa.

La necesidad de encontrar arquitecturas de gran ancho de banda, y redes de distribución de peso, pérdidas y volumen reducidos, para redes conformadoras de haces apunta irremediablemente hacia la tecnología fotónica. Dentro de este amplio campo de trabajo, sería conveniente encontrar nuevas arquitecturas que, además de aumentar considerablemente el ancho de banda, presenten como propiedades un peso reducido, una gran robustez, una alta fiabilidad y gran flexibilidad para el control de la alimentación de la agrupación y la conformación de los haces. En esta Tesis, se analizará la aplicación de redes de difracción sobre fibra como elementos retardadores, estudiando su aplicación a redes ópticas conformadoras de haces.

\subsection{Estructura de la Tesis}

Teniendo en cuenta el marco de la Tesis, presentado previamente, se pueden distinguir dos campos sobre los que se ha trabajado con un objetivo común, como es el de aplicar dentro del amplio campo de las redes conformadoras de haces nuevas ideas, conceptos o arquitecturas. Para ello, es necesario introducir brevemente en el capítulo 2 los conceptos, limitaciones y tendencias relacionados con las agrupaciones de antenas, incidiendo sobre aquellos que posteriormente serán de utilidad. Al final del citado capítulo se hace un breve resumen de lo presentado, realzando aquellos aspectos que los capítulos posteriores pretenden cubrir.

Una vez se ha introducido el campo de investigación y detectados diferentes 
entornos en los que se pueden aportar nuevas ideas y resultados, éstos se suceden en los capítulos siguientes, de forma que los capítulos 3 y 4 presentan los resultados obtenidos al aplicar tecnología monolítica a antenas desfasables electrónicamente y a antenas multihaz, respectivamente.

Por otro lado, en el capítulo 5, se ha analizado la introducción de un nuevo dispositivo óptico, como elemento retardador de gran ancho de banda, del cual se han establecido sus prestaciones y limitaciones. Este retardador se ha utilizado como base de una prometedora arquitectura de red óptica conformadora de haces.

Aunque cada uno de los tres capítulos presenta unas conclusiones propias de la aplicación sobre la que se ha trabajado, resumiendo los resultados más destacados, en el capítulo 6 se recogen unas conclusiones globales sobre los resultados de esta Tesis Doctoral, identificando las contribuciones originales de la misma. 



\section{Capítulo 2}

\section{Introducción a las redes conformadoras de haces}

\subsection{Introducción}

El objetivo de este capítulo es presentar el campo de investigación de la Tesis, mostrando el estado actual del mismo y haciendo especial hincapié a su vez, en los resultados básicos necesarios para el posterior desarrollo de la Tesis. Este capítulo pretende explicar brevemente, qué es una agrupación de antenas, qué es una red conformadora de haces y cuales son sus requerimientos típicos, sus aplicaciones y sus arquitecturas actuales. No se intenta cubrir todos los conceptos y variantes, sino aquellas más interesantes para el desarrollo de la Tesis.

El concepto de red conformadora de haces aparece íntimamente ligado al de agrupación de antenas, ya que la primera se encarga de alimentar a la segunda, con el fin de que la agrupación radíe uno o varios haces, con unas características determinadas en cuanto a dirección de apuntamiento, posición de los nulos, ganancia o lóbulos secundarios. De hecho, una de las principales ventajas de las agrupaciones de antenas es la posibilidad de controlar la iluminación de la agrupación, con el fin de obtener lóbulos secundarios reducidos, o incluso, un diagrama de radiación conformado de acuerdo a las especificaciones, función que se encarga de realizar la red conformadora de haces.

\subsection{Agrupación de antenas}

Una agrupación de antenas está formada por un conjunto de elementos radiantes, generalmente iguales o de características similares, distribuidos espacialmente de acuerdo con los requerimientos de radiación esperados. En general, la principal motivación para el uso de las agrupaciones de antenas es el conseguir mayor directi- 


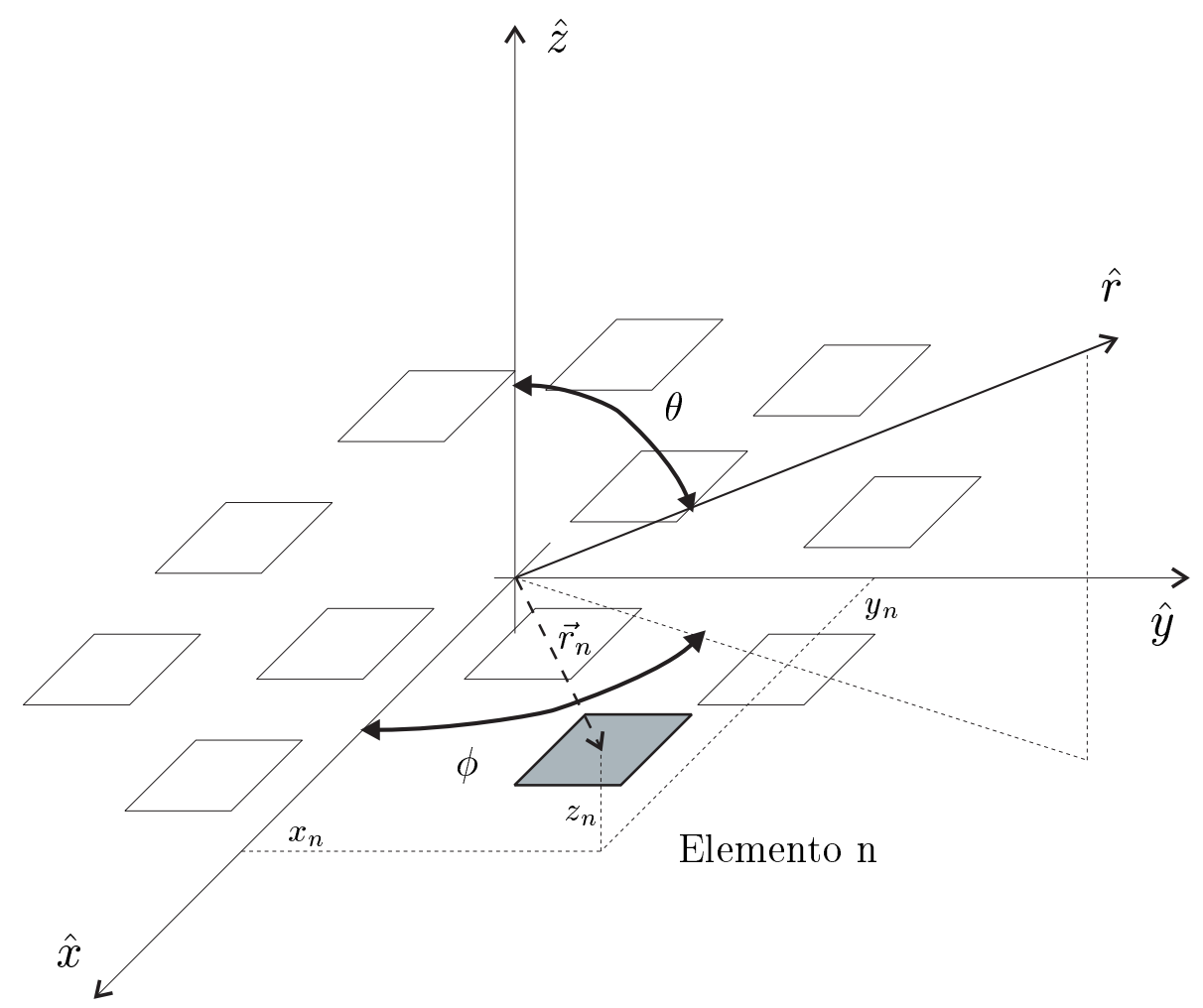

Figura 2.1: Geometría de una agrupación de antenas genérica.

vidad que la que ofrecería un único elemento radiante, gracias a la mayor dimensión geométrica de la agrupación.

El diagrama de radiación de la agrupación de antenas vendrá definido por la ponderación que se haga de la contribución de cada uno de los elementos y por la combinación temporal que se haga de esas mismas contribuciones; es decir por el coeficiente (siempre positivo) y el retardo que se le aplique a cada elemento de la agrupación. Las propiedades a tener en cuenta en el diagrama de radiación son, principalmente, la dirección de máxima radiación (apuntamiento de la antena), el ancho de haz en la citada dirección, el nivel y posición de los lóbulos secundarios, la posición de los lóbulos de difracción y la posición de los nulos de radiación.

Por ejemplo, para la geometría de la figura 2.1, y despreciando posibles acoplamientos entre los diversos elementos ${ }^{\mathrm{i}}$, supuestos iguales, el diagrama de radiación en campo de una agrupación de $\mathrm{N}$ elementos alimentados cada uno con la misma señal con amplitudes $A_{n}$ y fases $\alpha_{n}$ sería [Mai93a]:

$$
F_{\text {TOTAL }}(\theta, \phi)=F_{\text {TOTAL }}(\hat{r})=f_{e}(\theta, \phi) \sum_{n=1}^{N} A_{n} e^{j \alpha_{n}} e^{j k \vec{r}_{n} \hat{r}}
$$

donde $k=2 \pi / \lambda$ es el número de onda de la onda plana incidente o transmitida y

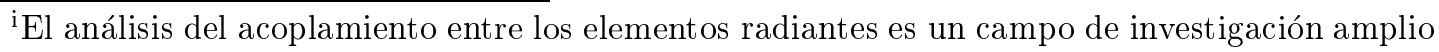
dentro del electromagnetismo, que queda fuera del objetivo de esta Tesis Doctoral.
} 
$f_{e}(\theta, \phi)$ es el diagrama de radiación de un elemento por separado. Además:

$$
\begin{aligned}
\hat{r} & =u \hat{x}+v \hat{y}+\cos (\theta) \hat{z} \\
u & =\sin (\theta) \cos (\phi) \\
v & =\sin (\theta) \sin (\phi) \\
\vec{r}_{n} & =x_{n} \hat{x}+y_{n} \hat{y}+z_{n} \hat{z}
\end{aligned}
$$

donde $u$ y $v$ se denominan cosenos directores. La dirección en la que se calcula el diagrama de radiación vendrá definida indistintamente por el vector director $\hat{r}$ o por el par de ángulos $(\theta, \phi)$.

Se puede comprobar en (2.1) que, despreciando acoplamientos entre elementos, el diagrama de radiación de una agrupación es el producto del diagrama de radiación de un elemento, por un sumatorio de términos dependientes de la ponderación y posición de cada elemento, a este sumatorio se le denomina factor de la agrupación:

$$
F_{o}(\theta, \phi)=F_{o}(\hat{r})=\sum_{n=1}^{N} A_{n} e^{j \alpha_{n}} e^{j k \vec{r}_{n} \hat{r}}
$$

Si se consideran los elementos radiantes como isotrópicos, el diagrama de radiación y el factor de la agrupación coinciden. En los resultados mostrados en la presente Tesis, se asumirá que los elementos son isotrópicos.

\subsubsection{Apuntamiento de la agrupación}

Si se desea obtener, para todas las frecuencias, un máximo del diagrama de radiación en la dirección $\left(\theta_{o}, \phi_{o}\right)$, definida por el vector director $\hat{r}_{o}$, la fase de la alimentación del elemento n-ésimo debería ser, independientemente de los coeficientes de amplitud $A_{n}$ :

$$
\begin{aligned}
\alpha_{n} & =-k \hat{r}_{o} \vec{r}_{n}=-k\left[\sin \left(\theta_{o}\right)\left(x_{n} \cos \left(\phi_{o}\right)+y_{n} \sin \left(\phi_{o}\right)\right)+\cos \left(\theta_{o}\right) z_{n}\right] \\
& =-k\left[u_{o} \sin \left(\theta_{o}\right)\left(x_{n} \cos \left(\phi_{o}\right)+y_{n} \sin \left(\phi_{o}\right)\right)+\cos \left(\theta_{o}\right) z_{n}\right]
\end{aligned}
$$

donde $u_{o}=\sin \left(\theta_{o}\right) \cos \left(\phi_{o}\right)$ y $v_{o}=\sin \left(\theta_{o}\right) \sin \left(\phi_{o}\right)$ son los cosenos directores en la dirección de máxima radiación.

El desfase calculado en (2.7) presenta una variación lineal con la frecuencia y por lo tanto, equivale a introducir el siguiente retardo en la distribución a cada elemento:

$$
\tau_{n}=\frac{\sin \left(\theta_{o}\right)\left(x_{n} \cos \left(\phi_{o}\right)+y_{n} \sin \left(\phi_{o}\right)\right)+\cos \left(\theta_{o}\right) z_{n}}{c}=\frac{u_{o} x_{n}+v_{o} y_{n}+\cos \left(\theta_{o}\right) z_{n}}{c}
$$

retardo, que compensa exactamente los diferentes caminos recorridos hacia/desde cada elemento, por una onda plana incidente/transmitida que viaje en la dirección $\left(\theta_{o}, \phi_{o}\right)$, obteniéndose en esa dirección el siguiente valor del factor de la agrupación:

$$
F_{o}\left(\theta_{o}, \phi_{o}\right)=F_{o}\left(\hat{r}_{o}\right)=\sum_{n=1}^{N} A_{n}
$$


En el caso de que se disponga de dispositivos que permitan obtener los retardos indicados en (2.8), para las señales de cada elemento radiante, se estará ante una agrupación cuyo máximo de radiación apuntará en la dirección $\hat{r}_{o}$, para todas las frecuencias, siendo la expresión de su diagrama de radiación:

$$
F_{o}(\theta, \phi)=F_{o}(\hat{r})=\sum_{n=1}^{N} A_{n} e^{-j k \hat{r}_{o} \vec{r}_{n}} e^{j k \hat{r} \vec{r}_{n}}
$$

Una vez se dispone de una agrupación de antenas fija (por ejemplo, con divisores de potencia y líneas de transmisión de diferentes longitudes), cuyo factor de la agrupación sea el mostrado en (2.10), apuntando en la dirección deseada, la variación de ese ángulo de apuntamiento, podría venir por un ajuste mecánico de la posición de la agrupación. Esta solución es conocida como agrupación de antenas con apuntamiento mecánico ${ }^{\mathrm{i}}$.

Otra posibilidad, con prestaciones muy superiores, es realizar un apuntamiento electrónico, es decir variar las características del diagrama de radiación, mediante la modificación de los coeficientes $\left(A_{n}\right)$ y los retardos $\left(\tau_{n}\right)$ o fases $\left(\alpha_{n}\right)$ aplicados a las contribuciones de cada uno de los elementos constituyentes de la agrupación, sin necesidad de alterar la posición de la antena, y por lo tanto, sin necesidad de movimientos mecánicos, lo cual redunda claramente en una mayor rapidez en la adaptación del diagrama.

Esta solución es conocida como agrupación de antenas apuntables electrónicamente ii, en la cual la contribución de cada uno de los elementos de la agrupación aparece ponderada por una amplitud y una fase determinadas, pero variables.

Las ventajas operacionales de las agrupaciones de antenas con apuntamiento electrónico, sobre las antenas con apuntamiento mecánico son, entre otras, la capacidad de seguimiento de blancos múltiples, la posibilidad de sintetizar y conformar el haz de forma adaptativa dirigiendo incluso los nulos de radiación en direcciones escogidas, una mayor superficie de antena (y por lo tanto ganancia) para un volumen dado del sistema y, finalmente, la capacidad de usar algoritmos de detección mejorados (una consecuencia de la agilidad de haz) [Coh94, Lis91].

Es interesante comentar que a la hora de realizar el apuntamiento electrónico, la compensación de los diferentes retardos en cada elemento debería realizarse, idealmente con retardadores, de forma que esta estructura sería, por construcción, de banda ancha. Por limitaciones tecnológicas, y debido a que hasta recientemente las aplicaciones eran de banda estrecha, se sustituían los retardadores por desfasadores equivalentes a una frecuencia dada $\left(f_{R F}\right)$, es decir:

$$
\alpha_{n}=-k_{R F} \hat{r}_{o} \vec{r}_{n}=-\omega_{R F} \tau_{n}
$$

donde $\alpha_{n}$ es el desfase a aplicar al elemento n-ésimo para que a la frecuencia $\omega_{R F}=$ $2 \pi c / \lambda_{R F}$ se obtenga un retardo $\tau_{n}$, el cual depende del ángulo de apuntamiento y

${ }^{\mathrm{i}}$ Mechanically Steered Array Antenna, en terminología inglesa.

ii Electronically Steered Array Antenna, en terminología inglesa. 
de la situación del elemento, según (2.8). Con esos valores de $\alpha_{n}$ la expresión del diagrama de radiación quedaría:

$$
F_{o}(\theta, \phi)=\sum_{n=1}^{N} A_{n} e^{-j k_{R F} \hat{r}_{o} \vec{r}_{n}} e^{j k \hat{r} \vec{r}_{n}}
$$

expresión que presenta un máximo en la dirección $\hat{r}_{o}$ únicamente para la frecuencia $k=k_{R F}$, por lo que la estructura de la agrupación desfasable, inicialmente de banda ancha, pasa a ser de banda estrecha. Cuando el apuntamiento se realiza de acuerdo con las fases de (2.11), en lugar de las indicadas en (2.7), se hablará de agrupación desfasable de antenas ${ }^{\mathrm{i}}$, término que suele abarcar a todas las agrupaciones de antenas con apuntamiento electrónico, por ser el caso más habitual.

Por lo que respecta a los coeficientes $A_{n}$, y al igual que ocurre en las antenas de apertura, la distribución de las amplitudes $A_{n}$ en la agrupación determina la forma del diagrama de radiación, especialmente el ancho de haz y el nivel de los lóbulos secundarios y por lo tanto se escogerán de acuerdo con las especificaciones del diagrama de radiación.

Una selección conjunta de los valores de amplitud $\left(A_{n}\right)$ y fase $\left(\alpha_{n}\right)$ permitirá la conformación del diagrama de radiación deseado, mediante técnicas de síntesis de agrupaciones; siendo posible incluso la sintetización adaptativa de los diagramas de radiación de cada haz [Har93, Gab76, Gri83]. Dentro de la síntesis adaptativa de diagramas, ha merecido especial interés investigador, la obtención de diagramas con nulos en direcciones específicas ${ }^{\mathrm{ii}}$, con el fin de eliminar o reducir interferencias propias o externas [Mai82].

\subsubsection{Propiedades de radiación}

Dependiendo de la disposición espacial de los elementos de la agrupación, se hablará de agrupación lineal, cuando todos los elementos estén situados sobre un eje, agrupación plana, cuando los elementos se distribuyan sobre un plano y, agrupación conformada, cuando los elementos se configuran siguiendo la forma del material que sirve de soporte a la agrupación; lo que, generalmente, implica que los elementos dejen de compartir una misma orientación.

A partir de este instante, los resultados presentados se limitarán al caso más habitual, de agrupaciones planas. A la hora de representar el diagrama de radiación de una agrupación plana, es habitual utilizar el plano $(u, v)$ [Aul60], que para el margen visible, pasa a ser la proyección del diagrama de radiación en la esfera unidad sobre el plano de la agrupación, y que, entre otras propiedades, cumple, que una variación del ángulo de apuntamiento, de acuerdo con (2.7), se traduce en una traslación del diagrama en el plano $(u, v)$ sin deformarlo (Fig. 2.2).

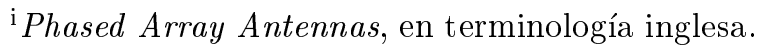

ii Nulling, en terminología inglesa.
} 


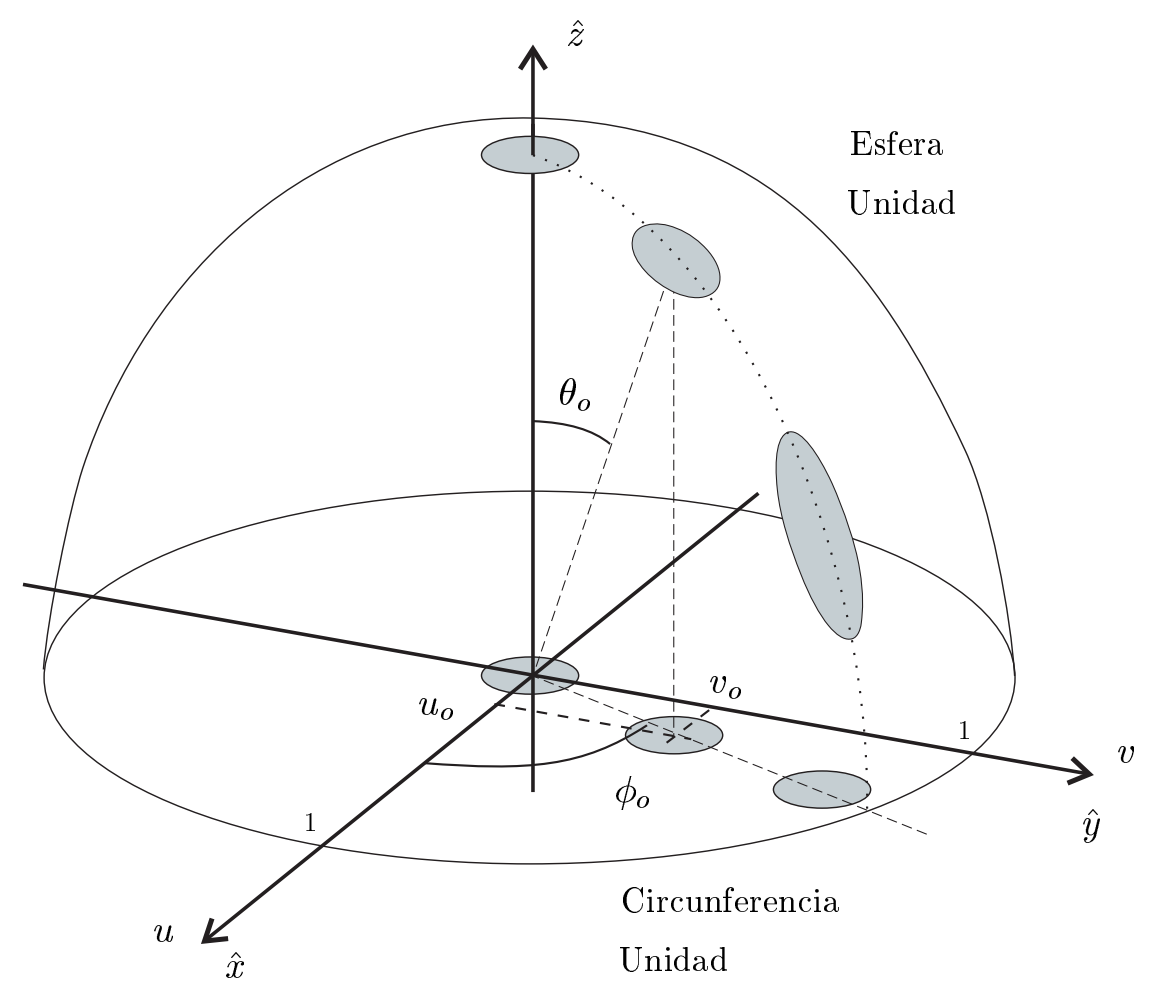

Figura 2.2: Ejemplo de proyección de contornos de haces sobre el plano $(u, v)$.

Para una agrupación plana, la expresión del factor de la agrupación será:

$$
F_{o}(u, v)=F_{o}(\theta, \phi)=F_{o}(\hat{r})=\sum_{n=1}^{N} A_{n} e^{j \alpha_{n}} e^{j k\left(u x_{n}+v y_{n}\right)}
$$

En el caso de una agrupación plana con $z_{n}=0$ se puede simplificar el resultado (2.7), de forma, que la expresión del factor de la agrupación (2.13), cuando se desea un máximo en la dirección $\left(\theta_{o}, \phi_{o}\right)$, será:

$$
F_{o}(u, v)=F_{o}(\theta, \phi)=F_{o}(\hat{r})=\sum_{n=1}^{N} A_{n} e^{j k\left(\left(u-u_{o}\right) x_{n}+\left(v-v_{o}\right) y_{n}\right)}
$$

Por cuestiones de simplicidad y eficiencia de radiación, es habitual distribuir los elementos de una agrupación plana, en filas y columnas con espaciado interelementos constante $\left(d_{x}\right.$ y $d_{y}$ en cada eje). En la figura 2.3 se muestran dos ejemplos de esta distribución. Para el caso de disponer los elementos de acuerdo con una rejilla rectangular, (Fig. 2.3a), las coordenadas del centro de cada elemento serán de la forma $\left[x_{n}, y_{n}\right]=\left(p d_{x}, q d_{y}\right)$ con $p, q \in \mathbb{Z}$. De acuerdo con (2.14), es fácil comprobar que el factor de la agrupación, tomará el valor máximo indicado en (2.9), siempre que el exponente de la función exponencial de (2.14) sea un múltiplo de $2 \pi$, para cualquier valor de $n$. En el caso de la agrupación plana con rejilla rectangular, esta 


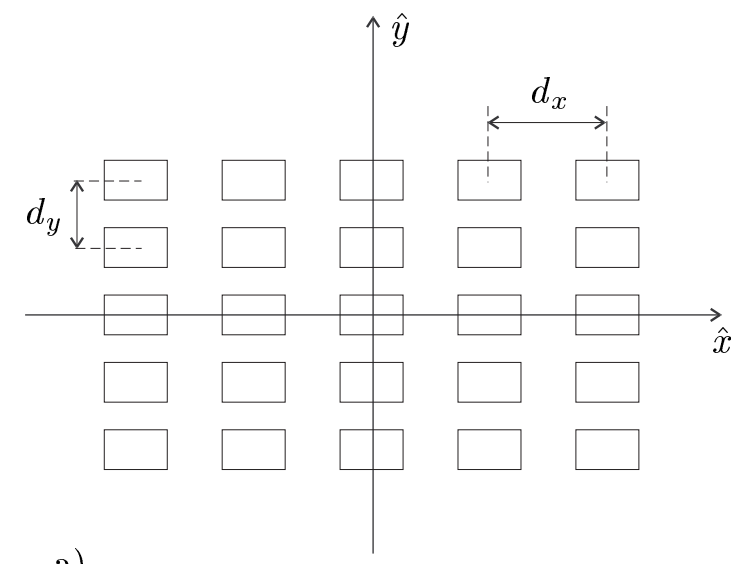

a)

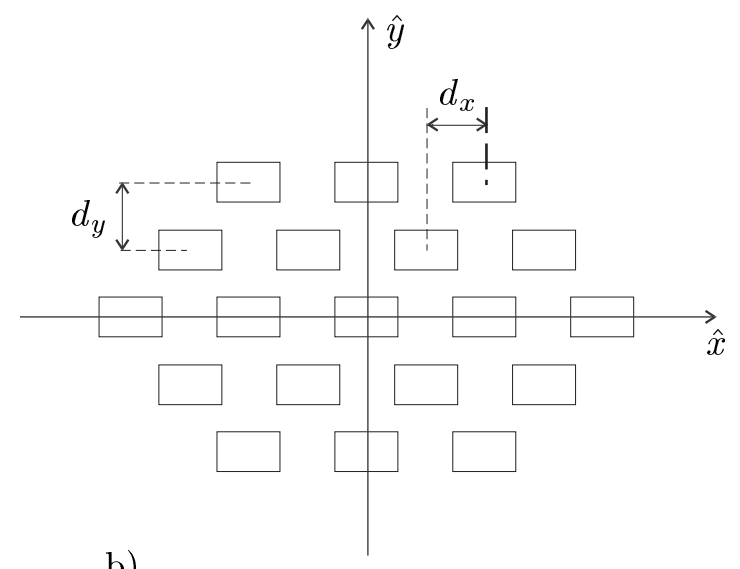

b)

Figura 2.3: Agrupaciones planas con distribución regular de los elementos. a) Rejilla rectangular. b) Rejilla triangular/hexagonal.

condición se traduce en:

$$
\begin{aligned}
& \frac{d_{x}}{\lambda}\left(u_{M A X}-u_{o}\right)=r ; r \in \mathbb{Z} \rightarrow u_{M A X}=u_{o}+\frac{r \lambda}{d_{x}} \\
& \frac{d_{y}}{\lambda}\left(v_{M A X}-v_{o}\right)=s ; s \in \mathbb{Z} \rightarrow v_{M A X}=v_{o}+\frac{s \lambda}{d_{y}}
\end{aligned}
$$

las coordenadas $\left[u_{M A X}, v_{M A X}\right]$ se suelen representar sobre el plano $(u, v)$ de la forma mostrada en la figura 2.4, donde se presentan los casos particulares, $u_{o}=0$ y $u_{o} \neq 0$.

Otra disposición habitual de los elementos en una agrupación plana es la rejilla triangular o hexagonal, mostrada en la figura $2.3 \mathrm{~b}$, en cuyo caso, las coordenadas del centro de cada elemento, en filas alternas, serán de la forma $\left[x_{n}, y_{n}\right]=$ $\left((2 p) d_{x},(2 q) d_{y}\right)$ con $p, q \in \mathbb{Z}$ o $\left[x_{n}, y_{n}\right]=\left((2 p+1) d_{x},(2 q+1) d_{y}\right)$ con $p, q \in \mathbb{Z}$. En el caso de la agrupación plana, con rejilla triangular/hexagonal, las condiciones que se deben cumplir, simultáneamente, para que el factor de agrupación (2.14) dé una valor máximo, se traduce en:

$$
\begin{aligned}
& \frac{d_{x}}{\lambda}\left(u_{M A X}-u_{o}\right)=\frac{2 r+1}{2} ; r \in \mathbb{Z} \rightarrow u_{M A X}=u_{o}+\frac{(2 r+1) \lambda}{2 d_{x}} \\
& \frac{d_{y}}{\lambda}\left(v_{M A X}-v_{o}\right)=\frac{2 s+1}{2} ; s \in \mathbb{Z} \rightarrow v_{M A X}=v_{o}+\frac{(2 s+1) \lambda}{2 d_{y}}
\end{aligned}
$$

o bien, el siguiente par de condiciones:

$$
\begin{aligned}
& \frac{d_{x}}{\lambda}\left(u_{M A X}-u_{o}\right)=r ; r \in \mathbb{Z} \rightarrow u_{M A X}=u_{o}+\frac{r \lambda}{d_{x}} \\
& \frac{d_{y}}{\lambda}\left(v_{M A X}-v_{o}\right)=s ; s \in \mathbb{Z} \rightarrow v_{M A X}=v_{o}+\frac{s \lambda}{d_{y}}
\end{aligned}
$$

las coordenadas $\left[u_{M A X}, v_{M A X}\right]$ para el caso de rejilla triangular se muestran en la figura 2.4 para $u_{o}=0$ y para $u_{o} \neq 0$. 

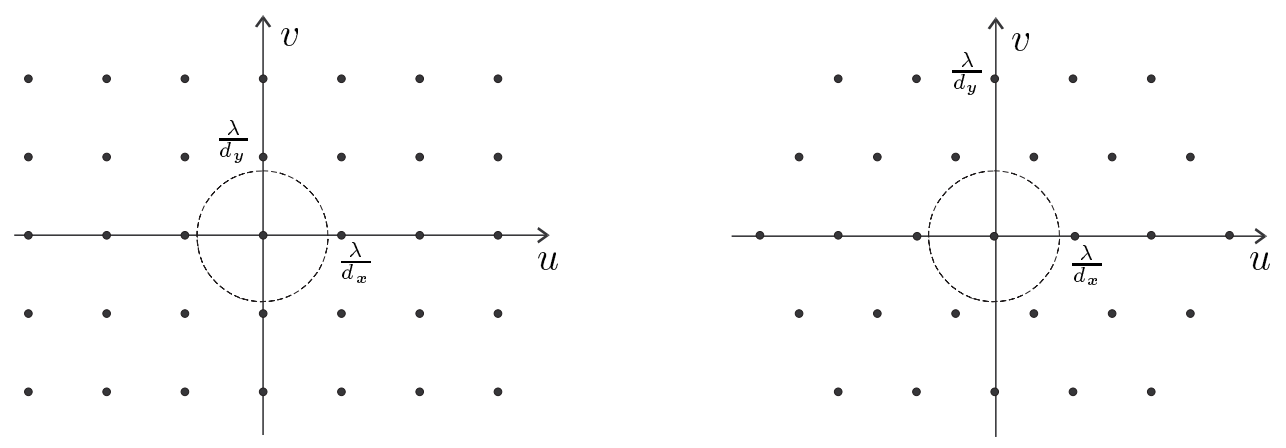

a)

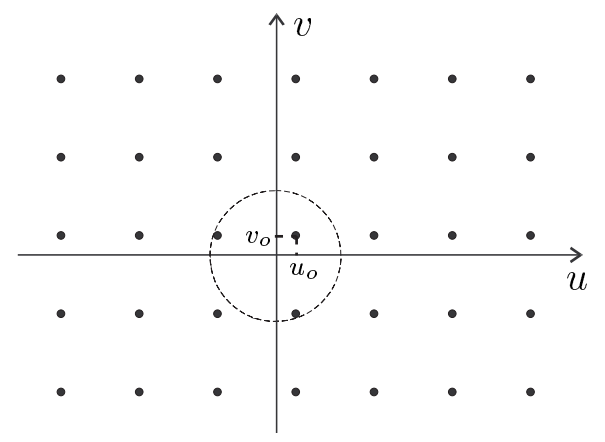

b)

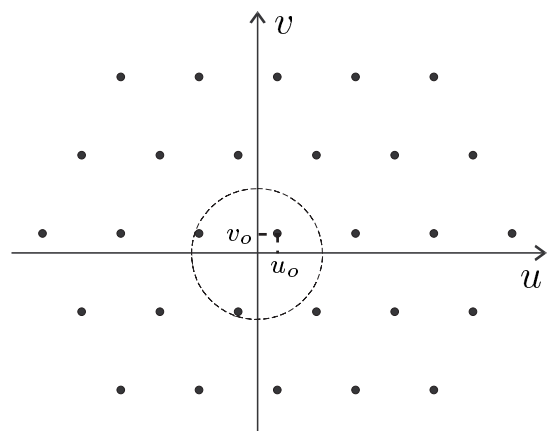

Figura 2.4: Posiciones de los máximos de radiación en el espacio $(u, v)$ sin apuntamiento $\left(u_{o}=v_{o}=0\right)$ y con apuntamiento. a) Distribución rectangular de los elementos. b) Distribución hexagonal de los elementos.

\section{Lóbulos de difracción}

De acuerdo con la definición de los cosenos directores, y como se puede observar en la figura 2.2, el margen visible corresponde dentro del espacio $(u, v)$ a la condición:

$$
u^{2}+v^{2} \leq 1
$$

Si el margen visible abarcara más de uno de los máximos del espacio $(u, v)$ se diría que existen lóbulos de difracción, que corresponden a máximos de radiación en direcciones diferentes a las deseadas. Tal y como se muestra en la figura 2.4, se debe tener en cuenta el margen de ángulos de apuntamiento de la agrupación, para que, en ningún caso, aparezcan lóbulos de difracción dentro del margen visible.

Si la zona de cobertura de la agrupación consiste en un cono de ángulo $\theta_{M A X}$ alrededor de la normal al plano de la agrupación, el espaciado de los elementos, distribuidos rectangularmente, deberá cumplir las siguientes condiciones para que no aparezcan lóbulos de difracción:

$$
\left(1+\operatorname{sen}\left(\theta_{M A X}\right)\right) \leq \frac{\lambda}{d_{x}} \quad y \quad\left(1+\operatorname{sen}\left(\theta_{M A X}\right)\right) \leq \frac{\lambda}{d_{y}}
$$

luego el área de cada elemento será como máximo de:

$$
A_{\text {rect }}=d_{x} \cdot d_{y} \leq \frac{\lambda^{2}}{\left(1+\operatorname{sen}\left(\theta_{M A X}\right)\right)^{2}}
$$


Para el caso de elementos distribuidos hexagonalmente, la condición que debe cumplir su espaciado se resume en:

$$
\left(1+\operatorname{sen}\left(\theta_{M A X}\right)\right) \leq \frac{\lambda}{d_{x}} \quad y \quad\left(1+\operatorname{sen}\left(\theta_{M A X}\right)\right) \leq \frac{\lambda}{\sqrt{3} d_{y}}
$$

siendo el área máxima que puede ocupar cada elemento de:

$$
A_{h e x}=2 d_{x} \cdot d_{y} \leq \frac{2 \lambda^{2}}{\sqrt{3}\left(1+\operatorname{sen}\left(\theta_{\text {MAX }}\right)\right)^{2}}
$$

Comparando (2.22) con (2.20), se concluye que para las mismas condiciones de cobertura cónica, una distribución hexagonal de los elementos permite cubrir la misma superficie total de la agrupación, con un 15.5\% menos elementos, lo cual inmediatamente implica menos controles y un menor coste para agrupaciones desfasable de antenas. En caso de trabajar con áreas de coberturas distintas de la cónica, las conclusiones sobre el dimensionamiento de la agrupación variarán [Sha61].

\section{Ancho de haz. Directividad}

El ancho de haz a mitad de potencia para una agrupación lineal uniforme de $N$ elementos isotrópicos, separados entre sí, una distancia $d$ en la dirección normal a la agrupación, depende del tamaño total de la agrupación $(N \cdot d)$ de la siguiente forma aproximada [Mai93a]:

$$
\Delta \theta_{-3 \mathrm{~dB}}(\mathrm{rad}) \approx \frac{0.886 \lambda}{N \cdot d}
$$

si se apunta el haz un ángulo $\theta_{o}$ respecto a la normal, el ancho de haz aumenta de acuerdo con la siguiente expresión:

$$
\Delta \theta_{-3 \mathrm{~dB}}(\mathrm{rad}) \approx \frac{0.886 \lambda}{N \cdot d \cos \left(\theta_{o}\right)}
$$

que no será válida en las direcciones longitudinales a la agrupación.

La directividad para la misma agrupación con distribución de amplitudes cualquiera será [Mai93a]:

$$
D=\frac{\left(\sum_{n=1}^{N} A_{n}\right)^{2}}{\sum_{n=1}^{N} \sum_{m=1}^{N} A_{n} A_{m} e^{-j k d(n-m) \cos \left(\theta_{o}\right)} \operatorname{sinc}(k d(n-m))}
$$


expresión que se puede simplificar para elementos espaciados media longitud de onda como:

$$
D=\frac{\left(\sum_{n=1}^{N} A_{n}\right)^{2}}{\sum_{n=1}^{N} A_{n}^{2}}=N \eta
$$

donde $\eta$ es la eficiencia por iluminación y se define como:

$$
\eta=\frac{\left(\sum_{n=1}^{N} A_{n}\right)^{2}}{N \sum_{n=1}^{N} A_{n}^{2}}
$$

Cuando la separación entre los elementos de la agrupación está entre 0 y $\lambda$ se puede aproximar (2.25) como [Mai93a]:

$$
D \approx \frac{2 d}{\lambda} \frac{\left(\sum_{n=1}^{N} A_{n}\right)^{2}}{\sum_{n=1}^{N} A_{n}^{2}}=\frac{2 d}{\lambda} N \eta
$$

\subsubsection{Distribución de amplitudes}

El ancho del haz principal y el nivel de los lóbulos secundarios, están gobernados por la distribución de amplitudes de la iluminación de la agrupación, de forma que, es posible reducir el nivel de los lóbulos secundarios, excitando más los elementos centrales de la agrupación frente a los elementos extremos. A cambio, el ancho del haz principal aumenta y la directividad se reduce de acuerdo con (2.25).

Para cada distribución de amplitudes se define un factor de ensanchado de haz $f_{\text {ens }} \mathrm{y}$ la ecuación (2.24) pasa a ser:

$$
\Delta \theta_{-3 \mathrm{~dB}}(\mathrm{rad}) \approx \frac{0.886 \lambda}{N \cdot d \cos \left(\theta_{o}\right) f_{\text {ens }}}
$$

donde $f_{\text {ens }}=1$ para la agrupación uniforme.

En cuanto a los diferentes tipos de distribuciones disponibles, es posible aplicar resultados conocidos de la teoría de filtros y del enventanado en sistemas discretos, teniendo en cuenta la relación transformada que existe entre la iluminación de la agrupación y el diagrama de radiación. En la tabla 2.1, se muestra un resumen de 


\begin{tabular}{|l|c|c|c|}
\hline Iluminación & Eficiencia, $\eta$ & NLSP $(\mathrm{dB})$ & Factor de ensanche, $f_{\text {ens }}$ \\
\hline \hline Uniforme & 1 & -13.3 & 1 \\
\hline Coseno & 0.81 & -23 & 0.75 \\
\hline Hanning & 0.67 & -32 & 0.62 \\
\hline Hamming & 0.73 & -43 & 0.68 \\
\hline Dolph-Chebyshev & 0.66 & -60 & 0.62 \\
\hline Taylor $\bar{n}=3$ & 0.9 & -26 & 0.85 \\
\hline Taylor $\bar{n}=5$ & 0.8 & -36 & 0.75 \\
\hline \hline Circular Uniforme & 1 & -17.6 & 0.87 \\
\hline Circular Taylor $\bar{n}=3$ & 0.91 & -26 & 0.79 \\
\hline
\end{tabular}

Tabla 2.1: Características de diversas funciones de iluminación para aperturas contínuas lineales y planas circulares [Sko90a].

características (eficiencia $(\eta)$, nivel del mayor lóbulo secundario respecto al lóbulo principal $(N L S P)$ y factor de ensanche $\left.\left(f_{\text {ens }}\right)\right)$ de diferentes distribuciones para aperturas contínuas lineales y circulares ${ }^{\mathrm{i}}$ [Sko90a]. Los resultados para aperturas contínuas son una aproximación muy buena respecto a las agrupaciones lineales, siempre y cuando el espaciado entre elementos sea menor de media longitud de onda.

Para aperturas lineales, se ha comprobado que el nivel de los lóbulos secundarios está directamente relacionado con la continuidad en los extremos, de la distribución de amplitud y sus derivadas [Mai93a].

Aunque los resultados aquí presentados corresponden a agrupaciones lineales, éstos son extrapolables a agrupaciones planas para planos normales a la agrupación, si se considera como distribución de la agrupación, la suma de las contribuciones en ejes normales al plano de la agrupación.

\subsubsection{Agrupaciones desfasables de antenas}

Como se comentó previamente, la utilización de agrupaciones desfasables de antenas conlleva innumerables ventajas en cuanto a la flexibilidad de uso de la antena, por su propiedad de conformar, adaptar y reconfigurar su diagrama de radiación.

La aplicación de agrupaciones desfasables de antenas se pueden dividir en dos grupos [Lis91]:

Antenas de Imagen ${ }^{\mathrm{ii}}$ Una agrupación desfasable de pequeño tamaño se magnifica a través de un reflector [Ass69] o de un sistema dual, con un subreflector y un reflector [Hay87, Ram87, Tun86, Yng88].

\footnotetext{
iPara aperturas circulares, el factor de ensanche hace referencia a una apertura lineal de tamaño igual al diámetro de la apertura circular.

ii Imaging antennas.
} 
a)
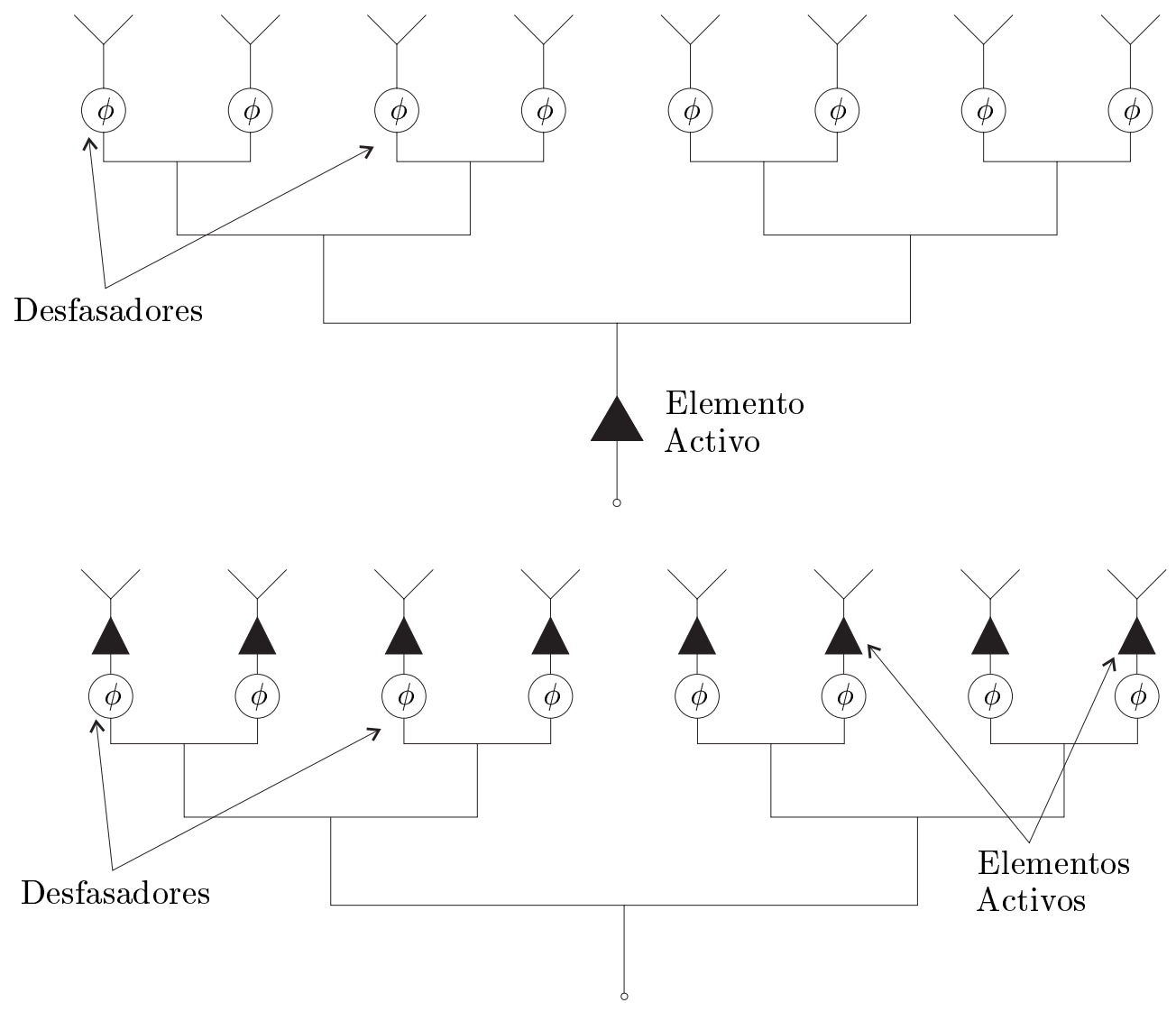

Figura 2.5: Agrupaciones desfasables en modo transmisión. a) Agrupación pasiva. b) Agrupación activa.

Agrupación de radiación directa $\left(\mathrm{DRA}^{\mathrm{i}}\right)$ El reflector parabólico más el conjunto de los alimentadores, se sustituye por una agrupación de elementos radiantes, con un tamaño total similar al tamaño del reflector. Generalmente, estos elementos radiantes son agrupados en subagrupaciones, alimentadas conjuntamente.

Otra clasificación, más interesante desde el punto de vista de las redes conformadoras de haces, es la distinción entre agrupaciones activas y pasivas. En una agrupación pasiva (Fig. 2.5a): en transmisión, la señal a transmitir se genera en una fuente de gran potencia y, mediante una red de distribución pasiva, se reparte esa señal a cada elemento con la amplitud y fase requerida; mientras que, en recepción, las señales recibidas en cada elemento son combinadas, pasivamente, con el factor y el retardo adecuados, y la salida de esta combinación es llevada al receptor.

Por otro lado, las agrupaciones desfasables activas (Fig. 2.5b): en transmisión, la señal a transmitir, ya sea en banda base o modulada a la frecuencia portadora, es repartida con bajos niveles de potencia a cada uno de los elementos radiantes, donde será amplificada al nivel adecuado para la transmisión mediante un amplificador de

i Direct Radiating Array. 
potencia en cada elemento o subconjunto de elementos; la conformación del haz, es decir la adecuación de la amplitud y la fase de la señal para cada elemento puede realizarse en el mismo elemento o mediante la red de distribución. En recepción, la señal recibida en cada elemento es detectada y amplificada con un amplificador de bajo ruido, previamente a la combinación de las contribuciones de cada uno de los elementos o subconjuntos de elementos radiantes.

Dentro de las ventajas de las agrupaciones activas, frente a las pasivas, conviene citar la degradación gradual de las agrupaciones activas, al trabajar con una multiplicidad de fuentes. Además, las agrupaciones activas, ofrecen una mayor sensibilidad en recepción y, en general, una mayor eficiencia [Coh94, Lis91].

Si se desea trabajar con $N$ haces independientes, será necesario repetir $N$ veces la estructura de la agrupación desfasable, lo que encarece considerablemente la agrupación total. Además del número de haces disponibles, otro criterio muy importante a la hora de comparar agrupaciones activas y pasivas es la eficiencia de la misma. En 1992 se propusieron una serie de soluciones mixtas, que trabajan con un conjunto de amplificadores en una etapa intermedia, entre el acceso de cada haz y los elementos radiantes, de forma que todos los amplificadores están trabajando siempre un su punto de máxima eficiencia [Roe92].

\subsubsection{Agrupación retardable de antenas frente a agrupación desfasable de antenas. Subagrupaciones}

A partir de (2.8), es posible calcular el efecto que provoca, la variación de la frecuencia de trabajo, en la posición del máximo del diagrama de radiación, obteniéndose para el sistema de referencia de la figura 2.1:

$$
\begin{aligned}
\frac{\Delta \theta_{o}}{\Delta \omega_{R F}} & =\frac{-1}{\omega_{R F}} \tan \left(\theta_{o}\right) \\
\frac{\Delta \phi_{o}}{\Delta \omega_{R F}} & =0
\end{aligned}
$$

como ejemplo, indicar que, para una aplicación que trabaje con un ancho de banda de la señal radiada de un $5 \%$, ésta limitación conllevaría un movimiento del haz principal de $\pm 1.5^{\circ}$ alrededor de $\theta_{o}=45^{\circ}$. El desapuntamiento del haz es mayor a medida que el haz apunta a direcciones más alejadas de la normal al plano de la agrupación.

Este efecto de variación del ángulo de apuntamiento en función de la frecuencia es conocido como estrabismo del haz $z^{\mathrm{i}}$, y constituye la principal limitación, en cuanto a ancho de banda, para las agrupaciones desfasables de antenas. En realidad, no sólo el ángulo de apuntamiento varía con la frecuencia, sino que la forma del diagrama de radiación de una antena de agrupación desfasable, deja de ser independiente de

\footnotetext{
${ }^{\mathrm{i} B e a m}$ squinting, en terminología inglesa.
} 
la frecuencia, obteniéndose para el caso de la figura 2.1, la siguiente expresión:

$$
F_{N}(\theta, \phi, f)=f_{e}(\theta, \phi) \sum_{n=1}^{N} A_{n} e^{-j \frac{\omega_{R F}}{\omega}\left(\omega \tau_{n}\right)} e^{j\left(k_{x} x_{i}+k_{y} y_{i}\right)}
$$

donde $\omega_{R F}$ es la frecuencia para la que se ha diseñado la antena de agrupación. Esta dependencia del diagrama de radiación con la frecuencia, puede afectar significativamente a los niveles de los lóbulos secundarios, a la posición de los nulos de radiación, además de al ángulo de apuntamiento.

Para un receptor situado en la dirección del máximo de radiación, el estrabismo del haz se traduce en una distorsión lineal de la señal recibida, debido, a la mayor atenuación sufrida por las componentes de mayor frecuencia de la señal en banda base, en función del menor valor del diagrama de radiación de la agrupación $F_{N}\left(\theta_{o}, \phi_{o}, f\right)$. Este efecto es la principal consecuencia del estrabismo del haz para agrupaciones de antenas con un haz principal ancho y un ancho de banda relativamente estrecho. De acuerdo con (2.30), el ancho de banda relativo a $-3 \mathrm{~dB}$, por efecto del estrabismo del haz, para una antena de agrupación desfasable apuntando a $\left(\theta_{o}, \phi_{o}\right)$, sería:

$$
\frac{\Delta \omega_{-3 \mathrm{~dB}}\left(\theta_{o}, \phi_{o}\right)}{\omega_{R F}}=\frac{\Delta \theta_{-3 \mathrm{~dB}}\left(\theta_{o}, \phi_{o}\right)}{\tan \left(\theta_{o}\right)}
$$

donde $\Delta \theta_{-3 \mathrm{~dB}}\left(\theta_{o}, \phi_{o}\right)$ es el ancho de haz a mitad de potencia, en radianes, de la antena de agrupación en la dirección $\left(\theta_{o}, \phi_{o}\right)$. En el caso particular en el que el ángulo máximo de apuntamiento del haz, respecto a la normal al plano de la agrupación, fuera $45^{\circ}\left(\theta_{o}<45^{\circ}, \forall \phi_{o}\right)$, éste constituiría el peor caso y el ancho de banda sería:

$$
\frac{\Delta \omega_{-3 \mathrm{~dB}}}{\omega_{R F}}=\Delta \theta_{-3 \mathrm{~dB}}
$$

Teniendo en cuenta la ecuación (2.29), se concluye que el ancho de banda relativo es inversamente proporcional al tamaño total de la agrupación, por serlo también el ancho de haz:

$$
\frac{\Delta \omega_{-3 \mathrm{~dB}}\left(\theta_{o}, \phi_{o}\right)}{\omega_{R F}}=\frac{0.886 \lambda}{N d \cdot \operatorname{sen}\left(\theta_{o}\right) f_{\text {ens }}}
$$

por consiguiente, la limitación en frecuencia, puede ser razonable para agrupaciones pequeñas, pero no para agrupaciones grandes.

El estrabismo del haz puede corregirse con una ligera amplificación de las frecuencias altas de la señal en banda base para agrupaciones de antenas con un haz principal bastante ancho o anchos de banda muy estrechos. Por otra parte, para aplicaciones que requieran diagramas de radiación muy directivos (anchos de haz de pocos grados, o incluso, décimas de grado), y anchos de banda grandes, el efecto del estrabismo del haz hace totalmente inviable la utilización de agrupaciones desfasables de antenas. Requerimientos más exigentes, tales como, la obtención de nulos 
selectivos en determinadas direcciones o de lóbulos secundarios por debajo de un cierto nivel, no hacen más que agravar el problema de la utilización de agrupaciones desfasables de antenas en aplicaciones de banda ancha.

A partir de las consideraciones anteriores, es evidente la necesidad de trabajar con retardadores temporales, en lugar de desfasadores, para la conformación del haz, en aplicaciones de banda ancha. A las agrupaciones basadas en retardadores se les denomina agrupaciones retardables de antenas ${ }^{\mathrm{i}} \mathrm{y}$ al método operativo en el que se basan, retardo verdadero ${ }^{\text {ii }}$, para diferenciar estos retardadores de aquellos basados en desfasadores que aprovechan la periodicidad de la señal, para únicamente implementar retardos menores de un periodo. Líneas de transmisión de diferentes longitudes constituyen ejemplos de retardos fijos, fácilmente implementables, pero para las aplicaciones habituales son necesarias líneas excesivamente largas y pesadas. Por lo tanto, conseguir dispositivos de retardo verdadero variables, compactos en tamaño, reducidos en peso y coste y de gran ancho de banda es uno de los retos actuales para la tecnología de agrupaciones de antenas.

Debido a consideraciones de coste, peso y volumen de las arquitecturas actualmente disponibles, para dispositivos de retardo verdadero, no se suelen implementar agrupaciones de antenas retardables propiamente dichas, sino una combinación de desfasadores y retardadores. En concreto, los dos enfoques más habituales a la hora de introducir retardadores en agrupaciones de antenas son:

- Introducción de unidades retardadoras en cada elemento de la agrupación (Fig. 2.6a), pero limitadas a $M$ posibles posiciones de retardo, correspondientes a $M$ posiciones de apuntamiento del haz en el espacio real. Para ajustar la posición del haz a un ángulo intermedio entre dos de esas $M$ posiciones, se utilizan desfasadores variables convencionales. De esta forma, se aumenta el ancho de banda en un factor $M$ [Mai93a].

- Dividir el conjunto de elementos de la antena de agrupación se divide en subagrupaciones $^{\mathrm{iii}}$ (Fig. 2.6b), generalmente iguales, de forma que la distribución de la señal a los diferentes subagrupaciones se lleva a cabo mediante retardadores, mientras que, cada subagrupación es tratada internamente como una agrupación desfasable de antenas. El menor tamaño de las subagrupaciones hace, que el ancho de haz correspondiente sea lo suficientemente ancho, como para permitir trabajar con un ancho de banda adecuado a la aplicación concreta, mejorando el ancho de banda de la antena $M$ veces.

En ambos casos, el diagrama de radiación de la antena de agrupación total, resulta ser el producto del diagrama de radiación de un elemento, del factor de la agrupación de una subagrupación, y del factor de la agrupación considerando cada subagrupación como un elemento. En el caso de que el tamaño de la subagrupación

\footnotetext{
i Timed Phased Array, en terminología inglesa.

ii True-Time Delay, en terminología inglesa.

iii Subarray, en terminología inglesa.
} 


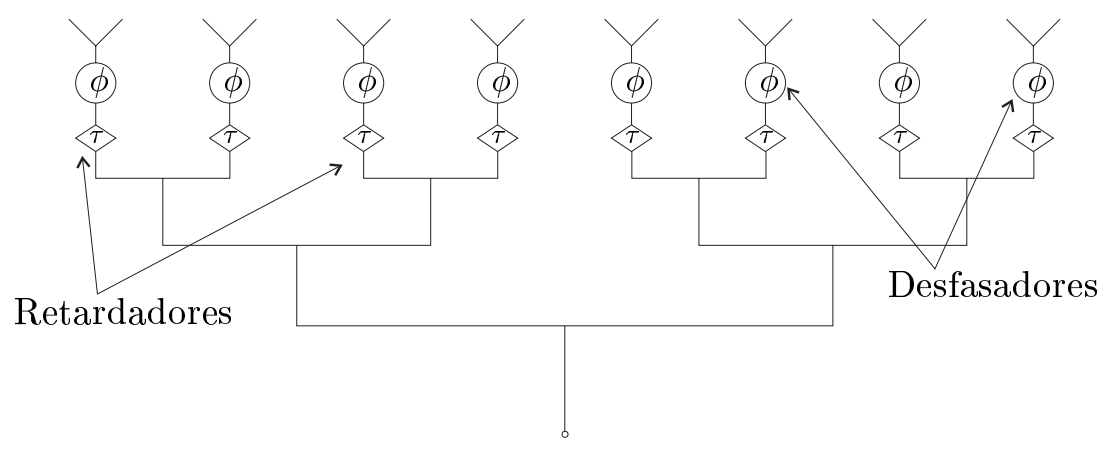

a)

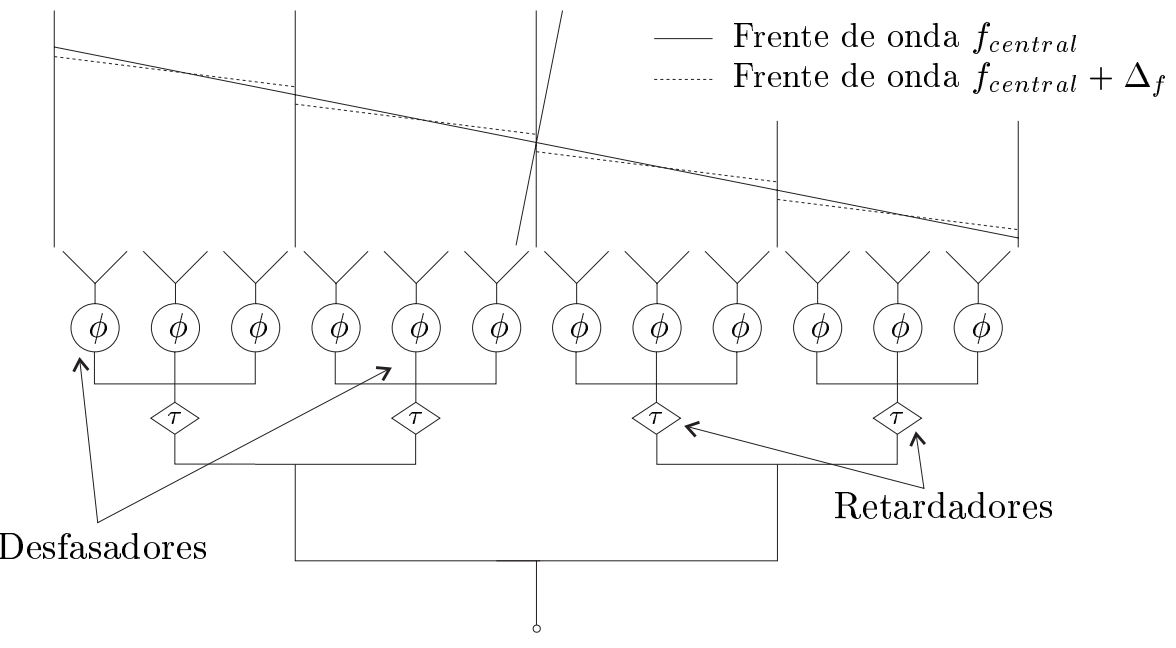

Figura 2.6: Aplicación de retardadores temporales a agrupaciones de antenas. a) Retardadores limitados en cada elemento. b) Retardadores completos a nivel de subagrupaciones.

fuera lo suficientemente reducido para que el factor de la subagrupación presentara un ancho de haz mayor que el margen de apuntamiento especificado para la agrupación, no sería necesario realizar el apuntamiento del haz de la subagrupación mediante el desfasaje relativo de sus elementos.

La división en subagrupaciones desfasables introduce nuevos problemas, como la aparición de los lóbulos de difracción debidos al factor de la agrupación de subagrupaciones, que al estar más separadas que los elementos, tienen lóbulos de difracción más cercanos, que a la frecuencia central se cancelan con los nulos del factor de la subagrupación, pero que al variar la frecuencia ya no se cancelan (Fig. 2.6b). Como soluciones a este problema se pueden citar, la utilización de subagrupaciones de diferentes tamaños, lo que distribuye la potencia de los lóbulos de difracción reduciendo su valor de pico, o bien, trabajar con subagrupaciones solapadas en lugar de contiguas [Tan72, Mai93a, Mai93b].

En agrupaciones planas es posible aumentar $N$ veces el ancho de banda mediante la utilización de un máximo de $N^{2}$ retardadores temporales [Fra72]; si se aprovecharan las simetrías de la agrupación, se podrían necesitar menos retardadores. 


\section{ELEMENTOS DE LA AGRUPACIÓN}

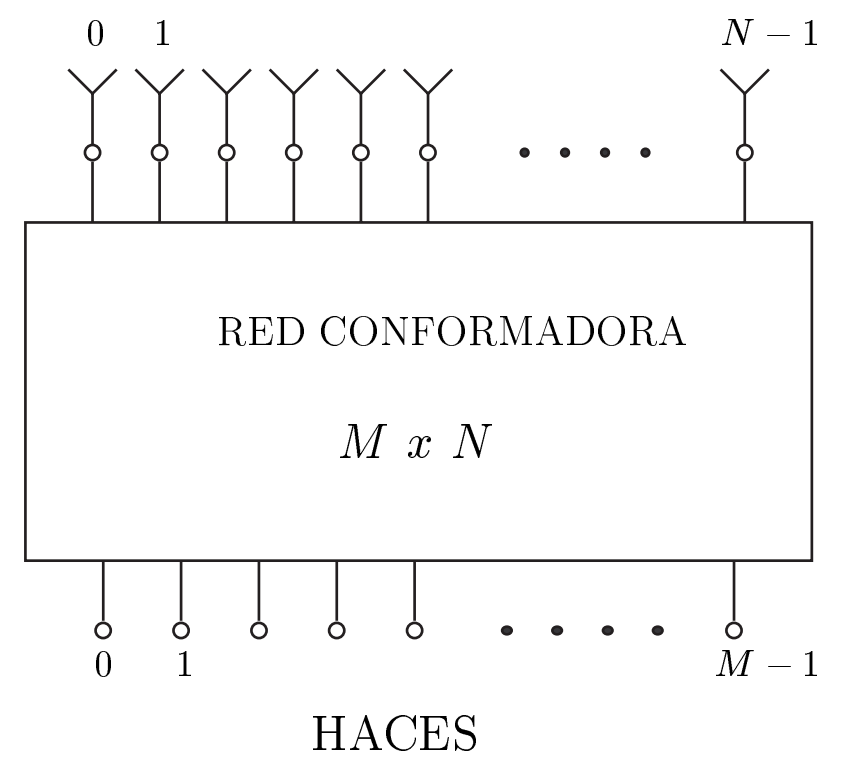

Figura 2.7: Ejemplo de red conformadora de haces para antena multihaz.

Otra razón importante para el uso de agrupaciones desfasables aparece en sistemas de RADAR pulsado [Fra72, Tan72], pues es necesario que la combinación de las contribuciones de todos los elementos de la agrupación se haga síncronamente, teniendo en cuenta el concepto de tiempo de llenado ${ }^{\mathrm{i}}$ :

$$
T=\frac{N d}{c \operatorname{sen}\left(\theta_{M A X}\right)}
$$

en relación con la duración del pulso, $\tau$. En estas aplicaciones, no suele ser conveniente utilizar la aproximación habitual, de trabajar con desfasadores limitados a un único periodo de desfases $\left(360^{\circ}\right)$.

\subsection{Antenas multihaz}

Opuesto al concepto de agrupaciones desfasables, aparecen las antenas multihaz $\left(\mathrm{MBA}^{\mathrm{ii}}\right)$ donde, un conjunto de $M$ haces diferentes, son radiados a partir de la selección de uno, entre $M$ puertos de acceso (Entrada/Salida) disponibles. A diferencia de las agrupaciones desfasables, en lugar de un haz (o varios) apuntables, se dispone de diversos haces fijos conmutables mediante la selección del puerto de acceso adecuado.

\footnotetext{
${ }^{\mathrm{i}}$ Fill-up time, en terminología inglesa.

ii Multiple beam antennas, en terminología inglesa.
} 
El concepto MBA, aparece en los reflectores con diferentes alimentadores en el foco; en cuanto a las agrupaciones de antenas, será necesario disponer de algún dispositivo (red con $M$ accesos para los haces y $N$ accesos para los elementos de antena, Fig. 2.7) que, a partir de las selección de uno de sus $M$ terminales de haces, sea capaz de presentar en los $N$ terminales de antena, las alimentaciones (amplitud y fase) adecuadas, para que los $N$ elementos de la agrupación radíen el haz m-ésimo. Ese dispositivo es una red conformadora de haces.

Además de conmutar entre los $M$ accesos disponibles, para seleccionar el haz deseado, es posible combinar dos o más de estos accesos para formar un haz combinado, cuyo diagrama de radiación sea la combinación de los diagramas de los haces seleccionados, buscando aumentar la cobertura o reducir el nivel de lóbulos secundarios.

A la hora de escoger las propiedades de los $M$ haces, se establecen los mismos criterios de alta ganancia y lóbulos secundarios reducidos que para las antenas desfasables. Un criterio muy importante en las MBA, es el nivel al que se cruzan o solapan los haces, pues este nivel de cruce ${ }^{\mathrm{i}}$ será el nivel mínimo que se puede asegurar que se recibirá en cualquier punto del área de cobertura al ir conmutando entre los diferentes haces disponibles. Este nivel de cruce, se define como el nivel del punto de intersección de dos haces contiguos, medido respecto al máximo de cada haz (supuestos con igual ganancia).

Otro parámetro importante en las MBA, son las pérdidas introducidas por la red conformadora de haces. A continuación se verá que existen ciertas condiciones bajo las cuales pueden existir redes conformadoras sin pérdidas para una MBA concreta.

\subsubsection{Haces ortogonales. Límite de Stein}

A la hora de diseñar una MBA es importante buscar la red conformadora de haces que sea óptima. Un criterio importante es la eficiencia que se puede obtener en una MBA partiendo de los haces que se desean sintetizar y de los factores de acoplamiento entre los mismos. A este eficiencia máxima se le conoce como límite de Stein. A continuación se muestra el método de cálculo del límite de Stein de una forma más compacta y sencilla que en la referencia original [Ste62].

Sea una agrupación con $N$ elementos con la que se desea construir una MBA con $M$ haces, utilizando para ello, la red pasiva mostrada en la figura 2.7. Cada uno de los $M$ haces, vendrá caracterizado por una cierta alimentación en cada uno de los $N$ elementos:

$$
B_{n m}=A_{n m} \cdot e^{j \alpha_{n m}}
$$

\footnotetext{
${ }^{\mathrm{i} C r o s s o v e r ~ l e v e l, ~ e n ~ t e r m i n o l o g i ́ a ~ i n g l e s a . ~}$
} 
de forma que el factor de la agrupación será:

$$
F_{o m}(\theta, \phi)=F_{o m}(\hat{r})=\sum_{n=1}^{N} B_{n m} e^{j k \vec{r}_{n} \hat{r}}
$$

si los pesos $B_{n m}$ se han obtenido a partir de introducir en la red conformadora una potencia unidad, y tanto la red conformadora como los elementos radiantes, son dispositivos sin pérdidas, la potencia total radiada sería:

$$
P_{o m}=\frac{1}{2 Z_{o}} \int_{0}^{2 \pi} \int_{0}^{\pi} F_{o m}^{*}(\theta, \phi) F_{o m}(\theta, \phi) d \theta d \phi=1
$$

donde $F_{o m}^{*}(\theta, \phi)$ representa el conjugado del factor de agrupación sin pérdidas y $Z_{o}$ es la impedancia del medio. En un caso real, la red conformadora podría introducir pérdidas, que se modelarán como una cierta eficiencia de haz, $\eta_{m}$, de forma que los pesos realmente conseguidos serían:

$$
S_{n m}=\sqrt{\eta_{m}} B_{n m}
$$

y el factor de agrupación real:

$$
F_{m}(\theta, \phi)=F_{m}(\hat{r})=\sqrt{\eta_{m}} \sum_{n=1}^{N} B_{n m} e^{j k \vec{r}_{n} \hat{r}}=\sqrt{\eta_{m}} F_{o m}(\theta, \phi)
$$

y, por lo tanto, la potencia realmente radiada por el haz m-ésimo será:

$$
P_{m}=\frac{\eta_{m}}{2 Z_{o}} \int_{0}^{2 \pi} \int_{0}^{\pi} F_{m}^{*}(\theta, \phi) F_{m}(\theta, \phi) d \theta d \phi=\eta_{m} P_{o m}=\eta_{m}
$$

Para el caso general en el que se tiene en cada uno de los $M$ accesos una señal $x_{p}$, se obtendrá un factor de agrupación total, formado como combinación lineal de los factores de cada haz:

$$
F(\theta, \phi)=\sum_{p=1}^{M} x_{p} F_{p}(\theta, \phi)
$$

de forma que, la potencia total radiada se puede expresar como:

$$
\begin{aligned}
& P_{\text {radiada }}=\frac{1}{2 Z_{o}} \int_{0}^{2 \pi} \int_{0}^{\pi} F^{*}(\theta, \phi) F(\theta, \phi) d \theta d \phi \\
& =\frac{1}{2 Z_{o}} \int_{0}^{2 \pi} \int_{0}^{\pi}\left(\sum_{p=1}^{M} x_{p}^{*} F_{p}^{*}(\theta, \phi)\right)\left(\sum_{q=1}^{M} x_{q} F_{q}(\theta, \phi)\right) d \theta d \phi \\
& =\frac{1}{2 Z_{o}} \sum_{p=1}^{M} \sum_{q=1}^{M} x_{p}^{*} x_{q}{\sqrt{\eta_{p}}}^{*} \sqrt{\eta_{q}}\left(\int_{0}^{2 \pi} \int_{0}^{\pi} F_{o p}^{*}(\theta, \phi) F_{o q}(\theta, \phi)\right) d \theta d \phi
\end{aligned}
$$


definiendo el factor de acoplamiento entre los haces p-ésimo y q-ésimo como:

$$
C_{p q}=\frac{1}{2 Z_{o}} \int_{0}^{2 \pi} \int_{0}^{\pi} F_{o p}^{*}(\theta, \phi) F_{o q}(\theta, \phi) d \theta d \phi
$$

factor de acoplamiento que cumple $C_{m m}=1, C_{p q}=C_{p q}^{*}$ y $\left|C_{p q}\right| \leq 1$; se puede introducir una matriz ${ }^{i}$ de acoplamiento C. En los casos en que $C_{p q}=0$ se dirá que los haces p-ésimo y q-ésimo son ortogonales entre sí, de forma que si todos los haces lo fueran, la matriz $\mathbf{C}$ sería la matriz identidad $\mathbf{I}$ y no existiría ningún acoplo entre los $M$ haces.

Si se define una matriz $\Gamma$ de tamaño $M x M$ cuyos elementos fueran:

$$
\Gamma_{p q}={\sqrt{\eta_{p}}}^{*} C_{p q} \sqrt{\eta_{q}}
$$

la expresión de la potencia radiada queda en formato matricial como:

$$
P_{\text {radiada }}=\sum_{q=1}^{M} \sum_{p=1}^{M} x_{q}^{*} \Gamma_{q p} x_{p}=\mathbf{x}^{*} \Gamma \mathbf{x}
$$

donde $\mathbf{x}$ es un vector de tamaño $M$ formado por las excitaciones de los accesos de $\operatorname{los} M$ haces.

La matrix $\Gamma$ tiene $M$ autovalores $\lambda_{m}$ y $M$ autofunciones $\mathbf{x}_{m}$ que cumplen:

$$
\Gamma \mathbf{x}_{m}=\lambda_{m} \mathbf{x}_{m}
$$

Teniendo en cuenta las propiedades de los coeficientes de acoplo $C_{p q}$ se puede demostrar, que la matriz $\Gamma$ es hermítica y semidefinida positiva y por lo tanto:

$$
0 \leq \lambda_{m} \leq 1
$$

Para el caso habitualii en que todos los haces tienen la misma eficiencia $\eta_{M B A}$, se cumple:

$$
\Gamma=\eta_{M B A} \mathbf{C}
$$

relación entre matrices, que en cuanto a los autovalores de la matriz $\mathbf{C}\left(\Lambda_{m}\right)$ se traduce en:

$$
\Lambda_{m}=\eta_{M B A} \lambda_{m}
$$

y la ecuación (2.48) se convierte en:

$$
\left(\lambda_{m}\right)_{\max }=\frac{\left(\Lambda_{m}\right)_{\max }}{\eta_{M B A}} \leq 1 \quad \text { ó } \quad \eta_{M B A} \leq \frac{1}{(\Lambda)_{\max }}
$$

\footnotetext{
${ }^{\mathrm{i} A}$ lo largo de este documento se aplica la nomenclatura habitual de representar los vectores en tipo minúscula negrita y las matrices en tipo mayúscula negrita.

${ }^{i}$ Se puede consultar en la literatura [DuF92] el caso de haces con eficiencia distintas, llegándose a conclusiones análogas.
} 
luego, la eficiencia de una MBA está limitada por el inverso del máximo autovalor de la matriz de acoplo (C). Esta afirmación se conoce como el límite de Stein. Para el caso particular de haces ortogonales, se ha visto que la matriz de acoplo es la matriz identidad y, por lo tanto, el valor máximo de los autovalores es $\lambda_{M A X}=1 \mathrm{y}$ es posible obtener una red conformadora con una eficiencia $\eta_{M B A}=1$, es decir una red sin pérdidas.

En el caso general, una vez se especifican los haces a sintetizar por una MBA, la ecuación (2.51) fija la máxima eficiencia que se puede esperar de una red conformadora de haces óptima. En la literatura se pueden encontrar métodos para sintetizar redes, a partir de la matriz de acoplo, que cumplan el límite de Stein [DuF92]. En cualquier caso, se debe comentar que existen múltiples soluciones, que éstas soluciones, que idealmente cumplen el límite de Stein, tendrán más pérdidas debidas a los componentes reales utilizados y que además existen otros criterios para definir que una red es la óptima para una MBA concreta, como pueden ser, el ancho de banda, el peso, el coste o la fiabilidad. La utilidad del límite de Stein consiste en establecer un objetivo ideal con el cual comparar las implementaciones bajo estudio.

Todas las consideraciones realizadas sobre el límite de Stein pierden su significado si se utiliza conformación digital, pues en el dominio digital deja de tener sentido hablar de eficiencia del conformador.

\subsubsection{Antenas multihaz en agrupaciones lineales}

El caso más estudiado de antenas multihaz corresponde a una agrupación lineal uniforme de $N$ elementos, equiespaciados una distancia $d_{x}$, alimentada por una red conformadora de haces que permite disponer de $M$ haces. Para ese caso particular, el coeficiente de acoplamiento entre dos haces se expresará, en función de la alimentación normalizada de los mismos $\left(B_{n p}\right.$ y $\left.B_{n q}\right)$ :

$$
\begin{aligned}
C_{p q} & =\frac{1}{2 Z_{o}} \int_{0}^{2 \pi} \int_{0}^{\pi}\left(\sum_{r=1}^{N} B_{r p} e^{j k r d_{x} u}\right)^{*} \sum_{s=1}^{N} B_{s q} e^{j k s d_{x} u} d \theta d \phi \\
& =\frac{\pi}{Z_{o}} \sum_{r=1}^{N} \sum_{s=1}^{N} B_{r p}^{*} B_{s q} \frac{\operatorname{sen}\left(k d_{x}(r-s)\right)}{k d_{x}(r-s)}
\end{aligned}
$$

Cuando la separación entre elementos es media longitud de onda $\left(d_{x}=\lambda / 2\right)$, (2.52) se reduce al producto escalar de las alimentaciones de los haces respectivos:

$$
C_{p q}=\frac{\pi}{Z_{o}} \sum_{r=1}^{N} B_{r p}^{*} B_{r q}=\frac{\pi}{Z_{o}} \mathbf{B}_{p}^{\dagger} \cdot \mathbf{B}_{q}
$$

ecuación que para el caso de agrupaciones uniformes con fase lineal queda simplifi- 


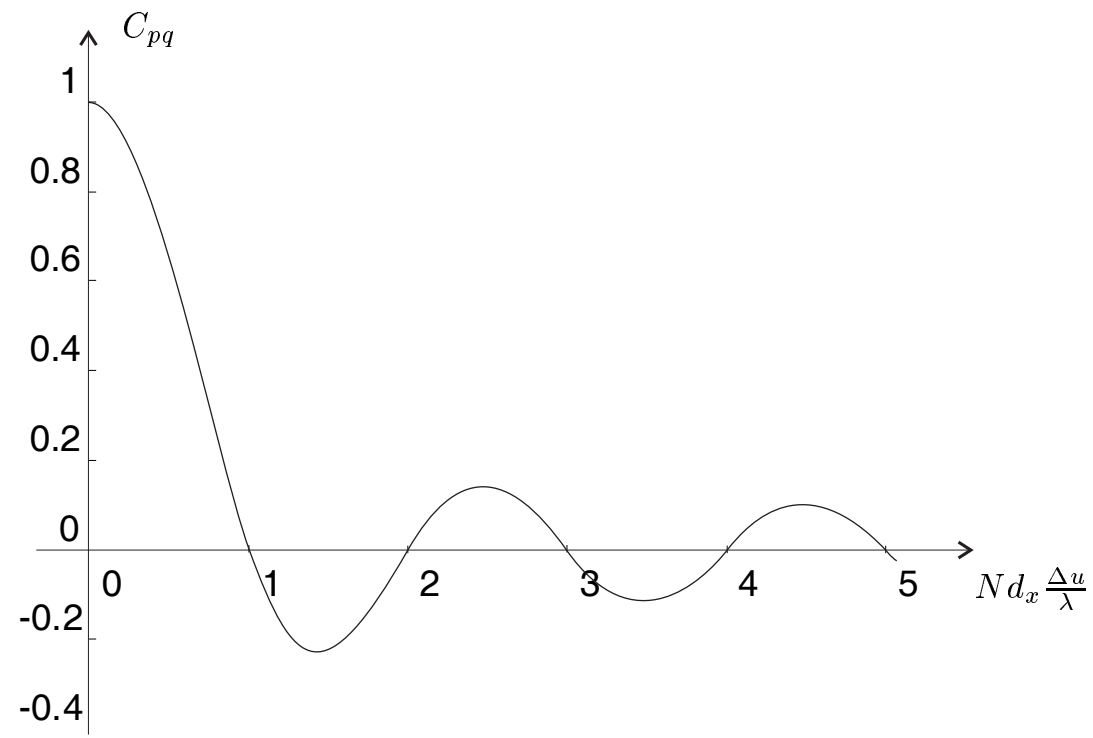

Figura 2.8: Coeficiente de acoplo $\left(C_{p q}\right)$ entre dos haces separados $\Delta u$ para una agrupación lineal de $N=10$ elementos.

cada como:

$$
C_{p q}=\frac{\operatorname{sen}\left(N \pi\left(u_{p}-u_{q}\right) \frac{d_{x}}{\lambda}\right)}{N \operatorname{sen}\left(\pi\left(u_{p}-u_{q}\right) \frac{d_{x}}{\lambda}\right)}
$$

siendo $u_{p}$ e $u_{q}$ las posiciones de los máximos de los haces respectivos. La ecuación (2.54) se representa en la figura 2.8 en función de la separación entre haces $\Delta u=$ $u_{p}-u_{q}$. Se puede observar en la citada figura, que si se desean haces desacoplados (máxima eficiencia) los haces deberán estar espaciados entre sí:

$$
\Delta u=\frac{q \lambda}{N \cdot d_{x}}
$$

donde $q \in \mathbb{Z}$.

Si se desean sintetizar $M=N$ haces con la máxima eficiencia, se puede comprobar a partir de la matriz de acoplo que los $N$ haces deben estar equiespaciados en el espacio $u$ y al ser la periodicidad del mismo, $\lambda / d_{x}$, los haces estarán separados entre sí según:

$$
\Delta u=\frac{\lambda}{N \cdot d_{x}}
$$

La separación mostrada en (2.56) indica la separación entre los haces, dejando un grado de libertad en cuanto a la posición absoluta de los mismos; en concreto es habitual escoger las siguientes posiciones absolutas de los $M$ haces:

$$
u_{m}=m \frac{\lambda}{M \cdot d_{x}}=m \frac{\lambda}{N \cdot d_{x}}
$$


a)
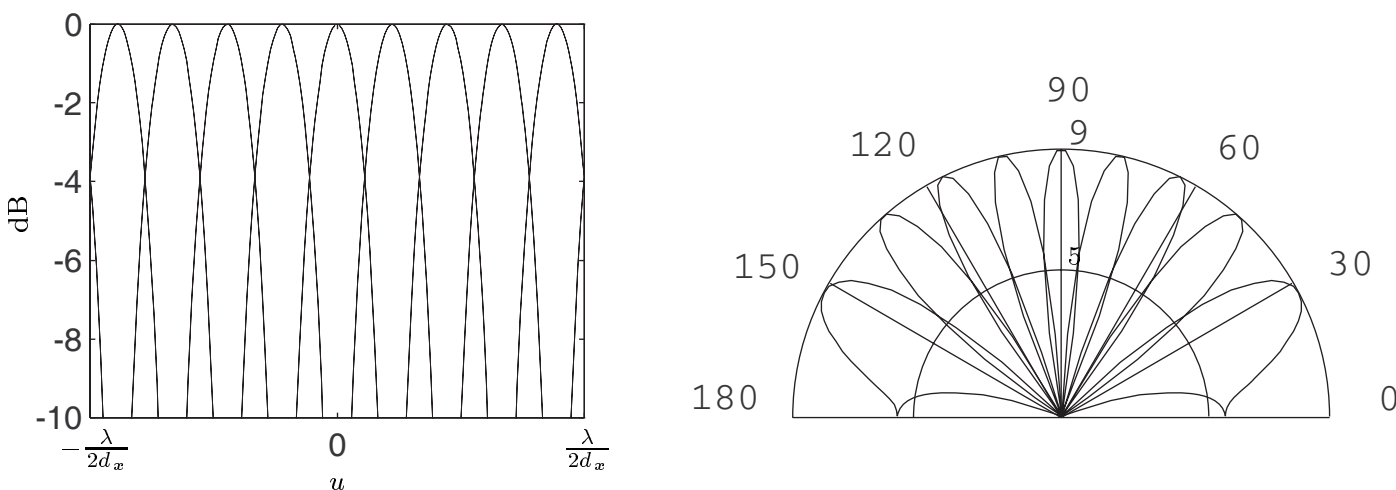

b)
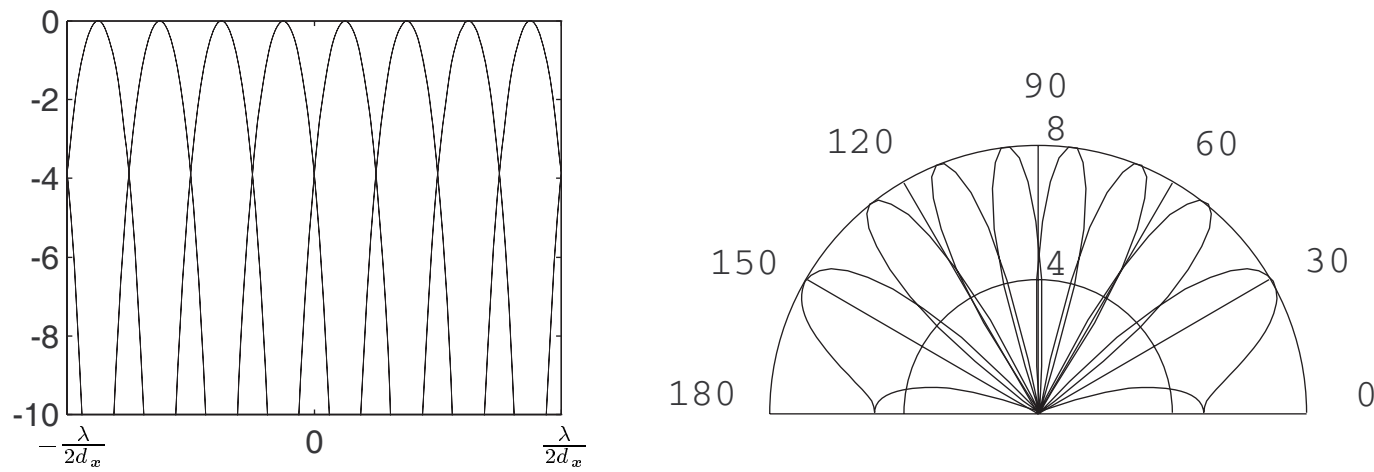

Figura 2.9: Factor de agrupación en plano $u$ y en espacio real para $\operatorname{los} N$ haces ortogonales de una MBA lineal. a) $N=8$. b) $N=9$.

con $m=0, \pm 1, \pm 2 \ldots$ si $N$ impar y $m= \pm 1 / 2, \pm 3 / 2 \ldots$ si $N$ par. Posiciones de los haces que corresponden a las siguientes fases en los elementos uniformes:

$$
\alpha_{n}=-n k d_{x} u_{m}=-2 \pi n \frac{m}{M}=-2 \pi n \frac{m}{N}
$$

y a los siguiente factores de agrupación normalizados:

$$
\left|F_{m}(u)\right|=\left|\sum_{n=1}^{N} e^{\alpha_{n}} e^{j k n\left(u d_{x}\right)}\right|=\left|\frac{\operatorname{sen}\left(N \pi\left(\frac{u d_{x}}{\lambda}-\frac{m}{N}\right)\right)}{N \operatorname{sen}\left(\pi\left(\frac{u d_{x}}{\lambda}-\frac{m}{N}\right)\right)}\right|
$$

En la figura 2.9, se muestran, superpuestos, en un periodo del espacio $u$, los factores de agrupación de los haces de (2.57), para los casos $N=8$ y $N=9$; también se muestra, el diagrama de radiación para elementos isotrópicos y separación entre elementos de media longitud de onda, teniendo en cuenta que, para separaciones mayores, aparecerán lóbulos de difracción de algunos de los haces y que para separaciones menores, no aparecerían todos los haces en el margen visible.

La elección habitual de los haces representados por (2.59) se debe a que son ortogonales entre sí, lo que implica que es posible sintetizarlos mediante una red conformadora sin pérdidas. Además, cumplen que son independientes, en el sentido de que cada uno de los haces, tiene nulos de radiación en las direcciones de los 
máximos del resto de haces. Los inconvenientes principales de trabajar con estos $N$ haces ortogonales son dos: el nivel de cruce entre haces contiguos y el nivel de los lóbulos secundarios.

\section{Nivel de cruce y NLPS}

En cuanto al nivel de cruce, corresponderá al punto medio entre el haz $m$ y el $m+1$, por lo tanto, normalizando respecto al máximo del haz:

$$
F_{\text {cruce }}=\left|F_{m}\left(u_{m}+\frac{\lambda}{2 N d_{x}}\right)\right|=\left|\frac{\operatorname{sen}\left(N \pi\left(\frac{1}{2 N}\right)\right)}{N \operatorname{sen}\left(\pi\left(\frac{1}{2 N}\right)\right)}\right|=\frac{1}{N \operatorname{sen}\left(\frac{\pi}{2 N}\right)}
$$

que para valores relativamente grandes de $N$, resulta $F_{\text {cruce }}=-3.92 \mathrm{~dB}$, tal y como se puede comprobar en la figura 2.9 .

El nivel de lóbulos principal a secundario de cada haz, corresponde al de una agrupación uniforme, por lo que tenderá a $13.26 \mathrm{~dB}$, a medida que aumente el número de elementos.

Para bastantes aplicaciones, tanto el nivel de cruce como el NLPS obtenidos no son adecuados, deseándose haces con menores lóbulos secundarios y que se crucen en niveles más altos.

\section{Mejoras en el nivel de cruce y NLPS}

Si se desea mejorar el nivel de cruce o el nivel de los lóbulos secundarios, es necesario modificar los haces, ya sea su número o su forma. En concreto existen tres posibilidades para ello, que se analizarán a continuación.

Sobredimensionamiento de la red conformadora En lugar de utilizar una red conformadora de tamaño $N \times N$ se aumenta el tamaño de la red a $M \times M$ (que correspondería a una antena de $M$ elementos) pero conectando únicamente $N$ de los $M$ accesos del lado de los elementos de antena, terminando el resto $(N-M)$ con cargas adaptadas. De esta forma el número de haces y su posición corresponden a una antena de $M$ elementos, mientras que la anchura y forma de los mismos vienen definidos por el tamaño de la agrupación $(N)$. Por lo tanto el factor de la agrupación normalizado de cada uno de los $M$ haces sería:

$$
\left|F_{m}(u)\right|=\frac{1}{N}\left|\sum_{n=1}^{N} e^{-j K n d_{x} \frac{m}{M}} e^{j k n\left(u d_{x}\right)}\right|=\left|\frac{\operatorname{sen}\left(N \pi\left(\frac{u d_{x}}{\lambda}-\frac{m}{M}\right)\right)}{N \operatorname{sen}\left(\pi\left(\frac{u d_{x}}{\lambda}-\frac{m}{M}\right)\right)}\right|
$$

conjunto de haces para los que el nivel de cruce respecto al máximo de cada haz será:

$$
\left|F_{\text {cruce }}\right|=\left|F_{m}\left(u_{m}+\frac{\lambda}{2 M d_{x}}\right)\right|=\left|\frac{\operatorname{sen}\left(\frac{N \pi}{2 M}\right)}{N \operatorname{sen}\left(\frac{\pi}{2 M}\right)}\right|
$$




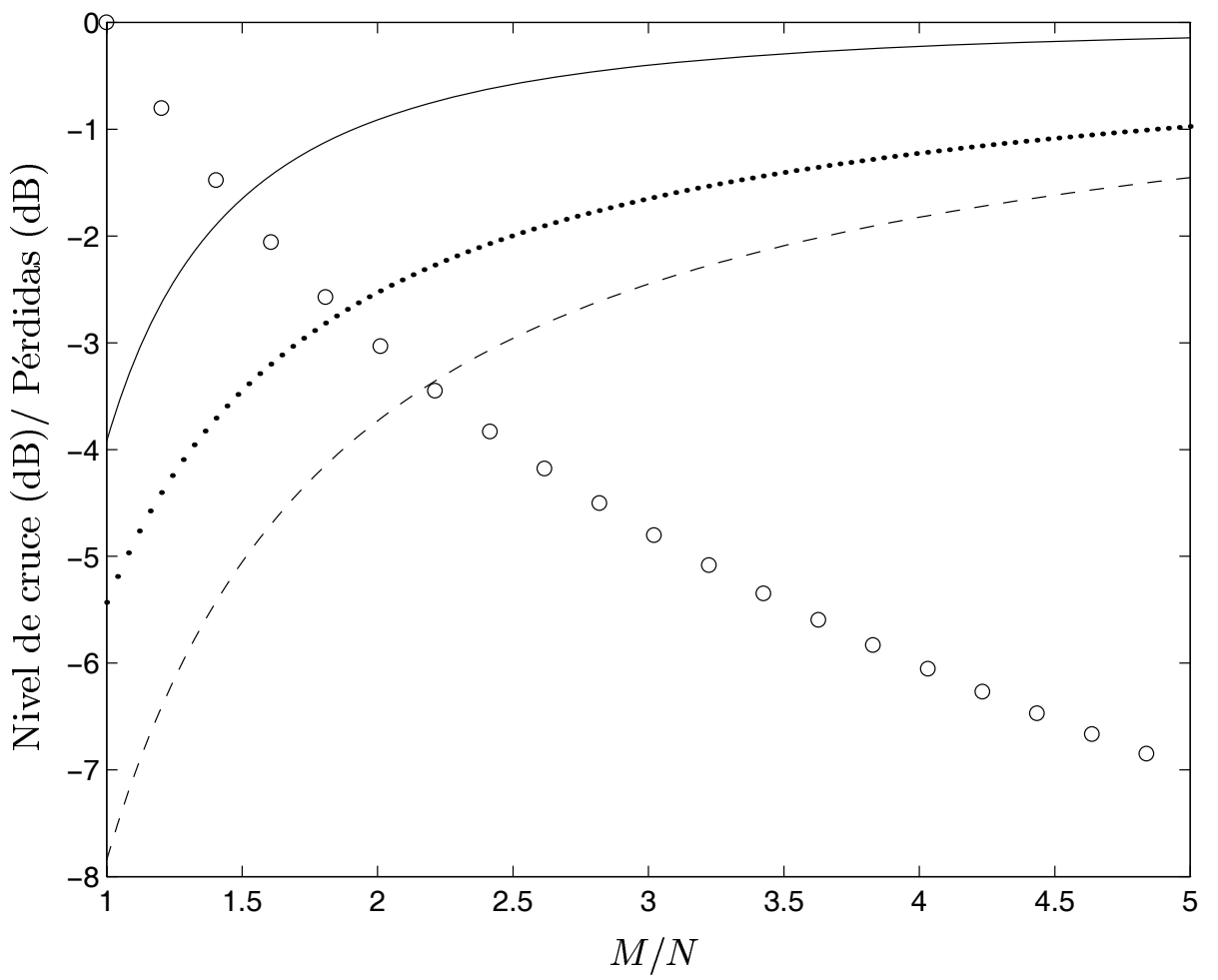

Figura 2.10: Nivel de cruce de los haces en agrupaciones lineales (trazo contínuo), planas rectangulares (trazo discontínuo) y hexagonales (trazo punteado) y pérdidas (circunferencias) en los accesos terminados para conformadores de haces sobredimensionados en un factor $M / N$.

que para valores relativamente grandes de $N$ y $M$, se representa en la figura 2.10. Como efecto indeseado del sobredimensionamiento de la red conformadora está la reducción de la eficiencia de la red en un factor $N / M$ debido a las pérdidas generadas en los accesos terminados cuando la red se utiliza en transmisión y a la potencia perdida en salidas distintas de la del haz correspondiente cuando se está en modo recepción; expresando las pérdidas en decibelios:

$$
L_{M / N}=10 \log \left(\frac{M}{N}\right)
$$

resultado que se muestra en la figura 2.10.

Además los $M$ haces dejan de ser ortogonales e independientes, por lo que los máximos de cada haz dejan de coincidir con los nulos de otros haces, lo cual en determinadas condiciones puede reducir la densidad del sistema.

Combinación de entradas Se ha visto que si una red pasiva y sin pérdidas ha de conformar $N$ haces desacoplados, para una MBA de $N$ elementos, entonces esos haces estarán equiespaciados $\lambda /\left(N d_{x}\right)$ en el espacio $u$; pero si se necesita un número de haces menor de $N$, entonces es posible combinar accesos de la red conformadora $N \times N$ para mejorar el NLPS [Kah62]. Por ejemplo, si se combinan dos entradas (el número de haces se reduce a $N / 2$ ) se obtiene una distribución tipo coseno con lo que 
se reduce el NLSP a -23 dB; pero si se desea mantener ortogonalidad la separación ha de aumentar $2 \lambda /\left(N d_{x}\right)$, reduciéndose simultáneamente el nivel de cruce a $-9.5 \mathrm{~dB}$. Para una distribución tipo coseno al cuadrado o coseno al cuadrado sobre pedestal se deben combinar tres entradas de la red uniforme ideal (el número de haces se reduce a $N / 3)$ y la separación en el espacio $u$ debe aumentar hasta $3 \lambda /\left(N d_{x}\right)$ para mantener la ortogonalidad de los haces, disminuyendo también el nivel de cruce.

Distribución de amplitud a la salida Si para una MBA de $N$ elementos, se dispone de una red conformadora sin pérdidas que genere las distribuciones uniformes de fases, adecuadas para sintetizar los $N$ haces ortogonales, la colocación de una etapa atenuadora (luego, con pérdidas) posterior a la red conformadora de haces es una solución óptima para conseguir la distribución de amplitudes deseada, y con ella el nivel de lóbulos secundarios y de ancho de haz correspondiente (Tabla 2.1) [DuF85]. Además se cumple que para cualquier distribución de amplitudes, $A_{n}$, la eficiencia máxima de acuerdo con el límite de Stein se puede expresar como [DuF85]:

$$
\eta_{M B A}=\frac{\overline{A_{n}^{2}}}{\left(A_{n}\right)_{\text {max }}^{2}}
$$

es decir la relación entre el valor medio y el valor de pico del cuadrado de la distribución. La eficiencia de (2.64) se refiere a agrupaciones con elementos separados media longitud de onda; si se reduce el espaciado, se acoplan los haces y las redes conformadoras aumentan sus pérdidas, siendo posible desacoplar los haces mediante la inserción de redes resistivas [Whi62].

\subsubsection{Antenas multihaz en agrupaciones planas}

Las agrupaciones planas rectangulares pueden ser alimentadas a través de una separación entre filas y columnas. A partir de esa división, es posible aplicar las redes conformadoras sin pérdidas para MBA lineales vistas previamente, que generaban $N$ haces ortogonales. De esta forma, una agrupación plana rectangular separable de $N \times N$ obtendría los haces mostrados en la figura 2.11a que presentan un nivel de cruce de $-3.92 \mathrm{~dB}$ en las direcciones de las filas y las columnas, pero un nivel de cruce de $-7.8 \mathrm{~dB}$ en las diagonales.

El trabajar en dos dimensiones introduce un mayor grado de libertad a la hora de escoger la posición de los haces en el plano $(u, v)$. La distribución rectangular de los haces de la figura 2.11 implica que los haces serán independientes si la iluminación es uniforme. Si en lugar de tomar una distribución rectangular de los haces, se escoge una red conformadora que genere los haces dentro de una rejilla hexagonal, pero manteniendo la distribución rectangular de los elementos, el nivel de cruce se reduce hasta $-5.4 \mathrm{~dB}$, pero los haces dejan de ser ortogonales [Mai93a].

Tanto si se escoge una distribución de los haces rectangular, como hexagonal, es posible reducir el nivel de cruce mediante la aplicación de un sobredimensionamiento de la red conformadora, como se puede ver en la figura 2.10, donde el factor $M / N$ hace referencia al número total de elementos de la agrupación. 


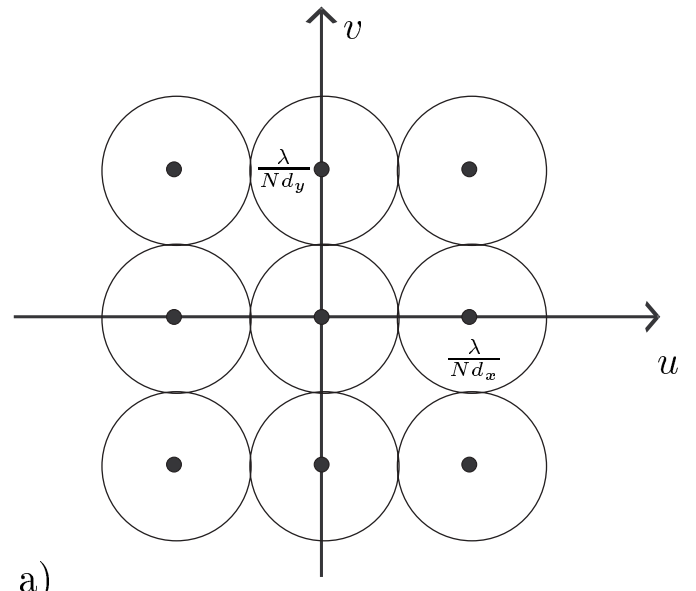

a)

Figura 2.11: a) Distribución rectangular de haces. b) Distribución hexagonal de haces.

En términos generales, comentar que para una MBA, la situación ideal consiste en obtener una mayor densidad de haces, y que éstos presenten unos lóbulos secundarios de menor amplitud. Por desgracia, estos implica que al implementarlos con un conformador pasivo se aumenten las pérdidas debidas a la no ortogonalidad de los haces, incluso para el caso ideal.

\subsection{Redes conformadoras de haces eléctricas}

La red conformadora de haces es el dispositivo que se encarga de alimentar los elementos de la agrupación con la distribución de amplitudes y retardos (o fases) necesarios para sintetizar el haz especificado. En las antenas de imagen, parte de la conformación se realiza en el espacio libre, de acuerdo con la posición de la agrupación alimentadora en el foco y la reflexión y combinación en el reflector o reflectores. Tanto este caso, como el de conformación a través de lentes [Ruz50, Jaz63], quedan fuera del campo de investigación de este Tesis Doctoral, pudiendo encontrarse excelentes recopilaciones de referencias en la literatura [Hal90]. El resto de redes conformadoras eléctricas, corresponden a un conjunto de líneas de transmisión, desfasadores, divisores de potencia y acopladores cuya arquitectura final dependerá de las especificaciones de la agrupación a construir.

Es habitual distinguir entre los casos de, redes conformadoras para antenas desfasables o redes de distribución, y redes conformadoras para antenas multihaz.

\subsubsection{Redes de distribución}

Una red de distribución está formada por divisores, líneas de transmisión y desfasadores, y se encarga de distribuir la señal a cada elemento de la agrupación mediante 
divisiones y desfases desde un punto común; en recepción, se encargará de concentran la señal en un punto. Suelen trabajar con un único haz desfasable a través de desfasadores variables para cada elemento o subagrupación de elementos.

Los desfasadores variables más habituales son digitales en el sentido de que el rango de desfases que pueden presentar corresponden a un conjunto de circuitos o dígitos, cada uno de los cuales es un desfasador fijo con dos estados. Generalmente se suele trabajar con desfasadores digitales de rango completo, es decir que cubren $360^{\circ}$ a partir de dígitos de $180^{\circ}, 90^{\circ}, 45^{\circ}, \ldots$ hasta el tamaño de dígito que sea inferior a la resolución deseada.

Las redes corporativas se pueden dividir entre pasivas y activas, de acuerdo con la definición realizada en 2.2.4. Además, otra clasificación habitual se basa en el tipo de distribución de señal que se escoja y así se habla de redes de distribución en serie, si la señal se va distribuyendo pasando de elemento en elemento, o en paralelo, si la llega a cada elemento a través de sucesivas divisiones (por ejemplo, Fig. 2.5). Las redes en serie suelen a su vez distinguirse según el punto de alimentación esté en el centro o en algún extremo.

\subsubsection{Redes conformadoras para antenas multihaz}

El diseño de una antena multihaz con $N$ elementos y $M$ haces requiere de una red conformadora con $M+N$ accesos (Fig. 2.7). La eficiencia de esa red, tiene como valor máximo el límite de Stein, de acuerdo con los $M$ haces que se deseen conformar.

Dentro de las arquitecturas típicas de conformadores para antenas multihaz se pueden citar, la matriz de Butler y la matriz de Blass. Tanto la matriz de Butler como la matriz de Blass permiten diseñar antenas con haces múltiples, pero no agrupaciones desfasables electrónicamente; de todas formas, pueden conseguirse unas prestaciones bastante similares si se consigue aumentar el nivel de cruce, aunque eso implique que los haces dejen de ser ortogonales y que aumenten las pérdidas de la red.

\section{Matriz de Butler}

La matriz de Butler consiste en una combinación de acopladores y desfasadores fijos interconectados entre sí, que permite sintetizar los haces ortogonales definidos en (2.57) para una agrupación lineal [But61]. En la figura 2.12 se muestra un ejemplo para $N=8$. Existen diferentes versiones de la misma, en función si se desea que el conjunto de los haces ortogonales incluya, o no incluya, un haz en la dirección normal a la agrupación. Fue inicialmente definida para un número de elementos que fuera potencia de dos, $N=2^{p}$, pero, como se verá en el capítulo 4 , se puede generalizar su estructura para cualquier tamaño, aún cuando será más eficiente para determinados tamaños. Asímismo, se puede generalizar su diseño para agrupaciones planas, tanto si la distribución de los elementos es rectangular como hexagonal 

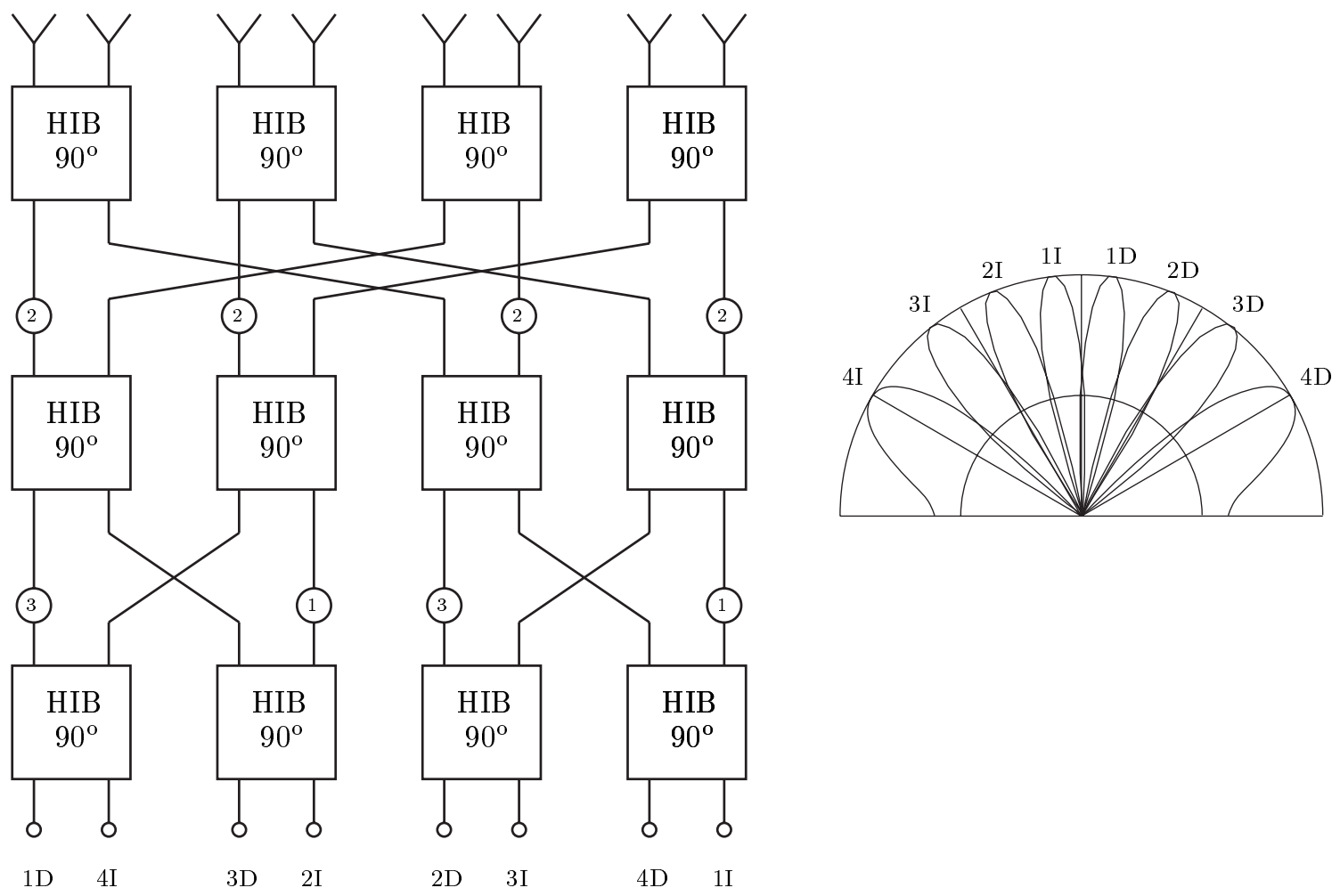

Figura 2.12: Ejemplo de matriz de Butler para $N=8$ (los desfasadores están indicados en múltiplos de $22.5^{\circ}$ ).

[But61, She68, McF79]. En la literatura existen algoritmos prácticos para escoger la arquitectura de diferentes tamaños de matrices de Butler, generalmente limitadas al caso de $N=2^{p}$ [Mac87, Moo64].

Debido al gran número de componentes requeridos a medida que aumenta el tamaño de la agrupación y a la complejidad en la interconexión de todos sus componentes, la utilización de la matiz de Butler está actualmente limitada por la tecnología a tamaños del orden de dieciseis a sesenta y cuatro elementos [Hal90, Lo88].

La matriz de Butler permite conseguir niveles de cruce reducidos mediante la elección de un conformador de un tamaño sobredimensionado, con el correspondiente bajo nivel de solapamiento (Fig. 2.10), y terminando los accesos no utilizados. El ancho de banda de la matriz de Butler está limitado, pues, se trata de una red basada en desfases y no en retardos, por lo que presenta el problema del estrabismo del haz.

Recientemente se ha implementado una matriz de Butler de tamaño $4 \times 4$ a frecuencias ópticas mediante diferentes longitudes de fibra óptica monomodo y acopladores en fibra [Gal97]. 


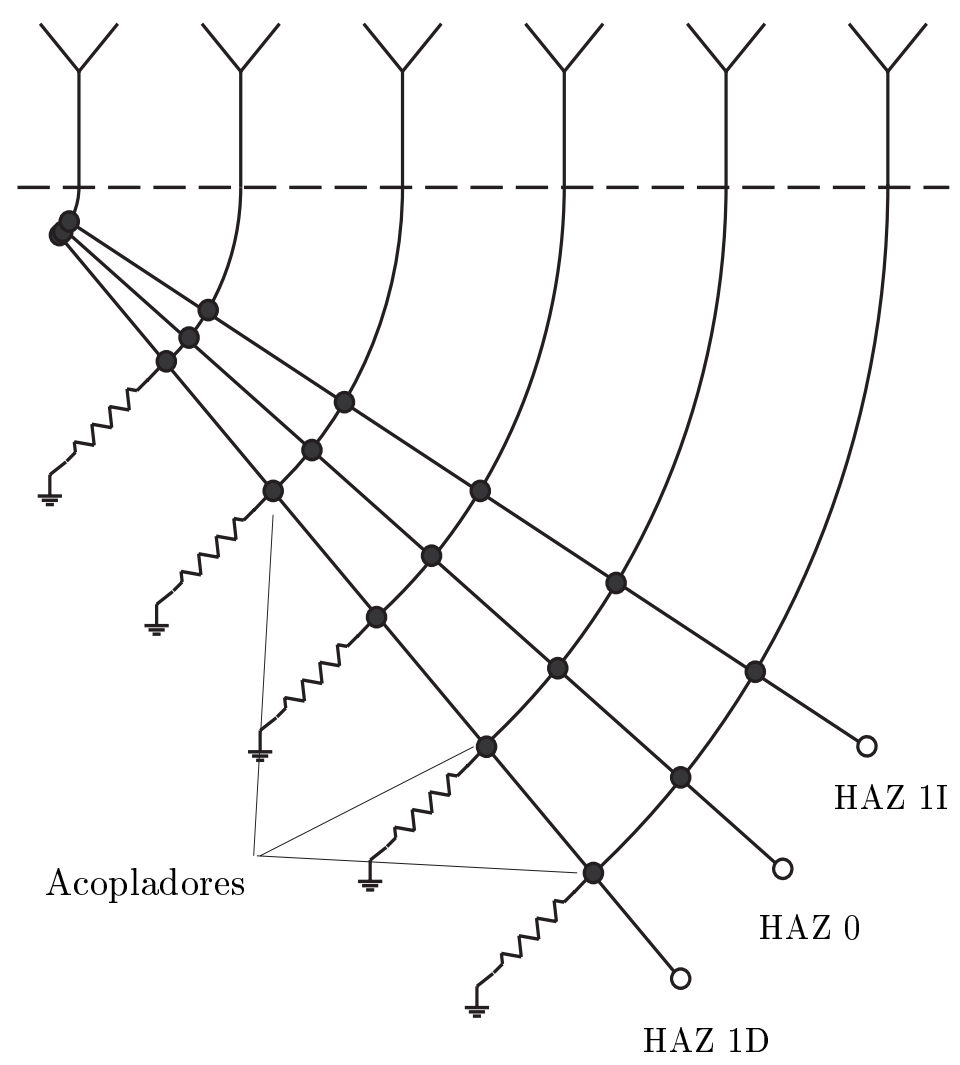

Figura 2.13: Ejemplo de matriz de Blass

\section{Matriz de Blass}

A diferencia de la matriz de Butler, la matriz de Blass (Fig. 2.13) se basa en diferentes logitudes de línea de transmisión más un conjunto de acopladores, de forma que para cada haz el camino recorrido hasta cada elemento varía linealmente y, por lo tanto, se trata de un conformador de banda ancha [Bla60]. A pesar de la flexibilidad que introduce a priori a la hora de escoger la posición de los haces, ésta se encuentra limitada por el acoplo entre los accesos de los diferentes haces a medida que la posición angular de los haces se aleja de las posiciones ortogonales. Otro punto clave en el diseño de una matriz de Blass es la directividad de los acopladores, pues una directividad baja conllevará acoplos entre diferentes haces que se traducirán en errores en la distribución en los elementos de la agrupación. Por consiguiente, la matriz de Blass está más limitada que la matriz de Butler, y ha tenido mucho menos éxito que ésta última.

\subsubsection{Impacto de la tecnología MMIC}

A pesar de las conocidas propiedades de las agrupaciones de antenas desfasables o de las antenas multihaz, el desarrollo de las mismas ha estado muy limitado a sistemas militares en tierra, de forma que el principal fabricante mundial de agrupaciones 
desfasables era la conocida empresa Raytheon, con su no menos conocido sistema PATRIOT del cual instaló hasta 173 sistemas, toda una marca de ventas.

Todos estos sistemas utilizan desfasadores de ferrita o de estado sólido dentro de módulos T/R. En la última década, ha comenzado a estudiarse la posibilidad de desarrollar agrupaciones desfasables o antenas multihaz basadas en circuitos integrados monolíticos de microondas (MMIC), aprovechando sus propiedades de repetibilidad, peso y tamaño reducido y bajo coste para grandes producciones [Str97, Kom91, Her87, Gup91, Gup90, Bha86, Dev91]. La disponibilidad de módulos $\mathrm{T} / \mathrm{R}$ en tecnología MMIC permite, por un lado, llevar las agrupaciones de antenas desfasable a sistemas aerotransportados (aviones o satélites) gracias a su reducido tamaño, mientras que su capacidad de producción en masa y bajo coste hacen posible que las agrupaciones de antenas se implanten en sistemas comerciales de comunicaciones. Dentro de estas aplicaciones comerciales, la que está dando, y puede dar un mayor impulso a la fabricación de MMIC es las de los sistemas de comunicaciones móviles via satélite, como pueden ser IRIDIUM, GLOBALSTAR o el sistema de banda ancha TELEDESIC. Todos ellos requieren la fabricación de miles de módulos $\mathrm{T} / \mathrm{R}$, para las constelaciones respectivas de satélites, siendo el más exigente, el sistema TELEDESIC que requeriría millones de MMIC a frecuencias de ondas milimétricas.

Actualmente, los únicos sistemas activos que se basan en módulos $\mathrm{T} / \mathrm{R}$ con tecnología MMIC son los sistemas militares en tierra THAAD y COBRA [Bro97a, Bro97b]; el primero de los cuales ha llegado a fabricar más de 60.000 unidades de módulos T/R MMIC. Otros sistemas militares aerotransportados están a punto de ver la luz (AMSAR en Francia, Alemania y el Reino Unido, AESA en Suecia), al igual que los sistemas de comunicaciones móviles por satélite.

\subsection{Redes ópticas conformadoras de haces}

Inicialmente, la aplicación de tecnología óptica a agrupaciones de antenas estaba enfocada a la utilización de fibra óptica para la distribución de señales a los diferentes elementos de agrupaciones desfasables aprovechando sus propiedades de bajas pérdidas, ancho de banda inmenso y poco peso [See93]. El razonamiento era que el desarrollo de MMICs haría posible disponer de elementos activos de bajo coste, permitiendo trabajar con agrupaciones con un gran número de elementos, y que una gran parte del coste de la agrupación residiría entonces en la función de distribución de señales. Las reducciones de peso y en pérdidas predichas harían a las agrupaciones desfasables de antenas significativamente más atractivas para un amplio rango de aplicaciones. Hoy en día, ya están disponibles módulos $\mathrm{T} / \mathrm{R}$ (Transmisor/Receptor) de bajo coste. Aún más, el uso de módulos $\mathrm{T} / \mathrm{R}$ activos hace apropiado el uso de técnicas ópticas en la arquitectura del sistema, ya que implica la distribución de señales de baja potencia hacia el plano de la agrupación.

Pero la introducción de tecnología fotónica en agrupaciones no se ha limitado 


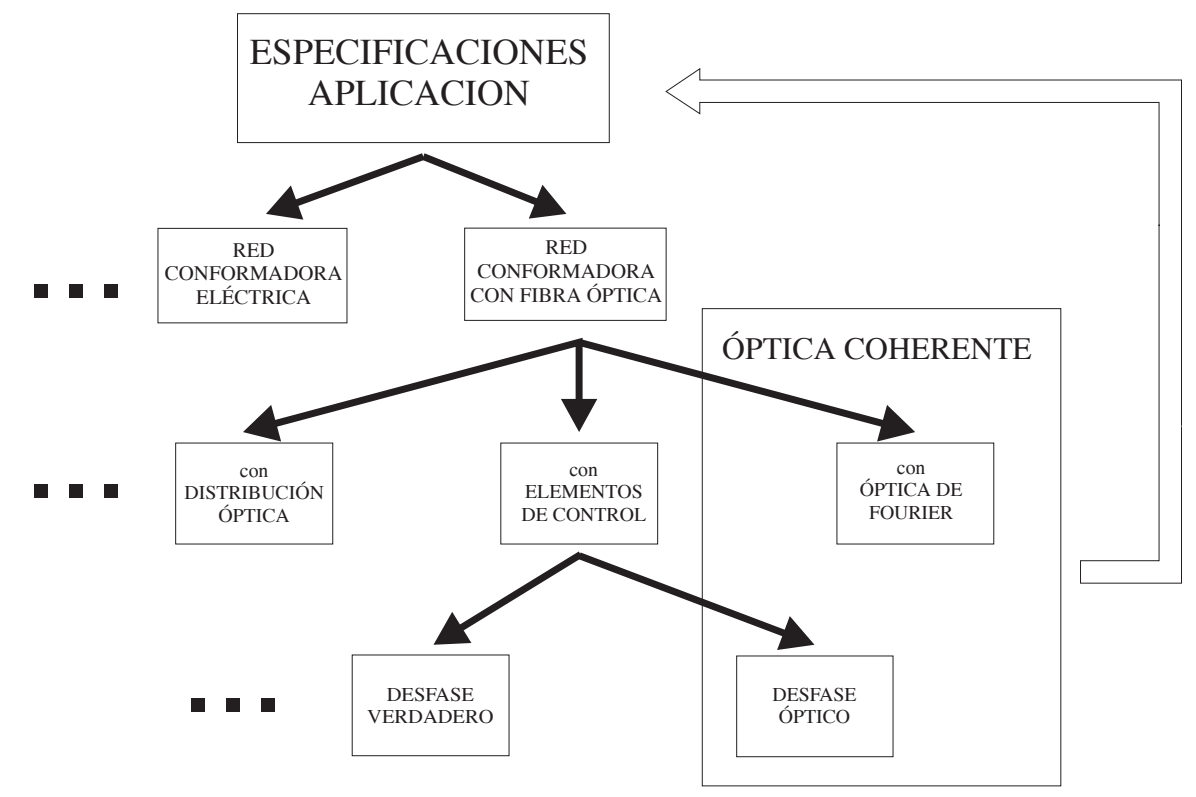

Figura 2.14: Diferentes posibilidades de aplicación de tecnología óptica a redes conformadoras de haces.

a las tareas de distribución de señales [Lev78, Her87, Law88], sino que comienza a introducirse en el campo de la conformación de haces.

La aplicación de componentes ópticos (Fig. 2.14), tanto si se consideran fibras ópticas como medio de transmisión, o componentes optoelectrónicos como elementos de control, introducirá nuevas características y requerimientos a los sistemas de distribución actualmente conocidos.

En el caso de una agrupación desfasable de antenas controlada ópticamente $e^{\mathrm{i}}$ las funciones de conformado de haces puede realizarse en diferentes etapas de la red de distribución. Se pueden distinguir tres categorías [Her88a, Sae93]:

1. Procesado en el plano de la antena de agrupación (Fig. 2.15) mediante el uso de dispositivos monolíticos de microondas (MMIC) controlados ópticamente (desfasadores y atenuadores) [Pao88, Ros86, Jes87, Soa83].

2. Conformación de los haces dentro de la propia red de distribución de fibra óptica a través del uso de elementos de retardo verdadero [Her87, Dar86, Her86, Li97].

3. Conformación en el plano de entrada de la red de distribución de fibra óptica, mediante esquemas de procesado óptico en espacio libre (procesadores de Fourier (Fig. 2.16)) [Koe84, And88, Vos89, Riz97].

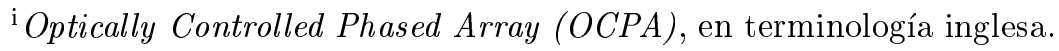




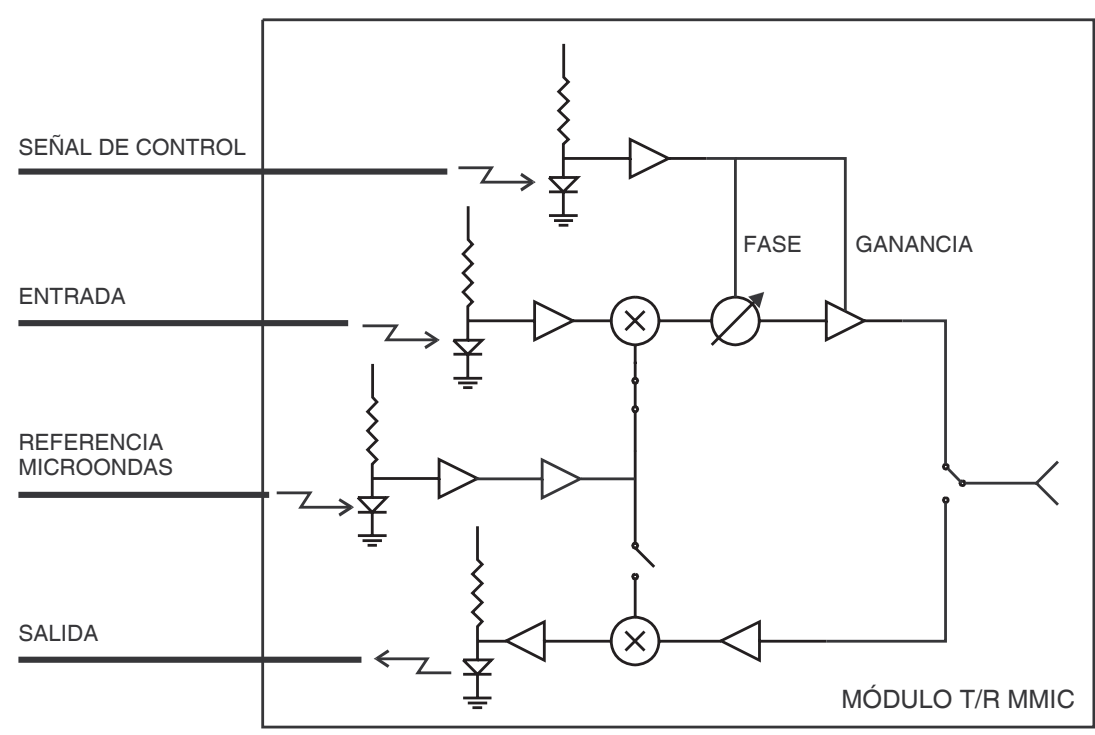

a)

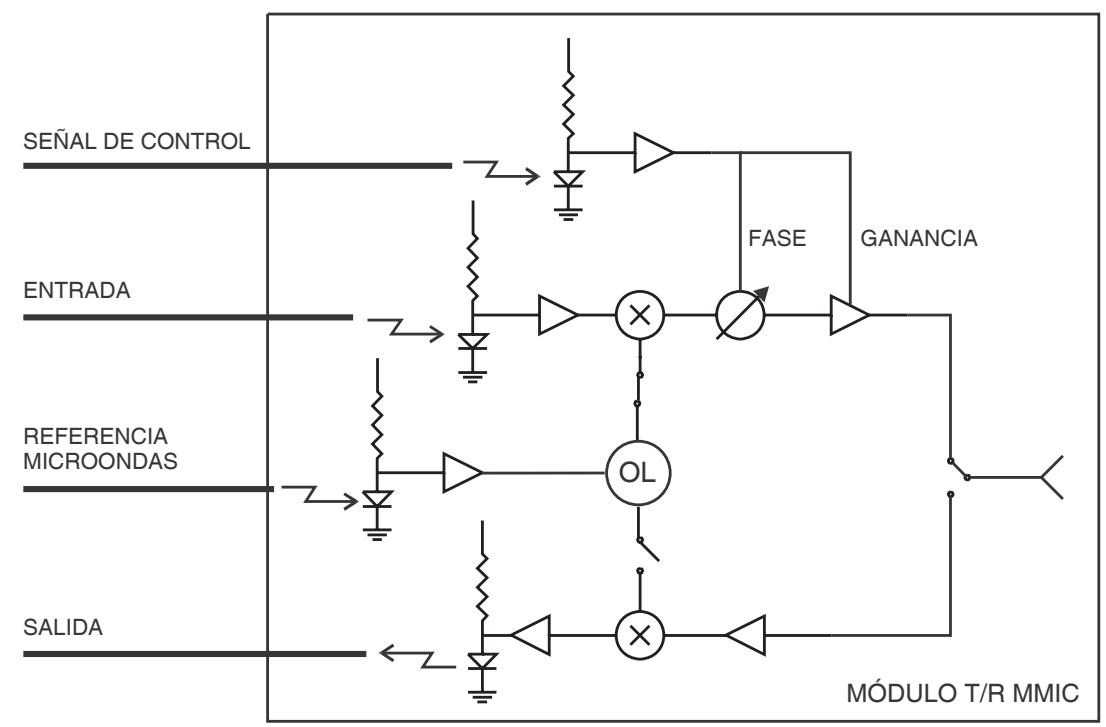

b)

Figura 2.15: Módulo T/R MMIC controlado ópticamente. a) Detección directa y amplificación de la señal de referencia. b) Oscilador local enganchado por inyección. 


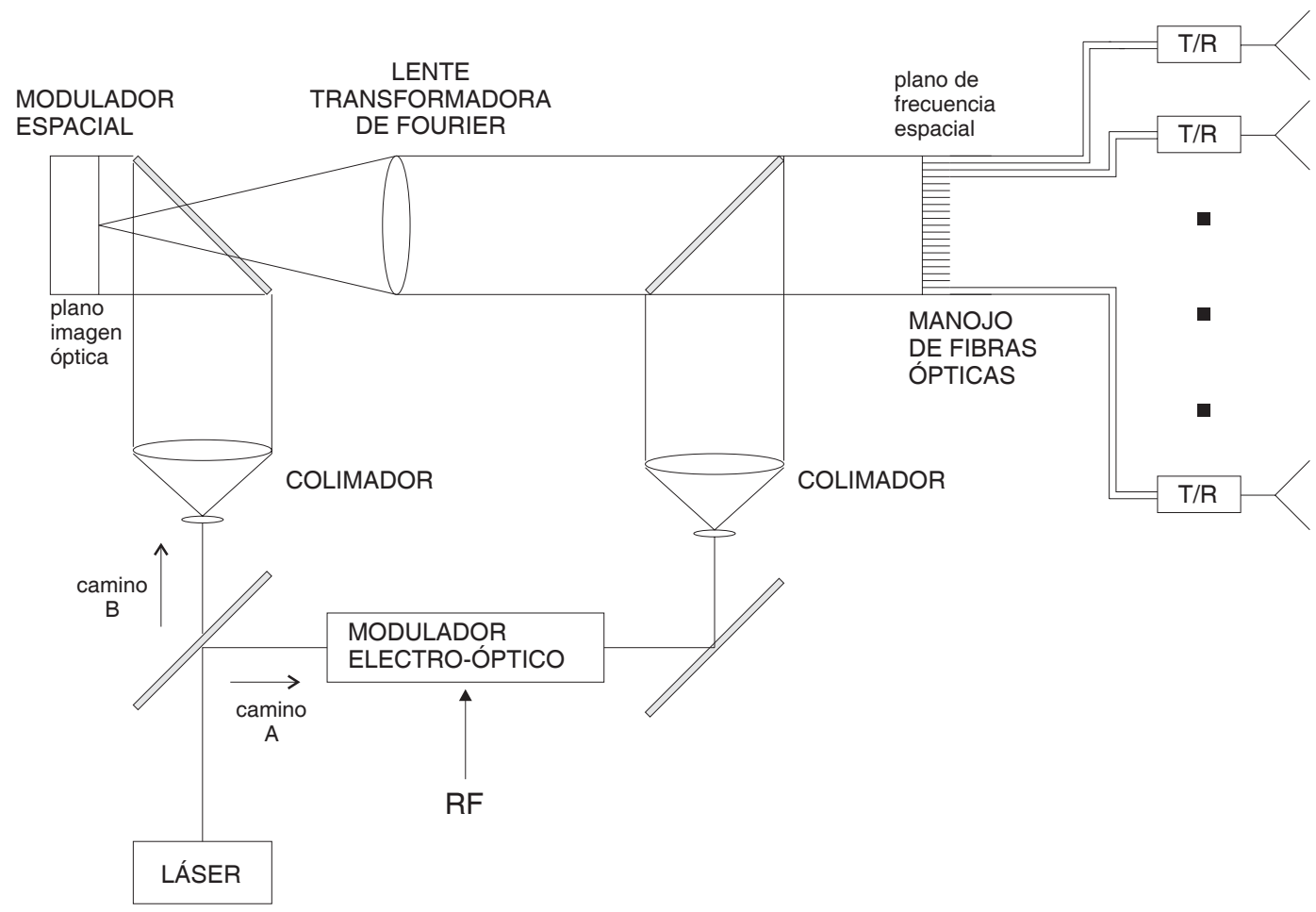

Figura 2.16: Ejemplo de conformador de haces mediante procesado óptico de Fourier en espacio libre.

La primera opción se convierte en la más conservadora y rápida en su implementación al partir de unas estructuras típicas de radiofrecuencia. De la misma forma que se reduce el riesgo, la variación en cuanto a prestaciones y características respecto a configuraciones tradicionales será menor que una opción como la tercera que conlleva un cambio radical de supuestos y arquitectura. El objetivo planteado con la primera opción es generar ópticamente las funciones de control necesarias para gobernar la ganancia y el desfase en cada módulo T/R [Her88b]. Esta opción permite además un alto grado de integración, un bajo coste, una alta fiabilidad y un cómodo interfaz entre sistemas ópticos y de microondas. La sincronización óptica de osciladores mediante técnicas de enganche por inyección ${ }^{\mathrm{i}}$ [Her86, Dar87b, Ber91, Dar87a] es otra posibilidad considerada dentro de esta opción.

La segunda opción, se basa en arquitecturas que conforman el haz mediante los retardos sufridos por la portadora, o portadoras ópticas, hasta cada uno de los elementos de la agrupación. Para ello, se dispone de líneas de retardo programables como pueden ser las basadas en fibra óptica altamente dispersiva que ofrecen un retardo sintonizable en longitud de onda [Joh93, Fra95, Fra96, Mat97, Esm93, Ton96a, Sor92]. Otro enfoque para las líneas de retardo con control óptico, ha sido la utilización de redes de difracción de banda estrecha y periodo constante, como elementos reflectores seleccionables en longitud de onda [Bal94, Lem94, Ton96b, Mol95, Zmu97, Sor96, Cha96]. Finalmente, otra posibilidad de implementar en el

\footnotetext{
${ }^{\mathrm{i}}$ Injection locking, en su acepción inglesa.
} 
dominio óptico líneas de retardo variables, consiste en la conmutación de diferentes longitudes de fibra óptica [Gou92].

\subsection{Efecto sobre el diagrama de radiación de los errores en amplitud y fase en la alimentación de la agrupación}

Dentro de las causas que pueden deteriorar el comportamiento ideal de una agrupación de antenas se puede citar las debidas al acoplamiento de los elementos radiantes que dan lugar a impedancias mutuas y desadaptaciones dependientes del ángulo de apuntamiento y a fenómenos de ceguera ${ }^{\mathrm{i}}$ debido a la aparición de ondas de superficie en la apertura [Mai82, Far66]. Esta fuente de errores está más relacionado con el fenómeno de radiación, que con la conformación de los haces, por lo que no entra dentro del campo de investigación de esta Tesis.

Otro causa de error importante en las agrupaciones de antenas y, más relacionada con el proceso de conformación, es la debida a los errores en la alimentación de los elementos de la agrupación. Estos errores pueden tener una naturaleza aleatoria debido a las tolerancias de fabricación de los componentes utilizados en la red conformadora, o bien, pueden aparecer de forma correlada como es el caso de los errores de cuantificación que aparecen en los desfasadores variables digitales.

En esta sección se resumirán los resultados más importantes relativos al efecto de los errores de alimentación de la agrupación sobre parámetros de radiación, como la ganancia, el nivel de los lóbulos secundarios o el ángulo de apuntamiento.

\subsubsection{Errores Aleatorios}

Partiendo de una agrupación de antenas donde el elemento enésimo de la figura 2.1 tiene un error relativo en la amplitud de iluminación de $\Delta_{n}$, un error en la fase de $\delta_{n}$ y donde la probabilidad de que un elemento de la agrupación funcione correctamente es $P$, se puede obtener la siguiente expresión para el factor de la agrupación:

$$
F(\theta, \phi)=\sum_{n=1}^{N} p(n) A_{n}\left(1+\Delta_{n}\right) e^{j \delta_{n}} e^{-j k \hat{r}_{o} \vec{r}_{n}} e^{j k \hat{r} \vec{r}_{n}}
$$

donde $p(n)$ es una función aleatoria que toma el valor 1 con una probabilidad $P, \mathrm{y}$ 0 con una probabilidad $(1-P)$.

A partir de la expresión (2.65) es posible obtener estimadores estadísticos para el diagrama de radiación, teniendo en cuenta que los resultados obtenidos hacen

\footnotetext{
${ }^{\mathrm{i} S c a n}$ blindness o Array blindness, en terminología inglesa.
} 
referencia a un conjunto de muestras de diagramas de radiación de diferentes agrupaciones con las mismas distribuciones estadísticas en amplitud y fase y no a un conjunto de muestras de direcciones del espacio dentro de un único diagrama de radiación ${ }^{\mathrm{i}}$.

Partiendo de que los posibles errores correlados han sido eliminados ${ }^{\mathrm{ii}}$, dejando únicamente errores aleatorios y asumiendo que los errores en amplitud y en fase de cada elemento, siguen una misma distribución estadística gaussiana de media nula y de varianzas $\overline{\Delta^{2}}$ y $\overline{\delta^{2}}$ respectivamente, y que los elementos fallidos se distribuyen espacialmente de una forma aleatoria, se puede obtener la siguiente expresión para el diagrama medio de radiación de potencia:

$$
\overline{|F(\theta, \phi)|^{2}}=P^{2} e^{-\overline{\delta^{2}}}\left|F_{o}(\theta, \phi)\right|^{2}+\left[P\left(1+\overline{\Delta^{2}}\right)-P^{2} e^{-\overline{\delta^{2}}}\right] \sum_{n=1}^{N} A_{n}^{2}
$$

donde $\left|F_{o}(\theta, \phi)\right|^{2}$ es el diagrama de radiación de potencia ideal a partir de (2.10). A partir del resultado (2.66), se puede concluir que el diagrama de radiación medio, consiste en el diagrama de radiación ideal reducido por un factor que tiene en cuenta los elementos fallidos y la dispersión introducida por los errores aleatorios en la fases, más un término constante para cualquier dirección espacial y que depende de todas las fuentes de error. El término constante corresponde a un nivel residual de radiación para cualquier dirección del espacio y permite obtener el nivel medio de los lóbulos secundarios; para ello se normalizará (2.66) dividiendo por el máximo de radiación calculado a partir de (2.66) despreciando el segundo término:

$$
\overline{\left|F\left(\theta_{o}, \phi_{o}\right)\right|^{2}} \approx P^{2} e^{-\overline{\delta^{2}}}\left|F_{o}\left(\theta_{o}, \phi_{o}\right)\right|^{2}=P^{2} e^{-\overline{\delta^{2}}}\left(\sum_{n=1}^{N} A_{n}\right)^{2}
$$

obteniéndose:

$$
\overline{\left|F_{N}(\theta, \phi)\right|^{2}} \approx\left|F_{o N}(\theta, \phi)\right|^{2}+\left[1-P+\overline{\Delta^{2}}+\overline{\delta^{2}}\right] \frac{\sum_{n=1}^{N} A_{n}^{2}}{P\left(\sum_{n=1}^{N} A_{n}\right)^{2}}
$$

por lo tanto el nivel medio de los lóbulos secundario normalizados respecto al lóbulo principal es:

$$
\overline{\sigma^{2}}=\frac{1-P+\overline{\Delta^{2}}+\overline{\delta^{2}}}{P N \eta}=\frac{\overline{\epsilon^{2}}}{P N \eta}
$$

donde $\eta$ es la eficiencia de iluminación de la agrupación, definida en (2.27).

\footnotetext{
${ }^{i}$ Un análisis más exhaustivo, puede encontrarse en el apéndice $\mathrm{A}$

${ }^{\text {ii }}$ Se asume por tanto que los errores de cada elemento son estadísticamente independientes entre sí.
} 


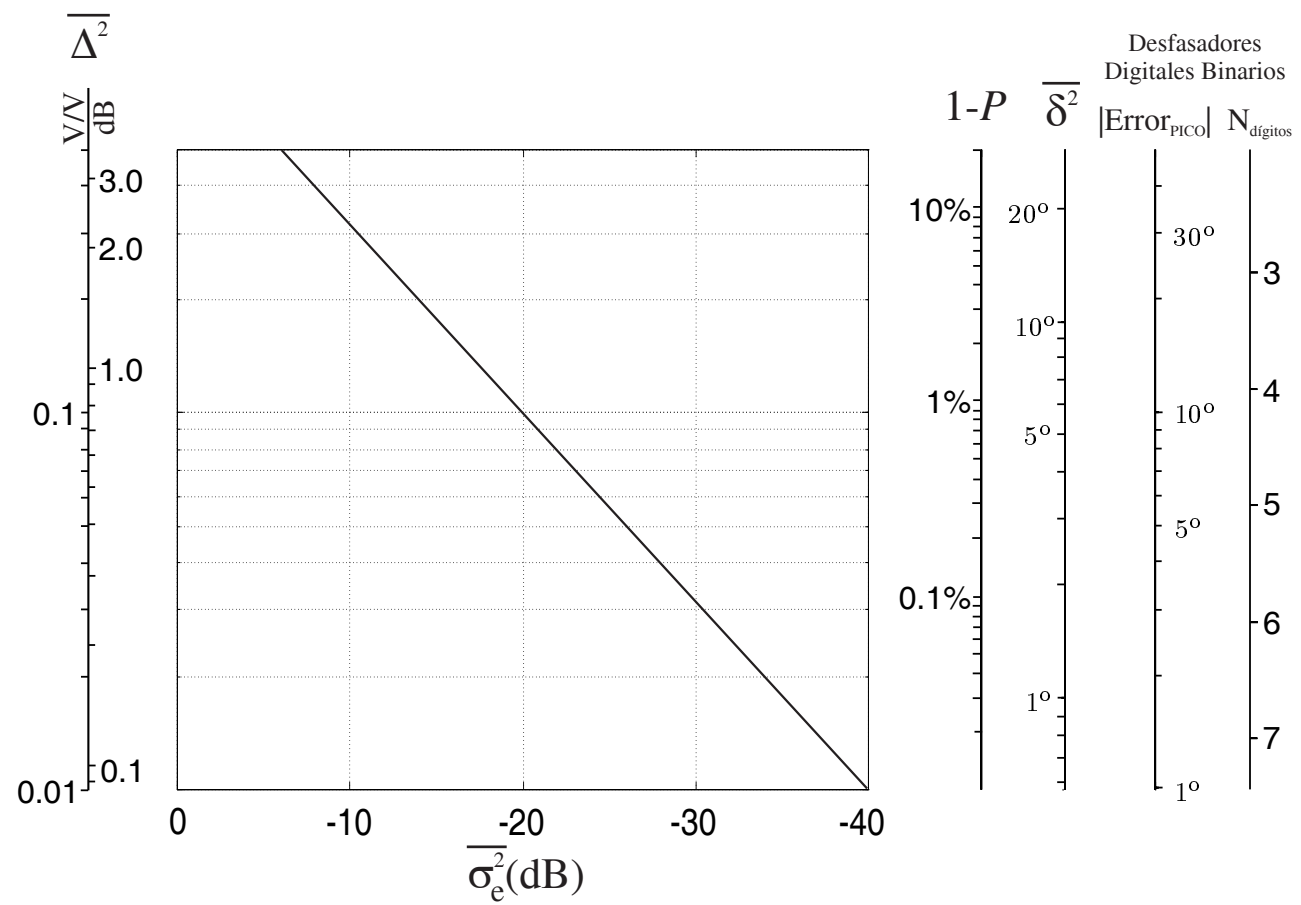

Figura 2.17: Nivel residual normalizado de los lóbulos secundarios de fondo.

En la figura 2.17 se representa gráficamente el nivel medio de los lóbulos secundarios de (2.69) normalizados para $N=1$ y $\eta=1$, cuando los errores provienen únicamente de los errores de fase, de amplitud o por elementos fallidos. Para una agrupación concreta habría que desnormalizar el nivel de lóbulo secundario obtenido de la gráfica, restándole $10 \cdot \log (N \eta)$.

La ecuación (2.69) calcula el nivel relativo medio del suelo de lóbulos secundarios presentes en un conjunto de diagramas de radiación. Generalmente, es más interesante disponer de información del nivel de pico de los lóbulos secundarios, para lo cual es necesario obtener la distribución estadística que sigue el diagrama de radiación. Se ha demostrado que para una dirección del espacio dada, el diagrama de radiación de campo de un conjunto de agrupaciones con las mismas estadísticas de errores sigue una distribución tipo Rice con una función densidad de probabilidad [Hsi85, All63]:

$$
p(F)=\frac{2 F}{\overline{\sigma^{2}}} I_{o}\left[\frac{2 F F_{o}}{\overline{\sigma^{2}}}\right] e^{\left[-\frac{\left(F^{2}+F_{o}^{2}\right)}{\overline{\sigma^{2}}}\right]}
$$

expresión que permite calcular la probabilidad, $p(F)$, de que el nivel del campo radiado en una dirección determinada del espacio, para un conjunto de diagramas de radiación, esté entre $F$ y $F+d F$, cuando $F_{o}$ es el nivel ideal del diagrama en esa dirección del espacio y $\overline{\sigma^{2}}$ es el nivel residual de fondo de los lóbulos secundarios a partir de (2.69).

Para el caso en el que los errores son lo suficientemente grandes comparados con 
los lóbulos secundarios o con las profundidades de los nulos, es posible despreciar la radiación teórica de los lóbulos menores y la estadística del diagrama de intensidad de campo seguirá una distribución Rayleigh, de forma que la probabilidad de que en un punto determinado se exceda un nivel $F_{d}$ será:

$$
\operatorname{Prob}\left(F>F_{d}\right)=e^{-\left(\frac{F_{d}^{2}}{\sigma^{2}}\right)}
$$

con $\sigma^{2}$ obtenida de (2.69).

En un caso general, la ecuación (2.70) permite estimar la probabilidad de que el campo radiado en cierta dirección esté por debajo de un cierto valor deseado $\left(F_{d}\right)$ teniendo en cuenta el nivel en esa dirección del diagrama de radiación sin errores:

$$
\operatorname{Prob}\left(F<F_{d}\right)=\int_{0}^{F_{d}} \frac{2 F}{\overline{\sigma^{2}}} I_{o}\left[\frac{2 F F_{o}}{\overline{\sigma^{2}}}\right] e^{\left[-\frac{\left(F^{2}+F_{o}^{2}\right)}{\overline{\sigma^{2}}}\right]} d F
$$

Por lo tanto, es posible relacionar el nivel de lóbulos secundario diseñado de acuerdo a la iluminación ideal escogida $\left(F_{o}\right)$, con el nivel finalmente obtenido $\left(F_{d}\right)$ con una cierta probabilidad $P$ y el nivel residual del diagrama $\left(\overline{\sigma^{2}}\right)$ debido a los errores en la iluminación. En la gráfica 2.18 se puede comprobar la relación entre estos cuatro parámetros.

La figura 2.18, da el nivel residual de lóbulos secundarios en función del nivel de lóbulo secundario ideal, donde ambos valores están normalizados respecto al nivel deseado. Como ejemplo de utilización de la citada figura, comentar que si se desea tener un diagrama de radiación real con lóbulos secundarios que no superen por ejemplo $-25 \mathrm{~dB}$ respecto al lóbulo principal $\left(F_{d}=-25 \mathrm{~dB}\right)$ con una probabilidad del $95 \%$, se puede partir de una iluminación que ofrezca un NLPS ideal de $28 \mathrm{~dB}$ $\left(F_{o}=-28 \mathrm{~dB}, F_{o} / F_{d}=-3 \mathrm{~dB}\right)$ dejando un margen para la tolerancia de los componentes de $\overline{\sigma^{2}}=0.057 * F_{d}^{2}=1.8 \cdot 10^{-4}$, lo que para una agrupación con 100 elementos y una eficiencia de $\eta=0.9$, equivaldría a un requerimiento para la precisión en la amplitud de la iluminación de $0.13 \mathrm{~V} / \mathrm{V}(1 \mathrm{~dB})$ o a una varianza en la fase de $7.3^{\circ}$ (aproximadamente la que ofrece un desfasador digital de cuatro dígitos) o a una probabilidad de supervivencia de los elementos de 98.4\%. Otra solución, menos restrictiva en cuanto a la tolerancia de los componentes, sería partir de una iluminación que ofrezca un NLPS ideal de de $33 \mathrm{~dB}\left(F_{o}=-33 \mathrm{~dB}, F_{o} / F_{d}=\right.$ $-8 \mathrm{~dB}$ ) aumentando el margen para errores de fabricación en los componentes de $\overline{\sigma^{2}}=0.2 \cdot F_{d}^{2}=6.4 \cdot 10^{-4}$, lo que para una agrupación con 100 elementos y una eficiencia de $\eta=0.8$ equivaldría a una varianza en la amplitud de la iluminación de $0.22 \mathrm{~V} / \mathrm{V}\left(1.76 \mathrm{~dB}\right.$ ), o a una varianza en la fase de $12.9^{\circ}$ (desfasador digital de tres dígitos), o a una probabilidad de supervivencia de los elementos del $95 \%$.

De esta forma, la gráfica 2.18, nos permite especificar la función de iluminación utilizada, teniendo en cuenta el nivel de lóbulos secundarios deseado y la tolerancia de los componentes que forman la red conformadora de haces.

Las probabilidades calculadas hasta este momento, hacen referencia a la probabilidad de que se supere un cierto nivel de campo en una dirección determinada del 


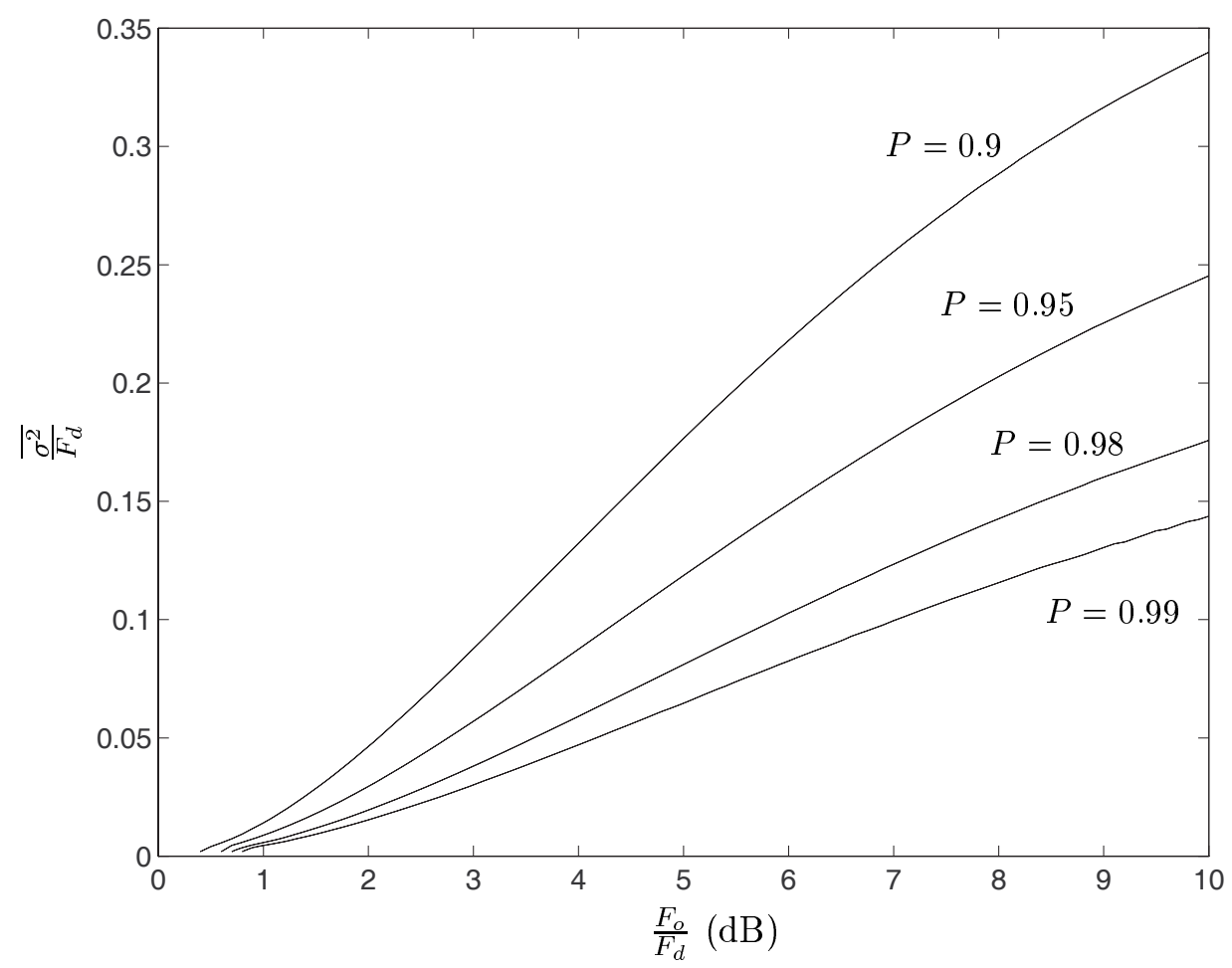

Figura 2.18: Nivel residual normalizado de lóbulos secundarios respecto a la relación entre nivel de pico de los lóbulos y nivel de diseño parametrizado respecto a la probabilidad de quedar por debajo del nivel de pico.

diagrama de radiación. Una vez conocido el número de lóbulos secundarios presentes en el diagrama de radiación es inmediato obtener una expresión para la probabilidad acumulada de que en alguno de ellos se supere un cierto nivel, o incluso expresiones para la probabilidad de que se supere en K de ellos [All63, Kap86].

Partiendo de la expresión del diagrama de radiación de potencia medio expresado en (2.66) se puede obtener fácilmente el siguiente resultado aproximado, para la variación de la ganancia de la agrupación respecto a la de la agrupación ideal:

$$
\frac{G}{G_{o}} \approx \frac{P}{\left(1+\overline{\Delta^{2}}\right) e^{\overline{\delta^{2}}}} \approx \frac{P}{1+\overline{\Delta^{2}}+\overline{\delta^{2}}}
$$

como se puede comprobar la reducción de la ganancia de la agrupación por efecto de los errores en la alimentación es pequeña, ya que, por ejemplo un error en la fase de $\overline{\delta^{2}}=20^{\circ}$ implica una reducción de sólo $0.5 \mathrm{~dB}$.

Por otro lado, el error de apuntamiento para una agrupación uniforme es [Car73]:

$$
\overline{\Delta_{\theta}^{2}}=\frac{12}{N^{3}} \overline{\delta^{2}}
$$




\subsubsection{Efecto de la cuantificación de la alimentación de la agrupación}

Aparte de los errores aleatorios estudiados, existen unos errores deterministas debidos a la utilización de desfasadores y atenuadores variables, que no permiten una variación contínua de su desfase o atenuación, sino que ofrecen una serie de valores discretos; es decir, desfasadores y atenuadores digitales.

Un desfasador digital consiste en una serie de desfasadores fijos conmutables (también llamados dígitos) en cascada. Estos dígitos conmutables tienen dos estados posibles de desfase: el estado cero y el correspondiente al peso del dígito. Los pesos de los dígitos suelen estar relacionadas en potencias de 2 , de forma que un desfasador digital con tres dígitos y que pretenda cubrir el rango completo de $360^{\circ}$ tendrá tres desfasadores con pesos $180^{\circ}, 90^{\circ}$ y $45^{\circ}$. Combinando estos tres desfasadores en sus dos estados posibles se obtienen ocho estados de fase para el desfasador total, a saber: $315^{\circ}, 270^{\circ}, 225^{\circ}, 180^{\circ}, 135^{\circ}, 90^{\circ}, 45^{\circ}$ y $0^{\circ}$.

Para el caso general de un desfasador digital con un rango de $360^{\circ}$ y $p$ dígitos, el número de estados posibles del mismo es de $2^{p}$; estados que para el caso de un desfasador con pesos binarios, estarán separados $2 * \pi / 2^{p}$ radianes. En ese caso y asumiendo que el desfasador debería proporcionar cualquier fase entre $0^{\circ}$ y $360^{\circ}$ con igual probabilidad, es conocido que los errores de fase en cada elemento $\left(\delta_{e}\right)$ presentan una distribución uniforme con los siguientes parámetros:

$$
\begin{aligned}
\overline{\delta_{e}} & =0 \\
\overline{\delta_{e}^{2}} & =\frac{\pi^{2}}{3 \cdot 2^{2 p}} \\
\delta_{r m s} & =\frac{\pi}{2^{p} \sqrt{3}} \\
\delta_{P I C O} & = \pm \frac{\pi}{2^{p}}
\end{aligned}
$$

En el apéndice A se demuestra que la pérdida en la ganancia de la agrupación (medida en la dirección del máximo de la agrupación sin errores), cuando los errores de fase siguen una distribución uniforme simétrica dependen del valor de pico según la ecuación (A.32), por lo tanto en el caso de un desfasador binario de $p$ dígitos:

$$
\frac{G}{G_{o}}=\operatorname{sinc}^{2}\left(\frac{\pi}{2^{p}}\right)+\left[1-\operatorname{sinc}^{2}\left(\frac{\pi}{2^{p}}\right)\right] \frac{1}{N \eta} \approx \operatorname{sinc}^{2}\left(\frac{\pi}{2^{p}}\right)
$$

donde $G_{o}$ es la ganancia de la agrupación sin errores de cuantificación, $N$ es el número de elementos y $\eta$ la eficiencia de la iluminación.

De acuerdo con los resultados del apéndice A, el nivel RMS de los lóbulos secundarios cuando la distribución de errores es uniforme será:

$$
\overline{\sigma^{2}} \approx \frac{1-\operatorname{sinc}^{2}\left(\frac{\pi}{2^{p}}\right)}{\operatorname{sinc}^{2}\left(\frac{\pi}{2^{p}}\right)} \cdot \frac{1}{N \eta} \approx \frac{\pi^{2}}{3 N \eta 2^{2 p}}
$$


Mientras que, la desviación del haz principal se puede expresar en función del ancho de haz como:

$$
\Delta \theta=\frac{\pi}{4 \cdot 2^{p}} \Delta \theta_{-3}
$$

El resultado (2.77) se basa en que el error de fase en cada elemento es una variable aleatoria independiente; pero asumiendo que a cada desfasador le llega la señal con la misma fase, no se puede afirmar en absoluto que los desfases introducidos en cada elemento sean independientes, al contrario, estarían relacionados de acuerdo con (2.7). Teniendo esto en cuenta se ha demostrado [Buc72, Mil64] que los errores en los desfases correlados implican que el diagrama de radiación total sea aproximadamente la suma de:

- El diagrama de radiación ideal con un máximo en $\left(\theta_{o}, \phi_{o}\right)$ de acuerdo con la iluminación $A_{n} \cdot e^{j \alpha_{n}}$.

- Un número infinito de diagramas de radiación con máximos en las direcciones $\left(\theta_{i}, \phi_{i}\right)$ y amplitudes $E_{i}$ de acuerdo con:

$-E_{i}=\frac{1}{i \cdot 2^{p}}$

$-\vec{r}_{n} \hat{r}_{i}=\vec{r}_{n} \hat{r}_{o}\left(1+i 2^{p}\right)+s / \lambda$, con $s \in \mathbb{Z}$

$-i= \pm 1, \pm 2, \pm 3 \ldots$

Por consiguiente, y como primera aproximación, se puede afirmar que aparecerán lóbulos de cuantificación de amplitud máxima aproximada:

$$
\sigma_{Q}=\frac{1}{2^{p}}
$$

como ejemplo, para un desfasador digital binario de cinco dígitos el nivel de los lóbulos de cuantificación podría llegar al entorno de los -30 dB.

En este análisis, se desprecia la interacción que pudiera existir entre esos infinitos diagramas de radiación y, en concreto, se ha obviado la posibilidad de que para alguna combinación de desfase $\alpha_{n}$ (es decir, para algún ángulo de apuntamiento, $\theta_{o}$ ), esos infinitos diagramas de radiación sumen sus máximos de forma coherente, caso, que para una agrupación lineal, tiene lugar cuando los errores de fase aparecen de forma periódica en la agrupación y el salto de fase entre elementos contiguos es $\Delta \alpha=\pi / 2^{p}+k\left(2 \pi / 2^{p}\right)$, con $k \in \mathbb{Z}$. En esos casos concretos el nivel de los lóbulos de cuantificación es de [Sko90b]:

$$
\sigma_{Q \max }=\frac{\pi}{2 \cdot 2^{p}}
$$

Con el fin de eliminar los lóbulos de cuantificación es necesario decorrelar los errores de fase de cada elemento. En los casos en los que la red de distribución 
produce una distribución de señales en fase a la entrada de cada elemento, la mejor solución es añadir una fase aleatoria, pero conocida, al camino recorrido por la señal hasta cada desfasador, reduciendo el nivel de los lóbulos de cuantificación hasta aproximadamente el nivel de fondo de los lóbulos secundarios de (2.77). De esta forma se elimina también el error medio de apuntamiento. Otra solución utilizada en sistemas RADAR que realicen promediado de diferentes pulsos, se denomina temblor de fase o temblor de haz ${ }^{\mathrm{i}}$ y consiste en introducir en cada pulso un ruido de fase aleatorio diferente sumándolo a la fase ideal a sintetizar en cada desfasador variable; el promediado de los errores de fase de cada pulso cancelan los lóbulos de cuantificación [Bro91].

Como conclusión comentar que, claramente, el efecto más importante de los errores en los coeficientes de iluminación de una agrupación es el aumento del nivel de los lóbulos secundarios, lo que implicará la necesidad de sobredimensionarlos teniendo en cuenta la tolerancia estimada de los componentes utilizados.

Por otro lado, es interesante constatar la flexibilidad que puede ofrecer una red conformadora de haces digital en cuanto a temas como la compensación en la alimentación de la agrupación ante la existencia de elementos fallidos conocidos [Mai93b].

\subsection{Resumen}

En este capítulo introductorio a las redes conformadoras de haces, se han presentado los conceptos básicos relacionados con las agrupaciones de antenas y las redes conformadoras de haces haciendo especial hincapié en las principales limitaciones que se presentan a la hora de trabajar con agrupaciones desfasables electrónicamente y antenas multihaz.

En el caso de las agrupaciones desfasables electrónicamente, la aparición de los MMIC ha permitido la combinación de varios integrados dentro de un módulo $\mathrm{T} / \mathrm{R}$ reduciendo el tamaño y el peso. Los circuitos integrados típicos a implementar, para poder conformar el haz, serán atenuadores y desfasadores. Además, si se trata de agrupaciones desfasable activas, estos módulos incluirán elementos activos como amplificadores de potencia o amplificadores de bajo ruido. En la mayoría de los casos, el diseño de estos circuitos integrados son traslación de las configuraciones equivalentes en tecnología híbrida. Una de las características contínuamente destacada a la hora de defender la integración de circuitos integrados monolíticos en sistemas de comunicaciones es su alta repetibilidad, cualidad que debe ser tenida muy en cuenta durante el diseño considerando las tolerancias propias del proceso de fabricación escogido. A medida que se aumenta en frecuencia, esta repetibilidad de los circuitos comienza a ponerse en entredicho, pues pequeña variaciones en las dimensiones o propiedades de las diversas capas y deposiciones que forman el MMIC afectan bastante al comportamiento de los elementos de dimensiones cada vez más

\footnotetext{
${ }^{\text {i } P h a s e ~ o ~ B e a m ~ d i t h e r i n g, ~ e n ~ t e r m i n o l o g i ́ a ~ i n g l e s a . ~}$
} 
reducidas. A la hora de escoger la topología del circuito, sería conveniente tener en cuenta esas variaciones del proceso, junto a los errores de cuantificación o aleatorios estudiados en este capítulo y analizados detalladamente en el apéndice A.

Las antenas multihaz presentan cualidades muy interesantes cuando se requiere trabajar con numerosos haces; lo que, en el caso de agrupaciones desfasables requiere la repetición múltiple de la red conformadora. Desgraciadamente, su utilización está limitada por la complejidad e imprecisión que conlleva la implementación de matrices de Butler o de Blass para antenas con un gran número de elementos. Sería deseable una mayor aplicación de tecnología integradas que redujeran el tamaño y la complejidad de las redes conformadoras de haces para antenas multihaz.

Aún cuando se disponga de un gran número de elementos $T / R$ diseñados con tecnología MMIC, con el fin de construir una agrupación de antenas de altas prestaciones, queda pendiente el problema de las distribución a cada elemento, de la señal de información (en frecuencia intermedia o directamente en radiofrecuencia) y las múltiples señales de control para los T/R. La utilización de redes ópticas conformadoras de haces, permite no sólo la distribución de la señal de información a cada módulo, si no, reducir o eliminar la necesidad de transmitir señales de control a los elementos de la antena, ya que la propia red se encarga de conformar el haz mediante el control de dispositivos, generalmente colocados en el lugar de la fuente de señal y no el lado de los elementos de la agrupación. 



\section{Capítulo 3}

\section{Aplicación de tecnología monolítica a agrupaciones desfasables de antenas}

\subsection{Introducción}

La aplicación de circuitos monolíticos (MMIC) a agrupaciones desfasables de antenas se concentra en el diseño de dispositivos para el control de la amplitud (amplificadores/atenuadores) y de la fase (desfasadores) de alimentación de cada elemento. La función de estos circuitos es la conformación del haz deseado y, por su reducido tamaño, se pueden situar cerca del elemento radiante o subagrupación correspondiente.

Además de la conformación del haz, las agrupaciones desfasables activas incluirán amplificadores de potencia en transmisión y amplificadores de bajo ruido en recepción; circuitos fácilmente implementables en tecnología monolítica. Es habitual combinar los citados elementos dentro de un módulo T/R (Transmisor/Receptor).

A diferencia de otras aplicaciones, las agrupaciones de antenas requieren un control muy preciso de la amplitud y la fase, por parte de las etapas amplificado-

ras/atenuadoras y desfasadoras; lo que suele implicar que cada etapa esté compuesta de diferentes módulos o dígitos conmutables habitualmente entre dos estados y capaces de introducir diferentes valores de ganancia o desfase. Teniendo en cuenta la relación inversa que existe entre el rendimiento de fabricación de un MMIC y su tamaño, y el gran número de señales de control y monitorización necesarias para seleccionar la amplitud y fase de cada elemento, es habitual combinar diferentes MMICs dentro de un módulo híbrido, llegando incluso a dividir la etapa desfasadora en diversos MMICs.

Para todos los casos considerados, el dispositivo común a todos ellos es siempre el elemento desfasador, pues consiste en el elemento básico para realizar el apunta- 
miento del haz de la agrupación.

A la hora de diseñar la etapa desfasadora, un aspecto básico es encontrar la topología más adecuada de los desfases de los diferentes dígitos que la forman para minimizar el efecto combinado de los errores de cuantificación y de los errores aleatorios debidos a variaciones del proceso de fabricación MMIC. La fabricación de desfasadores variables ${ }^{\mathrm{i}}$ con tecnología MMIC lleva implícita la presencia de imprecisiones en los desfases introducidos por cada dígito (ambos estados conmutables). Estos errores aleatorios en los pesos de cada dígito se traducen en un error en el desfase total del desfasador, que se combina con el error de cuantificación ya introducido por un desfasador digital, y que ha sido estudiado en la sección 2.6.2.

En la sección 3.2 se presenta un modelado original del efecto combinado de ambos errores teniendo en cuenta conceptos como los de calibración de los desfasadores o la introducción de cierta redundancia en el desfasador variable total. Además, se han llevado a la práctica las consideraciones realizadas en la sección 3.2, mediante el diseño de un desfasador variable de seis dígitos para una frecuencia de trabajo de $24 \mathrm{GHz}$, estableciéndose los siguientes objetivos para el diseño:

- Alta resolución junto con bajas pérdidas de inserción.

- Diseño adecuado para su producción en masa sin necesidad de caros ajustes manuales posteriores.

- Prestaciones poco, o nada sensibles, a variaciones del proceso de fabricación MMIC.

- Encontrar tecnologías de desfasadores adecuadas para MMIC para ondas milimétricas, identificando qué aspectos podrían mejorarse en el proceso de modelado, diseño y fabricación MMIC.

En la sección 3.3 se presenta el diseño en tecnología MMIC de un desfasador digital con seis dígitos ponderados de forma no binaria con una frecuencia de trabajo de $24 \mathrm{GHz}$. A continuación, todas las consideraciones sobre el diseño de los dígitos individuales aparecen en los apartados 3.3.3 a 3.3.5. En el apartado 3.3.6 se muestra el diseño final y su distribución espacial ${ }^{\mathrm{ii}}$. Finalmente, se establecen algunas conclusiones.

\subsection{Efecto combinado de los errores de fase alea- torios y de cuantificación. Calibración}

Asumiendo que los errores debidos a la correlación de los errores de cuantificación han sido eliminados, mediante el método de la adición de una fase cualquiera (fija

\footnotetext{
${ }^{\text {i }}$ Phase Shifter, en terminología inglesa.

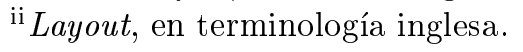


y conocida) al camino hacia cada desfasador variable, los posibles errores en la distribución de fases de la agrupación serán independientes para cada desfasador y se deberán a los errores de cuantificación y aleatorios en los desfasasadores variables.

El origen de los desfases aleatorios citados, corresponde a variaciones debidas a las tolerancias de los procesos de fabricación MMIC, que harán que los desfases introducidos en los estados de cada desfasador variable fluctúen alrededor de su valor medio. Los errores de diseño implicarían un valor medio para la diferencia de fases entre ambos estados de cada dígito, distinto del diseñado, y es de suponer que se corregirían en sucesivas iteraciones del diseño.

El efecto de los errores en las fases introducidas por los desfasadores variables puede corregirse mediante la introducción de un proceso de calibración. Esta calibración puede realizarse en diferentes grados, cubriendo un único desfasador o la totalidad de ellos, y para cada desfasador, considerando un estado, algunos o todos los estados del desfasador.

Cabría distinguir cuatro casos:

- A) No hay calibración. El estado de cada desfasador variable se escoge de acuerdo con los desfases ideales diseñados, de forma que se minimice la distancia entre el desfase deseado y el obtenido con el desfasador variable ideal.

- B) Se calibra el estado cero de cada desfasador para ajustar los retardos de los diferentes caminos hasta los elementos radiantes.

- C) Se calibra en función de un desfasador variable estándar/medio. Se corrigen las desviaciones medias frente a los valores de diseño, pero las desviaciones del proceso de un desfasador variable a otro se mantienen.

- D) Se calibra completamente cada desfasador variable por separado.

A continuación se analizan por separado cada uno de los cuatro casos, buscando caracterizar el error de un desfasador en función de los errores en los diferentes dígitos que lo componen.

\subsubsection{Sin calibración}

En el caso A) los errores aleatorios en el peso de cada dígito introducen un error que es estadísticamente independiente del ruido de cuantificación, de forma que el 
$\Phi_{T O T A L}=\Phi_{\text {TOTAL }}^{o}+\delta_{e}$

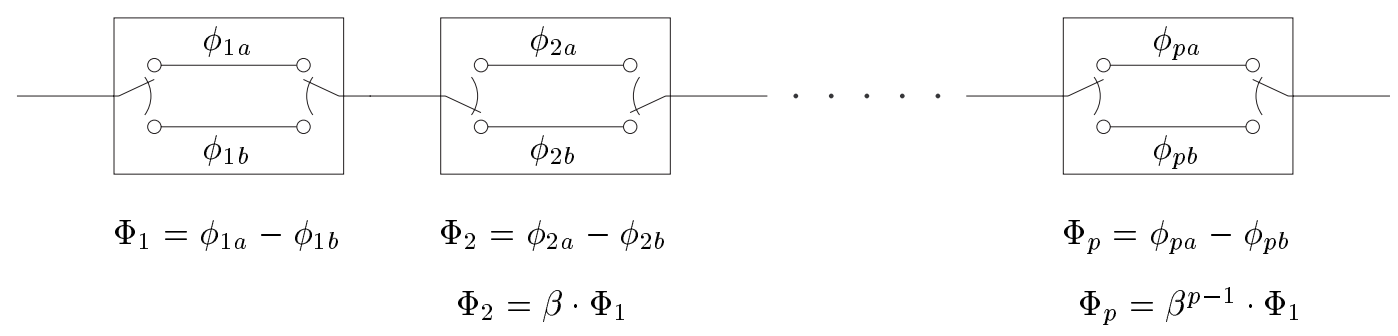

Figura 3.1: Esquema de un desfasador digital variable de $p$ dígitos y factor $\beta$.

error total en el desfasador $(\delta)$ cumplirá:

$$
\begin{aligned}
\delta & =\delta_{Q}+\delta_{e} \\
\bar{\delta} & =\overline{\delta_{Q}}+\overline{\delta_{e}} \\
\overline{\delta^{2}} & =\overline{\delta_{Q}^{2}}+\overline{\delta_{e}^{2}}+2 \overline{\delta_{e}} \cdot \overline{\delta_{Q}} \\
\sigma_{\delta}^{2} & =\sigma_{\delta_{Q}}^{2}+\sigma_{\delta_{e}}^{2} \\
\pm \delta_{P I C O} & = \pm\left[\left(\delta_{Q}\right)_{P I C O}+\left(\delta_{e}\right)_{P I C O}\right]
\end{aligned}
$$

donde $\delta_{Q}$ es el error de cuantificación, error que para el caso de un desfasador digital de rango completo $(2 \pi)$ y binario (pesos de los dígitos relacionados por un factor $\beta=2$ ) presentará una estadística uniforme con los estadísticos mostrados en las ecuaciones (2.75). Por otro lado, el error $\delta_{e}$ modela el efecto conjunto de las variaciones del desfase de todos los dígitos (ambos estados) sobre el desfase esperado total.

Se ha visto en la sección 2.6 que el principal efecto de los errores de fase en la iluminación es el aumento del nivel de los lóbulos secundarios y, en menor medida, la variación de la ganancia de la agrupación en la dirección del máximo de radiación. Como se demuestra en el apéndice A, un parámetro directamente relacionado con el nivel de los lóbulos secundarios y con la reducción de la ganancia, cuando los errores de cada elemento están incorrelados entre sí, es el producto $\overline{e^{j \delta}} \cdot \overline{e^{-j \delta}}$. Para el caso de errores independientes se demuestra en el citado apéndice que:

$$
\overline{e^{j \delta}} \cdot \overline{e^{-j \delta}}=\left(\overline{e^{j \delta_{Q}}} \cdot \overline{e^{-j \delta_{Q}}}\right) \cdot\left(\overline{e^{j \delta_{e}}} \cdot \overline{e^{-j \delta_{e}}}\right)
$$

El hecho de que los errores de cuantificación y aleatorio sean independientes, permite calcular por separado el efecto de ambos errores. El error de cuantificación de un desfasador digital binario de rango completo ya ha sido estudiado anteriormente, por lo que se pasa a estudiar el efecto de los errores aleatorios.

En la figura 3.1 se muestra esquemáticamente un desfasador digital variable de $p$ dígitos; dígitos ponderados con pesos que mantiene una razón geométrica $\beta$ entre sí. Cada uno de los $p$ dígitos tiene dos estados de desfases $\left(\phi_{i a}, \phi_{i b}\right)$ de forma que la 
diferencia entre ambos, es el peso del dígito correspondiente $\Phi_{i}=\phi_{i a}-\phi_{i b}$, siendo el desfase totali:

$$
\Phi_{T O T A L}=C_{1} \phi_{1 a}+\tilde{C}_{1} \phi_{1 b}+C_{2} \phi_{2 a}+\tilde{C}_{2} \phi_{2 b}+\cdots+C_{p} \phi_{p a}+\tilde{C}_{p} \phi_{p b}
$$

donde $C_{i}$ es un coeficiente cuyo valor es 1 o 0 según si el estado del dígito i-ésimo es el $a$ o el $b$, mientras que $\tilde{C}_{i}$ es el complementario ii de $C_{i}\left(\tilde{C}_{i}=1-C_{i}\right)$. Normalizando respecto al desfase introducido en el estado $\Phi_{00 \cdots 0}\left(C_{i}=0, \forall i=1 \cdots p\right)$, se obtiene el desfase diferencial:

$$
\Delta \Phi_{T O T A L}=\Phi_{T O T A L}-\Phi_{00 \cdots 0}=C_{1} \Phi_{1}+C_{2} \Phi_{2}+\cdots+C_{p} \Phi_{p}
$$

desfase diferencial que define al desfasador variable y que tiene $2^{p}$ estados posibles de acuerdo con los valores de $C_{i}$.

Si debido a causas como las tolerancias de los procesos de fabricación ambos estados de desfase de cada dígito $\left(\phi_{i a}, \phi_{i b}\right)$ presenta una variación aleatoria $\left(\delta_{i a}, \delta_{i b}\right)$ sobre su valor medio, el nuevo peso del dígito i-ésimo será:

$$
\Phi_{i}=\phi_{i a}-\phi_{i b}=\left(\phi_{i a}^{o}+\delta_{i a}\right)-\left(\phi_{i b}^{o}+\delta_{i b}\right)
$$

donde $\phi_{i a}^{o}-\phi_{i b}^{o}=\Phi_{i}^{o}$ es el peso diseñado para el dígito i-ésimo.

Introduciendo (3.5) en (3.3) y separando los términos constantes de los aleatorios se llega a:

$$
\begin{aligned}
\Phi_{T O T A L}= & \left(C_{1} \phi_{1 a}^{o}+\tilde{C}_{1} \phi_{1 b}^{o}+C_{2} \phi_{2 a}^{o}+\tilde{C}_{2} \phi_{2 b}^{o}+\cdots+C_{p} \phi_{p a}^{o}+\tilde{C}_{p} \phi_{p b}^{o}\right) \\
& +\left(C_{1} \delta_{1 a}+\tilde{C}_{1} \delta_{1 b}+C_{2} \delta_{2 a}+\tilde{C}_{2} \delta_{2 b}+\cdots+C_{p} \delta_{p a}+\tilde{C}_{p} \delta_{p b}\right) \\
= & \Phi_{\text {TOTAL }}^{o}+\delta_{e}
\end{aligned}
$$

donde $\Phi_{\text {TOTAL }}^{o}$ es el desfase introducido por el desfasador variable de acuerdo al diseño realizado mientras $\delta_{e}$ corresponde al error total introducido por las imprecisiones de los desfases de los diferentes dígitos.

Una vez se dispone de la expresión del error de fase total $\left(\delta_{e}\right)$ en función de los errores de fase en cada estado de cada dígito, es posible obtener estadísticos de $\delta_{e}$.

\footnotetext{
${ }^{\mathrm{i} E n}$ este punto se asume que la interacción entre los diferentes dígitos del desfasador variable es nula, de forma que el cambiar el estado de cualquiera de ellos no afecta a los desfases introducidos por el resto. Como se verá posteriormente, este punto es muy importante a la hora de diseñar un circuito real.

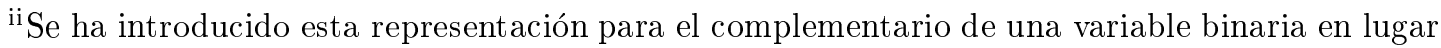
de la habitual $\overline{C_{i}}$ para evitar confusiones con el estimador estadístico valor medio.
} 
En concreto, el valor medio, $\overline{\delta_{e}}$, la varianza, $\sigma_{\delta_{e}}^{2}$, y el producto, $\overline{e^{j \delta_{e}}} \cdot \overline{e^{-j \delta_{e}}}$ :

$$
\begin{aligned}
& \overline{\delta_{e}}=\overline{C_{1} \delta_{1 a}}+\overline{\tilde{C}_{1} \delta_{1 b}}+\overline{C_{2} \delta_{2 a}}+\overline{\tilde{C}_{2} \delta_{2 b}}+\cdots+\overline{C_{p} \delta_{p a}}+\overline{\tilde{C}_{p} \delta_{p b}} \\
& =\overline{C_{1}} \cdot \overline{\delta_{1 a}}+\overline{\tilde{C}_{1}} \cdot \overline{\delta_{1 b}}+\cdots+\overline{C_{p}} \cdot \overline{\delta_{p a}}+\overline{\widetilde{C}_{p}} \cdot \overline{\delta_{p b}}
\end{aligned}
$$

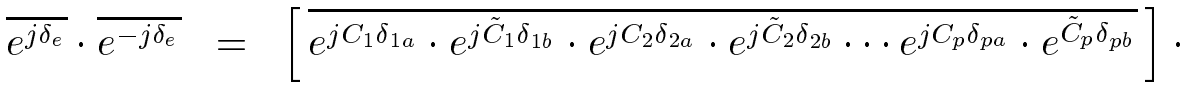

$$
\begin{aligned}
& {\left[\overline{e^{-j C_{1} \delta_{1 a}} \cdot e^{-j \tilde{C}_{1} \delta_{1 b}} \cdot e^{-j C_{2} \delta_{2 a}} \cdot e^{-j \tilde{C}_{2} \delta_{2 b}} \cdots e^{-j C_{p} \delta_{p a}} \cdot e^{-j \tilde{C}_{p} \delta_{p b}}}\right]} \\
& =\left[\prod_{i=1}^{p} \overline{e^{j C_{i} \delta_{i a}} \cdot e^{j \tilde{C}_{i} \delta_{i b}}}\right] \cdot\left[\prod_{i=1}^{p} \overline{e^{-j C_{i} \delta_{i a}} \cdot e^{-j \tilde{C}_{i} \delta_{i b}}}\right]
\end{aligned}
$$

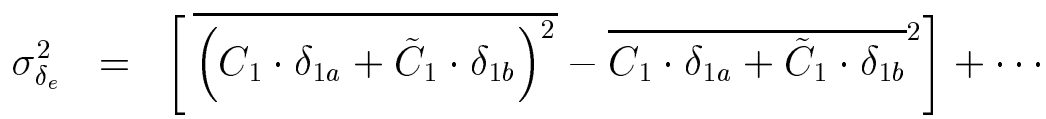

$$
\begin{aligned}
& \cdots+\left[\overline{\left(C_{p} \cdot \delta_{p a}+\tilde{C}_{p} \cdot \delta_{p b}\right)^{2}}-{\overline{C_{p} \cdot \delta_{p a}+\tilde{C}_{p} \cdot \delta_{p b}}}^{2}\right] \\
& =\sum_{i=1}^{p}\left[\overline{\left(C_{i} \cdot \delta_{i a}+\tilde{C}_{i} \cdot \delta_{i b}\right)^{2}}-{\overline{C_{i} \cdot \delta_{i a}+\tilde{C}_{i} \cdot \delta_{i b}}}^{2}\right]
\end{aligned}
$$

Asumiendo que todos los estados son equiprobables, la probabilidad de que cada coeficiente $C_{i}$ y $\tilde{C}_{i}$ tome el valor 1 , o el valor 0 , es $1 / 2$. Por consiguiente:

$$
\begin{aligned}
& \overline{C_{i}}=\overline{\tilde{C}_{i}}=\frac{1}{2} \\
& \overline{C_{i}^{2}}=\overline{\tilde{C}_{i}^{2}}=\frac{1}{2}
\end{aligned}
$$

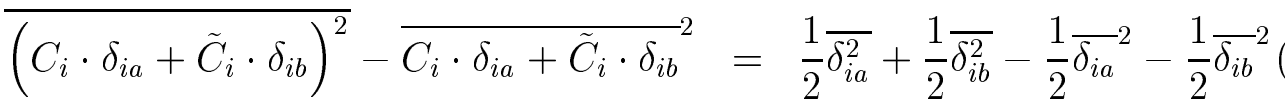

$$
\begin{aligned}
& \overline{e^{ \pm j C_{i} \delta_{i a}} \cdot e^{ \pm j \tilde{C}_{i} \delta_{i b}}}=\frac{1}{2} \overline{e^{ \pm j \delta_{i a}}}+\frac{1}{2} \overline{e^{ \pm j \delta_{i b}}}
\end{aligned}
$$

aplicando estos resultados a las ecuaciones (3.7):

$$
\begin{aligned}
& \overline{\delta_{e}}=\sum_{i=1}^{p}\left[\frac{\overline{\delta_{1 a}}+\overline{\delta_{1 b}}}{2}\right] \\
& \overline{e^{j \delta_{e}}} \cdot \overline{e^{-j \delta_{e}}}=\left[\prod_{i=1}^{p} \frac{\overline{e^{j \delta_{i a}}}+\overline{e^{j \delta_{i b}}}}{2}\right] \cdot\left[\prod_{i=1}^{p} \frac{\overline{e^{-j \delta_{i a}}}+\overline{e^{-j \delta_{i b}}}}{2}\right] \\
& \sigma_{\delta_{e}}^{2}=\sum_{i=1}^{p}\left[\frac{\overline{\delta_{i a}^{2}}-{\overline{\delta_{i a}}}^{2}+\overline{\delta_{i b}^{2}}-{\overline{\delta_{i b}}}^{2}}{2}\right]=\sum_{i=1}^{p}\left[\frac{\sigma_{i a}^{2}+\sigma_{i b}^{2}}{2}\right]
\end{aligned}
$$




\section{Errores en cada dígito iguales}

En el caso de que los errores en todos los dígitos (ambos estados) tengan los mismos estadísticos $\left(\bar{\delta}, \overline{\delta^{2}}, \sigma_{\delta}^{2}, \overline{e^{ \pm j \delta}}\right)$, los estadísticos del error de fase total son:

$$
\begin{aligned}
\overline{\delta_{e}} & =p \cdot \frac{\bar{\delta}+\bar{\delta}}{2}=p \cdot \bar{\delta} \\
\overline{e^{j \delta_{e}}} \cdot \overline{e^{-j \delta_{e}}} & =\left(\overline{e^{j \delta}}\right)^{p} \cdot\left(\overline{e^{-j \delta}}\right)^{p}=\left(\overline{e^{j \delta}} \cdot \overline{e^{-j \delta}}\right)^{p} \\
\sigma_{\delta_{e}}^{2} & =p \frac{\sigma_{\delta}^{2}+\sigma_{\delta}^{2}}{2}=p \cdot \sigma_{\delta}^{2}
\end{aligned}
$$

Estadística uniforme Para el caso de errores en cada dígito con estadística uniforme en el margen $[-E, E]$ y de acuerdo con los resultados del apéndice A se llega al resultado :

$$
\begin{aligned}
\overline{\delta_{e}} & =p \cdot \bar{\delta}=0 \\
\overline{e^{j \delta_{e}}} \cdot \overline{e^{-j \delta_{e}}} & =\left(\operatorname{sinc}^{2}(E)\right)^{p}=\operatorname{sinc}^{2 p}(E) \\
\sigma_{\delta_{e}}^{2} & =p \cdot \frac{E^{2}}{3} \\
\pm\left(\delta_{e}\right)_{P I C O} & = \pm(p \cdot E)
\end{aligned}
$$

Estadística gausiana Para el caso de errores en cada dígito con estadística gausiana con varianza $\sigma_{\delta}^{2}$ y media nula $(\bar{\delta}=0)$, teniendo en cuenta el resultado de la ecuación (A.21) se tiene:

$$
\begin{aligned}
\overline{\delta_{e}} & =p \cdot \bar{\delta}=0 \\
\overline{e^{j \delta_{e}}} \cdot \overline{e^{-j \delta_{e}}} & =\left(e^{-\sigma_{\delta}^{2}}\right)^{p}=e^{-p \sigma_{\delta}^{2}} \\
\sigma_{\delta_{e}}^{2} & =p \cdot \sigma_{\delta}^{2} \\
\pm\left(\delta_{e}\right)_{P I C O} & = \pm\left(2 p \cdot \sigma_{\delta}\right)
\end{aligned}
$$

donde los errores de pico se han calculado considerando que se cubre el $95.5 \%$ de los posibles errores de cada dígito.

\section{Errores en cada dígito proporcionales a su peso}

Otro caso interesante para analizar, es cuando los errores en cada dígito son un porcentaje del peso ideal del mismo, manteniéndose la misma estadística para todos

\footnotetext{
${ }^{\mathrm{i}}$ Se ha tenido en cuenta que para esa estadística, es conocido que $\bar{\delta}=0$ y $\sigma_{\delta}^{2}=\frac{E^{2}}{3}$.
} 
ellos. En ese caso:

$$
\begin{aligned}
\overline{\delta_{i a}} & =\overline{\delta_{i b}}=\overline{\delta_{i}}=\frac{\Phi_{i}^{o}}{\Phi_{1}^{o}} \overline{\delta_{1}}=\beta^{i-1} \cdot \overline{\delta_{1}} \\
\sigma_{\delta_{i a}}^{2} & =\sigma_{\delta_{i b}}^{2}=\sigma_{\delta_{i}}^{2}=\left(\frac{\Phi_{i}^{o}}{\Phi_{1}^{o}}\right)^{2} \sigma_{\delta_{1}}^{2}=\beta^{2 i-2} \cdot \sigma_{\delta_{1}}^{2} \\
\overline{e^{j \delta_{i a}}} & =\overline{e^{j \delta_{i b}}}=\overline{e^{j \delta_{i}}} \\
\overline{e^{-j \delta_{i a}}} & =\overline{e^{-j \delta_{i b}}}=\overline{e^{-j \delta_{i}}}
\end{aligned}
$$

Sustituyendo los resultados anteriores, en las ecuaciones (3.9) se obtienen los estadísticos del error total cuando los errores son proporcionales y no hay calibración:

$$
\begin{aligned}
\overline{\delta_{e}} & =\sum_{i=1}^{p} \frac{\Phi_{i}^{o}}{\Phi_{1}^{o}} \overline{\delta_{1}}=\overline{\delta_{1}}\left(1+\beta+\cdots+\beta^{p-1}\right)=\overline{\delta_{1}} \frac{\beta^{p}-1}{\beta-1} \\
\overline{e^{j \delta_{e}}} \cdot \overline{e^{-j \delta_{e}}} & =\prod_{i=1}^{p}\left[\overline{e^{j \delta_{i}}} \cdot \overline{e^{-j \delta_{i}}}\right] \\
\sigma_{\delta_{e}}^{2} & =\sum_{i=1}^{p}\left(\frac{\Phi_{i}^{o}}{\Phi_{1}^{o}}\right)^{2} \sigma_{\delta_{1}}^{2}=\sigma_{\delta_{1}}^{2}\left(1+\beta^{2}+\cdot+\beta^{2 p-2}\right)=\sigma_{\delta_{1}}^{2} \frac{\beta^{2 p}-1}{\beta^{2}-1}
\end{aligned}
$$

Estadística uniforme Para el caso de errores en cada dígito con estadística uniforme en los márgenes $\left[-s \Phi_{i}^{o}, s \Phi_{i}^{o}\right]=\left[-s \beta_{i-1} \Phi_{1}^{o}, s \beta^{i-1} \Phi_{1}^{o}\right]$ para el dígito i-ésimo $\left(\overline{\delta_{i}}=0\right.$, $\left.\sigma_{\delta_{i}}^{2}=\frac{\left(s \Phi_{i}^{o}\right)^{2}}{3}=\frac{\left(s \beta^{i-1} \Phi_{1}^{o}\right)^{2}}{3}\right)$, donde $s$ es el porcentaje de variación de cada dígito sobre su peso ideal. De acuerdo con los resultados del apéndice A se obtiene:

$$
\begin{aligned}
\overline{\delta_{e}} & =0 \cdot \frac{\beta^{p}-1}{\beta-1}=0 \\
\overline{e^{j \delta_{e}}} \cdot \overline{e^{-j \delta_{e}}} & =\prod_{i=1}^{p} \operatorname{sinc}^{2}\left(s \Phi_{i}^{o}\right)=\prod_{i=1}^{p} \operatorname{sinc}^{2}\left(s \beta^{i-1} \Phi_{1}^{o}\right) \\
\sigma_{\delta_{e}}^{2} & =\frac{\left(s \Phi_{1}^{o}\right)^{2}}{3} \cdot \frac{\beta^{2 p}-1}{\beta^{2}-1} \\
\pm\left(\delta_{e}\right)_{P I C O} & = \pm\left(s \cdot \Phi_{1}^{o} \cdot \frac{\beta^{p}-1}{\beta-1}\right)
\end{aligned}
$$

Estadística gausiana Cuando los errores en el dígito i-ésimo (ambos estados) siguen distribuciones gausianas de media nula $\overline{\delta_{i a}}=\overline{\delta_{i b}}=0$ y varianza $\sigma_{\delta_{i a}}^{2}=\sigma_{\delta_{i b}}^{2}=$ $\left(s \Phi_{i}^{o}\right)^{2}=\left(s \beta^{i-1} \Phi_{1}^{o}\right)^{2}$, donde $s$ es el porcentaje que la desviación estándar de cada dígito representa sobre su peso ideal, se obtienen los siguientes estadísticos para el 
error de fase total:

$$
\begin{aligned}
\overline{\delta_{e}} & =0 \cdot \frac{\beta^{p}-1}{\beta-1}=0 \\
\overline{e^{j \delta_{e}}} \cdot \overline{e^{-j \delta_{e}}} & =\prod_{i=1}^{p} e^{-\left(s \beta^{i-1} \cdot \Phi_{1}^{o}\right)^{2}}=\prod_{i=1}^{p}\left[e^{-\left(s \Phi_{1}^{o}\right)^{2}}\right]^{\beta^{2 i-2}}=\left[e^{-\left(s \Phi_{1}^{o}\right)^{2}}\right]^{\frac{\beta^{2 p}-1}{\beta^{2}-1}} \\
\sigma_{\delta_{e}}^{2} & =\left(s \Phi_{1}^{o}\right)^{2} \cdot \frac{\beta^{2 p}-1}{\beta^{2}-1} \\
\pm\left(\delta_{e}\right)_{P I C O} & = \pm\left(2 s \cdot \Phi_{1}^{o} \cdot \frac{\beta^{p}-1}{\beta-1}\right)
\end{aligned}
$$

\subsubsection{Calibración simple}

En una agrupación de antenas desfasable electrónicamente es habitual calibrar para cada camino, al menos, el estado cero de cada desfasador variable. En ese caso y sustituyendo (3.5) en (3.4) se obtiene:

$$
\begin{aligned}
\Delta \Phi_{\text {TOT AL }}= & \left(C_{1}\left(\phi_{1 a}^{o}-\phi_{1 b}^{o}\right)+C_{2}\left(\phi_{2 a}^{o}-\phi_{2 b}^{o}\right)+\cdots+C_{p}\left(\phi_{p a}^{o}-\phi_{p b}^{o}\right)\right) \\
& +\left(C_{1}\left(\delta_{1 a}-\delta_{1 b}\right)+C_{2}\left(\delta_{2 a}-\delta_{2 b}\right)+\cdots+C_{p}\left(\delta_{p a}-\delta_{p b}\right)\right) \\
= & \Delta \Phi_{\text {TOTAL }}^{o}+\delta_{e}
\end{aligned}
$$

A partir del resultado anterior se llega a las siguientes expresiones para los estadísticos del error de fase total del desfasador $\left(\delta_{e}\right)$ :

$$
\begin{aligned}
& \overline{\delta_{e}}=\overline{C_{1}}\left(\overline{\delta_{1 a}}-\overline{\delta_{1 b}}\right)+\overline{C_{2}}\left(\overline{\delta_{2 a}}-\overline{\delta_{2 b}}\right)+\cdots+\overline{C_{p}}\left(\overline{\delta_{p a}}-\overline{\delta_{p b}}\right) \\
& =\sum_{i=1}^{p} \overline{C_{i}}\left(\overline{\delta_{i a}}-\overline{\delta_{i b}}\right) \\
& \overline{e^{j \delta_{e}}} \cdot \overline{e^{-j \delta_{e}}}=\left[\overline{e^{j C_{1} \delta_{1 a}} \cdot e^{-j C_{1} \delta_{1 b}} \cdot e^{j C_{2} \delta_{2 a}} \cdot e^{-j C_{2} \delta_{2 b}} \cdots e^{j C_{p} \delta_{p a}} e^{-j C_{p} \delta_{p b}}}\right] . \\
& {\left[\overline{e^{-j C_{1} \delta_{1 a}} \cdot e^{j C_{1} \delta_{1 b}} \cdot e^{-j C_{2} \delta_{2 a}} \cdot e^{j C_{2} \delta_{2 b}} \cdots e^{-j C_{p} \delta_{p a}} \cdot e^{j C_{p} \delta_{p b}}}\right]} \\
& =\left[\prod_{i=1}^{p} \overline{e^{j C_{i} \delta_{i a}} \cdot e^{-j C_{i} \delta_{i b}}}\right] \cdot\left[\prod_{i=1}^{p} \overline{e^{-j C_{i} \delta_{i a}} \cdot e^{j C_{i} \delta_{i b}}}\right]
\end{aligned}
$$

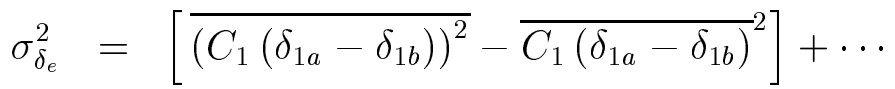

$$
\begin{aligned}
& \cdots+\left[\overline{\left(C_{p}\left(\delta_{p a}-\delta_{p b}\right)\right)^{2}}-{\overline{C_{p}\left(\delta_{p a}-\delta_{p b}\right)}}^{2}\right] \\
& =\sum_{i=1}^{p}\left[\overline{\left(C_{i}\left(\delta_{i a}-\delta_{i b}\right)\right)^{2}}-{\overline{C_{i}\left(\delta_{i a}-\delta_{i b}\right)}}^{2}\right]
\end{aligned}
$$


Teniendo en cuenta los resultados de las ecuaciones (3.8) se puede comprobar:

$$
\begin{aligned}
\overline{\left(C_{i}\left(\delta_{i a}-\delta_{i b}\right)\right)^{2}}-{\overline{C_{i}\left(\delta_{i a}-\delta_{i b}\right)}}^{2} & =\frac{1}{2} \overline{\delta_{i a}^{2}}+\frac{1}{2} \overline{\delta_{i b}^{2}}-\frac{1}{2}{\overline{\delta_{i a}}}^{2}-\frac{1}{2}{\overline{\delta_{i b}}}^{2} \\
\overline{e^{ \pm j C_{i} \delta_{i a}} \cdot e^{\mp j C_{i} \delta_{i b}}} & =\frac{1+\overline{e^{ \pm j \delta_{i a}}} \cdot \overline{e^{\mp j \delta_{i b}}}}{2}
\end{aligned}
$$

y por lo tanto las expresiones finales para los estadísticos de $\delta_{e}$ en el caso B serán:

$$
\begin{aligned}
\overline{\delta_{e}} & =\sum_{i=1}^{p} \frac{\overline{\delta_{i a}}-\overline{\delta_{i b}}}{2} \\
\overline{e^{j \delta_{e}}} \cdot \overline{e^{-j \delta_{e}}} & =\left[\prod_{i=1}^{p} \frac{1+\overline{e^{j \delta_{i a}}} \cdot \overline{e^{-j \delta_{i b}}}}{2}\right] \cdot\left[\prod_{i=1}^{p} \frac{1+\overline{e^{-j \delta_{i a}}} \cdot \overline{e^{j \delta_{i b}}}}{2}\right] \\
\sigma_{\delta_{e}}^{2} & =\sum_{i=1}^{p}\left[\frac{\overline{\delta_{i a}^{2}}-{\overline{\delta_{i a}}}^{2}+\overline{\delta_{i b}^{2}}-\overline{\delta_{i b}}}{2}\right]=\sum_{i=1}^{p}\left[\frac{\sigma_{i a}^{2}+\sigma_{i b}^{2}}{2}\right]
\end{aligned}
$$

Al igual que se realizó en el caso A, se pasa a particularizar las expresiones anteriores para los casos de errores uniforme y proporcionales, con estadísticas uniformes y gausianas para cada caso.

\section{Errores en cada dígito iguales}

En el caso de que los errores en todos los dígitos (ambos estados) tengan los mismos estadísticos $\left(\bar{\delta}, \overline{\delta^{2}}, \sigma_{\delta}^{2}, \overline{e^{ \pm j \delta}}\right)$, los estadísticos del error de fase total son:

$$
\begin{aligned}
\overline{\delta_{e}} & =p \cdot \frac{\bar{\delta}-\bar{\delta}}{2}=0 \\
\overline{e^{j \delta_{e}}} \cdot \overline{e^{-j \delta_{e}}} & =\left[\frac{1+\overline{e^{j \delta}} \cdot \overline{e^{-j \delta}}}{2}\right]^{2 p} \\
\sigma_{\delta_{e}}^{2} & =p \frac{\sigma_{\delta}^{2}+\sigma_{\delta}^{2}}{2}=p \cdot \sigma_{\delta}^{2}
\end{aligned}
$$

Estadística uniforme Para el caso de errores en cada dígito con estadística uniforme en el margen $[-E, E]$, y de acuerdo con los resultados del apéndice A se obtiene:

$$
\begin{aligned}
\overline{\delta_{e}} & =0 \\
\overline{e^{j \delta_{e}}} \cdot \overline{e^{-j \delta_{e}}} & =\left[\frac{1+\operatorname{sinc}^{2}(E)}{2}\right]^{2 p} \\
\sigma_{\delta_{e}}^{2} & =p \cdot \frac{E^{2}}{3} \\
\pm\left(\delta_{e}\right)_{P I C O} & = \pm(2 p \cdot E)
\end{aligned}
$$


Estadística gausiana Para el caso de errores en cada dígito con estadística gausiana con varianza $\sigma_{\delta}^{2}$ y media nula $(\bar{\delta}=0)$, teniendo en cuenta el resultado de la ecuación (A.21), se tendrá:

$$
\begin{array}{r}
\overline{\delta_{e}}=0 \\
\overline{e^{j \delta_{e}}} \cdot \overline{e^{-j \delta_{e}}}=\left[\frac{1+e^{-\sigma_{\delta}^{2}}}{2}\right]^{2 p} \\
\sigma_{\delta_{e}}^{2}=p \cdot \sigma_{\delta}^{2} \\
\pm\left(\delta_{e}\right)_{P I C O}= \pm\left(4 p \cdot \sigma_{\delta}\right)
\end{array}
$$

\section{Errores en cada dígito proporcionales a su peso}

Aplicando los resultados obtenidos en (3.13) a las ecuaciones (3.21) se llega a:

$$
\begin{aligned}
\overline{\delta_{e}} & =\sum_{i=1}^{p} \frac{\overline{\delta_{1}}-\overline{\delta_{1}}}{2}=0 \\
\overline{e^{j \delta_{e}}} \cdot \overline{e^{-j \delta_{e}}} & =\left[\prod_{i=1}^{p} \frac{1+\overline{e^{j \delta_{i}}} \cdot \overline{e^{-j \delta_{i}}}}{2}\right]^{2} \\
\sigma_{\delta_{e}}^{2} & =\sum_{i=1}^{p}\left(\frac{\Phi_{i}^{o}}{\Phi_{1}^{o}}\right)^{2} \sigma_{\delta_{1}}^{2}=\sigma_{\delta_{1}}^{2}\left(1+\beta^{2}+\cdot+\beta^{2 p-2}\right)=\sigma_{\delta_{1}}^{2} \frac{\beta^{2 p}-1}{\beta^{2}-1}
\end{aligned}
$$

Estadística uniforme Para el caso de errores en cada dígito con estadística uniforme en los márgenes $\left[-s \Phi_{i}^{o}, s \Phi_{i}^{o}\right]=\left[-s \beta_{i-1} \Phi_{1}^{o}, s \beta^{i-1} \Phi_{1}^{o}\right]$ para el dígito i-ésimo $\left(\overline{\delta_{i}}=0\right.$, $\left.\sigma_{\delta_{i}}^{2}=\frac{\left(s \Phi_{i}^{o}\right)^{2}}{3}=\frac{\left(s \beta^{i-1} \Phi_{1}^{o}\right)^{2}}{3}\right)$, donde $s$ es el porcentaje de variación de cada dígito sobre su peso ideal. De acuerdo con los resultados del apéndice A, se tendrá:

$$
\begin{aligned}
\overline{\delta_{e}} & =0 \\
\overline{e^{j \delta_{e}}} \cdot \overline{e^{-j \delta_{e}}} & =\left[\prod_{i=1}^{p} \frac{1+\operatorname{sinc}^{2}\left(s \Phi_{i}^{o}\right)}{2}\right]^{2}=\left[\prod_{i=1}^{p} \frac{1+\operatorname{sinc}^{2}\left(s \beta^{i-1} \Phi_{1}^{o}\right)}{2}\right]^{2} \\
\sigma_{\delta_{e}}^{2} & =\frac{\left(s \Phi_{1}^{o}\right)^{2}}{3} \cdot \frac{\beta^{2 p}-1}{\beta^{2}-1} \\
\pm\left(\delta_{e}\right)_{P I C O} & = \pm\left(2 s \cdot \Phi_{1}^{o} \cdot \frac{\beta^{p}-1}{\beta-1}\right)
\end{aligned}
$$

Estadística gausiana Cuando los errores en el dígito i-ésimo (ambos estados) siguen distribuciones gausianas de media nula $\overline{\delta_{i a}}=\overline{\delta_{i b}}=0$ y varianza $\sigma_{\delta_{i a}}^{2}=\sigma_{\delta_{i b}}^{2}=$ $\left(s \Phi_{i}^{o}\right)^{2}=\left(s \beta^{i-1} \Phi_{1}^{o}\right)^{2}$, donde $s$ es el porcentaje que la desviación estándar de cada dígito representa sobre su peso ideal, se obtienen los siguientes estadísticos para el 
error de fase total:

$$
\begin{aligned}
\overline{\delta_{e}} & =0 \\
\overline{e^{j \delta_{e}}} \cdot \overline{e^{-j \delta_{e}}} & =\left[\prod_{i=1}^{p} \frac{1+e^{-\left(s \beta^{i-1} \cdot \Phi_{1}^{o}\right)^{2}}}{2}\right]^{2} \\
\sigma_{\delta_{e}}^{2} & =\left(s \Phi_{1}^{o}\right)^{2} \cdot \frac{\beta^{2 p}-1}{\beta^{2}-1} \\
\pm\left(\delta_{e}\right)_{P I C O} & = \pm\left(4 s \cdot \Phi_{1}^{o} \cdot \frac{\beta^{p}-1}{\beta-1}\right)
\end{aligned}
$$

\subsubsection{Calibración según desfasador promedio}

En un primer diseño de un desfasador variable, puede ocurrir que debido a errores de diseño o modelado de componentes, los pesos medios de cada dígito no se correspondan con los esperados, por lo que, se debería utilizar una calibración completa del desfasador variable y utilizar una tabla de traslación para escoger el estado más cercano al valor de desfase deseado. En siguientes iteraciones del diseño, es de esperar que se corrijan los errores de modelado o de diseño $o^{i}$, obteniéndose un circuito con pesos medios iguales a los deseados. Por lo tanto, para un sistema real, este caso es equivalente al caso $\mathrm{A}$.

\subsubsection{Calibración completa de cada desfasador}

La caracterización completa de los $2^{p}$ estados de cada desfasador digital de la agrupación, permite reducir considerablemente el efecto de los errores aleatorios que aparecen en los pesos de cada dígito, de forma que los estadísticos del error de fase total de cada desfasador son mucho menores que los calculados para el caso A. Como contrapartida, este caso requiere una calibración completa de cada desfasador, además, de disponer en la agrupación de una tabla de traslación para cada desfasador ( $2^{p}$ posiciones de memoria por desfasador). Un diseño del desfasador digital que consiga minimizar la interacción entre los desfases de todos los dígitos cuando se conmuta el estado de uno de ellos, es decir, que consiga independizar los desfases de cada dígito de los estados en los que estén el resto de dígitos, sólo necesitaría tablas con $2 \cdot p$ posiciones para cada desfasador (dos posiciones por dígito).

Para el análisis de este caso se ha de resaltar, que ahora los procesos de cuantificación y de errores aleatorios en los pesos de los dígitos dejan de ser independientes y será necesario estudiarlos de forma conjunta, por lo que se ha recurrido a simulaciones con un número significativo de muestras para obtener los resultados estadísticos mostrados en el apartado siguiente ${ }^{\text {ii }}$.

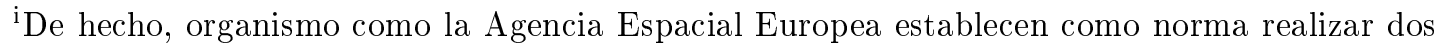
iteraciones para el diseño de cualquier MMIC con complejidad media o baja [Gat95].

${ }^{i i}$ Los resultados para el caso D corresponden a varios meses de simulaciones, de forma que el
} 


\subsubsection{Resultados combinados}

En este apartado se estudia el efecto combinado de los errores de cuantificación de un desfasador digital y de los errores aleatorios introducidos por los diferentes dígitos del mismo. En todos los casos, se consideran desfasadores digitales de rango completo $(2 \pi)$ y se analiza el efecto de la presencia o no de un proceso de calibración. A la hora de calcular los estadísticos del error total se ha tenido en cuenta las propiedades presentadas en (3.1) a (3.2) para los casos A y B. Los errores de cuantificación se han modelado de acuerdo a los resultados de las ecuaciones (2.75).

\section{Casos A y B}

En la tabla 3.1 se muestran los resultados del efecto combinado de los errores de cuantificación y aleatorios en los pesos de los dígitos para los casos A y B presentados previamente.

\begin{tabular}{|lr|cccc|cccc|}
\hline \multirow{2}{*}{ Dígitos } & $\begin{array}{r}\text { Error } \\
\text { Dígito }\end{array}$ & $\begin{array}{c} \pm \delta_{\text {max }} \\
\text { (grados) }\end{array}$ & $\begin{array}{c}\sigma_{e} \\
\text { (grados) }\end{array}$ & $\begin{array}{c}N L S P_{n} \\
(\mathrm{~dB})\end{array}$ & $\begin{array}{c}\Delta G \\
(\mathrm{~dB})\end{array}$ & $\begin{array}{c} \pm \delta_{\max } \\
\text { (grados) }\end{array}$ & $\begin{array}{c}\sigma_{e} \\
\text { (grados) }\end{array}$ & $\begin{array}{c}N L S P_{n} \\
(\mathrm{~dB})\end{array}$ & $\begin{array}{c}\Delta G \\
(\mathrm{~dB})\end{array}$ \\
\hline \hline 5 UI & $\delta_{i}= \pm 2^{\circ}$ & 15,63 & 4,15 & $-22,79$ & $-0,02$ & 25,63 & 4,15 & $-22,79$ & $-0,02$ \\
\hline 6 UI & $\delta_{i}= \pm 5^{\circ}$ & 32,81 & 7,26 & $-17,91$ & $-0,07$ & 62,81 & 7,26 & $-17,92$ & $-0,07$ \\
\hline \hline 5 GI & $\sigma_{\delta_{i}}=1^{\circ}$ & 15,63 & 3,94 & $-23,23$ & $-0,02$ & 25,63 & 3,94 & $-23,23$ & $-0,02$ \\
\hline 6 GI & $\sigma_{\delta_{i}}=2^{\circ}$ & 26,81 & 5,16 & $-20,89$ & $-0,04$ & 50,81 & 5,16 & $-20,89$ & $-0,04$ \\
\hline \hline 5 UP & $\delta_{i}= \pm 2 \% \Phi_{i}^{\circ}$ & 12,60 & 4,04 & $-23,03$ & $-0,02$ & 19,58 & 4,04 & $-23,03$ & $-0,02$ \\
\hline 6 UP & $\delta_{i}= \pm 5 \% \Phi_{i}^{\circ}$ & 20,53 & 6,22 & $-19,27$ & $-0,05$ & 38,25 & 6,22 & $-19,27$ & $-0,05$ \\
\hline \hline 5 GP & $\sigma_{\delta_{i}}=1 \% \Phi_{i}^{\circ}$ & 12,60 & 3,86 & $-23,43$ & $-0,02$ & 19,58 & 3,86 & $-23,43$ & $-0,02$ \\
\hline 6 GP & $\sigma_{\delta_{i}}=2 \% \Phi_{i}^{\circ}$ & 16,99 & 4,46 & $-22,16$ & $-0,03$ & 31,16 & 4,46 & $-22,16$ & $-0,03$ \\
\hline
\end{tabular}

Tabla 3.1: Comparativa de los estadísticos del error total para desfasadores digitales de cinco y seis dígitos para diferentes estadísticas de error en los dígitos individuales (UI=uniforme,iguales; $\mathrm{GI}=$ gausiana,iguales; $\mathrm{UP}=$ uniforme,proporcionales; $\mathrm{GP}=$ gausiana,proporcionales) y para los casos A (sin calibración) y B (calibración simple).

Se han escogido desfasadores digitales de cinco y seis dígitos y se ha analizado su comportamiento para diferentes estadísticas de errores de acuerdo con las ecuaciones previas. Los resultados incluyen el error de pico total, el nivel de lóbulo principal a secundario normalizado, $N L S P_{n}$, calculado de acuerdo con (A.26) y la reducción de la ganancia de la agrupación calculada según (A.27). Para una agrupación concreta se puede desnormalizar el valor de $N L S P_{n}$ obtenido de la tabla, añadiéndole -10 . $\log (N \eta)$, donde $N$ es el número de elementos/desfasadores de la agrupación y $\eta$ es la eficiencia de iluminación de la agrupación que se definió en (2.27).

muestreo estadístico se considera suficientemente bueno, aún cuando pudieran existir pequeñas imprecisiones en el último dígito de las estimaciones de los errores de pico, siendo prácticamente despreciables en el resto de parámetros. 


\begin{tabular}{|cc|cccc|cccc|}
\hline \multirow{2}{*}{ Dígitos } & \multirow{6}{*}{$\delta_{i}$} & \multicolumn{4}{|c|}{ CASO A } & \multicolumn{4}{c|}{ CASO D } \\
& & $\begin{array}{c} \pm \delta_{\text {max }} \\
\text { (grados) }\end{array}$ & $\begin{array}{c}\sigma_{e} \\
\text { (grados) }\end{array}$ & $\begin{array}{c}N L S P_{n} \\
(\mathrm{~dB})\end{array}$ & $\begin{array}{c}\Delta G \\
(\mathrm{~dB})\end{array}$ & $\begin{array}{c} \pm \delta_{\text {max }} \\
\text { (grados) }\end{array}$ & $\begin{array}{c}\sigma_{e} \\
\text { (grados) }\end{array}$ & $\begin{array}{c}N L S P_{n} \\
(\mathrm{~dB})\end{array}$ & $\begin{array}{c}\Delta G \\
(\mathrm{~dB})\end{array}$ \\
\hline \hline 4 & $\pm 2^{\circ}$ & 19,25 & 6,89 & $-18,36$ & $-0,06$ & 17,25 & 6,59 & $-18,75$ & $-0,06$ \\
\hline 4 & $\pm 5^{\circ}$ & 31,25 & 8,69 & $-16,33$ & $-0,10$ & 26,96 & 7,06 & $-18,13$ & $-0,07$ \\
\hline 4 & $\pm 10^{\circ}$ & 51,25 & 13,25 & $-12,60$ & $-0,23$ & 41,75 & 8,35 & $-16,59$ & $-0,09$ \\
\hline \hline 5 & $\pm 2^{\circ}$ & 15,63 & 4,15 & $-22,79$ & $-0,02$ & 12,82 & 3,44 & $-24,42$ & $-0,02$ \\
\hline 5 & $\pm 5^{\circ}$ & 30,63 & 7,23 & $-17,95$ & $-0,07$ & 23,89 & 4,20 & $-22,59$ & $-0,02$ \\
\hline 5 & $\pm 10^{\circ}$ & 55,63 & 13,31 & $-12,56$ & $-0,23$ & 40,51 & 5,54 & $-19,97$ & $-0,04$ \\
\hline \hline 6 & $\pm 2^{\circ}$ & 14,81 & 3,26 & $-24,89$ & $-0,01$ & 10,18 & 1,96 & $-29,25$ & $-0,01$ \\
\hline 6 & $\pm 5^{\circ}$ & 32,81 & 7,26 & $-17,91$ & $-0,07$ & 21,53 & 2,78 & $-25,96$ & $-0,01$ \\
\hline 6 & $\pm 10^{\circ}$ & 62,81 & 14,24 & $-11,96$ & $-0,27$ & 34,42 & 3,74 & $-23,05$ & $-0,02$ \\
\hline \hline 7 & $\pm 2^{\circ}$ & 15,41 & 3,16 & $-25,16$ & $-0,01$ & 9,94 & 1,27 & $-32,83$ & 0,00 \\
\hline 7 & $\pm 5^{\circ}$ & 36,41 & 7,68 & $-17,41$ & $-0,08$ & 21,63 & 1,89 & $-28,98$ & $-0,01$ \\
\hline 7 & $\pm 10^{\circ}$ & 71,41 & 15,30 & $-11,31$ & $-0,31$ & 35,44 & 2,43 & $-26,32$ & $-0,01$ \\
\hline
\end{tabular}

Tabla 3.2: Efecto combinado de los errores de cuantificación y errores aleatorios en cada dígito iguales y con estadística uniforme.

De los resultados de las tablas se puede comprobar que los casos A y B son prácticamente iguales, excepto en los errores de pico, donde el caso B ofrece un peor comportamiento, pues se ha considerado que los desfases de los dos estados de un dígito son independientes y, por lo tanto, sus desviaciones pueden dirigirse en sentidos contrarios y al normalizar los pesos de los dígitos respecto al error del uno de los dos estados, se puede doblar el error del peso del dígito; de forma que, dependiendo del error de cuantificación, el caso B puede llegar a doblar el valor de pico del caso A, tanto más cuanto menor sea el error de cuantificación (mayor número de dígitos). El resto de parámetros analizados en la tabla 3.1 (varianza, $N L S P_{n}$ y $\left.\Delta G\right)$ son prácticamente iguales para los casos A y B; siendo las variaciones despreciables para los tamaños de desfasadores y tipos de errores más comunes.

Como conclusión, la calibración sobre el estado cero debe hacerse sobre el desfasador promedio, si lo que se desea es calibrar el camino eléctrico recorrido, pero nunca sobre un desfasador cualquiera pues se aumentaría considerablemente el error de pico.

\section{Casos A y D}

Resulta mucho más interesante comparar los casos A y D, que corresponden a sistemas sin calibración o con calibración completa.

En las tablas 3.2 a 3.5 se muestran los mismos parámetros que se analizaron en la tabla 3.1, pero separados para las cuatro distribuciones estadísticas consideradas (errores en cada dígito iguales o proporcionales, distribución de los errores de los dígitos individuales uniforme o gausiana) y con un número mayor de tamaños de 


\begin{tabular}{|lc|cccc|cccc|}
\hline \multirow{2}{*}{ Dígitos } & & \multicolumn{5}{|c|}{ CASO A } & \multicolumn{4}{c|}{ CASO D } \\
& $\sigma_{\delta_{i}}$ & $\pm \delta_{\text {max }}$ & $\sigma_{e}$ & $N L S P_{n}$ & $\Delta G$ & $\pm \delta_{\max }$ & $\sigma_{e}$ & $N L S P_{n}$ & $\Delta G$ \\
& & $($ grados) & (grados) & $(\mathrm{dB})$ & $(\mathrm{dB})$ & $($ grados $)$ & (grados) & $(\mathrm{dB})$ & $(\mathrm{dB})$ \\
\hline \hline 4 & $1^{\mathrm{o}}$ & 19,25 & 6,80 & $-18,48$ & $-0,06$ & 14,73 & 6,57 & $-18,78$ & $-0,06$ \\
\hline 4 & $2^{\mathrm{o}}$ & 27,25 & 7,63 & $-17,47$ & $-0,08$ & 18,35 & 6,77 & $-18,51$ & $-0,06$ \\
\hline 4 & $5^{\circ}$ & 51,25 & 11,92 & $-13,54$ & $-0,19$ & 29,31 & 7,96 & $-17,02$ & $-0,09$ \\
\hline \hline 5 & $1^{\circ}$ & 15,63 & 3,94 & $-23,23$ & $-0,02$ & 9,58 & 3,39 & $-24,54$ & $-0,02$ \\
\hline 5 & $2^{\circ}$ & 25,63 & 5,53 & $-20,29$ & $-0,04$ & 13,63 & 3,77 & $-23,59$ & $-0,02$ \\
\hline 5 & $5^{\circ}$ & 55,63 & 11,64 & $-13,75$ & $-0,18$ & 25,01 & 5,13 & $-20,67$ & $-0,04$ \\
\hline \hline 6 & $1^{\circ}$ & 14,81 & 2,94 & $-25,79$ & $-0,01$ & 7,23 & 1,88 & $-29,61$ & 0,00 \\
\hline 6 & $2^{\circ}$ & 26,81 & 5,16 & $-20,89$ & $-0,04$ & 11,56 & 2,36 & $-27,49$ & $-0,01$ \\
\hline 6 & $5^{\circ}$ & 62,81 & 12,35 & $-13,22$ & $-0,20$ & 22,61 & 3,49 & $-23,71$ & $-0,02$ \\
\hline \hline 7 & $1^{\circ}$ & 15,41 & 2,77 & $-26,32$ & $-0,01$ & 6,15 & 1,19 & $-33,48$ & 0,00 \\
\hline 7 & $2^{\circ}$ & 29,41 & 5,35 & $-20,57$ & $-0,04$ & 10,32 & 1,60 & $-30,63$ & 0,00 \\
\hline 7 & $5^{\circ}$ & 71,41 & 13,25 & $-12,60$ & $-0,23$ & 20,26 & 2,32 & $-26,82$ & $-0,01$ \\
\hline
\end{tabular}

Tabla 3.3: Efecto combinado de los errores de cuantificación y errores aleatorios en cada dígito iguales y con estadística gausiana (el error en cada dígito corresponde a la desviación estándar del desfase de cada estado).

desfasadores (4, 5, 6 o 7 dígitos) y de errores ${ }^{\mathrm{i}}$.

Para el caso de errores en cada dígito, iguales y con distribución uniforme (Tabla 3.2), se puede destacar cómo la introducción de la calibración comienza a ser necesaria en cuanto la magnitud de los errores aleatorios introducidos $\left(\delta_{i}\right)$ pasa a ser comparable a la resolución del desfasador digital (Res $\left.=2 \pi / 2^{p}\right)$; en concreto, entre un medio y un tercio de la misma. Si los errores en cada dígito son iguales pero siguen una distribución gausiana en lugar de uniforme (Tabla 3.3), se puede establecer como umbral a partir del cual se debería considerar la calibración de los desfasadores una varianza en cada dígito $\left(\sigma_{\delta_{i}}\right)$ de entre la cuarta o la quinta parte de la resolución del desfasador.

Como comparación de los resultados de las tablas 3.2 y 3.3 con los de las tablas 3.4 y 3.5 se debe destacar que cuando los errores en cada dígito son proporcionales al peso de cada uno de ellos, se produce una mejora de los resultados al aumentar el número de dígitos, mientras que en el caso de errores iguales, ésto sólo ocurre cuando los errores aleatorios son de pequeña magnitud.

Además de los resultados tabulados, en las figuras 3.2 a 3.5 se representan los valores de pico y RMS del error total, así como el nivel normalizado de los lóbulos secundarios para los parámetros estudiados de los casos A y D.

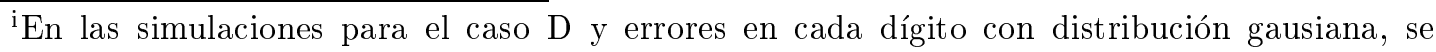
considera como error de pico total aquel valor que no se supera en el $99 \%$ de los casos.
} 


\begin{tabular}{|cc|cccc|cccc|}
\hline \multirow{2}{*}{ Dígitos } & \multirow{2}{*}{$\delta_{i} / \Phi_{i}^{\circ}$} & \multicolumn{5}{|c|}{ CASO A } & \multicolumn{4}{c|}{ CASO D } \\
& & $\begin{array}{l} \pm \delta_{\max } \\
\text { (grados) }\end{array}$ & $\begin{array}{c}\sigma_{e} \\
\text { (grados) }\end{array}$ & $\begin{array}{c}N L S P_{n} \\
(\mathrm{~dB})\end{array}$ & $\begin{array}{c}\Delta G \\
(\mathrm{~dB})\end{array}$ & $\begin{array}{c} \pm \delta_{\max } \\
\text { (grados) }\end{array}$ & $\begin{array}{c}\sigma_{e} \\
\text { (grados) }\end{array}$ & $\begin{array}{c}N L S P_{n} \\
(\mathrm{~dB})\end{array}$ & $\begin{array}{c}\Delta G \\
(\mathrm{~dB})\end{array}$ \\
\hline \hline 4 & $\pm 2 \%$ & 18,00 & 6,92 & $-18,32$ & $-0,06$ & 16,87 & 6,53 & $-18,82$ & $-0,06$ \\
\hline 4 & $\pm 5 \%$ & 28,13 & 8,83 & $-16,18$ & $-0,10$ & 25,41 & 6,73 & $-18,56$ & $-0,06$ \\
\hline 4 & $\pm 10 \%$ & 45,00 & 13,62 & $-12,34$ & $-0,25$ & 38,46 & 7,35 & $-17,77$ & $-0,07$ \\
\hline \hline 5 & $\pm 2 \%$ & 12,60 & 4,04 & $-23,03$ & $-0,02$ & 11,21 & 3,29 & $-24,82$ & $-0,01$ \\
\hline 5 & $\pm 5 \%$ & 23,06 & 6,82 & $-18,45$ & $-0,06$ & 19,30 & 3,48 & $-24,32$ & $-0,02$ \\
\hline 5 & $\pm 10 \%$ & 40,50 & 12,43 & $-13,16$ & $-0,20$ & 35,87 & 4,08 & $-22,84$ & $-0,02$ \\
\hline \hline 6 & $\pm 2 \%$ & 9,90 & 2,90 & $-25,92$ & $-0,01$ & 8,52 & 1,66 & $-30,74$ & 0,00 \\
\hline 6 & $\pm 5 \%$ & 20,53 & 6,22 & $-19,27$ & $-0,05$ & 17,25 & 1,85 & $-29,77$ & 0,00 \\
\hline 6 & $\pm 10 \%$ & 38,25 & 12,11 & $-13,39$ & $-0,19$ & 31,79 & 2,47 & $-26,93$ & $-0,01$ \\
\hline \hline 7 & $\pm 2 \%$ & 8,55 & 2,53 & $-27,08$ & $-0,01$ & 7,04 & 0,85 & $-36,56$ & 0,00 \\
\hline 7 & $\pm 5 \%$ & 19,27 & 6,05 & $-19,49$ & $-0,05$ & 15,00 & 1,06 & $-34,46$ & 0,00 \\
\hline 7 & $\pm 10 \%$ & 37,13 & 12,03 & $-13,45$ & $-0,19$ & 29,53 & 1,72 & $-29,64$ & 0,00 \\
\hline
\end{tabular}

Tabla 3.4: Efecto combinado de los errores de cuantificación y errores aleatorios en cada dígito proporcionales iguales y con estadística uniforme.

\begin{tabular}{|lc|cccc|cccc|}
\hline \multirow{2}{*}{ Dígitos } & $\sigma_{\delta_{i}} / \Phi_{i}^{o}$ & \multicolumn{5}{|c|}{ CASO A } & \multicolumn{4}{c|}{ CASO D } \\
& & $\begin{array}{c} \pm \delta_{\text {max }} \\
\text { (grados) }\end{array}$ & $\begin{array}{c}\sigma_{e} \\
\text { (grados) }\end{array}$ & $\begin{array}{c}N L S P_{n} \\
(\mathrm{~dB})\end{array}$ & $\begin{array}{c}\Delta G \\
(\mathrm{~dB})\end{array}$ & $\begin{array}{c} \pm \delta_{\max } \\
\text { (grados) }\end{array}$ & $\begin{array}{c}\sigma_{e} \\
\text { (grados) }\end{array}$ & $\begin{array}{c}N L S P_{n} \\
(\mathrm{~dB})\end{array}$ & $\begin{array}{c}\Delta G \\
\text { (dB) }\end{array}$ \\
\hline \hline 4 & $1 \%$ & 18,00 & 6,82 & $-18,45$ & $-0,06$ & 14,84 & 6,52 & $-18,84$ & $-0,06$ \\
\hline 4 & $2 \%$ & 24,75 & 7,71 & $-17,38$ & $-0,08$ & 18,69 & 6,61 & $-18,72$ & $-0,06$ \\
\hline 4 & $5 \%$ & 45,00 & 12,24 & $-13,31$ & $-0,20$ & 30,26 & 7,15 & $-18,02$ & $-0,07$ \\
\hline \hline 5 & $1 \%$ & 12,60 & 3,86 & $-23,43$ & $-0,02$ & 9,25 & 3,28 & $-24,84$ & $-0,01$ \\
\hline 5 & $2 \%$ & 19,58 & 5,27 & $-20,70$ & $-0,04$ & 13,14 & 3,36 & $-24,62$ & $-0,01$ \\
\hline 5 & $5 \%$ & 40,50 & 10,88 & $-14,35$ & $-0,16$ & 23,87 & 3,88 & $-23,29$ & $-0,02$ \\
\hline \hline 6 & $1 \%$ & 9,90 & 2,64 & $-26,73$ & $-0,01$ & 6,47 & 1,65 & $-30,79$ & 0,00 \\
\hline 6 & $2 \%$ & 16,99 & 4,46 & $-22,16$ & $-0,03$ & 10,11 & 1,74 & $-30,35$ & 0,00 \\
\hline 6 & $5 \%$ & 38,25 & 10,52 & $-14,65$ & $-0,15$ & 21,41 & 2,28 & $-27,69$ & $-0,01$ \\
\hline \hline 7 & $1 \%$ & 8,55 & 2,23 & $-28,19$ & $-0,01$ & 4,96 & 0,84 & $-36,66$ & 0,00 \\
\hline 7 & $2 \%$ & 15,69 & 4,24 & $-22,61$ & $-0,02$ & 8,87 & 0,93 & $-35,72$ & 0,00 \\
\hline 7 & $5 \%$ & 37,13 & 10,42 & $-14,73$ & $-0,14$ & 20,70 & 1,52 & $-30,74$ & 0,00 \\
\hline
\end{tabular}

Tabla 3.5: Efecto combinado de los errores de cuantificación y errores aleatorios en cada dígito proporcionales y con estadística gausiana (el error en cada dígito corresponde a la desviación estándar del desfase de cada estado). 


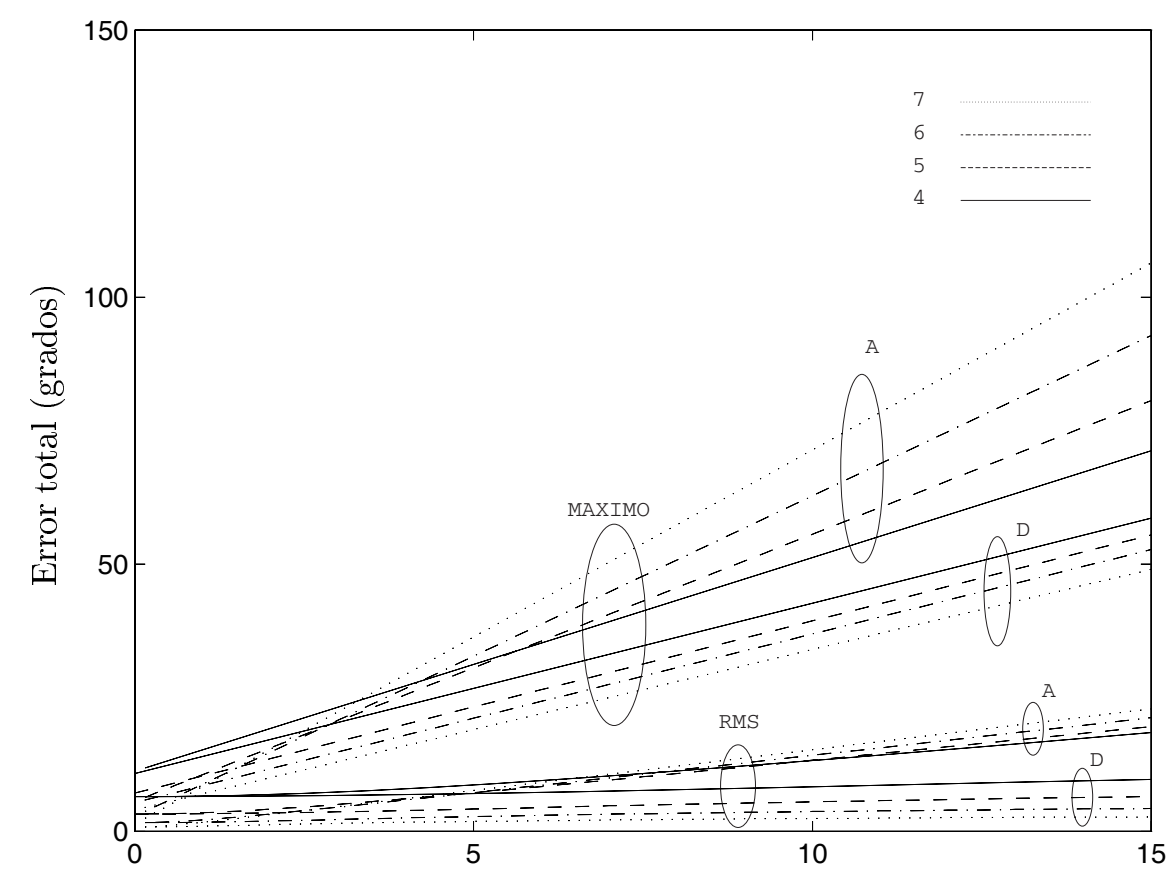

a)

Error dígito ( \pm grados)

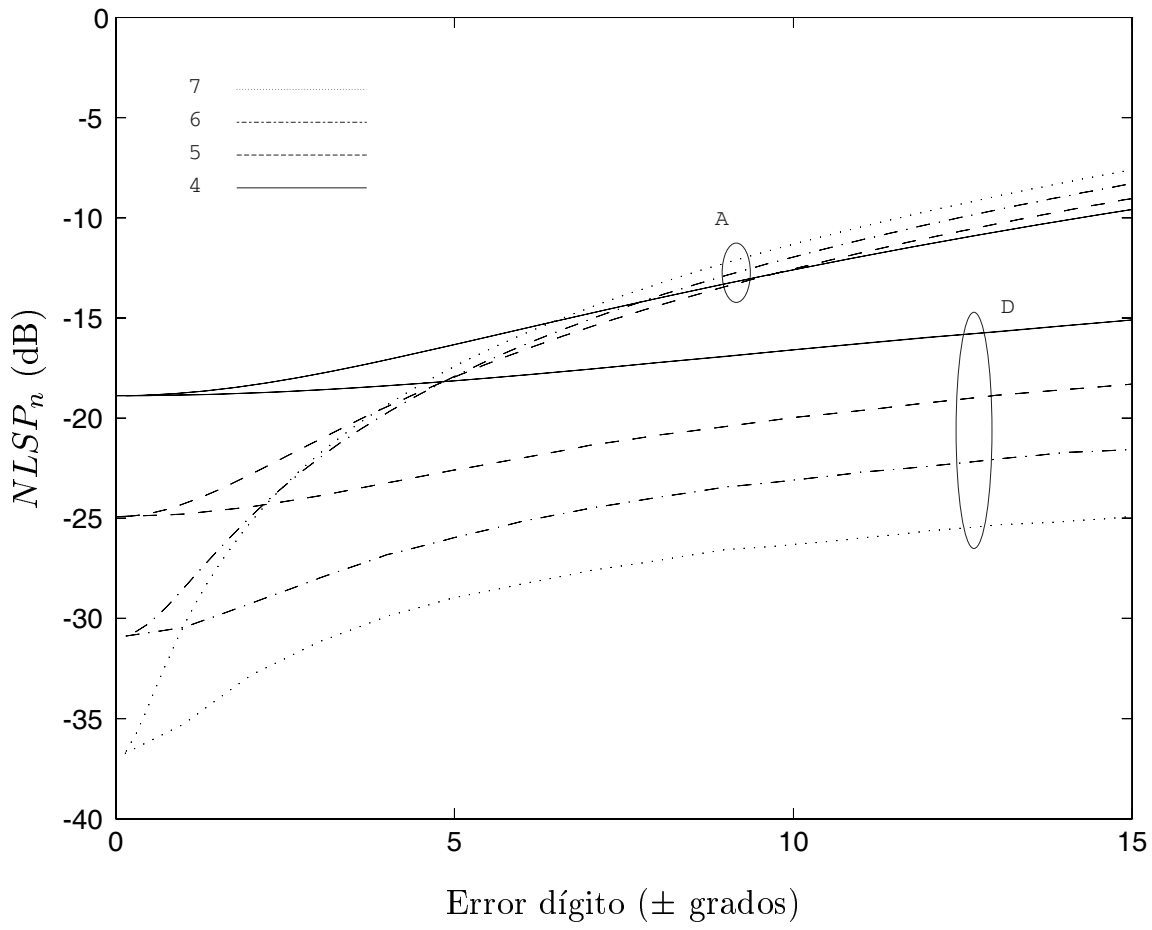

Figura 3.2: Comparativa de los casos A y D en función del error de cada dígito cuando los errores son iguales y con distribución uniforme. a) Error máximo y RMS. b) $N L S P_{n}$. 


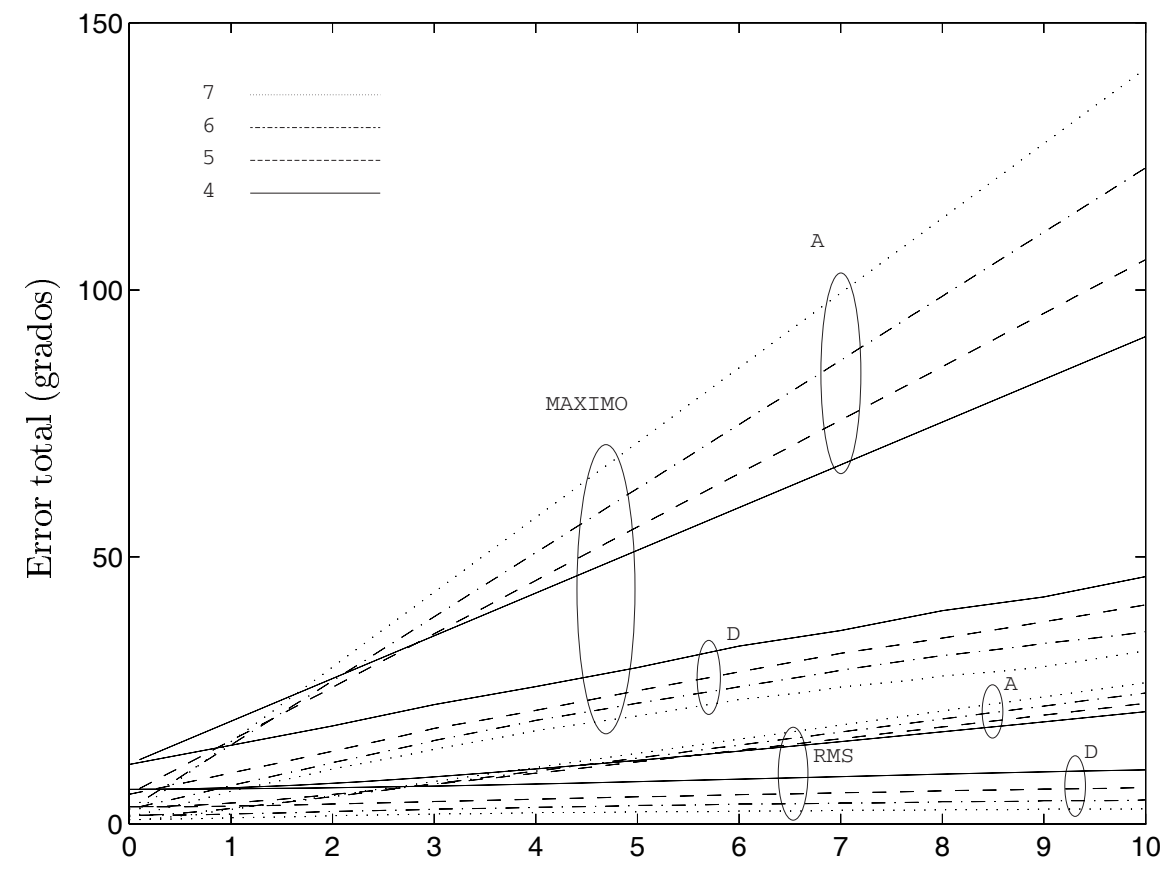

a)

Desviación estándar del error en cada dígito ( \pm grados)

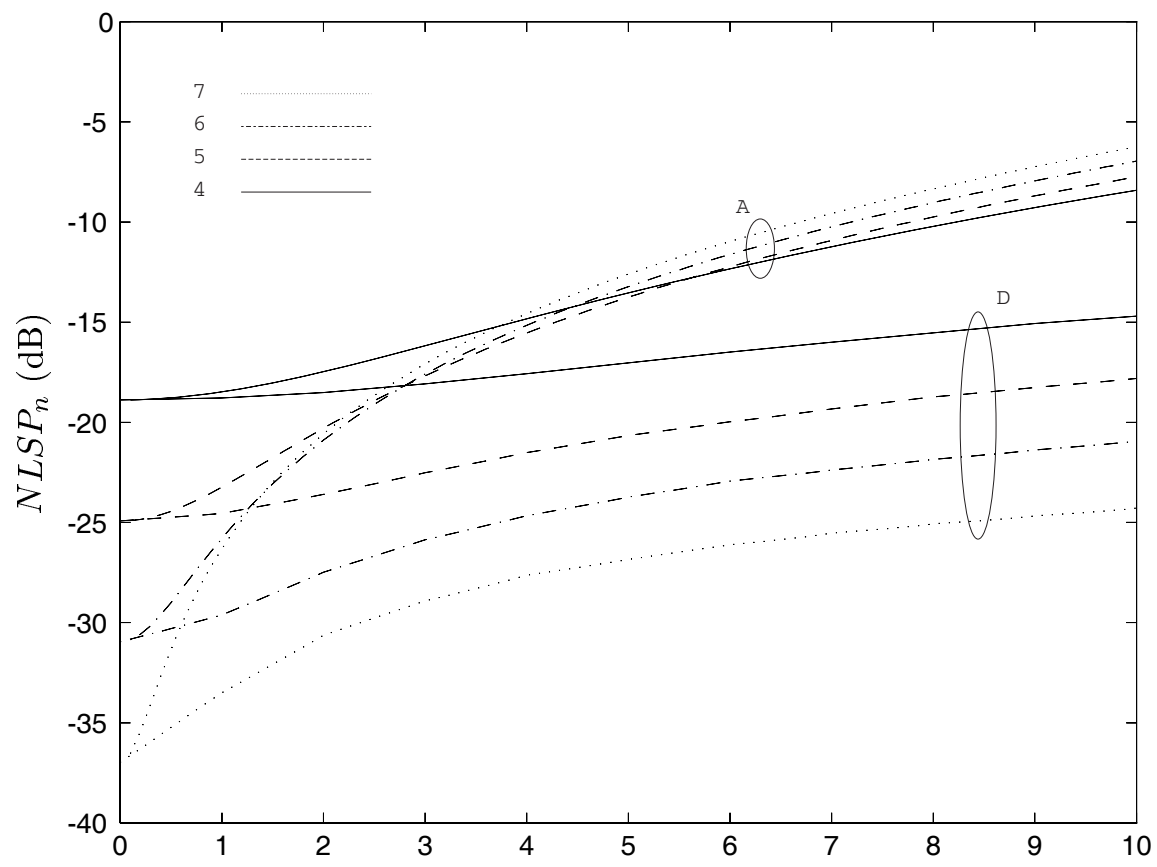

b)

Desviación estándar del error en cada dígito ( \pm grados $)$

Figura 3.3: Comparativa de los casos A y D en función del error de cada dígito cuando los errores son iguales y con distribución gausiana. a) Error máximo y RMS. b) $N L S P_{n}$. 


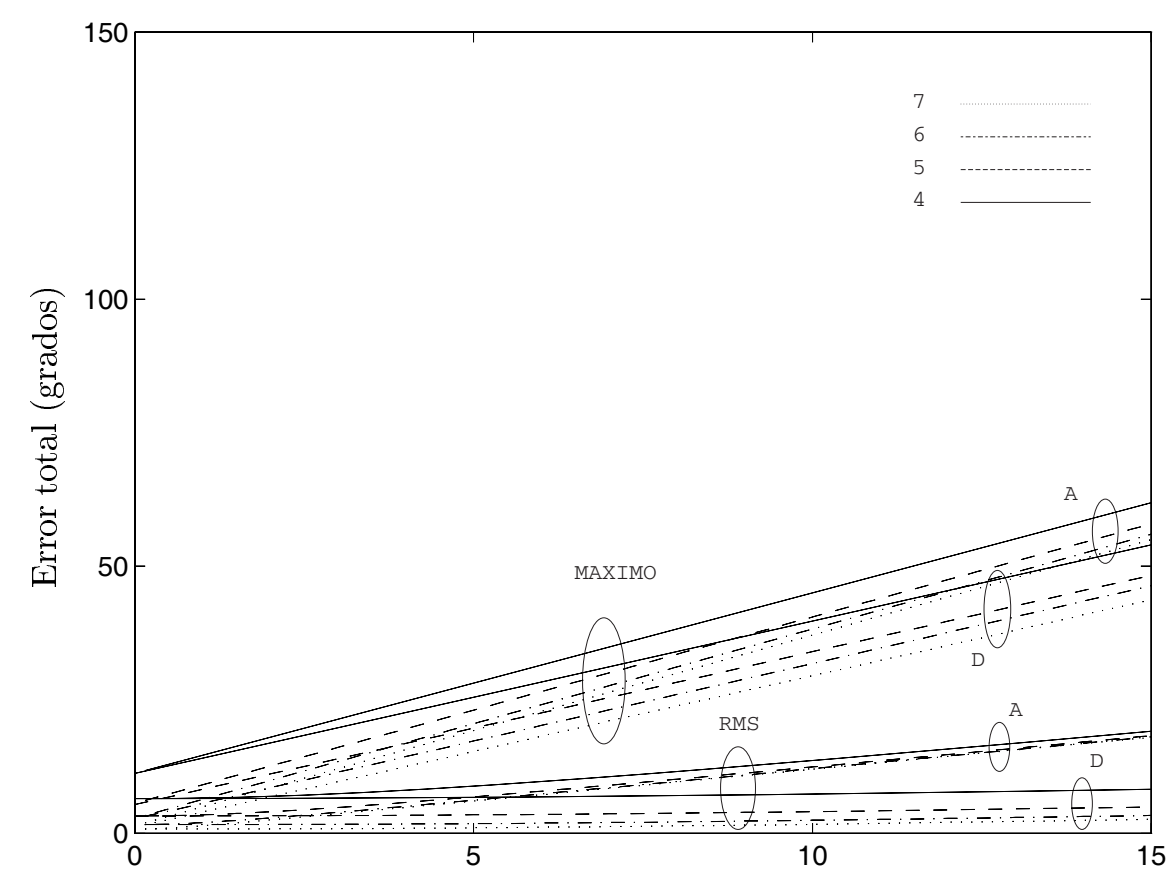

a)

Error dígito $( \pm \%)$

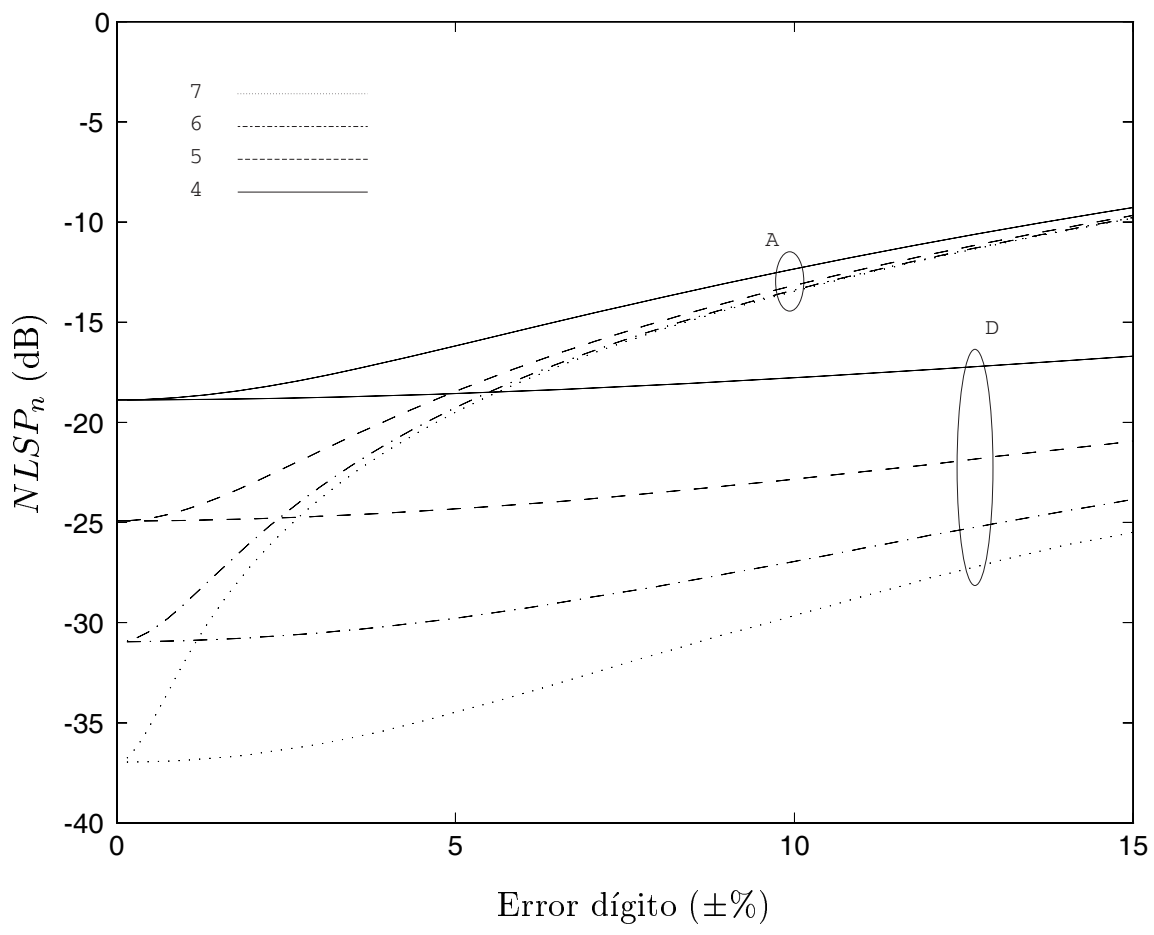

Figura 3.4: Comparativa de los casos A y D en función del error de cada dígito cuando los errores son proporcionales y con distribución uniforme. a) Error máximo y RMS. b) $N L S P_{n}$. 


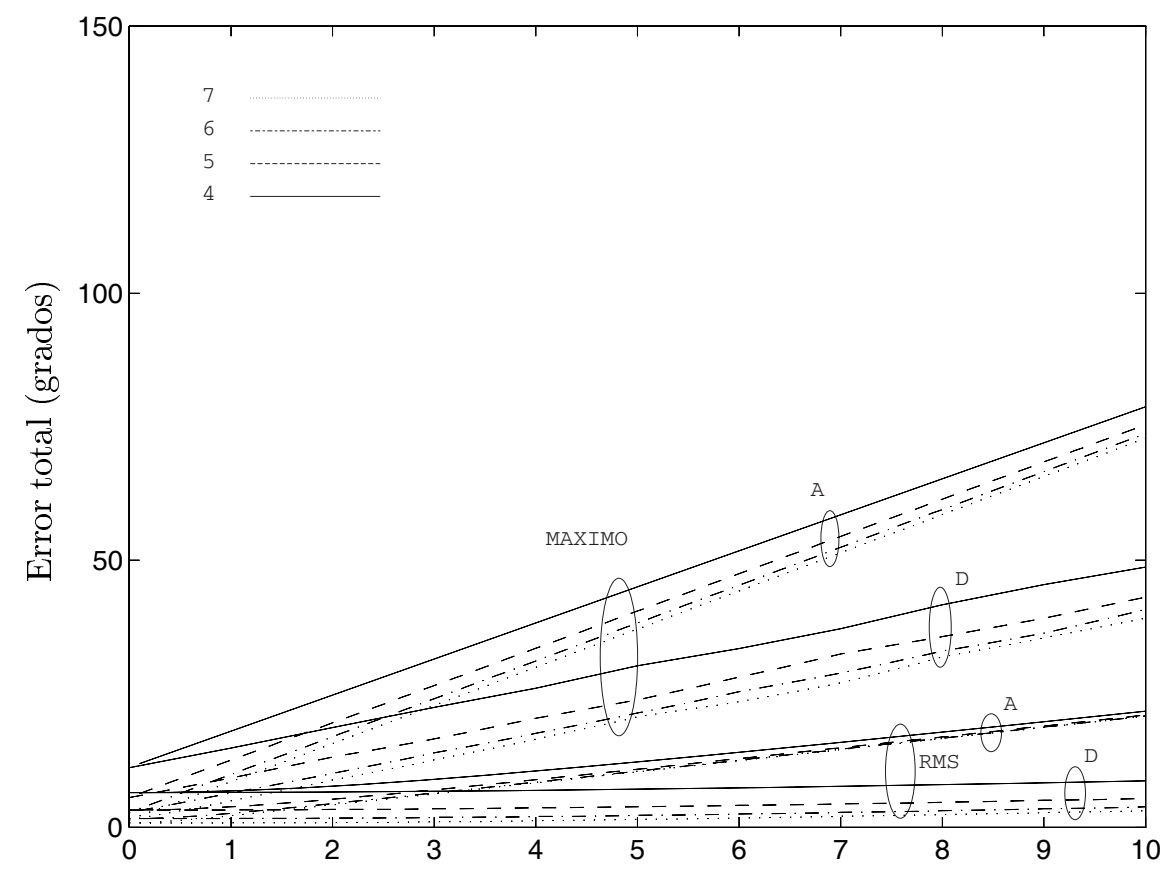

a)

Desviación estándar del error en cada dígito $( \pm \%)$

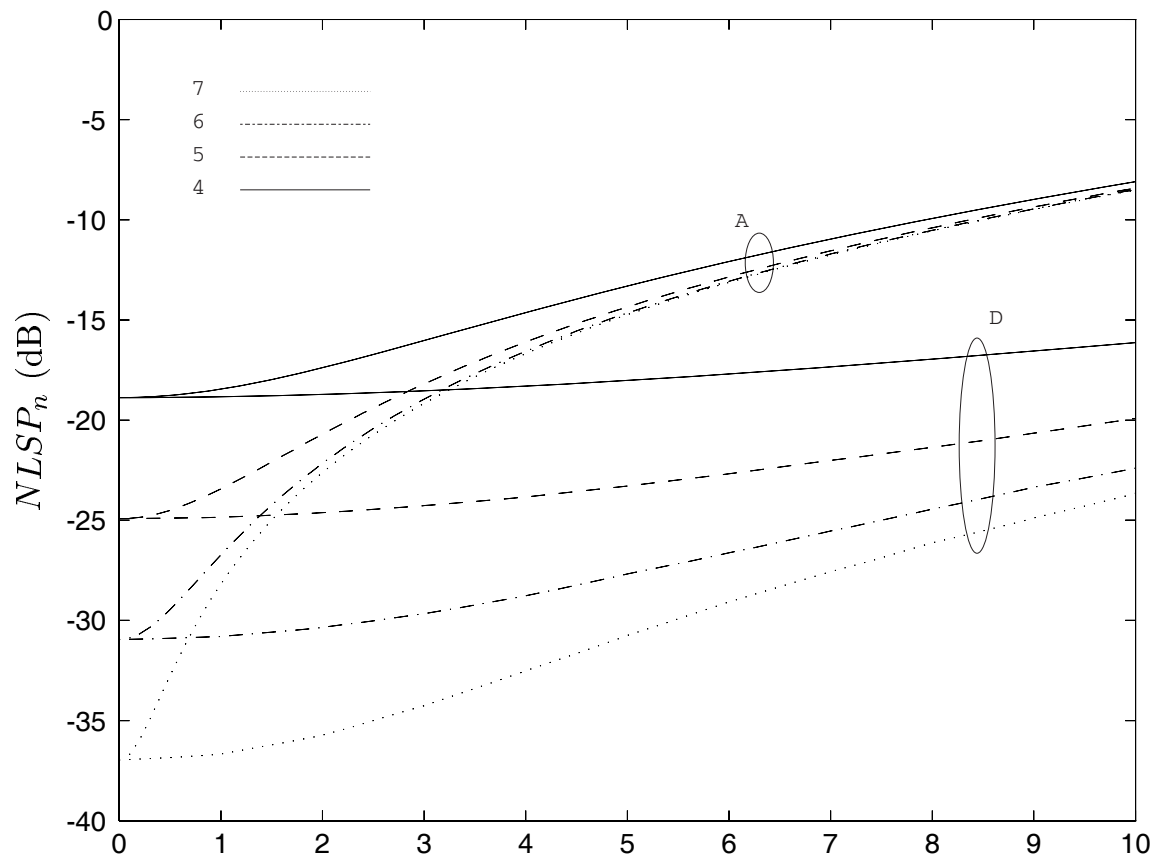

b)

Desviación estándar del error en cada dígito $( \pm \%)$

Figura 3.5: Comparativa de los casos A y D en función del error de cada dígito cuando los errores son proporcionales y con distribución gausiana. a) Error máximo y RMS. b) $N L S P_{n}$. 


\subsubsection{Redundancia. Desfasadores no binarios}

Tras comprobar el efecto que provoca la incertidumbre en los pesos de los dígitos y la importancia de la calibración, se hace evidente la necesidad de disponer de una tabla de traslación para escoger el estado del desfasador variable más adecuado al desfase requerido. La existencia de una tabla de traslación completa, permite, asímismo, tener en cuenta las posibles interacciones entre los desfases de los diferentes dígitos al conmutar el estado de alguno de ellos, ya que se calibran todos los estados del desfasador.

Una vez se hace necesaria la utilización de una tabla de traslación, desaparece una de las ventajas de los desfasadores digitales binarios (pesos de los dígitos ponderados con un factor $\beta=2$ ), como es que permiten obtener directamente el estado de cada dígito a partir de la cuantificación del desfase deseado.

Con elementos ponderados de forma binaria, el error máximo o la resolución están limitados por los errores en los pesos de cada dígito individual, como se ha visto anteriormente; este error máximo es igual a la suma de las incertidumbres de los pesos de los dígitos individuales más medio escalón de cuantificación. Si los errores son proporcionales al peso de los dígitos, el error total vendrá dominado por el error en el peso del dígito más significativo $\left(\mathrm{MSB}^{\mathrm{i}} ; \Phi_{p}\right.$ en la figura 3.1). Cuando la calibración completa de todos los desfasadores de la agrupación no es condición suficiente para asegurar la precisión especificada, se hace necesario introducir algún tipo de redundancia en los dígitos de cada desfasador; de forma que, incluso ante la presencia de incertidumbre en los pesos de los dígitos, se pueda cubrir el rango de desfase y la resolución especificados.

La duplicación del dígito menos significativo (LSB ${ }^{\mathrm{ii}} ; \Phi_{1}$ en la figura 3.1) suele ser la solución habitual para este problema; pero todavía sigue siendo necesaria una precisión en el MSB menor que el peso del LSB. En lugar de concentrar la redundancia añadida en un LSB repetido dejando el peso del resto de dígitos inalterados, parece más conveniente partir de que se dispone de $p+1$ dígitos y escoger los pesos de los mismos de forma óptima, para conseguir la precisión que se tendría con $p$ dígitos cuando no existe incertidumbre en los pesos de los dígitos. En este concepto se basa una solución alternativa, propuesta y patentada en los últimos años [Buc94]: reducir la razón geométrica entre los pesos de dígitos consecutivos $(\beta)$ a menos de 2 , obteniéndose elementos ponderados de forma no binaria. Al hablar de desfasadores con pesos no binarios, no se debe confundir con los desfasadores digitales ternarios, donde cada dígito puede presentar tres estados de fase distintos, con lo que un desfasador de $p$ dígitos ofrece $3^{p}$ estados de fase totales [Mag94].

\footnotetext{
${ }^{\mathrm{i}}$ Most Significant Bit, en terminología inglesa.

${ }^{i i}$ Least Significant Bit, en terminología inglesa.
} 


\section{El uso de pesos no binarios en desfasadores digitales con tolerancia a fallos}

Con elementos ponderados en forma binaria, el máximo error de operación o la resolución están limitados por los errores en los pesos de los dígitos individuales. El error, en el peor caso, puede darse al no alcanzar el rango requerido con todos los dígitos actuados si todos los pesos presentan un error tal que su valor es menor que el nominal; o, si los errores presentaran signos diferentes, en la transición de 0111 a 1000, es decir, cuando se complementan todos los dígitos. En ambos casos, el error máximo (sobre la supuesta precisión por cuantificación de medio LSB) es igual a la suma de las incertidumbres de los pesos de cada elemento, presumiblemente dominado por el error en el peso del dígito más significativo.

La duplicación del dígito menos significativo ayudaría, pero todavía sería necesaria una precisión de un LSB en el dígito más significativo. Una alternativa es reducir la razón geométrica entre los pesos de dígitos consecutivos a un valor menor que dos. De esta forma, se asegura una cobertura total del rango requerido, sin huecos entre estados contiguos, mayores que la resolución especificada.

Una vez se han evaluado los pesos finalmente conseguidos, se puede construir una tabla de traslación para traducir el valor de desfase deseado a la posición óptima de los dígitos, expresada como la palabra código más adecuada, que se traslada a las señales de control de los diferentes dígitos.

A continuación se muestran las ecuaciones que define las prestaciones de un desfasador con pesos no binarios y el criterio que se ha de seguir para escoger el valor de esos pesos.

Se desea una resolución equivalente a la que daría un desfasador digital ideal con $N$ dígitos binarios que tuviera que cubrir desfases en un rango ${ }^{\mathrm{i}} R$; entendiendo como resolución, la separación máxima entre dos estados de desfase contiguos $\left(\right.$ Res $_{\text {ideal }}=$ $\left.R /\left(2^{N}-1\right)\right)$. Ante la imposibilidad de obtener la citada resolución, debido a la incertidumbre de los pesos de los $N$ dígitos, se pretende obtener una expresión para el número de dígitos $p-N$ que se deben añadir, de forma que la resolución del desfasador no se vea afectada por una determinada incertidumbre en los pesos de los dígitos $(\partial)$. Para resolver estas cuestiones se asumirá que los errores son proporcionales al peso de los dígitos $\left(\left(\delta_{i a}\right)_{P I C O}=\left(\delta_{i b}\right)_{P I C O}=\partial \cdot \Phi_{i}^{o}, \forall i \in[1, p]\right) \mathrm{y}$ que los $p$ pesos se relacionan por un factor $\beta<2\left(\Phi_{i}^{o}=\beta^{i-1} \cdot \Phi_{1}^{o}\right)$.

La elección de los parámetros $(p, \beta)$ debe asegurar el cumplimiento de los siguientes tres criterios:

- Se debe cumplir la resolución especificada incluso si el LSB fuera mayor que

\footnotetext{
${ }^{i}$ Se define el rango como la diferencia entre los desfases máximo y mínimo que presenta el desfasador, sin tener en cuenta la periodicidad de la fase.
} 
su valor nominal:

$$
\Phi_{1}^{o}=\frac{\text { Res }_{\text {ideal }}}{1+\partial}
$$

- Se debe cubrir todo el rango especificado incluso si los pesos de todos los dígitos fueran menores que sus valores nominales:

$$
\begin{aligned}
\sum_{i=1}^{p} \frac{\Phi_{i}^{o}}{1+\partial} & =\left(2^{N}-1\right) \cdot \text { Res }_{\text {ideal }} \\
\sum_{i=1}^{p} \frac{\beta^{i-1} \Phi_{1}^{o}}{1+\partial} & =\frac{\Phi_{1}^{o}}{1+\partial} \sum_{i=1}^{p} \beta^{i-1}=\frac{\Phi_{1}^{o}}{1+\partial} \frac{\beta^{p}}{\beta-1}=\left(2^{N}-1\right) \cdot \text { Res }_{i d e a l}
\end{aligned}
$$

- La diferencia entre el dígito r-ésimo con un peso mayor de su valor nominal y la suma de los dígitos precedentes con pesos inferiores a los valores nominales debe ser igual a la resolución:

$$
\begin{gathered}
\Phi_{r}(1+\partial)-\sum_{i=1}^{r-1} \frac{\Phi_{i}^{o}}{1+\partial}=R_{e s} s_{\text {deal }} \\
\beta^{r-1} \Phi_{1}^{o}(1+\partial)-\frac{\beta^{r-1}-1}{\beta-1} \frac{\Phi_{1}^{o}}{1+\partial}=\text { Res }_{\text {ideal }}
\end{gathered}
$$

Combinando las ecuaciones (3.28) y (3.29):

$$
\frac{\operatorname{Res}_{\text {ideal }}}{(1+\partial)^{2}} \frac{\beta^{p}-1}{\beta-1}=\left(2^{N}-1\right) \cdot \text { Res }_{\text {ideal }}
$$

Por otro lado, combinando las ecuaciones (3.28) y (3.31):

$$
\beta^{r-1} \operatorname{Res}_{\text {ideal }}-\frac{\operatorname{Res}_{\text {ideal }}}{(1+\partial)^{2}} \frac{\beta^{r-1}-1}{\beta-1}=\operatorname{Res}_{\text {ideal }}
$$

o simplificando:

$$
\left(\beta^{r-1}-1\right)\left(1-\frac{1}{(\beta-1)(1+\partial)^{2}}\right)=0
$$

para que se cumpla la ecuación (3.34) para cualquier valor de $r$, es necesario que se cumpla:

$$
(\beta-1)(1+\partial)^{2}=1
$$

Finalmente, combinando (3.32) y (3.35):

$$
\beta^{p}=2^{N}
$$




\begin{tabular}{|c|c|c|}
\hline $\begin{array}{c}\text { Número de dígitos } \\
p\end{array}$ & $\begin{array}{c}\text { Razón entre pesos } \\
\beta\end{array}$ & $\begin{array}{c}\text { Error tolerable } \\
\partial(\%)\end{array}$ \\
\hline \hline 5 & 2.00 & 0 \\
\hline 6 & 1.78 & 13 \\
\hline 7 & 1.64 & 25 \\
\hline 8 & 1.54 & 36 \\
\hline
\end{tabular}

Tabla 3.6: Porcentaje de error tolerable $(\partial)$ en función del número de dígitos reales $(p)$ para cinco dígitos equivalentes $(N=5)$.

\begin{tabular}{|c|c|c|}
\hline $\begin{array}{c}\text { Incertidumbre } \\
\partial(\%)\end{array}$ & $\begin{array}{c}\text { Razón entre pesos } \\
\beta: 1\end{array}$ & $\begin{array}{c}\text { Dígitos equivalentes } \\
\mathrm{N}\end{array}$ \\
\hline \hline 0 & 2.00 & 6 \\
\hline 5 & 1.91 & 5.6 \\
\hline 10 & 1.83 & 5.2 \\
\hline 15 & 1.76 & 4.9 \\
\hline 20 & 1.69 & 4.5 \\
\hline
\end{tabular}

Tabla 3.7: Número de dígitos equivalentes $(N)$ en función del porcentaje de error tolerable $(\partial)$ para seis dígitos reales $(p=6)$.

Las ecuaciones (3.35) y (3.36) definen la relación entre el número de dígitos, $p$, el error relativo tolerable, $\partial$, la razón entre los pesos, $\beta$ y el número equivalente de dígitos binarios para la misma resolución, $N$; cifra que no tiene necesariamente que ser un número entero.

La tabla 3.6, muestra el impacto del número de dígitos escogido para obtener una resolución de cinco dígitos binarios equivalentes sobre el máximo error tolerable para el peso de cada dígito; mientras, que la tabla 3.7, presenta la relación entre el máximo error tolerable y la resolución garantizada cuando se usan seis dígitos reales.

A la hora de trabajar con pesos no binarios, el método de diseño partiría de la resolución deseada (expresada como el número de dígitos binarios $(N)$ con resolución equivalente) y de la incertidumbre que se tiene en el peso de los dígitos reales $(\partial)$. Introduciendo la incertidumbre en la ecuación (3.35), se puede obtener la razón geométrica entre los pesos de los dígitos reales $(\beta)$, y a continuación se calcula el número de dígitos reales $(p)$ a partir de (3.36). Finalmente, la ecuación (3.28) da el peso del dígito más pequeño $\left(\Phi_{1}^{o}\right)$.

El desarrollo anterior demuestra la utilidad de los desfasadores con pesos no binarios desde el punto de vista de la resolución, es decir de los errores de pico, de un único desfasador, sin tener en cuenta, la varianza de los errores o su efecto sobre los parámetros de radiación de una agrupación. En la figura 3.6 se compara el efecto de una incertidumbre uniforme y proporcional en los dígitos individuales en cuanto a valores de pico y RMS del error de fase total, y del nivel normalizado de los 


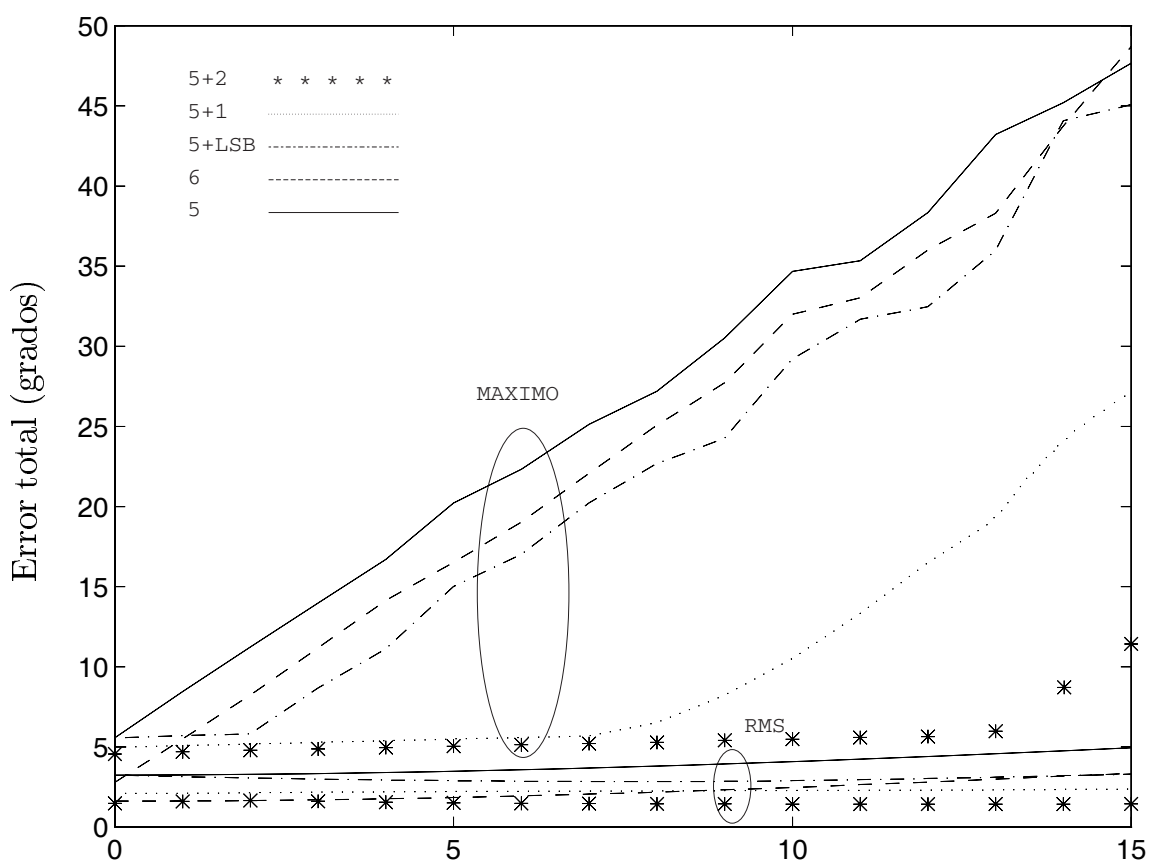

a)

Error dígito $( \pm \%)$

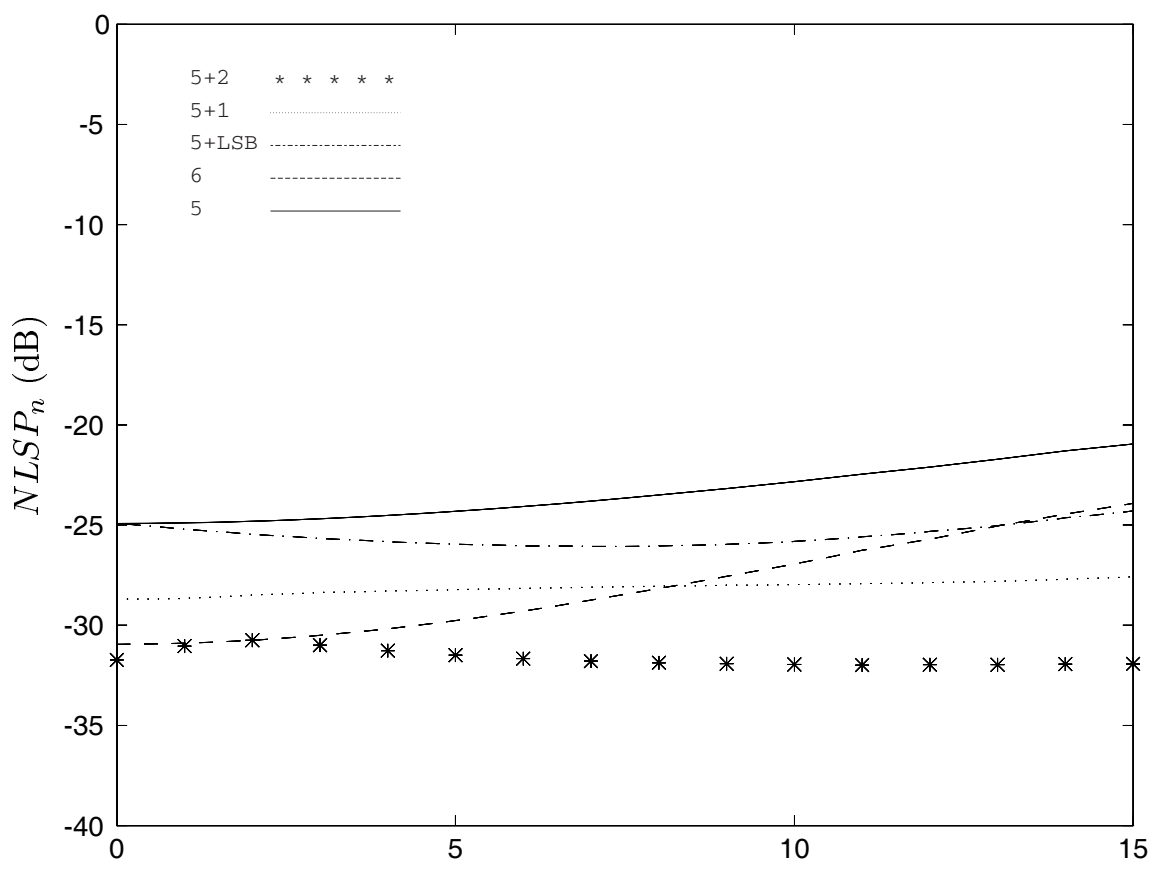

b)

Error dígito $( \pm \%)$

Figura 3.6: Comparativa en función del error de cada dígito cuando los errores son proporcionales y con distribución uniforme para desfasadores digitales: binario de cinco dígitos (5), binario de seis dígitos (6), binario de cinco dígitos con LSB repetido (5+LSB), no binario de seis dígitos con cinco equivalentes $(5+1)$ y no binario de siete dígitos con cinco equivalentes (5+2). a) Error máximo y RMS. b) $N L S P_{n}$. 
lóbulos secundarios respecto al lóbulo principal $\left(N L S P_{n}\right)$ para cinco casos distintos de desfasadores digitales. En concreto, se presentan datos sobre dos desfasadores binarios sin redundancia (cinco y seis dígitos), un desfasador binario con el LSB repetido y dos desfasadores (seis y siete dígitos) con pesos no binarios y resolución equivalente de un desfasador binario de cinco dígitos.

En cuanto al error de pico, el comportamiento se adecúa a lo esperado, pues el desfasador no binario de seis dígitos presenta una tolerancia mucho mayor que los desfasadores binarios de cinco y seis dígitos, y que el desfasador de cinco dígitos con el LSB repetido. Es interesante resaltar, que la tolerancia frente a los errores mostrada es inferior a la indicada en la ecuación (3.35), siendo del $8 \%$ en lugar del $13 \%$ reflejado en la tabla 3.6; debiéndose esta discordancia a que la ecuación (3.35) considera que el error máximo ocurre para el mismo caso que para los desfasadores con pesos binarios, cuando la distribución de los desfases deja de ser equiespaciada y pasa a ser mucho menos uniforme, por lo que el peor caso puede ocurrir entre dos desfases cualesquiera. Como se comprueba en la figura 3.6a, el comportamiento del desfasador de siete dígitos no binarios con cinco dígitos equivalentes presenta una tolerancia superior al $13 \%$.

Resulta más interesante comparar la varianza del error; en este caso, el desfasador no binario de seis dígitos presenta un comportamiento mejor que el de los desfasadores de cinco dígitos binarios y que el desfasador con el LSB repetido, e incluso, a partir de un cierto valor de incertidumbre (alrededor de 8.5\%) en los pesos, su comportamiento es mejor que el de un desfasador digital binario de seis dígitos.

Finalmente, en la figura 3.6b, se compara el comportamiento de los cinco mismos tipos de desfasadores, en cuanto al nivel normalizado de lóbulos secundarios respecto al lóbulo principal, $N L S P_{n}$, de una agrupación formada por cada tipo de desfasador. Como era de esperar, las agrupaciones con desfasadores con pesos binarios empeoran su comportamiento al aumentar el error de los desfasadores. Si la agrupación trabaja con desfasadores con el LSB repetido, el $N L S P_{n}$ se mantiene prácticamente constante en el rango de errores de la gráfica, pero, en cualquier caso, sus prestaciones frente a los errores son siempre peores que las correspondientes a una agrupación basada en desfasadores con seis dígitos y pesos no binarios; siendo para ambos casos el número total de dígitos de seis. Incluso a partir de un porcentaje de error del $8 \%$ se comporta mejor una agrupación con desfasadores de seis dígitos con pesos no binarios, que si se utilizaran desfasadores de seis dígitos binarios. Resulta muy interesante la comparación de los tres casos estudiados con un mismo número de dígitos en el desfasador, pues, es de suponer, que, presentarán las mismas características en cuanto a pérdidas de inserción y al tamaño del circuito integrado; dos parámetros muy importantes a la hora de diseñar un desfasador digital con tecnología MMIC. Sería posible obtener resultados similares para la reducción en la ganancia del haz principal, pero se omiten por su menor importancia frente al $N L S P_{n}$.

En resumen, queda demostrado el mejor comportamiento de los desfasadores no binarios frente a la presencia de errores en los pesos de los dígitos. Esta técnica también puede emplearse en el diseño de los circuitos de control de ganancia. 


\subsection{Diseño de un desfasador variable a $24 \mathrm{GHz}$ con tecnología MMIC}

\subsubsection{Introducción}

Esta sección presenta el diseño de un desfasador variable a $24 \mathrm{GHz}$, cuyas especificaciones fueron elegidas de acuerdo con los requisitos de un radar de lluvia con haz dual. Este radar de lluvia (RAIN RADAR) es un instrumento para la Observación de la Tierra que actualmente está en estudio para el programa METOP de la Agencia Espacial Europea.

\subsubsection{Datos generales}

Peso ligero, fiabilidad y bajas pérdidas son requisitos para desfasadores variables en las muy exigentes aplicaciones espaciales, tanto en aplicaciones de observación de la Tierra como en aplicaciones en comunicaciones.

La introducción de circuitos integrados monolíticos de microondas (MMIC), permite el uso de agrupaciones desfasables de antenas altamente flexibles. Con este concepto en mente, y con las especificaciones de la misión RAIN RADAR como objetivo, se comenzó el diseño de un desfasador variable MMIC a $24.15 \mathrm{GHz}$.

\section{Especificaciones del Desfasador Variable}

A partir de conversaciones con los responsables del proyecto RAIN RADAR (F. Coromina (XRM, ESTEC) y P.L. Mancini (XRI, ESTEC)) y de algunas publicaciones internas de la Agencia Espacial Europea [Dub92], se han definido las siguientes especificaciones:

- Frecuencia central: $24.15 \mathrm{GHz}$.

- Banda estrecha.

- Rango de desfase: $360^{\circ}$.

- Resolución: $11.25^{\circ}$ (equivalente a un desfasador digital de cinco dígitos).

- Pérdidas de inserción $<12$ dB.

- Diferencia entre las pérdidas de inserción de estados de desfase diferentes: $\pm 1 \mathrm{~dB}$

- Pérdidas de Retorno > 15 dB.

- Impedancia nominal: $50 \Omega$. 


\section{Características del proceso D02AH}

El proceso de fabricación utilizado ha sido el proceso D02AH de la fundición Philips Microwave Limeil, cuyas características más importantes pueden consultarse en el apéndice B. Se trata de un proceso basado en transistores con alta movilidad de los electrónes y heteroestructura pseudomórfica $\left(\mathrm{P}-\mathrm{HEMT}^{\mathrm{i}}\right)$.

\section{Herramientas de diseño}

La mayoría de las simulaciones llevadas a cabo durante este diseño fueron hechas con el paquete LIBRA Serie IV de HP-EESOF. El entorno de simulación LIBRA no es adecuado para manejar circuitos con múltiples estados, como es el caso de un desfasador variable digital, Por esta razón, tareas como la selección del orden de los dígitos del desfasador variable digital, fueron realizadas con el entorno MATLAB de The MathWorks Inc. en la forma indicada en el apartado 3.3.6.

\subsubsection{Primeros enfoques}

\section{Analógico frente a digital}

La primera decisión a tomar en el diseño de un desfasador variable debe ser la elección entre un diseño analógico (el desfase relativo de una red puede variarse de una forma contínua mediante la correspondiente variación contínua de una señal de control) y uno digital (diferentes circuitos (dígitos ${ }^{\mathrm{ii}}$ ) pueden conmutarse entre, generalmente, dos estados de desfase fijos, denominándose peso del dígito a la diferencia entre los desfases introducidos en ambos estados). La opción digital fue la elegida, debido a la mayor sensibilidad de los desfasadores variables analógicos a las variaciones de temperatura y al envejecimiento. Además, los desfasadores digitales permiten un control remoto mucho más adecuado para agrupaciones de antenas, al requerir señales digitales para su control.

\section{Binario frente a no binario}

La resolución especificada $\left(11.25^{\circ}\right)$ requiere idealmente cinco dígitos con ponderación binaria $\left(180^{\circ}, 90^{\circ}, 45^{\circ}, 22.5^{\circ}\right.$ y $\left.11.25^{\circ}\right)$. La precisión de estos cinco elementos viene afectada por diversos factores, como pueden ser, las incertidumbres del diseño, las variaciones del proceso debidas a la producción, la dispersión en frecuencia o las variaciones en el ensamblaje.

De acuerdo con los resultados de la sección 3.2.6 la principal ventaja de la ponderación no binaria de los pesos del desfasador digital, es la posibilidad de que todos

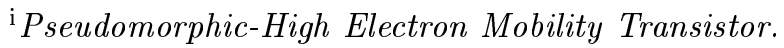

ii Bits, en terminología inglesa.
} 


\begin{tabular}{|l|c|c|c|c|}
\hline & $\begin{array}{c}\text { Binario } \\
5 \text { dígitos }\end{array}$ & $\begin{array}{c}\text { Binario } \\
\text { 5+LSB dígitos }\end{array}$ & $\begin{array}{c}\text { Binario } \\
6 \text { dígitos }\end{array}$ & $\begin{array}{c}\text { No binario } \\
6 \text { dígitos }\end{array}$ \\
\hline \hline$\delta_{\max }$ & $\pm 5.6^{\circ}$ & $\pm 5.6^{\circ}$ & $\pm 2.8^{\circ}$ & $\pm 5^{\circ}$ \\
$\delta_{\max }(\mathrm{err}=5 \%)$ & $\pm 20.2^{\circ}$ & $\pm 14.4^{\mathrm{o}}$ & $\pm 16.3^{\circ}$ & $\pm 5.5^{\circ}$ \\
$\delta_{\max }(\mathrm{err}=10 \%)$ & $\pm 32.1^{\circ}$ & $\pm 25.6^{\mathrm{o}}$ & $\pm 32^{\circ}$ & $\pm 10.5^{\circ}$ \\
\hline \hline$\sigma_{\delta}$ & $3.25^{\circ}$ & $3.25^{\circ}$ & $1.62^{\circ}$ & $2.11^{\circ}$ \\
$\sigma_{\delta}(\mathrm{err}=5 \%)$ & $3.48^{\circ}$ & $2.88^{\circ}$ & $1.85^{\mathrm{o}}$ & $2.22^{\circ}$ \\
$\sigma_{\delta}(\mathrm{err}=10 \%)$ & $4.07^{\circ}$ & $2.9^{\circ}$ & $2.48^{\mathrm{o}}$ & $2.29^{\circ}$ \\
\hline \hline$N L S P_{n}$ & $-24.9 \mathrm{~dB}$ & $-24.9 \mathrm{~dB}$ & $-30.9 \mathrm{~dB}$ & $-28.7 \mathrm{~dB}$ \\
$N L S P_{n}(\mathrm{err}=5 \%)$ & $-24.3 \mathrm{~dB}$ & $-25.9 \mathrm{~dB}$ & $-29.8 \mathrm{~dB}$ & $-28.2 \mathrm{~dB}$ \\
$N L S P_{n}(\mathrm{err}=10 \%)$ & $-22.8 \mathrm{~dB}$ & $-25.8 \mathrm{~dB}$ & $-26.9 \mathrm{~dB}$ & $-28.0 \mathrm{~dB}$ \\
\hline
\end{tabular}

Tabla 3.8: Comparación de resultados estadísticos para diversas configuraciones de desfasadores variables digitales.

y cada uno de los dígitos tuvieran un porcentaje de error determinado, $\mathrm{X} \%$, respecto a su peso nominal, mientras el desfasador completo contínua cubriendo el rango especificado cumpliendo el requisito de una resolución de cinco dígitos.

Con el fin de comparar las diferentes opciones de desfasador variable (binario de cinco dígitos, binario de cinco dígitos con LSB repetido, binario de seis dígitos y no binario de seis dígitos), se calculó el error máximo, la varianza del error y el parámetro $N L S P_{n}$, tanto para el caso nominal como cuando existe una incertidumbre del $5 \%$ y del $10 \%$ en cada dígito individual (Tabla 3.8). Es importante resaltar que para la configuración binaria, el peor caso tiene lugar cuando el dígito más significativo aumenta respecto al nominal y el resto de los dígitos disminuyen; pero esta afirmación deja de ser cierta para la configuración no binaria, tal y como se demostró previamente.

Como se puede observar en la tabla 3.8, el enfoque no binario es el mejor en los casos en los que se requiere algún tipo de redundancia. Teniendo en cuenta la frecuencia de trabajo y el hecho de trabajar con un proceso de fabricación MMIC todavía en fase pre--comercial, se necesitaba una buena tolerancia frente a posibles variaciones del proceso o errores de modelado. Los inconvenientes del enfoque no binario, si se compara con el desfasador variable de cinco dígitos, son, la necesidad de una tabla de traslación y el tiempo de calibración y las pérdidas de inserción añadidas, debido a la inclusión del sexto dígito; inconvenientes que, por otra parte, también estarían presentes si se optara por la solución de duplicar el dígito menos significativo.

Aplicando la técnica descrita en la sección 3.2.6 al diseño de un desfasador digital variable con cinco dígitos binarios equivalentes genera los pesos de los dígitos indicados en la tabla 3.9, donde se muestran además el rango mínimo y la resolución máxima. 


\begin{tabular}{|c|c|c|c|c|c|c|c|c|}
\hline Dígito & 6 & 5 & 4 & 3 & 2 & 1 & $\begin{array}{c}\text { Rango } \\
\text { Mínimo }\end{array}$ & $\begin{array}{c}\text { Resolución } \\
\text { Máxima }\end{array}$ \\
\hline Desfase & $180^{\circ}$ & $101^{\circ}$ & $56.7^{\circ}$ & $31.8^{\circ}$ & $17.9^{\circ}$ & $10.0^{\circ}$ & $348.75^{\circ}$ & $\pm 5.625^{\circ}$ \\
\hline
\end{tabular}

Tabla 3.9: Pesos para un desfasador digital variable no binario de seis dígitos con una resolución de cinco dígitos binarios equivalentes.

\section{Elección de la topología del circuito}

Una revisión completa sobre diferentes topologías de desfasadores variables, está fuera del alcance de esta Tesis y puede encontrarse en diversas referencias ([Whi74, Tem72, Sko90c]). En la figura 3.7 se muestra un diagrama con los casos más conocidos.

Las técnicas más prometedoras en tecnología MMIC son la basada en líneas-conmutadas $^{\mathrm{i}}$ (con las líneas situados dentro o fuera del circuito integrado dependiendo de la frecuencia de trabajo) y la de filtros-conmutados o redes-conmutadas ${ }^{\text {ii }}$. Los diseños del tipo reflexión fueron descartados, debido a consideraciones de tamaño (requieren un híbrido de $90^{\circ}$ ) y a la falta de un buen modelo de acoplador tipo Lange. Los diseños basados en amplificadores, han sido también descartados por su mayor consumo de potencia y peor estabilidad térmica. Los desfasadores tipo línea cargada son adecuados únicamente para valores de desfase pequeños, y generalmente, ofrecen un mayor tamaño que los diseños del tipo conmutación de filtros.

Teniendo en cuenta como primer criterio la sencillez y robustez del diseño, la primera opción, para todos los elementos de los desfasadores variables, fue la de la tecnología líneas-conmutadas. Debido a la alta frecuencia de trabajo, las longitudes de línea de transmisión a conmutar pueden incluirse dentro del circuito integrado, lo cual redundará en un menor tamaño del mismo, a la vez, que se aumenta la fiabilidad del desfasador y la repetibilidad del circuito monolítico. La impedancia, el retardo y las pérdidas de inserción de estas líneas son muy insensibles a variaciones del proceso y, por lo tanto, predecibles, a diferencia de los elementos activos o los condesadores.

El principal inconveniente del desfasador variable tipo líneas-conmutadas es el alto valor de las pérdidas de inserción, debido al hecho de que la señal debe pasar a través de dos conmutadores y una cierta longitud de línea de transmisión en cualquiera de los caminos (Fig. 3.8). Después de las primeras simulaciones, era evidente que la opción de utilizar seis dígitos tipo líneas-conmutadas introduciría unas pérdidas de inserción totales excesivamente altas, alrededor de $16 \mathrm{~dB}$, lo cual está obviamente fuera de consideración. Se decidió implementar los tres dígitos menos significativos, LSBs, con una configuración con menos pérdidas, como el tipo filtros-conmutados, el cual es además de banda ancha y presenta un reducido tamaño. En lugar de la típica estructura filtros-conmutados, se eligió un filtro reconfigurable. En este caso,

${ }^{\mathrm{i}}$ Switched-line, en terminología inglesa.

ii Switched-filter ó switched-network, en terminología inglesa. 


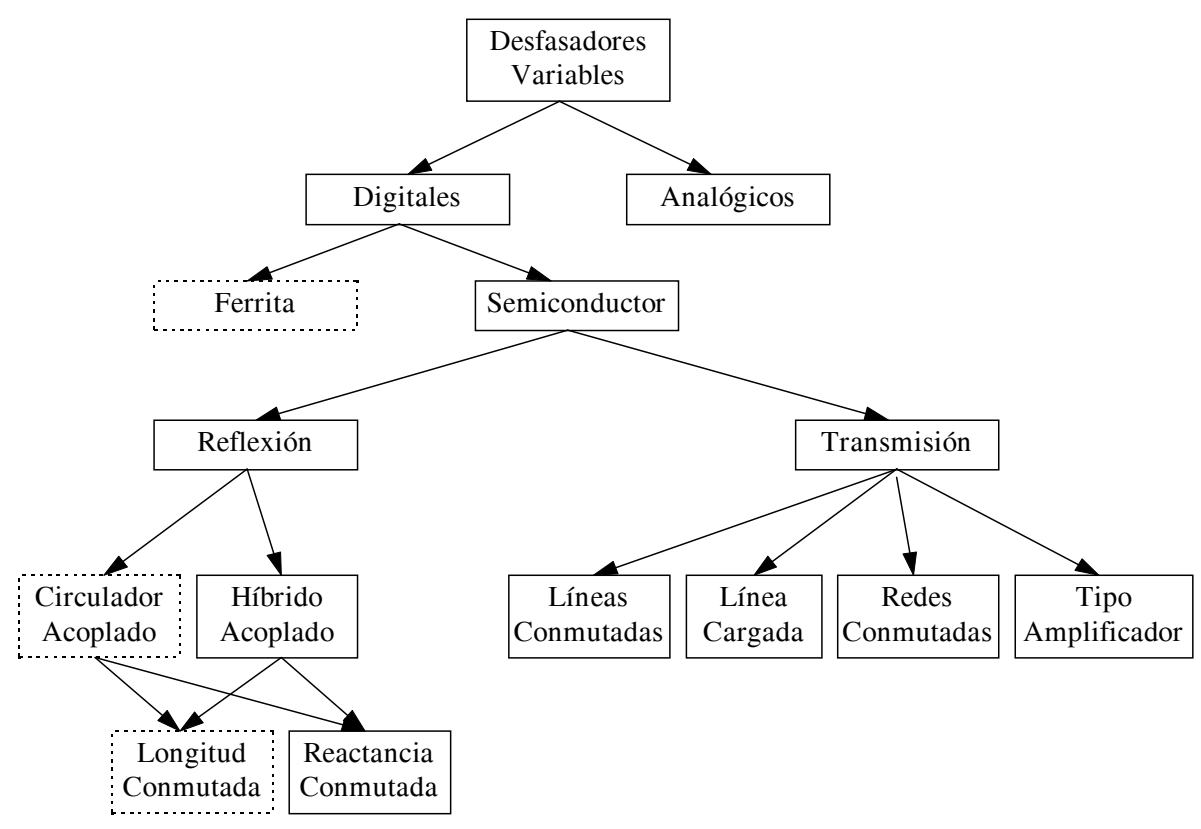

Figura 3.7: Diversos tipos de desfasadores variables de microondas.

los componentes parásitos de los conmutadores PHEMTs se usarán, en conjunción con los elementos pasivos, para crear un filtro paso-alto o paso-bajo dependiendo de las tensiones de control. De esta forma, la configuración filtros-conmutados de los tres dígitos menos significativos, permite mantener dentro de las especificaciones el valor medio de las pérdidas de inserción por cada dígito.

El enfoque adoptado para el diseño del desfasador variable completo, fue el de diseñar primero, cada dígito individual por separado, teniendo como objetivos:

- Una buena adaptación de impedancias (Relación de Onda Estacionaria (ROE) baja).

- Minimizar la variación de la ROE con el cambio de estado del dígito.

- Exactitud aceptable del desfase.

- Pérdidas de inserción reducidas y lo más parecidas posible para ambos estados de cada dígito.

La simulación y optimización de cada uno de los dígitos individuales, de acuerdo con los objetivos previamente indicados, puede llevarse a cabo con los programas comerciales de simulación de circuitos de microondas, ya que, únicamente, se consideran dos estados para cada dígito.

Por otra parte, la interacción de las diferentes adaptaciones (ROEs) de cada dígito, en sus dos estados, puede degradar seriamente la exactitud de los desfases 


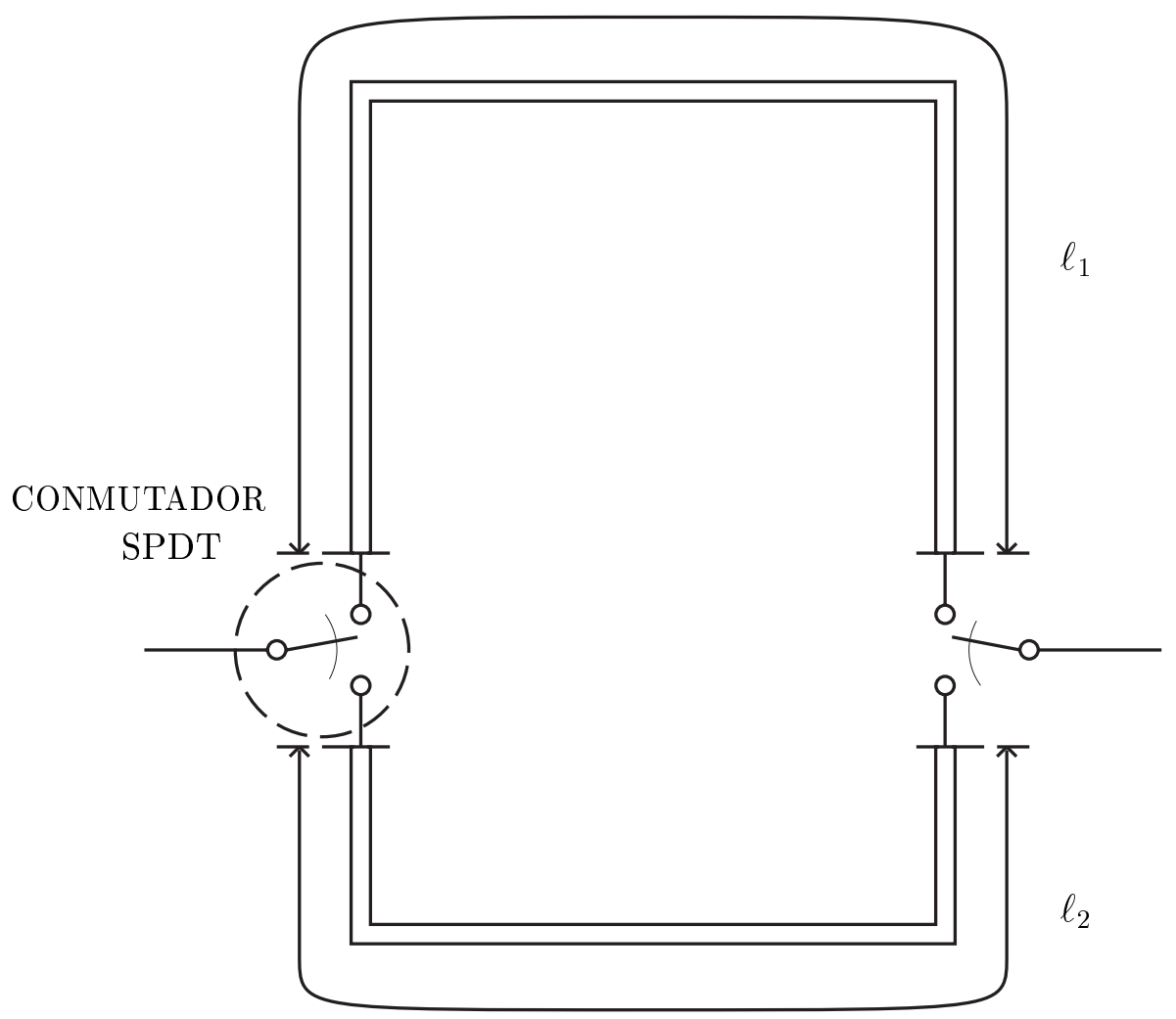

Figura 3.8: Desfasador variable de un dígito con configuración líneas-conmutadas.

y de las pérdidas de inserción. Consecuentemente, el rendimiento del desfasador variable de seis dígitos completo, ha sido analizado en todos sus 64 estados, para identificar tanto el posicionamiento óptimo de los seis dígitos individuales dentro del circuito completo, como una posible degradación de las prestaciones del desfasador variable al encadenar los diferentes dígitos individuales.

\subsubsection{Tres dígitos más significativos $\left(180^{\circ}, 101^{\circ}\right.$ y $\left.57^{\circ}\right)$}

La configuración típica de un desfasador variable tipo líneas-conmutadas de un único dígito, se muestra en la figura 3.8. Se utilizan dos conmutadores de dos vías $\left(\mathrm{SPDT}^{\mathrm{i}}\right)$ en ambos extremos del desfasador variable, con la función de enrutar la señal a través de una de las dos longitudes de línea de transmisión alternativas, que se denominarán, caminos LARGO y CORTO. Cuando la señal pasa a través del camino LARGO, la misma sufre un desfase adicional proporcional a la diferencia de longitudes de los caminos y también a la frecuencia.

Todos los desfasadores variables digitales requieren algún tipo de capacidad de conmutación. El dispositivo preferido de conmutación dentro de la tecnología MMIC actual es el MESFET sobre GaAs, usado como un dispositivo pasivo de dos accesos

\footnotetext{
${ }^{\mathrm{i}}$ Single-Pole Double-Throw.
} 
entre drenador y fuente, teniendo la puerta como acceso de control [Gas78, Aya82, Gop85, Gar89]. Las principales ventajas de este elemento son su bajo consumo de potencia, la alta velocidad de conmutación y la facilidad con la que permite el aislamiento entre radiofrecuencia (RF) y alimentación (DC).

El procedimiento de diseño para los tres dígitos más significativos fue el siguiente:

1. Definición del modelo del conmutador.

2. Selección de la configuración y las dimensiones del conmutador (serie, paralelo o serie y paralelo).

3. Elección de las longitudes de los caminos (para cada dígito).

4. Adaptación de la entrada y la salida (para cada dígito).

\section{Modelo del conmutador}

Al principio, se utilizó un modelo PHEMT en frío ${ }^{\mathrm{i}}$ con $V_{d s}=0 \mathrm{~V}$ y tensiones de conmutación de $V_{g s}=0 \mathrm{~V}$ en el estado ON y $V_{g s}=-0.9 \mathrm{~V}$ en el estado OFF (aunque $-0.9 \mathrm{~V}$ es justamente la tensión nominal de estrangulamiento ${ }^{\mathrm{ii}}$, era la tensión más baja disponible en el modelo de pequeña señal del PHEMT; siendo deseable utilizar una tensión de puerta muy por debajo de la tensión de estrangulamiento).

Después de diversas peticiones, PML suministró un modelo preliminar de conmutador que había sido extraído a partir de medidas de un conmutador serie $6 \times 15$ (con una resistencia de $2 \mathrm{k} \Omega$ en serie en la puerta). En este caso, los dos estados correspondían a unas tensiones de drenador a fuente $\left(V_{d s}\right)$ de $0 \mathrm{~V}(\mathrm{ON})$ y $-2 \mathrm{~V}$ (OFF), de forma que se mejoraba el aislamiento del conmutador. Desgraciadamente, las medidas difieren de las del PHEMT en frío para el estado ON (se encontró una diferencia bastante importante en la resistencia de fuente a drenador, $\left.R_{d s}\right)$. El modelo del PHEMT en frío se extrajo de unas medidas realizadas en configuración fuente común, mientras el conmutador medido era un dispositivo en configuración puerta común, con lo cual se decidió utilizar el modelo del conmutador (Fig. 3.9). Siguiendo las recomendaciones de PML, los parámetros del modelo del conmutador fueron escalados según las reglas de escalado definidas para el modelo PHEMT de pequeña señal [PML94], incluso aunque el modelo del conmutador fue definido a partir de medidas de un único conmutador $6 \times 15$.

Con el fin de utilizar correctamente el modelo, deberá tenerse cuidado de cortocircuitar en contínua (DC) los accesos del drenador y la fuente.

\footnotetext{
${ }^{\mathrm{i}}$ Cold PHEMT model, en terminología inglesa.

ii Pinch-off voltage, en terminología inglesa.
} 


\section{MODELO INTRÍNSECO DEL CONMUTADOR}

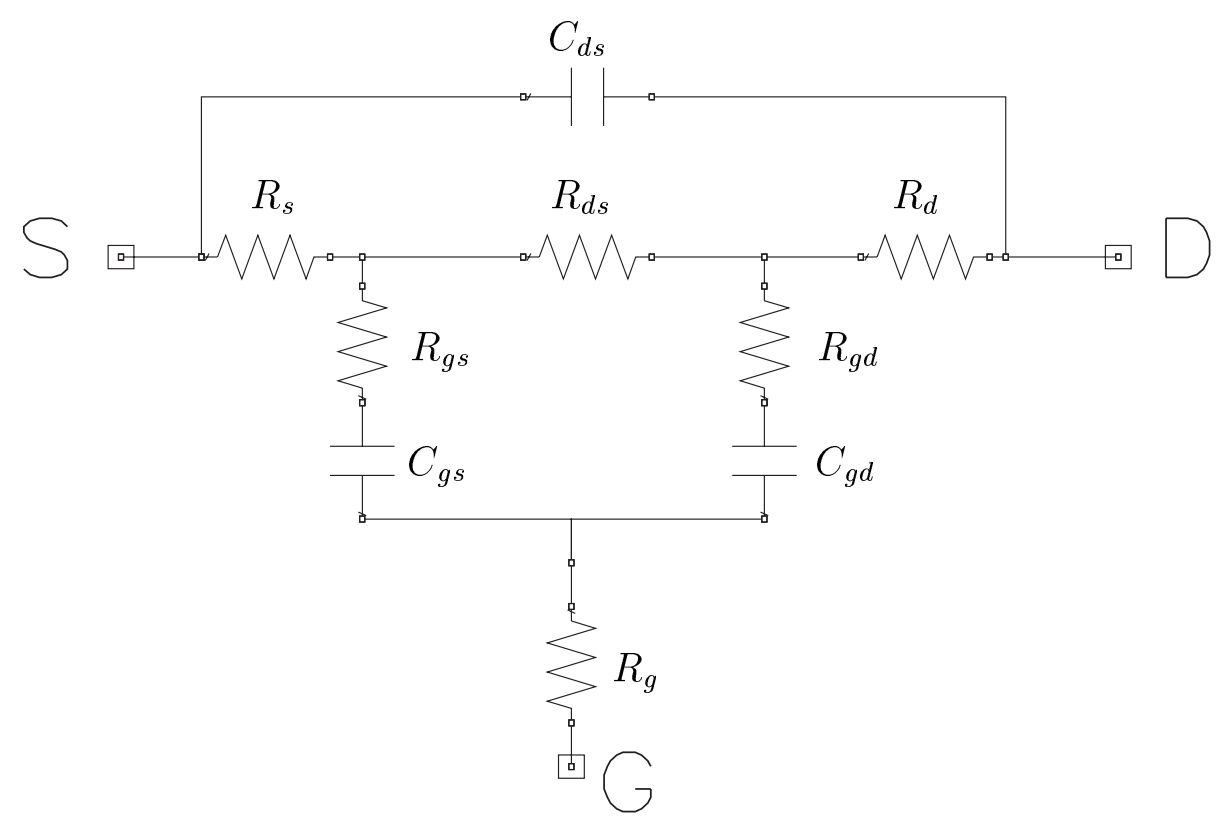

Figura 3.9: Modelo del conmutador P-HEMT del proceso D02AH.

\section{Configuración del conmutador}

Como se muestra en la figura 3.8, se necesita una estructura SPDT en ambos extremos del dígito tipo líneas-conmutadas. Con el fin de seleccionar la configuración correcta para el SPDT, fueron estudiadas tres posibilidades para el dispositivo conmutador: serie, paralelo y serie/paralelo (Fig. 3.10). Para los tres casos, las simulaciones se realizaron tanto con la puerta del PHEMT en circuito abierto, como en cortocircuito. La solución en circuito abierto (de hecho, una resistencia de alto valor, alrededor de $3 \mathrm{k} \Omega$ ) fue elegida por sus mejores prestaciones (los elementos del modelo situados entre drenador y puerta o entre fuente y puerta serán acabados también por un circuito abierto) y el aislamiento DC-RF que ofrece. En el apéndice $\mathrm{C}$ se han resumido los resultados comparativos de diversos SPDT con PHEMT de diversos tamaños y las tres configuraciones consideradas.

El parámetro más importante del dispositivo conmutador es la resistencia entre drenador y fuente $R_{d s}$, la cual es inversamente proporcional a la anchura de puerta; de forma que, cuanto más pequeña sea la anchura de puerta, menores serán las pérdidas de inserción, pero también menor es el aislamiento. Pero, cuando se tiene en cuenta la presencia de los SPDTs, el efecto de ambos conmutadores (caminos ON y OFF) se acumulan de forma que, cuando se aumenta la anchura de la puerta, las pérdidas de inserción del camino $\mathrm{ON}$ se reducen, pero también se reduce el aislamiento del camino no seleccionado. A medida que el aislamiento del camino no 
a)

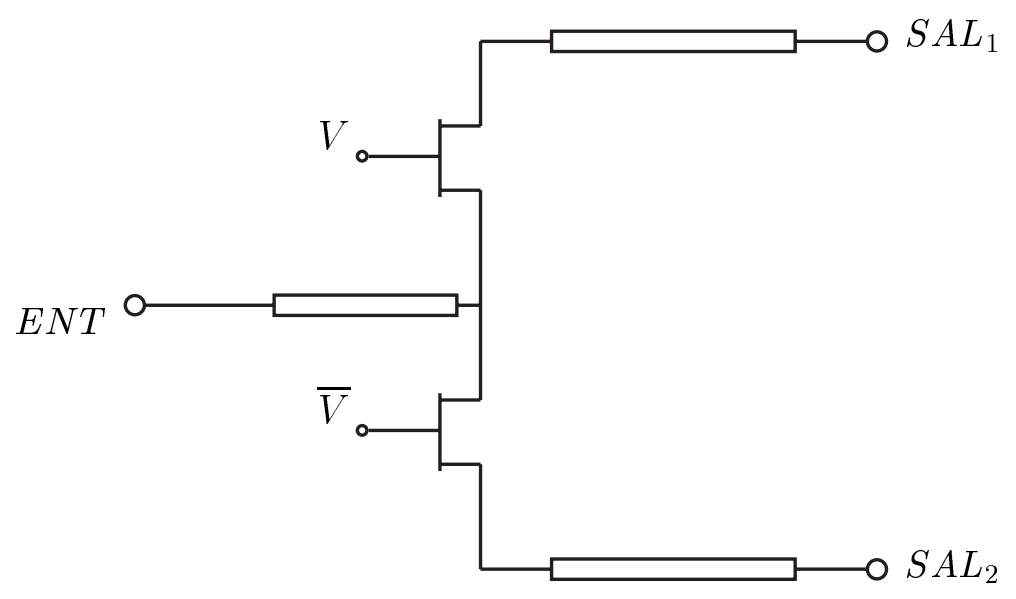

b)

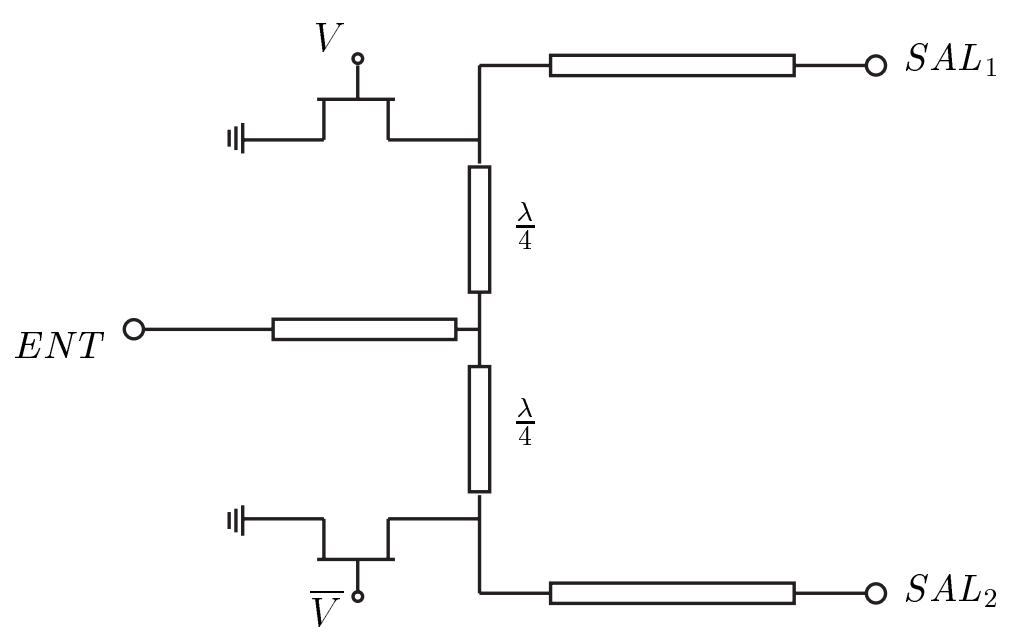

c)

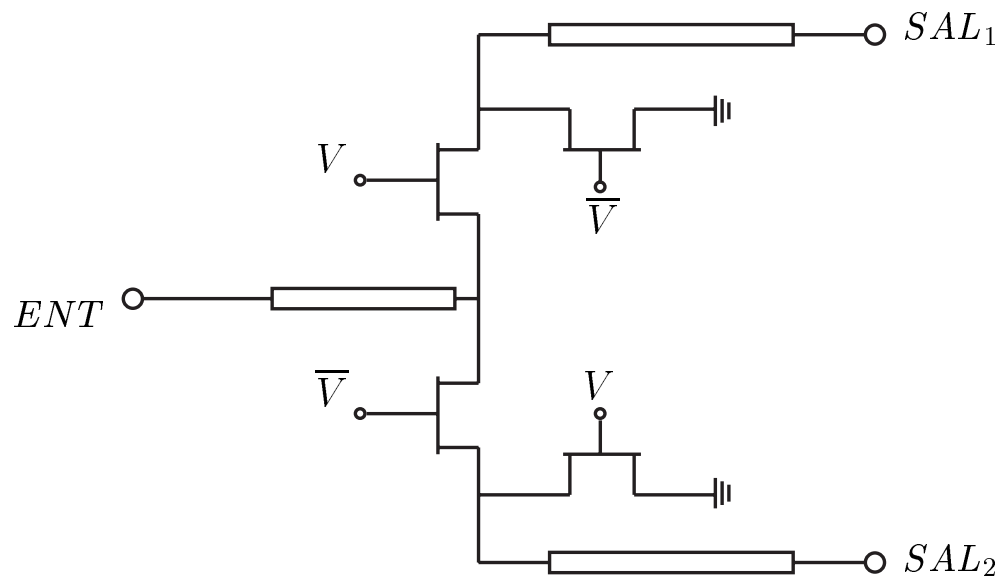

Figura 3.10: Diversas configuraciones SPDT a) Conmutadores en serie. b) Conmutadores en paralelo. c) Conmutadores en serie y paralelo. 
seleccionado disminuye, más potencia se desviará a través de esta vía, aumentándose, por tanto, las pérdidas de inserción; por consiguiente, hay un tamaño de PHEMT óptimo más allá del cual no es posible reducir más las pérdidas de inserción del camino ON.

Se realizaron diversas simulaciones para diferentes valores de anchura de puerta y la selección se hizo basándose en la relación (Pérdidas de inserción del camino $O N) /($ Aislamiento del camino OFF). Después de unas primeras simulaciones, era evidente que no era posible obtener unas buenas prestaciones, debido al bajo valor de la reactancia en serie para el camino OFF $\left(C_{d s}\right.$ a $\left.24 \mathrm{GHz}\right)$, la cual reduce considerablemente la impedancia del camino OFF. La inserción de una inductancia resonante en paralelo entre el drenador y la fuente [McL80, McL81], es el método habitual para evitar o, al menos reducir, este efecto. La introducción de una inductancia compensatoria en circuitos de conmutación basados en FETs, es mucho más fácil que en los basados en diodos, ya que no hay diferencia de potencial en contínua entre los terminales de drenador y de fuente; los circuitos de conmutación con diodos requerirían un condensador de bloqueo de contínua en serie con la inductancia compensadora. Además, esta inductancia asegura el cortocircuito en contínua entre el drenador y la fuente. En tecnología MMIC, esta inductancia se implementa generalmente con una cierta longitud de línea de transmisión de alta impedancia o bien con una bobina impresa dependiendo básicamente del valor de inductancia requerido.

Inicialmente, se estudió el SPDT con los conmutadores en serie (Fig. 3.10a). En el modo serie, la inserción de la inductancia resonante implica algunos beneficios en el camino OFF tales como un mejor aislamiento, lo cual también implica mejor desadaptación de este camino, según se ve desde la unión en 'T' del SPDT, así que las pérdidas de inserción del camino ON también mejoran. La selección del tamaño adecuado de conmutador, depende de un compromiso entre las bajas pérdidas de inserción de los PHEMTs con gran anchura de puerta y el alto aislamiento de los PHEMTs con valores pequeños de anchura de puerta.

Debido al alto valor de la inductancia resonante (mayor que $0.5 \mathrm{nH}$ para el tamaño de los conmutadores de interés), no puede utilizarse una línea de transmisión de alta impedancia ya que implicaría un gran tamaño y unas pérdidas altas. En su lugar se utilizó un inductor en espiral [PML94].

Después de diversas iteraciones, los SPDTs con conmutador en serie de tamaños $4 \times 50,4 \times 60$ o $4 \times 70$ mostraron las mejores prestaciones; en resumen, pérdidas de inserción de $1.4 \mathrm{~dB}$, aislamiento de $30 \mathrm{~dB}$ y adaptación a la entrada de $15 \mathrm{~dB}$. Se prefirió el conmutador de menor tamaño $(4 \times 50)$ ya que el modelo es más exacto (fue extraído de medidas de un conmutador serie $6 \times 15$ y se recomienda que la anchura de puerta unitaria esté por debajo de $50 \mu \mathrm{m})$, hay menos efectos distribuidos y, además, el SPDT $4 \times 50$ era más fácil de adaptar en el acceso de entrada, simplemente con una inductancia de pequeño valor que puede implementarse con una pequeña longitud de línea de alta impedancia. 


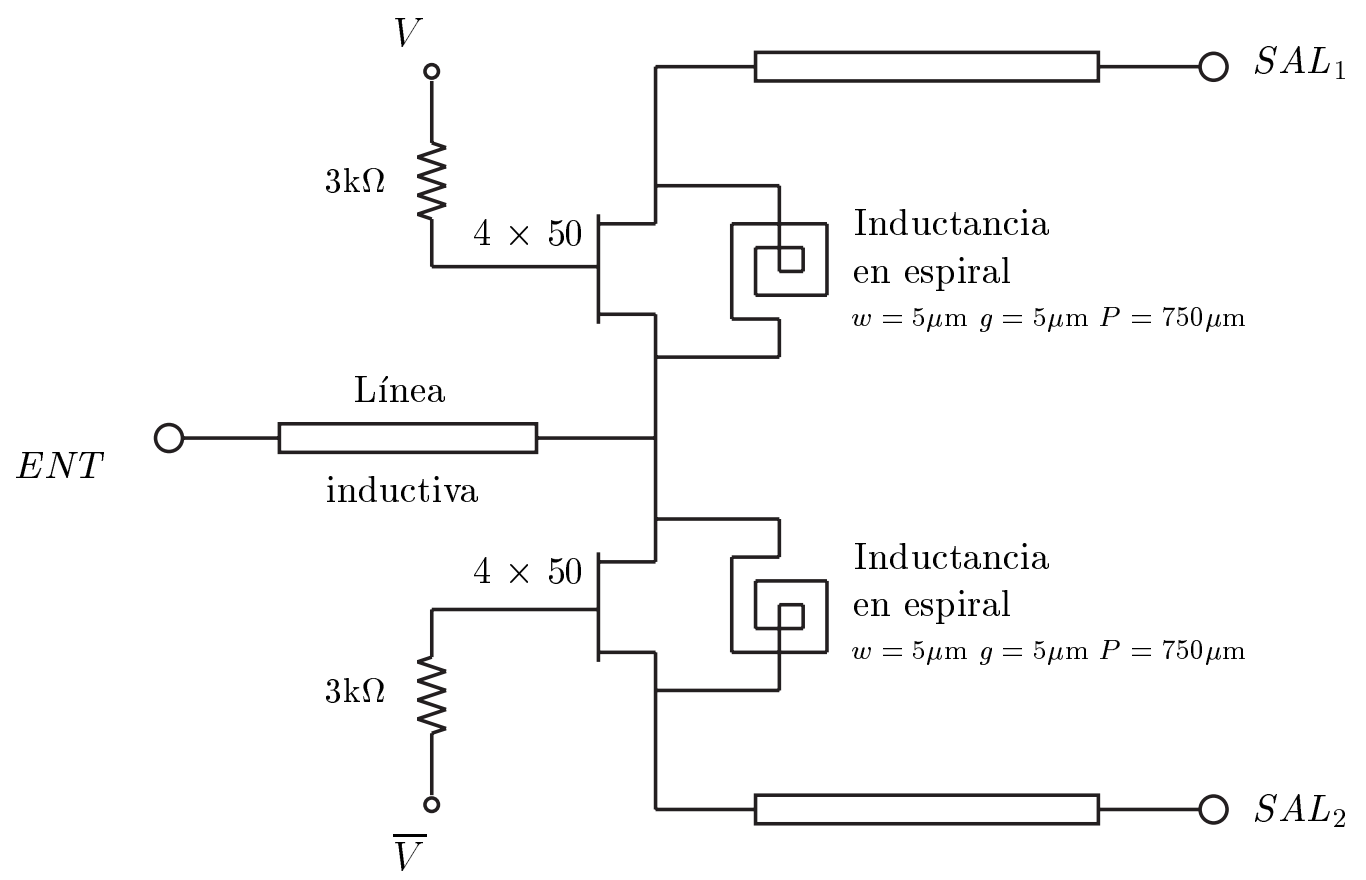

Figura 3.11: Configuración final del SPDT.

Aunque es posible conseguir prestaciones similares con el conmutador paralelo (Fig. 3.10b) que con el serie, el primero tiene un inconveniente bastante importante: se necesita una línea de transmisión de cuarto de longitud de onda desde la unión en ' $\mathrm{T}$ ' hasta cada conmutador, con lo cual se añaden más pérdidas $\mathrm{y}$, lo que es más importante, se necesita un tamaño mayor de circuito.

La opción serie/paralelo (Fig. 3.10c) no necesita esta línea de cuarto de onda, pero ninguna de las configuraciones estudiadas (siempre con el PHEMT serie más ancho que el PHEMT paralelo) pudo mejorar las prestaciones del SPDT $4 \times 50$ serie como se puede consultar en el apéndice $\mathrm{C}$.

La configuración final para el SPDT (Fig. 3.11) incluye dos PHEMTs $4 \times 50$ en configuración de conmutador serie con una inductancia espiral resonante entre drenador y fuente, y una resistencia de $3 \mathrm{k} \Omega$ en la puerta.

\section{Dígitos completos}

Con la topología del SPDT ya elegida, el siguiente paso fue, para cada dígito, coger dos SPDTs con las líneas de transmisión adecuadas para la adaptación a la entrada incluidas y dos líneas de transmisión, con la diferencia de longitudes correcta para conseguir el desfase requerido, intentando mantener las pérdidas de inserción y las pérdidas de retorno, en ambos estados, tan constantes como sean posible.

Como primera intención, se mantienen pérdidas de inserción iguales entre los dos estados de fase mediante el diseño de los caminos LARGO y CORTO del desfasador 
con diferentes valores de impedancia. Finalmente, existen otros efectos a tener en cuenta como las longitudes de ambos caminos, el valor de las inductancias resonantes, los elementos parásitos de los PHEMTs y posibles resonancias, con lo cual este efecto no es tan importante y es sobrepasado por el resto. Además, era deseable que ambos caminos presentaran la misma anchura y la misma forma, con el fin de minimizar el efecto de posibles errores de modelado en las curvas de las líneas microtira.

Uno de los problemas comunes de los desfasadores tipo líneas-conmutadas son las resonancias del camino no seleccionado (camino OFF) [Bah88]. La línea de transmisión del camino OFF en conjunción con la reactancia de los conmutadores en el estado OFF, muestra una resonancia para una frecuencia dada. Esta resonancia se traduce en un pico en la curva de pérdidas de inserción, así como por una variación rápida del desfase con la frecuencia alrededor de la frecuencia de resonancia. Con el fin de evitar este problema, se requiere una adecuada elección de las longitudes de ambos caminos, manteniendo fija la diferencia de longitudes.

Una vez se ha definido la topología, se optimizaron los paramétros de los elementos. Tanto la anchura como la longitud de ambos caminos eran paramétros optimizables, pero limitados para mantener una diferencia entre las anchuras no muy grande, con el fin de minimizar posibles efectos no modelados correctamente en las curvas o en los accesos a los PHEMTs. La mínima separación entre líneas paralelas (tres veces mayor que el grosor de la oblea, lo cual significa $300 \mu \mathrm{m}$ ) y la orientación de los PHEMTs (el ancho de la puerta siempre orientada según el eje X) limitan la longitud mínima de cada camino.

Los parámetros de las bobinas en espiral (anchura, separación entre líneas y perímetro total) y las líneas de adaptación se optimizaron también.

Los objetivos de optimización para cada uno de los tres dígitos fueron:

- Pérdidas de inserción tan bajas como sea posible.

- Pérdidas de inserción iguales para ambos estados.

- Respuesta de fase en función de la frecuencia tan plana como sea posible y con el valor correcto a la frecuencia central.

- Pérdidas de retorno por encima de $20 \mathrm{~dB}$ para ambos estados, de forma que no hubiera interacción entre los diferentes dígitos cuando estos fueran colocados en cascada en el desfasador variable completo; pues la interacción de las pérdidas de retorno de los dígitos cambiantes, puede degradar seriamente la exactitud del desfase y las pérdidas de inserción del desfasador variable completo.

Las restriciones por la distribución espacial no afectaron seriamente a los diseños, excepto en el dígito de $101^{\circ}$, el cual requiere un camino CORTO de longitud bastante pequeña, lo que hubiera implicado en el camino LARGO el incumplimiento de la regla de separación entre líneas. 


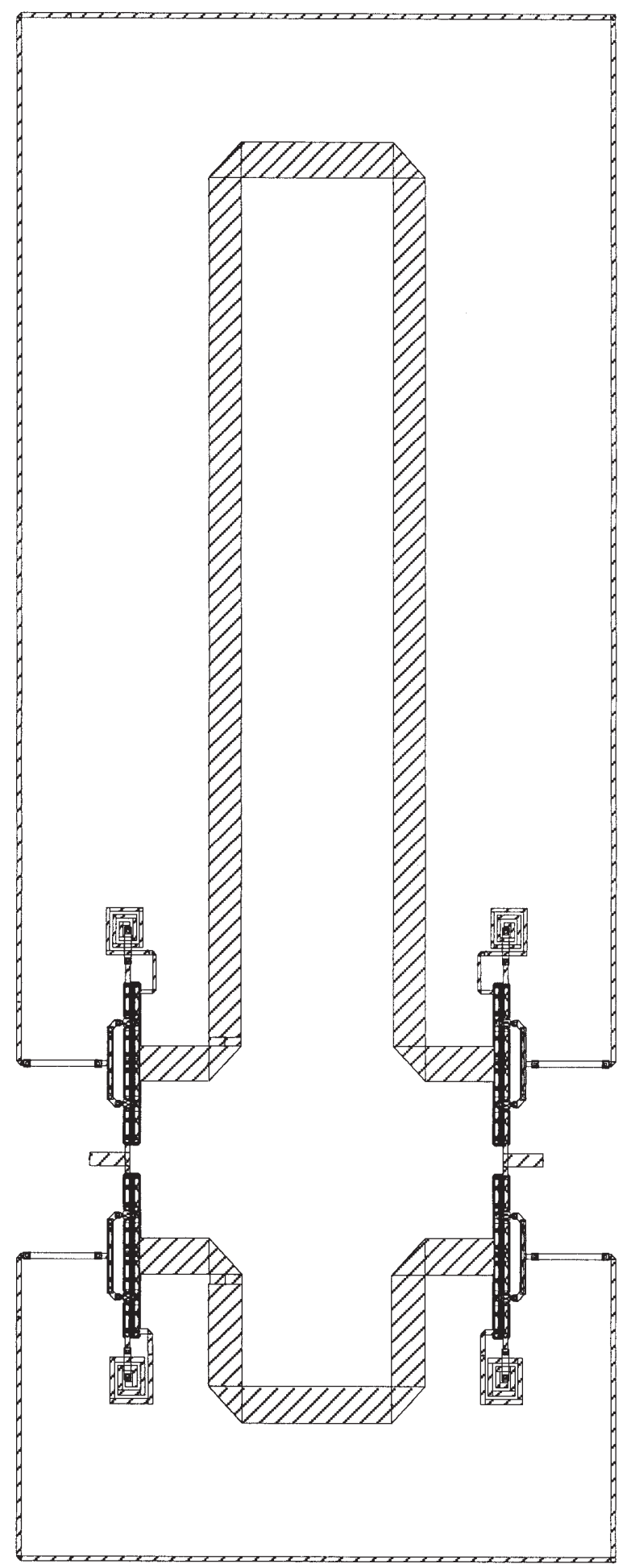

Figura 3.12: Distribución espacial del dígito de $180^{\circ}$ con configuración líneas-conmutadas. 


\begin{tabular}{|c|c|c|c|c|c|}
\hline \multirow[b]{2}{*}{$\begin{array}{c}\text { Frecuencia } \\
\qquad(\mathrm{GHz})\end{array}$} & \multicolumn{2}{|c|}{ Camino LARGO } & \multicolumn{2}{|c|}{ Camino CORTO } & \multirow[b]{2}{*}{ Desfase } \\
\hline & $\begin{array}{c}\text { Pérdidas de } \\
\text { retorno }\end{array}$ & $\begin{array}{c}\text { Pérdidas de } \\
\text { inserción }\end{array}$ & $\begin{array}{c}\text { Pérdidas de } \\
\text { retorno }\end{array}$ & $\begin{array}{c}\text { Pérdidas de } \\
\text { inserción }\end{array}$ & \\
\hline 23.15 & $18.5 \mathrm{~dB}$ & $2.9 \mathrm{~dB}$ & $21.5 \mathrm{~dB}$ & $2.8 \mathrm{~dB}$ & $171.5^{\circ}$ \\
\hline 24.15 & $26 \mathrm{~dB}$ & $2.9 \mathrm{~dB}$ & $22.5 \mathrm{~dB}$ & $2.8 \mathrm{~dB}$ & $180^{\circ}$ \\
\hline 25.15 & $22 \mathrm{~dB}$ & $3.0 \mathrm{~dB}$ & $21.5 \mathrm{~dB}$ & $2.85 \mathrm{~dB}$ & $190.5^{\circ}$ \\
\hline
\end{tabular}

Tabla 3.10: Resultados de simulación del dígito de $180^{\circ}$ tipo líneas-conmutadas.

\begin{tabular}{|c|c|c|c|c|c|}
\hline \multirow[b]{2}{*}{$\begin{array}{l}\text { Frecuencia } \\
\qquad(\mathrm{GHz})\end{array}$} & \multicolumn{2}{|c|}{ Camino LARGO } & \multicolumn{2}{|c|}{ Camino CORTO } & \multirow[b]{2}{*}{ Desfase } \\
\hline & $\begin{array}{l}\text { Pérdidas de } \\
\text { retorno }\end{array}$ & $\begin{array}{l}\text { Pérdidas de } \\
\text { inserción }\end{array}$ & $\begin{array}{l}\text { Pérdidas de } \\
\text { retorno }\end{array}$ & $\begin{array}{l}\text { Pérdidas de } \\
\text { inserción }\end{array}$ & \\
\hline 23.15 & $21.5 \mathrm{~dB}$ & $2.9 \mathrm{~dB}$ & $26 \mathrm{~dB}$ & $3 \mathrm{~dB}$ & $96^{\circ}$ \\
\hline 24.15 & $18.5 \mathrm{~dB}$ & $2.9 \mathrm{~dB}$ & $22 \mathrm{~dB}$ & $3.05 \mathrm{~dB}$ & $101^{\circ}$ \\
\hline 25.15 & $14 \mathrm{~dB}$ & $3.1 \mathrm{~dB}$ & $19.5 \mathrm{~dB}$ & $3.1 \mathrm{~dB}$ & $106^{\circ}$ \\
\hline
\end{tabular}

Tabla 3.11: Resultados de simulación del dígito de $101^{\circ}$ tipo líneas-conmutadas

El dígito de $180^{\circ}$ (Fig. 3.12) fue el de más difícil optimización y requirió la elección de un tamaño diferente $(4 \times 60)$ para los conmutadores PHEMT con el fin de mejorar las prestaciones del dígito completo. Por otro lado, este dígito fue el único donde fue posible mantener ambos caminos con la misma anchura de pista.

Ambos dígitos de $101^{\circ}$ (Fig. 3.13) y de $57^{\circ}$ (Fig. 3.14) usan el SPDT $4 \times 50$. Las anchuras de ambos caminos se mantuvieron tan iguales como fue posible sin que se degradase el comportamiento de los circuitos. El dígito de $57^{\circ}$ muestra mejores prestaciones con unas pérdidas de retorno de $20 \mathrm{~dB}$, una respuesta de desfase relativo bastante plana con la frecuencia y las resonancias bastante separadas de la frecuencia central.

Por todas estas razones, fue el dígito de $57^{\circ}$ el elegido para ser medido y analizado separadamente en un circuito de prueba independiente, cuyos datos se pueden consultar en [CG95a, CG95b].

Se muestra en las tablas 3.10, 3.11 y 3.12 un breve resumen de los resultados de los tres dígitos individuales de mayor peso [CG95a].

\begin{tabular}{|c|c|c|c|c|c|}
\hline \multirow[b]{2}{*}{$\begin{array}{l}\text { Frecuencia } \\
\qquad(\mathrm{GHz})\end{array}$} & \multicolumn{2}{|c|}{ Camino LARGO } & \multicolumn{2}{|c|}{ Camino CORTO } & \multirow[b]{2}{*}{ Desfase } \\
\hline & $\begin{array}{l}\text { Pérdidas de } \\
\text { retorno }\end{array}$ & $\begin{array}{c}\text { Pérdidas de } \\
\text { inserción }\end{array}$ & $\begin{array}{c}\text { Pérdidas de } \\
\text { retorno }\end{array}$ & $\begin{array}{c}\text { Pérdidas de } \\
\text { inserción }\end{array}$ & \\
\hline 23.15 & $23 \mathrm{~dB}$ & $3.05 \mathrm{~dB}$ & $28 \mathrm{~dB}$ & $3.0 \mathrm{~dB}$ & $54^{\circ}$ \\
\hline 24.15 & $24.5 \mathrm{~dB}$ & $2.9 \mathrm{~dB}$ & $28 \mathrm{~dB}$ & $3.0 \mathrm{~dB}$ & $56.7^{\circ}$ \\
\hline 25.15 & $18.5 \mathrm{~dB}$ & $2.95 \mathrm{~dB}$ & $26 \mathrm{~dB}$ & $3.0 \mathrm{~dB}$ & $59.5^{\circ}$ \\
\hline
\end{tabular}

Tabla 3.12: Resultados de simulación del dígito de $57^{\circ}$ tipo líneas-conmutadas. 


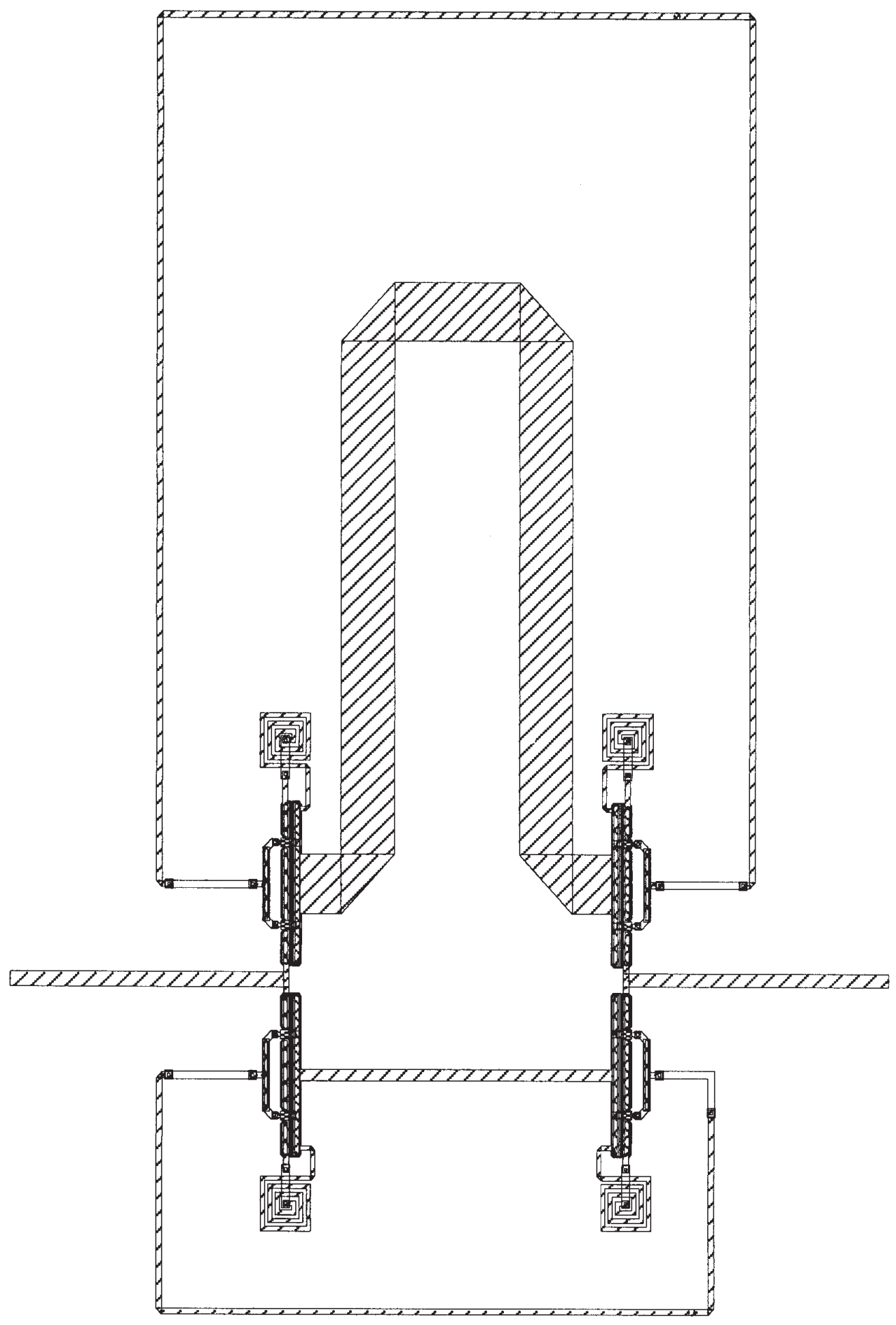

Figura 3.13: Distribución espacial del dígito de $101^{\circ}$ con configuración líneas-conmutadas. 


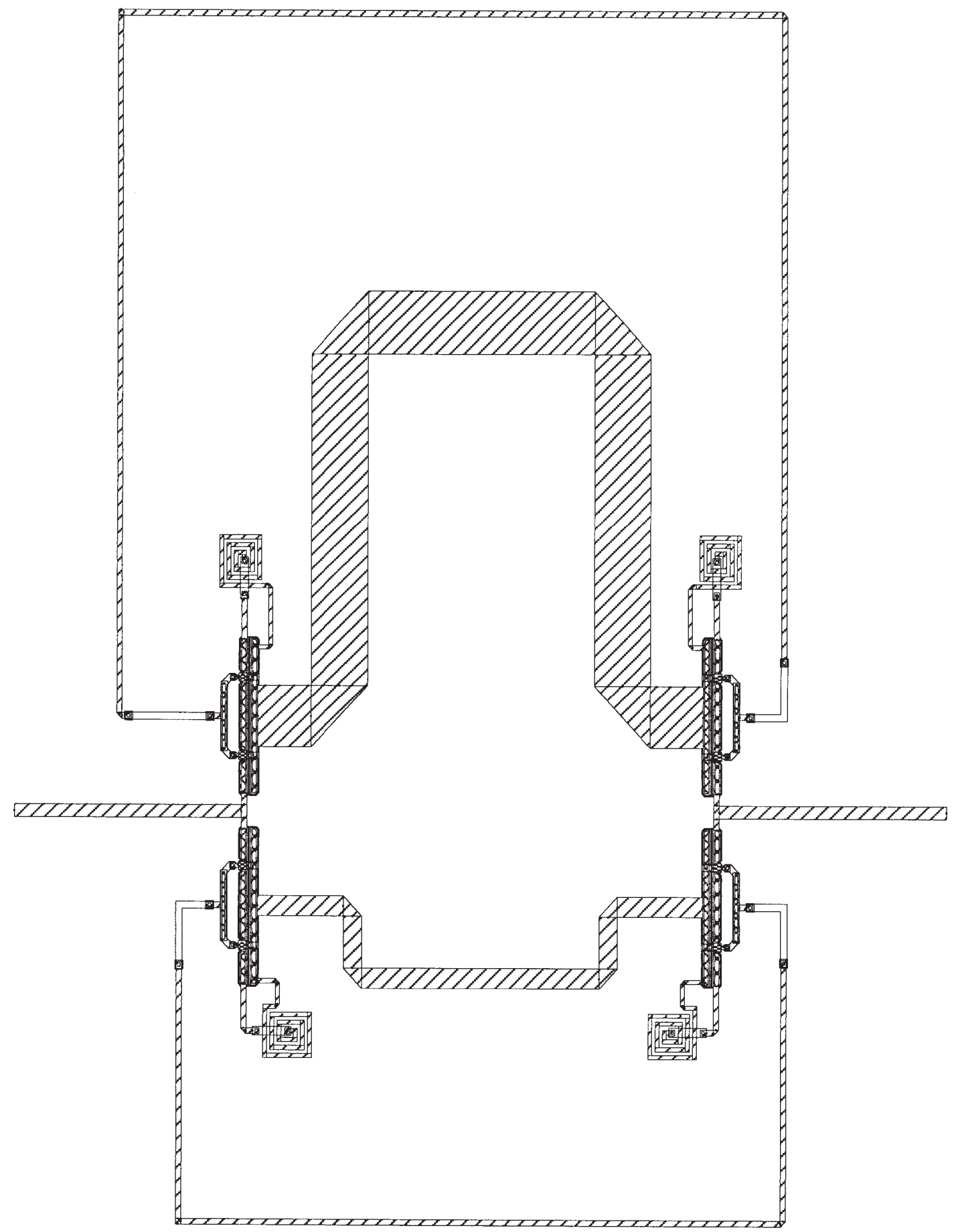

Figura 3.14: Distribución espacial del dígito de $57^{\circ}$ con configuración líneas-conmutadas. 
Los tres dígitos líneas-conmutadas muestran unas buenas prestaciones. En resumen:

- Pérdidas de inserción de alrededor de $3 \mathrm{~dB}$.

- Pérdidas de inserción diferenciales tan bajas como $\pm 0.1 \mathrm{~dB}$.

- Muy buena adaptación (18.5 dB de pérdidas de retorno para el peor caso).

- Respuesta de fase con la frecuencia típica para un desfasador tipo líneas-conmutadas.

\subsubsection{Tres dígitos menos significativos $\left(32^{\circ}, 18^{\circ}\right.$ y $\left.10^{\circ}\right)$}

\section{Configuración filtros-conmutados}

La configuración típica filtros-conmutados se parece bastante a la configuración líneas-conmutadas, pero en el primer caso, el desfase entre los dos estados se consigue mediante la conmutación entre una red paso-bajo (comportamiento de retardo de fase) y una red paso-alto (comportamiento de adelanto de fase), en lugar de líneas de transmisión con longitudes diferentes. La principal ventaja de este enfoque es el tamaño reducido, gracias a la implementación de las redes de los filtros con elementos concentrados.

Para el diseño aquí considerado, esta configuración no implicaría mejoras en cuanto a las pérdidas de inserción, ya que la principal contribución a las mismas viene de los SPDTs y con la configuración normal filtros-conmutados se necesitan el mismo número de SPDTs.

Por esta razón, se buscó una variante de la configuración filtros-conmutados [Dev93, Kom91]; se trata de una configuración tipo filtros-conmutados reconfigurable, donde se utilizan conmutadores PHEMTs para reconfigurar la red de un único filtro entre un estado paso-alto y un estado paso-bajo (Fig. 3.15). En lugar de conmutar la señal entre dos redes diferentes (paso-bajo/paso-alto), se dispone de una única red, en la cual, mediante una conmutación adecuada, se consigue una estructura paso-bajo o paso-alto.

Como se muestra en la figura 3.15a el circuito se compone de tres PHEMTs, dos inductancias de pequeño valor $\left(L_{1}\right.$ y $L_{2}$, implementadas como líneas de transmisión de alta impedancia, $L T_{1}$ y $\left.L T_{2}\right)$ más un inductor en espiral $\left(L_{3}\right)$. A continuación se explica el modo de funcionamiento:

Estado paso-alto Los conmutadores $\mathrm{PHEMT}_{1}$ y $\mathrm{PHEMT}_{2}$ están en estado ON, mientras el conmutador $\mathrm{PHEMT}_{3}$ está en estado OFF. La señal se enruta através de $\mathrm{PHEMT}_{1}$ y de $L T_{1}+L T_{2}$. El camino paralelo con $\mathrm{PHEMT}_{2}$, $\mathrm{PHEMT}_{3}$ y $L_{3}$ ofrecería un circuito abierto ya que el valor de $L_{3}$ se elige para que resuene en paralelo con la capacidad drenador-fuente $\left(C_{d s}\right)$ de $\mathrm{PHEMT}_{3}$. 
a)

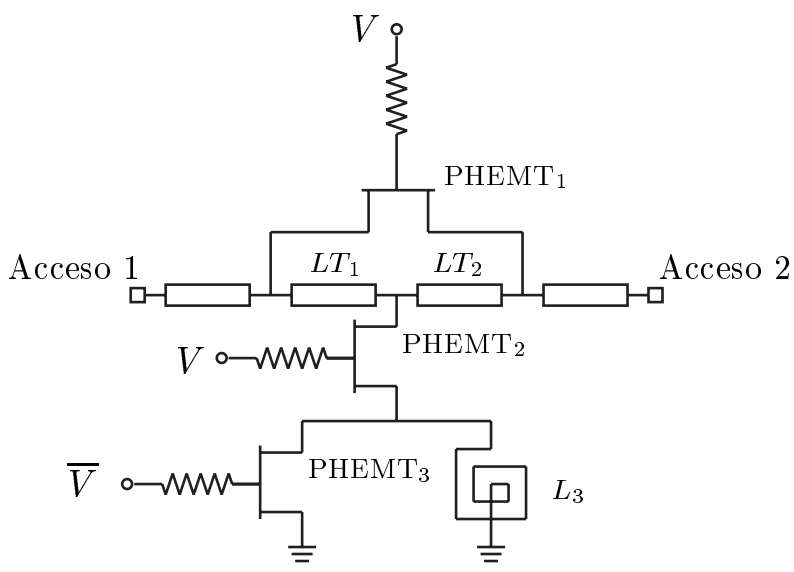

b)

c)

Acc. 1

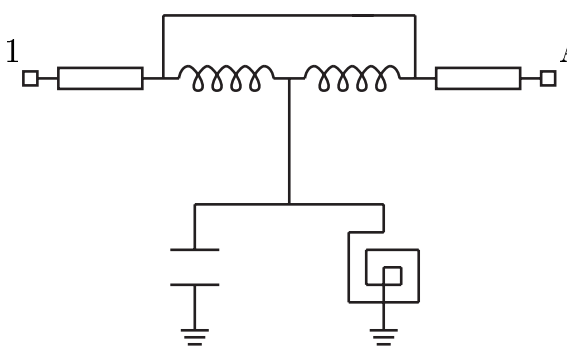

Acc. 2 Acc. 1

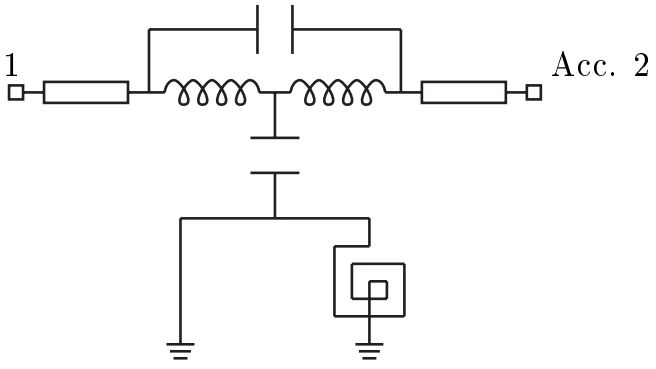

Figura 3.15: Desfasador variable con configuración filtros-conmutados reconfigurable. a) Circuito. b) Red equivalente en estado paso-alto. c) Red equivalente en estado paso-bajo.

Estado paso-bajo Los conmutadores PHEMT $_{1}$ y $\mathrm{PHEMT}_{2}$ están en estado OFF, mientras el conmutador $\mathrm{PHEMT}_{3}$ está en estado $\mathrm{ON}$. La señal se dirige a través del filtro paso-bajo formado por $L_{1}+L_{2}+C_{d s}\left(\mathrm{PHEMT}_{2}\right)$. El conmutador $\mathrm{PHEMT}_{3}$ suministra la conexión a tierra de esta red.

Se obtienen unas prestaciones peores que un desfasador filtros-conmutados típico y el diseño es bastante arriesgado debido a que los PHEMTs se usan como parte de los filtros, de forma que, los elementos parásitos, deben estar muy bien modelados. Además, como todos los elementos influyen en ambos estados, se dispone de menos parámetros libres para optimizar por separado ambos estados. Por otro lado, el diseño presenta un menor tamaño y las pérdidas de inserción son bastante más pequeñas.

\section{Diseño de cada dígito}

Todos los temas relacionados con el modelo del conmutador ya se han tratado en la sección 3.3.4. Con el fin de elegir el tamaño de los tres PHEMTs se deben tener en 
cuenta diversas consideraciones:

PHEMT1 En el estado paso-alto, este elemento permite el paso a través de toda la señal, mientras que en el estado paso-bajo debe mostrar un circuito abierto. Criterio de diseño: compromiso entre las bajas pérdidas para el estado pasoalto (dispositivo con anchura de puerta grande) y el alto aislamiento para el estado paso-bajo (dispositivo con anchura de puerta pequeña).

PHEMT2 En el estado paso-alto, el comportamiento de este elemento debe ser el de un resistor de pequeño valor, aún cuando no es importante si este valor no es excesivamente pequeño, ya que se encuentra en serie con una red LC paralela resonante, la cual deberá ofrecer un circuito abierto. Por otro lado, en el estado paso-bajo, la anchura de puerta es bastante critica, ya que en este elemento en conjunción con las dos líneas inductivas $\left(L T_{1}\right.$ y $\left.L T_{2}\right)$, es responsable de crear la red filtrante paso-bajo. Criterio de diseño: selección del tamaño de acuerdo con los requisitos de la estructura paso-bajo.

PHEMT3 En el estado paso-alto, este PHEMT se encuentra en estado OFF y su capacidad $C_{d s}$ deberá resonar con el inductor en espiral $L_{3}$. Por otro lado, en el estado paso-bajo, el estado de este PHEMT será ON y su función será la de suministrar la conexión a masa de la red filtrante paso-bajo, siendo por tanto preferible un dispositivo ancho en términos de tamaño de puerta. Criterio de diseño: su tamaño está bastante relacionado con la formación de la red resonante paralela, con lo cual se requerirán bajas pérdidas y un valor de acuerdo con $L_{3}$.

Todas estas consideraciones son una primera aproximación al problema. Debido a los elementos parásitos de los dispositivos activos, la diferencia entre la primera elección y el resultado final de la optimización pudiera ser bastante grande.

Además, se estudiaron otras configuraciones:

1. Ya que el papel del $\mathrm{PHEMT}_{3}$ es, principalmente, su uso como condensador para el estado filtro paso-bajo, y en el estado filtro paso-alto, su efecto debería ser aislado por el circuito abierto ofrecido por la red resonante formada por $L_{3}$ y $C_{d s}\left(\mathrm{PHEMT}_{3}\right)$, se consideró su posible sustitución por un condensador de pequeño valor (decenas de $\mathrm{fF}$ ), el cual sería implementado con el elemento del proceso D02AH condensador MIM de pequeño tamaño/alto rendimiento que usa ambas capas dieléctricas $\left(\mathrm{Si}_{3} \mathrm{~N}_{4}\right.$ y $\left.\mathrm{SiO}_{2}\right)$. Aunque los resultados de simulación a la frecuencia central eran más que prometedores, la aparición de una resonancia indeseada cercana a la frecuencia central impide el uso de esta opción más sencilla y de menor tamaño.

2. La sustitución de la red resonante $L_{3}-\mathrm{PHEMT}_{3}$ por un único PHEMT fue también considerada. Este PHEMT podría teóricamente ofrecer los valores 
de baja resistencia/alta resistencia necesarios en los estados paso-alto y pasobajo. Los resultados de simulación muestran que las prestaciones son peores que la configuración original, sobre todo en términos de adaptación.

Debido a que el modelo del conmutador no es un modelo distribuido, debe tenerse cuidado en no añadir longitudes eléctricas extras en alguno de los dos estados (en el dígito de $32^{\circ}$, el dispositivo PHEMT $_{1}$ mide $4 \times 60=240 \mu \mathrm{m}$ de largo, lo cual equivale a la frecuencia de diseño a un desfase de alrededor de $21^{\circ}$ ). Este tema ha tenido algunas consecuencias sobre la distribución espacial. En el modelo con elementos concentrados del conmutador (Fig. 3.9), el acceso de la fuente ofrece dos posibles planos de referencia. El enfoque para eliminar este efecto, fue el de seleccionar puntos de conexión adecuados tanto en el acceso de fuente como en el de drenador.

\section{Resultados y distribuciones espaciales de los dígitos completos}

Antes de comenzar la optimización, se requería un primer boceto de la distribución espacial del desfasador completo para así poder identificar posibles restriciones debidas a la distribución de los elementos.

La distribución espacial de los componentes ha tenido una gran importancia en el diseño de los desfasadores, ya que todos los elementos son bastante pequeños y deben evitarse los acoplamientos electromagnéticos indeseados. Por ejemplo, debido a las reglas de distribución espacial del proceso, no era posible mantener iguales las longitudes de ambas líneas de transmisión $L T_{1}$ y $L T_{2}$, por lo que se escogió una anchura menor para la línea más corta con el fin de mantener inductancias equivalentes lo más próximas posible. Además, estas dos líneas se mantuvieron tan separadas entre sí como fue posible con el fin de evitar acoplamientos no deseados.

Con respecto a los retardos extra en los PHEMTs, el peor caso corresponde al dispositivo $\mathrm{PHEMT}_{1}$, ya que presenta la puerta de mayores dimensiones. Como tanto la línea de entrada, como la de salida de cada dígito, están conectadas a los accesos de fuente y drenador de este PHEMT, la solución adoptada fue la de situar la línea de entrada y la de salida en extremos diferentes del PHEMT (Figs. 3.16 y 3.18), de forma que en ambos estados, la señal pasará a través de la longitud del PHEMT en su totalidad. Finalmente, este enfoque no pudo mantenerse en el dígito de $18^{\circ}$ (Fig. 3.16) debido a restricciones sobre la distribución espacial del desfasador variable completo.

En cuanto al $\mathrm{PHEMT}_{2}$, la figura 3.15a muestra que, tanto el transistor $\mathrm{PHEMT}_{3}$ como la inductancia $L_{3}$ están conectadas a uno de sus accesos (fuente o drenador). Se escogió el acceso fuente, de forma que estos dos elementos se mantenían apartados entre sí mediante la conexión de ambos a un plano de referencia de la fuente diferente. De esta forma, se evitaron posibles acoplamientos electromagnéticos inesperados.

Todos los parámetros de los elementos se consideraron optimizables, excepto la longitud de ambas líneas de alta impedancia ( $L T_{1}$ y $L T_{2}$ en Figura 3.15), los cuales 


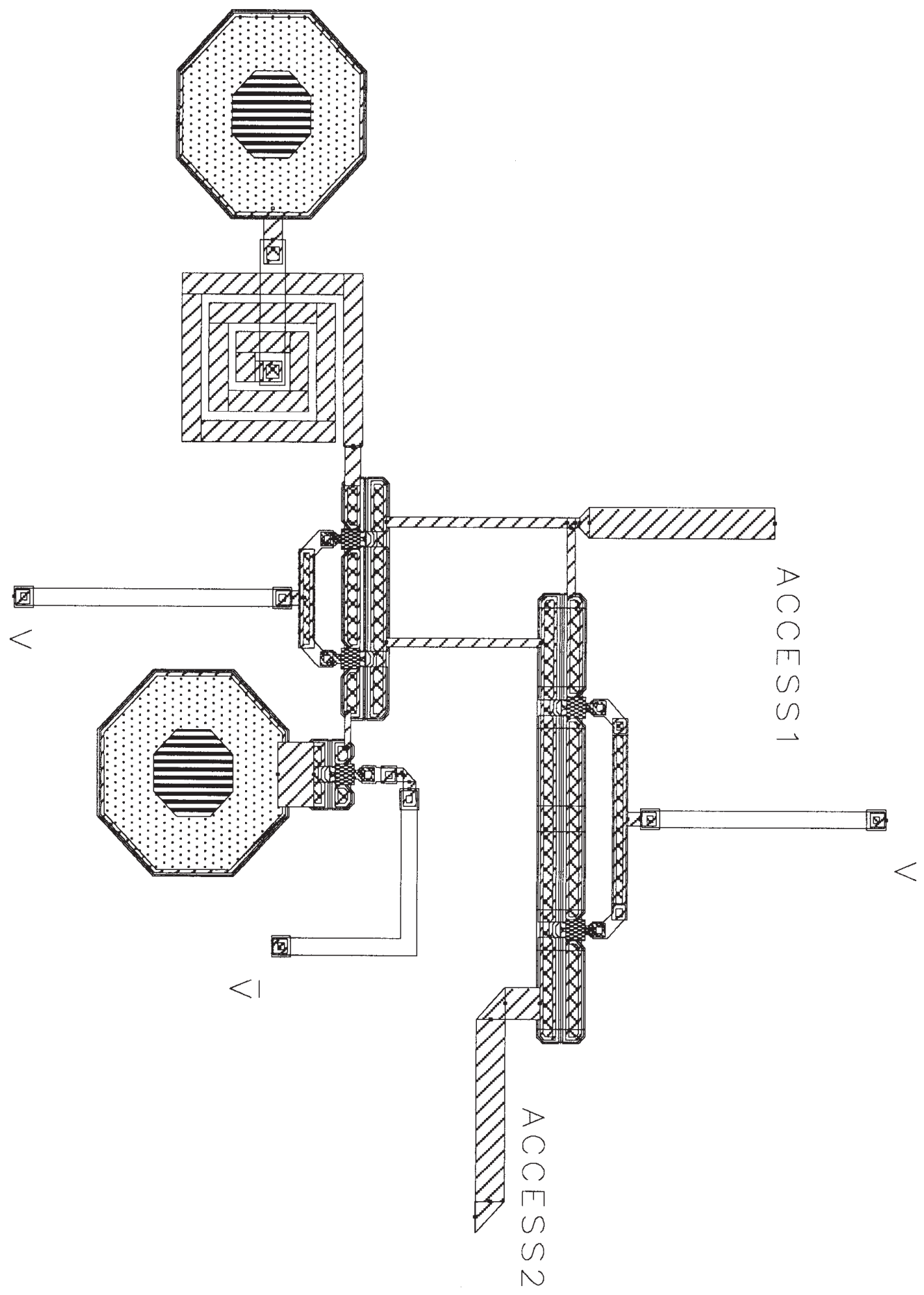

Figura 3.16: Distribución espacial del dígito de $32^{\circ}$ con configuración filtros-conmutados. 


\begin{tabular}{|c|c|c|c|c|c|}
\hline \multirow[b]{2}{*}{$\begin{array}{c}\text { Frecuencia } \\
\qquad(\mathrm{GHz})\end{array}$} & \multicolumn{2}{|c|}{ Estado PASO-ALTO } & \multicolumn{2}{|c|}{ Estado PASO-BAJO } & \multirow[b]{2}{*}{ Desfase } \\
\hline & $\begin{array}{l}\text { Pérdidas de } \\
\text { retorno }\end{array}$ & $\begin{array}{c}\text { Pérdidas de } \\
\text { inserción }\end{array}$ & $\begin{array}{l}\text { Pérdidas de } \\
\text { retorno }\end{array}$ & $\begin{array}{l}\text { Pérdidas de } \\
\text { inserción }\end{array}$ & \\
\hline 23.15 & $25.5 \mathrm{~dB}$ & $1.2 \mathrm{~dB}$ & $21.5 \mathrm{~dB}$ & $1.1 \mathrm{~dB}$ & $31^{\circ}$ \\
\hline 24.15 & $23.5 \mathrm{~dB}$ & $1.2 \mathrm{~dB}$ & $20 \mathrm{~dB}$ & $1.2 \mathrm{~dB}$ & $31.8^{\circ}$ \\
\hline 25.15 & $22 \mathrm{~dB}$ & $1.25 \mathrm{~dB}$ & $18.5 \mathrm{~dB}$ & $1.3 \mathrm{~dB}$ & $32.6^{\circ}$ \\
\hline
\end{tabular}

Tabla 3.13: Resultados de simulación del dígito de $32^{\circ}$ tipo filtros-conmutados.

venían limitados por la necesidad de una separación segura entre los transistores PHEMT $_{1}$ y PHEMT $_{2}$.

Los objetivos de optimización para todos los tres dígitos eran:

- Pérdidas de inserción tan bajas como sea posible.

- Pérdidas de inserción iguales para ambos estados.

- Respuesta de fase en función de la frecuencia tan plana como sea posible y con el valor correcto a la frecuencia central.

- Pérdidas de retorno por encima de $20 \mathrm{~dB}$ para ambos estados, de forma que no hubiera interacción entre los diferentes dígitos, cuando estos fueran colocados en cascada en el desfasador variable completo.

Cuanto mayor era el desfase requerido, más altos eran los valores de las inductancias $L_{1}$ y $L_{2}$, lo que permitía el uso de líneas estrechas y altamente inductivas. En cuanto al dígito de $10^{\circ}$, los valores para estas inductancias eran bastante pequeños, por lo que se necesitaron líneas más anchas (Fig. 3.18) para mantener una separación de seguridad entre los transistores $\mathrm{PHEMT}_{1}$ y $\mathrm{PHEMT}_{2}$. Estas líneas anchas pudieran implicar acoplamientos indeseados, aunque estos han sido evitados en la medida de lo posible.

El dígito de $32^{\circ}$ presenta los valores de inductancias más grandes, por lo que los elementos parásitos de los PHEMTs son relativamente menos importantes. Además, este dígito es el de mayor tamaño, lo que equivale a decir que se esperan menos acoplamientos electromagnéticos no previstos. Todas estas razones son las que han llevado a elegir el dígito de $32^{\circ}$ para ser incluido como circuito independiente en la oblea [CG95a, CG95b].

En las tablas 3.13, 3.14 y 3.15 se presenta un breve resumen de los resultados de los dígitos individuales con configuración filtros-conmutados, pudiéndose consultar unos gráficos más detallados en el informe [CG95a].

Los tres dígitos tipo filtros-conmutados muestran unas buenas prestaciones. Los resultados son bastante similares, con la única exception de las mayores pérdidas de inserción para los dígitos de mayor tamaño. Brevemente: 


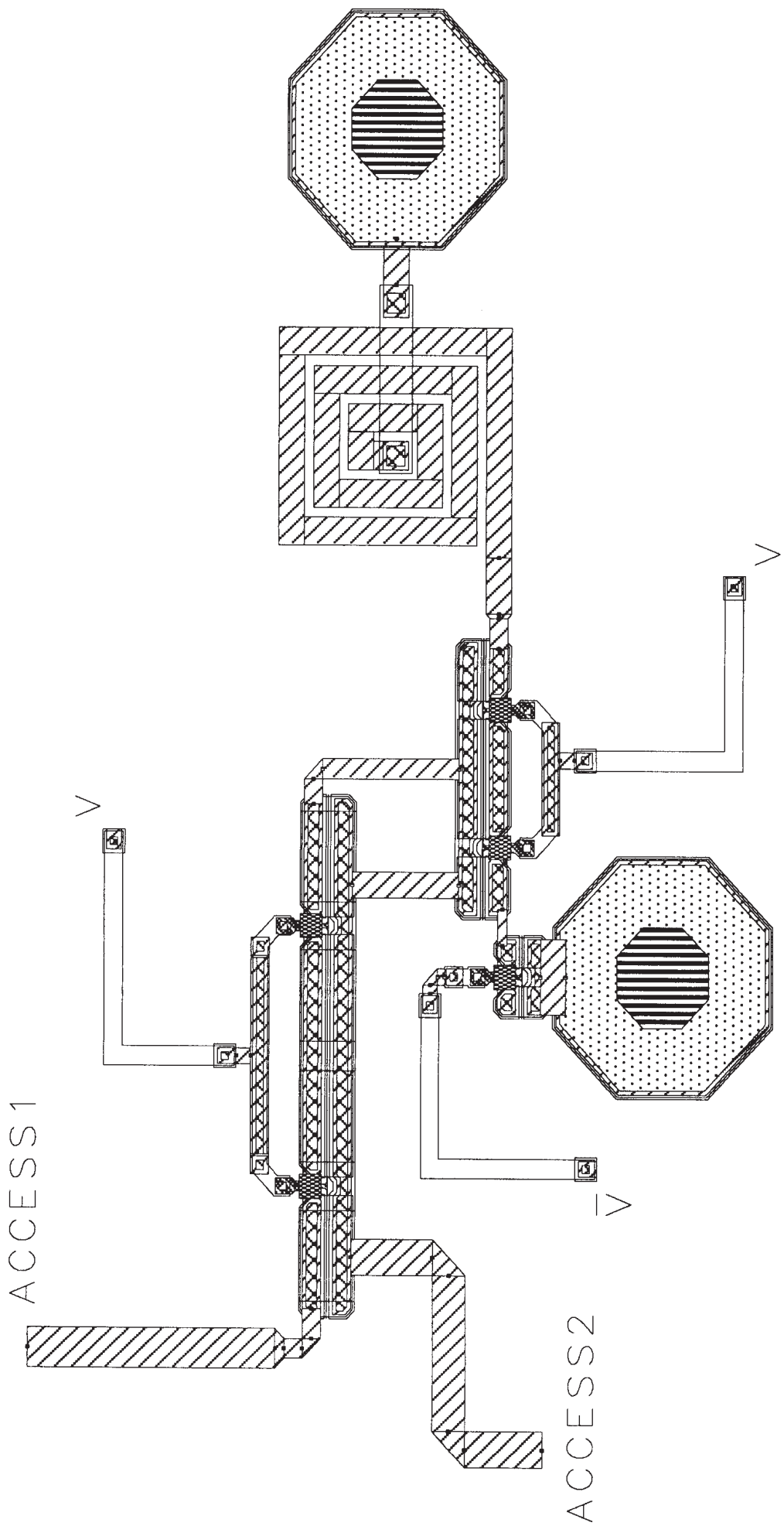

Figura 3.17: Distribución espacial del dígito de $18^{\circ}$ con configuración filtros-conmutados. 


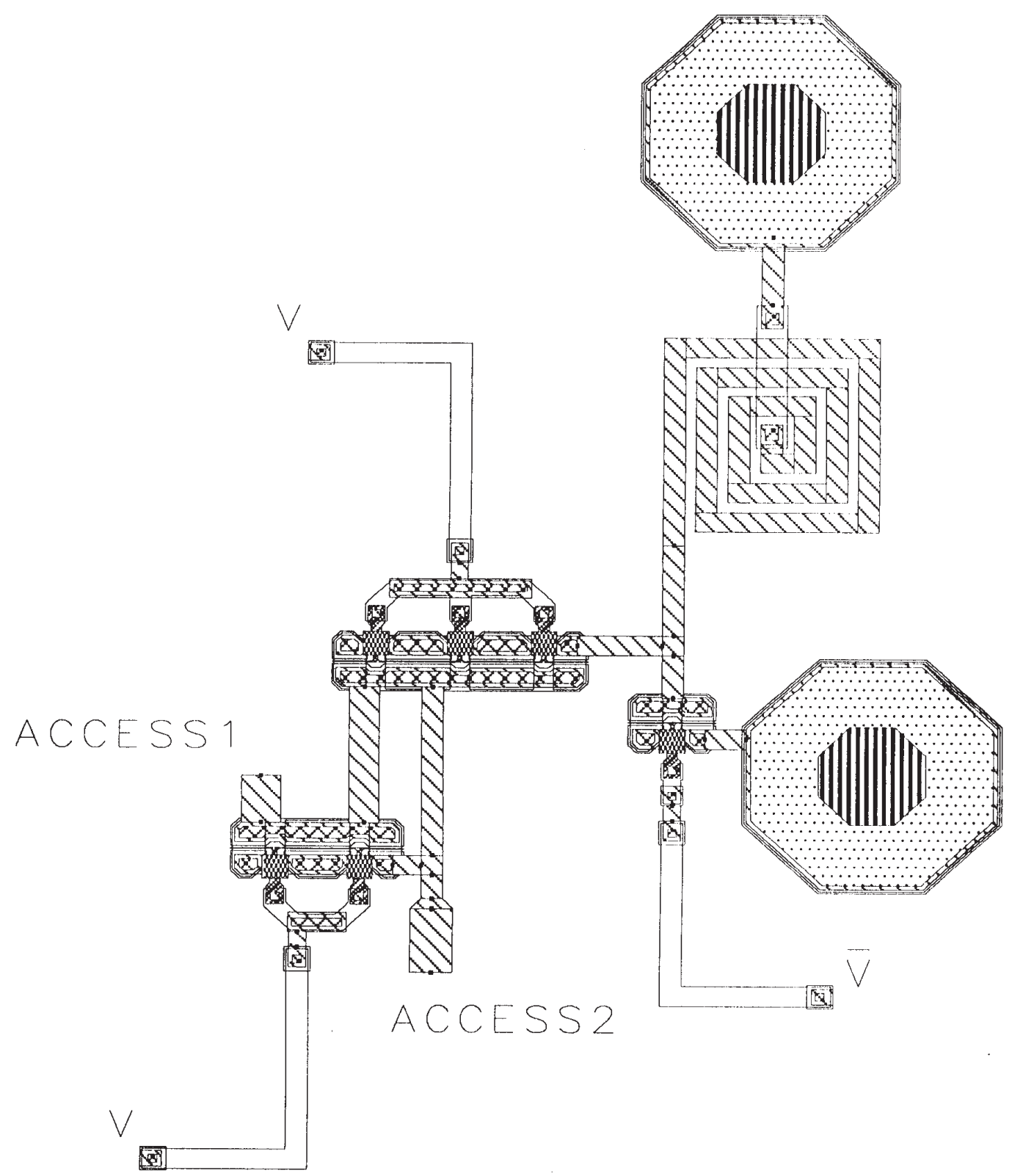

Figura 3.18: Distribución espacial del dígito de $10^{\circ}$ con configuración filtros-conmutados.

\begin{tabular}{|c|c|c|c|c|c|}
\hline \multirow[b]{2}{*}{$\begin{array}{c}\text { Frecuencia } \\
\qquad(\mathrm{GHz})\end{array}$} & \multicolumn{2}{|c|}{ Estado PASO-ALTO } & \multicolumn{2}{|c|}{ Estado PASO-BAJO } & \multirow[b]{2}{*}{ Desfase } \\
\hline & $\begin{array}{c}\text { Pérdidas de } \\
\text { retorno }\end{array}$ & $\begin{array}{l}\text { Pérdidas de } \\
\text { inserción }\end{array}$ & $\begin{array}{l}\text { Pérdidas de } \\
\text { retorno }\end{array}$ & $\begin{array}{c}\text { Pérdidas de } \\
\text { inserción }\end{array}$ & \\
\hline 23.15 & $25 \mathrm{~dB}$ & $0.95 \mathrm{~dB}$ & $21 \mathrm{~dB}$ & $0.9 \mathrm{~dB}$ & $18.4^{\circ}$ \\
\hline 24.15 & $22 \mathrm{~dB}$ & $0.95 \mathrm{~dB}$ & $21 \mathrm{~dB}$ & $0.95 \mathrm{~dB}$ & $17.9^{\circ}$ \\
\hline 25.15 & $19.5 \mathrm{~dB}$ & $1 \mathrm{~dB}$ & $21 \mathrm{~dB}$ & $1 \mathrm{~dB}$ & $17.5^{\circ}$ \\
\hline
\end{tabular}

Tabla 3.14: Resultados de simulación del dígito de $18^{\circ}$ tipo filtros-conmutados. 


\begin{tabular}{|c|c|c|c|c|c|}
\hline \multirow[b]{2}{*}{$\begin{array}{c}\text { Frecuencia } \\
\qquad(\mathrm{GHz})\end{array}$} & \multicolumn{2}{|c|}{ Estado PASO-ALTO } & \multicolumn{2}{|c|}{ Estado PASO-BAJO } & \multirow[b]{2}{*}{ Desfase } \\
\hline & $\begin{array}{l}\text { Pérdidas de } \\
\text { retorno }\end{array}$ & $\begin{array}{c}\text { Pérdidas de } \\
\text { inserción }\end{array}$ & $\begin{array}{c}\text { Pérdidas de } \\
\text { retorno }\end{array}$ & $\begin{array}{c}\text { Pérdidas de } \\
\text { inserción }\end{array}$ & \\
\hline 23.15 & $23.5 \mathrm{~dB}$ & $0.7 \mathrm{~dB}$ & $20 \mathrm{~dB}$ & $0.65 \mathrm{~dB}$ & $10.9^{\circ}$ \\
\hline 24.15 & $23.5 \mathrm{~dB}$ & $0.7 \mathrm{~dB}$ & $20 \mathrm{~dB}$ & $0.7 \mathrm{~dB}$ & $10^{\circ}$ \\
\hline 25.15 & $28 \mathrm{~dB}$ & $0.7 \mathrm{~dB}$ & $20 \mathrm{~dB}$ & $0.7 \mathrm{~dB}$ & $9.4^{\circ}$ \\
\hline
\end{tabular}

Tabla 3.15: Resultados de simulación del dígito de $10^{\circ}$ tipo filtros-conmutados.

- Bajas pérdidas de inserción.

- Pérdidas de inserción diferenciales muy bajas.

- Muy buena adaptación (20 dB de pérdidas de retorno para el peor caso).

- Respuesta de fase bastante plana como se esperaba en un desfasador tipo filtros-conmutados.

\subsubsection{Diseño completo}

\section{Selección de la configuración del Desfasador Variable}

Después de completar el diseño de los dígitos individuales, se determinó la configuración óptima para el desfasador variable de seis dígitos mediante el análisis del comportamiento del desfasador para la totalidad de sus 64 estados de fase; considerando todas las posibles ordenaciones de los dígitos, lo que significa 360 configuraciones diferentes. El propósito de este análisis es la determinación de los efectos sobre el comportamiento del desfasador variable completo, en términos de pérdidas y de desfase de las complejas interacciones que se dan entre las diversas desadaptaciones de los dígitos individuales, en los 64 estados de cada configuración; incluso, aunque se tuvo gran cuidado en el diseño de cada dígito individual persiguiéndose una buena adaptación para ambos estados de fase.

La comparación de los resultados de simulación para las 360 diferentes configuraciones, con 64 estado cada una de ellas, conlleva una ingente cantidad de cálculo numérico. Para ello, se partió de los datos de simulación en formato de parámetros $\mathrm{S}$ para cada uno de los seis dígitos individuales (para ambos estados en cada caso), limitados a cinco valores de frecuencia $(22.15 \mathrm{GHz}, 23.15 \mathrm{GHz}, 24.15 \mathrm{GHz}, 25.15 \mathrm{GHz}$ y $26.15 \mathrm{GHz}$ ), a partir de los cuales se obtuvo la matriz de parámetros $\mathrm{S}$ de la red total para cada combinación de las 360 configuraciones y los 64 estados posibles, seleccionando la configuración más adecuada.

Para cada una de las 360 ordenaciones de los seis dígitos individuales, se calcularon, a la frecuencia de diseño, los siguientes datos para el desfasador variable completo: 


\begin{tabular}{|c|c|c|c|c|}
\hline & $\overline{S_{11}}$ & $\overline{S_{22}}$ & $\begin{array}{c}S_{21} \\
\text { Diferencial }\end{array}$ & $\begin{array}{c}\text { Error de fase } \\
\text { (respecto nominal) }\end{array}$ \\
\hline \hline Mejor caso & $-27.4 \mathrm{~dB}$ & $-28.5 \mathrm{~dB}$ & $0.17 \mathrm{~dB}$ & $0.6^{\circ}$ \\
\hline Peor caso & $-17.7 \mathrm{~dB}$ & $-18.5 \mathrm{~dB}$ & $0.57 \mathrm{~dB}$ & $1.1^{\circ}$ \\
\hline
\end{tabular}

Tabla 3.16: Comparativa de los resultados medios del desfasador variable completo, para la mejor y la peor ordenación de los seis dígitos individuales.

- Valores máximos de $S_{11}$ y $S_{22}$.

- Media de los parámetros $S_{11}$ y $S_{22}$.

- Máxima diferencia de las pérdidas de inserción, considerando los 64 estados.

- Error de fase (respecto al valor nominal).

Las 360 configuraciones se ordenaron según los parámetros arriba mencionados y la selección fue hecha mediante un compromiso entre los mismos. Todas las simulaciones necesarias tuvieron una duración total de varias horas, al ejecutar las funciones al efecto sobre una estación de trabajo SPARCstation 10 de SUN Microsystems.

La configuración elegida fue:

$$
180^{\circ}-101^{\circ}-10^{\circ}-18^{\circ}-32^{\circ}-57^{\circ}
$$

Como podía esperarse, los dígitos con pérdidas de inserción mayores $\left(180^{\circ}\right.$ y $57^{\circ}$ respectivamente) aparecen a ambos extremos del desfasador variable, de forma que, posibles señales reflejadas internamente sufrirán una atenuación mínima de $6 \mathrm{~dB}$ (pérdidas asociadas al paso de ida y vuelta de la señal a través de los dígitos de $180^{\circ}$ o de $\left.57^{\circ}\right)$.

Como demostración de la importancia de una correcta ordenación de los dígitos del desfasador variable, se muestran, en la tabla 3.16, el mejor y el peor caso para cada uno de los parámetros que han sido utilizados como criterios de selección. La diferencia observada en cuanto a los valores medios (referidos a los 64 diferentes estados) de los parámetros de adaptación, es bastante importante (alrededor de 10 dB) y también lo es en el máximo valor de pérdidas de inserción diferenciales. En cuanto al error de fase, éste no es tan importante gracias a la robustez del diseño y la pequeña diferencia entre el peor y mejor caso.

\section{Distribución espacial del desfasador variable y resultados de simulación}

Una vez la configuración estaba ya elegida, el siguiente paso consistía en la modificación de las redes de adaptación de entrada en los dígitos situados en los extremos del 
desfasador (dígitos de $180^{\circ}$ y $57^{\circ}$ ), con el fin de tener en cuenta los efectos debidos al recuadro de conexión ${ }^{\mathrm{i}}$ y a los hilos de conexión ${ }^{\mathrm{ii}}$.

El modelo del recuadro de conexión suministrado por PML no es distribuido, por lo que se ha añadido al modelo un trozo de línea de transmisión, con la anchura del recuadro y la longitud de medio recuadro (asumiendo que los hilos de conexión van a ser soldados sobre el centro del recuadro). Asímismo, se ha eliminado de la fórmula de la capacidad total del recuadro, la capacidad correspondiente a la línea añadida. También, la parte proporcional del périmetro del recuadro que está en contacto con la línea que accede al mismo, ha sido eliminado del valor del perímetro total, con el fin de calcular el valor de la capacidad equivalente total.

Algunas modificaciones menores sobre la distribución espacial de todos los dígitos fueron necesarias para interconectarlos correctamente, evitando discontinuidades en las anchuras de línea, acoplos indeseados y líneas de interconexión demasiado largas. Una vez los dígitos externos estaban fijados completamente, se volvieron a ordenar los cuatro dígitos restantes.

El orden final es:

$$
180^{\circ}-101^{\circ}-18^{\circ}-10^{\circ}-32^{\circ}-57^{\circ}
$$

Después de todas las modificaciones, los resultados de simulación del desfasador variable completo (incluido el efecto de los hilos de conexión) se muestran en las figuras 3.19 y 3.20 para todos los 64 estados de fase. La distribución espacial final del desfasador variable, puede observarse en la figura 3.21.

De acuerdo con las simulaciones, todas las especificaciones han sido cubiertas:

- Las pérdidas de retorno a la entrada y a la salida del circuito son mejores que $15 \mathrm{~dB}$ para todos los 64 estados, con valores medios de $21 \mathrm{~dB}$ y $23 \mathrm{~dB}$ respectivamente. El ancho de banda a $10 \mathrm{~dB}$ de pérdidas de retorno es de alrededor de $4 \mathrm{GHz}$, ofreciendo una cierta banda de seguridad, frente a posibles variaciones del proceso.

- Las pérdidas de inserción son menores de $12 \mathrm{~dB}$, incluso para el peor estado. La diferencia en pérdidas de inserción entre el peor y el mejor caso es de sólo $0.6 \mathrm{~dB}$.

- El rango de fases y la resolución en desfase, se cumplen perfectamente gracias al enfoque no binario del diseño y al pequeño error de desfase medio.

- El diseño es un desfasador variable de banda estrecha centrado a $24.15 \mathrm{GHz}$, con una impedancia nominal de $50 \Omega$.

\footnotetext{
${ }^{\mathrm{i}}$ Bonding Pad, en terminología inglesa.

ii Bonding Wires, en terminología inglesa.
} 


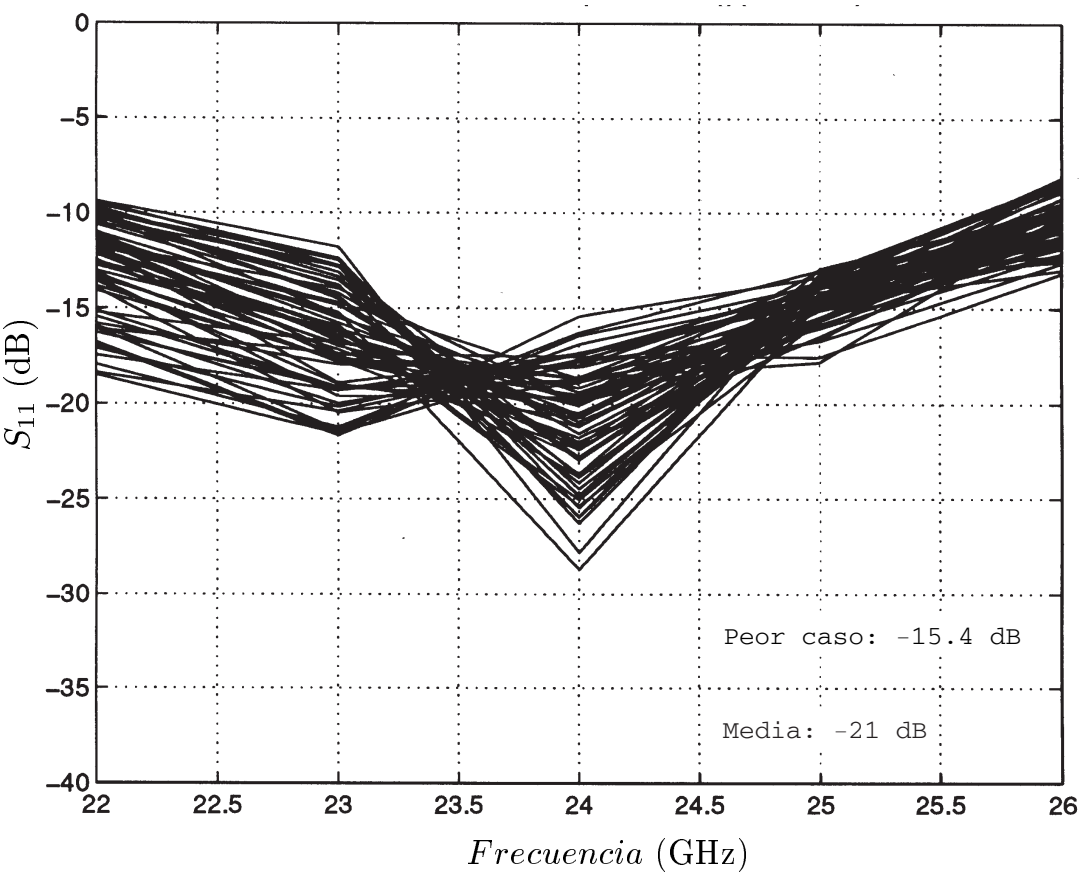

a)

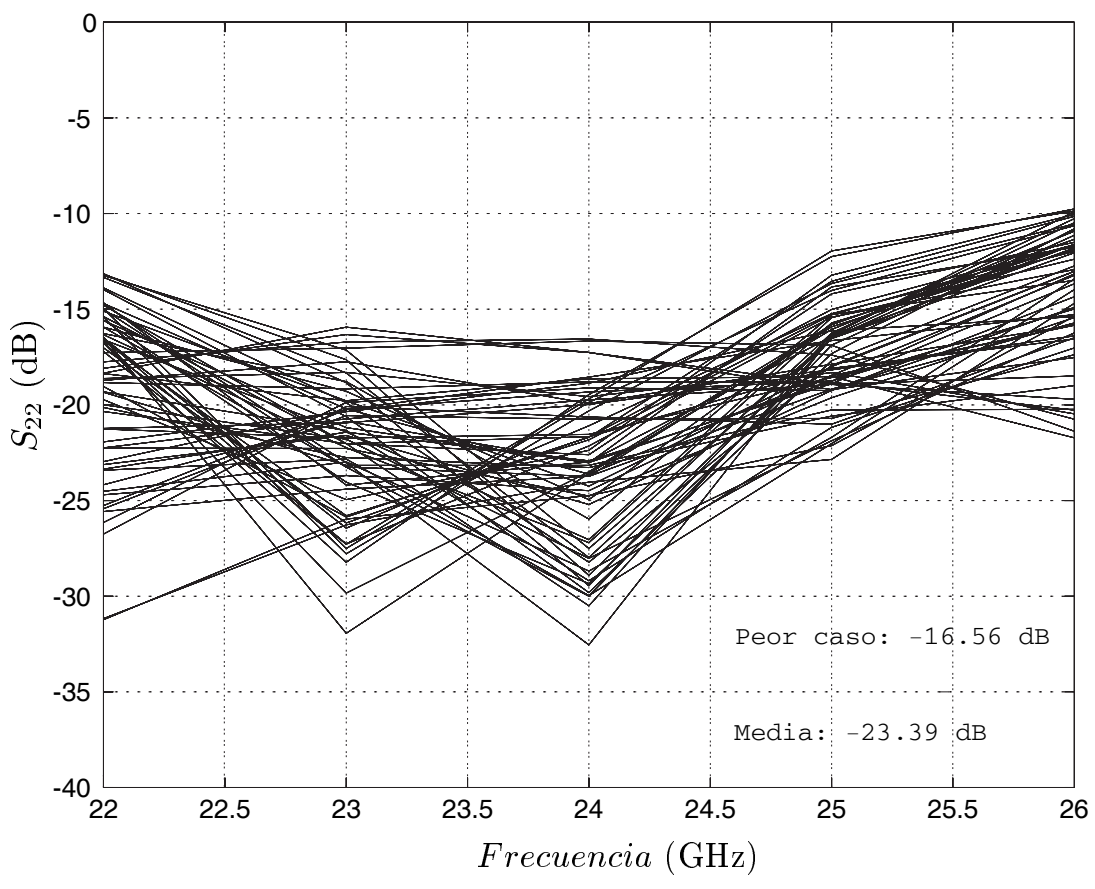

Figura 3.19: Adaptación del desfasador variable completo, incluyendo el efecto de los hilos de conexión (64 estados). a) Adaptación a la entrada. b) Adaptación a la salida. 


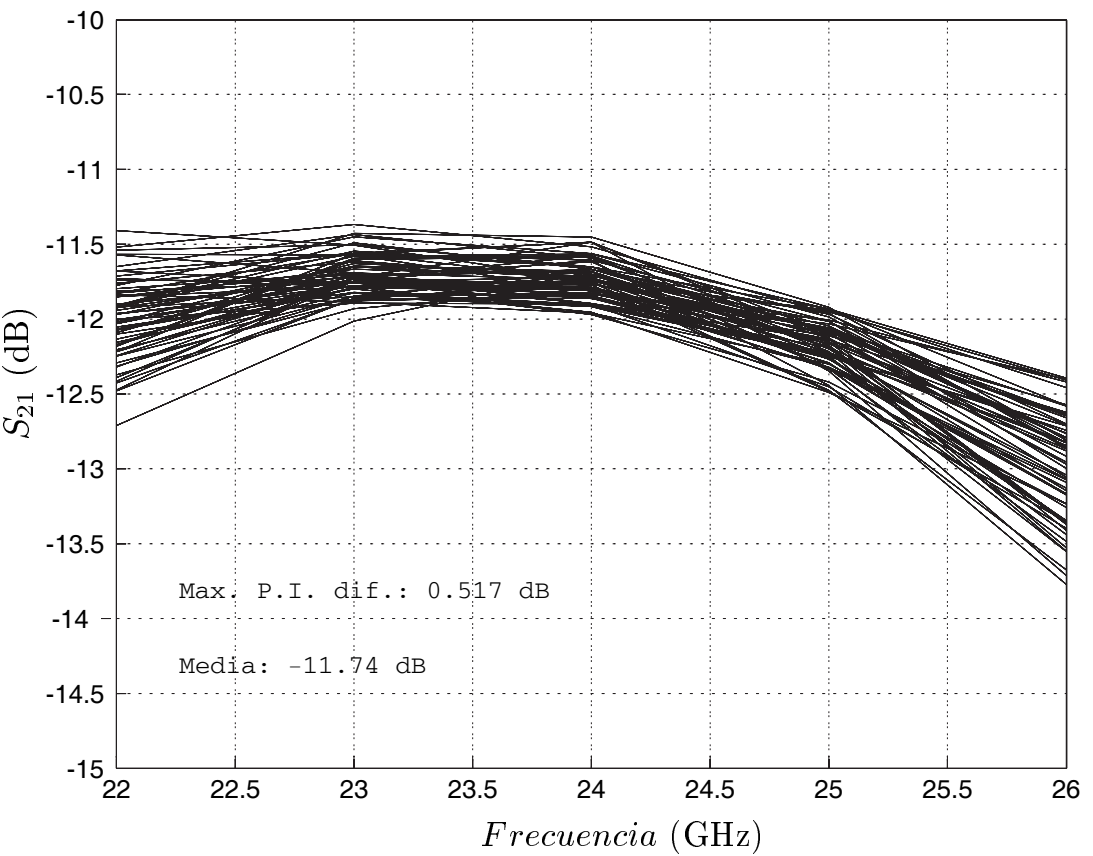

a)

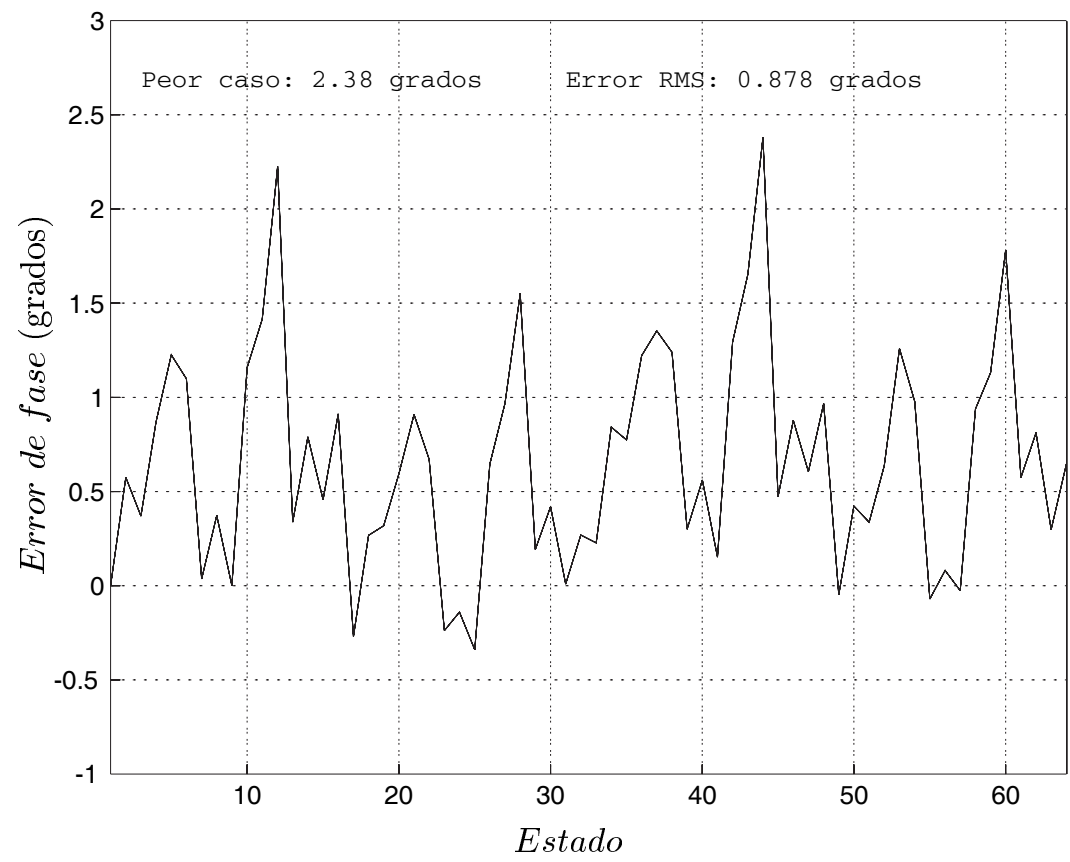

b)

Figura 3.20: Resultados de simulacion del desfasador variable completo incluyendo el efecto de los hilos de conexión (64 estados). a) Pérdidas de inserción. b) Error de fase respecto al desfase nominal para la frecuencia central. 


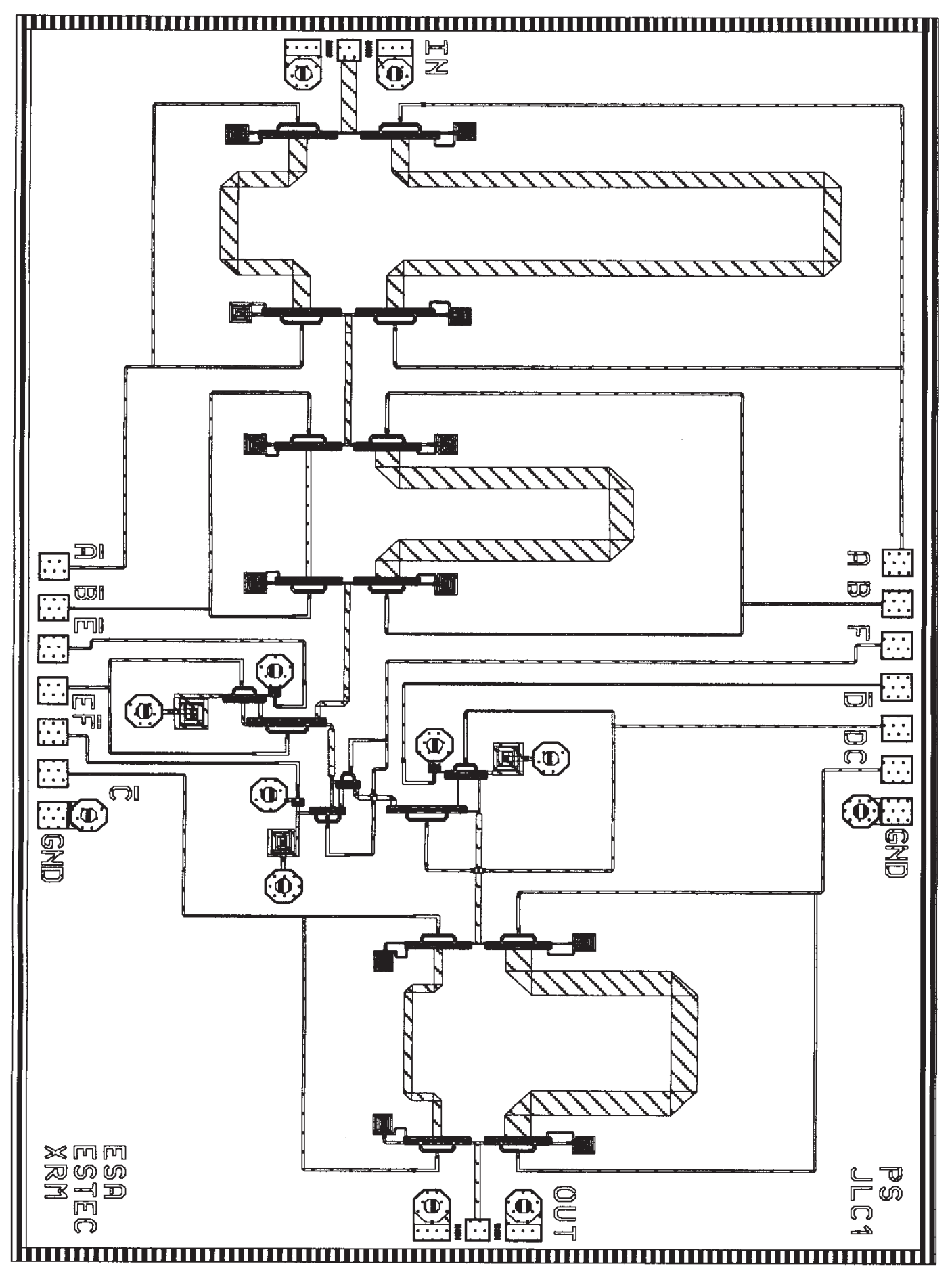

Figura 3.21: Distribución espacial del desfasador variable a 24 GHz. 


\section{Montaje}

A continuación se ofrece una serie de datos generales, concernientes al montaje del circuito integrado.

Tamaño $4.5 \mathrm{~mm}$ x $3 \mathrm{~mm}$ (incluyendo los márgenes para el corte establecidos en el proceso, es decir un tamaño de $\mathbf{4 . 4} \mathbf{~ m m ~ x ~} 2.9 \mathrm{~mm}$ para el circuito cortado).

Hilos de conexión Un único hilo sobre cada recuadro de conexión.

diámetro $25 \mu \mathbf{m}$

longitud $250 \mu \mathbf{m}$

(Para estos valores, no se necesita compensación externa de la inductancia del hilo).

Polarización Para cada dígito las tensiones de control son:

ON) 0 Voltios.

OFF) -2 Voltios.

(No son necesarios circuitos de bloqueo de la radiofrecuencia, pues se han incluido en el circuito integrado resistencias de $3 \mathrm{k} \Omega$ para cada línea de polarización). 


\subsubsection{Resultados de simulación para las medidas sobre la oblea del desfasador variable}

Los datos de simulación del desfasador variable sin los hilos de conexión, se muestran a continuación para los catorce estados especificados en la tabla 3.17, para ser medidos sobre la oblea. Los estados seleccionados corresponden al estado de todos los dígitos en su estado 0, al estado de todos los dígitos en su estado 1, a los seis casos de que un único dígito esté a 1 y, finalmente, los seis casos en los que un único dígito está a 0 .

\begin{tabular}{|c|c|c|c|c|c|c|c|}
\hline Dígito & $180^{\circ}$ & $-101^{\circ}$ & $-57^{\circ}$ & $32^{\circ}$ & $18^{\circ}$ & $10^{\circ}$ & Desfase Ideal \\
\hline \hline estado 0 & 0 & 0 & 0 & 0 & 0 & 0 & $0^{\circ}$ \\
\hline estado 1 & 0 & 0 & 0 & 0 & 0 & 1 & $10.02^{\circ}$ \\
\hline estado 2 & 0 & 0 & 0 & 0 & 1 & 0 & $17.86^{\circ}$ \\
\hline estado 4 & 0 & 0 & 0 & 1 & 0 & 0 & $31.82^{\circ}$ \\
\hline estado 8 & 0 & 0 & 1 & 0 & 0 & 0 & $-56.69^{\circ}$ \\
\hline estado 16 & 0 & 1 & 0 & 0 & 0 & 0 & $-101.02^{\circ}$ \\
\hline estado 32 & 1 & 0 & 0 & 0 & 0 & 0 & $180^{\circ}$ \\
\hline estado 63 & 1 & 1 & 1 & 1 & 1 & 1 & $81.99^{\circ}$ \\
\hline estado 62 & 1 & 1 & 1 & 1 & 1 & 0 & $71.97^{\circ}$ \\
\hline estado 61 & 1 & 1 & 1 & 1 & 0 & 1 & $64.13^{\circ}$ \\
\hline estado 59 & 1 & 1 & 1 & 0 & 1 & 1 & $50.17^{\circ}$ \\
\hline estado 55 & 1 & 1 & 0 & 1 & 1 & 1 & $138.68^{\circ}$ \\
\hline estado 47 & 1 & 0 & 1 & 1 & 1 & 1 & $-176.99^{\circ}$ \\
\hline estado 31 & 0 & 1 & 1 & 1 & 1 & 1 & $-98.01^{\circ}$ \\
\hline
\end{tabular}

Tabla 3.17: Selección de catorce estados del desfasador variable para su medida sobre la oblea.

Los resultados de simulación para los catorce estados a la frecuencia central aparecen resumidos en la tabla 3.18, presentándose en las figuras 3.22 y 3.23 los resultados gráficos, dentro del rango de frecuencias de $22.15 \mathrm{GHz}$ a $26.15 \mathrm{GHz}$. Comparando los resultados de simulación para el circuito con los hilos de conexión (gráficas 3.19 y 3.20) con los resultados de simulación sin ellos aquí mostrados, se puede comprobar la gran influencia de la inductancia introducida por estos hilos de conexión en la adaptación del circuito integrado, para estas frecuencias de trabajo. 


\begin{tabular}{|c|c|c|c|c|}
\hline & $S_{11}$ & $S_{22}(d B)$ & $S_{21}(d B)$ & Desfase \\
\hline \hline estado 0 & $-15.2 \mathrm{~dB}$ & $-13.4 \mathrm{~dB}$ & $-12 \mathrm{~dB}$ & $0^{\circ}$ \\
\hline estado 1 & $-16 \mathrm{~dB}$ & $-15.2 \mathrm{~dB}$ & $-11.9 \mathrm{~dB}$ & $9.5^{\circ}$ \\
\hline estado 2 & $-16.1 \mathrm{~dB}$ & $-12.1 \mathrm{~dB}$ & $-12.1 \mathrm{~dB}$ & $17.7^{\circ}$ \\
\hline estado 4 & $-14.2 \mathrm{~dB}$ & $-13.3 \mathrm{~dB}$ & $-12 \mathrm{~dB}$ & $31.4^{\circ}$ \\
\hline estado 8 & $-15.4 \mathrm{~dB}$ & $-16 \mathrm{~dB}$ & $-12 \mathrm{~dB}$ & $-56.8^{\circ}$ \\
\hline estado 16 & $-16.6 \mathrm{~dB}$ & $-14.8 \mathrm{~dB}$ & $-12 \mathrm{~dB}$ & $-101.3^{\circ}$ \\
\hline estado 32 & $-13.8 \mathrm{~dB}$ & $-13.3 \mathrm{~dB}$ & $-11.8 \mathrm{~dB}$ & $179.2^{\circ}$ \\
\hline estado 63 & $-13.5 \mathrm{~dB}$ & $-21.6 \mathrm{~dB}$ & $-12 \mathrm{~dB}$ & $80.9^{\circ}$ \\
\hline estado 62 & $-14.7 \mathrm{~dB}$ & $-17.8 \mathrm{~dB}$ & $-11.9 \mathrm{~dB}$ & $70^{\circ}$ \\
\hline estado 61 & $-14.6 \mathrm{~dB}$ & $-24.9 \mathrm{~dB}$ & $-11.9 \mathrm{~dB}$ & $63.7^{\circ}$ \\
\hline estado 59 & $-12.7 \mathrm{~dB}$ & $-16.3 \mathrm{~dB}$ & $-11.8 \mathrm{~dB}$ & $48.1^{\circ}$ \\
\hline estado 55 & $-13.4 \mathrm{~dB}$ & $-12.7 \mathrm{~dB}$ & $-11.8 \mathrm{~dB}$ & $138.1^{\circ}$ \\
\hline estado 47 & $-15.2 \mathrm{~dB}$ & $-18.3 \mathrm{~dB}$ & $-11.7 \mathrm{~dB}$ & $-177.9^{\circ}$ \\
\hline estado 31 & $-15.2 \mathrm{~dB}$ & $-21.3 \mathrm{~dB}$ & $-11.9 \mathrm{~dB}$ & $-98.3^{\circ}$ \\
\hline
\end{tabular}

Tabla 3.18: Resultados de simulación (adaptación a la entrada y a la salida, pérdidas de inserción e inserción de fase), para catorce estados del desfasador variable completo, correspondientes a medidas RFOW sin hilos de conexión para una frecuencia de $24.15 \mathrm{GHz}$. 
a)
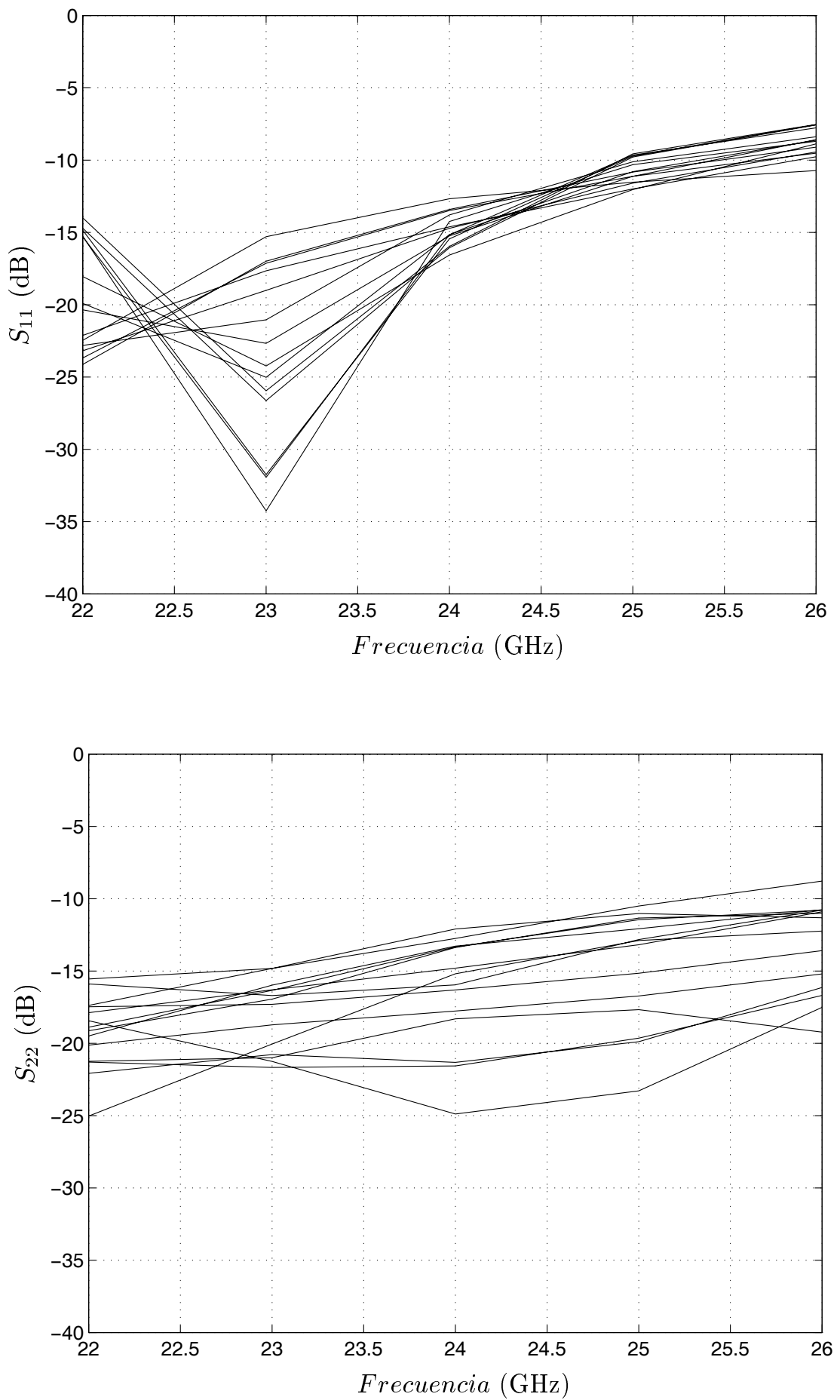

Figura 3.22: Simulación RFOW del desfasador variable para catorce estados. a) Adaptación a la entrada. b) Adaptación a la salida. 


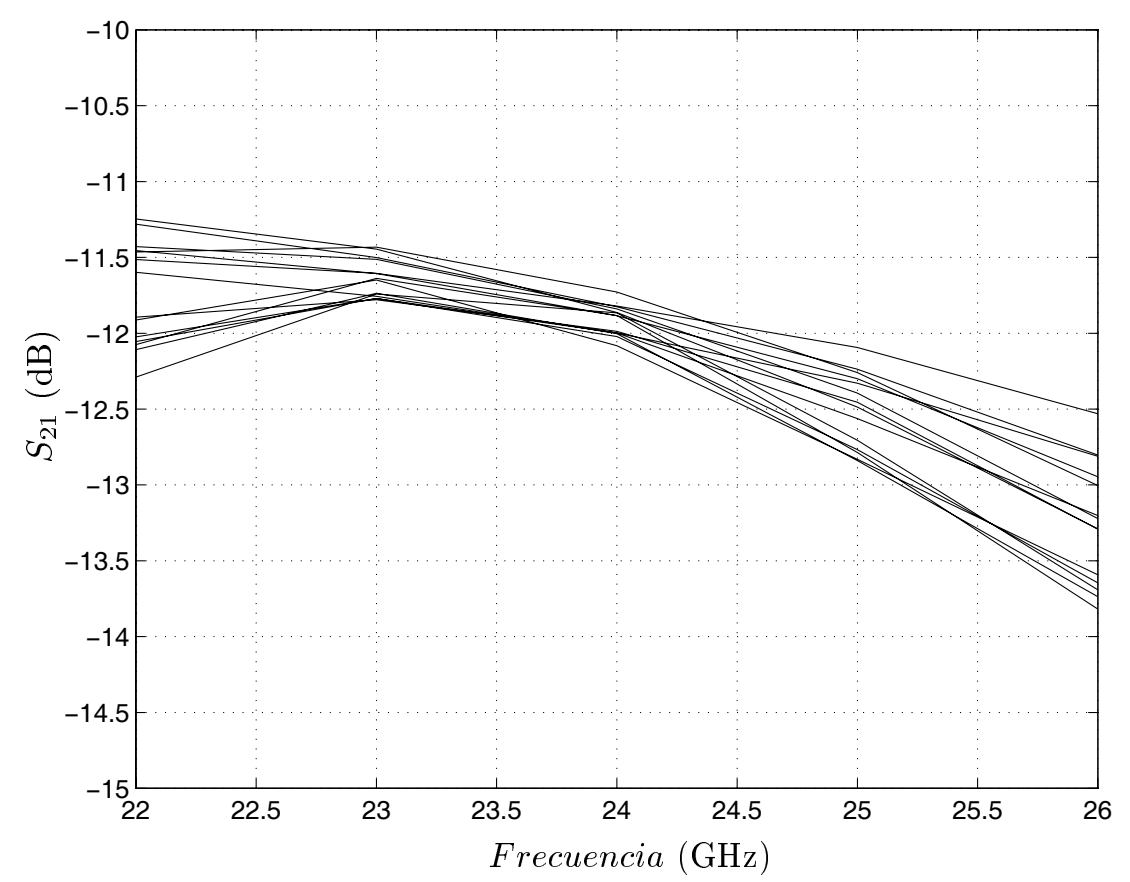

a)

b)

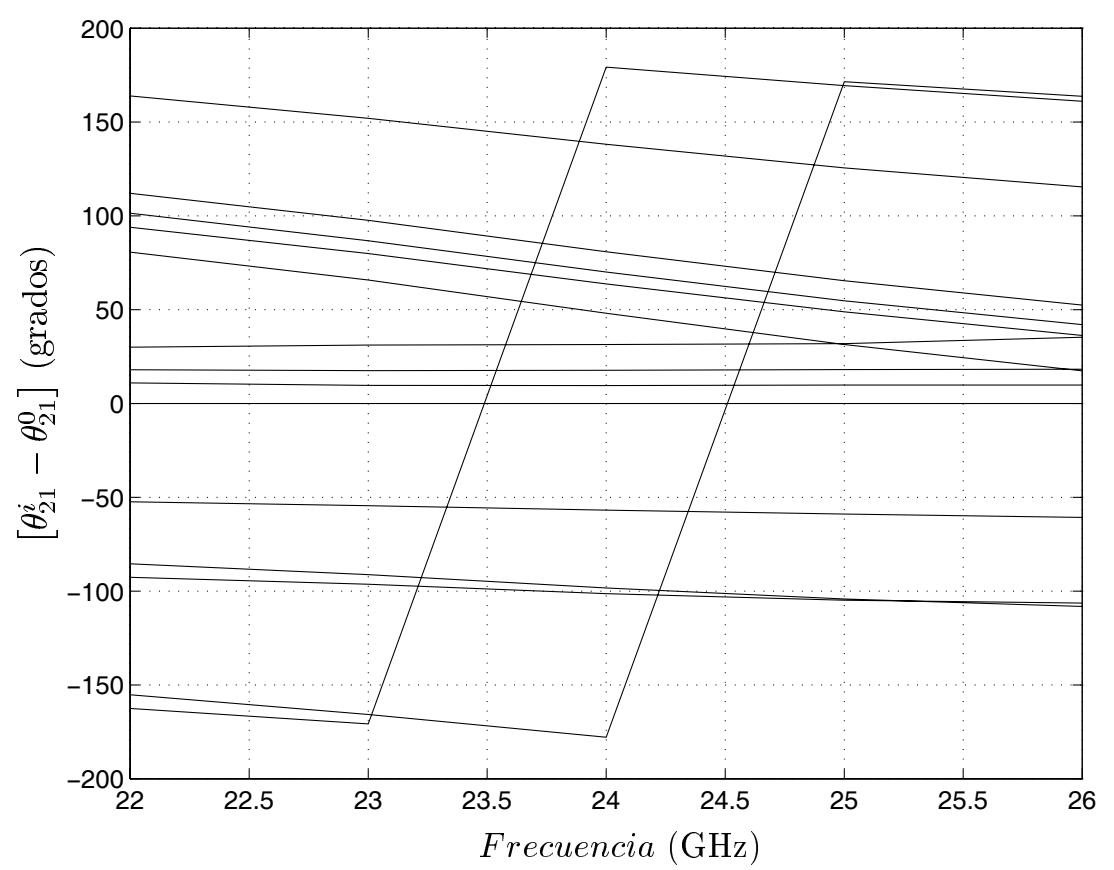

Figura 3.23: Simulación RFOW del desfasador variable para catorce estados. a) Pérdidas de inserción. b) Desfase respecto al estado 0. 


\subsection{Medidas RFOW de un desfasador variable a $24 \mathrm{GHz}$}

\subsubsection{Introducción}

En los laboratorios internos de la división XR de ESTEC, se llevaron a cabo algunas medidas de radiofrecuencia sobre la oblea $\left(\mathrm{RFOW}^{\mathrm{i}}\right)$, sobre diferentes iteraciones del desfasador variable no binario MMIC de $24 \mathrm{GHz}$. Los principales resultados se muestran en esta sección, con el fin de ser comparados con las simulaciones del apartado 3.3.7.

Para cada iteración y cada uno de los estados ${ }^{\mathrm{ii}}$ indicados en la tabla 3.17 , se midieron las pérdidas de adaptación a la entrada y a la salida del circuito integrado $\left(S_{11}, S_{22}\right)$, las pérdidas de inserción $\left(S_{21}\right)$ y la inserción de fase, en el rango de frecuencias desde 22.15 a $26.15 \mathrm{GHz}$.

\subsubsection{Medidas}

Basándose en el esquema de medidas RFOW propuesto, se midieron sobre la oblea tres iteraciones diferentes $\left(\mathrm{CCT}_{1}, \mathrm{CCT}_{2}\right.$ y $\left.\mathrm{CCT}_{3}\right)$ del mismo circuito. Los resultados de desfase, relativo al estado 0, obtenidos para estos circuitos se presentan en la tabla 3.19 y son comparados con los ideales.

Los tres dígitos más significativos (MSB) (estados: 8, 16 y 32), muestran un comportamiento realmente excelente, confirmando la elección hecha de una configuración líneas-conmutadas.

Por otro lado, los tres dígitos menos significativos (estados: 1, 2 y 4) muestran un error importante en el desfase. La falta de un modelo distribuido del PHEMT (usado como un conmutador serie), parece ser el principal motivo para este desacuerdo entre medidas y simulación. Este error confirma el riesgo asumido con la elección de la estructura reconfigurable filtros-conmutados, la cual requiere un modelo de conmutador mucho más exacto. El error en el desfase es mayor para el dígito de $18^{\circ}$, comparado con los dígitos de $32^{\circ}$ y $10^{\circ}$, debido a la diferente distribución espacial tal y como se explica en el apartado 3.3.5.

Los resultados de desfase para ambas iteraciones del desfasador son bastante

\footnotetext{
${ }^{\mathrm{i}}$ Radio-Frequency On Wafer measurements, en terminología inglesa.

${ }^{\mathrm{ii}}$ Los estados 1 y 0 de cada dígito corresponden a las siguientes tensiones de conmutación en los recuadros de alimentación de cada dígito:
}

\footnotetext{
1 Tensión en la puerta igual a 0 Voltios

0 Tensión en la puerta igual a -2 Voltios
} 


\begin{tabular}{|c|c|c|c|c|}
\hline & $\begin{array}{c}\text { Desfase } \\
\mathrm{CCT}_{1}\end{array}$ & $\begin{array}{c}\text { Desfase } \\
\mathrm{CCT}_{2}\end{array}$ & $\begin{array}{c}\text { Desfase } \\
\mathrm{CCT}_{3}\end{array}$ & $\begin{array}{c}\text { Desfase } \\
\text { Ideal }\end{array}$ \\
\hline \hline estado 0/ref. & $0^{\circ}$ & $0^{\circ}$ & $0^{\circ}$ & $0^{\circ}$ \\
\hline estado 1 & $17^{\circ}$ & $19^{\circ}$ & $17.5^{\circ}$ & $10.02^{\circ}$ \\
\hline estado 2 & $37^{\circ}$ & $39^{\circ}$ & $37^{\circ}$ & $17.86^{\circ}$ \\
\hline estado 4 & $40^{\circ}$ & $41^{\circ}$ & $40^{\circ}$ & $31.82^{\circ}$ \\
\hline estado 8 & $-57.5^{\circ}$ & $-57^{\circ}$ & $-57^{\circ}$ & $-56.69^{\circ}$ \\
\hline estado 16 & $-95^{\circ}$ & $-95^{\circ}$ & $-95^{\circ}$ & $-101.02^{\circ}$ \\
\hline estado 32 & $178^{\circ}$ & $175^{\circ}$ & $175^{\circ}$ & $180^{\circ}$ \\
\hline estado 63 & $115^{\circ}$ & $115^{\circ}$ & $114^{\circ}$ & $81.99^{\circ}$ \\
\hline estado 62 & $100^{\circ}$ & $100^{\circ}$ & $98^{\circ}$ & $71.97^{\circ}$ \\
\hline estado 61 & $79.5^{\circ}$ & $79.5^{\circ}$ & $78^{\circ}$ & $64.13^{\circ}$ \\
\hline estado 59 & $76^{\circ}$ & $76^{\circ}$ & $74^{\circ}$ & $50.17^{\circ}$ \\
\hline estado 55 & $172^{\circ}$ & $172^{\circ}$ & $170^{\circ}$ & $138.68^{\circ}$ \\
\hline estado 47 & $-146^{\circ}$ & $-146^{\circ}$ & $-147^{\circ}$ & $-176.99^{\circ}$ \\
\hline estado 31 & $-61.5^{\circ}$ & $-61.5^{\circ}$ & $-62^{\circ}$ & $-98.01^{\circ}$ \\
\hline
\end{tabular}

Tabla 3.19: Medidas RFOW de desfase para catorce estados del desfasador variable completo correspondientes a tres circuitos integrados distintos y comparadas con los resultados ideales esperados.

similares; de forma que si se comparan para los tres circuitos los estados en los que únicamente hay un dígito a ' 1 ', la diferencia máxima en valor absoluto es de $3^{\circ}$, mientras que porcentualmente es del 8.6\%. Se debe resaltar que la variación de los desfases es casi nula para los tres dígitos más significativos, gracias a la repetibilidad esperada de los desfasadores tipo líneas-conmutadas. Sería necesario realizar una campaña exhaustiva de medidas de multitud de iteraciones del mismo circuito, tanto de la misma oblea como de otras obleas, para poder extraer conclusiones definitivas sobre la repetibilidad del diseño.

Con el fin de comprobar los efectos de las interacciones de las adaptaciones entre los diferentes dígitos individuales, los estados de fase medidos con más de un dígito activo (puesto a ' 1 '), se han comparado con el valor esperado calculado como la suma de las contribuciones de los dígitos individuales (Tabla 3.20).

\begin{tabular}{|l|c|c|c|c|c|c|c|c|c|}
\hline & \multicolumn{3}{|c|}{ CCT $_{1}$} & \multicolumn{3}{c|}{$\mathrm{CCT}_{2}$} & \multicolumn{3}{c|}{$\mathrm{CCT}_{3}$} \\
& Medido & Esperado & Error & Medido & Esperado & Error & Medido & Esperado & Error \\
\hline \hline est. 63 & $115^{\circ}$ & $119.5^{\circ}$ & $-4.5^{\circ}$ & $115^{\circ}$ & $122^{\circ}$ & $-7^{\circ}$ & $114^{\circ}$ & $117.5^{\circ}$ & $-3.5^{\circ}$ \\
\hline est. 62 & $100^{\circ}$ & $102.5^{\circ}$ & $-2.5^{\circ}$ & $100^{\circ}$ & $103^{\circ}$ & $-3^{\circ}$ & $98^{\circ}$ & $100^{\circ}$ & $-2^{\circ}$ \\
\hline est. 61 & $79.5^{\circ}$ & $82.5^{\circ}$ & $-3^{\circ}$ & $79.5^{\circ}$ & $83^{\circ}$ & $-3.5^{\circ}$ & $78^{\circ}$ & $80.5^{\circ}$ & $-2.5^{\circ}$ \\
\hline est. 59 & $76^{\circ}$ & $79.5^{\circ}$ & $-3.5^{\circ}$ & $76^{\circ}$ & $81^{\circ}$ & $-5^{\circ}$ & $74^{\circ}$ & $77.5^{\circ}$ & $-3.5^{\circ}$ \\
\hline est. 55 & $172^{\circ}$ & $177^{\circ}$ & $-5^{\circ}$ & $172^{\circ}$ & $179^{\circ}$ & $-7^{\circ}$ & $170^{\circ}$ & $174.5^{\circ}$ & $-4.5^{\circ}$ \\
\hline est. 47 & $-146^{\circ}$ & $-145.5^{\circ}$ & $-0.5^{\circ}$ & $-146^{\circ}$ & $-143^{\circ}$ & $-3^{\circ}$ & $-147^{\circ}$ & $-147.5^{\circ}$ & $-0.5^{\circ}$ \\
\hline est. 31 & $-61.5^{\circ}$ & $-58.5^{\circ}$ & $-3^{\circ}$ & $-61.5^{\circ}$ & $-53^{\circ}$ & $-8.5^{\circ}$ & $-62^{\circ}$ & $-57.5^{\circ}$ & $-4.5^{\circ}$ \\
\hline
\end{tabular}

Tabla 3.20: Interacciones entre dígitos al conmutar estados. 


\begin{tabular}{|c|c|c|c|}
\hline & $S_{21}(d B)$ & $S_{11}(d B)$ & $S_{22}(d B)$ \\
\hline \hline estado 0 & 12.6 & 22.3 & 21.5 \\
\hline estado 1 & 12.7 & 22.4 & 11.6 \\
\hline estado 2 & 13 & 26.4 & 17 \\
\hline estado 4 & 13.2 & 18.5 & 9.4 \\
\hline estado 8 & 12.6 & 24.2 & 19.3 \\
\hline estado 16 & 13.4 & 18.4 & 10.7 \\
\hline estado 32 & 12.2 & 21.8 & 26.6 \\
\hline estado 63 & 12.9 & 22.3 & 12.9 \\
\hline estado 62 & 12.6 & 20 & 13.5 \\
\hline estado 61 & 12.3 & 25 & 30.8 \\
\hline estado 59 & 13 & 14.7 & 17.2 \\
\hline estado 55 & 12.9 & 29.3 & 11.4 \\
\hline estado 47 & 12.9 & 24.2 & 21.1 \\
\hline estado 31 & 12.4 & 26.7 & 11.4 \\
\hline Media & 12.76 & 22.59 & 16.74 \\
\hline Varianza $\sigma)$ & 0.33 & 3.70 & 6.22 \\
\hline
\end{tabular}

Tabla 3.21: Medidas RFOW de pérdidas de inserción y de adaptación para catorce estados del desfasador variable completo $\mathrm{CCT}_{3}$.

Teniendo en cuenta la precisión de las medidas $\left( \pm 0.25^{\circ}\right)$, la interacción entre dígitos es bastante baja en los circuitos $\mathrm{CCT}_{1}$ y $\mathrm{CCT}_{3}$ y ligeramente peor en el caso del circuito $\mathrm{CCT}_{2}$. Teniendo en cuenta los errores de modelado detectados en los dígitos menos significativos, se puede afirmar que, el comportamiento del desfasador en cuanto a interacción entre dígitos es realmente bueno.

El circuito $\mathrm{CCT}_{3}$ fue elegido para realizar más medidas. Las medidas de las pérdidas de inserción y la adaptación a la frecuencia de diseño $(24.15 \mathrm{GHz})$ se muestran en la tabla 3.21 .

Las pérdidas de inserción son aproximadamente $0.5 \mathrm{~dB}$ superiores a lo esperado. Por otro lado, es interesante destacar que la varianza de las pérdidas de inserción presenta un valor muy bajo, aún cuando se hayan medido únicamente catorce estados.

La adaptación del circuito es realmente buena para el acceso 1 y ligeramente peor para el acceso 2. Ambos accesos tienen como primer elemento un dígito con configuración líneas-conmutadas (los cuales han mostrado unas mejores prestaciones que los dígitos con configuración filtros-conmutados reconfigurable), pero el acceso 2 tiene como segundo elemento un dígito filtros-conmutados reconfigurable, lo cual pudiera ser la causa de las peores prestaciones. Es importante destacar que, la red de adaptación fue diseñada teniendo en cuenta el efecto de los hilos de conexión, por lo que se pueden esperar unos mejores resultados del circuito una vez montado.

En las figuras 3.24 y 3.25 se muestran algunas gráficas tomadas del analizador 
de redes. Las adaptaciones a la entrada y a la salida, las pérdidas de inserción y la inserción de fase para los catorce estados considerados se muestran para el circuito $\mathrm{CCT}_{3}$. El rango de frecuencias es de $22.15 \mathrm{GHz}$ a $26.15 \mathrm{GHz}$. Estas gráficas deben compararse con los resultados de simulación mostrados en las figuras 3.22 a 3.23.

La mayoría de los estados medidos muestran una resonancia en el parámetro $S_{11}$ (Fig. 3.24a) a una frecuencia mayor que la frecuencia de diseño, con únicamente cuatro estados con una resonancia por debajo de $24.15 \mathrm{GHz}$, como aparecían en los resultados de simulación. Los mismos comentarios pueden hacerse respecto al parámetro $S_{22}$ (Fig. 3.24b). Al añadir los hilos de conexión, los cuales está previsto que desplacen las resonancias hacia arriba en frecuencia, la adaptación final para el circuito montado podría llegar a ser ligeramente peor.

La respuesta de las pérdidas de inserción (Fig. 3.25) es tan buena como se esperaba, presentando una curva similar a la simulada con unos valores $0.5 \mathrm{~dB}$ menores y unas pérdidas incrementales sólo ligeramente superiores, pero cumpliendo las especificaciones.

Las medidas de la inserción de fase (Fig. 3.25) confirman la esperada buena linearidad, pero las curvas presentan un desplazamiento vertical con respecto a los resultados de las simulaciones, debido al diferente desfase de los tres dígitos menos significativos.

El enfoque no binario adoptado permite al desfasador variable soportar ciertos errores de desfase, mientras se mantiene la resolución deseada al mismo tiempo. Es por ello, que se han llevado a cabo algunas simulaciones, para valorar el efecto del error mayor que lo inicialmente permitido (8.5\%) en los desfases de los tres dígitos menos significativos. Como solamente se midieron catorce estados, se han tomado los desfases de los dígitos individuales medidos para el circuito $\mathrm{CCT}_{3}$, y a partir de ellos, se han calculado los sesenta y cuatro estados mediante la suma de las contribuciones de los dígitos individuales. Se asume una interacción nula entre los dígitos, lo cual no está muy alejado de la realidad para el caso del circuito $\mathrm{CCT}_{3}$, tal y como se comprobó anteriormente.

Ya que la resolución especificada era de cinco dígitos, los 32 estados de fase con un separación entre los mismos de $11.25^{\circ}$ se consideraron como objetivo; para cada uno de ellos se tomó de los 64 estados del desfasador variable, aquel que ofrecía un desfase más cercano al ideal, y se calculó el error de fase cometido. El mismo procedimiento se siguió con el desfasador variable ideal no binario de cinco dígitos con resolución de seis dígitos, es decir, aquel que se hubiera conseguido en un circuito sin errores.

Los resultados para todas estas simulaciones se muestran en la tabla 3.22 , donde también se indican los errores de desfase máximo $\left(\left(\delta_{e}\right)_{\text {pico }}\right)$, medio $\left(\overline{\delta_{e}}\right)$ y $\operatorname{RMS}\left(\sigma_{e}\right)$. El valor RMS es realmente bueno, confirmando la robustez del enfoque no binario. De hecho, el resultado es solamente $1.2^{\circ}$ peor que el diseño nominal no binario. Únicamente tres de los 32 estados exceden la precisión de un desfasador variable binario de cinco dígitos $\left( \pm 5.625^{\circ}\right)$. 


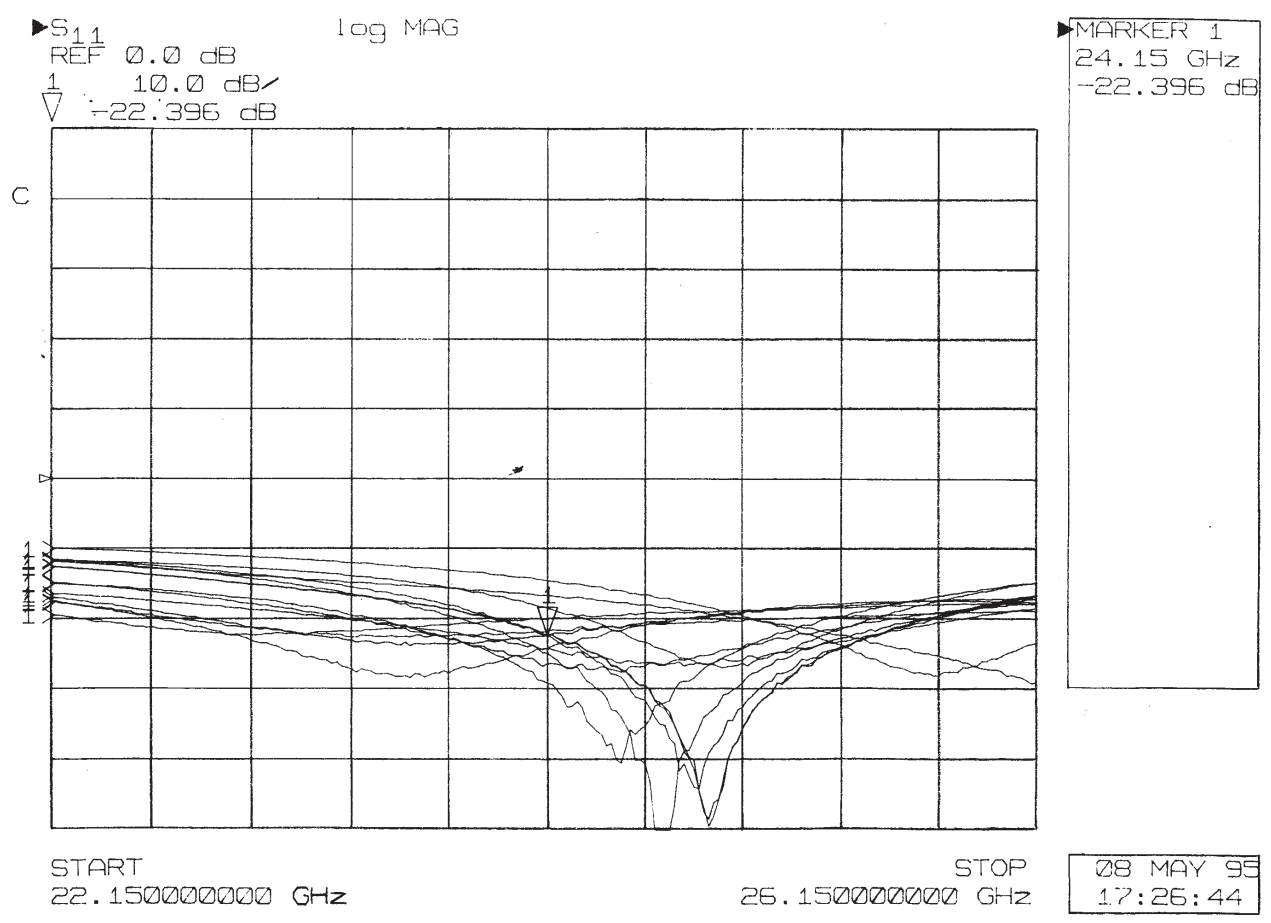

a)

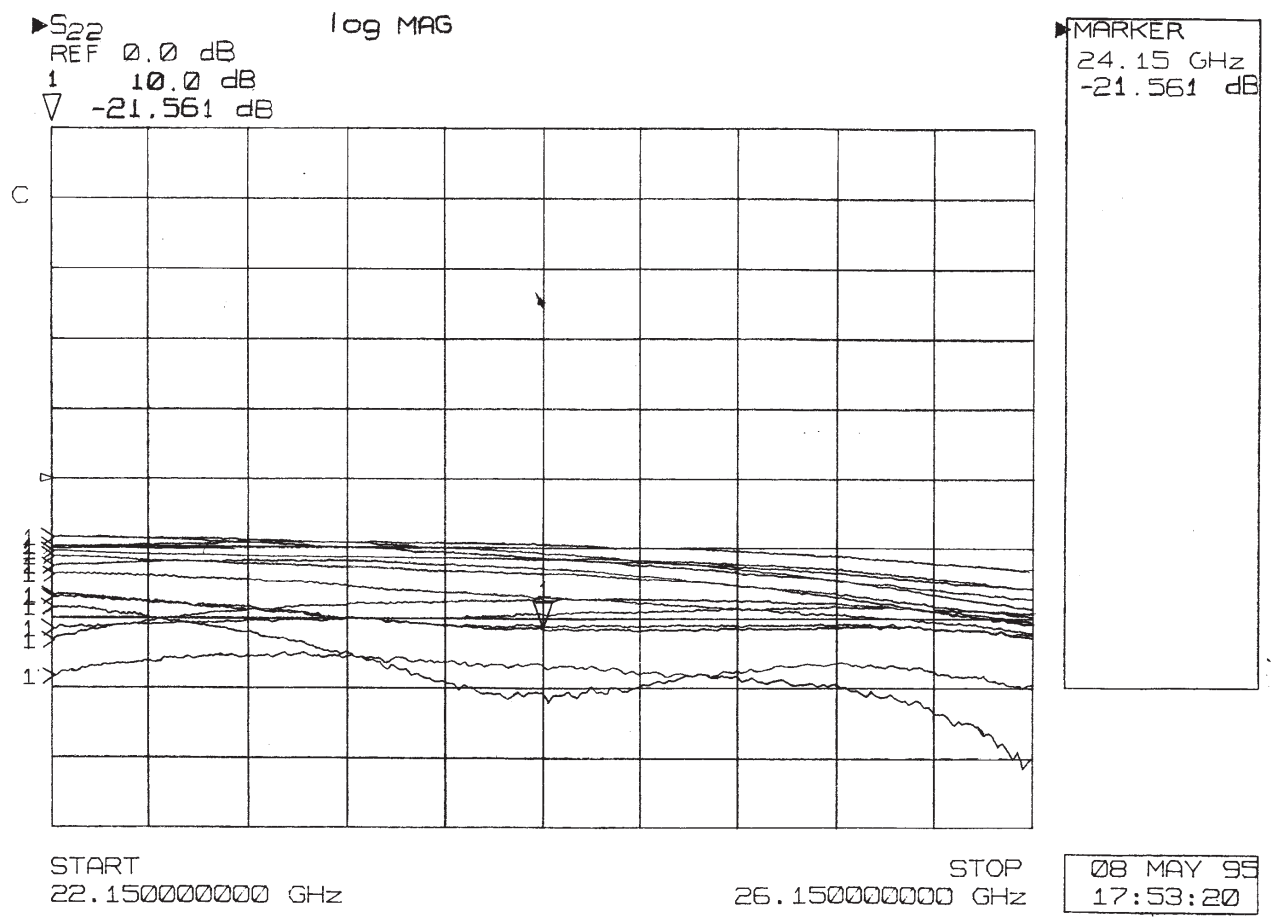

b)

Figura 3.24: Medidas RFOW del desfasador variable para catorce estados. a) Adaptación a la entrada. b) Adaptación a la salida. 


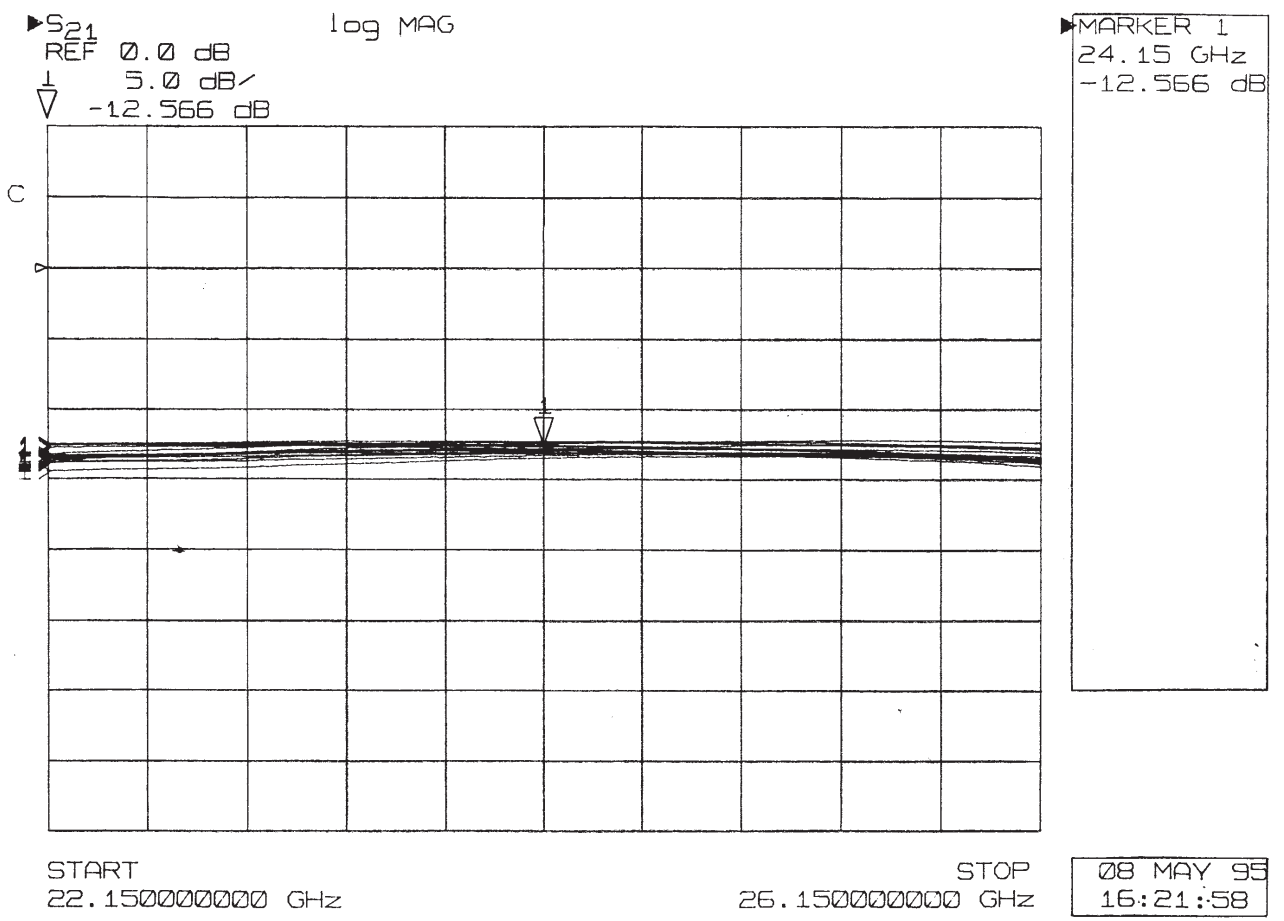

a)

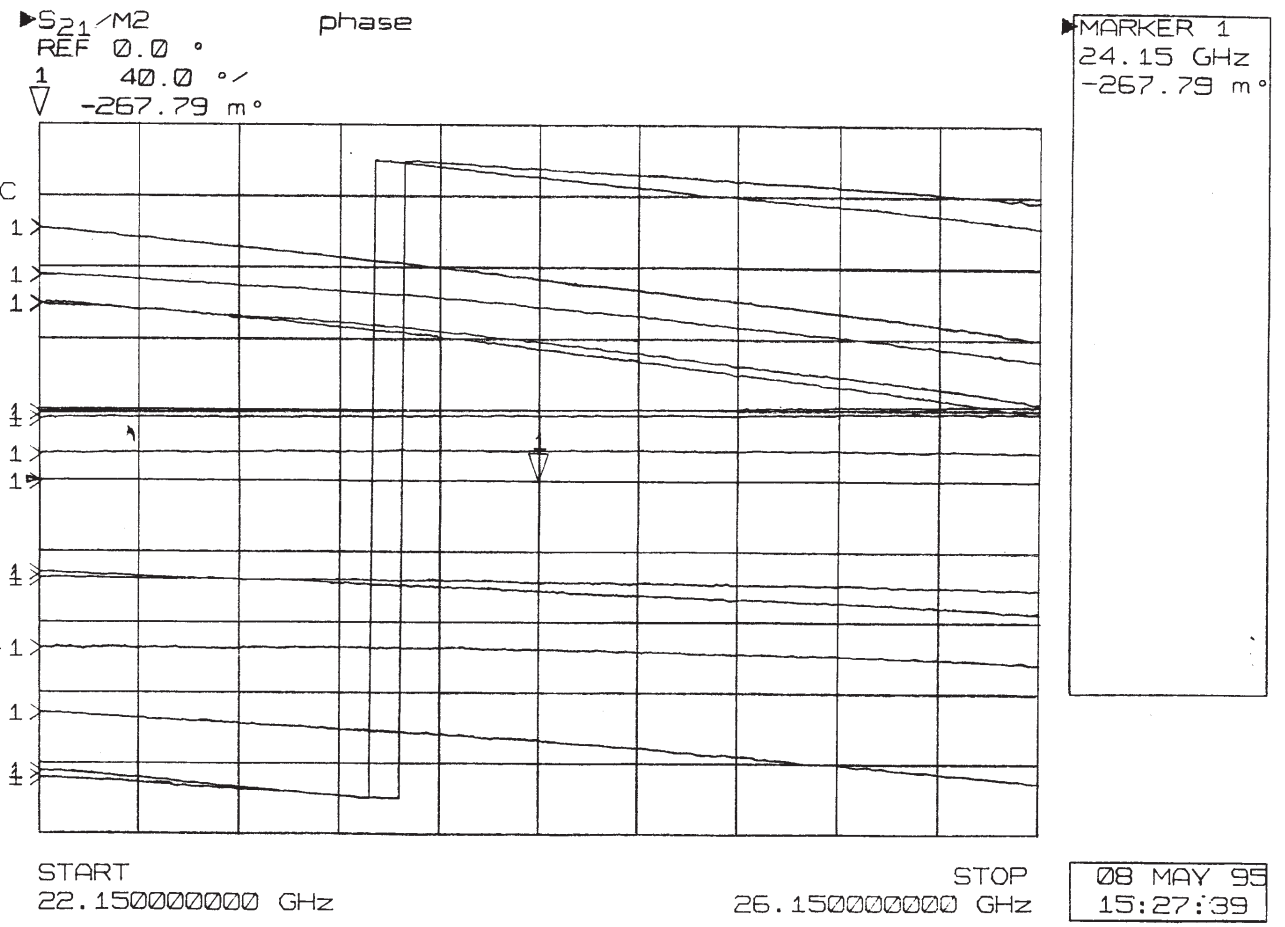

b)

Figura 3.25: Medidas RFOW del desfasador variable para catorce estados. a) Pérdidas de inserción. b) Desfase. 


\begin{tabular}{|c|c|c|c|c|c|}
\hline & \multirow{2}{*}{$\begin{array}{c}\text { Binario ideal } \\
5 \text { dígitos } \\
\end{array}$} & \multicolumn{2}{|c|}{$\mathrm{CCT}_{3}$} & \multicolumn{2}{|c|}{ No binario $6 / 5$ ideal } \\
\hline & & Más cercano & Error fase & Más cercano & Error fase \\
\hline estado 0 & $0^{\circ}$ & $0^{\circ}$ & $0^{\circ}$ & $0^{\circ}$ & $0^{\circ}$ \\
\hline estado 1 & $11.25^{\circ}$ & $7^{\circ}$ & $4.25^{\circ}$ & $10^{\circ}$ & $1.25^{\circ}$ \\
\hline estado 2 & $22.5^{\mathrm{o}}$ & $21.5^{\mathrm{o}}$ & $1^{\mathrm{o}}$ & $19^{\circ}$ & $3.5^{\mathrm{o}}$ \\
\hline estado 3 & $33.75^{\circ}$ & $37^{\circ}$ & $3.25^{\circ}$ & $36^{\circ}$ & $2.25^{\circ}$ \\
\hline estado 4 & $45^{\circ}$ & $44^{\circ}$ & $1^{\mathrm{O}}$ & $48^{\circ}$ & $3^{\circ}$ \\
\hline estado 5 & $56.25^{\circ}$ & $57^{\circ}$ & $0.75^{\circ}$ & $57^{\circ}$ & $0.75^{\circ}$ \\
\hline estado 6 & $67.5^{\circ}$ & $61.5^{\circ}$ & $6^{\circ}$ & $67^{\circ}$ & $0.5^{\mathrm{o}}$ \\
\hline estado 7 & $78.75^{\circ}$ & $77^{\circ}$ & $1.75^{\circ}$ & $74^{\circ}$ & $4.75^{\circ}$ \\
\hline estado 8 & $90^{\circ}$ & $94^{\circ}$ & $4^{\circ}$ & $88^{\circ}$ & $2^{\circ}$ \\
\hline estado 9 & $101.25^{\circ}$ & $97^{\circ}$ & $4.25^{\circ}$ & $101^{\circ}$ & $0.25^{\circ}$ \\
\hline estado 10 & $112.5^{\circ}$ & $112.5^{\circ}$ & $0^{\circ}$ & $111^{\circ}$ & $1.5^{\mathrm{o}}$ \\
\hline estado 11 & $123.75^{\circ}$ & $132^{\circ}$ & $8.25^{\circ}$ & $128^{\circ}$ & $4.25^{\circ}$ \\
\hline estado 12 & $135^{\circ}$ & $135^{\circ}$ & $0^{\circ}$ & $132^{\circ}$ & $3^{\circ}$ \\
\hline estado 13 & $146.25^{\circ}$ & $149.5^{\circ}$ & $3.25^{\circ}$ & $149^{\circ}$ & $2.75^{\circ}$ \\
\hline estado 14 & $157.5^{\circ}$ & $152.5^{\circ}$ & $5^{\circ}$ & $158^{\circ}$ & $0.5^{\circ}$ \\
\hline estado 15 & $168.75^{\circ}$ & $169.5^{\circ}$ & $0.75^{\circ}$ & $168^{\circ}$ & $0.75^{\circ}$ \\
\hline estado 16 & $180^{\circ}$ & $175^{\circ}$ & $5^{\circ}$ & $180^{\circ}$ & $0^{\circ}$ \\
\hline estado 17 & $191.25^{\circ}$ & $192^{\circ}$ & $0.75^{\circ}$ & $190^{\circ}$ & $1.25^{\circ}$ \\
\hline estado 18 & $202.5^{\circ}$ & $206.5^{\circ}$ & $4^{\circ}$ & $199^{\circ}$ & $3.5^{\circ}$ \\
\hline estado 19 & $213.75^{\circ}$ & $215^{\circ}$ & $1.25^{\circ}$ & $216^{\circ}$ & $2.25^{\circ}$ \\
\hline estado 20 & $225^{\circ}$ & $229^{\circ}$ & $4^{\mathrm{O}}$ & $228^{\circ}$ & $3^{\circ}$ \\
\hline estado 21 & $236.25^{\circ}$ & $232.5^{\circ}$ & $3.75^{\circ}$ & $237^{\circ}$ & $0.75^{\circ}$ \\
\hline estado 22 & $247.5^{\circ}$ & $246.5^{\circ}$ & $1^{\circ}$ & $247^{\circ}$ & $0.5^{\circ}$ \\
\hline estado 23 & $258.75^{\circ}$ & $252^{\circ}$ & $6.75^{\circ}$ & $254^{\circ}$ & $4.75^{\circ}$ \\
\hline estado 24 & $270^{\circ}$ & $270^{\circ}$ & $0^{\circ}$ & $268^{\circ}$ & $2^{\circ}$ \\
\hline estado 25 & $281.25^{\circ}$ & $286.5^{\circ}$ & $5.25^{\circ}$ & $281^{\circ}$ & $0.25^{\circ}$ \\
\hline estado 26 & $292.5^{\circ}$ & $289.5^{\circ}$ & $3^{\mathrm{O}}$ & $291^{\circ}$ & $1.5^{\circ}$ \\
\hline estado 27 & $303.75^{\circ}$ & $307^{\circ}$ & $3.25^{\circ}$ & $308^{\circ}$ & $4.25^{\circ}$ \\
\hline estado 28 & $315^{\circ}$ & $310^{\circ}$ & $5^{\mathrm{O}}$ & $312^{\circ}$ & $3^{\circ}$ \\
\hline estado 29 & $326.25^{\circ}$ & $326.5^{\circ}$ & $0.25^{\circ}$ & $329^{\circ}$ & $2.75^{\circ}$ \\
\hline estado 30 & $337.5^{\circ}$ & $344.5^{\circ}$ & $7^{\circ}$ & $338^{\circ}$ & $0.5^{\circ}$ \\
\hline estado 31 & $348.75^{\circ}$ & $347^{\circ}$ & $1.75^{\circ}$ & $348^{\circ}$ & $0.75^{\circ}$ \\
\hline$\left(\delta_{e}\right)_{p i c o}$ & - & - & $8.25^{\circ}$ & - & $4.75^{\circ}$ \\
\hline$\overline{\delta_{e}}$ & - & - & $2.98^{\circ}$ & - & $1.94^{\circ}$ \\
\hline$\overline{\sigma_{e}}$ & - & - & $3.76^{\circ}$ & - & $2.41^{\circ}$ \\
\hline
\end{tabular}

Tabla 3.22: Comparación entre las medidas del desfasador $\mathrm{CCT}_{3}$ con un desfasador digital binario de cinco dígitos y con un desfasador digital, no binario, de seis dígitos y cinco equivalentes $(6 / 5)$. 


\begin{tabular}{|c|c|c|c|c|}
\hline & $\pm\left(\delta_{e}\right)_{\text {pico }}$ & $\sigma_{e}$ & $N L S P_{n}$ & $\Delta G$ \\
\hline \hline $\mathrm{CCT}_{1}$ & $8.7^{\circ}$ & $3.5^{\circ}$ & $-24.3 \mathrm{~dB}$ & $-0.02 \mathrm{~dB}$ \\
\hline $\mathrm{CCT}_{2}$ & $8.4^{\circ}$ & $3.7^{\circ}$ & $-23.7 \mathrm{~dB}$ & $-0.02 \mathrm{~dB}$ \\
\hline $\mathrm{CCT}_{3}$ & $8.6^{\circ}$ & $3.8^{\circ}$ & $-23.5 \mathrm{~dB}$ & $-0.02 \mathrm{~dB}$ \\
\hline
\end{tabular}

Tabla 3.23: Valor máximo y RMS del error total, nivel normalizado de los lóbulos secundarios respecto al lóbulo principal y variación de la ganancia del haz principal, para cada una de las tres iteraciones del desfasador variable MMIC a $24 \mathrm{GHz}$; resultados obtenidos a partir de las medidas de los desfases de cada uno de los seis dígitos.

Los resultados de la tabla 3.22 reflejan únicamente los errores debidos a las desviaciones, respecto a los desfases nominales, de un desfasador binario de cinco dígitos y su efecto se puede asimilar a los errores aleatorios de la sección 3.2. Para hacer un análisis completo de las prestaciones del desfasador, es necesario tener en cuenta el efecto conjunto de los errores aleatorios y los errores de cuantificación, incluyendo el proceso de calibración.

Como se ha visto, las tres iteraciones medidas del desfasador variable presentan una interacción entre dígitos bastante baja, por lo que se puede asumir que, los desfases obtenidos en los 64 posibles estados no diferirán mucho de los obtenidos como combinación de los seis estados con un único desfasador a ' 1 '. Haciendo esta suposición, es posible analizar el comportamiento del desfasador variable completo, como se hace en la sección 3.2, donde se analizan los parámetros estadísticos de los errores de fase, cuando se desea una fase cualquiera dentro del rango del desfasador $\left(360^{\circ}\right)$. Operando de esta forma con las tres iteraciones del desfasador, se obtienen los resultados mostrados en la tabla 3.23.

Si se comparan los resultados de la tabla 3.23 con los presentados en la tabla 3.8 , se puede constatar que, a pesar de los errores de desfase obtenidos en los dígitos tipo filtros-conmutados, el desfasador variable total presenta unas prestaciones similares a las obtenidas con un desfasador ideal binario de cinco dígitos, si se comparan los parámetros más representativos, como son el error total RMS y el nivel de lóbulos principal a secundario.

\subsection{Conclusiones}

De acuerdo con los objetivos propuestos, se ha modelado el efecto que las tolerancias de fabricación de los procesos MMIC pueden tener sobre la precisión de desfasadores digitales, combinándolo con el efecto de los errores de cuantificación inherentes a los desfasadores digitales. Se han tenido en cuenta diferentes grados de calibración de los desfasadores, a partir de medidas de uno o de todos los desfasadores en una agrupación. 
Partiendo del análisis anterior, se han comparado las opciones binaria y no binaria, a la hora de escoger los pesos de los diferentes dígitos que forman un desfasador digital variable; estableciéndose fórmulas de diseño y criterios de selección. Mediante el análisis de supuestos prácticos, se ha demostrado la utilidad de los pesos no binarios como protección frente a la tolerancia de los desfases de cada dígito. Esta robustez frente a los errores, puede incluir también errores de diseño o variaciones con la frecuencia, permitiendo, en este último caso, utilizar el mismo desfasador en un mayor ancho de banda.

Se ha diseñado un desfasador variable con una frecuencia central de $24 \mathrm{GHz}$, en tecnología MMIC con el proceso D02AH de Philips Microwaves Limeil. Todas las especificaciones se han cumplido en las simulaciones, obteniéndose un tamaño de circuito integrado de $4.5 \mathrm{~mm}$ por $3 \mathrm{~mm}$.

Se ha tomado un enfoque no binario en el diseño, lo cual permite obtener una resolución de cinco dígitos con un diseño de seis dígitos reales y una tolerancia de seguridad de un $8.5 \%$ sobre el desfase de cada dígito.

Una configuración simple y fiable, líneas-conmutadas, ha sido elegida para los tres dígitos mayores. Por otro lado, y debido a consideraciones de tamaño y pérdidas, se ha seleccionado una estructura reconfigurable filtros-conmutados para los tres dígitos menos significativos. Es importante destacar la necesidad de disponer de un modelo de conmutador para el PHEMT suficientemente fiable. La disponibilidad de un modelo de conmutador más desarrollado o, incluso, de un modelo distribuido, hará menos arriesgada la opción filtros-conmutados.

Las medidas sobre la oblea llevadas a cabo a $24 \mathrm{GHz}$ con el desfasador variable no binario de seis dígitos (cinco dígitos binarios equivalentes de resolución), muestran unas buenas prestaciones en todos los aspectos, como adaptación, pérdidas de inserción, varianza en las pérdidas de inserción y baja interacción entre dígitos, excepto el desfase de los tres elementos menos significativos, los cuales fueron diseñados siguiendo una arquitectura reconfigurable filtros-conmutados. Esta configuración requiere un modelo de conmutador bastante exacto y fiable, el cual no estaba disponible en el momento en el que el diseño se realizó. Sin embargo, y gracias a la robustez del enfoque no binario adoptado, las prestaciones finales del circuito en términos de error de fase total y del efecto de los errores sobre el diagrama de radiación, confirman un desfasador variable totalmente operativo. 


\section{Capítulo 4}

\section{Aplicación de tecnologías monolíticas a redes conformadoras fijas para agrupaciones de antenas multihaz}

\subsection{Introducción}

Los requerimientos de las nuevas aplicaciones RADAR y de comunicaciones a bordo de satélites, tales como áreas de cobertura variables o acceso de múltiple usuarios, hace evidente la necesidad de disponer de antenas con múltiples haces, fiables, de bajo coste y con un tamaño reducido. Si además, se consideran sistemas con órbitas elípticas, como las diseñadas para comunicaciones móviles via satélite, se hace necesario obtener antenas multihaz, eficientes en cuanto a una menor complejidad de la antena, y con un denso conjunto de haces dentro del área de cobertura deseada.

El objetivo de este capítulo es el análisis de una nueva arquitectura para un conformador de haces de radiofrecuencia para agrupaciones de antenas multihaz propuesta recientemente [ESA94], y que se encuentra en periodo de estudio en la Agencia Espacial Europea. Esta arquitectura parte de una distribución hexagonal tanto de los elementos sobre el plano de la agrupación, como de los haces sobre la zona de cobertura en el espacio real. Su estructura está basada en el concepto de la matriz de Butler, ya presentada en la sección 2.4.2, y que ha sido adaptada y generalizada para agrupaciones con rejilla hexagonal.

La generalización de la matriz de Butler, permite construir redes conformadoras de tamaños diversos, a partir de la repetición e interconexión de multitud de módulos de pequeño tamaño. Aprovechando esta propiedad, en la fabricación de la red conformadora de haces, se combinan tecnología monolítica integrada para los 
módulos repetitivos y tecnología triplaca ${ }^{\mathrm{i}}$ multicapa para la interconexión de los mismos; dando lugar a un módulo de microondas con múltiples circuitos integrados $\left(\mathrm{MMCM}^{\mathrm{ii}}\right)$ que permite minimizar el tamaño y peso de la red conformadora.

En este capítulo se presenta la arquitectura de la red conformadora, estableciendo el método de diseño para la misma, así como recomendaciones sobre la obtención de arquitecturas eficientes en cuanto a la reutilización de un mismo módulo en toda la red conformadora. A partir de los resultados obtenidos, se diseña un módulo MMIC de tamaño $3 \times 3$, que es el elemento básico para implementar conformadores de diversos tamaños $(9 \times 9,27 \times 27$ o $81 \times 81)$. Para el diseño de este módulo se analizan las tolerancias del proceso de fabricación, presentando una solución para la sintonización del mismo. Una vez diseñado la celda básica, se estudia el comportamiento de redes conformadoras de mayor tamaño basadas en esa celda básica. Gracias a la construcción de un prototipo de red conformadora $9 \times 9$, se han podido contrastar las prestaciones del mismo.

\subsection{Arquitectura eficiente de red conformadora de haces de radiofrecuencia para agrupacio- nes hexagonales de antenas}

\subsubsection{Introducción}

Para aplicaciones con un gran número de haces sintetizados y de elementos radiantes en la antena, las redes conformadoras de haces de radiofrecuencia ( $\mathrm{RF}$ ) actuales son pesadas, mientras que los conformadores de haces de frecuencia intermedia (FI) o digitales, presentan un consumo de potencia muy elevado. Una discusión completa sobre técnicas de conformación de haces en radiofrecuencia puede encontrarse en la literatura [Hal90].

Como se vió en la sección 2.4.2, una matriz de Butler es un conformador de haces consistente en desfasadores fijos y acopladores, interconectados entre sí. Una matriz $N \times N$ junto a una agrupación uniforme de $N$ elementos, produce $N$ haces ortogonales iguales que se solapan a un nivel de $-3.9 \mathrm{~dB}$ respecto al máximo, para el caso de una agrupación lineal, y a niveles de $-7.8 \mathrm{~dB}$ y $-5.4 \mathrm{~dB}$, para agrupaciones planas, con distribución de los haces rectangular y hexagonal, respectivamente.

Los sistemas de comunicación requieren antenas multihaz, con haces que tengan menores lóbulos secundarios que los correspondientes a una agrupación uniforme y, simultáneamente, se necesita que los haces se solapen entre sí, a niveles mucho menores de los que corresponden a un conjunto de haces ortogonales. Como se vió en la sección 2.3, una solución óptima para la reducción de los lóbulos secundarios

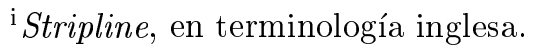

ii Microwave Multi-Chip Module, en terminología inglesa.
} 
es la introducción de una distribución de amplitud posterior a la conformación de los haces uniformes a partir de una matriz de Butler. En cuanto al nivel de cruce de los haces, se ha comprobado (sección 2.3) que, una solución idealmente óptima consiste en aumentar el tamaño del conformador de haces, mientras se mantiene fijo el número de elementos radiantes (Fig. 2.10) y se terminan los accesos no utilizados de la matriz. De esta forma, es posible obtener antenas multihaz que cumplan los requisitos de los modernos sistemas de comunicaciones, siempre y cuando se disponga de una arquitectura compacta, fiable y eficiente para la generación de los haces ortogonales.

En aplicaciones espaciales, se suelen considerar agrupaciones planas con distribución hexagonal de los elementos radiantes, en lugar de distribución rectangular, ya que por su mejor comportamiento en cuanto a los lóbulos de difracción se consigue, para una misma zona de cobertura, una reducción del $16 \%$ en el número de elementos necesarios (sección 2.2.2), que se traduce en la misma reducción en cuanto al número elementos de control o al tamaño de la red conformadora.

Independientemente de la distribución espacial de los elementos de la agrupación, es posible escoger la distribución de los haces, según una rejilla rectangular o hexagonal dentro del plano de los cosenos directores $(u, v)$. Como se vió en el apartado 2.2.2, la distribución hexagonal de los haces permite una mayor densidad de haces, con unas características más homogéneas en cuanto a niveles de cruces entre haces que las que se pueden conseguir con una distribución rectangular, debido a las diferencias que ésta presenta en las diagonales respecto a los ejes de la distribución (Fig. 2.11).

Con el fin de trabajar con agrupaciones con distribuciones hexagonal en los elementos y en las posiciones de los haces, es necesario modificar ligeramente la típica matriz de Butler, para agrupaciones con distribución espacial rectangular.

\section{Estructura de la red conformadora de radiofrecuencia}

La matriz de Butler para agrupaciones lineales, está formada por un conjunto de híbridos y desfasadores fijos [But61]. Este concepto se puede extender a agrupaciones planas con distribución hexagonal. Así como en la matriz de Butler original, diseñada para agrupaciones lineales con un número par de elementos $\left(N=2^{p}\right)$, los elementos que la formaban eran híbridos (redes $2 \times 2$ ) y desfasadores, al generalizar la matriz de Butler aparecen redes de otros tamaños $(3 \times 3,4 \times 4, \ldots)$, dependiendo de la descomposición de $N$ en factores.

Actualmente, el tamaño de las matrices de Butler está limitado por la complejidad en la interconexión de los diversos híbridos y desfasadores; habiéndose diseñado matrices de hasta 64 elementos [Hal90, Lo88]. Generalmente, la tecnología utilizada para su fabricación consiste en una mezcla de líneas de transmisión planares y líneas coaxiales para la interconexión de híbridos fabricados en tecnología planar (microtira o triplaca), lo cual complica excesivamente la estructura, siendo además muy 
propensa a introducir errores de desfase en las interconexiones.

El uso de tecnologías MMIC y MIC multicapa, permite conseguir una reducción muy significativa del peso y del tamaño del conformador de haces de radiofrecuencia, con respecto a soluciones previamente conocidas. Además, la elección del tamaño de la matriz como múltiplo de un entero pequeño (i.e: 3 ó 4), hace posible que toda la matriz sea construida a partir de la repetición de una única celda básica $(3 \times 3$ ó $4 \times 4)$.

Estas celdas básicas serían implementadas usando tecnologías miniaturizadas (MMIC). Gracias al uso de tecnología MMIC y su propiedad de repetibilidad, puede esperarse un comportamiento eléctrico similar para todas las células. Además, debido a la naturaleza simple de estos circuitos integrados, se puede esperar un rendimiento ${ }^{i}$ muy alto en la fabricación de los mismos, con la consiguiente reducción del precio de fabricación. El encaminamiento interno entre las células básicas sería realizado mediante tecnología planar multicapa, lo cual contribuiría aún más al objetivo de tamaño y peso reducidos.

\subsubsection{Análisis de la matriz de Butler generalizada para mues- treo hexagonal}

\section{Matriz de Butler generalizada para agrupaciones lineales}

En la figura 4.1 se muestra una matriz de Butler generalizada para una agrupación lineal de $N=10$ elementos. Si la alimentación de la agrupación de la figura corresponde a $B_{n}=A_{n} e^{j \alpha_{n}}$, su factor de la agrupación será:

$$
F(u)=\sum_{n=0}^{N-1} B_{n} e^{j k u\left(n d_{x}\right)}
$$

que si se compara con la expresión de una transformada de Fourier discreta en el tiempo $\left(\mathrm{DTFT}^{\mathrm{ii}}\right)$ :

$$
X\left(e^{j \omega}\right)=\sum_{n=-\infty}^{\infty} x[n] e^{-j \omega n}
$$

se puede comprobar que el factor de agrupación es la DTFT de la alimentación de la agrupación:

$$
F\left(-k u d_{x}\right)=\operatorname{DTFT}\left[B_{n}\right]
$$

donde $-k u d_{x}$ hace el papel de pulsación en el dominio discreto $(\omega)$ y $d_{x}$ el periodo de muestreo; comprobándose la periodicidad en el dominio transformado de acuerdo con $F\left(-k u d_{x}\right)=F\left(-k u d_{x}+2 \pi\right)$ o bien $F(-u)=F\left(-u+\lambda / d_{x}\right)$; propiedad ya

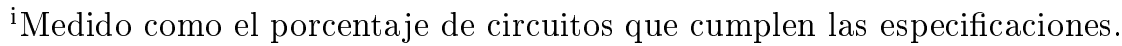

${ }^{i i}$ Discrete-Time Fourier Transform.
} 

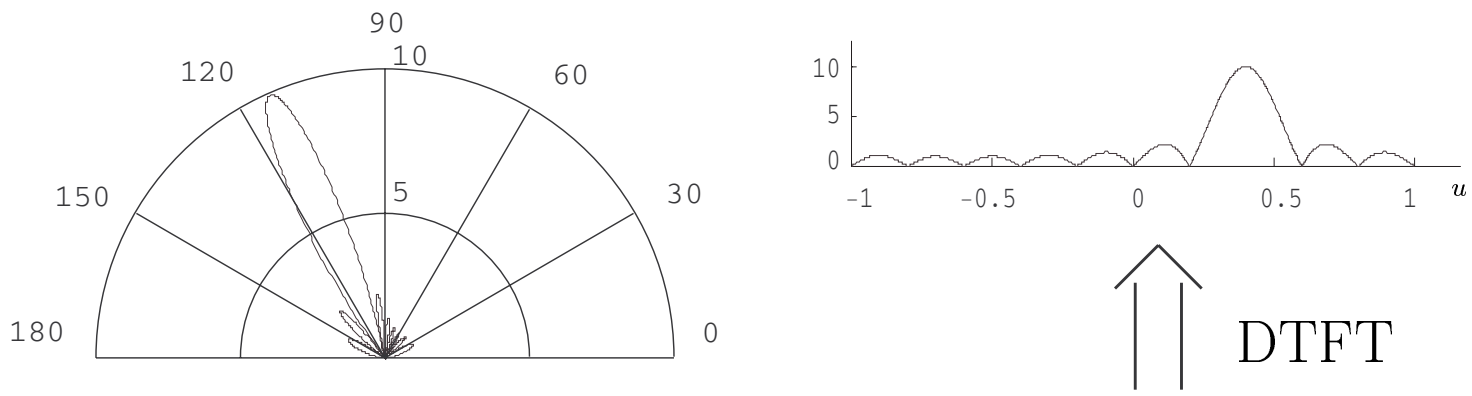

ELEMENTOS DE LA AGRUPACION
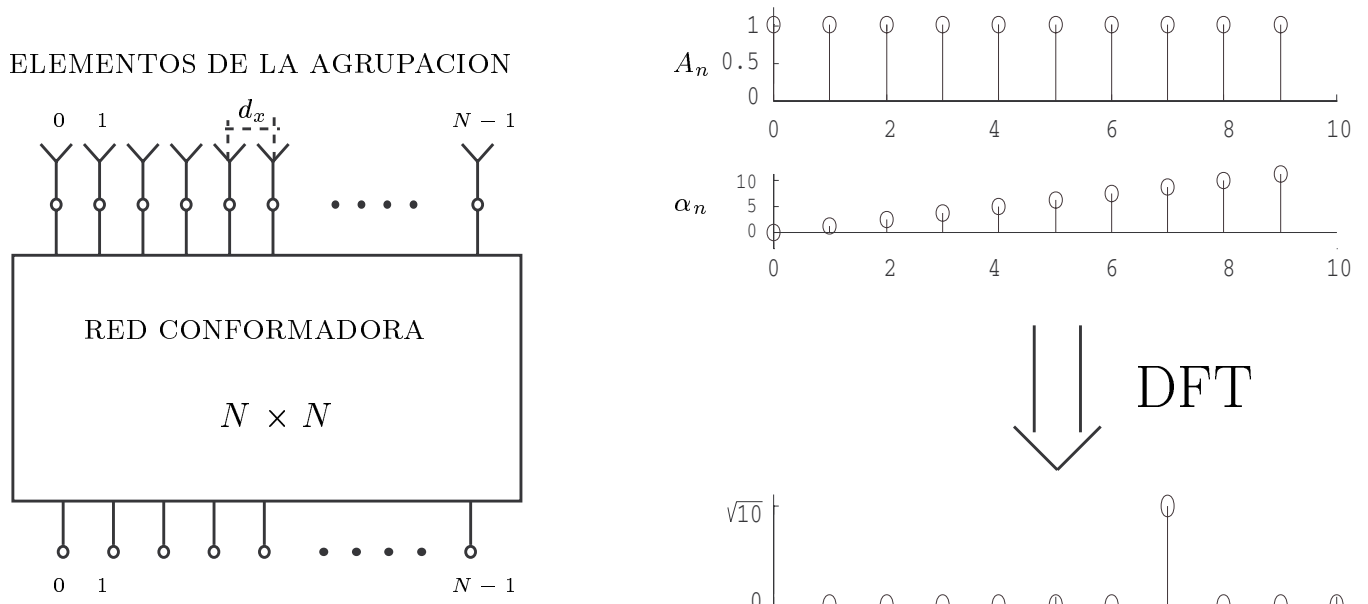

HACES

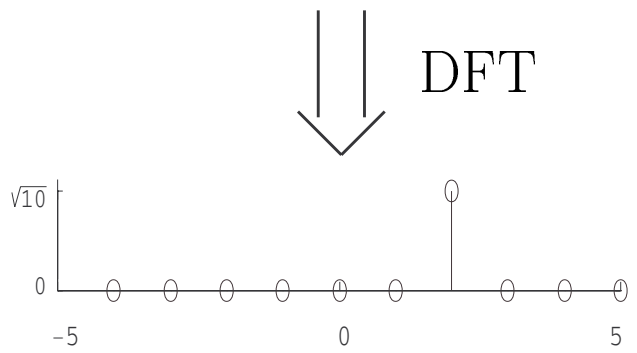

Figura 4.1: Ejemplo de matriz de Butler aplicada a una agrupación lineal de 10 elementos, mostrando claramente las relaciones entre los dominios espacial discreto (elementos de la agrupación), frecuencial contínuo (factor de agrupación) y frecuencial discreto (accesos haces).

introducida en el capítulo 2. Si se construye una señal periódica a partir de la alimentación $B_{n}$, repitiéndola con una periodicidad $N$, es posible calcular la transformada de Fourier discreta $\left(\mathrm{DFT}^{\mathrm{i}}\right)$ de la alimentación $\left(B_{n}\right)$, cuyos $N$ valores $X\left[-k u_{n} d_{x}\right]$ corresponden a muestras del factor de agrupación (dominio transformado) en las siguientes posiciones:

$$
\left(-u_{n}\right)=n \frac{\lambda}{d_{x} N}
$$

donde $n=0 \ldots N-1$; es decir son muestras equiespaciadas:

$$
\Delta u=\frac{\lambda}{d_{x} N}
$$

de acuerdo con la definición de matriz de Butler que se hizo en la sección 2.3. Por lo tanto, si la red conformadora de la figura 4.1 se encarga de realizar la DFT inversa $\left(\mathrm{DFT}^{-1}\right)$, cuando a la entrada de la misma todos los accesos sean nulos, excepto el correspondiente al haz n-ésimo, la salida de la red (la alimentación de la agrupación)

\footnotetext{
${ }^{\mathrm{i}}$ Discrete Fourier Transform.
} 
será tal que, el factor de la agrupación tenga un máximo en la dirección $u_{n}$. Por consiguiente, se puede generalizar que la matriz de Butler es la implementación analógica de la transformada discreta de Fourier inversa [Mai93a]. Por otro lado, la red conformadora deberá ser recíproca, para poder actuar tanto en modo transmisión como en recepción.

La posición de los máximos para una agrupación lineal, mostrados en (4.4) puede modificarse añadiendo un cierto desplazamiento $\delta u$ a todos ellos, mediante la inserción de una fase progresiva fija a la salida de la $\mathrm{DFT}^{-1}$, de forma que se consiga, por ejemplo, distribuir los haces de una agrupación con $N$ par, simétricamente respecto a la dirección normal a la agrupacióni.

La construcción de una matriz de Butler generalizada para agrupaciones lineales se traduce en la búsqueda de una arquitectura que realice la transformada discreta de Fourier inversa. Ya que habitualmente los algoritmos de cálculo de DFT hacen referencia a la transformada directa, será interesante utilizar las propiedades de dualidad de la DFT:

$$
\left\{\begin{array}{l}
x[n] \stackrel{D F T}{\longrightarrow} X[k] \\
X[n] \stackrel{D F T}{\longrightarrow} N x\left[((-k))_{N}\right]
\end{array}\right\}
$$

se concluye, que bastará con buscar una implementación de la DFT directa de $N$ puntos $(\mathrm{DFT}-N)$ y reordenar la salida de acuerdo con $(4.6)$, donde $((-k))_{N}$ significa $-k$ en módulo $\mathrm{N}$; en cuanto al factor $N$ que aparece en la relación dual de (4.6), no tiene importancia alguna, pues ese término constante en la relación entre entradas y salidas de la red, viene determinado por la división de la potencia de la señal de entrada entre las $N$ salidas. De hecho, teniendo en cuenta el criterio de conservación de la energía, la implementación analógica ideal de la DFT- $N$ corresponde a lo que en el campo del procesado de imagen, se conoce como DFT unitaria $\left(\mathrm{DFT}_{u}\right)$ :

$$
X\left(e^{j \omega}\right)=\frac{1}{\sqrt{N}} \sum_{n=-\infty}^{\infty} x[n] e^{-j \omega n}
$$

A la hora de realizar físicamente la red que implemente analógicamente la $\mathrm{DFT}^{-1}$, se pueden utilizar descomposiciones similares a las realizadas en el campo del procesado de señal, con el fin de simplificar su cálculo. Así, cuando el número de elementos y de haces fuera una potencia de dos $\left(N=2^{p}\right)$, se puede aplicar el algoritmo FFT $^{\text {ii }}$ base-2 que reduce significativamente el número de operaciones, quedando una arquitectura final formado por $p=\log _{2}(N)$ capas con $N / 2$ DFT-2 (redes $2 \times 2$, conocidas en el campo del procesado de señal como mariposas) y $N / 2$ multiplicaciones por fasores en cada capa. En la figura 4.2, se muestra la estructura de una DFT-2, cuya implementación analógica es un híbrido de $180^{\circ}$; mientras que los productos

\footnotetext{
${ }^{\mathrm{i}}$ Algunas implementaciones de matrices de Butler incluyen los citados desfases fijos internamente, por lo que en esos casos no se podría hablar estrictamente de que la matriz fuera una red $\mathrm{DFT}^{-1}$.

${ }^{\text {ii }}$ Fast Fourier Transform.
} 

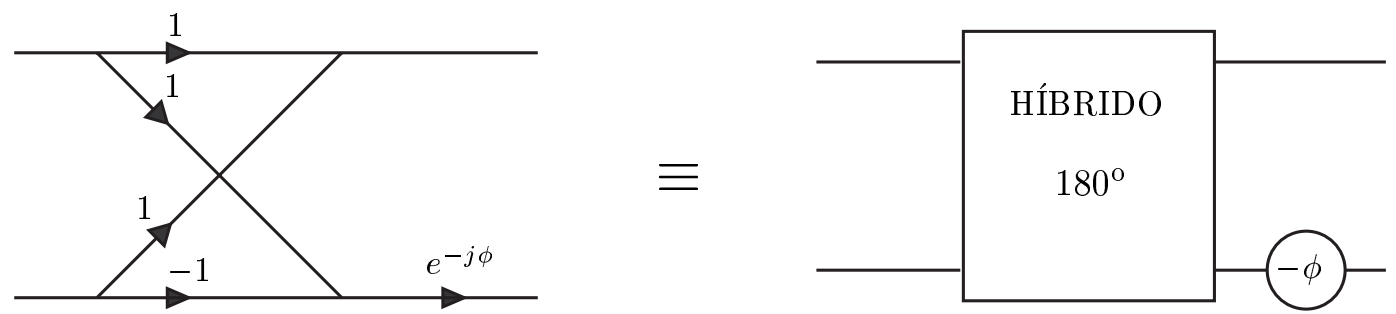

Figura 4.2: Esquema de DFT de 2 puntos $(\phi=0)$ e implementación analógica de la misma.

por fasores se traducirán en desfasadores fijos. Por consiguiente, la implementación física de una DFT de $\mathrm{N}$ puntos, cuando $N=2^{p}$, requiere $N / 2 \cdot \log _{2}(N)$ híbridos y $N / 2 \cdot \log _{2}(N)$ desfasadores fijos, de forma análoga a la mostrada en la figura 2.12.

Para los casos en los que $N$ no sea una potencia de dos, será necesario recurrir a otros algoritmos óptimos, distintos de la FFT, para el cálculo de la DFT [Opp89]. Todos los algoritmos eficientes se basan en la descomposición de $N$ en factores; básicamente, se puede hablar de dos grupos de algoritmos: algoritmos Cooley-Tukey y algoritmos de factores primos $\left(\mathrm{PFA}^{\mathrm{i}}\right)$. El primer grupo incluye los algoritmos base$\mathrm{R}$, adecuados para los casos en que $N$ es expresable como una potencia de un número entero $\left(N=R^{v}\right)$ y que de forma similar al caso de la FFT se componen de diferentes capas formados por redes $R \times R$ y desfasadores; también incluye los algoritmos de ratio mezclado cuando $N$ es expresable como producto de factores no iguales. El algoritmo de factores primos puede utilizarse cuando $N=N_{1} \cdot N_{2}$ donde $N_{1}$ y $N_{2}$ son factores primos entre sí y tiene como característica más importante e interesante que la DFT de $N$ puntos (DFT- $N$ ) está formada únicamente por una capa de $N_{1}$ redes de tamaño $N_{2} \times N_{2}$ interconectada con otra capa de $N_{2}$ redes de $N_{1} \times N_{1}$, sin necesidad de desfasadores entre ambas capas, cualidad muy interesante desde el punto de vista de modularidad de la red.

Para cualquiera de los algoritmos que se desee implementar, es muy importante tener en cuenta el reordenamiento de entrada o salida impuesto por el mismo, además del debido a la propiedad de dualidad expresada en (4.6). Este reordenamiento partirá del orden inicial impuesto por los elementos de antena situados en $n d_{x}$, $n=0 \ldots N-1$ y la posición de los haces siguiendo el orden $u_{n}, n=0 \ldots N-1$ indicado en (4.4).

Conviene resaltar que todos los algoritmos se basan en dividir el cálculo de la DFT- $N$ en varias DFT- $N_{i}$ mas pequeñas, que consitituirán las celdas básicas. Un criterio muy importante a la hora de buscar la implementación más adecuada, será escoger aquella que utilice un menor número de tamaños de celdas básicas distintas.

En la figura 4.3, se muestra el esquema de una celda básica de tamaño $N \times N$, donde se indican los desfases $\left(-\phi_{m n}\right)$ que sufre una señal desde la entrada n-ésima hasta la salida m-ésima. Estos desfases coinciden con los obtenidos en una red que

\footnotetext{
${ }^{\text {i }}$ Prime Factor Algorithms.
} 
Celda Básica $N \times N$

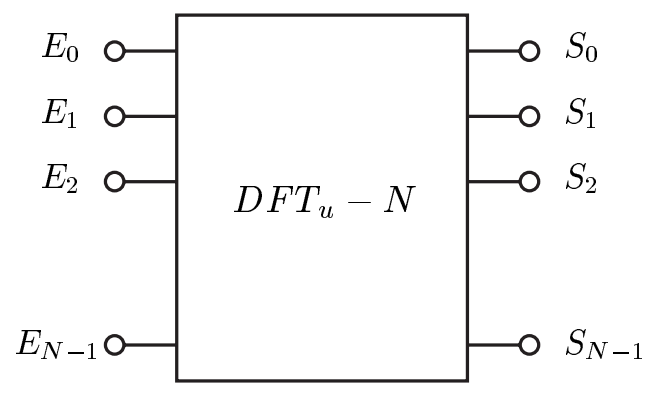

\begin{tabular}{|c|c|c|c|c|c|}
\hline$-\phi_{m n}$ & $E_{0}$ & $E_{1}$ & $E_{2}$ & $\cdots$ & $E_{N-1}$ \\
\hline$S_{0}$ & 0 & 0 & 0 & $\cdots$ & 0 \\
\hline$S_{1}$ & 0 & $\frac{2 \pi}{N}$ & $\frac{4 \pi}{N}$ & $\cdots$ & $\frac{2 \pi(N-1)}{N}$ \\
\hline$S_{2}$ & 0 & $\frac{4 \pi}{N}$ & $\frac{8 \pi}{N}$ & $\cdots$ & $\frac{4 \pi(N-1)}{N}$ \\
\hline & $\cdot$ & $\cdot$ & $\cdot$ & & $\cdot$ \\
\hline & $\cdot$ & $\cdot$ & $\cdot$ & & $\cdot$ \\
\hline$S_{N-1}$ & 0 & $\frac{2 \pi(N-1)}{N}$ & $\frac{4 \pi(N-1)}{N}$ & $\cdots$ & $\frac{2 \pi(N-1)^{2}}{N}$ \\
\hline
\end{tabular}

Figura 4.3: Esquema general de una celda básica $N \times N$.

implemente una DFT; además, en el caso de una celda ideal analógica, las señales a la salida vendrían afectadas por un factor $1 / \sqrt{N}$, debido a las pérdidas por división de la señal, y, por lo tanto, la red ideal cumplirá:

$$
\frac{S_{m}}{E_{n}}=\frac{1}{\sqrt{N}} e^{-j \phi_{m n}}=\frac{1}{\sqrt{N}} e^{-j \frac{2 \pi m n}{N}}
$$

Se ha visto que una red conformadora que realice una operación $\mathrm{DFT}_{u}^{-1}$ de $N$ puntos, es adecuada para conformar los $N$ haces equiespaciados típicos de una matriz de Butler (haces que para el caso particular $d_{x}=\lambda / 2$ y distribución uniforme son ortogonales entre sí, como se vió en 2.3). Sin embargo, en condiciones habituales, y salvo que se deseara combinar haces (para aumentar el ancho de haz o reducir el NLPS), la red conformadora va a ser utilizada siempre con entradas independientes, de forma que para cada haz se tiene una única entrada activa y el resto a cero; lo cual permite en algunos casos simplificar la estructura de la $\mathrm{DFT}_{u}^{-1}$.

Un ejemplo de estas simplificaciones es la inserción de algún desfase fijo en el camino recorrido por una señal, desde el acceso del haz n-ésimo pero no en el resto; en este caso el efecto sobre los factores de agrupación es nulo, pero la red deja de ser una $\mathrm{DFT}_{u}^{-1}$, en sentido estricto. A cambio, esta modificación puede redundar en una simplificación de la arquitectura de la red conformadora, por medio de una reducción de elementos (i.e., híbridos, desfasadores, elementos concentrados), lo cual se traduce en una reducción de costes, pérdidas y peso.

\section{Matriz de Butler generalizada para agrupaciones planas}

Las agrupaciones de antenas planas presentan una mayor libertad a la hora de situar los elementos radiantes, pues éstos se pueden distribuir de forma regular en un plano tanto con una rejilla rectangular como con una triangular/hexagonal.

A continuación se analizan ambas distribuciones, con el objetivo de encontrar arquitecturas eficientes para la conformación de haces. 
Rejilla rectangular El factor de agrupación de una agrupación plana rectangular con $N \times M$ elementos es:

$$
F(u, v)=\sum_{n=0}^{N-1} \sum_{m=0}^{M-1} B_{n m} e^{j k u\left(n d_{x}\right)} e^{j k v\left(m d_{y}\right)}
$$

que si se compara con la expresión de una transformada de Fourier discreta en el tiempo para dos dimensiones con muestreo rectangular (DTFT-2D rec $)$ :

$$
X\left(e^{j \omega_{1}}, e^{j \omega_{2}}\right)=\sum_{n=-\infty}^{\infty} \sum_{m=-\infty}^{\infty} x[n, m] e^{-j \omega_{1} n} e^{-j \omega_{2} m}
$$

se puede comprobar que el factor de agrupación es la DTFT-2D $\mathrm{D}_{\text {rec }}$ de los alimentación de la agrupación:

$$
F\left(-k u d_{x},-k v d_{y}\right)=D T F T\left[B_{n m}\right]
$$

Si se construye una señal periódica a partir de la alimentación $B_{n m}$, es posible calcular la transformada de Fourier discreta en dos dimensiones (DFT-2D rec $)$ de la alimentación $\left(B_{n m}\right)$, cuyos $N \times M$ valores $\left(X\left[-k u_{p} d_{x},-k v_{q} d_{y}\right]\right)$ corresponden a muestras del factor de agrupación en las siguientes posiciones del dominio transformado:

$$
\left(-u_{p},-v_{q}\right)=\left[p \frac{\lambda}{d_{x} N}, q \frac{\lambda}{d_{y} M}\right]
$$

donde $p=0 \ldots N-1$ y $q=0 \ldots M-1$. Se puede observar en (4.12) que las muestras del dominio transformado siguen una rejilla rectangular con espaciado entre muestras:

$$
\begin{aligned}
\Delta u & =\frac{\lambda}{d_{x} N} \\
\Delta v & =\frac{\lambda}{d_{y} M}
\end{aligned}
$$

Para implementar la $\mathrm{DFT}_{u}-2 \mathrm{D}_{r e c}$, es interesante descomponerla de la siguiente forma:

$$
\begin{aligned}
X[p, q] & =\frac{1}{\sqrt{N M}} \sum_{n=0}^{N-1} \sum_{m=0}^{M-1} x[n, m] e^{-j 2 \pi p \frac{1}{N} n} e^{-j 2 \pi q \frac{1}{M} m} \\
& =\frac{1}{\sqrt{N}}\left(\sum_{n=0}^{N-1}\left(\frac{1}{\sqrt{M}} \sum_{m=0}^{M-1} x[n, m] e^{-j 2 \pi q \frac{1}{M} m}\right) e^{-j 2 \pi p \frac{1}{N} n}\right)
\end{aligned}
$$

donde el paréntesis interior resulta ser una $\mathrm{DFT}_{u}-1 \mathrm{D}$ de $M$ puntos para cada una de las $N$ filas, $\left(X_{a u x}[n, q]\right)$; a continuación, se cogen las $N$ salidas correspondientes a la columna q-ésima de esas $\mathrm{DFT}_{u}-1 \mathrm{D}$ y se realiza otra tanda de $\mathrm{DFT}_{u}-1 \mathrm{D}$ :

$$
X[p, q]=\frac{1}{\sqrt{N}}\left(\sum_{n=0}^{N-1} X_{a u x}[n, q] e^{-j 2 \pi k \frac{1}{N} n}\right)
$$




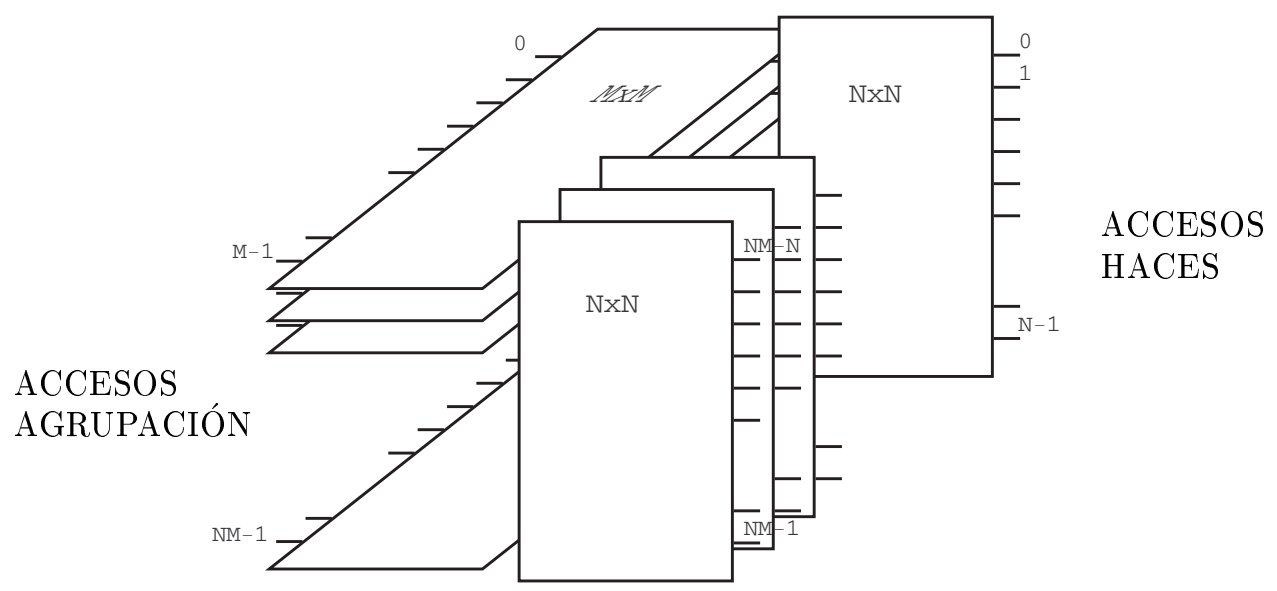

Figura 4.4: Matriz de Butler para agrupaciones planas rectangulares de $N \times M$ elementos.

es decir, la $\mathrm{DFT}_{u}-2 \mathrm{D}_{\text {rec }}$ se puede calcular a partir de $N \mathrm{DFT}_{u}-1 \mathrm{D}$ de $M$ puntos y $M \mathrm{DFT}_{u}$-1Dde $N$ puntos. Finalmente, aplicando la propiedad de dualidad de la DFT-2D, se pueden reordenar los accesos de la red $\mathrm{DFT}_{u}-2 \mathrm{D}$, para que realice la $\mathrm{DFT}_{u}^{-1}-2 \mathrm{D}$ deseada:

$$
\left\{\begin{array}{l}
x_{u}[n, m] \stackrel{D F T_{u}-2 D}{\longrightarrow} X_{u}[p, q] \\
X_{u}[p, q] \stackrel{D F T_{u}-2 D}{\longrightarrow} x_{u}\left[((-p))_{N},((-q))_{M}\right]
\end{array}\right\}
$$

Por consiguiente, para el caso particular de agrupaciones planas con $N \times M$ elementos dispuestos en una rejilla rectangular de $N$ filas y $M$ columnas se pueden utilizar $N$ matrices de Butler de tamaño $M \times M\left(\mathrm{DFT}_{u}^{-1}-1 \mathrm{D}-\mathrm{M}\right)$, más $M$ matrices de Butler de tamaño $N \times N\left(\mathrm{DFT}_{u}^{-1}-1 \mathrm{D}-\mathrm{N}\right)$, dispuestas de acuerdo con la descomposición en filas y columnas, mostrada en la figura 4.4, sin necesidad de desfasadores entre filas y columnas; resultado, por otra parte, ya conocido en la práctica [But61, She68].

Para el cálculo de las $\mathrm{DFT}_{u^{-}} 1 \mathrm{D}$, se utilizará el algoritmo más adecuado de los presentados en la sección precedente, para el caso de agrupaciones lineales.

Rejilla Hexagonal Como se ha comentado en el apartado 4.2.1, se pretende utilizar distribución hexagonal de los elementos de la agrupación, con el fin de reducir el número de elementos necesarios; además, la distribución de los haces en el dominio $(u, v)$, será también hexagonal para conseguir una mejor cobertura. Una vez se ha escogido el conjunto de haces (al menos su posición relativa) y la situación de los elementos de la agrupación, queda por encontrar una arquitectura de red conformadora que sea óptima y eficiente.

Antes de comenzar a analizar la estructura de la red conformadora, es necesario presentar el teorema del muestreo para rejillas hexagonales [Mer79, Mer83], con el fin de introducir una serie de términos y conceptos, imprescindibles para la posterior discusión sobre la arquitectura del conformador. Muchos de los términos aquí 


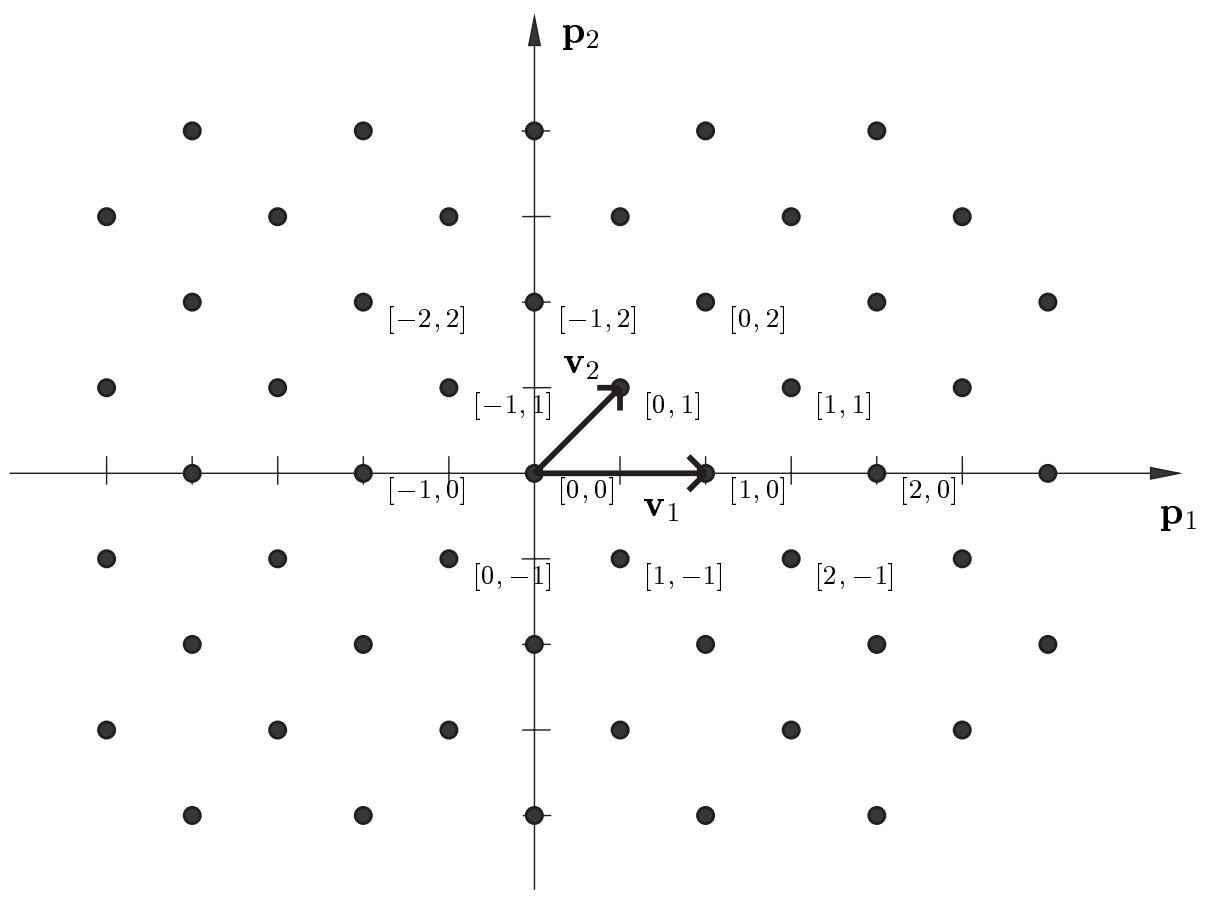

Figura 4.5: Rejilla hexagonal $L_{V}$ generada por la matriz de muestreo $\mathbf{V}=\left[\mathbf{v}_{1}, \mathbf{v}_{2}\right]$.

incluidos corresponden al campo de la teoría de los números.

En la figura 4.5, se muestra sobre unos ejes cartesianos un conjunto de elementos sobre una rejilla hexagonal; rejilla que se puede caracterizar, entre otras, por la siguiente base $\mathbf{V}=\left[\mathbf{v}_{1}, \mathbf{v}_{2}\right]=\left[(2,0)^{\dagger},(1,1)^{\dagger}\right]$, expresada en coordenadas euclídeas $\left(p_{1}, p_{2}\right)$. Cada elemento de la rejilla viene caracterizado por un vector de dos enteros $\mathbf{n}=\left[n_{1}, n_{2}\right]^{\dagger}$, de forma que respecto a los ejes cartesianos, la posición de cada elemento es:

$$
\mathbf{p}=\left[\begin{array}{l}
p_{1} \\
p_{2}
\end{array}\right]=\mathbf{V n}=\left[\begin{array}{ll}
v_{11} & v_{21} \\
v_{12} & v_{22}
\end{array}\right]\left[\begin{array}{l}
n_{1} \\
n_{2}
\end{array}\right]=\left[\begin{array}{ll}
2 & 1 \\
0 & 1
\end{array}\right]\left[\begin{array}{l}
n_{1} \\
n_{2}
\end{array}\right]
$$

donde $\mathbf{V}$ es la matriz de muestreo, que para el caso de muestreo rectangular sería una matriz diagonal. A partir de la matriz de muestreo, se define la densidad de muestreo en dos dimensiones como la inversa del módulo de su determinante, det $|\mathbf{V}|$.

Se define la rejilla $L_{V}$, generada por la matriz de muestreo $\mathbf{V}$, como todos aquellos puntos $\mathbf{p}$ generados a partir de (4.17) con $\mathbf{n}$ cualquier vector de enteros.

Sea $x[\mathbf{n}]$ un conjunto finito de muestras tomadas de acuerdo a la matriz de muestreo $\mathbf{V}$ en los puntos del espacio cartesiano $\mathbf{p}=\mathbf{V n}$, se define la DTFT en dos dimensiones con muestreo $\mathbf{V}$ (DTFT-2D) como:

$$
X(\boldsymbol{\omega})=X\left(\left[\begin{array}{l}
\omega_{1} \\
\omega_{2}
\end{array}\right]\right)=\sum_{n_{1}=-\infty}^{\infty} \sum_{n_{2}=-\infty}^{\infty} x(\mathbf{n}) e^{-j \boldsymbol{\omega}^{\dagger} \mathbf{p}}=\sum_{n_{1}=-\infty}^{\infty} \sum_{n_{2}=-\infty}^{\infty} x(\mathbf{n}) e^{-j \boldsymbol{\omega}^{\dagger} \mathbf{V} \mathbf{n}}
$$

donde si se particulariza para el caso de muestreo hexagonal, para la matriz de 
muestreo de la figura 4.5 se obtiene:

$$
\boldsymbol{\omega}^{\dagger} \mathbf{V n}=\left[\begin{array}{l}
\omega_{1} \\
\omega_{2}
\end{array}\right]\left[\begin{array}{ll}
2 & 1 \\
0 & 1
\end{array}\right]\left[\begin{array}{l}
n_{1} \\
n_{2}
\end{array}\right]
$$

siendo $\boldsymbol{\omega}$ el vector de frecuencias discretas referido a los ejes cartesianos $\left(p_{1}, p_{2}\right)$.

Si se compara la expresión de la DTFT (4.18), con la correspondiente al factor de agrupación, de una agrupación plana con $N$ elementos distribuidos en el plano $X Y$ de acuerdo con la rejilla $L_{V}$, considerando $d_{x}$ y $d_{y}$, como las unidades de medida en los ejes $(\hat{x}, \hat{y})$ :

$$
F(u, v)=\sum_{n=0}^{N-1} B_{n} e^{j k[u v]\left[\begin{array}{l}
x_{n} \\
y_{n}
\end{array}\right]}=\sum_{n=0}^{N-1} B_{n} e^{j k\left[\begin{array}{ll}
u d_{x} & v d_{y}
\end{array}\right]\left[\begin{array}{l}
\frac{x_{n}}{d_{x}} \\
\frac{y_{n}}{d_{y}}
\end{array}\right]}
$$

se comprueba que, el factor de la agrupación es la DTFT en dos dimensiones de la alimentación de la agrupación $B_{n}$ :

$$
F(\mathbf{s})=\operatorname{DTFT}\left[B_{n}\right]
$$

donde:

$$
\mathbf{s}=\left[\begin{array}{l}
s_{1} \\
s_{2}
\end{array}\right]=\left[\begin{array}{l}
k u d_{x} \\
k v d_{y}
\end{array}\right]=\left[\begin{array}{l}
-\omega_{1} \\
-\omega_{2}
\end{array}\right]=-\boldsymbol{\omega}
$$

Por consiguiente, y al igual que ocurría para los casos anteriores, la red conformadora para rejillas hexagonales deberá implementar la $\mathrm{DFT}_{u}^{-1}-2 \mathrm{D}_{\text {hex }}$; pero antes, deberán hacerse una serie de consideraciones sobre el cálculo de la misma. Para definir la DFT de una secuencia bidimensional, ésta debe ser finita dentro de una cierta superficie y además debe poderse cubrir todo el espacio bidimensional definido por la matriz de muestreo, $L_{V}$, mediante la repetición periódica de la misma; lo que se denomina propiedad de cobertura de la secuencia; de forma que si no se cumpliera, se debería rellenar con elementos nulos, hasta conseguir una nueva secuencia que si la cumpliera.

Para la repetición periódica de la secuencia se introduce una matriz de periodicidad, $\mathbf{N}$, formada por dos vectores independientes ( $\operatorname{det} \mathbf{N} \neq 0$ ) de números enteros, que indican cuánto hay que moverse en el sistema de coordenadas $\left(\mathbf{v}_{1}, \mathbf{v}_{2}\right)$ para encontrar dos muestras repetidas, de forma que la secuencia periódica, $\tilde{x}[\mathbf{n}]$, obtenida de repetir $x[\mathbf{n}]$, cumplirá:

$$
\tilde{x}[\mathbf{n}+\mathbf{N r}]=\tilde{x}[\mathbf{n}]
$$

para cualesquiera vectores de enteros $\mathbf{n}$ y $\mathbf{r}$. El módulo del determinante de la matriz de periodicidad, es igual al número de muestras de la secuencia finita. En la figura 4.6, se muestra un ejemplo de secuencia $x[\mathbf{n}]$ muestreada sobre la rejilla $L_{V}$ con la matriz de periodicidad $\mathbf{N}=\left[\begin{array}{cc}2 & -2 \\ 2 & 4\end{array}\right]$. En general, para cualquier secuencia finita la matriz de periodicidad $\mathbf{N}$ no es única. 


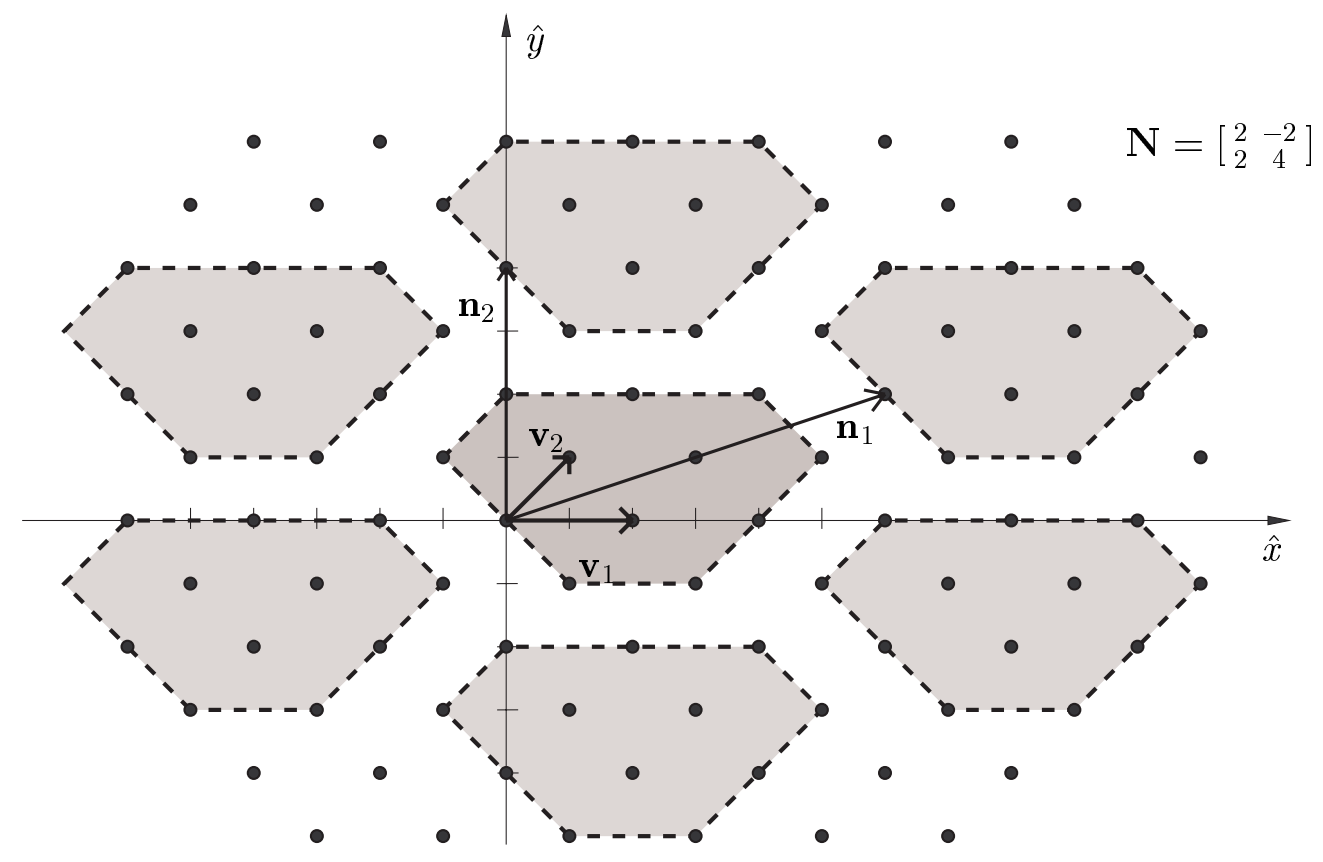

Figura 4.6: Ejemplo de secuencia finita con doce elementos (trama más oscura) y que cumple la propiedad de cobertura con la matriz de repetición $\mathbf{N}=\left[\mathbf{n}_{\mathbf{1}}, \mathbf{n}_{\mathbf{2}}\right]$ en la rejilla hexagonal $L_{V}, \mathbf{V}=\left[\mathbf{v}_{\mathbf{1}}, \mathbf{v}_{\mathbf{2}}\right]$.

Una vez se dispone de una secuencia finita, que cumple la propiedad de cobertura, se ha encontrado una matriz de periodicidad adecuada y se ha generado una secuencia periódica, se puede descomponer la secuencia periódica $\tilde{x}[\mathbf{n}]$ en sus series discretas de Fourier (DFS ${ }^{\mathrm{i}}$ :

$$
\tilde{X}[\mathbf{k}]=\sum_{\mathbf{n} \in I_{N}} \tilde{x}[\mathbf{n}] e^{-j \mathbf{k}^{\dagger}\left(2 \pi \mathbf{N}^{-1}\right) \mathbf{n}}
$$

donde $I_{N}$ es un periodo de $\tilde{x}[\mathbf{n}]$. Por otro lado, la expresión de la $\mathrm{DFS}^{-1}$ es:

$$
\tilde{x}[\mathbf{n}]=\frac{1}{|\operatorname{det} \mathbf{N}|} \sum_{\mathbf{k} \in J_{N}} \tilde{X}[\mathbf{k}] e^{j \mathbf{k}^{\dagger}\left(2 \pi \mathbf{N}^{-1}\right) \mathbf{n}}
$$

donde $J_{N}$ es un periodo de $\tilde{X}[\mathbf{k}]$, secuencia cuyo matriz de periodicidad es $\mathbf{N}^{\dagger}$, como se puede comprobar fácilmente en (4.24).

Comparando (4.24) con (4.18), se puede comprobar que los valores de la DFS corresponden a muestras de la DTFT en:

$$
\mathbf{k}^{\dagger}\left(2 \pi \mathbf{N}^{-1}\right)=\boldsymbol{\omega}^{\dagger} \mathbf{V}
$$

despejando, se obtiene los valores de frecuencia $\boldsymbol{\omega}$ a los que corresponden las muestras de la DFS:

$$
\boldsymbol{\omega}=\left(\mathbf{V}^{-1}\right)^{\dagger}\left(2 \pi \mathbf{N}^{-1}\right)^{\dagger} \mathbf{k}=\mathbf{U}\left(\mathbf{N}^{-1}\right)^{\dagger} \mathbf{k}
$$

\footnotetext{
${ }^{\mathrm{i} D i s c r e t e}$ Fourier Series.
} 
donde se ha introducido la matriz $\mathbf{U}=2 \pi\left(\mathbf{V}^{-1}\right)^{\dagger}$, que define la repetición de la DTFT en el dominio transformado contínuo y por lo tanto, define la posición de los lóbulos de difracción. Como la citada matriz U sólo depende de la matriz de muestreo $\mathbf{V}$, se comprueba que la posición de los lóbulos de difracción depende únicamente de la rejilla sobre la que se coloquen los elementos y no de su número o posición dentro de la rejilla.

Observando el resultado (4.27), se deduce que la matriz $\mathbf{R}=\mathbf{U}\left(\mathbf{N}^{-1}\right)^{\dagger}$ rige el muestreo en el dominio transformado, que, para el caso de la red conformadora de la agrupación, implica que define la posición y distribución de los máximos de los haces a conformar:

$$
\left[\begin{array}{c}
\frac{u d_{x}}{\lambda} \\
\frac{v d_{y}}{\lambda}
\end{array}\right]=-\left(\mathbf{N}^{-1} \mathbf{V}^{-1}\right)^{\dagger} \cdot\left[\begin{array}{l}
k_{1} \\
k_{2}
\end{array}\right]
$$

Por lo tanto, en los casos en los que la matriz $\mathbf{N}$ no es única, uno de los criterios a la hora de escoger la solución más adecuada, será observar a qué distribución de los haces corresponde ${ }^{\mathrm{i}}$.

Finalmente, el cálculo de la DFT, consiste en la elección de un periodo de $\tilde{x}[\mathbf{n}]$ $\left(x_{p}[\mathbf{n}]\right.$ definida en $\left.I_{N}\right)$ y un periodo de $\tilde{X}[\mathbf{k}]\left(X_{p}[\mathbf{k}]\right.$ definida en $\left.J_{N}\right)$, de forma que la DFT queda definida como:

$$
\begin{array}{rc}
X_{p}[\mathbf{k}]=\sum_{\mathbf{n} \in I_{N}} x_{p}[\mathbf{n}] e^{-j \mathbf{k}^{\dagger}\left(2 \pi \mathbf{N}^{-1}\right) \mathbf{n}} & \mathbf{k} \in J_{N} \\
x_{p}[\mathbf{n}]=\frac{1}{|\operatorname{det} \mathbf{N}|} \sum_{\mathbf{k} \in J_{N}} X_{p}[\mathbf{k}] e^{j \mathbf{k}^{\dagger}\left(2 \pi \mathbf{N}^{-1}\right) \mathbf{n}} & \mathbf{n} \in I_{N}
\end{array}
$$

el resultado de la DFT no depende del periodo escogido; pues, en cualquier caso se obtienen las $N$ muestras no repetidas de las secuencias periódicas en ambos dominios. En cambio, el algoritmo de cálculo, sí depende del periodo escogido y por lo tanto, su complejidad.

Comparando las expresiones (4.29) y (4.30), se observa que, salvo un término constante, que no tiene ninguna importancia para el diseño de la red conformadora, el cálculo de la $\mathrm{DFT}^{-1}-2 \mathrm{D}$ requiere los mismos cálculos que la $\mathrm{DFT}^{-1}-2 \mathrm{D}$, más una reordenación a la salida en función de observar a qué puntos del periodo $I_{N}$ corresponden los índices $-\mathbf{n}$, teniendo en cuenta la matriz de periodicidad $\mathbf{N}$.

\section{Simplificación de la DFT-2D $\mathrm{D}_{h e x}$}

Se ha presentado de una forma genérica la ecuación que permite el cálculo de la DFT para muestreo hexagonal. Partiendo de esa ecuación, se pueden aplicar di-

\footnotetext{
${ }^{\text {i}}$ Por ejemplo, la elección adecuada de la matriz de periodicidad, $\mathbf{N}$ (rellenando con ceros la secuencia $x[\mathbf{n}]$ si fuera necesario para cumplir la propiedad de cobertura), permite escoger conformadores que generen una distribución de los haces rectangular o hexagonal, independientemente de la rejilla utilizada para la posición de los elementos [Mer83].
} 
versos algoritmos para implementarla de una forma eficiente [Gue86]. Los citados algoritmos para el cálculo eficiente de la DFT, se basan en que en la ecuación (4.29) se necesita hacer un sumatorio sobre el conjunto de las $N$ muestras de $x[\mathbf{n}]$, pero realmente es lo mismo coger una muestra concreta o algunos de sus equivalentes periódicos en la secuencia repetida $\tilde{x}[\mathbf{n}]$, lo que importa, es que el conjunto escogido represente a las $N$ posibles muestras distintas que hay en la rejilla total $L_{V}$. Una selección adecuada del citado conjunto de $N$ muestras dispersas, permitirá simplificar considerablemente el cálculo de la DFT.

Antes de establecer criterios para la selección de ese conjunto de muestras, es necesario introducir algunos conceptos de la teoría de los números. Se denomina clase de equivalencia $\left[\mathbf{n}_{i}\right]$, a todas las posiciones dentro de la rejilla $L_{V}$, que se puede obtener desplazándose desde la posición $\mathbf{n}_{i}$, sobre la citada rejilla con la matriz de periodicidad $\mathbf{N}$, es decir todas las posiciones periódicas respecto a $\mathbf{n}_{i}$. Evidentemente, hay tantas clases de equivalencia como muestras tenga la señal $x[\mathbf{n}]$, es decir $N$; al conjunto de las $N$ clases de equivalencias se le denomina residuo módulo $\mathbf{N}$, y se representa por $L_{\mathbf{I} / \mathbf{N}}$. Al escoger una posición de cada una de las $N$ clases, se obtiene un conjunto de representantes, que es lo que se necesita para realizar el sumatorio de (4.29). Haciendo el mismo razonamiento en el dominio transformado, pero teniendo en cuenta que ahora la periodicidad la fija la matriz $\mathbf{N}^{\dagger}$, se hablará de residuo módulo $\mathbf{N}^{\dagger}$ representado como $L_{\mathbf{I} / \mathbf{N}^{\dagger}}$. Teniendo en cuenta ambas definiciones se pueden reescribir las ecuaciones transformadas como:

$$
\begin{array}{cc}
X[\mathbf{k}]=\sum_{\mathbf{n} \in L_{\mathbf{I} / \mathbf{N}}} x[\mathbf{n}] e^{-j \mathbf{k}^{\dagger}\left(2 \pi \mathbf{N}^{-1}\right) \mathbf{n}} & \mathbf{k} \in L_{\mathbf{I} / \mathbf{N}^{\dagger}} \\
x[\mathbf{n}]=\frac{1}{|\operatorname{det} \mathbf{N}|} \sum_{\mathbf{k} \in L_{\mathbf{I} / \mathbf{N}^{\dagger}}} X[\mathbf{k}] e^{j \mathbf{k}^{\dagger}\left(2 \pi \mathbf{N}^{-1}\right) \mathbf{n}} & \mathbf{n} \in L_{\mathbf{I} / \mathbf{N}}
\end{array}
$$

Los únicos parámetros que se pueden variar para optimizar el cálculo de la DFT son, la matriz de periodicidad, cuando ésta no es única, y la elección de los conjuntos de representativos para los sumatorios de (4.31) y (4.32).

Criterios de optimización A la hora de optimizar la arquitectura es necesario disponer de algunos criterios para realizar una correcta selección; teniendo en cuenta que la estructura del conformador será similar a la obtenida para los casos de agrupaciones lineales y planas rectangulares, los criterios a tener en mente serán:

- Buscar una descomposición en filas y columnas al estilo de la figura 4.4.

- Maximizar la modularidad del diseño, con el menor número posible de celdas básicas diferentes.

- Reducir o eliminar los desfasadores fijos que interconectan las diversas capas, tal y como se consigue con el algoritmo PFA en una dimensión. 
Conversión a DFT-2D rectangular Como primer paso, se descompondrá la matriz de periodicidad $\mathbf{N}$ en su forma normal de Smith:

$$
\mathrm{N}=\mathrm{CDE}
$$

donde $\mathbf{C}$ y $\mathbf{E}$ son matrices de enteros con determinante unidad, mientras que $\mathbf{D}$ es una matriz diagonal de números enteros donde $D_{11}$ es divisor de $D_{22}$. En general, existen múltiples soluciones para (4.33).

Introduciendo (4.33) en (4.31) se obtiene:

$$
X[\mathbf{k}]=\sum_{\mathbf{n} \in L_{\mathbf{I} / \mathbf{N}}} x[\mathbf{n}] e^{-j \mathbf{k}^{\dagger}\left(\mathbf{E}^{-1}\left(2 \pi \mathbf{D}^{-1}\right) \mathbf{C}^{-1} \mathbf{n}\right.} \quad \mathbf{k} \in L_{\mathbf{I} / \mathbf{N}^{\dagger}}
$$

definiendo:

$$
\begin{aligned}
& \mathbf{n}_{r}=\mathbf{C}^{-1} \mathbf{n} \\
& \mathbf{k}_{r}=\left(\mathbf{E}^{-1}\right)^{\dagger} \mathbf{k}
\end{aligned}
$$

se puede simplificar:

$$
X\left[(\mathbf{E})^{\dagger} \mathbf{k}_{r}\right]=\sum_{\mathbf{n}_{r} \in L_{\mathbf{I} / \mathbf{D}}} x\left[\mathbf{C n}_{r}\right] e^{-j \mathbf{k}_{r}^{\dagger}\left(2 \pi \mathbf{D}^{-1}\right) \mathbf{n}_{r}} \quad \quad \mathbf{k}_{r} \in L_{\mathbf{I} / \mathbf{D}}
$$

resultado que define una DFT-2 $\mathrm{D}_{\text {rec }}$ de tamaño $D_{11} \times D_{22}$ típica, ya resuelta en el apartado anterior (Fig. 4.4). Teniendo en cuenta que el determinante de $\mathbf{D}$ es igual a $D_{11} \cdot D_{22}$ y también igual al determinante de $\mathbf{N}$, que a su vez es el número de elementos de la secuencia $x[\mathbf{n}]$, se puede comprobar, que dada una secuencia, existe un número finito de posibles matrices $D$, que cumplan todos los requisitos; por ejemplo si det $|\mathbf{N}|=27$, las únicas posibilidades son $\mathbf{D}=\left[\begin{array}{ll}3 & 0 \\ 0 & 9\end{array}\right]$ y $\mathbf{D}=\left[\begin{array}{ll}1 & 0 \\ 0 & 27\end{array}\right]$, que equivalen respectivamente a un conformador para una agrupación plana de tamaño $3 \times 9$ y un conformador para una agrupación lineal de 27 elementos.

Algoritmo bidimensional de factores primos El algoritmo PFA se puede generalizar a $M$ dimensiones, partiendo del teorema del residuo chino $\left(\mathrm{CRT}^{\mathrm{i}}\right)$ para número enteros [Gue86], cuyo enunciado permite dividir el residuo $\left(\mathbf{n} \in L_{\mathbf{I} / \mathbf{N}}\right)$ en dos residuos de menor tamaño $\left(\mathbf{n} \in L_{\mathbf{I} / \mathbf{P}_{1}}\right.$ y $\left.\mathbf{n} \in L_{\mathbf{I} / \mathbf{Q}_{2}}\right)$, de forma que, para obtener $n$ representantes de las clases equivalentes, se pueden tomar $p$ representantes de $\mathbf{n} \in L_{\mathbf{I} / \mathbf{P}_{1}}$ y $q$ representantes de $\mathbf{n} \in L_{\mathbf{I} / \mathbf{Q}_{2}}$, donde $p$ y $q$ son dos números primos entre sí, cumpliéndose además que el determinante de la matriz $\mathbf{N}$ es el producto $p q$, además de ser el número de elementos $N$. La relación entre las matrices $\mathbf{N}, \mathbf{P}_{1}$ y $\mathbf{Q}_{2}$ debe ser:

$$
\mathbf{N}=\mathbf{P}_{1} \mathbf{Q}_{1}=\mathbf{Q}_{2} \mathbf{P}_{2}
$$

Teniendo en cuenta (4.33), D también tiene como determinante $p q$, por lo que fácilmente se puede descomponer en el producto de dos matrices diagonales con determinantes respectivos $p$ y $q$ :

$$
\mathbf{D}=\mathbf{D}_{1} \mathbf{D}_{2}
$$

\footnotetext{
${ }^{\mathrm{i} C h i n e s e ~ R e m a i n d e r}$ Theorem.
} 
en ese caso, es inmediato realizar la descomposición de (4.37) como:

$$
\begin{aligned}
& \mathbf{P}_{1} \mathbf{Q}_{1}=\left(\mathbf{C} \mathbf{D}_{1}\right)\left(\mathbf{D}_{2} \mathbf{E}\right) \\
& \mathbf{Q}_{2} \mathbf{P}_{2}=\left(\mathbf{C} \mathbf{D}_{2}\right)\left(\mathbf{D}_{1} \mathbf{E}\right)
\end{aligned}
$$

Una vez se ha encontrado la descomposición matricial necesaria para aplicar el CRT, falta el último paso: realizar una asignación de cada elemento de $\mathbf{n} \in L_{\mathbf{I} / \mathbf{N}}$ a un par $\left(\mathbf{n}_{1}, \mathbf{n}_{2}\right)$, donde $\mathbf{n}_{1} \in L_{\mathbf{I} / \mathbf{P}_{1}}$ y $\mathbf{n}_{2} \in L_{\mathbf{I} / \mathbf{Q}_{2}}$. Análogamente, se debe realizar otra asignación en el dominio transformado: cada elemento de $\mathbf{k} \in L_{\mathbf{I} / \mathbf{N}^{\dagger}}$ a un par $\left(\mathbf{k}_{1}, \mathbf{k}_{2}\right)$ donde $\mathbf{k}_{1} \in L_{\mathbf{I} / \mathbf{Q}_{1}^{\dagger}}$ y $\mathbf{k}_{2} \in L_{\mathbf{I} / \mathbf{P}_{2}^{\dagger}}$. Una de las posibles soluciones es:

$$
\begin{aligned}
& \mathbf{n} \equiv \mathbf{D}_{2} \mathbf{C}^{-1} \mathbf{n}_{1}+\left(\left(\mathbf{D}_{1} \mathbf{C}^{-1} \mathbf{n}_{2}\right)\right)_{\mathbf{N}} \\
& \mathbf{k} \equiv \mathbf{E}^{\dagger} \mathbf{D}_{2}\left(\mathbf{E}^{-1}\right)^{\dagger} \mathbf{k}_{1}+\left(\left(\mathbf{E}^{\dagger} \mathbf{D}_{2}\left(\mathbf{E}^{-1}\right)^{\dagger} \mathbf{k}_{2}\right)\right)_{\mathbf{N}^{\dagger}}
\end{aligned}
$$

Definiendo la nuevas variables

$$
\begin{aligned}
& \mathbf{n}_{\mathbf{r} \mathbf{1}} \equiv\left(\left(\mathbf{C}^{-1} \mathbf{n}_{1}\right)\right)_{\mathbf{D}_{\mathbf{1}}} \\
& \mathbf{n}_{\mathbf{r} \mathbf{2}} \equiv\left(\left(\mathbf{C}^{-1} \mathbf{n}_{2}\right)\right)_{\mathbf{D}_{\mathbf{2}}} \\
& \mathbf{k}_{\mathbf{r} \mathbf{1}} \equiv\left(\left(\mathbf{D}_{\mathbf{2}} \mathbf{E}^{-1} \mathbf{k}_{1}\right)\right)_{\mathbf{D}_{\mathbf{1}}} \\
& \mathbf{k}_{\mathbf{r} \mathbf{2}} \equiv\left(\left(\mathbf{D}_{\mathbf{1}} \mathbf{E}^{-1} \mathbf{k}_{2}\right)\right)_{\mathbf{D}_{\mathbf{2}}}
\end{aligned}
$$

que únicamente implican una nueva permutación de los índices, se puede simplificar la expresión de la DFT-2D hex como:

$$
X\left[\mathbf{k}_{\mathbf{r} \mathbf{1}}, \mathbf{k}_{\mathbf{r} \mathbf{2}}\right]=\sum_{\mathbf{n}_{\mathbf{r}} \mathbf{1}} \sum_{\mathbf{n}_{\mathbf{r}} \mathbf{2}} x\left[\mathbf{n}_{\mathbf{r} \mathbf{1}}, \mathbf{n}_{\mathbf{r} \mathbf{2}}\right] e^{-j 2 \pi \mathbf{k}_{\mathbf{r} \mathbf{1}}^{\dagger} \mathbf{D}_{\mathbf{1}}{ }^{-1} \mathbf{n}_{\mathbf{r}} \mathbf{1}} e^{-j 2 \pi \mathbf{k}_{\mathbf{r} 2} \mathbf{D}^{\dagger} \mathbf{D}^{-1} \mathbf{n}_{\mathbf{r}} \mathbf{2}}
$$

expresión que se puede descomponer fácilmente en dos DFT-2D rec, de tamaños $p$ y $q$ evaluables de acuerdo con lo indicado en el apartado anterior, o incluso se puede volver a aplicar el algoritmo MDFA.

Resumen DFT-2D hex A la hora de implementar una matriz de Butler generalizada para rejilla hexagonal, tanto en el dominio de los elementos de la agrupación como en el dominio de la posición de los haces, los pasos a seguir para su diseño serán los indicados a continuación, que se muestran gráficamente en la figura 4.7:

1. Escoger la secuencia inicial, $x[\mathbf{n}]$, teniendo en cuenta que se debe cumplir la propiedad de cobertura; lo que puede obligar a rellenar la secuencia y que ésta tenga un número de puntos, $H$, mayor que el número de elementos de la agrupación, $E$.

2. Escoger la matriz de periodicidad $\mathbf{N}$ y descomponerla según (4.33).

3. Aplicar uno de los métodos siguientes:

- De acuerdo con la matriz D, descomponer como en el caso de DFT-2D rec para una rejilla rectangular $D_{11} \times D_{22}$. 


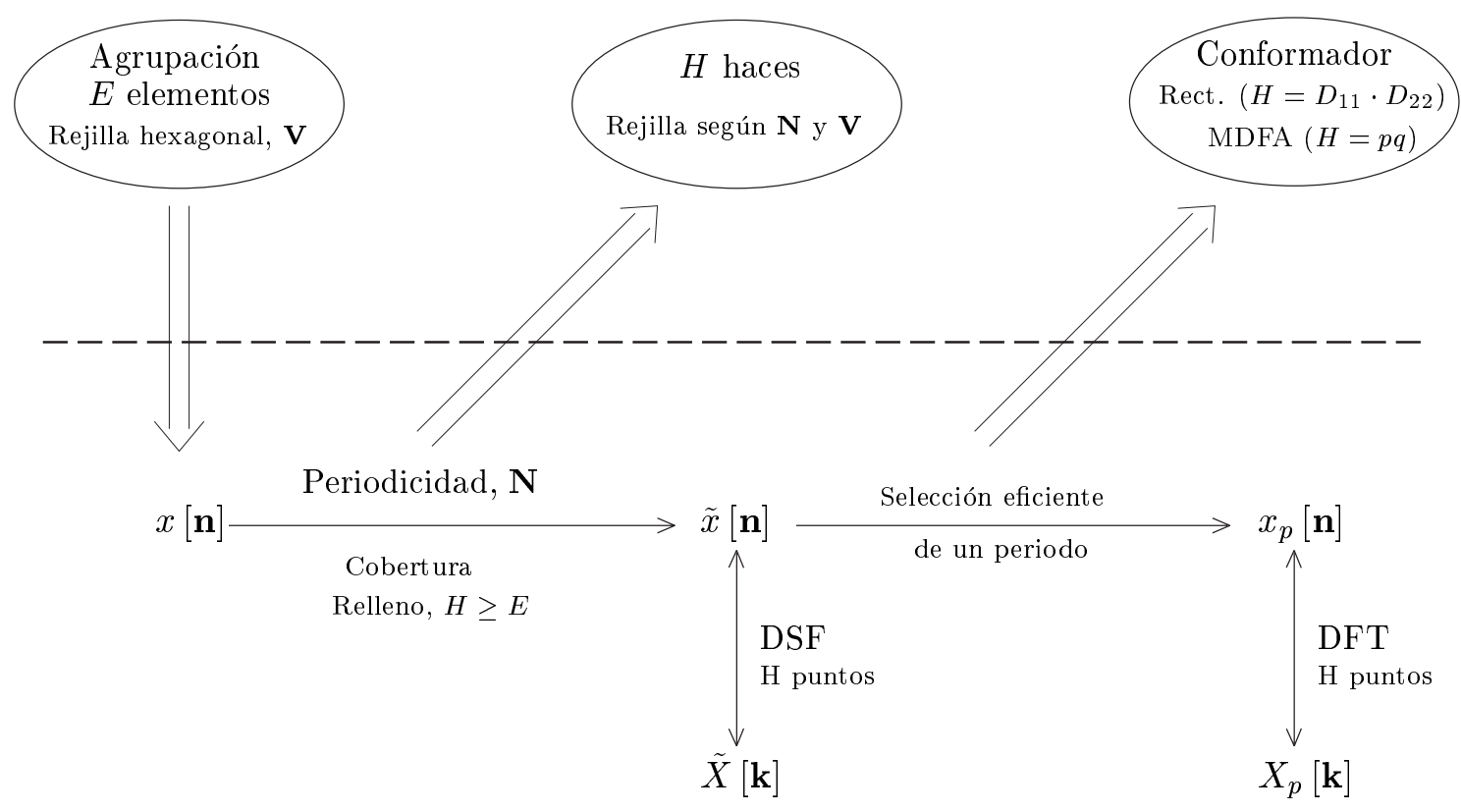

Figura 4.7: Resumen del cálculo de la arquitectura óptima de red conformadora para agrupaciones con rejilla hexagonal.

- Si el número de elementos de la secuencia, $H$, se puede expresar como el producto de dos factores primos entre sí $(H=p q)$, aplicar el método MDFA, que descompone la DFT-2D hex original en un total de $p+q$ DFT-2D $\mathrm{D}_{r e c}^{s}$ de menor tamañoi en concreto $p$ DFT-2D ${ }_{r e c}^{s}$ de $q$ puntos y $q$ DFT-2D rec $\mathrm{D}_{\text {re }} p$ puntos. Posteriormente, se puede volver a aplicar el método MDFA, o bien descomponer en filas y columnas de acuerdo con lo indicado previamente para DFT-2D rec .

4. Tener en cuenta las asignaciones de índices realizadas en cada paso, para ordenar finalmente, tanto los accesos de los haces como los de los elementos de antena. Además, se debe tener en cuenta una reordenación final de los accesos de los elementos de antena, para que la red DFT-2 $\mathrm{D}_{h e x}$ implemente la DFT-2D $\mathrm{D}_{\text {hex }}^{-1}$.

- Si se utilizó la descomposición rectangular, en el plano de los elementos se debe aplicar la siguiente relación entre la numeración de la red conformadora rectangular y la numeración de los elementos de la antena:

$$
\mathbf{n}_{\text {antena }}=-\mathbf{C n}_{\text {red }}
$$

mientras que los accesos de la red en el plano de los haces, corresponden a las siguientes posiciones de los haces en el dominio frecuencial:

$$
\mathbf{k}_{h a z}=\mathbf{E}^{\dagger} \mathbf{k}_{\text {red }}
$$

posiciones que se pueden traducir al dominio $(u, v)$ de acuerdo con (4.22).

\footnotetext{
${ }^{\mathrm{i}}$ Alguna de las dos DFT-2D rec podría ser de tamaño $S \times 1$, es decir una DFT-1D.
} 


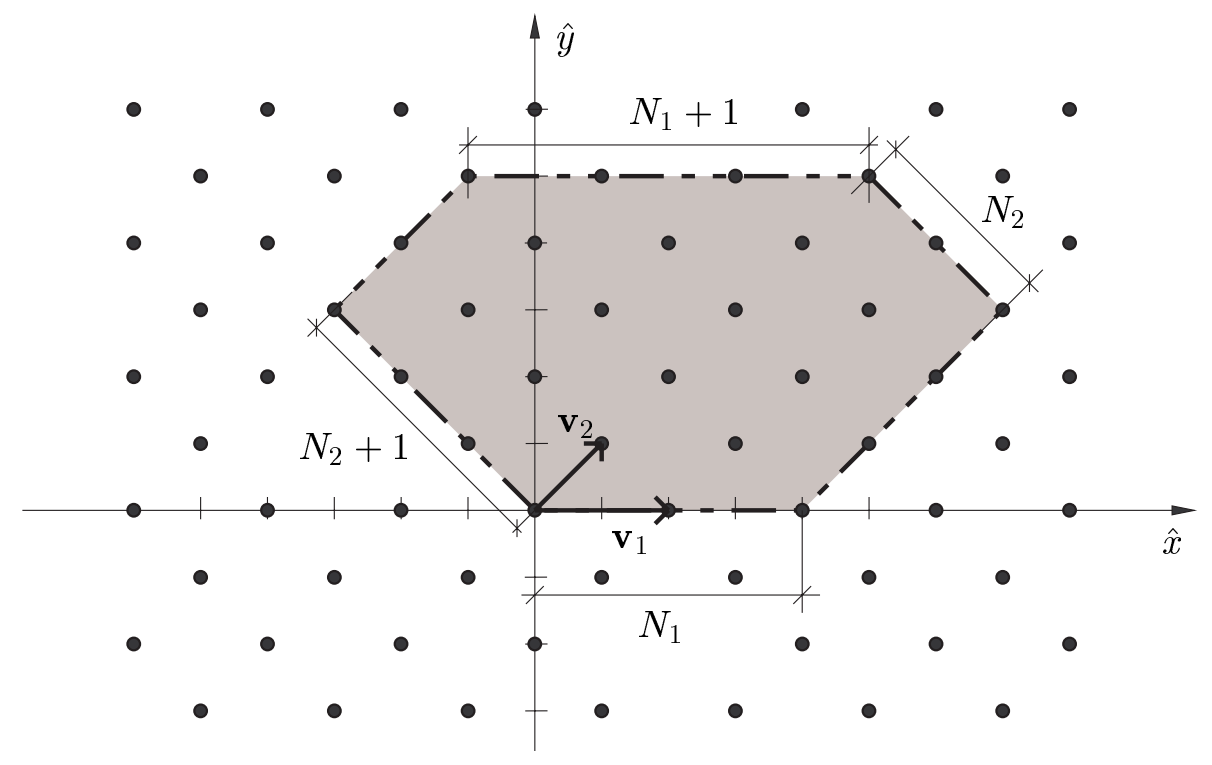

Figura 4.8: Ejemplo de secuencia bidimensional sobre rejilla hexagonal, que cumple la propiedad de cobertura.

- Si se aplicó el método MDFA, se deberán invertir las ecuaciones (4.41) para obtener las ordenaciones en el plano de los elementos y en el plano de los haces, teniendo en cuenta, para el caso de los elementos, el cambio de signo para implementar la DFT-2D hex . En el plano de los haces se deberá utilizar (4.22) para pasar las coordenadas de frecuencias $\left(\mathbf{k}_{h a z}\right)$ al dominio $(u, v)$.

Ejemplos de aplicación Como se ha visto previamente, todo el proceso de simplificación de la red conformadora de haces, descansa sobre la descomposición factorial del número de elementos de la secuencia original. Es habitual que la distribución hexagonal de la agrupación presente un contorno hexagonal regular, con $L$ elementos por lado, lo cual implica una agrupación de $E=3 L^{2}-3 L+1$ elementos. Una secuencia formada por esos $E$ puntos, cumple la propiedad de cobertura dando lugar a un conjunto de $E$ haces distribuidos en el plano $(u, v)$, de acuerdo con una rejilla hexagonal. Desgraciadamente, esa secuencia no permite una simplificación adecuada de la red conformadora, ya que el número de elementos resulta ser un número primo o producto de números primos $(7,19,37,61,91=7 \times 13,127,169=13 \times 13$, $217=7 \times 13,271, \ldots)$, demasiado grande para permitir una descomposición en factores simples, que permitan utilizar de forma intensiva redes de pequeño tamaño. Por consiguiente, convendrá rellenar la secuencia de los elementos de la agrupación con $H-E$ elementos ficticios extra, de forma que, se cumpla simultáneamente la propiedad de cobertura, que el número total de elementos $H$ sea factorizable en factores pequeños y que la matriz de periodicidad de la nueva secuencia, dé lugar a una distribución hexagonal de haces. Dos soluciones adecuadas para estos tres requisitos son las secuencias rectangulares y las secuencias hexagonales, no regulares (Fig. 4.8); éstas últimas vienen definidas por dos números $N_{1}$ y $N_{2}$, con un número total de 
elementos $H=2 N_{1} N_{2}+N_{2}^{2}$, de forma que, para el caso particular $N_{1}=N_{2}$, el número de elementos será $H=3 N_{2}^{2}$, que permite una fácil descomposición factorial.

En la figura 4.9 se muestra como, para una agrupación hexagonal regular de 19 elementos, se puede utilizar tanto una red conformadora de 27 elementos (relleno a secuencia rectangular no regular), como una de 36 elementos (relleno a secuencia rectangular) añadiendo adecuadamente elementos ficticios extra. Ambos conformadores admiten la descomposición a DFT-2D rectangular, de forma que a la primera le corresponde una descomposición $3 \times 9$, mientras que a la segunda le corresponde una $6 \times 6$. Posteriormente, los conformadores de tamaño 9 y 6 se pueden descomponer en conformadores de 2 y 3 elementos.

En concreto, si se escoge el conformador de 27 elementos, la descomposición $3 \times 9$ implica que la red conformadora se puede obtener mediante tres redes $9 \times 9$, interconectadas con nueve redes $3 \times 3$, tal y como se muestra en la figura 4.10 . Como posterior simplificación, en la figura 4.10 se ha descompuesto cada red $9 \times 9$ en seis redes $3 \times 3$ aplicando el algoritmo Cooley-Tukey en base 3 [Opp89]. El paso final, consiste en la reordenación de los accesos, de acuerdo con la posición real de los elementos de la agrupación, siendo la numeración indicada en la figura 4.10, la correspondiente a la geometría de la figura 4.13. Otra posible arquitectura de red conformadora, que hace un uso intensivo de DFT-1D de tamaño $3 \times 3$, es la mostrada en la figura 4.11, que corresponde a una secuencia rectangular similar a la de la figura $4.9 \mathrm{~b}$.

\subsubsection{Características de las celdas básicas del conformador de haces de radiofrecuencia con peso reducido}

En resumen, se está considerando una red conformadora de haces de radiofrecuencia de peso reducido, para la generación de múltiples haces distribuidos hexagonalmente en el dominio $(u, v)$, con una agrupación plana de antenas, con elementos distribuidos también de forma hexagonal.

Sus principales ventajas serían:

- Peso reducido, gracias a la utilización de tecnología MMIC, estimándose un peso aproximado de $2 \mathrm{~kg}$ para un conformador de haces de dimensiones $256 \times 256$ [Cor96].

- Tamaño reducido (limitado por el tamaño de los conectores).

- Número reducido de desfasadores fijos.

- Alta modularidad: la red completa puede construirse usando un único tipo de celda constructora.

Por otro lado, las celdas básicas MMIC, presentan unas pérdidas de inserción mayores que las del diseño equivalente en tecnología MIC, pero, a cambio, ofrecen 
a)
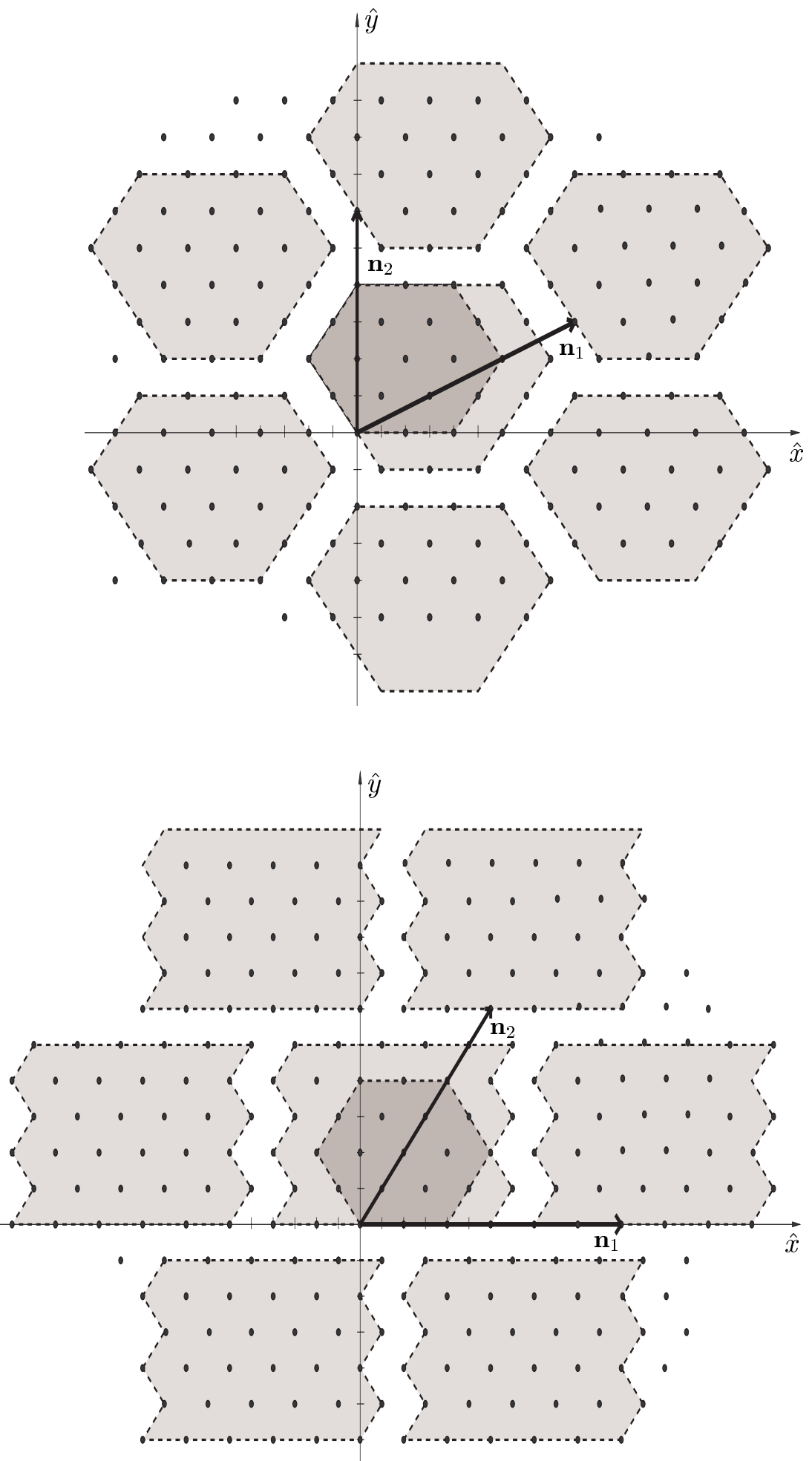

b)

Figura 4.9: Relleno de la secuencia correspondiente a una agrupación de $E=19$ elementos, con contorno hexagonal (trama más oscura) cumpliendo cobertura y distribución hexagonal de los haces. a) Secuencia hexagonal no regular con $H=27$ elementos. b) Secuencia rectangular con $H=36$ elementos. 
Accesos

Accesos

Haces

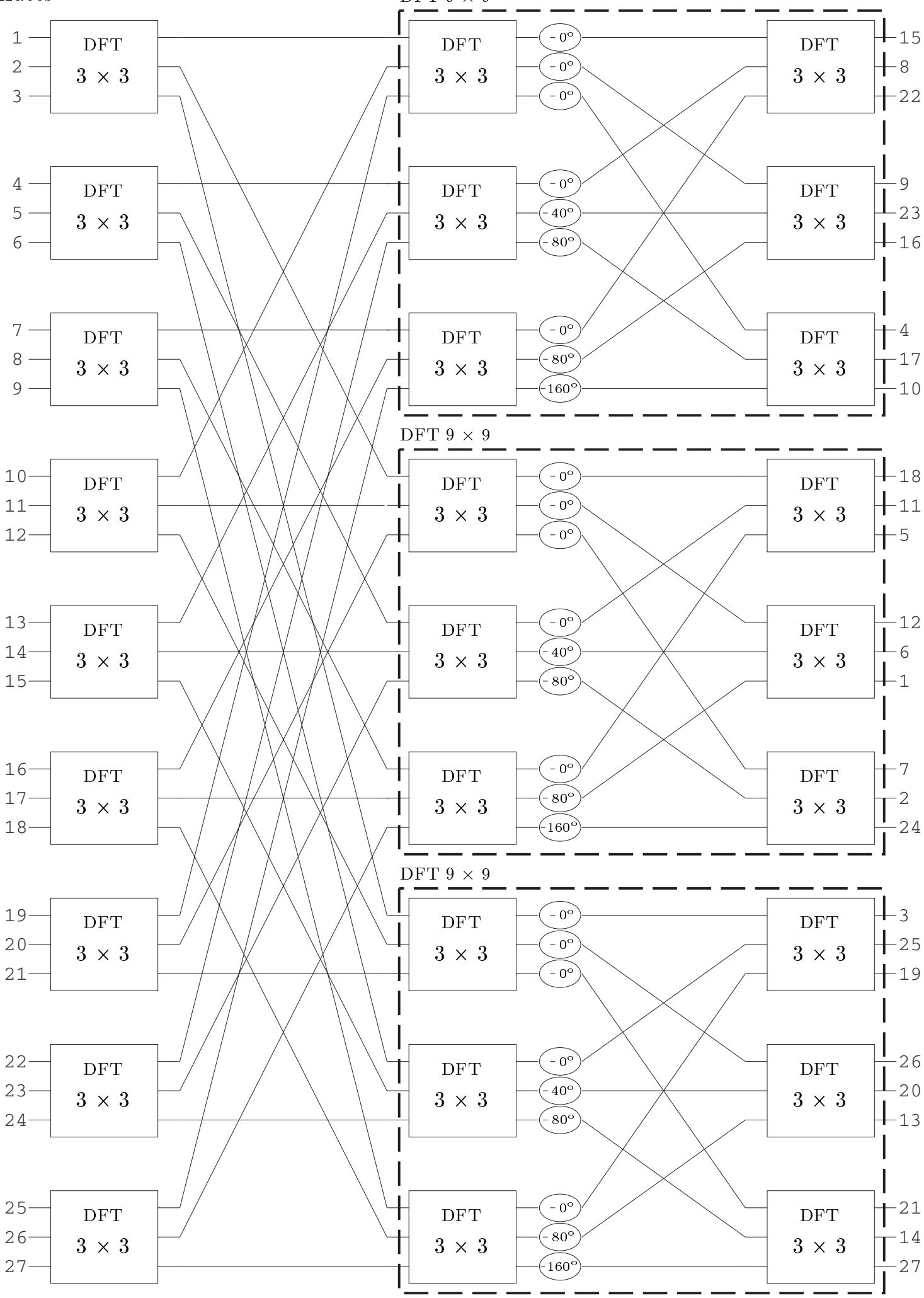

Figura 4.10: Arquitectura funcional del conformador de haces de Butler $27 \times 27$ para agrupaciones planas hexagonales. 

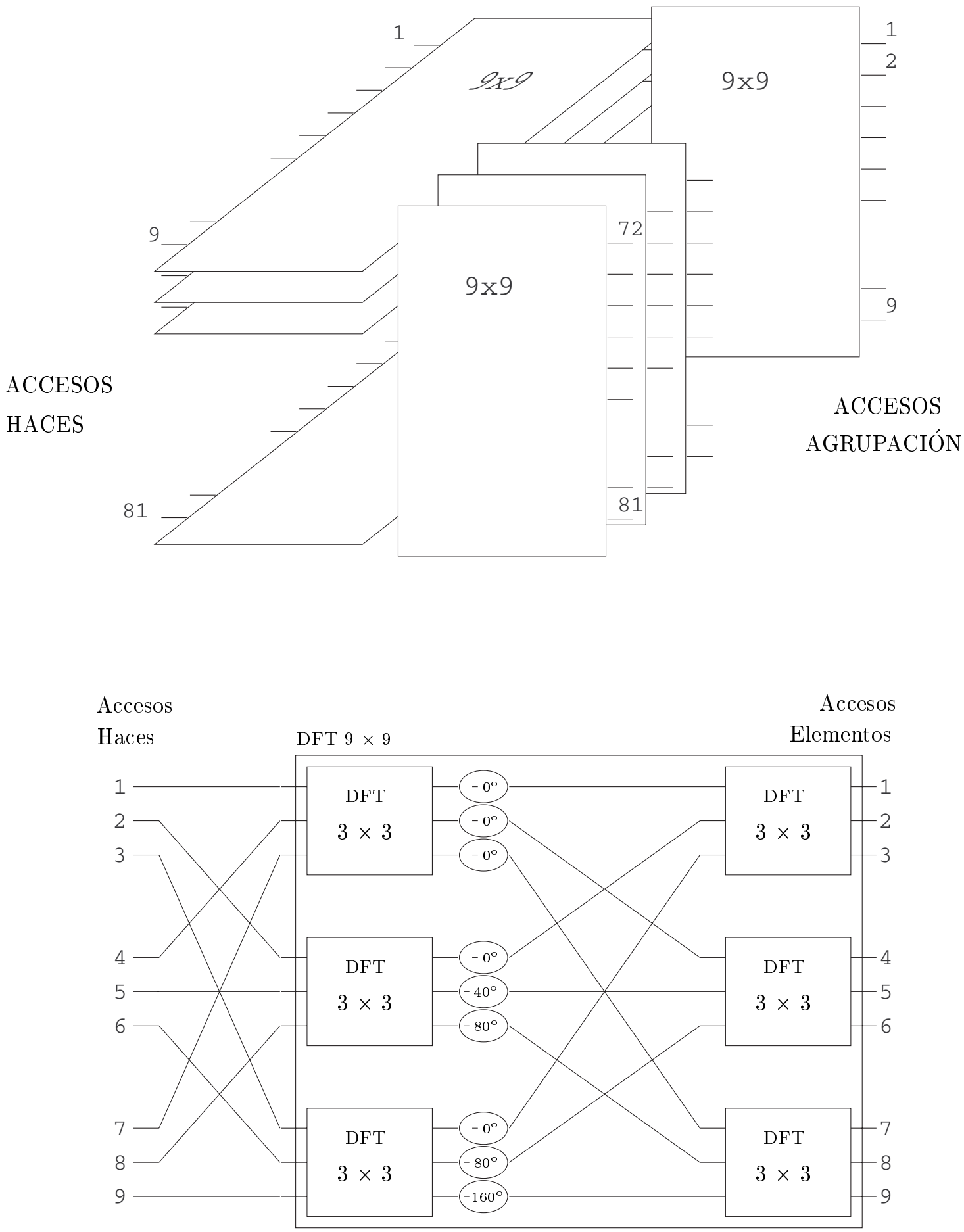

Figura 4.11: Arquitectura funcional del conformador de haces de Butler $81 \times 81$ para agrupaciones planas hexagonales. 
un tamaño reducido, una fiabilidad más alta y un menor tiempo de desarrollo. El efecto de las variaciones del proceso MMIC, puede evitarse mediante alguna forma de sintonización.

El diseño en estudio corresponde a una DFT de tres puntos, que de acuerdo con la figura 4.3, tendrá el diagrama de bloques funcional de la figura 4.12 y sería la celda básica para las redes conformadoras de haces de tamaño $27 \times 27$ (Fig. 4.10) y $81 \times 81$ (Fig. 4.11).

Las salidas de la red conformadora de haces $27 \times 27$ de la figura 4.10, serán conectadas a los 27 elementos de antena, correspondientes según la numeración mostrada en la figura 4.13, o al subconjunto de 19 elementos de la misma figura si el resto de salidas estuvieran terminadas.

Por otro lado, es de esperar que el circuito MMIC, que implemente la DFT de tres puntos, esté compuesto de elementos pasivos concentrados (bobinas y condensadores), de forma similar a como se puede implementar una DFT de dos puntos, compuesta de un híbrido y un desfasador, ambos elementos realizables a partir de elementos pasivos.

En resumen, un circuito pasivo muy simple, únicamente con bobinas y condensadores, es la celda básica para un conformador de haces de radiofrecuencia. Este circuito será implementado en tecnología MMIC. Para un proceso típico de una fundición MMIC, se puede esperar una varianza muy pequeña de los valores de las inductancias. Por otro lado, se puede esperar una varianza bastante significativa de los valores de las capacidades (principalmente debido a la varianza geométrica en la anchura del dieléctrico de los condensadores MIM). Siempre y cuando todos los MMICs de cada conformador de haces vengan de la misma oblea, todos los condensadores presentarán el mismo error sistemático, con una pequeña varianza entre ellos (menor del 2\%). Con el fin de solventar esta variación sistemática del proceso, en cuanto a la capacidad, se ha considerado un diseño sintonizable mediante diodos varactores. La tensión de control para estos varactores, sería compartida entre todos los dispositivos que formaran el conformador de haces, ya que la misma variación en la capacidad es esperable para la misma oblea.

\subsection{Diseño de la celda básica $3 \times 3$ con tecnología MMIC}

El objeto de esta sección es el diseño de una matriz $3 \times 3 \mathrm{MMIC}$, para una red conformadora de haces de radiofrecuencia, con peso reducido en banda $\mathrm{S}$.

Aunque el proceso D02AH puede no ser el óptimo para el diseño de circuito pasivo en banda S, la oportunidad de usar el proceso que se encontraba en ese momento bajo prueba en la sección XRM de ESTEC, lo hacía muy interesante para comprobar la validez de la nueva arquitectura de conformador de haces de radiofrecuencia. 
DFT $3 \times 3$

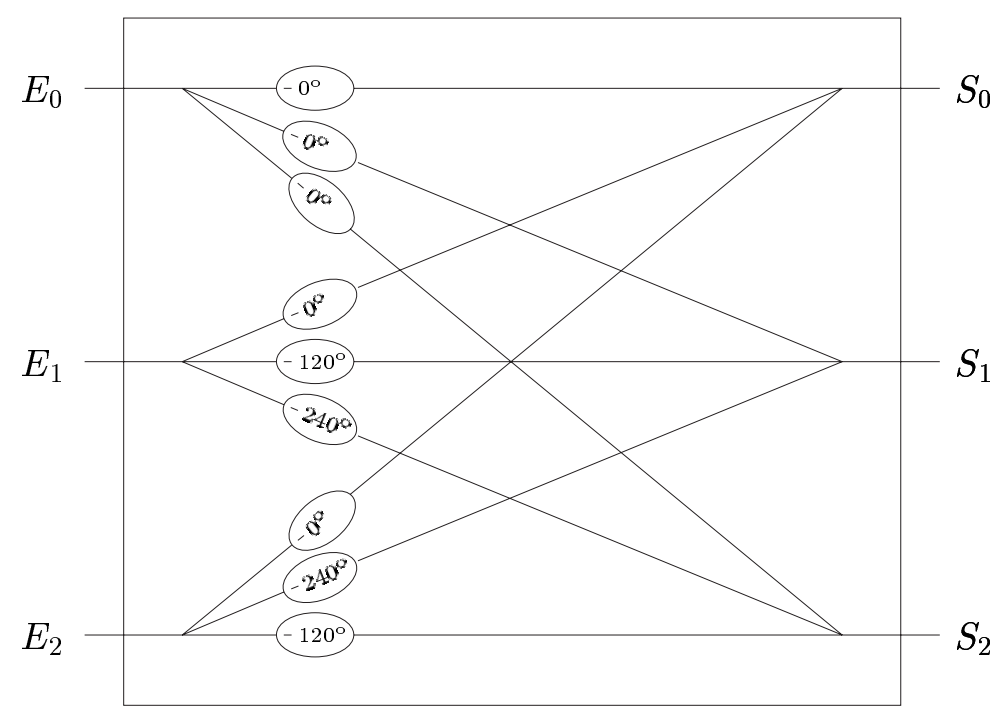

Figura 4.12: Diagrama de bloques funcional de la DFT unidimensional $3 \times 3$.

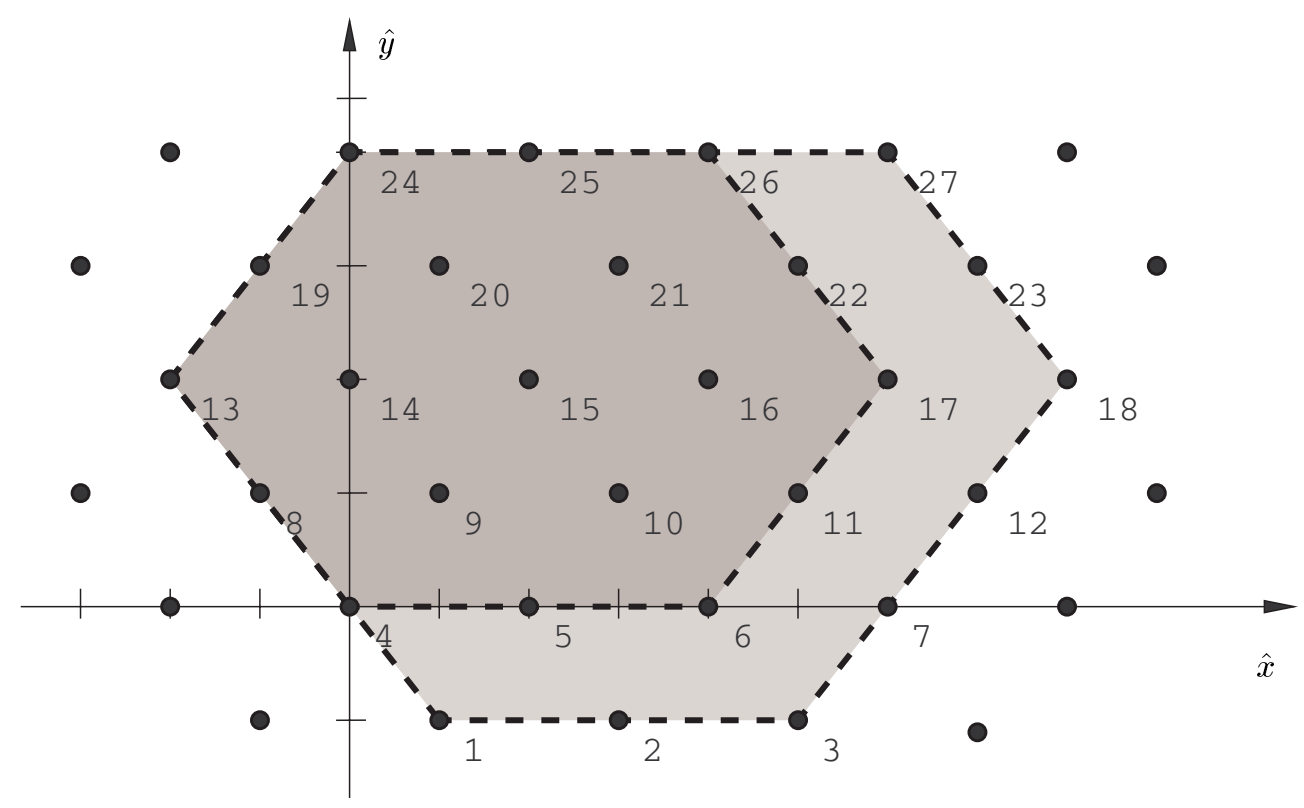

Figura 4.13: Numeración de los 27 elementos de la secuencia hexagonal no regular y de los 19 elementos de la agrupación hexagonal regular. 


\subsubsection{Datos generales}

Algunos datos generales sobre el circuito, el proceso de fabricación y las herramientas de diseño se desglosan a continuación.

\section{Especificaciones del circuito}

De acuerdo con los requisitos del sistema global se han definido las siguientes especificaciones:

- Frecuencia: $2.3 \mathrm{GHz}$.

- Un ancho de banda inferior al $1 \%$.

- Pérdidas de inserción tan bajas como sea posible.

- Diferencia entre las pérdidas de inserción de las salidas, para una misma entrada: $\pm 0.5 \mathrm{~dB}$.

- Diferencia entre las fases de inserción de las salidas, para una misma entrada: $\pm 0.5^{\circ}$.

- Pérdidas de Retorno $>15 \mathrm{~dB}$.

- Impedancia nominal: $50 \Omega$.

El diseño debe ser capaz de satisfacer todas las especificaciones, teniendo en cuenta posibles variaciones debidas a las tolerancias del proceso de fabricación [PML94].

\section{Características del proceso D02AH}

Al igual que en el caso del diseño del desfasador variable del capítulo 3, el proceso de fabricación utilizado ha sido el proceso D02AH, de la fundición Philips Microwave Limeil, cuyas características más importantes pueden consultarse en el apéndice B. Se trata de un proceso basado en transistores con alta movilidad de los electrones y heteroestructura pseudomórfica (P-HEMT $\left.{ }^{\mathrm{i}}\right)$.

\section{Herramientas de diseño}

La mayoría de las simulaciones llevadas a cabo durante este diseño, fueron hechas con el paquete LIBRA Serie IV de HP-EESOF. El entorno MATLAB de The MathWorks Inc., ha sido usado para simular la red conformadora de haces en su totalidad, debido

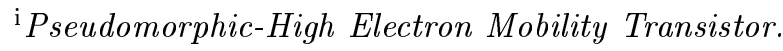




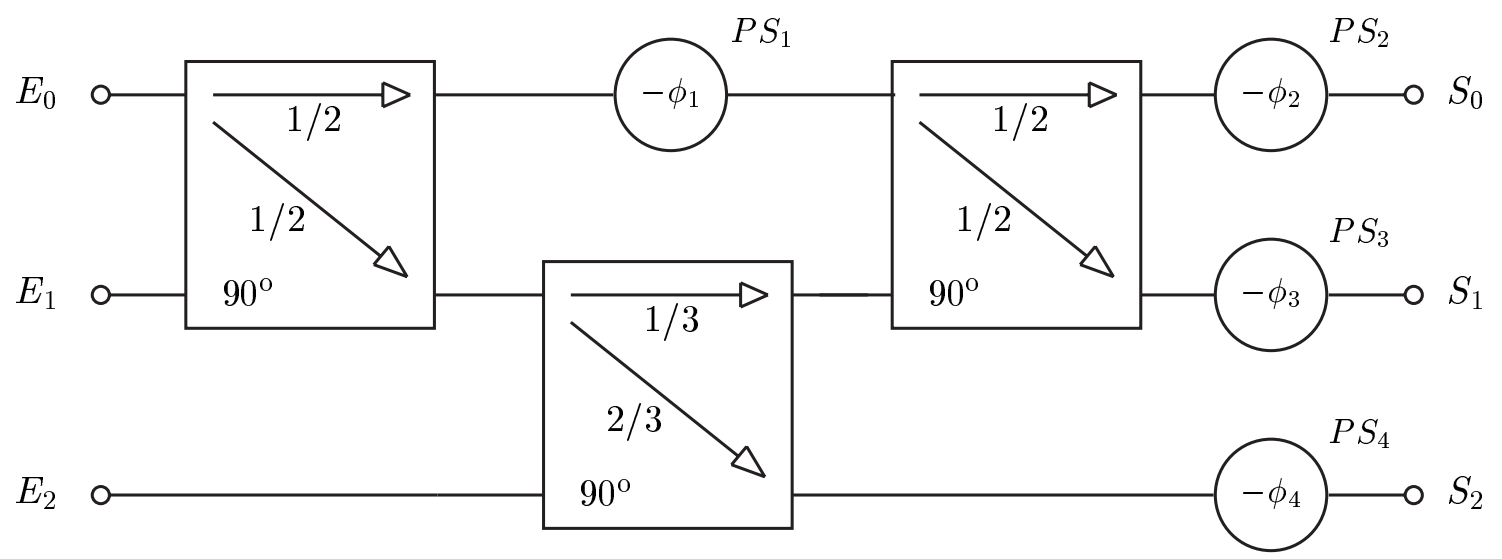

Figura 4.14: Arquitectura de la matriz fija $3 \times 3$ (Topología 1).

a las limitaciones en cuanto a número de accesos del paquete LIBRA. MATLAB también se ha utilizado para evaluar el diseño, en cuanto a su comportamiento en término de parámetros de antena.

\subsubsection{Matriz $3 \times 3$ fija}

\section{Selección de la topología del circuito}

Se estudiaron dos topologías para el diseño del circuito, con el diagrama de bloques funcional mostrado en la figura 4.12.

- Topología 1:

La primera estructura (Fig. 4.14) usa tres acopladores de $90^{\circ}$ y cuatro desfasadores fijos. Esta arquitectura es la típica mostrada en la literatura para matrices de Butler de tres puntos [CSE].

En esta topología, el valor de desfase de los desfasadores fijos $P S_{1}$, debe ser $0^{\circ}$ ó $180^{\circ}$, si se consideran acopladores de $90^{\circ}$ reales típicos (inserción de fase de $-90^{\circ}$ y $-180^{\circ}$ para las ramas directa y acoplada, respectivamente) y se requieren niveles iguales de amplitud en las tres salidas, independientemente de los valores de fase a la salida. En ambos casos, los valores de $P S_{2}, P S_{3}$ y $P S_{4}$ serán elegidos de acuerdo a una misma inserción de fase para las tres salidas $\left(S_{0}, S_{1}\right.$ y $\left.S_{2}\right)$ cuando la entrada activa sea $E_{0}$. Ambos casos se muestran en la figura 4.15, junto a sus diagrama de bloques funcionales.

Obviamente, la figura 4.15 a es la solución correcta, pero la figura 4.15b podría ser útil si las entradas $E_{1}$ y $E_{2}$ se conmutasen.

El acoplador de $90^{\circ}$, se implementaría con el equivalente concentrado del acoplador direccional con ramas paralelas ${ }^{\mathrm{i}}$. En este circuito equivalente, las sec-

\footnotetext{
${ }^{\mathrm{i}}$ Branch-line coupler, en terminología inglesa.
} 

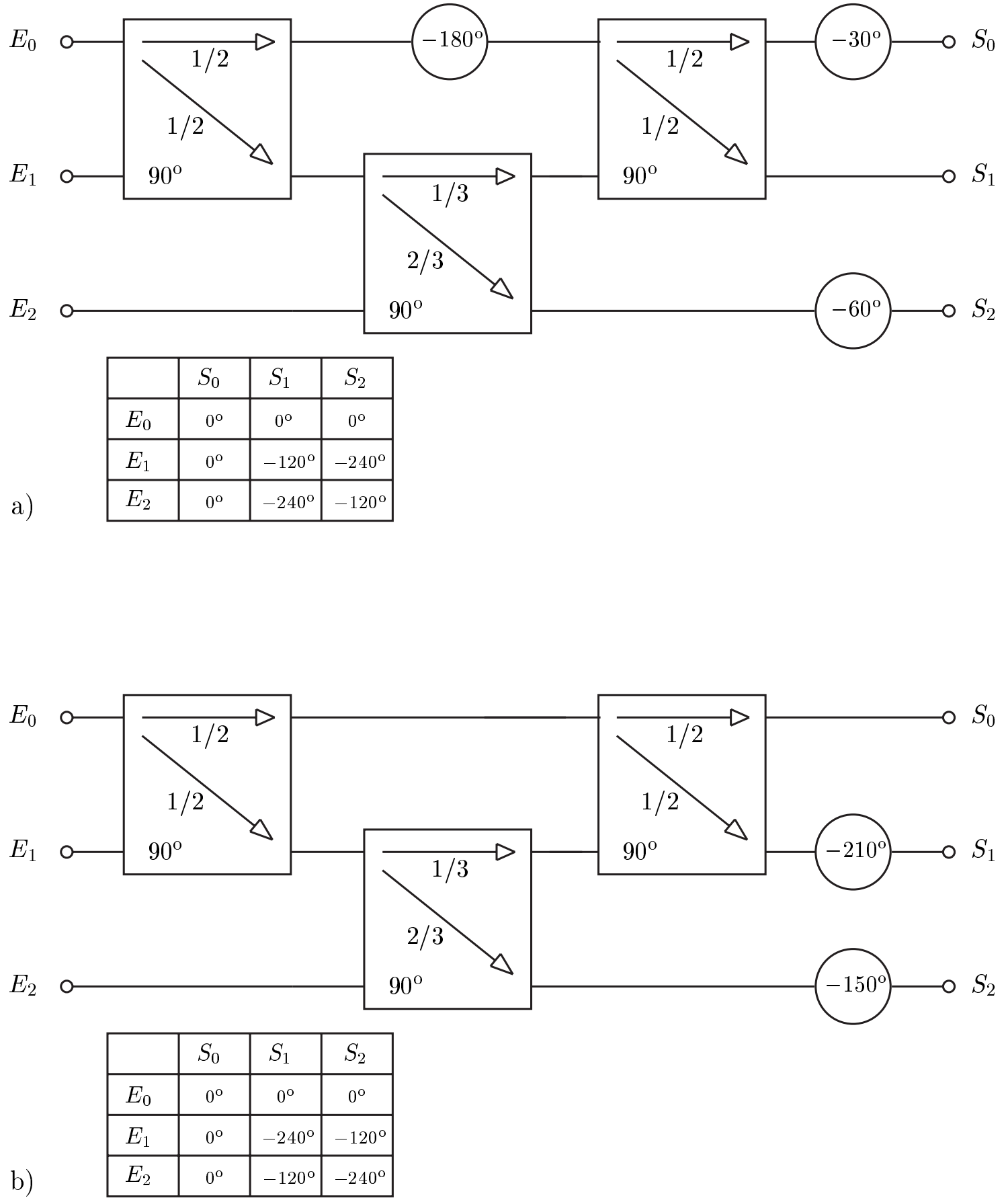

Figura 4.15: Implementación real de la topología 1. a) Opción 1. b) Opción 2. 

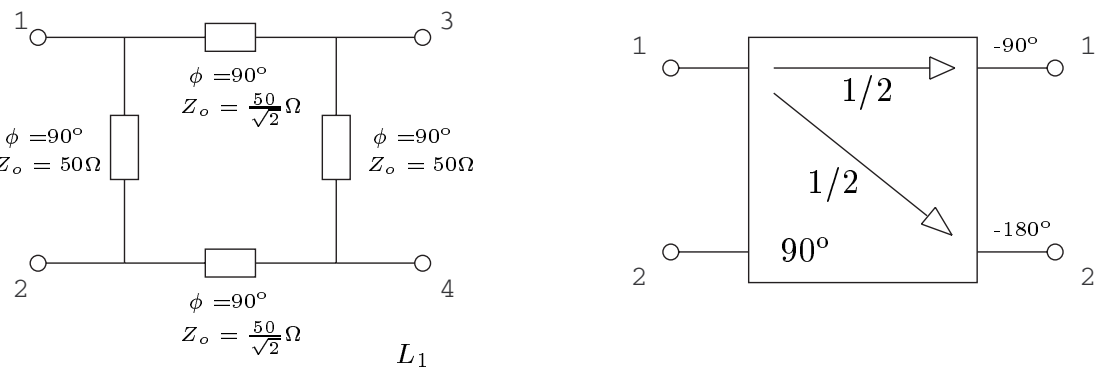

a)
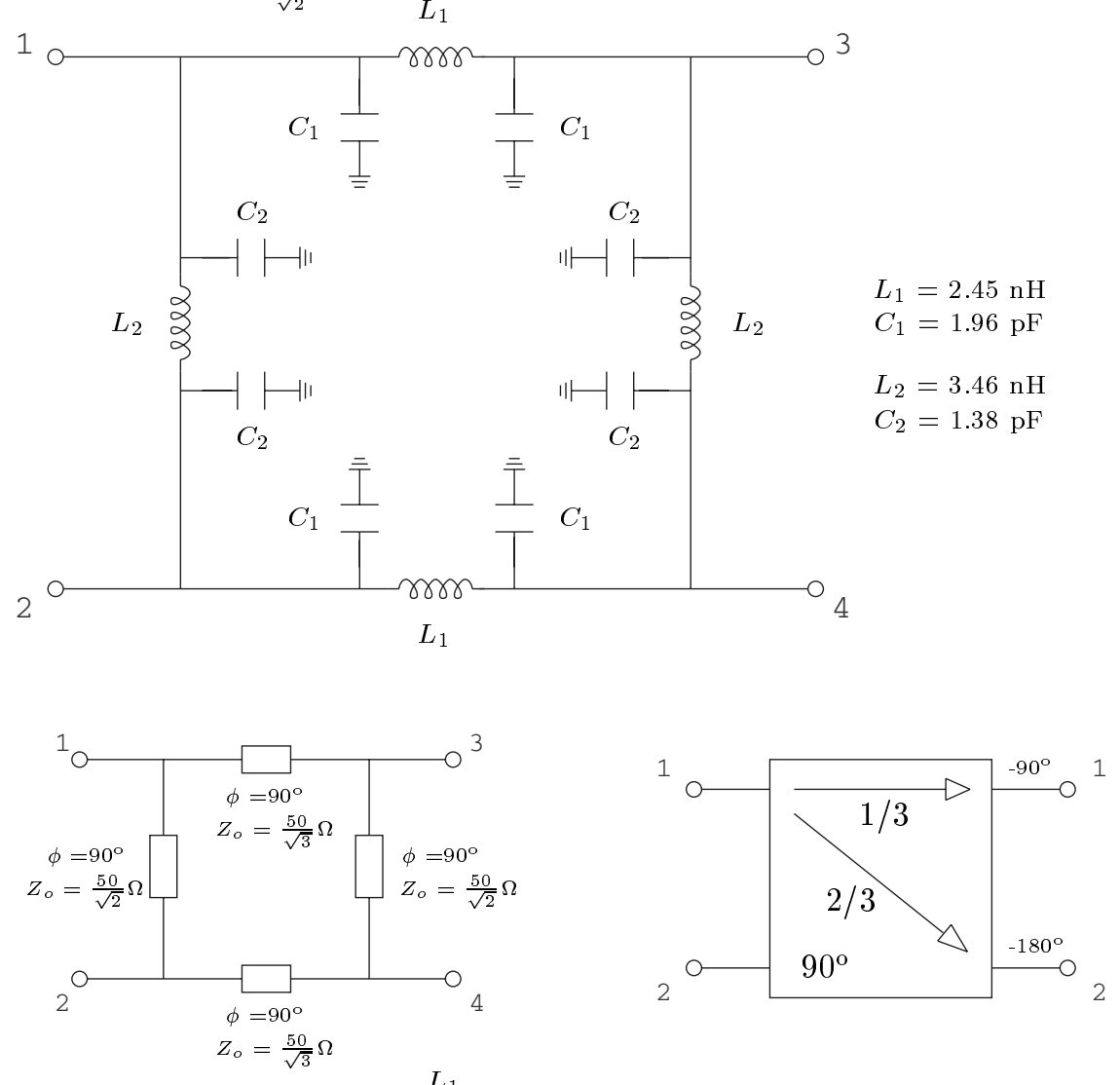

b)

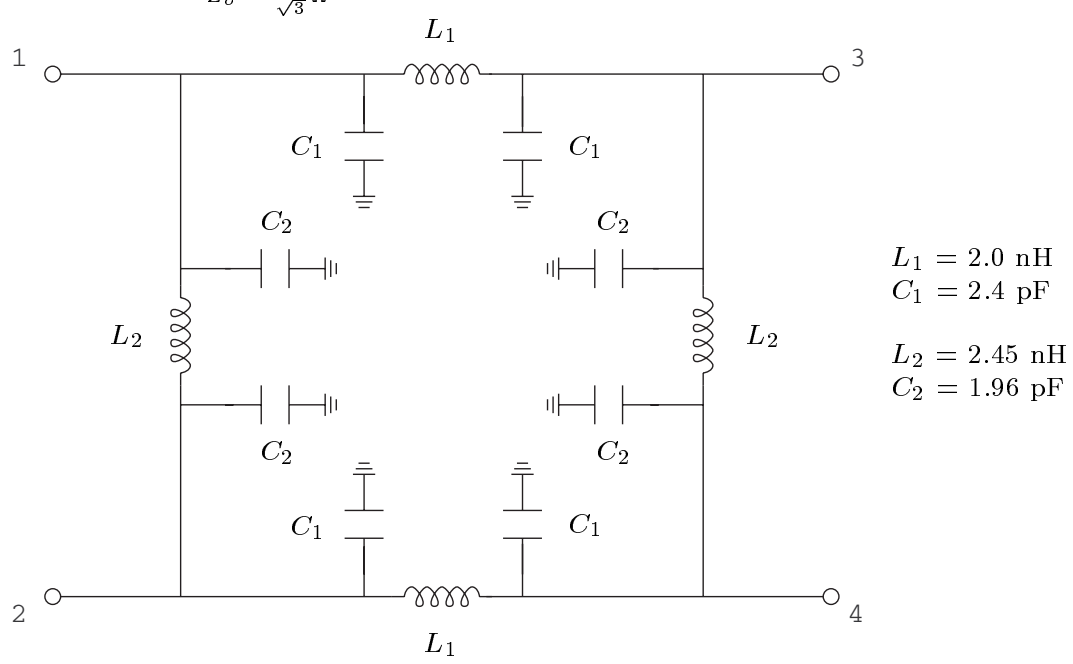

Figura 4.16: Circuitos equivalentes concentrados de acopladores direccionales con ramas laterales para una frecuencia de $2.3 \mathrm{GHz}$. a) Acoplamiento $-3 \mathrm{~dB}$ y desfase entre salidas $90^{\circ}$. b) Acoplamiento $-1.76 \mathrm{~dB}$ y desfase entre salidas $90^{\circ}$. 


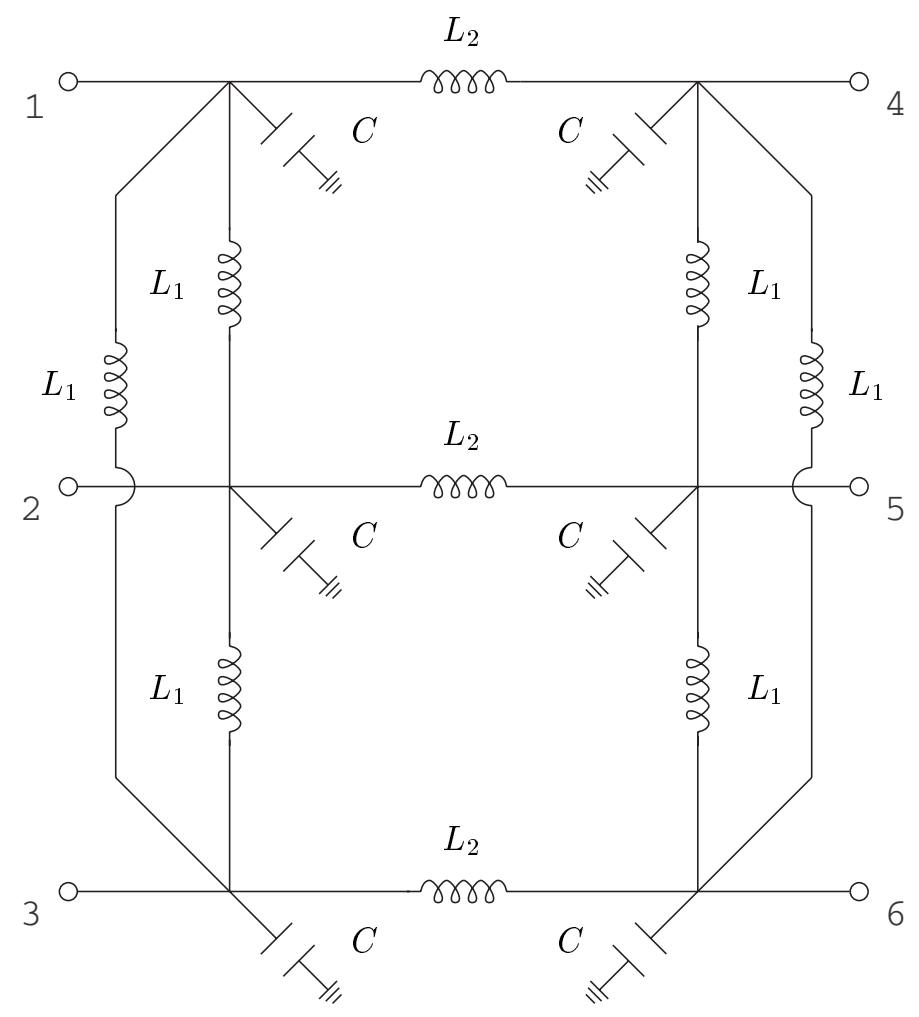

Figura 4.17: Arquitectura de la matriz fija $3 \times 3$ (topología 2).

ciones de cuarto de onda son reemplazadas por redes equivalentes en $\pi$. Los acopladores con ramas paralelas a $-3 \mathrm{~dB}$ y $-1.76 \mathrm{~dB}$ (ambos a $90^{\circ}$ ), se muestran en la figura 4.16 con sus redes en $\pi$ equivalentes, para $2.3 \mathrm{GHz}$ incluidas.

Cada acoplador incluye 4 bobinas y 4 condensadores (cada par $C_{1}-C_{2}$ están en paralelo y puede sustituirse por su equivalente). El desfasador fijo de $180^{\circ}$ sería implementado con dos redes en T desfasadoras de $90^{\circ}$, con lo cual se requieren 4 bobinas y 2 condensadores más. En total, 16 bobinas y 14 condensadores serían necesarios, sin tener en cuenta los desfasadores situados a las salidas $\left(P S_{1}, P S_{2}\right.$ y $\left.P S_{3}\right)$, cuya contribución puede ser incluida en las líneas de transmisión de interconexión de la red multicapa.

- Topología 2:

Con el objetivo de reducir el número total de elementos necesarios, se tomó un enfoque diferente. A partir de la teoría de redes, se obtuvo la celosía que se muestra en la figura 4.17 y se tomó como punto de partida ${ }^{\mathrm{i}}$. Esta red ofrece el comportamiento esperado, siempre y cuando se añada una red desfasadora fija de $120^{\circ}$ en el acceso número 4 .

Este desfase de $120^{\circ}$ puede ser incluido en las líneas de interconexión de las

${ }^{\mathrm{i} C}$ Comparando las figuras 4.14 y 4.17 se puede destacar que la celosía de la topología 2 es la extrapolación al caso de tres entradas y tres salidas del acoplador de ramas paralelas que implementa un híbrido de $90^{\circ}$. 
celdas básicas, en la estructura multicapa, de la misma forma que los desfasadores fijos de la figura 4.14. Sería tambíen posible implementar ese desfase sobre el circuito integrado, con una red en $\mathrm{T}$ formada por dos bobinas y un condensador, lo cual aumentaría ligeramente el tamaño total de la celda básica.

Con este enfoque, el número de elementos necesarios por cada celda básica se reduce a 7 bobinas y 6 condensadores, menos de la mitad de los elementos de la topología 1, reduciéndose el tamaño del circuito integrado considerablemente; esta reducción en el tamaño del circuito, implica directamente una reducción en el coste y un aumento en el rendimiento de fabricación del mismo.

\section{Diseño y resultados de simulación}

La topología 2, fue la elegida para ser diseñada. Para una frecuencia de diseño de $2.3 \mathrm{GHz}$, los valores obtenidos para los elementos concentrados de la figura 4.17 son:

- $L_{1}=2.997 \mathrm{nH}$

- $L_{2}=1.73 \mathrm{nH}$

- $C=5.164 \mathrm{pF}$

Los cuales se encuentran dentro del rango de valores posibles del proceso D02AH (de 0.1 a $50 \mathrm{pF}$ para los condensadores $S i_{3} N_{4}$ y de 0.5 a $15 \mathrm{nH}$ para las bobinas en espiral) y podrían ser implementados con los siguientes tamaños:

- $L_{1}$ : anchura $=15 \mu \mathrm{m}$, separación $=5 \mu \mathrm{m}$, perímetro=2.7 $\mathrm{mm}$.

- $L_{2}:$ anchura $=15 \mu \mathrm{m}$, separación=5 $\mu \mathrm{m}$, perímetro=1.8 $\mathrm{mm}$.

- $C: 115 \times 115 \mu \mathrm{m}^{2}$.

Después del proceso de optimización y de la obtención de la distribución espacial del circuito, estos valores fueron modificados ligeramente.

Durante la generación de la distribución espacial de los componentes, se tuvo como objetivo prioritario el mantener ésta lo más simétrica posible. Todos los condensadores tienen el mismo tamaño. Por otro lado, todas las bobinas fueron diseñadas con la máxima anchura de pista posible $(15 \mu \mathrm{m})$, con el fin de minimizar pérdidas, y con una separación lo más reducida posible $(5 \mu \mathrm{m})$ con el fin de reducir el tamaño.

Debido a que los caminos inductivos entre los accesos 1 a 3 y 4 a 6 son de mayor longitud que el resto, las líneas de acceso son más anchas y el valor de la bobina correspondiente se ha reducido, pretendiéndose con esto el equalizar las pérdidas 


\begin{tabular}{|l||c|c|c|}
\hline $\begin{array}{l}\text { Desfase relativo } \\
\text { al acceso 4 }\end{array}$ & Acceso 4 & Acceso 5 & Acceso 6 \\
\hline \hline Acceso 1 & $0^{\circ}$ & $-120.07^{\circ}$ & $-120.04^{\circ}$ \\
\hline Acceso 2 & $0^{\circ}$ & $120.44^{\circ}$ & $-0.02^{\circ}$ \\
\hline Acceso 3 & $0^{\circ}$ & $-0.04^{\circ}$ & $120.24^{\circ}$ \\
\hline
\end{tabular}

Tabla 4.1: Resultados de simulación de inserción de fase para la matriz fija MMIC $3 \times 3$.

\begin{tabular}{|l||c|c|c|}
\hline $\begin{array}{l}\text { Pérdidas de Inserción } \\
\text { Absolutas/Relativas acc. 4 }\end{array}$ & Acceso 4 & Acceso 5 & Acceso 6 \\
\hline \hline Acceso 1 & $8.89 \mathrm{~dB} / 0 \mathrm{~dB}$ & $8.89 \mathrm{~dB} / 0 \mathrm{~dB}$ & $8.89 \mathrm{~dB} / 0 \mathrm{~dB}$ \\
\hline Acceso 2 & $8.88 \mathrm{~dB} / 0 \mathrm{~dB}$ & $8.95 \mathrm{~dB} /+0.07 \mathrm{~dB}$ & $8.93 \mathrm{~dB} /+0.05 \mathrm{~dB}$ \\
\hline Acceso 3 & $8.87 \mathrm{~dB} / 0 \mathrm{~dB}$ & $8.93 \mathrm{~dB} /+0.05 \mathrm{~dB}$ & $8.94 \mathrm{~dB} /+0.06 \mathrm{~dB}$ \\
\hline
\end{tabular}

Tabla 4.2: Resultados de simulación de pérdidas de inserción para la matriz fija MMIC $3 \times 3$.

totales y el valor de la inductancia total del camino. También el recorrido entre los accesos 2 y 5 fue ligeramente modificado, con respecto a los caminos 1 a 4 y 3 a 6 .

En el diseño de la distribución espacial, es bastante importante minimizar los posibles acoplamientos electromagnéticos indeseados, y al mismo tiempo, se debe reducir el tamaño del circuito para así mejorar el rendimiento y reducir el coste.

En las tablas 4.1 a 4.3 se muestra un resumen de los resultados de simulación a la frecuencia central. Los resultados en términos de fase cumplen las especificaciones con un error en el peor caso de solamente $0.44^{\circ}$. Las pérdidas de inserción absolutas son bastante más altas que en el caso ideal (4.77 dB), debido a las mayores pérdidas de los elementos concentrados en tecnología MMIC. Por otro lado, las pérdidas de inserción incrementales cumplen sobradamente las especificaciones.

Los parámetros de aislamiento entre accesos del mismo lado (entradas o salidas), podría ser mejorado en siguientes diseños. Por otra parte, las pérdidas de retorno cumplen las especificaciones más que satisfactoriamente.

\begin{tabular}{|c|c|c|c|}
\hline \multicolumn{2}{|c|}{ Pérdidas de Retorno } & \multicolumn{2}{|c|}{ Aislamiento } \\
\hline \hline$S_{11}$ & $19.8 \mathrm{~dB}$ & $S_{21}$ & $13.8 \mathrm{~dB}$ \\
\hline$S_{22}$ & $21.4 \mathrm{~dB}$ & $S_{31}$ & $13.8 \mathrm{~dB}$ \\
\hline$S_{33}$ & $20.1 \mathrm{~dB}$ & $S_{32}$ & $13.8 \mathrm{~dB}$ \\
\hline$S_{44}$ & $19.8 \mathrm{~dB}$ & $S_{54}$ & $13.9 \mathrm{~dB}$ \\
\hline$S_{55}$ & $21.3 \mathrm{~dB}$ & $S_{64}$ & $13.9 \mathrm{~dB}$ \\
\hline$S_{66}$ & $19.9 \mathrm{~dB}$ & $S_{65}$ & $13.7 \mathrm{~dB}$ \\
\hline
\end{tabular}

Tabla 4.3: Resultados de simulación de adaptación y aislamiento para la matriz fija MMIC $3 \times 3$. 
Algunas gráficas de simulación se muestran en las figuras 4.18 y 4.19, para un ancho de banda del $20 \%$ alrededor de $2.3 \mathrm{GHz}$. De los resultados de estas gráficas se puede comprobar como la limitación en cuanto a ancho de banda se cumplen sobradamente, pues a partir de las pérdidas de retorno se obtiene un ancho de banda de $250 \mathrm{MHz}$ (11\%), mientras que la especificación de pérdidas de inserción diferenciales entre accesos de salida, implica una reducción del ancho de banda hasta $100 \mathrm{MHz}$ (4\%), el cual sigue siendo suficiente para las aplicaciones en estudio y cumple sobradamente las especificaciones.

\section{Distribución espacial y montaje}

La distribución espacial final se puede observar en la figura 4.20. A continuación, se ofrecen una serie de datos necesarios para el montaje del dispositivo:

Tamaño $1.5 \mathrm{~mm}$ x $3 \mathrm{~mm}$ (1.4 mm x $2.9 \mathrm{~mm}$. restando los márgenes para el corte de los circuitos).

Este tamaño venía forzado por el tamaño de los circuitos integrados disponible en la loseta ${ }^{i}$ y por lo tanto podría ser reducido en posteriores diseños.

Hilos de Conexión No se requieren elementos de compensación fuera del circuito integrado. Un único hilo de conexión sobre cada recuadro:

- Diámetro $=25 \mu \mathrm{m}$.

- Longitud $=250 \mu \mathrm{m}$.

Todas las dimensiones necesarias para el montaje del dispositivo se muestran en la figura 4.20 .

\footnotetext{
${ }^{\text {i } T i l e, ~ e n ~ t e r m i n o l o g i ́ a ~ i n g l e s a . ~}$
} 


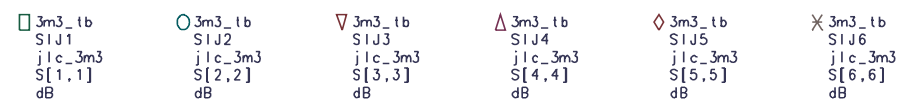

a)
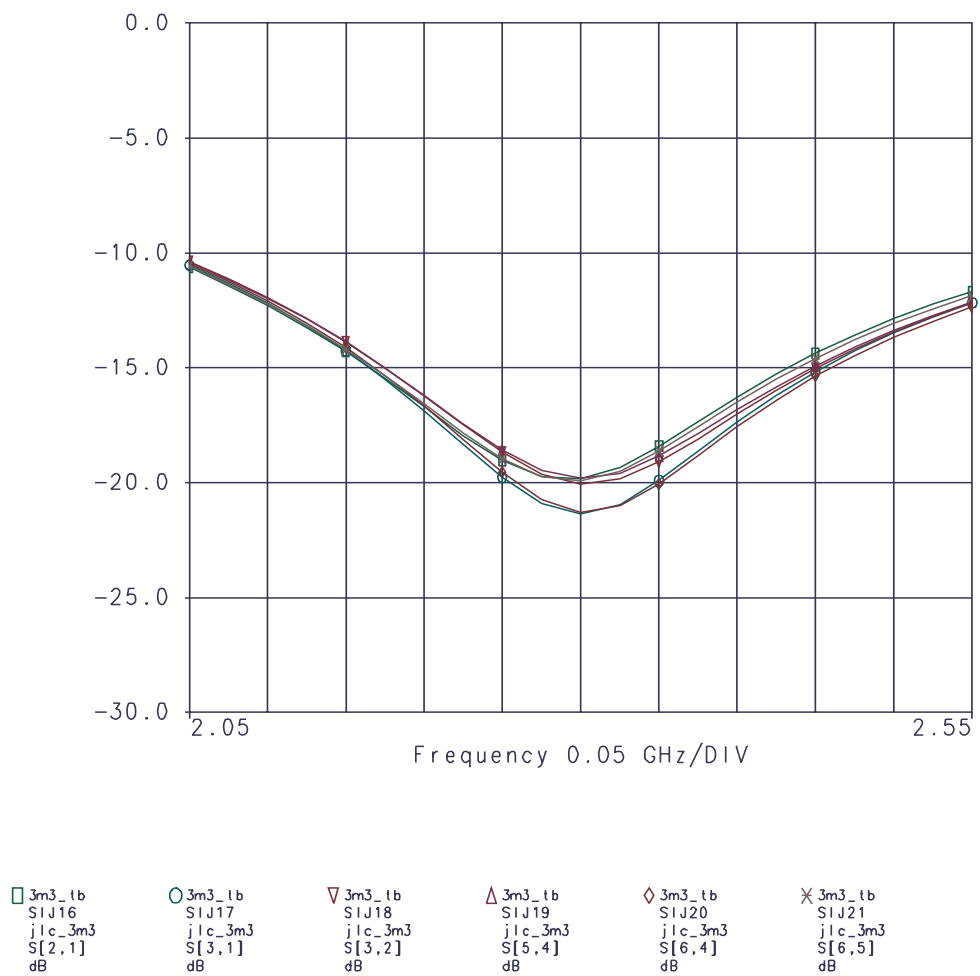

b)

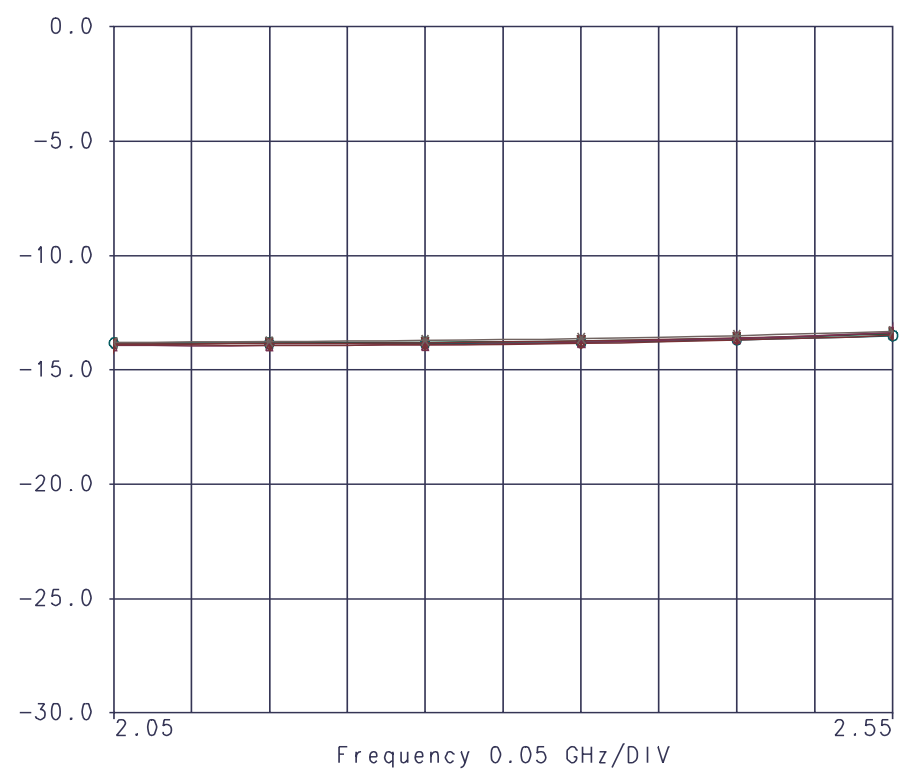

Figura 4.18: Resultados de simulación de la matriz fija $3 \times 3$. a) Adaptación. b) Aislamiento. 


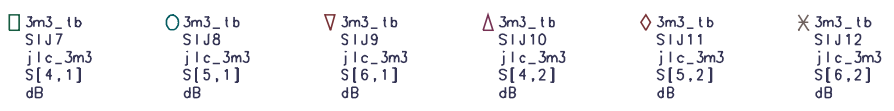

a)
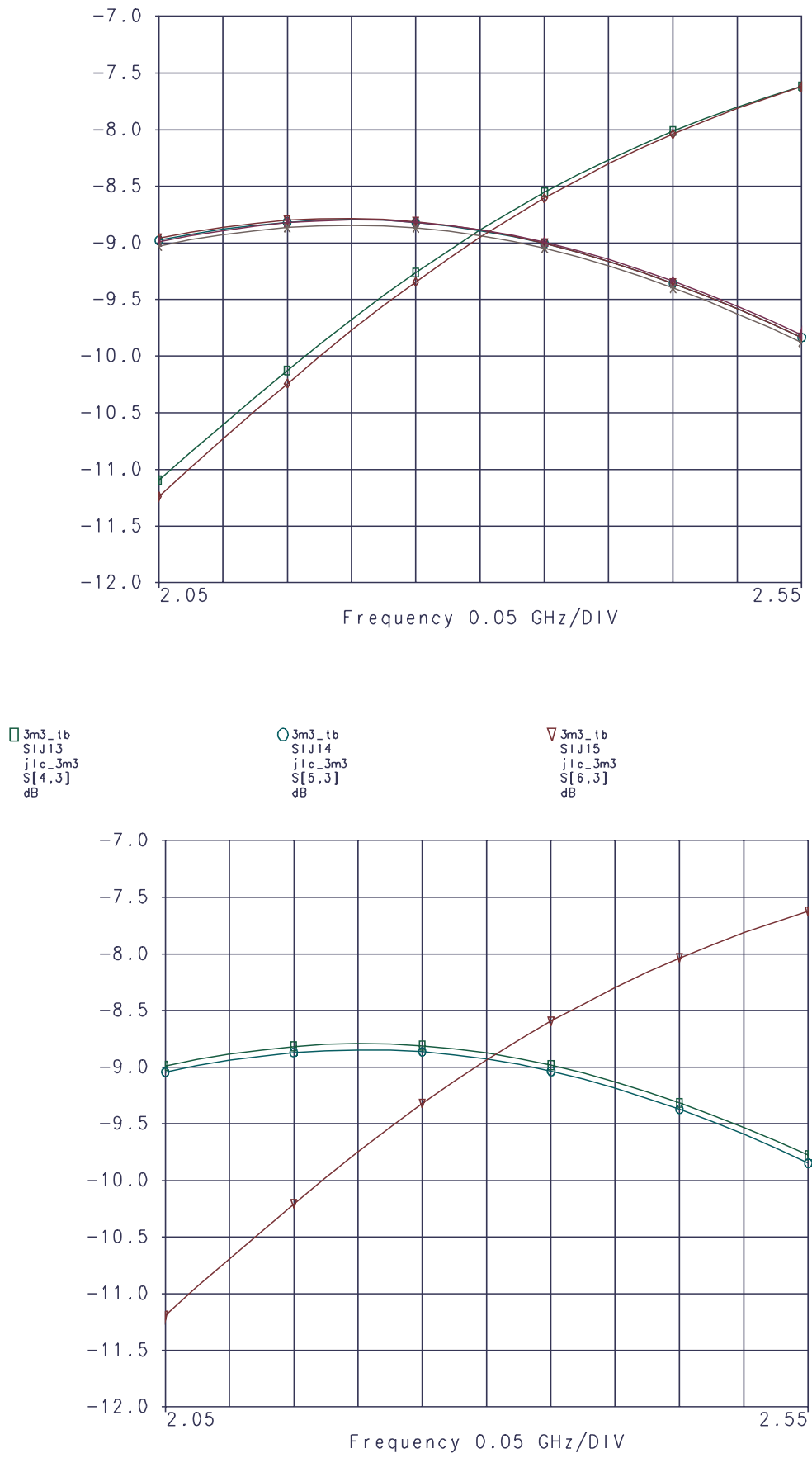

Figura 4.19: Resultados de simulación para las pérdidas de inserción de la matriz fija $3 \times 3$. 


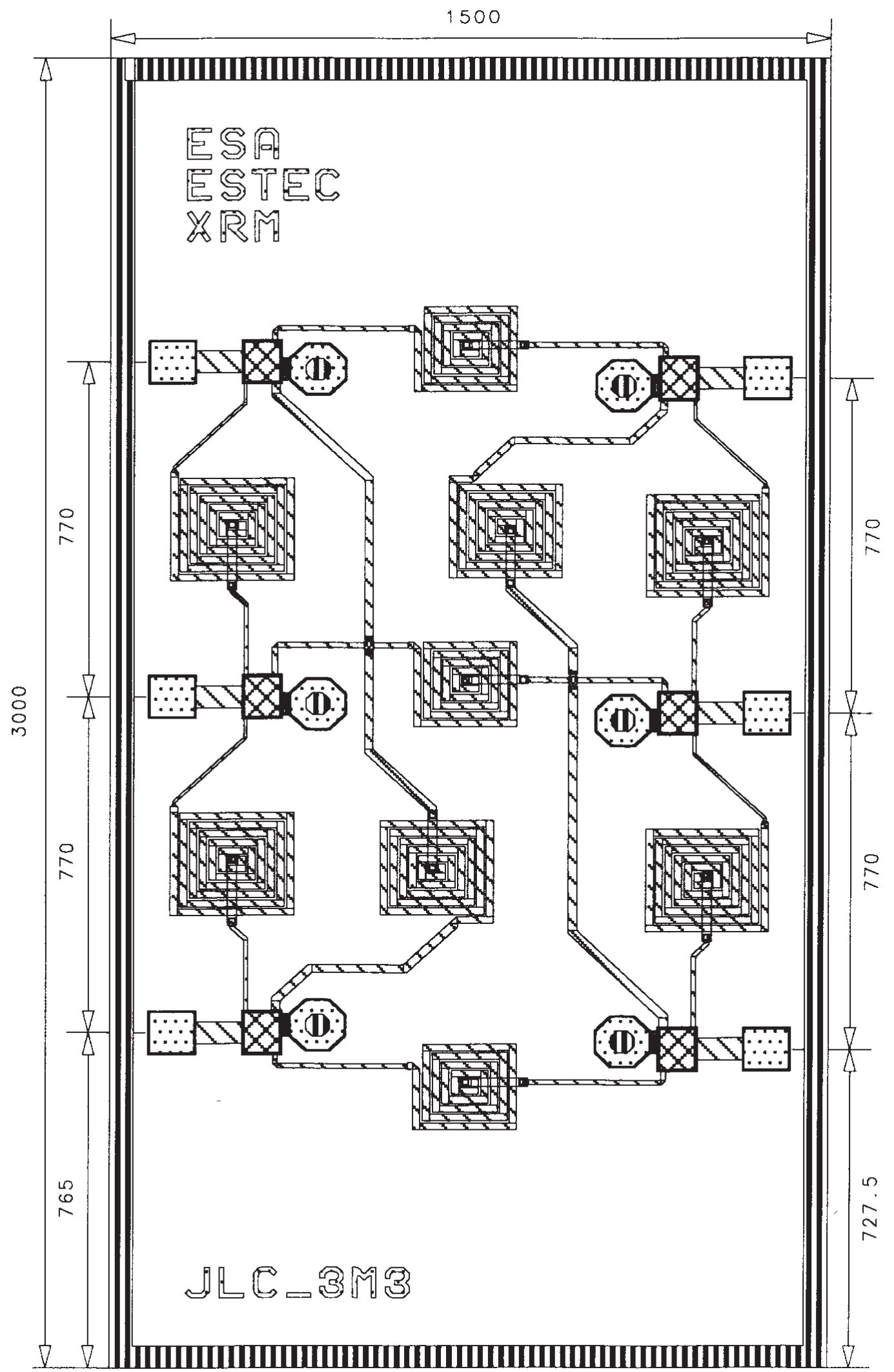

Figura 4.20: Distribución espacial del MMIC de la matriz fija $3 \times 3$. 


\subsubsection{Matriz $3 \times 3$ sintonizable}

El comportamiento nominal de la matriz $3 \times 3$, se verá afectado por las tolerancias del proceso de fabricación. En concreto, de los componentes que forman la matriz (bobinas, condensadores y líneas de transmisión), los más sensibles a variaciones del proceso son los condensadores, al depender su capacidad de los espesores de las diferentes capas dieléctricas depositadas. De hecho, la tolerancia sobre los valores de capacidad, especificada para el proceso MMIC D02AH es tan alta como un 20\% [PML94].

Con el fin de evaluar el efecto de las variaciones del proceso ,sobre el comportamiento de una red conformadora basada en la matriz $3 \times 3$, se han realizado una serie de simulaciones sobre el conformador de haces $27 \times 27$ de la figura 4.10, tal y como se explica en el apartado 4.3.4 y usando datos de simulación obtenidos para la matriz fija $3 \times 3$. Los errores cuadráticos medios en fase y en amplitud, han sido calculados para las 27 salidas del conformador de haces de tamaño $27 \times 27$, para cada uno de los 27 haces que ofrece el conformador; posteriormente, se ha determinado la media de estos errores.

Los resultados para el diseño nominal de la matriz fija $3 \times 3$, se muestran en la tabla 4.4, junto con los resultados para una variación de la capacidad de un $5 \%$, $10 \%, 15 \%,-5 \%,-10 \%$ y $-15 \%$ respecto a los valores nominales. Para cada caso se incluyen los valores RMS medios de los errores de fase y de amplitud; además, a partir de estos resultados, se han obtenido el nivel de los lóbulos secundarios de fondo y la variación en la ganancia de la agrupación en la dirección de máxima radiación utilizando las ecuaciones (A.22) y (A.23), y asumiendo una distribución gausiana de los errores y una agrupación uniforme de 27 elementos, sin elementos fallidos.

Respecto a los resultados para la matriz $3 \times 3$ nominal, es importante destacar que, con simplemente una mejora de $3 \mathrm{~dB}$ en los parámetros de aislamiento, el error de fase medio se podría haber reducido hasta $2.9^{\circ}$, e incluso hasta $1.7^{\circ}$ para una mejora de 6 dB. En cualquier caso, estos resultados son bastante dependientes de las longitudes de las líneas de transmisión, utilizadas para interconectar las diversas

\begin{tabular}{|c||c|c|c|c|c|c|c|}
\hline & Nominal & $+5 \%$ & $+10 \%$ & $+15 \%$ & $-5 \%$ & $-10 \%$ & $-15 \%$ \\
\hline \hline$\overline{\delta^{2}}$ & $5.2^{\mathrm{o}}$ & $6.1^{\mathrm{o}}$ & $7.9^{\mathrm{o}}$ & $10.1^{\mathrm{o}}$ & $6.1^{\mathrm{o}}$ & $8.5^{\mathrm{o}}$ & $11.8^{\mathrm{o}}$ \\
\hline$\overline{\Delta^{2}}$ & $0.86 \mathrm{~dB}$ & $0.95 \mathrm{~dB}$ & $1.19 \mathrm{~dB}$ & $1.5 \mathrm{~dB}$ & $0.95 \mathrm{~dB}$ & $1.19 \mathrm{~dB}$ & $1.5 \mathrm{~dB}$ \\
\hline$N L S P_{\text {fondo }}$ & $-20.7 \mathrm{~dB}$ & $-20.2 \mathrm{~dB}$ & $-19.0 \mathrm{~dB}$ & $-17.7 \mathrm{~dB}$ & $-20.2 \mathrm{~dB}$ & $-18.9 \mathrm{~dB}$ & $-17.6 \mathrm{~dB}$ \\
\hline$\Delta G$ & $-0.9 \mathrm{~dB}$ & $-1.0 \mathrm{~dB}$ & $-1.3 \mathrm{~dB}$ & $-1.6 \mathrm{~dB}$ & $-1.0 \mathrm{~dB}$ & $-1.3 \mathrm{~dB}$ & $-1.7 \mathrm{~dB}$ \\
\hline
\end{tabular}

Tabla 4.4: Efecto de las variaciones de la capacidad del proceso D02AH sobre las prestaciones del conformador $27 \times 27$, basado en la matriz $3 \times 3$ fija (para los 27 haces): error de fase RMS medio $\left(\overline{\delta^{2}}\right)$, error de amplitud RMS medio $\left(\overline{\Delta^{2}}\right)$, nivel de fondo de los lóbulos secundarios respecto al principal $\left(N L S P_{\text {fondo }}\right)$ y variación en la ganancia $(\Delta G)$. 
celdas básicas $3 \times 3$, las cuales afectan a los desfases de las diversas señales reflejadas. Por otro lado, la mejora de las pérdidas de retorno no hubiera comportado ninguna mejora en términos de error de fase medio.

En cuanto al efecto de las variaciones en las capacidades de los condensadores, debidas al proceso de fabricación, éstas pueden conllevar errores RMS en fase y en amplitud de hasta dos veces los valores nominales, un aumento en $3 \mathrm{~dB}$ del nivel de fondo de los lóbulos secundarios y una reducción en la ganancia de la agrupación de casi 2 dB. Con el fin de evitar o, al menos, limitar el efecto de la tolerancia de fabricación en la capacidad, se requiere alguna forma de sintonización del circuito, una vez fabricado. La opción de colocar un diodo varactor en paralelo con cada condensador, fue escogida como la mejor solución para sintonizar el valor de la capacidad conjunta.

En cuanto a la medición de los circuitos para su sintonización, es interesante resaltar que el proceso de fabricación D02AH, introduce en cada oblea nueve módulos de control PCM ${ }^{\mathrm{i}}$, repartidos por la superficie de la misma, que permiten monitorizar y controlar diversos parámetros eléctricos del proceso de fabricación, entre ellos la capacidad por unidad de superficie $C_{s}\left(F / \mathrm{mm}^{2}\right)$, de forma que, teniendo esos datos (que el fabricante suministra junto a los circuitos), no haría falta medir todos los circuitos de la oblea, sino simplemente basarse en las medidas de los PCM.

\section{Datos del varactor}

En el manual de diseño del proceso D02AH, únicamente se dispone de un diodo a partir de la capa metálica GM $(0.2 \mu \mathrm{m}$ de longitud), cuya capacidad (con polarización inversa) es muy pequeña [PML94]. Por lo tanto, no es posible utilizar este dispositivo como diodo varactor. Simultáneamente al diseño de las matrices $3 \times 3$, Philips Microwave Limeil estaba desarrollando, como nueva opción para el proceso D02AH, un diodo a partir de la capa metálica BE.

Para sintonizar los condensadores de la matriz $3 \times 3$ fija, se necesitaba un varactor con un rango de variación de su capacidad de alrededor de $1 \mathrm{pF}$, para lo que PML suministró el diodo en capa BE de tamaño $4 \times 30$. En la figura 4.21, se muestran conjuntamente, la capacidad medida en función de la tensión de polarización del diodo para cinco dispositivos distintos y las simulaciones de un modelo preliminar desarrollado por PML. Se comprueba en la figura 4.21, la posibilidad de conseguir un margen de variación de $1 \mathrm{pF}$ en la capacidad del varactor mediante una sintonización de la tensión de polarización de alrededor de 1 Voltio.

Como no estaba disponible en el manual de diseño del proceso ningún modelo del diodo BE y con el fin de reducir el tiempo de desarrollo, PML suministró datos de simulación del diodo $\mathrm{BE} 4 \times 30$, en forma de parámetros $\mathrm{S}$, para el rango de frecuencias adecuado y para diferentes tensiones de polarización del diodo $(-1.6 \mathrm{~V}$, $-1.4 \mathrm{~V},-1.2 \mathrm{~V},-1 \mathrm{~V},-0.8 \mathrm{~V},-0.6 \mathrm{~V},-0.4 \mathrm{~V},-0.2 \mathrm{~V}$ y $0 \mathrm{~V})$.

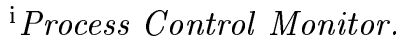




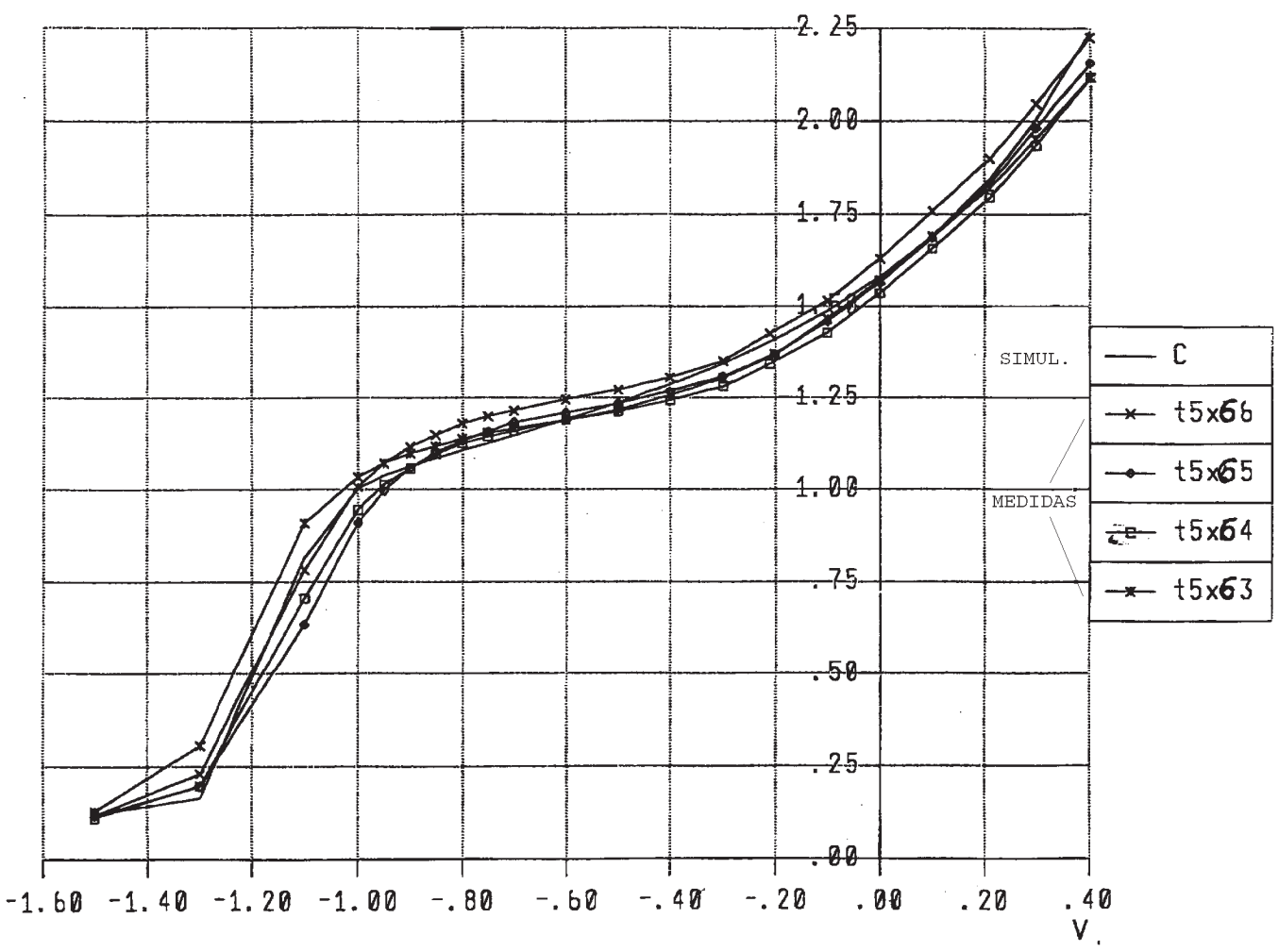

Figura 4.21: Capacidad serie (medidas y simulación) del diodo BE $4 \times 30$ del proceso D02AH.

La selección del punto de alimentación se basó en el comportamiento aproximadamente lineal de la capacidad del varactor frente a la tensión de polarización (Fig. 4.21) en el rango de $-1 \mathrm{~V}$ a $0 \mathrm{~V}$. Además, la baja pendiente de la curva, hace que el circuito sea menos sensible a variaciones en la tensión de alimentación. Dentro de este rango de tensiones, $-0.4 \mathrm{~V}$ es el valor más centrado de los que se dispone datos de simulación y, por lo tanto, este fue el valor escogido como polarización nominal de los varactores.

\section{Diseño y resultados de simulación}

El diseño de la matriz $3 \times 3$ sintonizable, es una modificación del diseño de la matriz fija. El tamaño del condensador MIM se reduce, de forma que el valor de capacidad total equivalente al varactor en paralelo más el condensador MIM, mantenga el valor esperado, para la tensión nominal de polarización del diodo $(-0.4 \mathrm{~V})$.

Después de incluir el varactor polarizado a la tensión nominal, se reoptimizaron todos los parámetros del circuito, intentando mantener las mismas consideraciones de simetría que en el diseño fijo.

En cuanto a la polarización de los diodos, todo el circuito integrado está interconectado en cuanto a señales de contínua, de forma que se necesita un único 


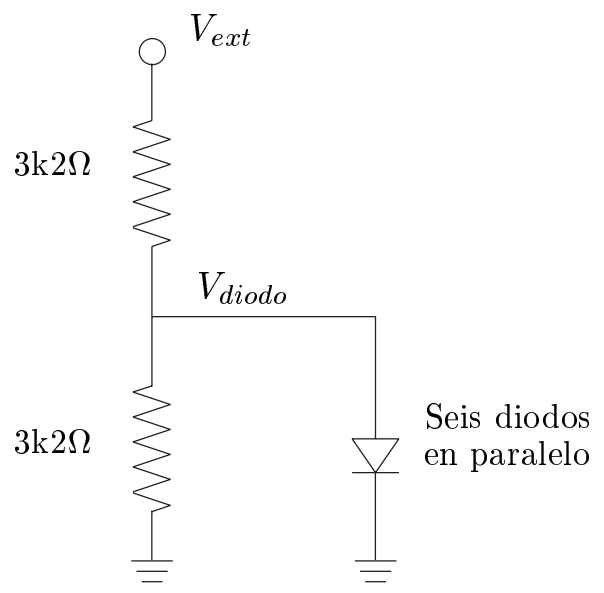

Figura 4.22: Red de polarización de la matriz sintonizable dentro del circuito integrado.

\begin{tabular}{|l||c|c|c|}
\hline $\begin{array}{l}\text { Desfase relativo } \\
\text { al acceso 4 }\end{array}$ & Acceso 4 & Acceso 5 & Acceso 6 \\
\hline \hline Acceso 1 & $0^{\circ}$ & $-119.95^{\circ}$ & $-119.96^{\circ}$ \\
\hline Acceso 2 & $0^{\circ}$ & $120.33^{\circ}$ & $0.13^{\circ}$ \\
\hline Acceso 3 & $0^{\circ}$ & $0.14^{\circ}$ & $120.24^{\circ}$ \\
\hline
\end{tabular}

Tabla 4.5: Resultados de simulación de inserción de fase, para la matriz sintonizable MMIC $3 \times 3$. Tensión de polarización de los diodos: $-0.4 \mathrm{~V}$.

recuadro de conexión y una única línea para la alimentación del circuito. Debido a consideraciones de seguridad y de aislamiento entre las señales de radiofrecuencia (RF) y de contínua (DC), se han añadido dos resistores de valores altos $(3 \mathrm{k} 2 \Omega$ ) a la línea de alimentación, tal y como se muestra en la figura 4.22. De esta forma, la tensión de alimentación necesaria en el recuadro de DC, debe ser el doble de la tensión requerida en el ánodo de los varactores.

Los seis recuadros de radiofrecuencia no están aislados en contínua (no presentan condensadores de desacoplo en serie), lo que permitiría que una única línea fuera suficiente para la alimentación de todo el conformador de haces $27 \times 27$, asumiendo que todos los circuitos integrados del mismo provienen de una única oblea, y, por lo tanto, presentan un valor similar de capacidad y requieren una misma tensión de polarización en el varactor sintonizador.

Los resultados de simulación para una tensión de alimentación nominal para el diodo y a la frecuencia central, se muestra en las tablas 4.5, 4.6 y 4.7.

Los resultados son similares a los del diseño fijo sin varactores. Los valores de desfase siguen cumpliendo las especificaciones, mientras que las pérdidas de inserción 


\begin{tabular}{|l||c|c|c|}
\hline $\begin{array}{l}\text { Pérdidas de Inserción } \\
\text { Absolutas/Relativas acc. } 4\end{array}$ & Acceso 4 & Acceso 5 & Acceso 6 \\
\hline \hline Acceso 1 & $10.07 \mathrm{~dB} / 0 \mathrm{~dB}$ & $10.03 \mathrm{~dB} /-0.04 \mathrm{~dB}$ & $10.07 \mathrm{~dB} / 0 \mathrm{~dB}$ \\
\hline Acceso 2 & $9.97 \mathrm{~dB} / 0 \mathrm{~dB}$ & $9.90 \mathrm{~dB} /-0.06 \mathrm{~dB}$ & $9.98 \mathrm{~dB} /+0.02 \mathrm{~dB}$ \\
\hline Acceso 3 & $10.05 \mathrm{~dB} / 0 \mathrm{~dB}$ & $10.02 \mathrm{~dB} /-0.03 \mathrm{~dB}$ & $10.03 \mathrm{~dB} /-0.01 \mathrm{~dB}$ \\
\hline
\end{tabular}

Tabla 4.6: Resultados de simulación de pérdidas de inserción, para la matriz sintonizable MMIC $3 \times 3$. Tensión de polarización de los diodos: $-0.4 \mathrm{~V}$.

\begin{tabular}{|c|c|c|c|}
\hline \multicolumn{2}{|c|}{ Pérdidas de Retorno } & \multicolumn{2}{c|}{ Aislamiento } \\
\hline \hline$S_{11}$ & $17.7 \mathrm{~dB}$ & $S_{21}$ & $13.5 \mathrm{~dB}$ \\
\hline$S_{22}$ & $19.2 \mathrm{~dB}$ & $S_{31}$ & $13.6 \mathrm{~dB}$ \\
\hline$S_{33}$ & $17.7 \mathrm{~dB}$ & $S_{32}$ & $13.5 \mathrm{~dB}$ \\
\hline$S_{44}$ & $17.7 \mathrm{~dB}$ & $S_{54}$ & $13.5 \mathrm{~dB}$ \\
\hline$S_{55}$ & $18.9 \mathrm{~dB}$ & $S_{64}$ & $13.4 \mathrm{~dB}$ \\
\hline$S_{66}$ & $17.9 \mathrm{~dB}$ & $S_{65}$ & $13.4 \mathrm{~dB}$ \\
\hline
\end{tabular}

Tabla 4.7: Resultados de simulación de adaptación y aislamiento, para la matriz sintonizable MMIC $3 \times 3$. Tensión de polarización de los diodos: $-0.4 \mathrm{~V}$.

son $1 \mathrm{~dB}$ peores que para el diseño de la matriz fija, pasando a ser de $10 \mathrm{~dB}$, aproximadamente. En cambio, las pérdidas de inserción diferenciales mantienen su buen comportamiento, cumpliéndose sobradamente las especificaciones realizadas.

Por lo que respecta a la adaptación del circuito, las pérdidas de retorno son $2 \mathrm{~dB}$ peores, pero aún así satisfacen las especificaciones. El comportamiento en cuanto al aislamiento de la matriz sintonizable, es similar al obtenido para el diseño fijo y podría mejorarse en futuros diseños.

En las figuras 4.23 y 4.24 se muestran unas gráficas de simulación para el valor de tensión de alimentación nominal, representadas para un ancho de banda de $500 \mathrm{MHz}$ alrededor de $2.3 \mathrm{GHz}$. La curva de respuesta de los parámetos de aislamiento es claramente similar a la obtenida para la matriz $3 \times 3$ fija. Los parámetros de adaptación han sufrido una desviación en frecuencia de aproximadamente $50 \mathrm{MHz}$, pero se siguen cumpliendo las especificaciones dentro de un ancho de banda de $250 \mathrm{MHz}$. De la misma forma que ocurría en el caso de la matriz fija, la especificación de las pérdidas de inserción diferenciales constituyen el criterio más restrictivo a la hora de calcular el ancho de banda del circuito, que pasa a ser en este caso de aproximadamente $75 \mathrm{MHz}$, es decir, un $3 \%$ de la frecuencia central. 


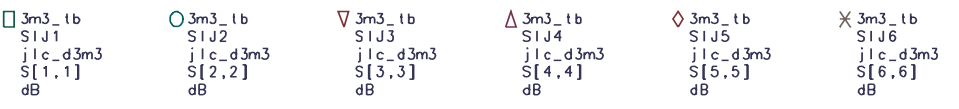

a)
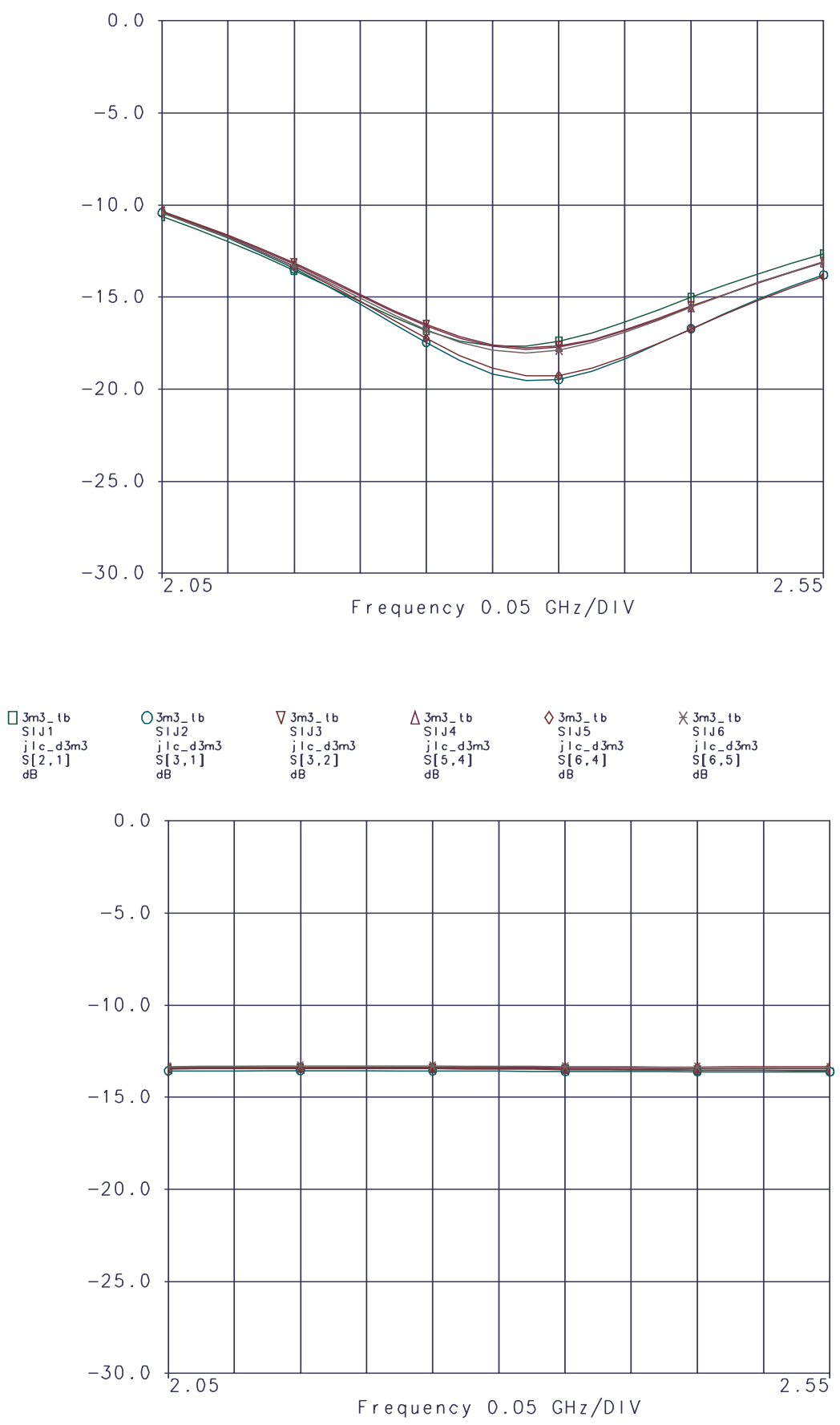

b)

Figura 4.23: Resultados de simulación de la matriz sintonizable $3 \times 3$. a) Adaptación. b) Aislamiento. 


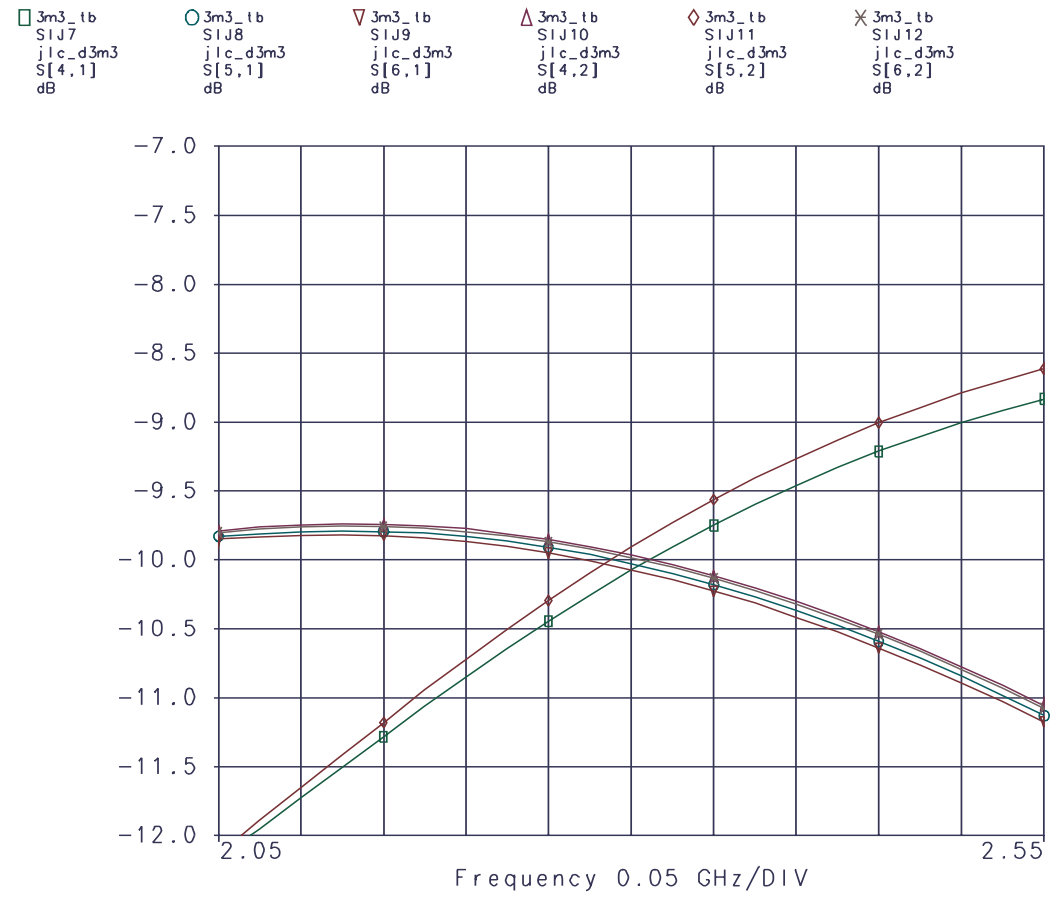

a)
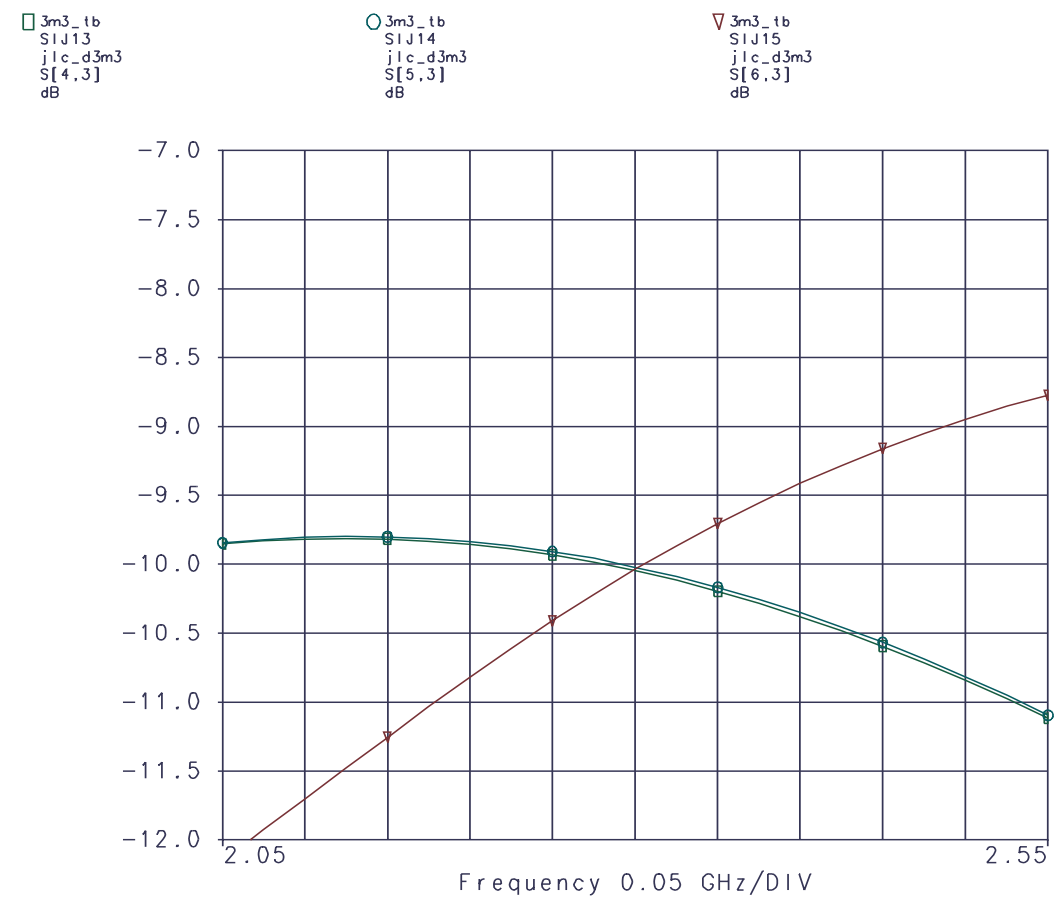

b)

Figura 4.24: Resultados de simulación para las pérdidas de inserción de la matriz sintonizable $3 \times 3$. 


\begin{tabular}{|l||c|c|c|}
\hline $\begin{array}{l}\text { Desfase relativo } \\
\text { al acceso 4 }\end{array}$ & Acceso 4 & Acceso 5 & Acceso 6 \\
\hline \hline Acceso 1 & $0^{\circ}$ & $-119.60^{\circ}$ & $-119.58^{\circ}$ \\
\hline Acceso 2 & $0^{\circ}$ & $119.93^{\circ}$ & $0.13^{\circ}$ \\
\hline Acceso 3 & $0^{\circ}$ & $0.11^{\circ}$ & $119.48^{\circ}$ \\
\hline
\end{tabular}

Tabla 4.8: Resultados de simulación de inserción de fase, para la matriz sintonizable MMIC $3 \times 3$. Tensión de polarización de los diodos: $-0.8 \mathrm{~V}$. Variación de capacidad corregida: $12.8 \%$.

\begin{tabular}{|l||c|c|c|}
\hline $\begin{array}{l}\text { Pérdidas de Inserción } \\
\text { Absolutas/Relativas acc. 4 }\end{array}$ & Acceso 4 & Acceso 5 & Acceso 6 \\
\hline \hline Acceso 1 & $10.19 \mathrm{~dB} / 0 \mathrm{~dB}$ & $10.34 \mathrm{~dB} /+0.15 \mathrm{~dB}$ & $10.39 \mathrm{~dB} /+0.2 \mathrm{~dB}$ \\
\hline Acceso 2 & $10.28 \mathrm{~dB} / 0 \mathrm{~dB}$ & $10.03 \mathrm{~dB} /-0.25 \mathrm{~dB}$ & $10.30 \mathrm{~dB} /+0.02 \mathrm{~dB}$ \\
\hline Acceso 3 & $10.36 \mathrm{~dB} / 0 \mathrm{~dB}$ & $10.34 \mathrm{~dB} /-0.03 \mathrm{~dB}$ & $10.15 \mathrm{~dB} /-0.21 \mathrm{~dB}$ \\
\hline
\end{tabular}

Tabla 4.9: Resultados de simulación de pérdidas de inserción, para la matriz sintonizable MMIC $3 \times 3$. Tensión de polarización de los diodos: $-0.8 \mathrm{~V}$. Variación de capacidad corregida: $12.8 \%$.

Con el fin de evaluar la capacidad de sintonización de la matriz $3 \times 3$ con los diodos varactores, se realizaron una serie de simulaciones. Debido al limitado rango de tensiones de alimentación para los que se disponía de datos de simulación del diodo en forma de parámetros $\mathrm{S}$, en lugar de fijar un porcentaje de variación en los valores de capacidad del proceso y alterar la tensión de alimentación de los varactores con el fin de compensar esta variación, se realizó el procedimiento inverso, fijando el valor de tensión del diodo. Por lo tanto, se tomaron unos valores de 0 Voltios y -0.8 Voltios y se encontraron unos valores de variaciones de capacidad que serían corregidos, de $-15.4 \%$ y $+12.8 \%$ respectivamente.

Un resumen de los datos de simulación obtenidos para estos dos casos se presenta en las tablas 4.8 a 4.13. De los resultados obtenidos, se puede comprobar que el

\begin{tabular}{|c|c|c|c|}
\hline \multicolumn{2}{|c|}{ Pérdidas de Retorno } & \multicolumn{2}{|c|}{ Aislamiento } \\
\hline \hline$S_{11}$ & $16.9 \mathrm{~dB}$ & $S_{21}$ & $13.6 \mathrm{~dB}$ \\
\hline$S_{22}$ & $18.2 \mathrm{~dB}$ & $S_{31}$ & $13.7 \mathrm{~dB}$ \\
\hline$S_{33}$ & $16.8 \mathrm{~dB}$ & $S_{32}$ & $13.6 \mathrm{~dB}$ \\
\hline$S_{44}$ & $16.9 \mathrm{~dB}$ & $S_{54}$ & $13.6 \mathrm{~dB}$ \\
\hline$S_{55}$ & $18.0 \mathrm{~dB}$ & $S_{64}$ & $13.5 \mathrm{~dB}$ \\
\hline$S_{66}$ & $17.1 \mathrm{~dB}$ & $S_{65}$ & $13.5 \mathrm{~dB}$ \\
\hline
\end{tabular}

Tabla 4.10: Resultados de simulación de adaptación y aislamiento, para la matriz sintonizable MMIC $3 \times 3$. Tensión de polarización de los diodos: $-0.8 \mathrm{~V}$. Variación de capacidad corregida: $12.8 \%$. 


\begin{tabular}{|l||c|c|c|}
\hline $\begin{array}{l}\text { Desfase relativo } \\
\text { al acceso 4 }\end{array}$ & Acceso 4 & Acceso 5 & Acceso 6 \\
\hline \hline Acceso 1 & $0^{\circ}$ & $-119.62^{\circ}$ & $-119.60^{\circ}$ \\
\hline Acceso 2 & $0^{\circ}$ & $119.95^{\circ}$ & $0.13^{\circ}$ \\
\hline Acceso 3 & $0^{\circ}$ & $0.11^{\circ}$ & $119.5^{\circ}$ \\
\hline
\end{tabular}

Tabla 4.11: Resultados de simulación de inserción de fase, para la matriz sintonizable MMIC $3 \times 3$. Tensión de polarización de los diodos: $0 \mathrm{~V}$. Variación de capacidad corregida: $-15.4 \%$.

\begin{tabular}{|l||c|c|c|}
\hline $\begin{array}{l}\text { Pérdidas de Inserción } \\
\text { Absolutas/Relativas acc. 4 }\end{array}$ & Acceso 4 & Acceso 5 & Acceso 6 \\
\hline \hline Acceso 1 & $10.18 \mathrm{~dB} / 0 \mathrm{~dB}$ & $10.33 \mathrm{~dB} /+0.14 \mathrm{~dB}$ & $10.37 \mathrm{~dB} /+0.19 \mathrm{~dB}$ \\
\hline Acceso 2 & $10.27 \mathrm{~dB} / 0 \mathrm{~dB}$ & $10.02 \mathrm{~dB} /-0.25 \mathrm{~dB}$ & $10.29 \mathrm{~dB} /+0.02 \mathrm{~dB}$ \\
\hline Acceso 3 & $10.35 \mathrm{~dB} / 0 \mathrm{~dB}$ & $10.32 \mathrm{~dB} /-0.03 \mathrm{~dB}$ & $10.15 \mathrm{~dB} /-0.21 \mathrm{~dB}$ \\
\hline
\end{tabular}

Tabla 4.12: Resultados de simulación de pérdidas de inserción, para la matriz sintonizable MMIC $3 \times 3$. Tensión de polarización de los diodos: $0 \mathrm{~V}$. Variación de capacidad corregida: $-15.4 \%$.

\begin{tabular}{|c|c|c|c|}
\hline \multicolumn{2}{|c|}{ Pérdidas de Retorno } & \multicolumn{2}{|c|}{ Aislamiento } \\
\hline \hline$S_{11}$ & $16.9 \mathrm{~dB}$ & $S_{21}$ & $13.6 \mathrm{~dB}$ \\
\hline$S_{22}$ & $18.3 \mathrm{~dB}$ & $S_{31}$ & $13.7 \mathrm{~dB}$ \\
\hline$S_{33}$ & $16.8 \mathrm{~dB}$ & $S_{32}$ & $13.6 \mathrm{~dB}$ \\
\hline$S_{44}$ & $16.9 \mathrm{~dB}$ & $S_{54}$ & $13.6 \mathrm{~dB}$ \\
\hline$S_{55}$ & $18.1 \mathrm{~dB}$ & $S_{64}$ & $13.5 \mathrm{~dB}$ \\
\hline$S_{66}$ & $17.2 \mathrm{~dB}$ & $S_{65}$ & $13.5 \mathrm{~dB}$ \\
\hline
\end{tabular}

Tabla 4.13: Resultados de simulación de adaptación y aislamiento, para la matriz sintonizable MMIC $3 \times 3$. Tensión de polarización de los diodos: $0 \mathrm{~V}$. Variación de capacidad corregida: $-15.4 \%$. 
diseño de la matriz $3 \times 3$ sintonizable permite corregir variaciones en la capacidad del proceso de hasta un $\pm 15 \%$. Después de la corrección, mediante la aplicación de una misma tensión de alimentación a todos los diodos de la matriz, las prestaciones del circuito son muy cercanas a las del circuito nominal, comentándose a continuación las principales diferencias. Las pérdidas de inserción son únicamente $0.3 \mathrm{~dB}$ peores que en el caso nominal, manteniéndose por otro lado un incremento diferencial entre ellas de alrededor de $\pm 0.2 \mathrm{~dB}$, resultado que cumple perfectamente las especificaciones. En cuanto a las pérdidas de retorno, éstas son $1 \mathrm{~dB}$ peores que las obtenidas para la tensión nominal, pero se siguen satisfaciendo las especificaciones, al igual que ocurre con los valores de desfase.

\section{Distribución espacial y montaje}

La distribución espacial final con todas las dimensiones necesarias para el montaje del dispositivo se muestra en la figura 4.25.

Tamaño $2 \mathrm{~mm}$ x $3 \mathrm{~mm}$ (1.9 mm x $2.9 \mathrm{~mm}$. excluyendo los márgenes para el cortado de los circuitos).

Este tamaño venía forzado por el tamaño de los recuadros disponibles en la loseta y por lo tanto podría ser reducido en posteriores diseños.

Hilos de conexión No se requieren elementos de compensación fuera del circuito integrado. Un único hilo sobre cada recuadro:

- Diámetro $=25 \mu \mathrm{m}$.

- Longitud $=250 \mu \mathrm{m}$.

Polarización del circuito Valores de tensión de alimentación del circuito:

- Nominal: -0.8 V (-0.4 V en el ánodo del diodo).

- Para un valor de capacidad del proceso distinto del nominal: Valor de Tensión doble del seleccionado según la figura 4.21.

Bloqueo de contínua Si se desea medir la matriz $3 \times 3$ en solitario, deberán colocarse fuera de la misma condensadores de bloqueo de contínua con el fin de eliminar las cargas de $50 \Omega$ en paralelo con las redes de alimentación. 


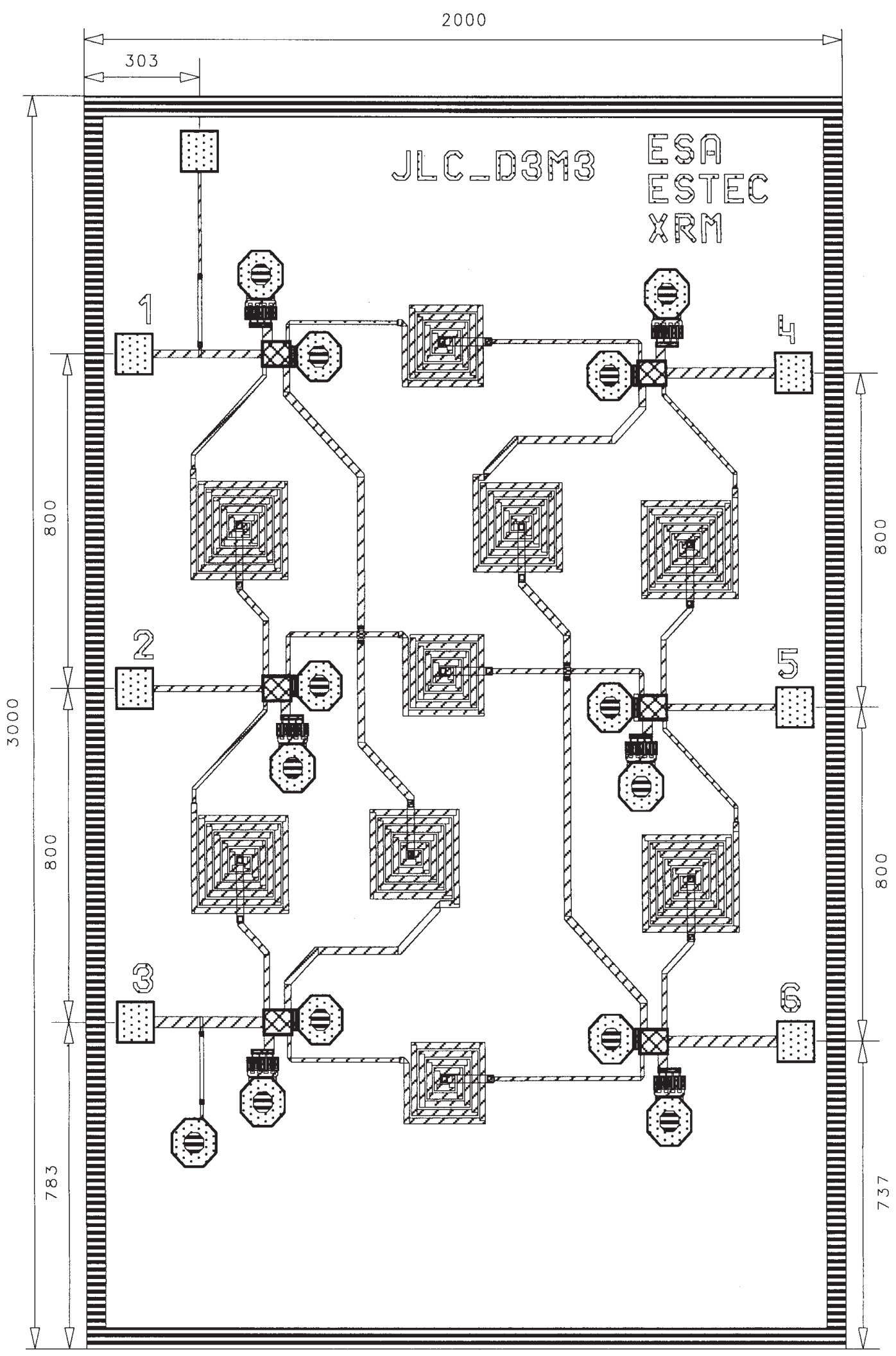

Figura 4.25: Distribución espacial del MMIC de la matriz sintonizable $3 \times 3$. 


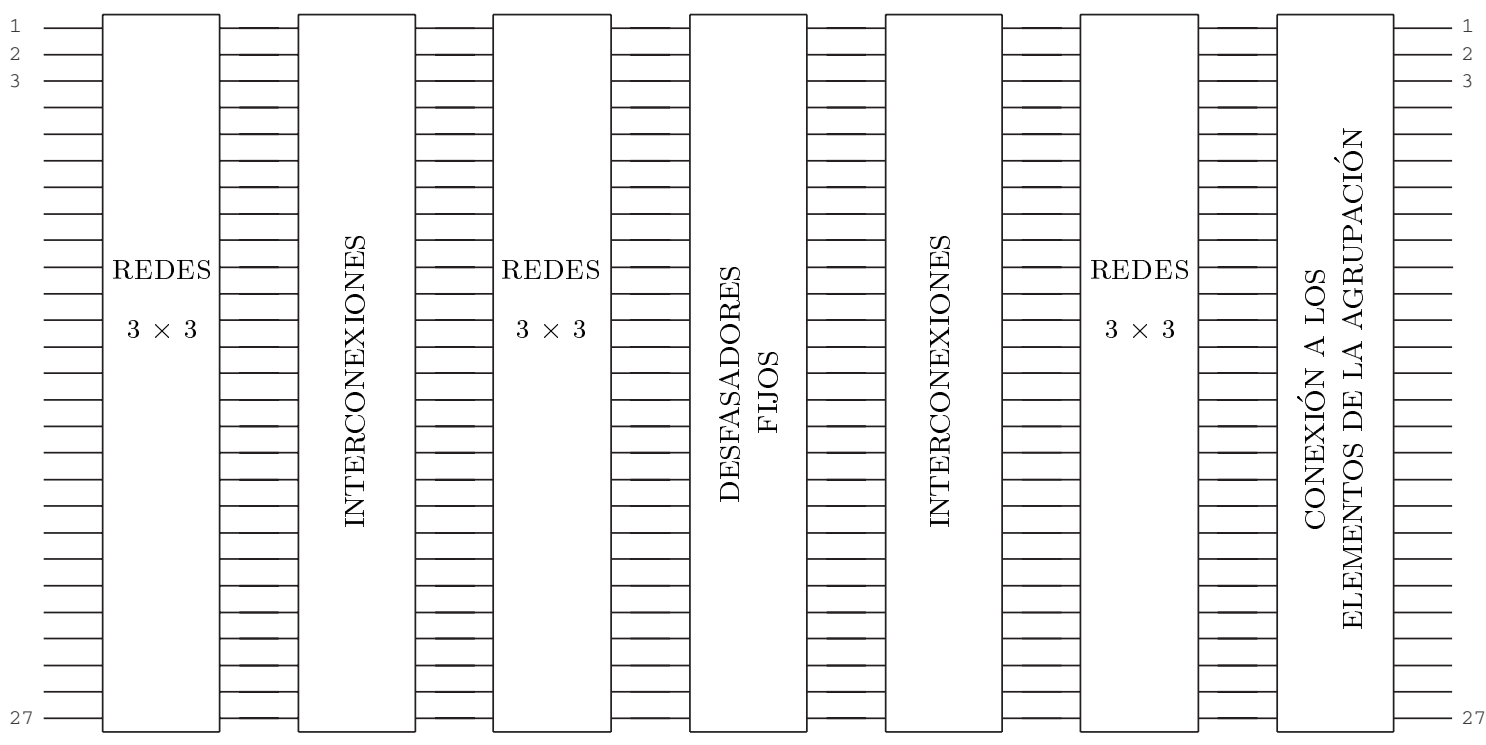

Figura 4.26: Conformador de haces $27 \times 27$ como subredes en cascada.

\subsubsection{Simulaciones de la red conformadora de haces}

Una vez ha sido diseñada una matriz $3 \times 3$, con unas buenas prestaciones como circuito independiente, se necesitaba realizar una serie de análisis más profundos para poder evaluar el nuevo concepto de red conformadora de haces y la robustez de su arquitectura, frente a tolerancias del proceso de fabricación o a desconexiones involuntarias.

Debido al número de accesos de las redes en estudio, el paquete de simulación LIBRA ya no era adecuado, al estar limitado a redes con pocos accesos. En su lugar, se han desarrollado una multitud de funciones para MATLAB, con la finalidad de evaluar las prestaciones de la arquitectura de red conformadora de haces, tanto a nivel de subsistema, como en términos de parámetros de antena, tales como anchos de haces o niveles de lóbulos principales a secundarios.

\section{Simulaciones de redes en cascada con múltiples accesos}

Con el fin de tener en cuenta todas las reflexiones internas y las interacciones entre los diversos bloques que forman la estructura de un conformador de haces completo, se decidió calcular y trabajar con los parámetros S de la red entera. Aprovechando la distribución en filas y columnas que presenta la arquitectura de los conformadores (figuras 4.10 y 4.11), la red se dividió en diversas redes en cascada; estando cada una de ellas compuesta de celdas básicas $(3 \times 3$ ó $4 \times 4)$, desfasadores fijos o simplemente interconexiones. Como ejemplo, la división de la red conformadora de haces $27 \times 27$, se puede ver en la figura 4.26 y puede compararse con la arquitectura real de la figura 4.10 . 


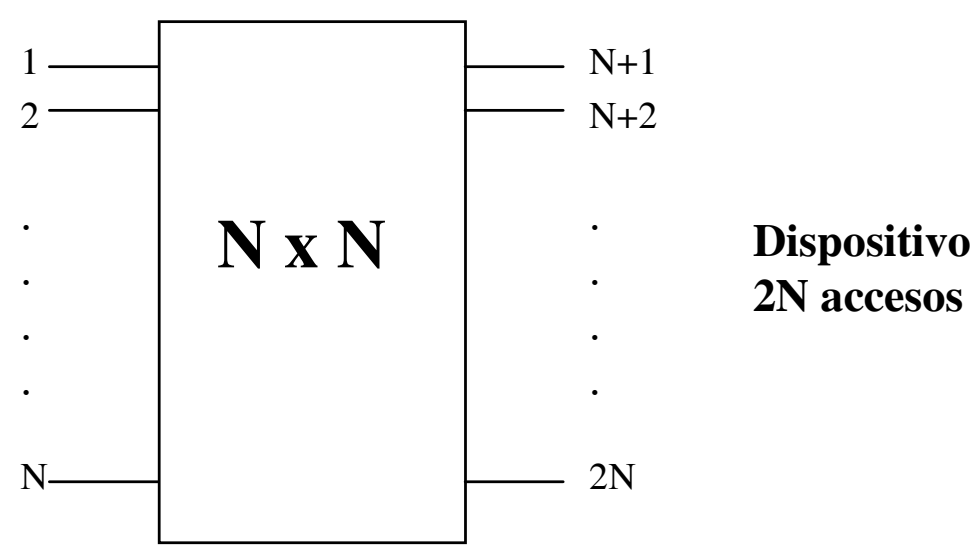
Acceso $1^{\mathrm{g}}$
Acceso $2^{\mathrm{g}}$
Generalizado
Generalizado

Figura 4.27: Red $2 \times 2$ generalizada para una red con $2 N$ accesos.

Las matrices de parámetros $\mathrm{S}$ de cada subred, han sido calculadas y convertidas en matrices de parámetros $\mathrm{T}$, gracias a una representación de las mismas en forma de matrices generalizadas. Todas las subredes consideradas tienen como característica común, el disponer de un número par de accesos, $2 N$ y presentar una estructura simétrica con $\mathrm{N}$ accesos a la entrada y $\mathrm{N}$ accesos a la salida, tal y como se muestra en la figura 4.27. La matriz de parámetros $\mathrm{S}$ de una red de estas características (ec. $(4.45))$, con un tamaño $2 \mathrm{~N} \times 2 \mathrm{~N}$, puede dividirse en cuatro subredes de tamaños $N \times N$, asimilándose, cada una de estas subredes, a uno de los cuatro parámetros de dispersión de una red de dos accesos, donde el acceso generalizado $1^{g}$ incluye los $\mathrm{N}$ accesos de entrada de la subred, y el acceso generalizado $2^{g}$ incluye los $\mathrm{N}$ accesos de salida de la subred.

$$
[S]=\left[\begin{array}{ccc|ccc}
S_{1,1} & \ldots & S_{1, N} & S_{1, N+1} & \ldots & S_{1,2 N} \\
\vdots & & \vdots & \vdots & & \vdots \\
S_{N, 1} & \ldots & S_{N, N} & S_{N, N+1} & \ldots & S_{N, 2 N} \\
\hline S_{N+1,1} & \ldots & S_{N+1, N} & S_{N+1, N+1} & \ldots & S_{N+1,2 N} \\
\vdots & & \vdots & \vdots & & \vdots \\
S_{2 N, 1} & \ldots & S_{2 N, N} & S_{2 N, N+1} & \ldots & S_{2 N, 2 N}
\end{array}\right]=\left[\begin{array}{ccc}
{\left[S_{1^{g}, 1^{g}}\right]} & {\left[S_{1^{g}, 2^{g}}\right]} \\
{\left[S_{2^{g}, 1^{g}}\right]} & {\left[S_{2^{g}, 2^{g}}\right]}
\end{array}\right]
$$

El significado de los cuatro parámetros $\mathrm{S}$ de la matriz generalizada, se explica a continuación:

$S_{1^{g}, 1^{g}}$ Submatriz $N \times N$, que tiene en cuenta todas las reflexiones e interconexiones 
entre los accesos 1 al $\mathrm{N}$ de la matriz original.

$S_{1^{g}, 2^{g}}$ Submatriz $N \times N$, que contiene todos los parámetros de transmisión, desde los accesos del lado derecho ( $\mathrm{N}+1$ a $2 \mathrm{~N}$, acceso generalizado $2^{g}$ ), a los accesos del lado izquierdo (1 a $\mathrm{N}$, acceso generalizado $1^{g}$ ).

$S_{2^{g}, 1^{g}}$ Submatriz $N \times N$, que contiene todos los parámetros de transmisión, desde los accesos del lado izquierdo ( 1 a $\mathrm{N}$, acceso generalizado $1^{g}$ ), a los accesos del lado derecho $\left(\mathrm{N}+1\right.$ a $2 \mathrm{~N}$, acceso generalizado $\left.2^{g}\right)$.

$S_{2^{g}, 2^{g}}$ Submatriz $N \times N$, que tiene en cuenta todas las reflexiones e interconexiones entre los accesos $\mathrm{N}+1$ al $2 \mathrm{~N}$, de la matriz original.

A partir de este momento, las operaciones con matrices de parámetros $\mathrm{S}$, o de parámetros $\mathrm{T}$, u operaciones de conversión entre las mismas, pasan a ser las mismas que corresponderían a dispositivos de dos únicos accesos, pero recordando, que cada uno de los cuatro parámetros es ahora una matriz.

Las matrices de parámetros $\mathrm{T}$ de cada subred, previa multiplicación directa entre las mismas en el orden adecuado, permiten obtener como resultado la matriz de parámetros $\mathrm{T}$ de la red completa. Una última conversión a parámetros $\mathrm{S}$, es lo único necesario para obtener el resultado esperado.

\section{Programas de evaluación desarrollados}

A continuación se presenta una breve explicación de las posibilidades que ofrece las funciones de MATLAB desarrolladas. Puede encontrarse más información en el manual de usuario del programa desarrollado [CG95c].

- Las redes conformadoras de haces de tamaños $27 \times 27,81 \times 81$ y $256 \times 256$, se encuentran ya implementadas, utilizando como celdas básicas una matriz de tamaño $3 \times 3$ (bien sea la matriz ideal o la implementación real en tecnología MMIC -fija o sintonizable-), para los dos primeros casos y una matriz de tamaño $4 \times 4$, para el último caso. También se han desarrollado funciones de adaptación de los resultados de simulación de estas redes conformadoras, a agrupaciones de antenas con distribución hexagonal y contorno hexagonal regular, de tres tamaños diferentes (número total de elementos radiantes de 19, 37 y 127). Los efectos de una desconexión accidental en un nodo interno de la red, pueden estimarse fácilmente para todos los conformadores de haces.

- El factor de la agrupación para cualquier geometría de antena y cualquier distribución de alimentación de los elementos de la agrupación, puede calcularse y mostrarse gráficamente en tres dimensiones, y en diagramas de contorno en dos dimensiones. También son posibles gráficos de diagrama de radiación, con superposición de contornos de múltiples haces. Además se pueden estimar los 
valores de nivel de lóbulo principal a secundario, posiciones de los centros de los haces y anchos de haces para cualquier tipo de antena.

- Definida una antena, un tamaño de conformador de haces y una posición orbital de un satélite es posible dibujar, para un haz, los contornos a nivel constante de ganancia (ISO-GAIN) o a nivel constante de densidad de flujo de potencia (ISO-PFD) sobre la superficie de la Tierra. Además, es posible dibujar sobre la superficie terrestre, los contornos de diversos haces para un único nivel ganancia y para cualquier posición orbital.

- Otra posibilidad de las funciones desarrolladas, consiste en escoger un área de cobertura (inicialmente sobre Europa), de forma que, se selecciona el mejor haz, dentro de los suministrados por la red conformadora de haces seleccionada, para diversas posiciones de órbita y estaciones del año (se tiene en cuenta la posición del sol y el satélite es convenientemente rotado). De esta forma, es posible conocer qué subconjunto de haces son necesarios para un área de cobertura dada, reduciéndose el número de entradas de haces en el conformador de haces.

\section{Estudios de aplicación}

Las funciones desarrolladas se han utilizado para evaluar la aplicación del nuevo concepto de red conformadora de haces de radiofrecuencia, a dos aplicaciones; en concreto, la misión ARCHIMEDES de la Agencia Espacial Europea y el sistema de comunicaciones móviles via satélite P21 ${ }^{\mathrm{i}}$, del organismo INMARSAT. A continuación se muestran varios ejemplos de análisis del comportamiento, a nivel de sistema de la arquitectura de red conformadora bajo estudio.

EJEMPLO 1.- $\quad$ El error medio en la fase y amplitud de salida para cada uno de los 27 haces de un conformador de haces $27 \times 27$, con la matriz sintonizable $3 \times 3$ como celda básica, han sido obtenidos mediante simulación y los resultados se muestran en la tabla 4.14, para los tres valores de tensión de alimentación de los diodos varactores:

- -0.4 Voltios (Polarización nominal).

- -0.8 Voltios (Compensando una variación en la capacidad del proceso de un $12.8 \%)$.

- 0 Voltios (Compensando una variación en la capacidad del proceso de un $-15.4 \%)$.

\footnotetext{
${ }^{\text {i }}$ Project 21, proyecto iniciado por INMARSAT en Septiembre de 1991 para dar servicio global a teléfonos móviles via satélite y que en Enero de 1995 dio lugar al nacimiento de la empresa ICO Global Communications Limited.
} 


\begin{tabular}{|c|c|c|c|}
\hline & $\begin{array}{c}V_{\text {sintonia }}=-0.4 \mathrm{~V} \\
\text { Nominal }\end{array}$ & $\begin{array}{c}V_{\text {sintonía }}=-0.8 \mathrm{~V} \\
\text { Variación Cap: } 12.8 \%\end{array}$ & $\begin{array}{c}V_{\text {sintonía }}=0 \mathrm{~V} \\
\text { Variación Cap }-15.4 \%\end{array}$ \\
\hline HAZ 1 & $6.3^{\circ} / 1.2 \mathrm{~dB}$ & $6.1^{\circ} / 1.2 \mathrm{~dB}$ & $6.1^{\circ} / 1.2 \mathrm{~dB}$ \\
\hline HAZ 2 & $4.9^{\circ} / 1.2 \mathrm{~dB}$ & $4.9^{\circ} / 1.1 \mathrm{~dB}$ & $4.9^{\circ} / 1.1 \mathrm{~dB}$ \\
\hline HAZ 3 & $4.9^{\circ} / 1.2 \mathrm{~dB}$ & $5.0^{\circ} / 1.1 \mathrm{~dB}$ & $5.0^{\circ} / 1.2 \mathrm{~dB}$ \\
\hline HAZ 4 & $6.8^{\circ} / 0.8 \mathrm{~dB}$ & $6.7^{\circ} / 0.8 \mathrm{~dB}$ & $6.7^{\circ} / 0.8 \mathrm{~dB}$ \\
\hline HAZ 5 & $7.3^{\circ} / 0.9 \mathrm{~dB}$ & $7.4^{\circ} / 0.9 \mathrm{~dB}$ & $7.4^{\circ} / 0.9 \mathrm{~dB}$ \\
\hline HAZ 6 & $7.4^{\circ} / 0.9 \mathrm{~dB}$ & $7.4^{\circ} / 0.9 \mathrm{~dB}$ & $7.4^{\circ} / 0.9 \mathrm{~dB}$ \\
\hline HAZ 7 & $7.5^{\circ} / 0.8 \mathrm{~dB}$ & $7.4^{\circ} / 0.8 \mathrm{~dB}$ & $7.4^{\circ} / 0.9 \mathrm{~dB}$ \\
\hline HAZ 8 & $7.2^{\circ} / 0.8 \mathrm{~dB}$ & $7.2^{\circ} / 0.8 \mathrm{~dB}$ & $7.2^{\circ} / 0.8 \mathrm{~dB}$ \\
\hline HAZ 9 & $7.2^{\circ} / 0.8 \mathrm{~dB}$ & $7.2^{\circ} / 0.8 \mathrm{~dB}$ & $7.2^{\circ} / 0.8 \mathrm{~dB}$ \\
\hline HAZ 10 & $6.6^{\circ} / 1.2 \mathrm{~dB}$ & $6.7^{\circ} / 1.4 \mathrm{~dB}$ & $6.7^{\circ} / 1.4 \mathrm{~dB}$ \\
\hline HAZ 11 & $5.1^{\circ} / 1.0 \mathrm{~dB}$ & $5.2^{\circ} / 1.1 \mathrm{~dB}$ & $5.2^{\circ} / 1.1 \mathrm{~dB}$ \\
\hline HAZ 12 & $5.2^{\circ} / 1.0 \mathrm{~dB}$ & $5.2^{\circ} / 1.1 \mathrm{~dB}$ & $5.2^{\circ} / 1.1 \mathrm{~dB}$ \\
\hline HAZ 13 & $7.5^{\circ} / 0.7 \mathrm{~dB}$ & $7.5^{\circ} / 0.8 \mathrm{~dB}$ & $7.5^{\circ} / 0.8 \mathrm{~dB}$ \\
\hline HAZ 14 & $6.3^{\circ} / 0.7 \mathrm{~dB}$ & $6.2^{\circ} / 0.8 \mathrm{~dB}$ & $6.2^{\circ} / 0.8 \mathrm{~dB}$ \\
\hline HAZ 15 & $6.4^{\circ} / 0.8 \mathrm{~dB}$ & $6.3^{\circ} / 0.8 \mathrm{~dB}$ & $6.3^{\circ} / 0.8 \mathrm{~dB}$ \\
\hline HAZ 16 & $6.2^{\circ} / 0.9 \mathrm{~dB}$ & $6.1^{\circ} / 0.9 \mathrm{~dB}$ & $6.1^{\circ} / 0.9 \mathrm{~dB}$ \\
\hline HAZ 17 & $5.5^{\circ} / 0.8 \mathrm{~dB}$ & $5.4^{\circ} / 0.8 \mathrm{~dB}$ & $5.4^{\circ} / 0.9 \mathrm{~dB}$ \\
\hline HAZ 18 & $5.6^{\circ} / 0.8 \mathrm{~dB}$ & $5.4^{\circ} / 0.8 \mathrm{~dB}$ & $5.4^{\circ} / 0.9 \mathrm{~dB}$ \\
\hline HAZ 19 & $6.9^{\circ} / 1.2 \mathrm{~dB}$ & $6.9^{\circ} / 1.3 \mathrm{~dB}$ & $7.0^{\circ} / 1.3 \mathrm{~dB}$ \\
\hline HAZ 20 & $5.1^{\circ} / 0.9 \mathrm{~dB}$ & $5.1^{\circ} / 1.0 \mathrm{~dB}$ & $5.1^{\circ} / 1.0 \mathrm{~dB}$ \\
\hline HAZ 21 & $5.1^{\circ} / 1.0 \mathrm{~dB}$ & $5.1^{\circ} / 1.1 \mathrm{~dB}$ & $5.1^{\circ} / 1.1 \mathrm{~dB}$ \\
\hline HAZ 22 & $6.5^{\circ} / 0.9 \mathrm{~dB}$ & $6.3^{\circ} / 0.9 \mathrm{~dB}$ & $6.4^{\circ} / 0.9 \mathrm{~dB}$ \\
\hline HAZ 23 & $4.9^{\circ} / 1.0 \mathrm{~dB}$ & $4.9^{\circ} / 1.0 \mathrm{~dB}$ & $4.9^{\circ} / 1.0 \mathrm{~dB}$ \\
\hline HAZ 24 & $5.0^{\circ} / 1.0 \mathrm{~dB}$ & $4.9^{\circ} / 1.0 \mathrm{~dB}$ & $4.9^{\circ} / 1.0 \mathrm{~dB}$ \\
\hline HAZ 25 & $6.7^{\circ} / 1.0 \mathrm{~dB}$ & $6.6^{\circ} / 1.0 \mathrm{~dB}$ & $6.6^{\circ} / 1.1 \mathrm{~dB}$ \\
\hline HAZ 26 & $6.4^{\circ} / 0.9 \mathrm{~dB}$ & $6.4^{\circ} / 0.9 \mathrm{~dB}$ & $6.4^{\circ} / 0.9 \mathrm{~dB}$ \\
\hline HAZ 27 & $6.5^{\circ} / 0.9 \mathrm{~dB}$ & $6.5^{\circ} / 0.9 \mathrm{~dB}$ & $6.2^{\circ} / 1.0 \mathrm{~dB}$ \\
\hline MEDIA $_{27}$ & $6.2^{\circ} / 0.9 \mathrm{~dB}$ & $6.2^{\circ} / 1.0 \mathrm{~dB}$ & $6.2^{\circ} / 1.0 \mathrm{~dB}$ \\
\hline
\end{tabular}

Tabla 4.14: Error de fase medio, y error de amplitud medio para la red conformadora de tamaño $27 \times 27$, basada en la matriz $3 \times 3$ sintonizable.

Se puede resaltar que, la correción de las variaciones de las capacidades debidas a las tolerancias del proceso de fabricación, son perfectamente compensadas mediante la sintonización de las matrices $3 \times 3$, obteniéndose unas prestaciones similares a las del caso nominal.

EJEMPLO 2.- $\quad$ La red conformadora de haces $27 \times 27$, construida a partir de la matriz $3 \times 3$ fija como celda básica, ha sido evaluada en términos de parámetros de antena; estimándose, para cada uno de los 27 haces, las variaciones en la ganancia de haz, la adaptación a la entrada y a la salida, el nivel de los lóbulos secundarios, el ancho de haz y la posición del máximo del haz.

La agrupación utilizada dispone de 27 elementos, distribuidos de acuerdo con una rejilla espacial hexagonal. Los datos han sido calculados para los casos nominales y con seis variaciones diferentes en la capacidad del proceso. Los valores medio, RMS 


\begin{tabular}{|c||c|c|c|c|c|c|c|}
\hline & Nominal & $\Delta \mathrm{C}+5 \%$ & $\Delta \mathrm{C}+10 \%$ & $\Delta \mathrm{C}+15 \%$ & $\Delta \mathrm{C}-5 \%$ & $\Delta \mathrm{C}-10 \%$ & $\Delta \mathrm{C}-15 \%$ \\
\hline \hline$\Delta G_{\text {HAZ }}$ & $0.19 \mathrm{~dB}$ & $0.24 \mathrm{~dB}$ & $0.3 \mathrm{~dB}$ & $0.36 \mathrm{~dB}$ & $0.15 \mathrm{~dB}$ & $0.12 \mathrm{~dB}$ & $0.11 \mathrm{~dB}$ \\
\hline $\bar{S}_{11}$ & $19.1 \mathrm{~dB}$ & $17.1 \mathrm{~dB}$ & $14.9 \mathrm{~dB}$ & $13.1 \mathrm{~dB}$ & $18.6 \mathrm{~dB}$ & $16.5 \mathrm{~dB}$ & $14.2 \mathrm{~dB}$ \\
\hline $\bar{S}_{22}$ & $19.1 \mathrm{~dB}$ & $17.1 \mathrm{~dB}$ & $15.0 \mathrm{~dB}$ & $13.3 \mathrm{~dB}$ & $18.9 \mathrm{~dB}$ & $16.2 \mathrm{~dB}$ & $14.0 \mathrm{~dB}$ \\
\hline$\overline{N L P S}$ & $14.9 \mathrm{~dB}$ & $14.8 \mathrm{~dB}$ & $14.2 \mathrm{~dB}$ & $13.1 \mathrm{~dB}$ & $14.5 \mathrm{~dB}$ & $13.8 \mathrm{~dB}$ & $13.0 \mathrm{~dB}$ \\
\hline$N L P S_{\text {MAX }}$ & $13.8 \mathrm{~dB}$ & $13.2 \mathrm{~dB}$ & $12.7 \mathrm{~dB}$ & $12.1 \mathrm{~dB}$ & $13.0 \mathrm{~dB}$ & $12.0 \mathrm{~dB}$ & $11.0 \mathrm{~dB}$ \\
\hline
\end{tabular}

Tabla 4.15: Resultados de simulación de una agrupación hexagonal de 27 elementos alimentada por una red conformadora de tamaño $27 \times 27$ fabricada a partir de la matriz $3 \times 3$ fija.

\begin{tabular}{|c||c|c|c|}
\hline & $\begin{array}{c}V_{\text {sintonia }}=-0.4 \mathrm{~V} \\
\Delta C 0 \%\end{array}$ & $\begin{array}{c}V_{\text {sintonia }}=-0.8 \mathrm{~V} \\
\Delta C 12.8 \%\end{array}$ & $\begin{array}{c}V_{\text {sintonia }}=0 \mathrm{~V} \\
\Delta C-15.4 \%\end{array}$ \\
\hline \hline$\Delta G_{H A Z}$ & $0.2 \mathrm{~dB}$ & $0.22 \mathrm{~dB}$ & $0.22 \mathrm{~dB}$ \\
\hline$\overline{S_{11}}$ & $17.3 \mathrm{~dB}$ & $16.5 \mathrm{~dB}$ & $16.5 \mathrm{~dB}$ \\
\hline$\overline{S_{22}}$ & $17.8 \mathrm{~dB}$ & $17.0 \mathrm{~dB}$ & $17.0 \mathrm{~dB}$ \\
\hline$\overline{N L P S}$ & $14.7 \mathrm{~dB}$ & $14.7 \mathrm{~dB}$ & $14.7 \mathrm{~dB}$ \\
\hline$N L P S_{M A X}$ & $13.6 \mathrm{~dB}$ & $13.5 \mathrm{~dB}$ & $13.5 \mathrm{~dB}$ \\
\hline
\end{tabular}

Tabla 4.16: Resultados de simulación de una agrupación hexagonal de 27 elementos alimentada por una red conformadora de tamaño $27 \times 27$ fabricada a partir de la matriz $3 \times 3$ sintonizable.

o de pico, entre los 27 haces, para todos esos parámetros se muestran en la tabla 4.15 .

No se han detectado efectos de importancia sobre el apuntamiento de los haces o la anchura de los mismos. Los parámetros más sensibles a las variaciones de la capacidad, son las pérdidas de retorno (hasta $6 \mathrm{~dB}$ peores, en el peor caso). El nivel de lóbulo secundario también se ve afectado, pero en una menor medida. Los mismos resultados han sido obtenidos para el conformador $27 \times 27$ construido a partir de la matriz $3 \times 3$ sintonizable como celda básica.

En la tabla 4.16, se muestran los resultados para el caso nominal y variación nula en la capacidad del proceso y, para dos valores de tensión de alimentación con sus respectivas variaciones de las capacidades. Al igual que ocurría con la matriz fija nominal, no se han detectado efectos de importancia sobre los centros de los haces o la anchura de los mismos.

Aunque los valores iniciales para el caso nominal son ligeramente peores en el diseño sintonizable, con respecto al diseño fijo, la consistencia de los resultados frente a variaciones de la capacidad, claramente resalta las ventajas del enfoque sintonizable. En cualquier caso, los valores iniciales para el caso nominal de la matriz $3 \times 3$ sintonizable, podrían mejorarse en siguientes diseños. 


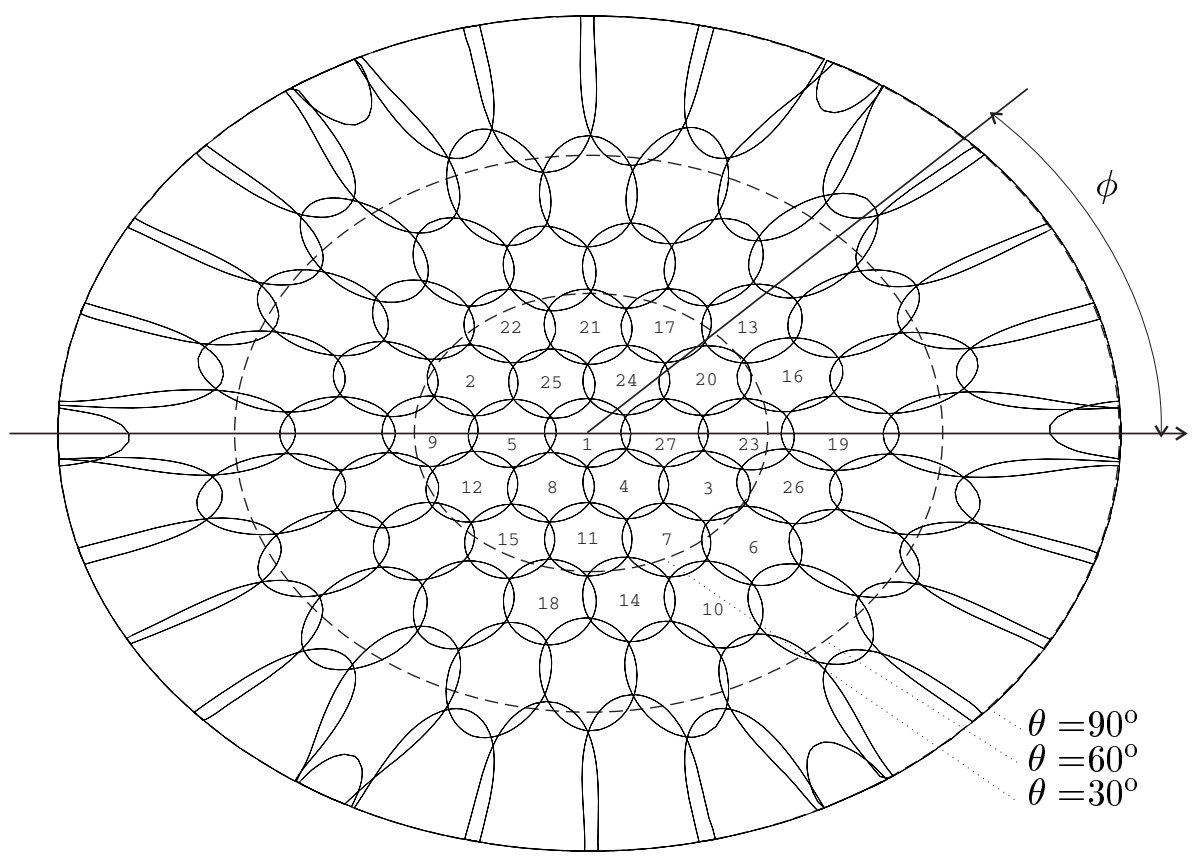

Figura 4.28: Contornos del factor de agrupación en espacio real $(\theta, \phi)$ para los 27 haces (lóbulos de difracción incluidos) al nivel de cruce (-3.8 dB), para una agrupación de 19 elementos, separados entre sí, una longitud de onda sobre una rejilla hexagonal y utilizando el conformador de haces de tamaño $27 \times 27$, construido con matrices $3 \times 3$ sintonizables y con la numeración de haces y de elementos indicada en la figura 4.10 .

EJEMPLO 3.- Conjunto de haces para el conformador de haces $27 \times 27$, mostrando para cada haz el contorno a un nivel fijo. En concreto, se trata de un conformador $27 \times 27$, alimentando una agrupación de 19 elementos con la distribución mostrada en la figura 4.13. Este sobredimensionamiento de la red conformadora, implicaría, según la figura 2.10, un nivel de cruce de $-3.8 \mathrm{~dB}$, como se confirma en la figura 4.28.

De la figura 4.28 se puede resaltar la buena cobertura que ofrece la agrupación alimentada por la red conformadora de $27 \times 27$, basada en los datos de simulación de la celda $3 \times 3$ sintonizable.

EJEMPLO 4.- Contornos de un haz sobre la superficie de la Tierra, para la misión ARCHIMEDES.

Datos de la antena de ARCHIMEDES [Esp94]:

- Agrupación de Radiación Directa (DRA ${ }^{\mathrm{i}}$.

- Distribución espacial hexagonal, con 37 elementos radiantes formando un hexágono regular.

\footnotetext{
${ }^{\mathrm{i}}$ Direct Radiating Array.
} 
- Elementos radiantes circulares con un diámetro de 2.2 veces la longitud de onda.

- Factor de relleno (elementos circulares sobre rejilla hexagonal): 0.907 .

- Frecuencia 1.5 GHz (Los datos de simulación para la matriz sintonizable a 2.3 $\mathrm{GHz}$ son utilizados, ya que no se esperan diferencias importantes).

- Potencia Transmitida: 90 Watios.

- Eficiencia de los elementos: $90 \%$.

Datos de la órbita de ARCHIMEDES:

- Órbita Altamente Elíptica Multi-Regional (M-HEOi).

- Altura en el apogeo: $26784 \mathrm{~km}$.

- Altura en el punto de traspaso ${ }^{\mathrm{ii}}: 20565 \mathrm{~km}$.

- Altura en el perigeo: $1000 \mathrm{~km}$.

- Periodo orbital: 8 horas.

- Pérdidas atmosféricas: $0.2 \mathrm{~dB}$.

Datos del conformador de haces:

- Red conformadora de haces de tamaño $81 \times 81$, consiguiéndose un nivel de cruce de $2.5 \mathrm{~dB}$.

Se han realizado simulaciones para el conformador de haces $81 \times 81$ ideal, y también para el conformador de haces $81 \times 81$, construido con la matriz sintonizable $3 \times 3$ como celda básica (Fig. 4.11). En la figura 4.29 se muestran, para ambos casos, los contornos de densidad de flujo de potencia sobre Europa en el apogeo (altura: $26784 \mathrm{~km}$ ), con un apuntamiento hacia el centro del área de cobertura (Longitud: 12E; Latitud: $50 \mathrm{~N}$ ).

Comparando ambos resultados, se puede destacar las pequeñas diferencias en la forma y la posición del lóbulo principal, aunque los lóbulos secundarios aparecen ligeramente modificados.

\footnotetext{
${ }^{\mathrm{i}}$ Multi-Regional High Elliptic Orbit.

ii Handover, en terminología inglesa.
} 
a)

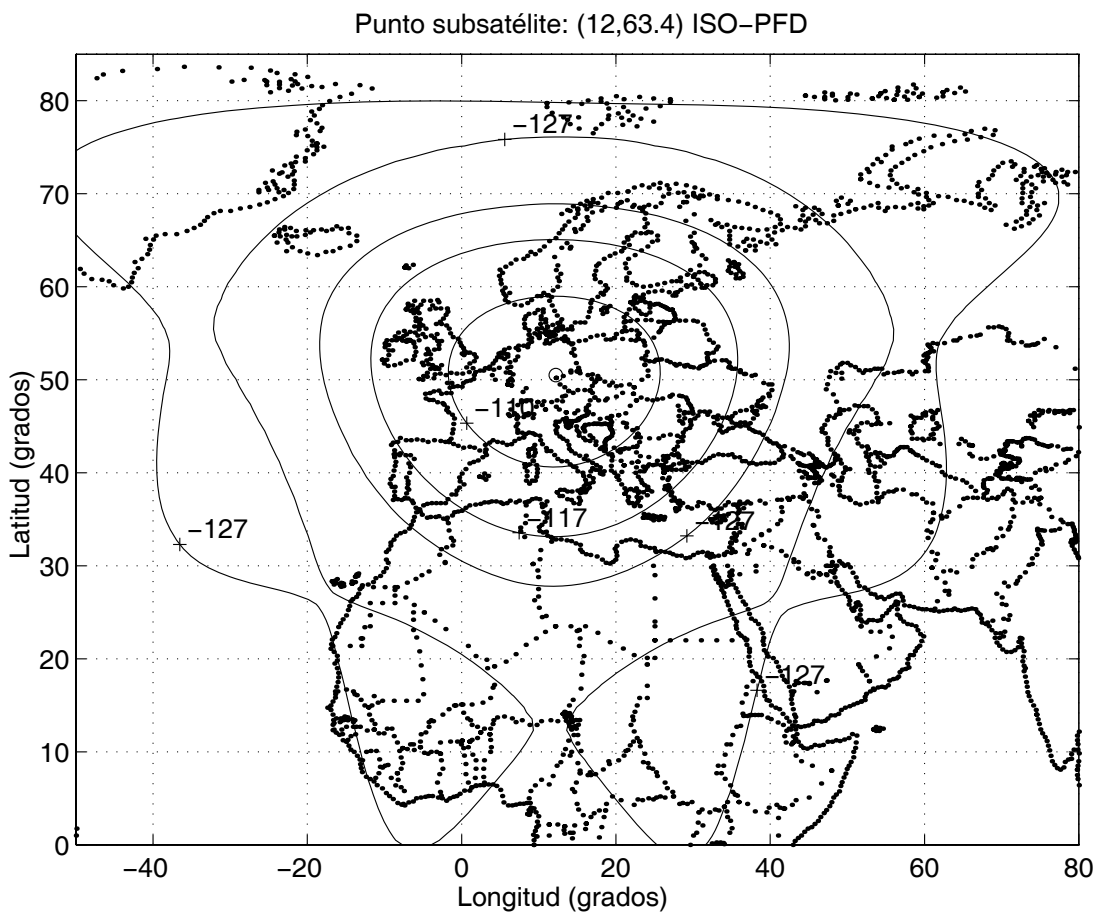

b)

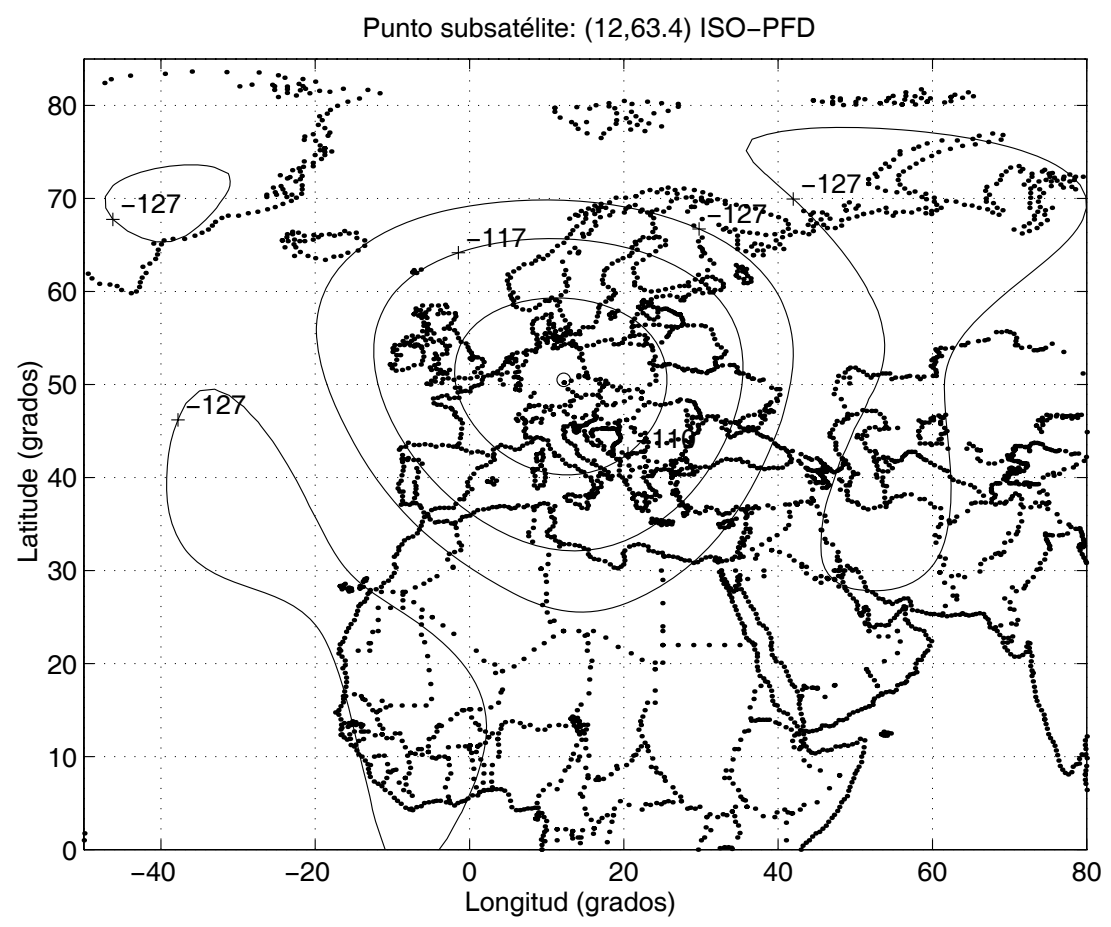

Figura 4.29: Contornos Iso-PFD para la agrupación de 37 elementos con rejilla hexagonal de la misión ARCHIMEDES. a) Antena alimentada con el conformador de haces $81 \times 81$ ideal. b) Antena alimentada con el conformador de haces $81 \times 81$ construido con matrices sintonizables $3 \times 3$. 


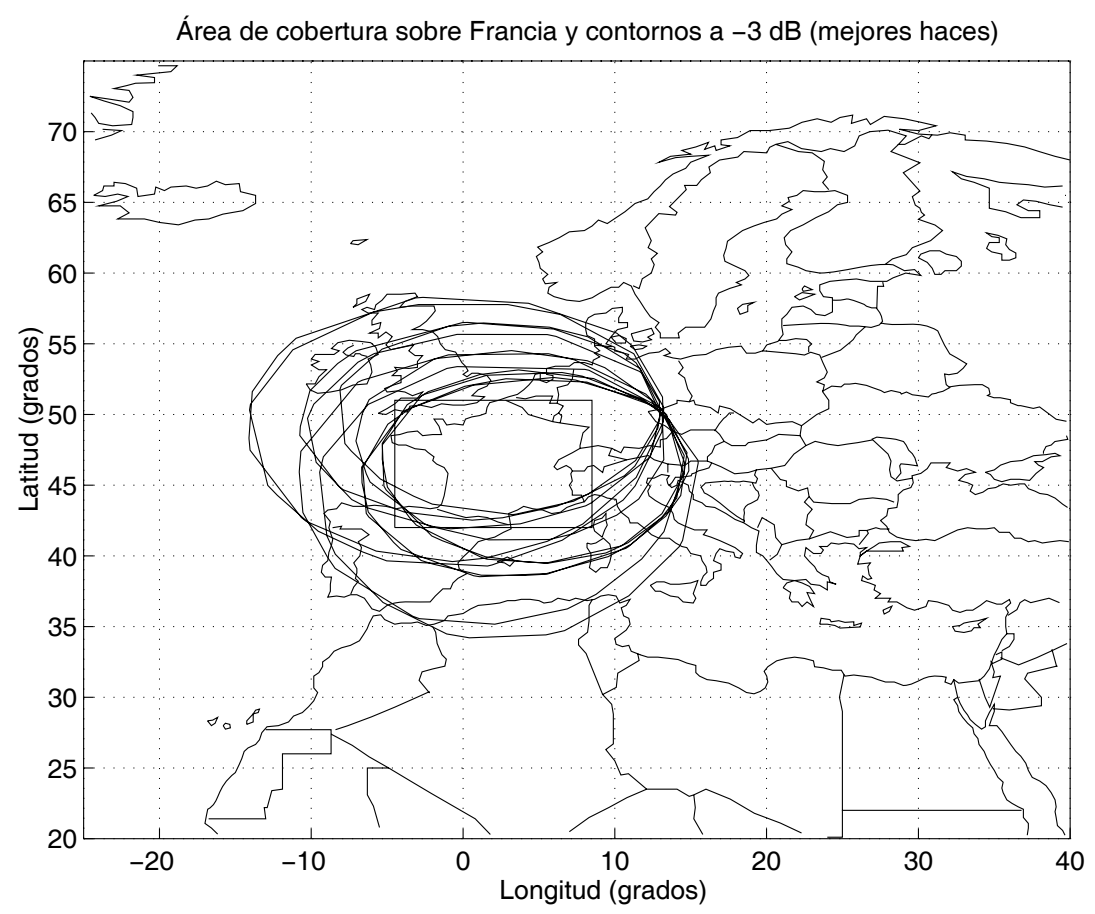

Figura 4.30: Ejemplo de área de cobertura para la misión ARCHIMEDES de Difusión de Audio Digital (DAB).

EJEMPLO 5.- $\quad$ Se ha seleccionado un área (Fig. 4.30) sobre Europa (Francia, Luxemburgo y Bélgica) como una de las cuatro áreas de cobertura a definir en la misión de difusión de audio digital ${ }^{\mathrm{i}}$ en ARCHIMEDES, estudiándose doce casos diferentes (tres posiciones orbitales por cuatro estaciones en el año). Los parámetros utilizados han sido los mismos que en el ejemplo previo, pero ahora el tamaño del conformador de haces elegido es de $256 \times 256$, lo que permite un nivel de solapamiento entre haces bastante mejor $(-0.8 \mathrm{~dB})$.

Para cada uno de los doce casos, se calculó la densidad de flujo de potencia sobre el área de cobertura para los 256 haces y se escogió el mejor haz, de acuerdo con el criterio del mejor peor caso. Los resultados de las simulaciones se pueden ver en la tabla 4.17, en la que se presentan para cada uno de los doce casos a estudiar, el número correspondiente al mejor haz y los niveles máximo y mínimo del flujo de densidad de potencia dentro del área de cobertura.

A partir de los datos anteriores, es evidente que, debido a la posición más cercana del satélite en el punto de traspaso, los haces son más estrechos sobre la superficie terrestre, pero, en cambio las pérdidas por propagación son menores, con lo cual se consigue un nivel mínimo similar al obtenido en apogeo. Las especificaciones para esta misión [Gal95] de la densidad de flujo de potencia, eran de $-111.5 \mathrm{dBW} / \mathrm{m}^{2}$.

\footnotetext{
${ }^{\mathrm{i}}$ Digital Audio Broadcasting, en terminología inglesa.
} 


\begin{tabular}{|c||c|c|c|c|}
\hline & Mitad invierno & Mitad primavera & Inicio Verano & Mitad Otoño \\
\hline \hline Punto de & HAZ 256 & HAZ 73 & HAZ 69 & HAZ 252 \\
traspaso 1 & $-105.1 \mathrm{dBW} / \mathrm{m}^{2}$ & $-105.1 \mathrm{dBW} / \mathrm{m}^{2}$ & $-105.1 \mathrm{dBW} / \mathrm{m}^{2}$ & $-105.1 \mathrm{dBW} / \mathrm{m}^{2}$ \\
& $-109.2 \mathrm{dBW} / \mathrm{m}^{2}$ & $-109.4 \mathrm{dBW} / \mathrm{m}^{2}$ & $-110.9 \mathrm{dBW} / \mathrm{m}^{2}$ & $-109.3 \mathrm{dBW} / \mathrm{m}^{2}$ \\
\hline Apogeo & $\mathrm{HAZ} 256$ & $\mathrm{HAZ} 5$ & $\mathrm{HAZ} 73$ & $\mathrm{HAZ} 252$ \\
& $-107.4 \mathrm{dBW} / \mathrm{m}^{2}$ & $-107.5 \mathrm{dBW} / \mathrm{m}^{2}$ & $-107.5 \mathrm{dBW} / \mathrm{m}^{2}$ & $-107.4 \mathrm{dBW} / \mathrm{m}^{2}$ \\
& $-110.5 \mathrm{dBW} / \mathrm{m}^{2}$ & $-109.9 \mathrm{dBW} / \mathrm{m}^{2}$ & $-109.2 \mathrm{dBW} / \mathrm{m}^{2}$ & $-110.7 \mathrm{dBW} / \mathrm{m}^{2}$ \\
\hline Punto de & $\mathrm{HAZ} 252$ & $\mathrm{HAZ} 256$ & $\mathrm{HAZ} 73$ & $\mathrm{HAZ} 16$ \\
traspaso 2 & $-105.2 \mathrm{dBW} / \mathrm{m}^{2}$ & $-105.2 \mathrm{dBW} / \mathrm{m}^{2}$ & $-105.2 \mathrm{dBW} / \mathrm{m}^{2}$ & $-105.2 \mathrm{dBW} / \mathrm{m}^{2}$ \\
& $-109.1 \mathrm{dBW} / \mathrm{m}^{2}$ & $-109.1 \mathrm{dBW} / \mathrm{m}^{2}$ & $-110.4 \mathrm{dBW} / \mathrm{m}^{2}$ & $-109.8 \mathrm{dBW} / \mathrm{m}^{2}$ \\
\hline
\end{tabular}

Tabla 4.17: Mejor haz y niveles de densidad de potencia mínimo y máximo para el área de cobertura de la figura 4.30, para doce situaciones distintas, correspondientes a tres posiciones orbitales del satélite y cuatro periodos del año.

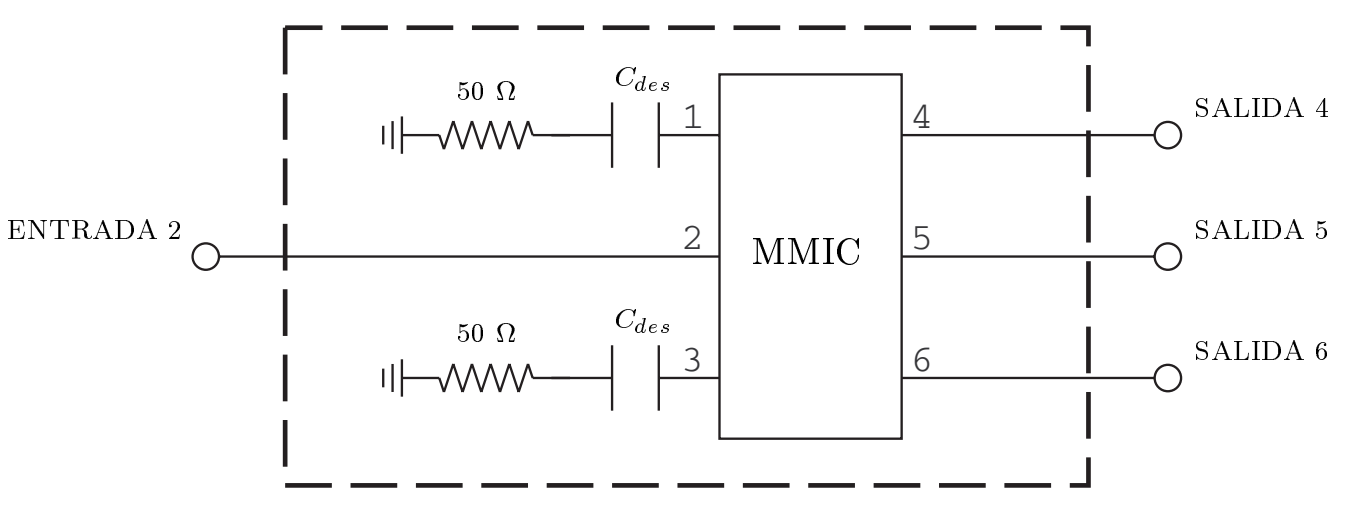

Soporte de prueba

Figura 4.31: Esquemático del soporte de prueba usado en las medidas de las matrices $3 \times 3$ fija y sintonizable.

\subsection{Medidas de las matrices $3 \times 3$}

\subsubsection{Matriz $3 \times 3$ fija}

Las medidas de la matriz fija $3 \times 3$ se llevaron a cabo en las instalaciones propias de la Agencia Espacial Europea. Para ello se usó un soporte de prueba ${ }^{i}$ (Fig. 4.31) con cuatro accesos: una de las tres entradas (acceso 2) y las tres salidas (accesos 4, 5 y 6); los otros dos accesos se terminaron con cargas SMD de $50 \Omega$ y condensadores de desacoplo de contínua $\left(C_{d e s}\right)$.

En la tabla 4.18, se pueden observar los resultados en cuanto a pérdidas de inserción entre la entrada 2 y las salidas 4,5 y 6 , el desfase relativo entre la salida

\footnotetext{
i Test fixture, en terminología inglesa.
} 


\begin{tabular}{|ccc||cc||c|}
\hline \multicolumn{2}{|c||}{ Pérdidas de inserción } & \multicolumn{2}{c||}{ Desfases relativos } & Aislamiento \\
$S_{24}$ & $S_{25}$ & $S_{26}$ & $\Delta \phi_{45}$ & $\Delta \phi_{65}$ & $S_{56}$ \\
\hline $9.20 \mathrm{~dB}$ & $9.60 \mathrm{~dB}$ & $9.36 \mathrm{~dB}$ & $-111.3^{\circ}$ & $-110.0^{\circ}$ & $15.8 \mathrm{~dB}$ \\
\hline
\end{tabular}

Tabla 4.18: Medidas de la matriz $3 \times 3$ fija.

5 y las salidas 4 y 6 y el aislamiento entre los accesos 5 y 6 . Todos los valores mostrados se refieren a la frecuencia de diseño de la matriz $(2.3 \mathrm{GHz})$.

Las gráficas del analizador de redes de las medidas de pérdidas de inserción, desfase relativo y aislamiento, se muestran en las figuras 4.32 y 4.33 para un rango de frecuencias entre 2 y $3 \mathrm{GHz}$.

Las pérdidas de inserción son aproximadamente $0.5 \mathrm{~dB}$ mayores de lo esperado, cantidad que puede ser debida al propio soporte de prueba y a los conectores. Las diferencias entre las pérdidas de inserción, cumplen las especificaciones, aunque resultan ser $0.3 \mathrm{~dB}$ mayores de lo simulado.

El aislamiento mejora en $2 \mathrm{~dB}$ los resultados obtenidos en las simulaciones. En cuanto al desfase entre salidas, las medidas presentan un valor bastante menor de lo esperado $\left(-9^{\circ}\right)$.

\subsubsection{Matriz $3 \times 3$ sintonizable}

La matriz $3 \times 3$ sintonizable, fue medida en las instalaciones de Dassault Electronique con el mismo soporte de prueba que la matriz fija. Los seis accesos fueron desacoplados en contínua, mediante el uso de circuitos de bloqueo de contínua, con el fin de eliminar las cargas de $50 \Omega$ en paralelo con la red de polarización. Como se explicó en el apartado 4.3.3, la tensión en el ánodo del varactor, $V_{\text {diodo }}$, es la mitad de la tensión disponible en el exterior del circuito integrado, $V_{\text {ext }}$.

Los resultados de las medidas, para siete niveles diferentes de tensión externa al circuito integrado, se muestran en la tabla 4.19. En la figura 4.34, se presenta gráficamente la diferencia de fase y las pérdidas de inserción en función de la tensión al exterior del circuito integrado.

A la hora de escoger la tensión de sintonización para la matriz $3 \times 3$ más adecuada, debe tenerse en cuenta el comportamiento de la misma, respecto a las pérdidas de inserción, la adaptación y sobre todo el desfase entre las salidas. En cuanto a las pérdidas de inserción, el mejor nivel de tensión estaría alrededor $V_{\text {ext }}=0 \mathrm{~V}$. Pero si se considera la respuesta en fase, deberían aplicarse tensiones mayores que $V_{\text {ext }} \approx 1 \mathrm{~V}$. El nivel de tensión considerado como el mejor compromiso entre la precisión del desfase y las pérdidas de inserción diferenciales, era de $V_{e x t}=0.7 \mathrm{~V}$.

Debería ser destacado que, debido al menor desfase conseguido para un valor de alimentación nominal (el mismo efecto que fue detectado en el diseño fijo), se ha 
a)
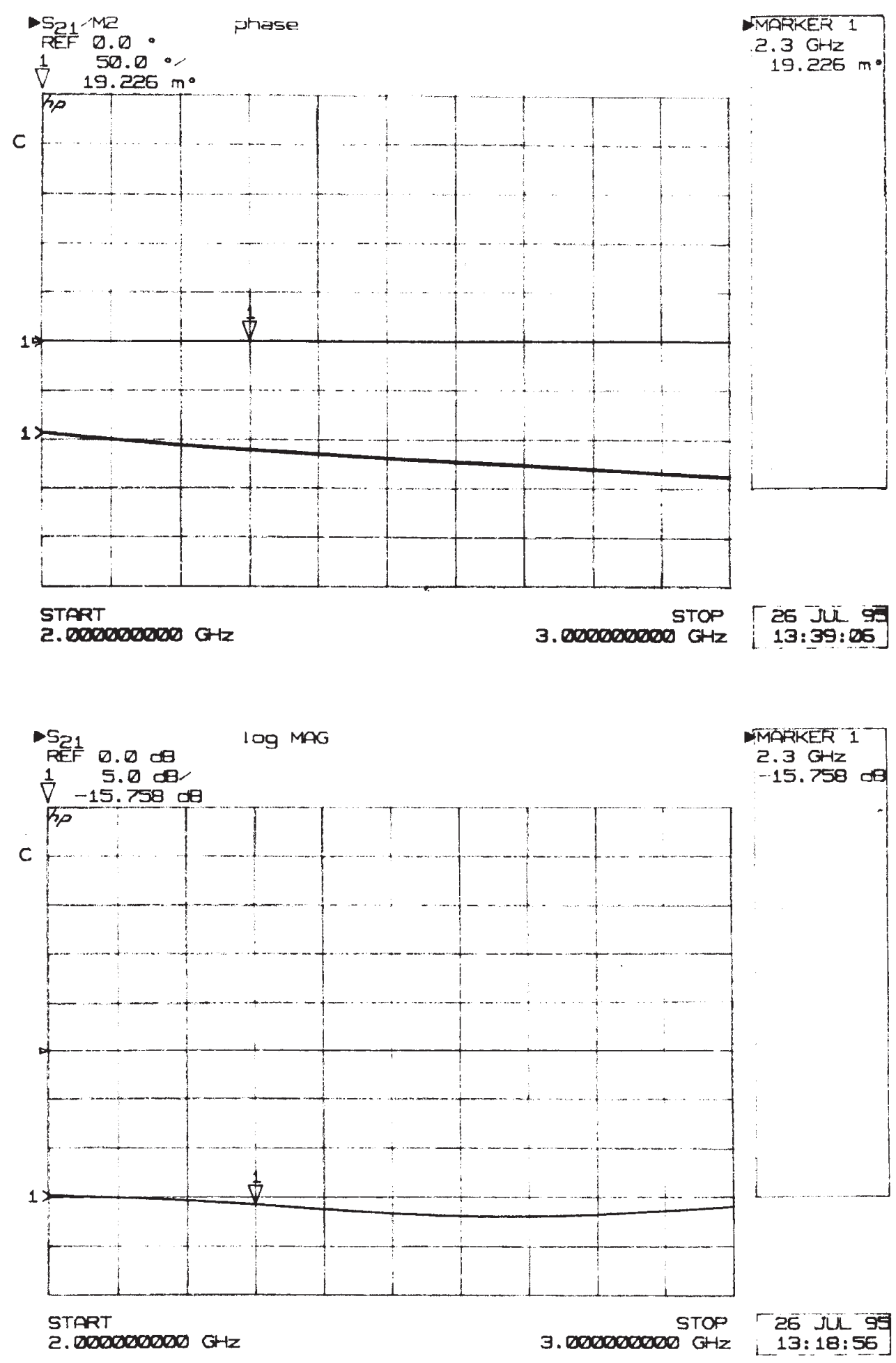

Figura 4.32: Medidas de la matriz $3 \times 3$ fija. a) Respuesta en fase, relativa al camino 2 a 4, para los caminos 2 a 4, 2 a 5 y 2 a 6 . b) Aislamiento entre los accesos 5 y 6. 


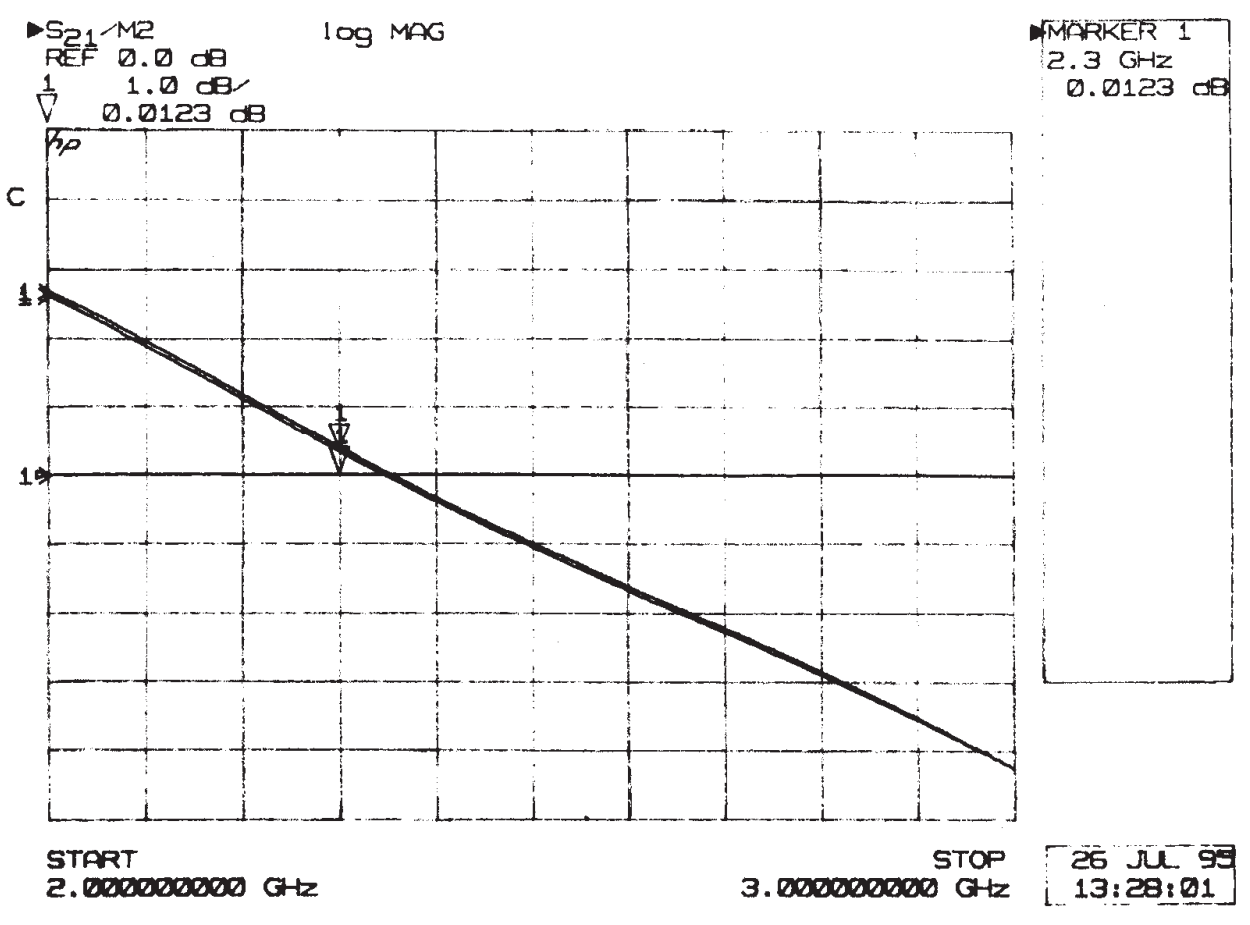

a)

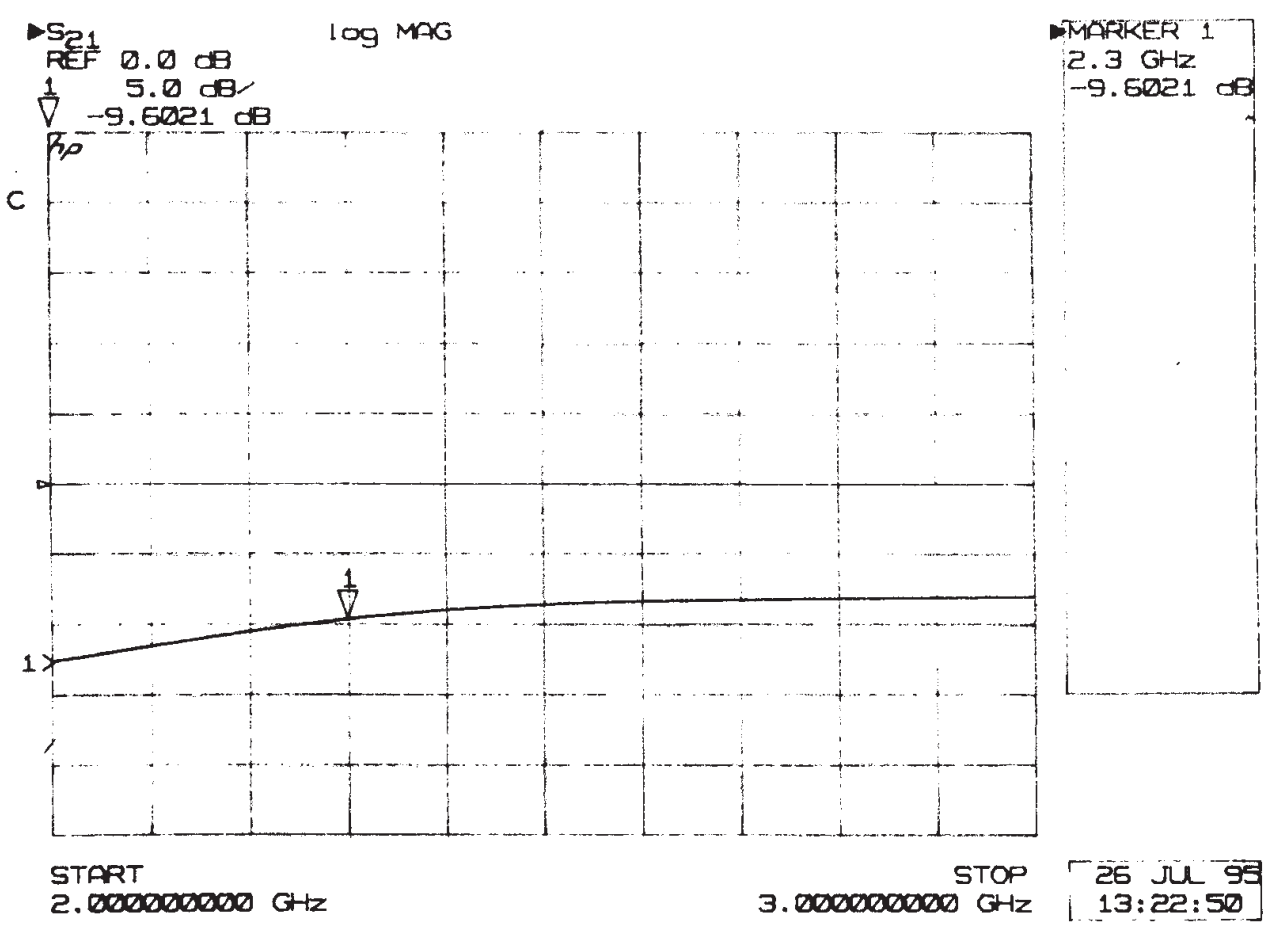

b)

Figura 4.33: Medidas de la matriz $3 \times 3$ fija. a) Pérdidas de inserción, relativas al camino 2 a 5, para los caminos 2 a 4,2 a 5 y 2 a 6 . b) Pérdidas de inserción para el camino 2 y 4. 


\begin{tabular}{|c||c|c||c|c|c||c|c|c|}
\hline \multicolumn{1}{|c||}{} & \multicolumn{2}{c||}{ Desfases } & \multicolumn{4}{c||}{} & \multicolumn{3}{c|}{ Pérdidas de retorno } \\
\multicolumn{1}{|c||}{$V_{e x t}$} & $\Delta \phi_{45}$ & $\Delta \phi_{65}$ & $S_{24}$ & $S_{25}$ & $S_{26}$ & $S_{44}$ & $S_{55}$ & $S_{66}$ \\
\hline \hline$-2 \mathrm{~V}$ & $-93^{\circ}$ & $-85^{\circ}$ & $-10.5 \mathrm{~dB}$ & $-12.4 \mathrm{~dB}$ & $-10.8 \mathrm{~dB}$ & $-8.1 \mathrm{~dB}$ & $-8 \mathrm{~dB}$ & $-8.1 \mathrm{~dB}$ \\
\hline$-1 \mathrm{~V}$ & $-109^{\circ}$ & $-106^{\circ}$ & $-10 \mathrm{~dB}$ & $-10.7 \mathrm{~dB}$ & $-10.3 \mathrm{~dB}$ & $-13.6 \mathrm{~dB}$ & $-13.3 \mathrm{~dB}$ & $-12 \mathrm{~dB}$ \\
\hline$-0.5 \mathrm{~V}$ & $-111^{\circ}$ & $-108^{\circ}$ & $-10 \mathrm{~dB}$ & $-10.5 \mathrm{~dB}$ & $-10.2 \mathrm{~dB}$ & $-14.2 \mathrm{~dB}$ & $-13.9 \mathrm{~dB}$ & $-14.2 \mathrm{~dB}$ \\
\hline $0 \mathrm{~V}$ & $-112^{\circ}$ & $-110^{\circ}$ & $-10.2 \mathrm{~dB}$ & $-10.3 \mathrm{~dB}$ & $-10.4 \mathrm{~dB}$ & $-14.6 \mathrm{~dB}$ & $-14.4 \mathrm{~dB}$ & $-14.6 \mathrm{~dB}$ \\
\hline $0.5 \mathrm{~V}$ & $-116^{\circ}$ & $-114^{\circ}$ & $-10.9 \mathrm{~dB}$ & $-10.2 \mathrm{~dB}$ & $-11.1 \mathrm{~dB}$ & $-13.4 \mathrm{~dB}$ & $-13.3 \mathrm{~dB}$ & $-13.4 \mathrm{~dB}$ \\
\hline $1 \mathrm{~V}$ & $-120^{\circ}$ & $-117^{\circ}$ & $-11.9 \mathrm{~dB}$ & $-10.4 \mathrm{~dB}$ & $-12.1 \mathrm{~dB}$ & $-11.2 \mathrm{~dB}$ & $-11.2 \mathrm{~dB}$ & $-11.2 \mathrm{~dB}$ \\
\hline $1.5 \mathrm{~V}$ & $-120^{\circ}$ & $-118^{\circ}$ & $-12.4 \mathrm{~dB}$ & $-10.7 \mathrm{~dB}$ & $-12.6 \mathrm{~dB}$ & $-10.4 \mathrm{~dB}$ & $-10.5 \mathrm{~dB}$ & $-10.4 \mathrm{~dB}$ \\
\hline
\end{tabular}

Tabla 4.19: Medidas de la matriz $3 \times 3$ sintonizable.

a)
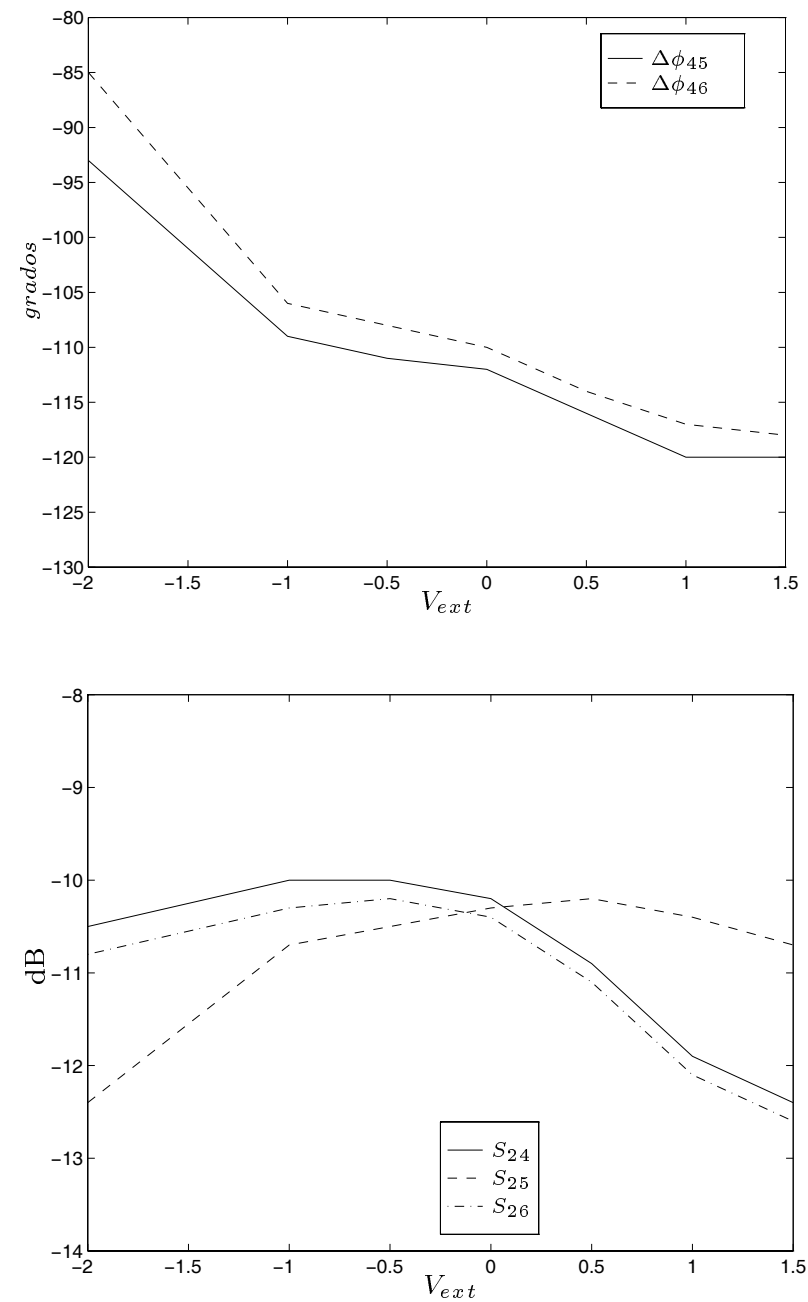

b)

Figura 4.34: Medidas de la matriz $3 \times 3$ sintonizable en función de la tensión de polarización. a) Desfase relativo entre las salidas 4 y 5 y las salidas 6 y 5 . b) Pérdidas de inserción para las salidas 4, 5 y 6 cuando la entrada es el acceso 2. 
necesitado un nivel mayor del esperado para conseguir la diferencia de fase de $-120^{\circ}$ deseada. Por otro lado, esta elección de la tensión de sintonización implica peores pérdidas de inserción diferenciales entre las diferentes salidas.

\subsubsection{Comentarios a los resultados de las medidas de las celdas básicas}

Los resultados de las medidas para las matrices $3 \times 3$ fija y sintonizable, presentan claras divergencias respecto a los resultados obtenidos en la simulación. Con el fin de detectar las posibles causas de estas diferencias y asegurarse que se obtienen los resultados esperados en sucesivos diseños, se ha llevado a cabo un análisis de ingeniería inversa sobre las simulaciones de la matriz fija.

Dentro de las posibles fuentes de error, se deben considerar, las tolerancias del proceso de fabricación, los errores en el modelado de los elementos utilizados y posibles acoplamientos, no esperados, entre elementos.

En cuanto a las tolerancias del proceso, la matriz $3 \times 3$ fija, dispone únicamente de bobinas en espiral, condensadores y líneas de transmisión; de estos tres elementos, los más sensibles a variaciones del proceso son los condensadores, cuya capacidad tiene una tolerancia del $20 \%$. Todos los circuitos considerados en este capítulo provienen de la misma oblea, por lo que se pueden utilizar los módulos de control PCM repartidos por la oblea para supervisar las variaciones producidas sobre la capacidad por área de los condensadores. A partir de las medidas de los módulos PCM para la capacidad, se ha obtenido un valor medio de $403 \mathrm{pF} / \mathrm{mm}^{2}$, en lugar del valor nominal de $400 \mathrm{pF} / \mathrm{mm}^{2}$, con una varianza de la distribución sobre el valor medio de $\sigma_{C}=3 \mathrm{pF} / \mathrm{mm}^{2}$. Por lo tanto, sólo se puede considerar una pequeña variación de la capacidad (menos del $2 \%$ ), con el fin de averiguar las razones de los medidas que se han obtenido.

Por lo que respecta a errores en el modelado, una primera aproximación es suponer que, los valores de las bobinas y condensadores presentan un cierta desviación sobre el valor modelado, proporcional al mismo. Sin embargo, ni una variación porcentual fija en las capacidades, ni en las inductancias podría explicar los resultados obtenidos, ya que ambas posibilidades implicarían una mayor variación en los valores de amplitud, si los porcentajes de desviación son fijados de acuerdo con los resultados de fase medidos.

Como los seis condensadores tienen el mismo valor, puede esperarse que sufran la misma variación con respecto al valor nominal; por consiguiente, las únicas razones que podrían explicar los resultados obtenidos serían, o bien una variación en los valores de las inductancias, o bien efectos de acoplamientos no considerados. Como se puede observar en la figura 4.20, los elementos fueron distribuidos espacialmente lo suficientemente separados como para evitarse acoplamientos indeseados, por lo que su efecto debería ser pequeño y despreciable, salvo quizás en el cruce de líneas a 


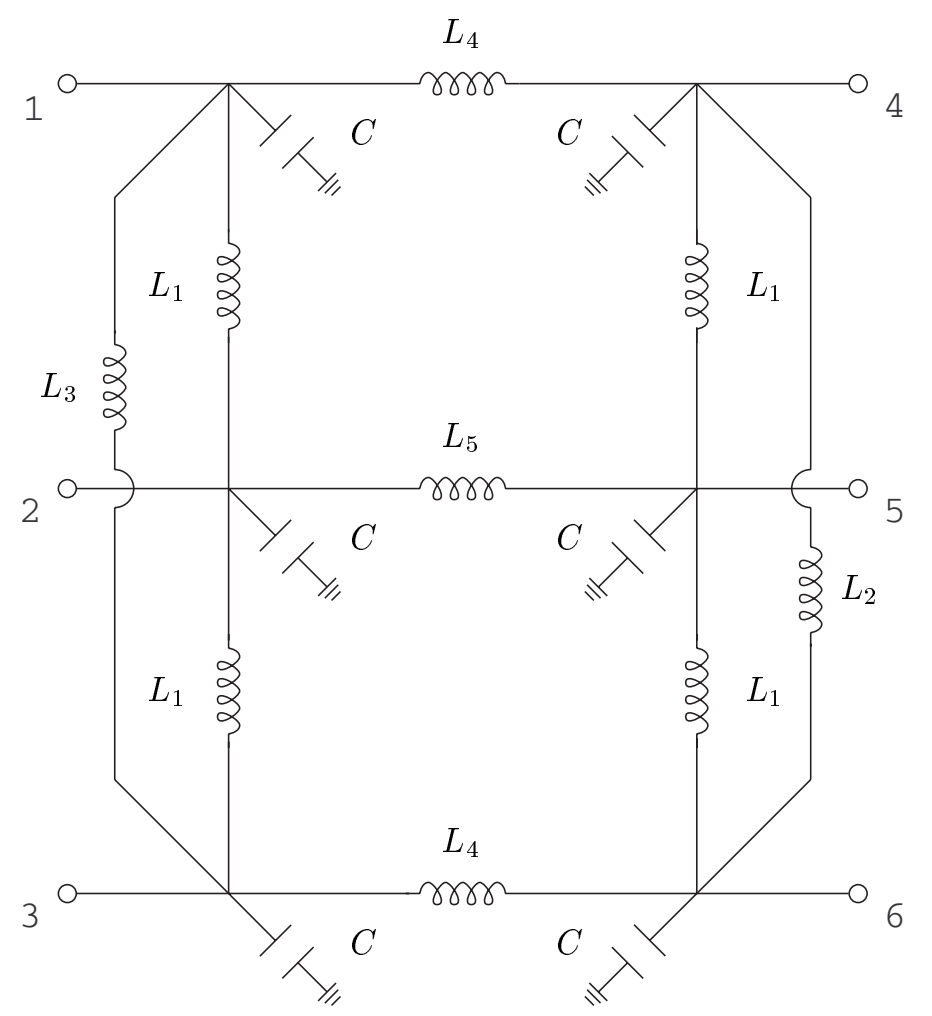

Figura 4.35: Esquemático de la matriz $3 \times 3$ mostrando los cinco diferentes valores de inductancia.

dos niveles; cruce que se ha realizado de acuerdo con las recomendaciones de PML.

Restringiendo las fuentes de error a las variaciones de la inductancias, se han dividido las nueve inductancias del diseño de la matriz $3 \times 3$ fija (Fig. 4.35) en dos grupos $\left(L_{1}, L_{2}, L_{3}\right)$ y $\left(L_{4}, L_{5}\right)$. $L_{2}$ y $L_{3}$ son aproximadamente elementos idénticos, y se han unido a $L_{1}$ para así mantener la simetría del circuito. El inductor $L_{5}$ es simplemente un poco más grande que $L_{4}$, por lo que pueden considerarse similares. Una vez agrupadas las bobinas, se variaron las inductancias dentro de un mismo grupo con un único coeficiente porcentual. Posteriormente, se variaron independientemente los coeficientes de ambos grupos, con el fin de intentar simular los resultados de las medidas. Los valores finales para estos coeficientes fueron:

- Grupo $L_{1}, L_{2}, L_{3}:-6.5 \%$.

- Grupo $L_{4}, L_{5}:-13.1 \%$.

Con los valores de inductancia alterados según estos coeficientes, se realizaron nuevas simulaciones de la matriz sintonizable, intentando obtener los resultados de las medidas.

En las tablas 4.20 y 4.21 se muestran los resultados de simulación para la matriz sintonizable (a la frecuencia central), sin modificaciones y con las inductancias corregidas respectivamente. 


\begin{tabular}{|c||c|c||c|c|c||c|c|c|}
\hline \multicolumn{1}{|c||}{} & \multicolumn{2}{c||}{ Desfases } & \multicolumn{3}{c||}{ Pérdidas de inserción } & \multicolumn{3}{c|}{ Pérdidas de retorno } \\
\multicolumn{1}{|c||}{$V_{\text {ext }}$} & $\Delta \phi_{45}$ & $\Delta \phi_{65}$ & $S_{24}$ & $S_{25}$ & $S_{26}$ & $S_{44}$ & $S_{55}$ & $S_{66}$ \\
\hline \hline$-3.2 \mathrm{~V}$ & $-87.3^{\circ}$ & $-87.3^{\circ}$ & $-9 \mathrm{~dB}$ & $-11.9 \mathrm{~dB}$ & $-9 \mathrm{~dB}$ & $-8.0 \mathrm{~dB}$ & $-7.8 \mathrm{~dB}$ & $-8 \mathrm{~dB}$ \\
\hline$-2.4 \mathrm{~V}$ & $-89^{\circ}$ & $-89^{\circ}$ & $-9.2 \mathrm{~dB}$ & $-11.9 \mathrm{~dB}$ & $-9.2 \mathrm{~dB}$ & $-8.3 \mathrm{~dB}$ & $-8.1 \mathrm{~dB}$ & $-8.3 \mathrm{~dB}$ \\
\hline$-1.6 \mathrm{~V}$ & $-115^{\circ}$ & $-114.9^{\circ}$ & $-10.1 \mathrm{~dB}$ & $-10.5 \mathrm{~dB}$ & $-10.1 \mathrm{~dB}$ & $-15.9 \mathrm{~dB}$ & $-16.4 \mathrm{~dB}$ & $-16.1 \mathrm{~dB}$ \\
\hline$-0.8 \mathrm{~V}$ & $-120.3^{\circ}$ & $-120.2^{\circ}$ & $-9.97 \mathrm{~dB}$ & $-9.9 \mathrm{~dB}$ & $-9.98 \mathrm{~dB}$ & $-17.7 \mathrm{~dB}$ & $-18.9 \mathrm{~dB}$ & $-17.9 \mathrm{~dB}$ \\
\hline $0 \mathrm{~V}$ & $-125^{\circ}$ & $-124.8^{\circ}$ & $-10.6 \mathrm{~dB}$ & $-9.6 \mathrm{~dB}$ & $-10.6 \mathrm{~dB}$ & $-15.3 \mathrm{~dB}$ & $-16.4 \mathrm{~dB}$ & $-15.4 \mathrm{~dB}$ \\
\hline
\end{tabular}

Tabla 4.20: Resultados de simulación de la matriz $3 \times 3$ sintonizable.

\begin{tabular}{|c||c|c||c|c|c||c|c|c|}
\hline \multicolumn{1}{|c||}{} & \multicolumn{2}{c||}{ Desfases } & \multicolumn{3}{c||}{} & \multicolumn{3}{c|}{} \\
\multicolumn{1}{|c||}{ relativos } & \multicolumn{3}{c|}{ Pérdidas de inserción } & \multicolumn{3}{c|}{ Pérdidas de retorno } \\
$V_{\text {ext }}$ & $\Delta \phi_{45}$ & $\Delta \phi_{65}$ & $S_{24}$ & $S_{25}$ & $S_{26}$ & $S_{44}$ & $S_{55}$ & $S_{66}$ \\
\hline \hline$-3.2 \mathrm{~V}$ & $-80.4^{\circ}$ & $-80.3^{\circ}$ & $-9.3 \mathrm{~dB}$ & $-12.3 \mathrm{~dB}$ & $-9.3 \mathrm{~dB}$ & $-6.4 \mathrm{~dB}$ & $-6.2 \mathrm{~dB}$ & $-6.4 \mathrm{~dB}$ \\
\hline$-2.4 \mathrm{~V}$ & $-82^{\circ}$ & $-82^{\circ}$ & $-9.5 \mathrm{~dB}$ & $-12.3 \mathrm{~dB}$ & $-9.6 \mathrm{~dB}$ & $-6.6 \mathrm{~dB}$ & $-6.5 \mathrm{~dB}$ & $-6.7 \mathrm{~dB}$ \\
\hline$-1.6 \mathrm{~V}$ & $-107^{\circ}$ & $-106.9^{\circ}$ & $-10 \mathrm{~dB}$ & $-10.9 \mathrm{~dB}$ & $-10.1 \mathrm{~dB}$ & $-11.6 \mathrm{~dB}$ & $-11.5 \mathrm{~dB}$ & $-11.7 \mathrm{~dB}$ \\
\hline$-0.8 \mathrm{~V}$ & $-112.3^{\circ}$ & $-112.2^{\circ}$ & $-9.8 \mathrm{~dB}$ & $-10.4 \mathrm{~dB}$ & $-9.8 \mathrm{~dB}$ & $-13.5 \mathrm{~dB}$ & $-13.5 \mathrm{~dB}$ & $-13.6 \mathrm{~dB}$ \\
\hline $0 \mathrm{~V}$ & $-117.2^{\circ}$ & $-117.1^{\circ}$ & $-10.2 \mathrm{~dB}$ & $-10.0 \mathrm{~dB}$ & $-10.2 \mathrm{~dB}$ & $-14.3 \mathrm{~dB}$ & $-15.0 \mathrm{~dB}$ & $-14.5 \mathrm{~dB}$ \\
\hline
\end{tabular}

Tabla 4.21: Resultados de simulación para la matriz sintonizable $3 \times 3$ (inductancias corregidas).

Como los niveles de tensión en las simulaciones eran aquellos para los cuales existían datos de simulación disponibles (suministrados por PML), y son diferentes a los utilizados en las medidas, resulta ser más complicado realizar una comparación entre los resultados de las simulaciones y las medidas. Las respuestas en fase y pérdidas de inserción diferenciales entre las tres salidas se muestran gráficamente en las figuras 4.36 a 4.37 .

La concordancia entre las medidas y las simulaciones es claramente mejor cuando los valores de las inductancias han sido corregidos. De todas formas, el comportamiento del circuito no está totalmente explicado con esta corrección. Otra pequeña causa de error podría venir de la contribución del efecto de bordes, a la capacidad total de los condensadores MIM. En este caso, su contribución es de alrededor de un $6 \%$ según la fórmula del manual de diseño del proceso D02AH [PML94], pero más de un tercio del perímetro de los condensadores está ocupado por líneas de acceso, reduciéndose el efecto de bordes en la misma proporción.

Para el caso de la matriz $3 \times 3$ sintonizable, la parte final de la discordancia entre las medidas y las simulaciones, podría venir de la incertidumbre de un $\pm 5 \%$ en los valores de capacidad del varactor (Fig. 4.21). De todas formas, como la curva de capacidad en función de la tensión del varactor es contínua, el valor requerido de capacidad siempre puede encontrarse finalmente mediante el ajuste de la tensión de alimentación. De igual manera que en las consideraciones previas sobre las variaciones de la capacidad del proceso, la influencia de los dos últimos efectos sería la misma para todos los condensadores. 


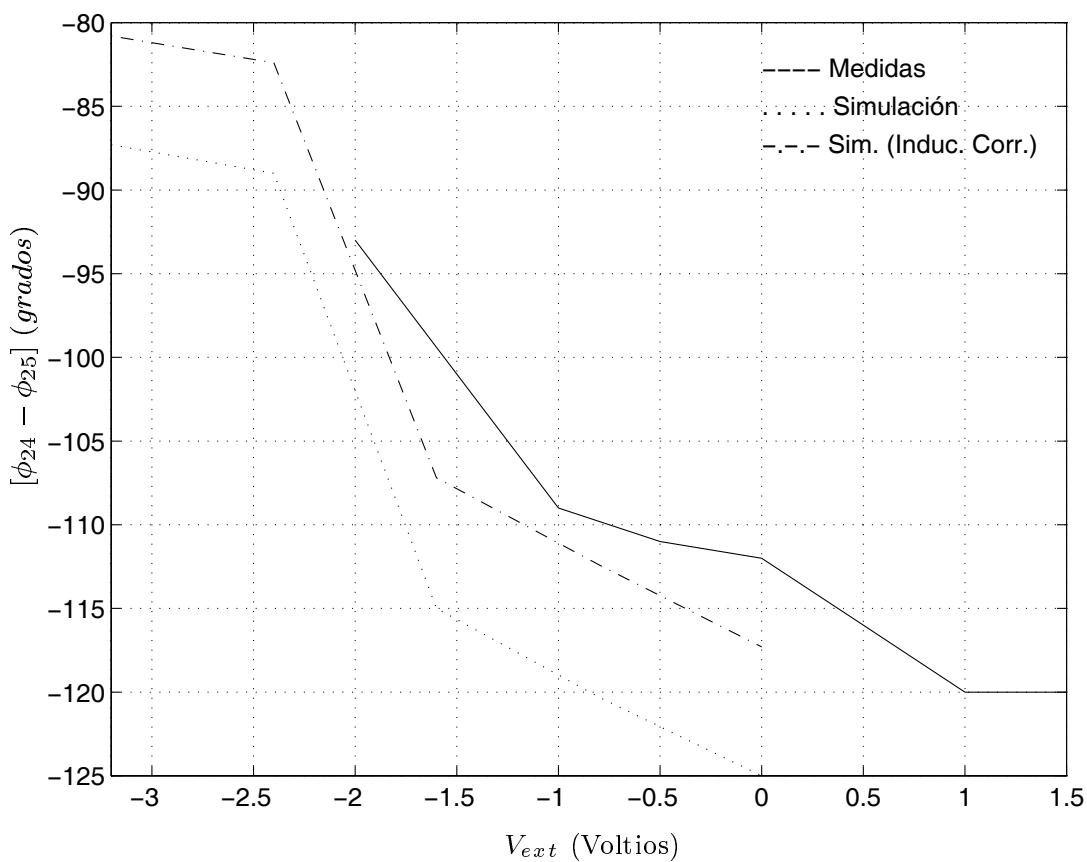

a)

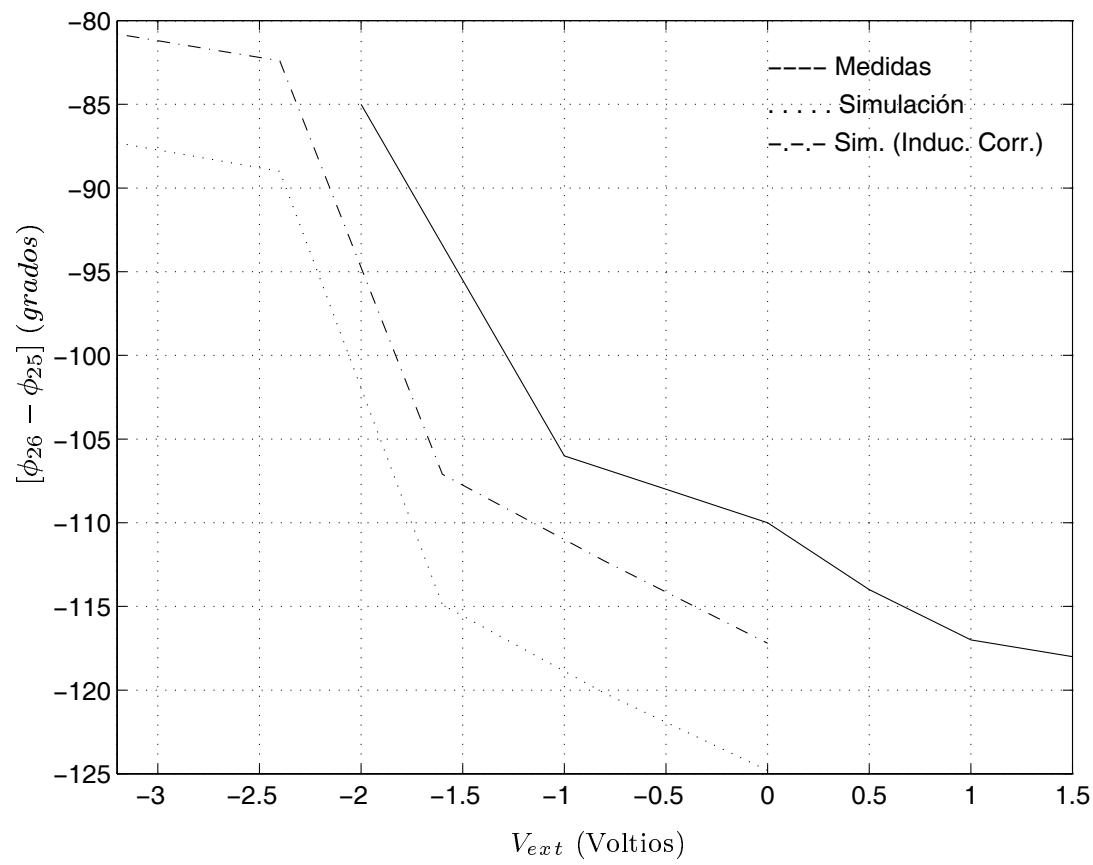

b)

Figura 4.36: Inserción de fase diferencial para la matriz sintonizable $3 \times 3$, en función de la tensión de polarización fuera del circuito integrado. a) Diferencia de desfases entre las salidas 4 y 5 , cuando la entrada es el acceso $2\left(\phi_{24}-\phi_{25}\right)$. b) Diferencia de desfases entre las salidas 6 y 5 , cuando la entrada es el acceso $2\left(\phi_{26}-\phi_{25}\right)$. 


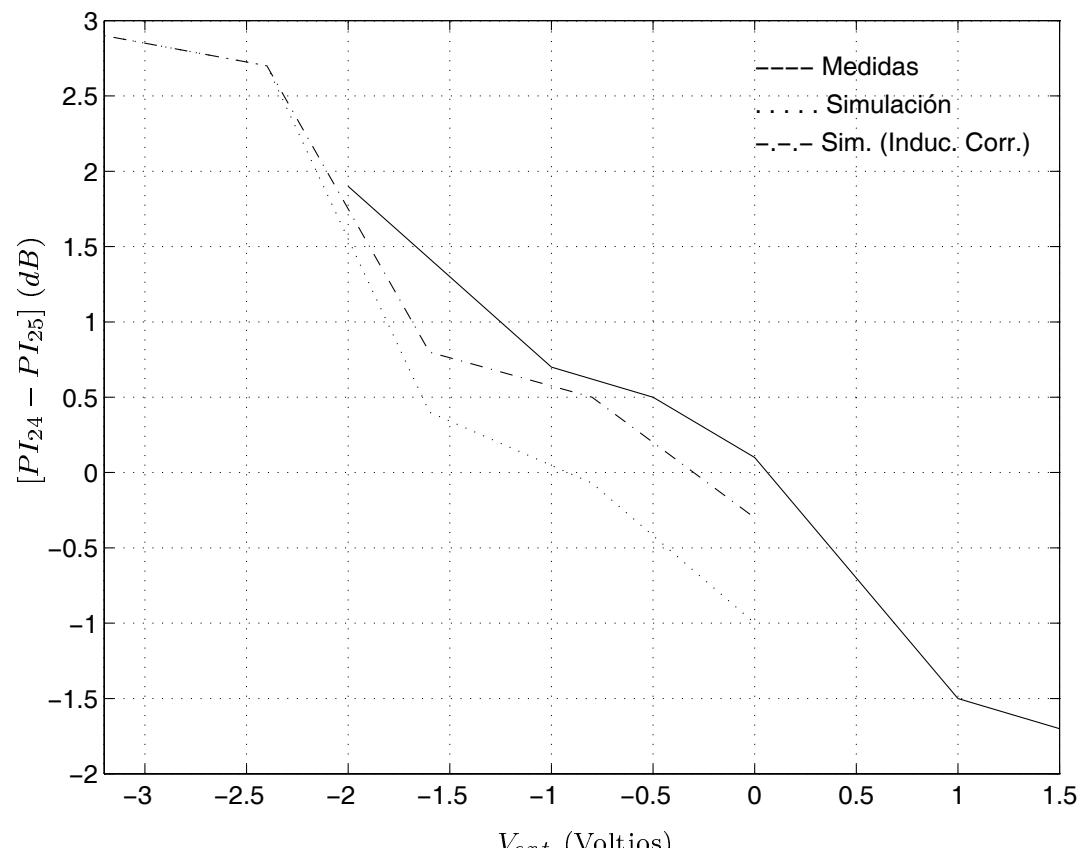

a)

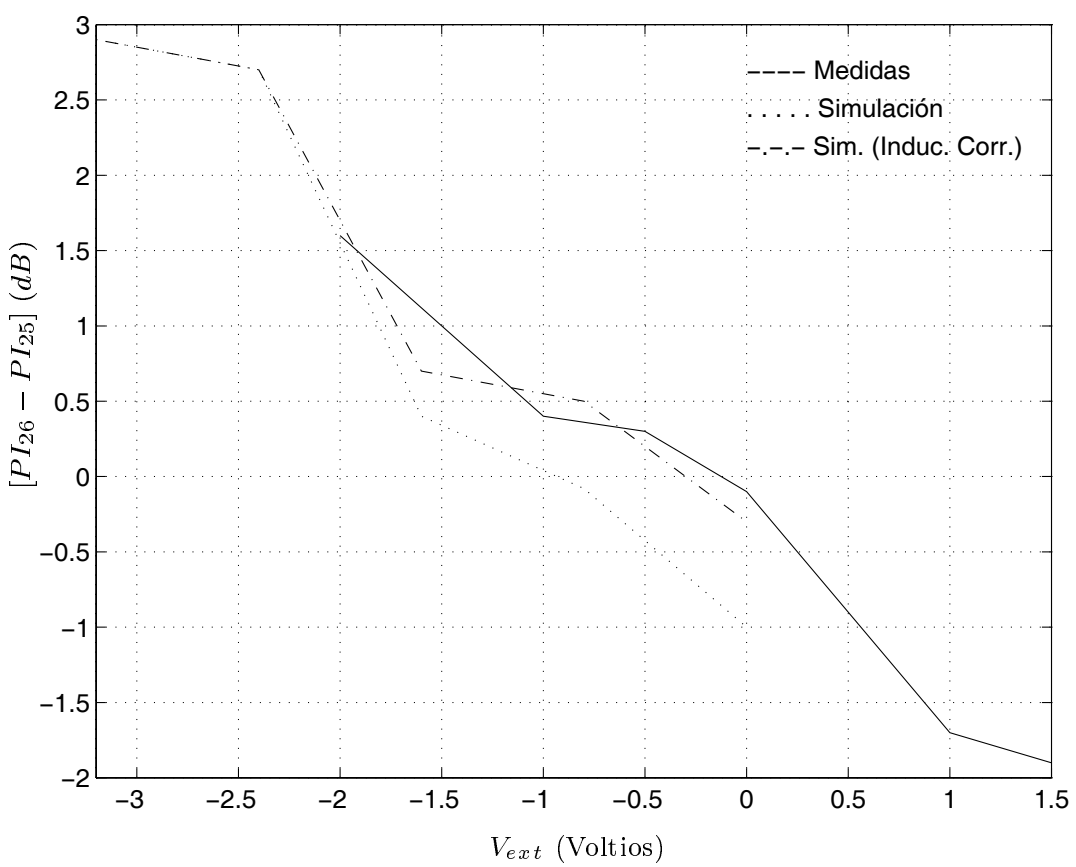

b)

Figura 4.37: Diferencia de pérdidas de inserción para la matriz sintonizable $3 \times 3$, en función de la tensión de polarización fuera del circuito integrado. a) Entre las salidas 4 y 5 , cuando la entrada es el acceso $2\left(P I_{24}-P I_{25}\right)$. a) Entre las salidas 6 y 5 , cuando la entrada es el acceso $2\left(P I_{26}-P I_{25}\right)$. 


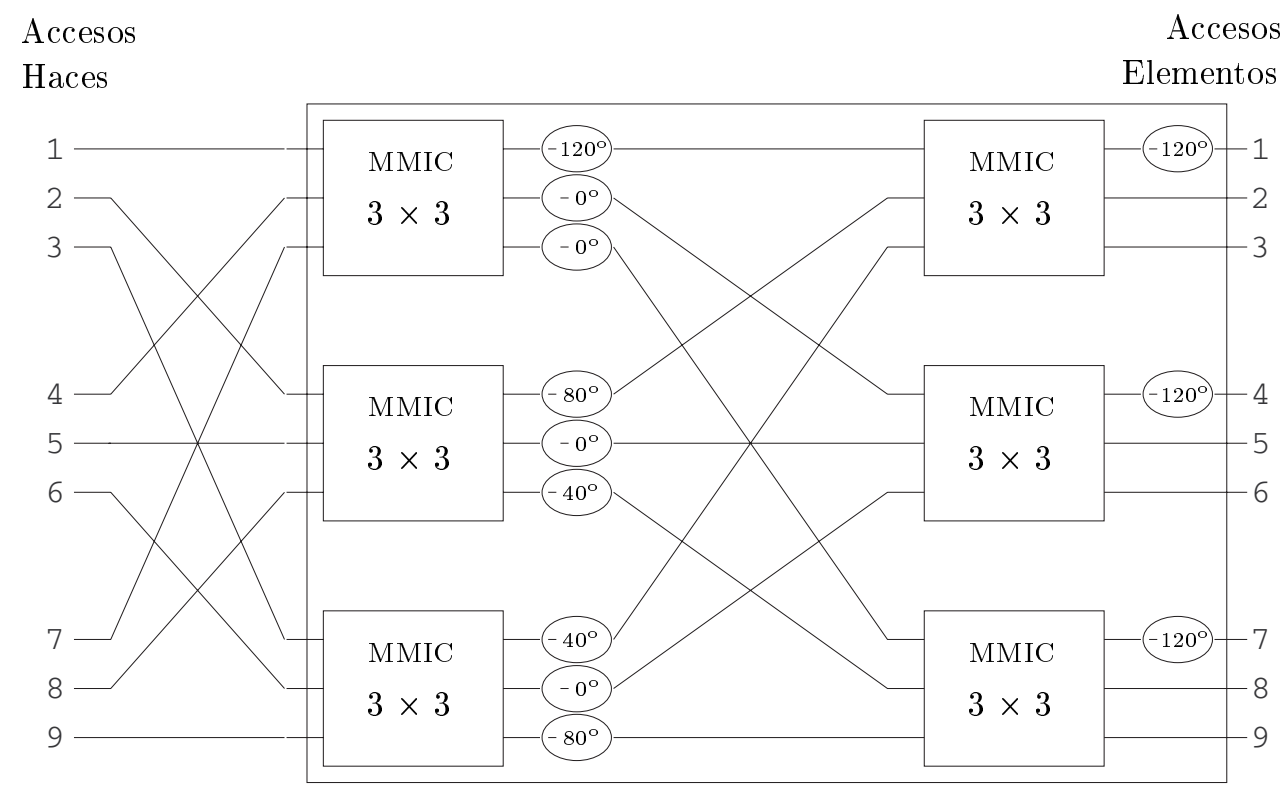

Figura 4.38: Esquemático de la red conformadora de haces $9 \times 9$, usando las matrices $3 \times 3$ MMIC como celdas básicas.

En resumen, el diseño sintonizable con varactores para compensar las variaciones de la capacidad del proceso, se ha comprobado que es una opción de sintonización adecuada para la matriz $3 \times 3$, incluso aunque el diseño nominal no mostrara el comportamiento esperado. Deberían hacerse algunas modificaciones al diseño de la matriz fija, y comprobarse los modelos del inductor, del condensador y del varactor con el fin de asegurar un segundo diseño completamente satisfactorio, una vez el proceso D02AH pasara a su etapa comercial.

\subsection{La matriz $3 \times 3$ como celda básica. Confor- madores de haces $9 \times 9$ y $81 \times 81$}

El bloque $3 \times 3$, mostrado en la figura 4.12, es equivalente a las matrices $3 \times 3$ ya diseñadas en tecnología MMIC, más un desfase extra de $120^{\circ}$ en el acceso número 4 (en el lado de los elementos de antena), de forma que la estructura de una red $9 \times 9$ construida a partir de las celdas $3 \times 3 \mathrm{MMIC}$, será la mostrada en la figura 4.38; donde, posteriormente a la adición del desfase de $120^{\circ}$, se han reducido los valores de los desfasadores a la salida de cada una de las tres celdas más cercanas a los accesos de los haces, pues únicamente se deben mantener los desfases relativos entre las salidas de cada celda. Los retardos fijos en la figura se implementarán como distintas longitudes de líneas de transmisión triplaca.

Para la fabricación y montaje de la red $9 \times 9$ mostrada en la figura 4.38, se encargó a la empresa francesa Dassault Electronique una estructura multicapa en tecnología triplaca. Esta red permitiría el diseño de una red conformadora de tamaño 


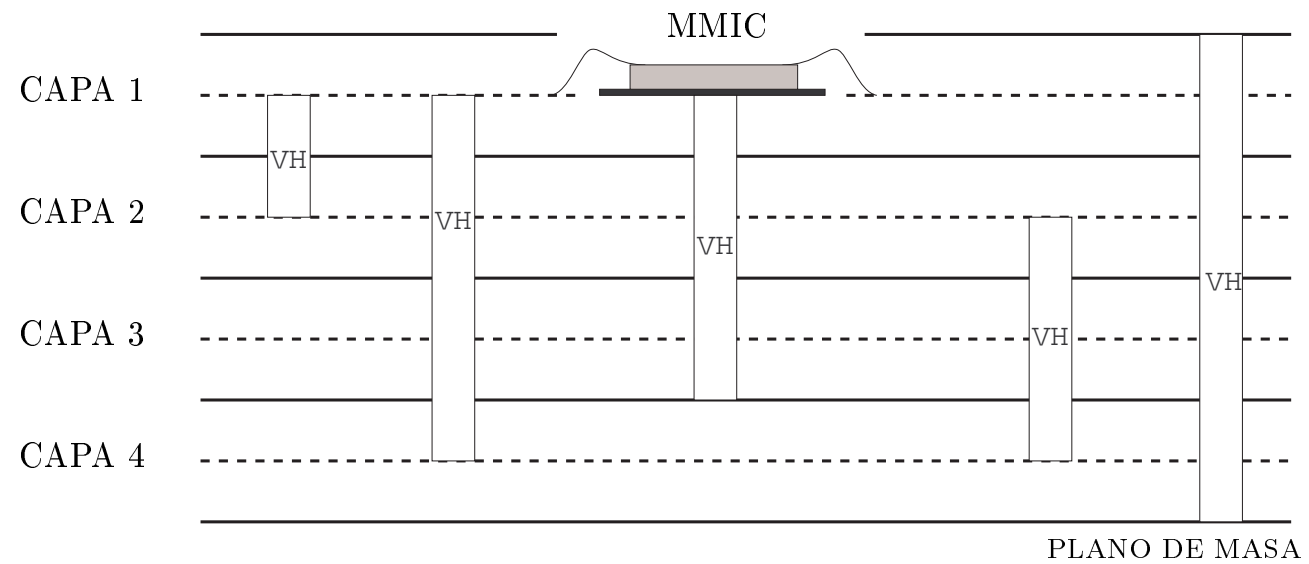

Figura 4.39: Sección transversal de la estructura multicapa diseñada por Dassault Electronique.

$81 \times 81$ como la presentada en la figura 4.11. Esta estructura está compuesta de cuatro capas triplaca fabricadas a partir del substrato 6002 Duroïd de Rogers Corp [Cor96]. Las interconexiones entre las diferentes capas, así como los conexiones a tierra se realizan a través de huecos pasantes metalizados ${ }^{\mathrm{i}}$ como se muestra en la figura 4.39. Las celdas MMIC se conectan a líneas de transmisión de la capa triplaca superior, mediante hilos de termocompresión, estando el MMIC pegado al plano de masa con un material epoxy conductor.

El tamaño de esta estructura multicapa es de $1.2 \mathrm{~cm}$ x $10 \mathrm{~cm}$ x $0.15 \mathrm{~cm}$, donde la dimensión mayor viene impuesta por el tamaño de los 18 conectores tipo SMA necesarios para su interconexionado. Esta estructura se insertó en un soporte de prueba diseñado por la Agencia Espacial Europea. El tamaño total del conformador de haces $9 \times 9$ completo, con el soporte de prueba incluido, es de $3.8 \mathrm{~cm}(1.9 \mathrm{~cm}$ sin conectores) x $11.5 \mathrm{~cm}$. x $1 \mathrm{~cm}$. Su peso es de 30 gramos.

Las prestaciones de la estructura triplaca multicapa en sí, se midieron mediante

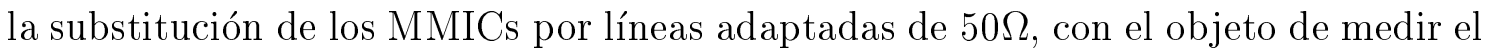
aislamiento entre accesos no conectados y la inserción de fase, debida a las líneas de transmisión triplaca y a los conectores. Se midió un aislamiento medio de $50 \mathrm{~dB}$ y un valor máximo de pérdidas de inserción de 2 dB [Bon95]. Algunos de los caminos entrada/salida mostraban un error en la inserción de fase tan grande como $8^{\circ}-9^{\circ}$, lo cual debería corregirse en siguiente diseños; de todas formas el valor RMS del error de fase era de $1.7^{\circ}$. Es importante destacar la dificultad del diseño con múltiples líneas de transmisión, curvas e interconexiones entre las diversas capas, totalizando una longitud de $1.5 \mathrm{~m}$ de líneas de transmisión.

\footnotetext{
${ }^{\mathrm{i}}$ Via hole (VH), en terminología inglesa.
} 


\begin{tabular}{|c||c|c|c|c|c|c|c|c|c|}
\hline & $S_{1}$ & $S_{2}$ & $S_{3}$ & $S_{4}$ & $S_{5}$ & $S_{6}$ & $S_{7}$ & $S_{8}$ & $S_{9}$ \\
\hline \hline$E_{1}$ & $0^{\circ}$ & $-1^{\circ}$ & $8^{\circ}$ & $1^{\circ}$ & $3^{\circ}$ & $2^{\circ}$ & $-5^{\circ}$ & $2^{\circ}$ & $-6.3^{\circ}$ \\
\hline$E_{2}$ & $0^{\circ}$ & $-10^{\circ}$ & $2^{\circ}$ & $-1^{\circ}$ & $-11^{\circ}$ & $-3^{\circ}$ & $-6^{\circ}$ & $-16^{\circ}$ & $-12^{\circ}$ \\
\hline$E_{3}$ & $0^{\circ}$ & $-1^{\circ}$ & $-2^{\circ}$ & $0^{\circ}$ & $-4^{\circ}$ & $-6^{\circ}$ & $-7^{\circ}$ & $-4^{\circ}$ & $-17^{\circ}$ \\
\hline$E_{4}$ & $0^{\circ}$ & $-4^{\circ}$ & $8^{\circ}$ & $5^{\circ}$ & $-13^{\circ}$ & $-12^{\circ}$ & $-2^{\circ}$ & $-12^{\circ}$ & $-7^{\circ}$ \\
\hline$E_{5}$ & $0^{\circ}$ & $-16^{\circ}$ & $-2^{\circ}$ & $-5^{\circ}$ & $-21^{\circ}$ & $-10^{\circ}$ & $-6^{\circ}$ & $-22^{\circ}$ & $-15^{\circ}$ \\
\hline$E_{6}$ & $0^{\circ}$ & $-17^{\circ}$ & $-8^{\circ}$ & $-11^{\circ}$ & $-16^{\circ}$ & $-18^{\circ}$ & $-9^{\circ}$ & $-17^{\circ}$ & $-\mathbf{- 2 3 ^ { \circ }}$ \\
\hline$E_{7}$ & $0^{\circ}$ & $8^{\circ}$ & $\mathbf{2 5}^{\mathrm{o}}$ & $6^{\circ}$ & $3^{\circ}$ & $20^{\circ}$ & $-3^{\circ}$ & $-6^{\circ}$ & $4^{\circ}$ \\
\hline$E_{8}$ & $0^{\circ}$ & $-16^{\circ}$ & $12^{\circ}$ & $0^{\circ}$ & $-15^{\circ}$ & $6^{\circ}$ & $-10^{\circ}$ & $-18^{\circ}$ & $0^{\circ}$ \\
\hline$E_{9}$ & $0^{\circ}$ & $-10^{\circ}$ & $7^{\circ}$ & $-1^{\circ}$ & $-9^{\circ}$ & $2^{\circ}$ & $-16^{\circ}$ & $-18^{\circ}$ & $-3^{\circ}$ \\
\hline
\end{tabular}

Tabla 4.22: Errores de fase relativos a la fase de la salida $S_{1}$ (Tensión de sintonización: 0.7 Voltios).

\subsubsection{Medidas y simulaciones del conformador $9 \times 9$}

El soporte de prueba $9 \times 9$ diseñado en la Agencia Espacial Europea incluyendo una estructura multicapa con seis celdas MMIC sintonizables montadas, fue medido en las instalaciones de Dassault Electronique para diferentes valores de tensión del varactor. Estas medidas confirmaron la elección hecha de acuerdo a los resultados de la matriz $3 \times 3$ sintonizable, por la que la tensión de alimentación externa al circuito integrado óptima era de $V_{\text {ext }}=0.7$ Voltios. A continuación se presentan los resultados de las medidas para ese valor de tensión de sintonización, junto con un resumen de medidas realizadas para unas tensiones de $V_{\text {ext }}=0.5$ Voltios y $V_{\text {ext }}=0$ Voltios, más otro resumen de medidas para una segunda iteración de la estructura multicapa montada sobre un soporte de prueba distinto.

\section{Medidas de fase}

Los valores de la inserción de fase en los nueve accesos de salida del soporte de prueba $\left(S_{i}, i=1 \ldots 9\right)$ relativos al obtenido en la salida $S_{1}$ han sido medidos para cada una de las nueve entradas de haz $\left(E_{i}, i=1 \ldots 9\right)$ y las diferencias con respecto a la nominal se muestran en la tabla 4.22 .

Los valores de error de fase en negrita corresponden a los errores máximo y mínimo. Si los errores de fase se normalizan frente al valor de error medio para cada haz (un haz corresponde a una fila $E_{i}, i=1 \ldots 9$ ), se obtendrían los valores indicados en la tabla 4.23 , donde también se presentan los errores medios para cada una de las nueve salidas $\left(S_{i}, i=1 \ldots 9\right)$.

En la tabla 4.24 se pueden ver los valores RMS del error de fase para cada una de las nueve entradas de haz. El valor de error de fase medio en cada salida $\left(S_{i}, i=1 \ldots 9\right)$, puede corregirse por medio de una pequeña longitud de línea de transmisión. Después de añadir estas líneas, se obtuvo un nuevo conjunto de errores 


\begin{tabular}{|c||c|c|c|c|c|c|c|c|c|}
\hline & $S_{1}$ & $S_{2}$ & $S_{3}$ & $S_{4}$ & $S_{5}$ & $S_{6}$ & $S_{7}$ & $S_{8}$ & $S_{9}$ \\
\hline \hline$E_{1}$ & $-0.4^{\circ}$ & $-1.4^{\circ}$ & $7.6^{\circ}$ & $0.6^{\circ}$ & $2.6^{\circ}$ & $1.6^{\circ}$ & $-5.4^{\circ}$ & $1.6^{\circ}$ & $-6.7^{\circ}$ \\
\hline$E_{2}$ & $6.3^{\circ}$ & $-3.7^{\circ}$ & $8.3^{\circ}$ & $5.3^{\circ}$ & $-4.7^{\circ}$ & $3.3^{\circ}$ & $0.3^{\circ}$ & $-9.7^{\circ}$ & $-5.7^{\circ}$ \\
\hline$E_{3}$ & $4.6^{\circ}$ & $3.6^{\circ}$ & $2.6^{\circ}$ & $4.6^{\circ}$ & $0.6^{\circ}$ & $-1.4^{\circ}$ & $-2.4^{\circ}$ & $0.6^{\circ}$ & $-12.4^{\circ}$ \\
\hline$E_{4}$ & $4.1^{\circ}$ & $0.1^{\circ}$ & $12.1^{\circ}$ & $9.1^{\circ}$ & $-8.9^{\circ}$ & $-7.9^{\circ}$ & $2.1^{\circ}$ & $-7.9^{\circ}$ & $-2.9^{\circ}$ \\
\hline$E_{5}$ & $10.8^{\circ}$ & $-5.2^{\circ}$ & $8.8^{\circ}$ & $5.8^{\circ}$ & $-10.2^{\circ}$ & $0.8^{\circ}$ & $4.8^{\circ}$ & $-11.2^{\circ}$ & $-4.2^{\circ}$ \\
\hline$E_{6}$ & $13.2^{\circ}$ & $-3.8^{\circ}$ & $5.2^{\circ}$ & $2.2^{\circ}$ & $-2.8^{\circ}$ & $-4.8^{\circ}$ & $4.2^{\circ}$ & $-3.8^{\circ}$ & $-9.8^{\circ}$ \\
\hline$E_{7}$ & $-6.3^{\circ}$ & $1.7^{\circ}$ & $\mathbf{1 8 . 7 ^ { \circ }}$ & $-0.3^{\circ}$ & $-3.3^{\circ}$ & $13.7^{\circ}$ & $-9.3^{\circ}$ & $-12.3^{\circ}$ & $-2.3^{\circ}$ \\
\hline$E_{8}$ & $4.6^{\circ}$ & $-11.4^{\circ}$ & $16.6^{\circ}$ & $4.6^{\circ}$ & $-10.4^{\circ}$ & $10.6^{\circ}$ & $-5.4^{\circ}$ & $-\mathbf{- 1 3 . 4 ^ { \circ }}$ & $4.6^{\circ}$ \\
\hline$E_{9}$ & $5.3^{\circ}$ & $-4.7^{\circ}$ & $12.3^{\circ}$ & $4.3^{\circ}$ & $-3.7^{\circ}$ & $7.3^{\circ}$ & $-10.7^{\circ}$ & $-12.7^{\circ}$ & $2.3^{\circ}$ \\
\hline Media & $4.7^{\circ}$ & $-2.8^{\circ}$ & $10.2^{\circ}$ & $4.0^{\circ}$ & $-4.5^{\circ}$ & $2.6^{\circ}$ & $-2.4^{\circ}$ & $-7.7^{\circ}$ & $-4.1^{\circ}$ \\
\hline
\end{tabular}

Tabla 4.23: Errores de fase relativos al error de fase medio de cada haz (Tensión de sintonización: 0.7 Voltios).

\begin{tabular}{|c||c|c|c|c|c|c|c|c|c||c|}
\hline & $E_{1}$ & $E_{2}$ & $E_{3}$ & $E_{4}$ & $E_{5}$ & $E_{6}$ & $E_{7}$ & $E_{8}$ & $E_{9}$ & TOTAL \\
\hline$\sigma_{\delta}$ & $4^{\circ}$ & $5.9^{\circ}$ & $5^{\circ}$ & $7.2^{\circ}$ & $7.6^{\circ}$ & $6.5^{\circ}$ & $9.6^{\circ}$ & $10^{\circ}$ & $7.9^{\circ}$ & $\mathbf{7 . 3}^{\circ}$ \\
\hline$\sigma_{\delta}^{c}$ & $4.7^{\circ}$ & $1.6^{\circ}$ & $5.6^{\circ}$ & $4.6^{\circ}$ & $4.1^{\circ}$ & $5.3^{\circ}$ & $6.9^{\circ}$ & $6.1^{\circ}$ & $4.3^{\circ}$ & $\mathbf{5 . 0}^{\mathbf{o}}$ \\
\hline
\end{tabular}

Tabla 4.24: Valor RMS de los errores de fase, en todas las salidas, para cada una de las nueve entradas de haz antes $\left(\sigma_{\delta}\right)$ y después $\left(\sigma_{\delta}^{c}\right)$ de la inserción de las líneas de corrección en cada salida (Tensión de sintonización: 0.7 Voltios).

de fase normalizados, los cuales se muestran en la tabla 4.25. Tras añadir las líneas de transmisión correctoras en cada una de las nueve salidas de elementos de antena, los valores RMS de error de fase, para cada uno de los haces, se han reducido como se puede comprobar en la tabla 4.24, obteniéndose un valor rms total de $5.0^{\circ}$.

\section{Medidas de Amplitud}

Las pérdidas de inserción, han sido medidas (tabla 4.26) para el mismo nivel de tensión de alimentación utilizado en las medidas de desfase. En la tabla 4.27 se muestran las desviaciones máximas y mínimas respecto a las pérdidas de inserción medias, para cada una de las nueve entradas de haz, así como el valor RMS de estas desviaciones. La tabla 4.28 ofrece un resumen de las medidas más importantes, referentes a las pérdidas de inserción del conformador $9 \times 9$. 


\begin{tabular}{|c||c|c|c|c|c|c|c|c|c|}
\hline & $S_{1}$ & $S_{2}$ & $S_{3}$ & $S_{4}$ & $S_{5}$ & $S_{6}$ & $S_{7}$ & $S_{8}$ & $S_{9}$ \\
\hline \hline$E_{1}$ & $-5.1^{\circ}$ & $1.4^{\circ}$ & $-2.6^{\circ}$ & $-3.4^{\circ}$ & $7.1^{\circ}$ & $-1.0^{\circ}$ & $-3.0^{\circ}$ & $9.2^{\circ}$ & $-2.6^{\circ}$ \\
\hline$E_{2}$ & $1.7^{\circ}$ & $-0.9^{\circ}$ & $-1.9^{\circ}$ & $1.3^{\circ}$ & $-0.1^{\circ}$ & $0.8^{\circ}$ & $2.8^{\circ}$ & $-2.0^{\circ}$ & $-1.5^{\circ}$ \\
\hline$E_{3}$ & $-0.1^{\circ}$ & $6.3^{\circ}$ & $-7.7^{\circ}$ & $0.5^{\circ}$ & $5.1^{\circ}$ & $-4.0^{\circ}$ & $0.0^{\circ}$ & $8.2^{\circ}$ & $-8.3^{\circ}$ \\
\hline$E_{4}$ & $-0.6^{\circ}$ & $2.9^{\circ}$ & $1.9^{\circ}$ & $5.1^{\circ}$ & $-4.3^{\circ}$ & $-10.5^{\circ}$ & $4.5^{\circ}$ & $-0.2^{\circ}$ & $1.2^{\circ}$ \\
\hline$E_{5}$ & $6.1^{\circ}$ & $-2.5^{\circ}$ & $-1.5^{\circ}$ & $1.8^{\circ}$ & $-5.7^{\circ}$ & $-1.8^{\circ}$ & $7.2^{\circ}$ & $-3.6^{\circ}$ & $-0.1^{\circ}$ \\
\hline$E_{6}$ & $8.5^{\circ}$ & $-1.0^{\circ}$ & $-5.0^{\circ}$ & $-1.8^{\circ}$ & $1.8^{\circ}$ & $-7.3^{\circ}$ & $6.7^{\circ}$ & $3.9^{\circ}$ & $-5.6^{\circ}$ \\
\hline$E_{7}$ & $-\mathbf{1 1 . 0}^{\mathrm{o}}$ & $4.4^{\circ}$ & $8.4^{\circ}$ & $-4.3^{\circ}$ & $1.2^{\circ}$ & $\mathbf{1 1 . 1}^{\mathrm{o}}$ & $-6.9^{\circ}$ & $-4.7^{\circ}$ & $1.8^{\circ}$ \\
\hline$E_{8}$ & $-0.1^{\circ}$ & $-8.7^{\circ}$ & $6.3^{\circ}$ & $0.5^{\circ}$ & $-5.9^{\circ}$ & $8.0^{\circ}$ & $-3.0^{\circ}$ & $-5.8^{\circ}$ & $8.7^{\circ}$ \\
\hline$E_{9}$ & $0.7^{\circ}$ & $-1.9^{\circ}$ & $2.1^{\circ}$ & $0.3^{\circ}$ & $0.9^{\circ}$ & $4.8^{\circ}$ & $-8.2^{\circ}$ & $-5.0^{\circ}$ & $6.4^{\circ}$ \\
\hline
\end{tabular}

Tabla 4.25: Errores de fase, relativos al error de fase medio de cada haz, después de la inserción de las líneas de corrección en cada salida de antena (Tensión de sintonización: 0.7 Voltios).

\begin{tabular}{|c||c|c|c|c|c|c|c|c|c|}
\hline & $S_{1}$ & $S_{2}$ & $S_{3}$ & $S_{4}$ & $S_{5}$ & $S_{6}$ & $S_{7}$ & $S_{8}$ & $S_{9}$ \\
\hline \hline$E_{1}$ & $21 \mathrm{~dB}$ & $22.7 \mathrm{~dB}$ & $22.1 \mathrm{~dB}$ & $20.5 \mathrm{~dB}$ & $21.3 \mathrm{~dB}$ & $21.9 \mathrm{~dB}$ & $21.1 \mathrm{~dB}$ & $21.3 \mathrm{~dB}$ & $21.9 \mathrm{~dB}$ \\
\hline$E_{2}$ & $20.7 \mathrm{~dB}$ & $20.7 \mathrm{~dB}$ & $20.4 \mathrm{~dB}$ & $21.7 \mathrm{~dB}$ & $21.3 \mathrm{~dB}$ & $22.1 \mathrm{~dB}$ & $22.2 \mathrm{~dB}$ & $21.1 \mathrm{~dB}$ & $23 \mathrm{~dB}$ \\
\hline$E_{3}$ & $20 \mathrm{~dB}$ & $21.4 \mathrm{~dB}$ & $20.4 \mathrm{~dB}$ & $21.9 \mathrm{~dB}$ & $21.9 \mathrm{~dB}$ & $21.7 \mathrm{~dB}$ & $22.1 \mathrm{~dB}$ & $22.3 \mathrm{~dB}$ & $21.8 \mathrm{~dB}$ \\
\hline$E_{4}$ & $21.7 \mathrm{~dB}$ & $22 \mathrm{~dB}$ & $22.4 \mathrm{~dB}$ & $20.2 \mathrm{~dB}$ & $22.3 \mathrm{~dB}$ & $21.9 \mathrm{~dB}$ & $20.7 \mathrm{~dB}$ & $21.2 \mathrm{~dB}$ & $21.7 \mathrm{~dB}$ \\
\hline$E_{5}$ & $22.8 \mathrm{~dB}$ & $21.6 \mathrm{~dB}$ & $22.4 \mathrm{~dB}$ & $21.2 \mathrm{~dB}$ & $20.8 \mathrm{~dB}$ & $21.3 \mathrm{~dB}$ & $21.4 \mathrm{~dB}$ & $20.4 \mathrm{~dB}$ & $22.3 \mathrm{~dB}$ \\
\hline$E_{6}$ & $22.3 \mathrm{~dB}$ & $22.3 \mathrm{~dB}$ & $22 \mathrm{~dB}$ & $20.5 \mathrm{~dB}$ & $22.2 \mathrm{~dB}$ & $20.4 \mathrm{~dB}$ & $21.6 \mathrm{~dB}$ & $22 \mathrm{~dB}$ & $21 \mathrm{~dB}$ \\
\hline$E_{7}$ & $22.1 \mathrm{~dB}$ & $21.9 \mathrm{~dB}$ & $22.2 \mathrm{~dB}$ & $21 \mathrm{~dB}$ & $21.6 \mathrm{~dB}$ & $21.1 \mathrm{~dB}$ & $19.9 \mathrm{~dB}$ & $22.8 \mathrm{~dB}$ & $20.7 \mathrm{~dB}$ \\
\hline$E_{8}$ & $22.9 \mathrm{~dB}$ & $21.2 \mathrm{~dB}$ & $21.6 \mathrm{~dB}$ & $21.8 \mathrm{~dB}$ & $21.9 \mathrm{~dB}$ & $21.6 \mathrm{~dB}$ & $21.4 \mathrm{~dB}$ & $20.7 \mathrm{~dB}$ & $21.4 \mathrm{~dB}$ \\
\hline$E_{9}$ & $22.1 \mathrm{~dB}$ & $21.9 \mathrm{~dB}$ & $21 \mathrm{~dB}$ & $21.5 \mathrm{~dB}$ & $22 \mathrm{~dB}$ & $20.7 \mathrm{~dB}$ & $21.6 \mathrm{~dB}$ & $22.3 \mathrm{~dB}$ & $20.6 \mathrm{~dB}$ \\
\hline
\end{tabular}

Tabla 4.26: Pérdidas de inserción para el conformador $9 \times 9$ (Tensión de sintonización: 0.7 Voltios).

\begin{tabular}{|c||c|c|c|c|c|c|c|c|c|}
\hline & $E_{1}$ & $E_{2}$ & $E_{3}$ & $E_{4}$ & $E_{5}$ & $E_{6}$ & $E_{7}$ & $E_{8}$ & $E_{9}$ \\
\hline \hline$\Delta_{\max }$ & $1.1 \mathrm{~dB}$ & $1.5 \mathrm{~dB}$ & $0.8 \mathrm{~dB}$ & $0.9 \mathrm{~dB}$ & $1.2 \mathrm{~dB}$ & $0.7 \mathrm{~dB}$ & $0.8 \mathrm{~dB}$ & $1.3 \mathrm{~dB}$ & $0.8 \mathrm{~dB}$ \\
& $-1 \mathrm{~dB}$ & $-1.1 \mathrm{~dB}$ & $-1.5 \mathrm{~dB}$ & $-1.4 \mathrm{~dB}$ & $-0.8 \mathrm{~dB}$ & $-1.2 \mathrm{~dB}$ & $-1.5 \mathrm{~dB}$ & $-0.9 \mathrm{~dB}$ & $-0.9 \mathrm{~dB}$ \\
\hline$\sigma_{\Delta}$ & $0.6 \mathrm{~dB}$ & $0.8 \mathrm{~dB}$ & $0.7 \mathrm{~dB}$ & $0.7 \mathrm{~dB}$ & $0.7 \mathrm{~dB}$ & $0.7 \mathrm{~dB}$ & $0.8 \mathrm{~dB}$ & $0.6 \mathrm{~dB}$ & $0.6 \mathrm{~dB}$ \\
\hline
\end{tabular}

Tabla 4.27: Desviaciones de pico $\left(\Delta_{\max }\right)$ y valor RMS $\left(\sigma_{\Delta}\right)$ de las pérdidas de inserción sobre el valor medio para cada haz (Tensión de sintonización: 0.7 Voltios).

\begin{tabular}{|c||c|}
\hline Valor medio & $21.54 \mathrm{~dB}$ \\
\hline Varianza & $0.7 \mathrm{~dB}$ \\
\hline Mínimo & $19.9 \mathrm{~dB}$ \\
\hline Máximo & $23.0 \mathrm{~dB}$ \\
\hline
\end{tabular}

Tabla 4.28: Resumen de las medidas de pérdidas de inserción del conformador $9 \times 9$ (Tensión de sintonización: 0.7 Voltios). 
a)

\begin{tabular}{|c||c|c|c|c|c|c|c|c|c|}
\hline & $E_{1}$ & $E_{2}$ & $E_{3}$ & $E_{4}$ & $E_{5}$ & $E_{6}$ & $E_{7}$ & $E_{8}$ & $E_{9}$ \\
\hline \hline$\Delta_{\max }$ & $1.7 \mathrm{~dB}$ & $0.9 \mathrm{~dB}$ & $0.7 \mathrm{~dB}$ & $1.2 \mathrm{~dB}$ & $1.2 \mathrm{~dB}$ & $1.0 \mathrm{~dB}$ & $1.9 \mathrm{~dB}$ & $1.0 \mathrm{~dB}$ & $1.5 \mathrm{~dB}$ \\
& $-0.9 \mathrm{~dB}$ & $-1.0 \mathrm{~dB}$ & $-1.3 \mathrm{~dB}$ & $-1.1 \mathrm{~dB}$ & $-1.2 \mathrm{~dB}$ & $-1.2 \mathrm{~dB}$ & $-0.8 \mathrm{~dB}$ & $-0.8 \mathrm{~dB}$ & $-1.3 \mathrm{~dB}$ \\
\hline$\sigma_{\Delta}$ & $0.9 \mathrm{~dB}$ & $0.6 \mathrm{~dB}$ & $0.6 \mathrm{~dB}$ & $0.6 \mathrm{~dB}$ & $0.7 \mathrm{~dB}$ & $0.7 \mathrm{~dB}$ & $0.8 \mathrm{~dB}$ & $0.5 \mathrm{~dB}$ & $0.7 \mathrm{~dB}$ \\
\hline
\end{tabular}

b)

\begin{tabular}{|c||c|c|c|c|c|c|c|c|c|}
\hline & $E_{1}$ & $E_{2}$ & $E_{3}$ & $E_{4}$ & $E_{5}$ & $E_{6}$ & $E_{7}$ & $E_{8}$ & $E_{9}$ \\
\hline \hline$\Delta_{\max }$ & $1.4 \mathrm{~dB}$ & $1.2 \mathrm{~dB}$ & $0.7 \mathrm{~dB}$ & $1.1 \mathrm{~dB}$ & $0.8 \mathrm{~dB}$ & $0.8 \mathrm{~dB}$ & $1.7 \mathrm{~dB}$ & $0.8 \mathrm{~dB}$ & $1.0 \mathrm{~dB}$ \\
& $-0.9 \mathrm{~dB}$ & $-1.1 \mathrm{~dB}$ & $-1.5 \mathrm{~dB}$ & $-1.2 \mathrm{~dB}$ & $-1.1 \mathrm{~dB}$ & $-1.0 \mathrm{~dB}$ & $-1.1 \mathrm{~dB}$ & $-1.1 \mathrm{~dB}$ & $-1.9 \mathrm{~dB}$ \\
\hline$\sigma_{\Delta}$ & $0.8 \mathrm{~dB}$ & $0.7 \mathrm{~dB}$ & $0.6 \mathrm{~dB}$ & $0.8 \mathrm{~dB}$ & $0.6 \mathrm{~dB}$ & $0.7 \mathrm{~dB}$ & $0.9 \mathrm{~dB}$ & $0.6 \mathrm{~dB}$ & $0.8 \mathrm{~dB}$ \\
\hline
\end{tabular}

Tabla 4.29: Desviaciones de pico $\left(\Delta_{\max }\right)$ y valor $\operatorname{RMS}\left(\sigma_{\Delta}\right)$ de las pérdidas de inserción sobre el valor medio para cada haz. a) Tensión de sintonización: 0 Voltios. b) Tensión de sintonización: 0.5 Voltios.

\begin{tabular}{|c||c|c||c|}
\hline \multicolumn{2}{|c|}{$V_{\text {ext }}=0 \mathrm{~V}$} & \multicolumn{2}{c|}{$V_{\text {ext }}=0.5 \mathrm{~V}$} \\
\hline \hline Valor medio & $20.73 \mathrm{~dB}$ & Valor medio & $21.06 \mathrm{~dB}$ \\
\hline Varianza & $0.71 \mathrm{~dB}$ & Varianza & $0.74 \mathrm{~dB}$ \\
\hline Mínimo & $19.2 \mathrm{~dB}$ & Mínimo & $19.1 \mathrm{~dB}$ \\
\hline Máximo & $22.6 \mathrm{~dB}$ & Máximo & $22.7 \mathrm{~dB}$ \\
\hline
\end{tabular}

Tabla 4.30: Resumen de las medidas de pérdidas de inserción del conformador $9 \times 9$ para las tensiones de sintonización $V_{e x t}=0$ Voltios y $V_{\text {ext }}=0.5$ Voltios.

\section{Otras medidas}

El conformador de haces $9 \times 9$, con el soporte de prueba diseñado por la Agencia Espacial Europea, fue también medido para otras tensiones de sintonización. En las tablas $4.29,4.30$ y 4.31 , se muestra un resumen de los resultados para unas tensiones de sintonización de 0.5 Voltios y 0 Voltios.

Por otra parte, Dassault Electronique ha desarrollado un soporte de prueba $9 \times 9$ diferente, para otra iteración de la misma estructura triplaca multicapa con seis matrices $3 \times 3 \mathrm{MMIC}$, sintonizables diferentes. Este soporte fue medido para una tensión de control de 0.7 Voltios, ofreciendo unos resultados bastante similares a los del soporte de prueba $9 \times 9$, diseñado por la ESA, confirmando la esperada buena repetibilidad de los diseños. Los resultados de estas medidas pueden observarse en las tablas $4.32,4.33$ y 4.34

Los errores en amplitud y fase obtenidos para la red $9 \times 9$, se traducen, en cuanto a los efectos sobre el diagrama de radiación, en los niveles de lóbulos secun-

\begin{tabular}{|cc||c|c|c|c|c|c|c|c|c||c|}
\hline & & $E_{1}$ & $E_{2}$ & $E_{3}$ & $E_{4}$ & $E_{5}$ & $E_{6}$ & $E_{7}$ & $E_{8}$ & $E_{9}$ & TOTAL \\
\hline \hline$V_{e x t}=0 \mathrm{~V}$ & $\sigma_{\delta}$ & $5.9^{\circ}$ & $6.0^{\circ}$ & $3.8^{\circ}$ & $7.9^{\circ}$ & $10.2^{\circ}$ & $6.8^{\circ}$ & $11.6^{\circ}$ & $12.6^{\circ}$ & $8.3^{\circ}$ & $\mathbf{8 . 6}^{\mathrm{o}}$ \\
\hline$V_{e x t}=0.5 \mathrm{~V}$ & $\sigma_{\delta}$ & $4^{\mathrm{o}}$ & $6.7^{\circ}$ & $5.4^{\mathrm{o}}$ & $6.8^{\mathrm{o}}$ & $8.3^{\circ}$ & $6.4^{\mathrm{o}}$ & $15.2^{\mathrm{o}}$ & $15.9^{\mathrm{o}}$ & $7.5^{\mathrm{o}}$ & $\mathbf{9 . 4}^{\mathbf{o}}$ \\
\hline
\end{tabular}

Tabla 4.31: Valor RMS de los errores de fase en todas las salidas para cada una de las nueve entradas de haz para las tensiones de sintonización $V_{\text {ext }}=0$ Voltios y $V_{\text {ext }}=0.5$ Voltios. 


\begin{tabular}{|c||c|c|c|c|c|c|c|c|c|}
\hline & $E_{1}$ & $E_{2}$ & $E_{3}$ & $E_{4}$ & $E_{5}$ & $E_{6}$ & $E_{7}$ & $E_{8}$ & $E_{9}$ \\
\hline \hline$\Delta_{\max }$ & $1.0 \mathrm{~dB}$ & $2.5 \mathrm{~dB}$ & $1.0 \mathrm{~dB}$ & $0.7 \mathrm{~dB}$ & $0.9 \mathrm{~dB}$ & $0.7 \mathrm{~dB}$ & $1.4 \mathrm{~dB}$ & $1.8 \mathrm{~dB}$ & $1.3 \mathrm{~dB}$ \\
& $-1.4 \mathrm{~dB}$ & $-1.4 \mathrm{~dB}$ & $-1.7 \mathrm{~dB}$ & $-1.1 \mathrm{~dB}$ & $-0.9 \mathrm{~dB}$ & $-1.1 \mathrm{~dB}$ & $-2.0 \mathrm{~dB}$ & $-1.5 \mathrm{~dB}$ & $-1.5 \mathrm{~dB}$ \\
\hline$\sigma_{\Delta}$ & $0.7 \mathrm{~dB}$ & $1.3 \mathrm{~dB}$ & $1.0 \mathrm{~dB}$ & $0.5 \mathrm{~dB}$ & $0.7 \mathrm{~dB}$ & $0.6 \mathrm{~dB}$ & $0.9 \mathrm{~dB}$ & $1.1 \mathrm{~dB}$ & $0.8 \mathrm{~dB}$ \\
\hline
\end{tabular}

Tabla 4.32: Desviaciones de pico $\left(\Delta_{\max }\right)$ y valor RMS $\left(\sigma_{\Delta}\right)$ de las pérdidas de inserción sobre el valor medio para cada haz (Tensión de sintonización: 0.7 Voltios). Soporte de prueba diseñado por Dassault Electronique.

\begin{tabular}{|c||c|}
\hline Valor medio & $21.58 \mathrm{~dB}$ \\
\hline Varianza & $0.89 \mathrm{~dB}$ \\
\hline Mínimo & $19.6 \mathrm{~dB}$ \\
\hline Máximo & $24.3 \mathrm{~dB}$ \\
\hline
\end{tabular}

Tabla 4.33: Resumen de las medidas de pérdidas de inserción del conformador $9 \times 9$ (Tensión de sintonización: 0.7 Voltios). Soporte de prueba diseñado por Dassault Electronique.

darios de fondo y las variaciones en la ganancia del haz principal mostradas en la tabla 4.35 para una agrupación de nueve elementos, alimentada por el conformador correspondiente. Los resultados obtenidos confirman el buen comportamiento de la red conformadora de haces.

\section{Simulaciones del conformador de haces $9 \times 9$}

Con el fin de comprobar las propiedades de conformación de haces de la estructura medida $9 \times 9$, en términos de prestaciones de antena, se llevaron a cabo algunas simulaciones a partir de las medidas realizadas a 0.7 Voltios del soporte de prueba diseñado por la ESA, con las errores de fase medios a las salidas corregidos por medio de líneas de transmisión. Una matriz de párametros $\mathrm{S}$, para una red de 18 accesos, se llenó con los datos medidos de pérdidas de inserción de fase y pérdidas de inserción, dejándose los parámetros de aislamiento y adaptación iguales a cero. Por lo tanto, solamente se consideran datos de transmisión.

La red $9 \times 9$, puede usarse como red conformadora de una agrupación lineal de $N=9$ elementos, y, en el caso de que la separación entre elementos sea $d_{x}=\lambda / 2$, la matriz generará 9 haces ortogonales e independientes. Usando la red en modo recepción, una onda con un gradiente de fase fijo, se consideró como la onda incidente en el lado de los elementos de antena, y se calculó la respuesta en los accesos de cada

\begin{tabular}{|c||c|c|c|c|c|c|c|c|c||c|}
\hline & $E_{1}$ & $E_{2}$ & $E_{3}$ & $E_{4}$ & $E_{5}$ & $E_{6}$ & $E_{7}$ & $E_{8}$ & $E_{9}$ & TOTAL \\
\hline$\sigma_{\delta}$ & $5.2^{\circ}$ & $3.6^{\circ}$ & $4.1^{\circ}$ & $4.4^{\circ}$ & $3.8^{\circ}$ & $5.8^{\circ}$ & $5.9^{\circ}$ & $5.7^{\circ}$ & $4.0^{\circ}$ & $\mathbf{4 . 8}^{\circ}$ \\
\hline
\end{tabular}

Tabla 4.34: Valor RMS de los errores de fase en todas las salidas para cada una de las nueve entradas de haz (Tensión de sintonización: 0.7 Voltios). Soporte de prueba diseñado por Dassault Electronique. 


\begin{tabular}{|l||c|c|c|c|}
\hline Conformador & $\sigma_{\Delta}$ & $\sigma_{\delta}$ & $N L S P_{\text {fondo }}$ & $\Delta G$ \\
\hline \hline ESA $V_{\text {ext }}=0.7 \mathrm{~V}$ & $0.7 \mathrm{~dB}$ & $5^{\circ}$ & $-27.9 \mathrm{~dB}$ & $-0.06 \mathrm{~dB}$ \\
\hline ESA $V_{\text {ext }}=0.5 \mathrm{~V}$ & $0.71 \mathrm{~dB}$ & $8.6^{\circ}$ & $-24.7 \mathrm{~dB}$ & $-0.13 \mathrm{~dB}$ \\
\hline ESA $V_{\text {ext }}=0 \mathrm{~V}$ & $0.74 \mathrm{~dB}$ & $9.4^{\circ}$ & $-24.5 \mathrm{~dB}$ & $-0.15 \mathrm{~dB}$ \\
\hline $\mathrm{DE} V_{e x t}=0.7 \mathrm{~V}$ & $0.89 \mathrm{~dB}$ & $4.8^{\circ}$ & $-26.8 \mathrm{~dB}$ & $-0.08 \mathrm{~dB}$ \\
\hline
\end{tabular}

Tabla 4.35: Efecto sobre el diagrama de radiación de los errores de amplitud y fase de los conformadores $9 \times 9$ de la Agencia Espacial Europea (ESA) y de Dassault Electronique (DE) para diferentes valores de tensión de sintonización.

uno de los nueve haces, como se puede observar en la figura 4.40; de esta forma, el nivel de potencia en cada uno de los accesos de cada haz, es conocido en función del gradiente de fase de la onda incidente.

Los niveles máximos aparecen en cada acceso de haz, exactamente para el valor de gradiente esperado. El nivel de los lóbulos secundarios respecto al nivel del lóbulo principal para cada haz está bastante cerca del ideal $(-12.9 \mathrm{~dB})$, con un valor medio de $-12.4 \mathrm{~dB}$ y un peor caso de $-11.7 \mathrm{~dB}$.

Debido a la propiedad de ortogonalidad e independencia de los haces generados por una matriz de Butler, cuando el nivel de señal recibida es máximo en la entrada (salida al trabajar en modo recepción) de uno de los haces, las entradas de los otros deberían ofrecer un nivel nulo de señal recibida, de forma que toda la potencia incidente debería aparecer en el acceso del haz considerado. Estos esperados ceros, pasan a tener un valor finito con una red conformadora real, de forma que no son tan profundos como los ideales. Su valor medio es de $27.5 \mathrm{~dB}$, con un peor caso de $19.6 \mathrm{~dB}$; para este caso en concreto, la no nulidad de esos valores implica que, cuando una onda con un gradiente de fase de $-160^{\circ}$ incida sobre la antena, la señal que debería, en un caso ideal, aparecer unicamente en el acceso del haz $E_{8}$, aparece también en el acceso del haz $E_{7}$, con un nivel de potencia $19.6 \mathrm{~dB}$ por debajo del nivel de señal en el acceso $E_{8}$.

\subsubsection{Simulaciones del conformador de haces $81 \times 81$}

Tal y como se muestra en la figura 4.11, es posible construir un conformador de haces de dimensiones $81 \times 81$, mediante la interconexión de 18 conformadores de tamaño $9 \times 9$. De acuerdo con esta consideración, se ha simulado una red conformadora de haces de tamaño $81 \times 81$, a partir de las medidas del conformador $9 \times 9$ presentadas previamente.

Esta red conformadora de haces, ha sido caracterizada en términos de parámetros de radiacion, para lo que se ha utilizado la agrupación de 37 elementos con distribución espacial hexagonal y contorno hexagonal, que ha sido diseñada para la misión ARCHIMEDES. Más detalles sobre esta misión se dan en el ejemplo 4 (apartado 
a)

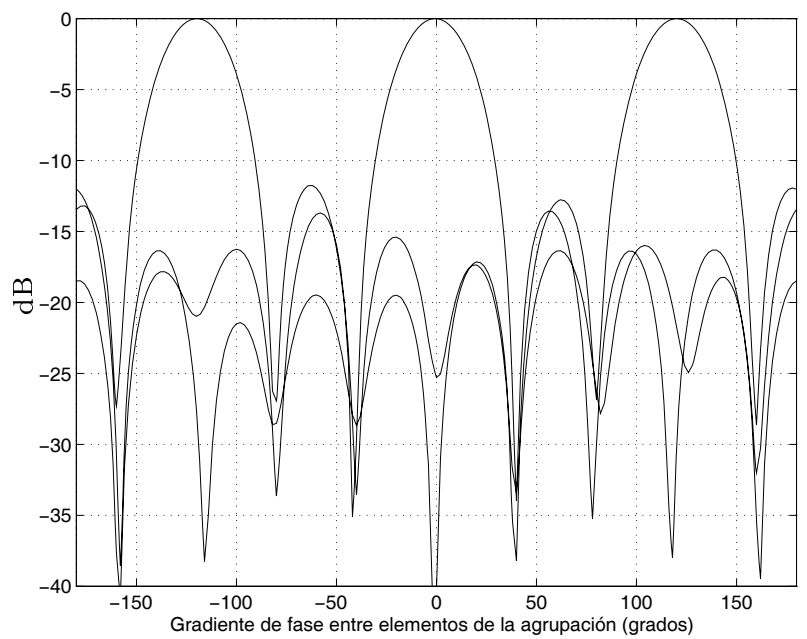

b)

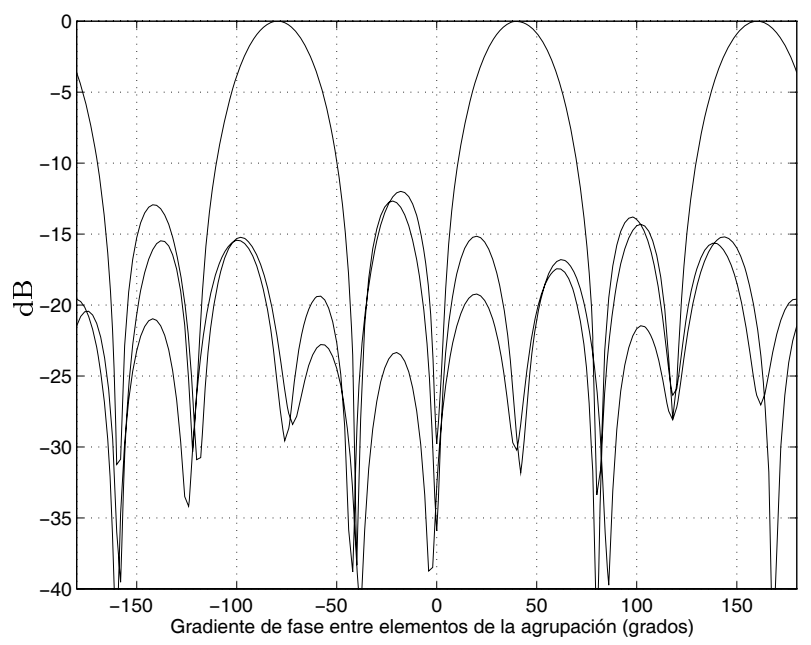

a)

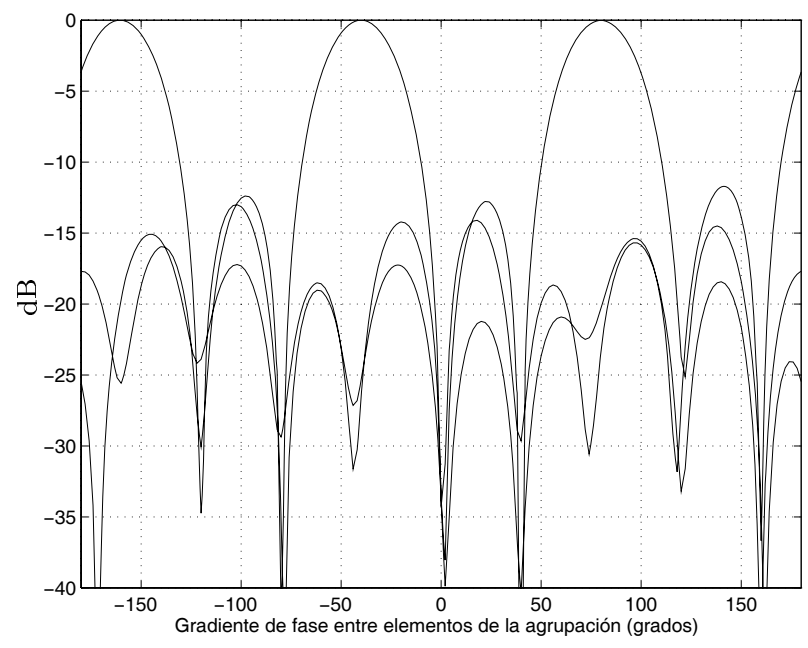

Figura 4.40: Nivel de haz en función del gradiente de fase de la onda incidente, para el conformador $9 \times 9$ alimentando una agrupación lineal de nueve elementos. a) Accesos 1, 2 y 3. b) Accesos 4, 5 y 6. c) Accesos 7, 8 y 9. 
4.3.4).

Como ejemplo, se muestran las gráficas de los contornos en dos dimensiones ${ }^{\mathrm{i}}$ del factor de la agrupación para el peor y el mejor haz (Figs. 4.42a y 4.41a respectivamente), los cuales se pueden comparar con los obtenidos con un conformador de haces $81 \times 81$ ideal (Figs. $4.42 \mathrm{~b}$ y $4.41 \mathrm{~b}$ respectivamente).

La forma y la posición del lóbulo principal son prácticamente iguales a los obtenidos en los casos ideales para ambos haces. El nivel de los lóbulos secundarios presenta la diferencia más apreciable, en comparación con la situación ideal. De todas formas, estas diferencias son bastante pequeñas para el mejor caso.

Un conformador de haces $81 \times 81$, permite disponer de 81 haces diferentes; para cada uno de estos haces, se han calculado los niveles de lóbulos secundarios, la ganancia, la posición y los anchos de haz. Los resultados estadísticos para los 81 haces aparecen resumidos en las tablas 4.36 a 4.39 .

Con el fin de analizar la distinta importancia de los errores de fase y de amplitud, se han considerado tres situaciones independientes para la estructura $9 \times 9$ :

- Pérdidas de inserción medidas e inserciones de fase medidas (Se tienen en cuenta todos los errores).

- Pérdidas de inserción ideales e inserciones de fase medidas (Se consideran solamente los errores de fase).

- Pérdidas de inserción medidas e inserciones de fase ideales (Se consideran solamente los errores de amplitud).

El efecto de la inserción de una distribución de amplitud con iluminación no uniforme, ha sido también considerada para los tres casos. La distribución de amplitud elegida, divide la antena en tres subconjuntos como se muestra en la figura 4.43.

Cuando se considera iluminación uniforme, los valores medios de los lóbulos secundarios están bastante cerca de los ideales (una diferencia de $1.3 \mathrm{~dB}$ para las medidas reales del conformador $9 \times 9$ ). La introducción de una distribución de amplitud no uniforme, hace que la diferencia con el caso ideal aumente considerablemente.

A partir del conjunto de los resultados, se puede concluir que, en términos de nivel de lóbulos secundarios, los errores de fase son más importantes que los errores de amplitud.

Por lo que respecta a la ganancia de los haces, los resultados obtenidos confirman unas prestaciones realmente buenas en términos de varianza de la ganancia de los haces, con un peor caso de una desviación de $0.5 \mathrm{~dB}$ sobre el valor medio de los

\footnotetext{
${ }^{\mathrm{i}}$ Los contornos se representan respecto a los ángulos $\theta_{x}$ y $\theta_{y}$ medidos en los planos XZ y YZ respecto a la normal a la agrupación situada sobre el plano XY.
} 


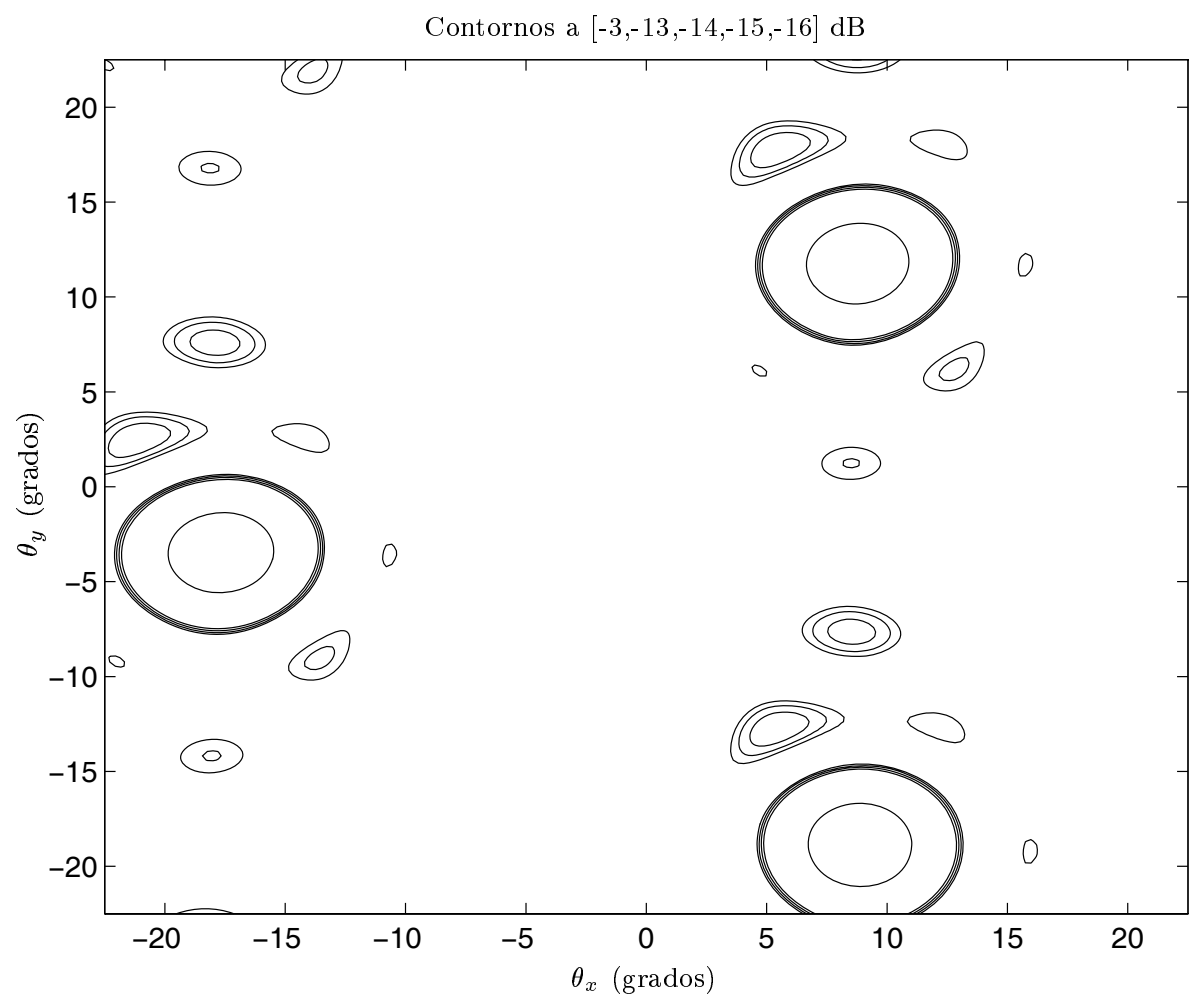

a)

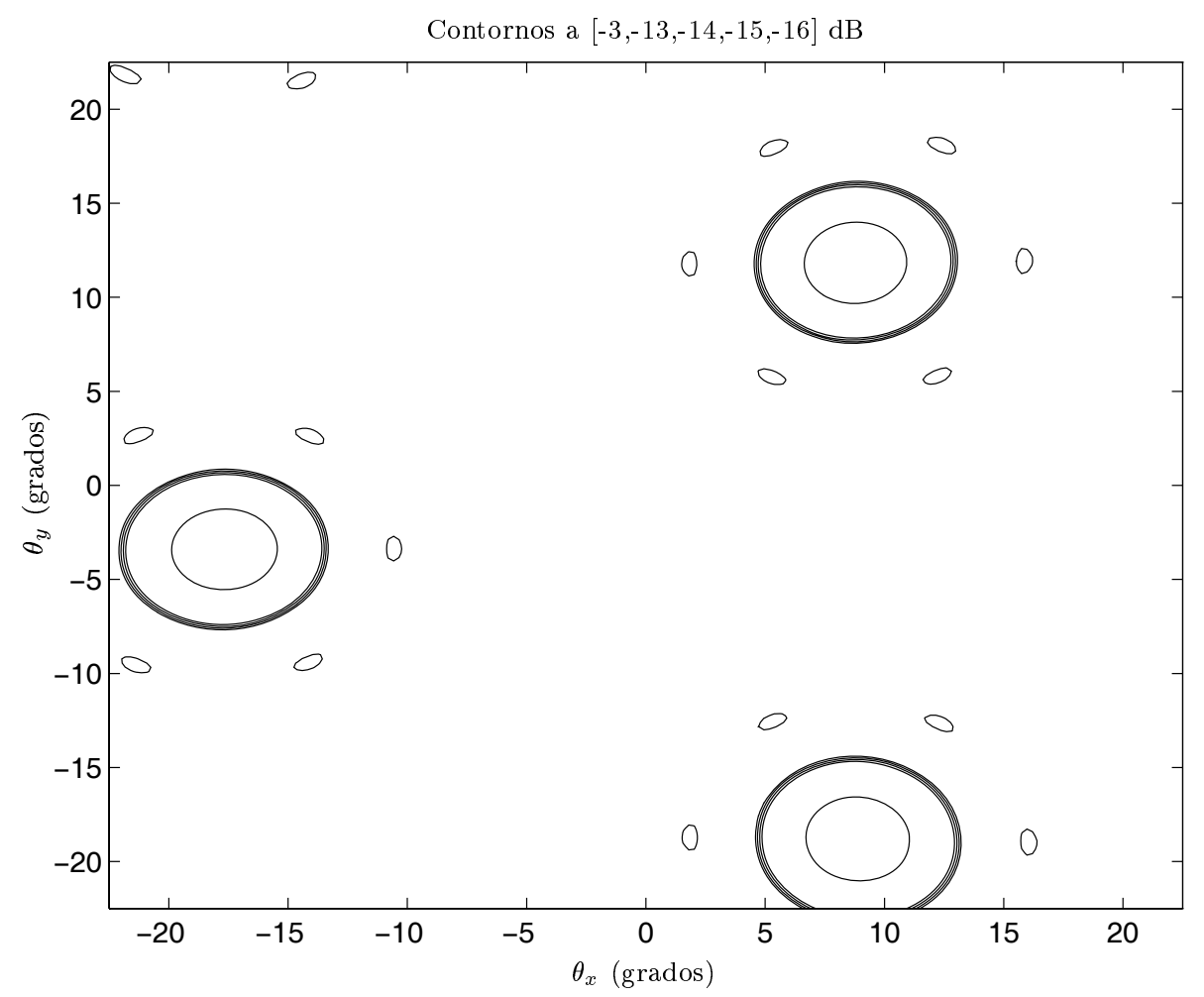

Figura 4.41: Contornos del factor de la agrupación para el haz 22 para la antena ARCHIMEDES alimentada por un conformador $81 \times 81$. a) Simulación a partir de medidas del conformador $9 \times 9$. b) Según conformador $81 \times 81$ ideal. 


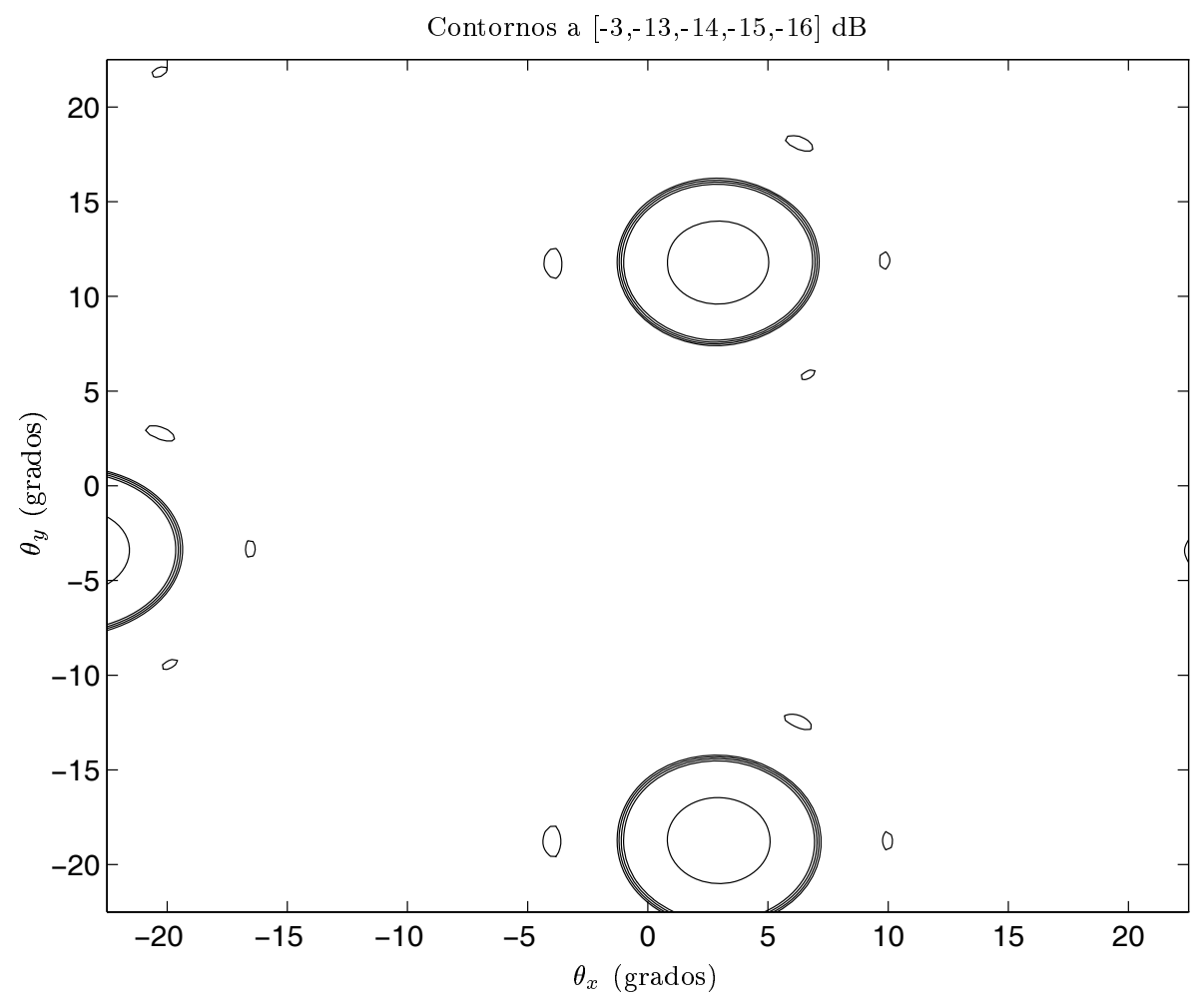

a)

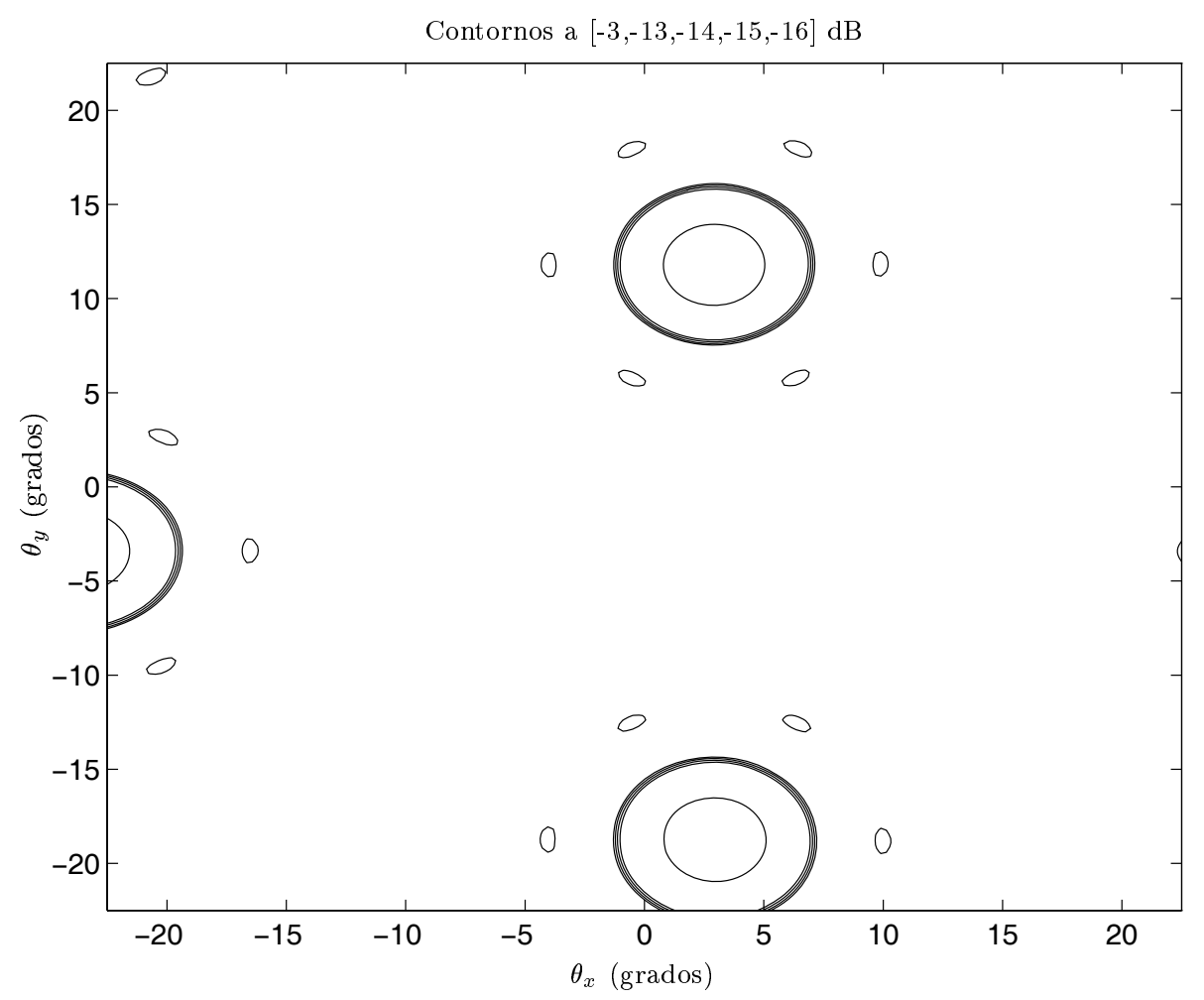

Figura 4.42: Contornos del factor de la agrupación para el haz 77 para la antena ARCHIMEDES alimentada por un conformador $81 \times 81$. a) Simulación a partir de medidas del conformador $9 \times 9$. b) Según conformador $81 \times 81$ ideal. 


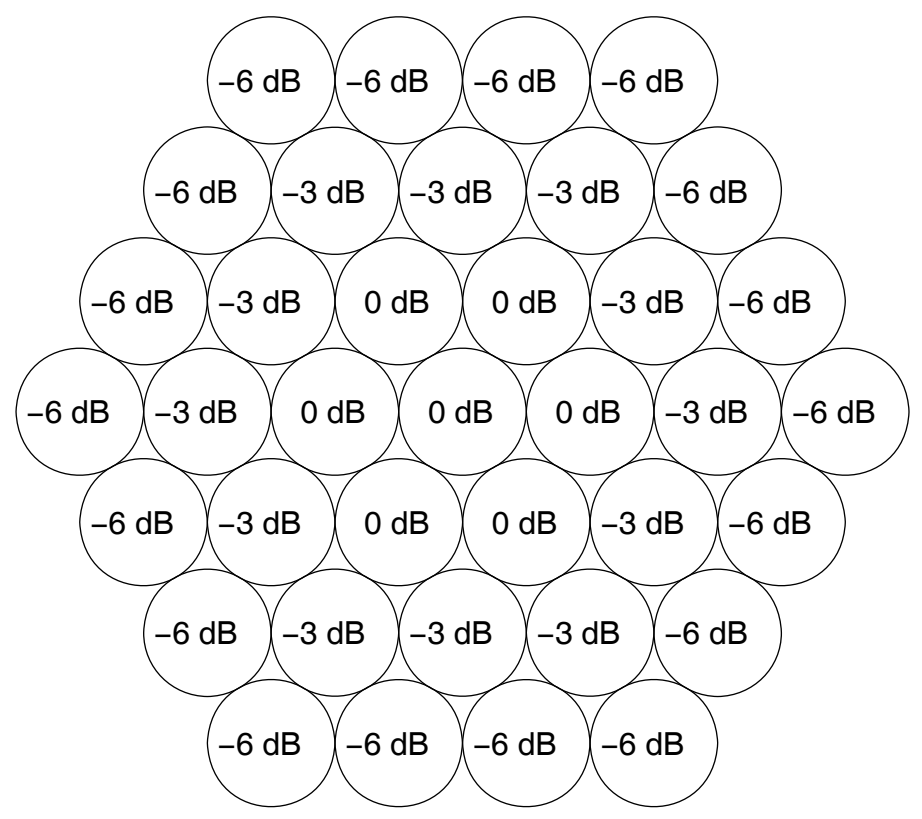

Figura 4.43: Ejemplo de iluminación no uniforme en amplitud para la agrupación hexagonal de ARCHIMEDES.

\begin{tabular}{|c|c|c|c|c|c|c|}
\hline & \multicolumn{2}{|c|}{$\begin{array}{c}\text { Todos los errores } \\
\text { considerados }\end{array}$} & \multicolumn{2}{|c|}{$\begin{array}{c}\text { Sólo errores } \\
\text { de fase }\end{array}$} & \multicolumn{2}{|c|}{$\begin{array}{l}\text { Sólo errores } \\
\text { de amplitud }\end{array}$} \\
\hline & $\begin{array}{l}\text { Ilumin. } \\
\text { uniforme }\end{array}$ & $\begin{array}{l}\text { Ilumin. } \\
(-6,-3,0)\end{array}$ & $\begin{array}{l}\text { Ilumin. } \\
\text { uniforme }\end{array}$ & $\begin{array}{l}\text { Ilumin. } \\
(-6,-3,0)\end{array}$ & $\begin{array}{l}\text { Ilumin. } \\
\text { uniforme }\end{array}$ & $\begin{array}{l}\text { Ilumin. } \\
(-6,-3,0)\end{array}$ \\
\hline Media & $14.5 \mathrm{~dB}$ & $17.0 \mathrm{~dB}$ & $14.9 \mathrm{~dB}$ & $18.2 \mathrm{~dB}$ & $15.2 \mathrm{~dB}$ & $19.1 \mathrm{~dB}$ \\
\hline Mejor & $15.7 \mathrm{~dB}$ & $20.8 \mathrm{~dB}$ & $15.6 \mathrm{~dB}$ & $20.8 \mathrm{~dB}$ & $15.94 \mathrm{~dB}$ & $21.3 \mathrm{~dB}$ \\
\hline Peor & $13.1 \mathrm{~dB}$ & $14.9 \mathrm{~dB}$ & $13.7 \mathrm{~dB}$ & $16.0 \mathrm{~dB}$ & $13.7 \mathrm{~dB}$ & $16.0 \mathrm{~dB}$ \\
\hline$\sigma$ & $0.7 \mathrm{~dB}$ & $1.6 \mathrm{~dB}$ & $0.5 \mathrm{~dB}$ & $1.2 \mathrm{~dB}$ & $0.7 \mathrm{~dB}$ & $1.6 \mathrm{~dB}$ \\
\hline
\end{tabular}

Tabla 4.36: Prestaciones del conformador $81 \times 81$ simulado a partir de las medidas del conformador $9 \times 9$. NLPS (dB) (Valores ideales: 15.8 dB (iluminación uniforme) $21.1 \mathrm{~dB}$ (iluminación -6/-3/0 dB).

\begin{tabular}{|c||c|c|c|c|c|c|}
\hline \multicolumn{1}{|c||}{} & \multicolumn{2}{c|}{ Todos los errores } & \multicolumn{2}{c|}{ Sólo errores } & \multicolumn{2}{c|}{ Sólo errores } \\
& \multicolumn{2}{c|}{ considerados } & \multicolumn{2}{c|}{ de fase } & \multicolumn{2}{c|}{ de aAmplitud } \\
& Ilumin. & Ilumin. & Ilumin. & Ilumin. & Ilumin. & Ilumin. \\
& uniforme & $(-6,-3,0)$ & uniforme & $(-6,-3,0)$ & uniforme & $(-6,-3,0)$ \\
\hline \hline Desviación & $+0.5 \mathrm{~dB}$ & $+0.4 \mathrm{~dB}$ & $+0.06 \mathrm{~dB}$ & $+0.05 \mathrm{~dB}$ & $+0.4 \mathrm{~dB}$ & $+0.3 \mathrm{~dB}$ \\
Máxima & $-0.4 \mathrm{~dB}$ & $-0.4 \mathrm{~dB}$ & $-0.09 \mathrm{~dB}$ & $-0.07 \mathrm{~dB}$ & $-0.4 \mathrm{~dB}$ & $-0.3 \mathrm{~dB}$ \\
\hline$\sigma$ & $0.17 \mathrm{~dB}$ & $0.14 \mathrm{~dB}$ & $0.03 \mathrm{~dB}$ & $0.02 \mathrm{~dB}$ & $0.16 \mathrm{~dB}$ & $0.13 \mathrm{~dB}$ \\
\hline
\end{tabular}

Tabla 4.37: Prestaciones del conformador $81 \times 81$ simulado a partir de las medidas del conformador $9 \times 9$. Ganancia de los haces. 


\begin{tabular}{|c||c|c|c|c|c|c|}
\hline \multicolumn{1}{||||}{} & \multicolumn{2}{c|}{$\begin{array}{c}\text { Todos los errores } \\
\text { considerados }\end{array}$} & \multicolumn{2}{c|}{$\begin{array}{c}\text { Sólo errores } \\
\text { de fase }\end{array}$} & \multicolumn{2}{c|}{$\begin{array}{c}\text { Sólo errores } \\
\text { de amplitud }\end{array}$} \\
& Ilumin. & Ilumin. & Ilumin. & Ilumin. & Ilumin. & Ilumin. \\
& uniforme & $(-6,-3,0)$ & uniforme & $(-6,-3,0)$ & uniforme & $(-6,-3,0)$ \\
\hline \hline Media & $0.05^{\circ}$ & $0.05^{\circ}$ & $0.05^{\circ}$ & $0.05^{\circ}$ & $0.03^{\circ}$ & $0.03^{\circ}$ \\
\hline Max & $0.1^{\circ}$ & $0.16^{\circ}$ & $0.13^{\circ}$ & $0.17^{\circ}$ & $0.06^{\circ}$ & $0.06^{\circ}$ \\
\hline
\end{tabular}

Tabla 4.38: Prestaciones del conformador $81 \times 81$ simulado a partir de las medidas del conformador $9 \times 9$. Error en el apuntamiento del centro de los haces. La rejilla usada para las simulaciones es de $0.09^{\circ}$.

\begin{tabular}{|c|c|c|c|c|c|c|}
\hline & \multicolumn{2}{|c|}{$\begin{array}{c}\text { Todos los errores } \\
\text { considerados }\end{array}$} & \multicolumn{2}{|c|}{$\begin{array}{c}\text { Sólo errores } \\
\text { de fase }\end{array}$} & \multicolumn{2}{|c|}{$\begin{array}{l}\text { Sólo errores } \\
\text { de amplitud }\end{array}$} \\
\hline & $\begin{array}{l}\text { Ilumin. } \\
\text { uniforme }\end{array}$ & $\begin{array}{l}\text { Ilumin. } \\
(-6,-3,0)\end{array}$ & $\begin{array}{l}\text { Ilumin. } \\
\text { uniforme }\end{array}$ & $\begin{array}{l}\text { Ilumin. } \\
(-6,-3,0)\end{array}$ & $\begin{array}{l}\text { Ilumin. } \\
\text { uniforme }\end{array}$ & $\begin{array}{l}\text { Ilumin. } \\
(-6,-3,0)\end{array}$ \\
\hline Media & $4.19^{\circ}$ & $4.58^{\circ}$ & $4.18^{\circ}$ & $4.57^{\circ}$ & $4.19^{\circ}$ & $4.6^{\circ}$ \\
\hline$\sigma$ & $0.05^{\circ}$ & $0.06^{\circ}$ & $0.04^{\circ}$ & $0.05^{\circ}$ & $0.05^{\circ}$ & $0.06^{\circ}$ \\
\hline
\end{tabular}

Tabla 4.39: Prestaciones del conformador $81 \times 81$ simulado a partir de las medidas del conformador $9 \times 9$. Anchos de haz. La rejilla usada en las simulaciones es de $0.09^{\circ}$, siendo el ancho de haz ideal de $4.18^{\circ}$.

81 haces. Como era de esperar, las ganancias de los haces son más sensibles a los errores de amplitud que a los errores de fase. En cuanto al efecto de aplicar una iluminación no uniforme, las prestaciones mejoran ligeramente.

El error de apuntamiento de los centros de los haces, puede considerarse despreciable, mientras que los anchos de haz obtenidos con la red conformadora $81 \times 81$ basada en las medidas del conformador $9 \times 9$, son prácticamente iguales a los ideales.

Todas las simulaciones llevadas a cabo con el conformador de haces $81 \times 81$, realizadas a partir de las medidas de la estructura real $9 \times 9$, muestran unas prestaciones realmente buenas y claramente prueban la viabilidad de su uso en aplicaciones reales. Por otro lado, los resultados mostrados para el conformador de haces de tamaño $81 \times 81$ no son completamente realistas ya que en una implementación real las 18 estructuras $9 \times 9$ no serían exactamente iguales, con lo que su comportamiento sería ligeramente distinto. Estas pequeñas diferencias probablemente mejorarían los resultados estadisticos del conformador de haces.

\subsubsection{Comentarios a las medidas del conformador $9 \times 9$}

El conformador de haces $9 \times 9$, construido con una estructura triplaca multicapa y seis matrices MMIC $3 \times 3$, sintonizable mediante una tensión de control, ha mostrado unas prestaciones ciertamente buenas, demostrando todas las características 
esperadas, como bajo peso, tamaño reducido y capacidad de sintonización.

Las prestaciones de la celda básica $3 \times 3$, podrían mejorarse en siguientes diseños. También la estructura multicapa podría mejorarse, con el fin de reducir los errores en la inserción de fase. Sus pérdidas de inserción y aislamiento son ya suficientemente buenos. Por lo tanto, se espera que una segunda iteración del conformador de haces $9 \times 9$, ofrezca unos resultados aún mejores.

La simulación del conformador de haces $81 \times 81$ basada en las medidas de la estructura $9 \times 9$, ha confirmado la viabilidad de esta nueva arquitecura para redes conformadoras de haces, con buenas prestaciones en términos de parámetros de antena. Los niveles de lóbulos secundarios y el efecto sobre los mismos de la inserción de una distribución de amplitudes de alimentación no uniforme en los elementos de la antena, son los temas que podrían mejorar en una segunda iteración del diseño.

\subsection{Conclusiones}

En este capítulo, se ha generalizado la matriz de Butler para agrupaciones planas, con cualquier distribución, tanto en el plano de la agrupación, como en el dominio transformado de la posición de los haces; mostrando la relación que existe entre ambas distribuciones, el número de elementos de la agrupación y el número y posición de los haces a conformar. Se ha particularizado los resultados para distribución en rejilla hexagonal en ambos dominios, presentando un método rápido para el diseño del conformador, destacando las características necesarias para que, la implementación del mismo permita una descomposición eficiente en celdas de menor tamaño.

Además de analizar la arquitectura, era necesario probar la viabilidad de la misma, para lo cual se han diseñado dos matrices $3 \times 3 \mathrm{MMIC}$, con el proceso D02AH de Philips microondas Limeil. Con el fin de resolver el problema de las tolerancias del proceso, se ha diseñado una matriz sintonizable mediante diodos varactores.

Las prestaciones de los circuitos cumplen las especificaciones, excepto por una menor inserción de fase, comportamiento que parece ser debido a errores en el modelado de las bobinas del proceso. La capacidad de sintonización gracias a los diodos varactores ha sido demostrada en uno de los diseños. De esta forma, el circuito es muy robusto frente a las variaciones del proceso.

Seis matrices $3 \times 3$ sintonizables, desarrolladas con tecnología MMIC fueron montadas en una estructura triplaca multicapa (desarrollada por Dassault Electronique, Francia) con el fin de construir una matriz $9 \times 9$, la cual podría ser usada como parte de los conformadores de tamaño $27 \times 27$ o $81 \times 81$. Este conformador de haces $9 \times 9$ fue medido en las instalaciones de Dassault Electronique, mostrando unas buenas prestaciones. Incluso, aunque el diseño nominal presentaba una diferencia de inserción de fase menor de lo esperado, la capacidad de sintonización de la arquitectura consiguió resolver parcialmente el problema, a costa de aumentar las 


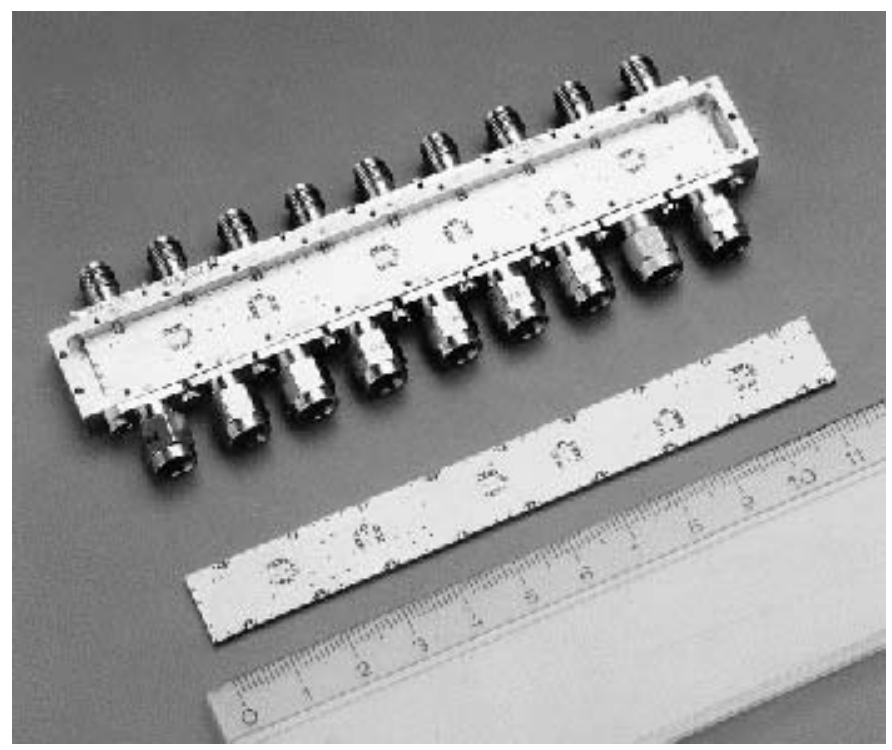

Figura 4.44: Fotografía del conformador $9 \times 9$ montado en un soporte de prueba junto a otra copia de la estructura triplaca multicapa con los circuitos integrados montados.

diferencias en las pérdidas de inserción.

El tiempo de desarrollo para el conformador $9 \times 9$ ha sido bastante corto. El diseño de los MMICs, comenzó en Septiembre de 1994, obteniéndose los circuitos listos para ser medidos en Mayo de 1995. El conformador de haces $9 \times 9$ completo se midió en Junio de 1995.

Con el fin de comprobar cómo los errores de fase y de amplitud del conformador de haces $9 \times 9$, afectarían al diagrama de radiación de la antena, se han llevado a cabo una serie de simulaciones para un conformador de tamaño $81 \times 81$ construido con estructuras $9 \times 9$ reales. Los resultados prueban, totalmente, la viabilidad de la nueva arquitectura de conformador de haces de radiofrecuencia.

Aunque el primer diseño ha dado unos resultados bastante buenos, un segundo diseño con unas matrices $3 \times 3$ y una estructura triplaca multicapa mejoradas, podría ser la prueba final para este nuevo concepto de red conformadora de haces.

Este dispositivo $\left(9 \times 9\right.$, Fig. 4.44) fue presentado en el pabellón de $230 \mathrm{~m}^{2}$ de la Agencia Espacial Europea (ESA) en la feria de telecomunicaciones más importante del mundo TELECOM'95, que tuvo lugar en Ginebra (Suiza) del 3 al 11 de Octubre de 1995, feria organizada cada cuatro años por la Unión Internacional de Telecomunicaciones (ITU ${ }^{\mathrm{i}}$ ) [ESA95].

\footnotetext{
${ }^{\mathrm{i} I n t e r n a t i o n a l ~ T e l e c o m m u n i c a t i o n}$ Union, en terminología inglesa.
} 



\section{Capítulo 5}

\section{Diseño, implementación y medida de una red conformadora de haces controlada ópticamente mediante el uso de redes de difracción en fibra}

\subsection{Introducción}

La aplicación de fibras ópticas como medio de transmisión en redes conformadoras de haces, permite disfrutar de características tan deseadas como, peso reducido, bajas pérdidas, gran ancho de banda o inmunidad frente a interferencias electromagnéticas. Para poder disponer de todas estas prestaciones, se necesitan elementos de control adecuados para conformar el haz, mediante la variación de las amplitudes y los retardos asociados a cada elemento de la agrupación. La integración de los métodos de conformación de haz con la red de distribución óptica a partir de dispositivos sobre fibra óptica permitirá conjugar todos estos requisitos.

En este capítulo se describe la utilización de redes de difracción en fibra ${ }^{\mathrm{i}}$ como líneas de retardo para aplicaciones de redes conformadoras de haces controladas ópticamente.

El uso de las redes de difracción sobre fibra óptica para el procesamiento de señales ópticas se ha centrado hasta en sistemas de comunicaciones para la compensación de la dispersión de la fibra monomodo y para el filtrado de canal en sistemas con multiplexación en frecuencia óptica $\left(\mathrm{OFDM}^{\mathrm{ii}}\right)$ y en sistema de sensores basados en la variación de las propiedades ópticas de la red de difracción con la temperatura

\footnotetext{
${ }^{\mathrm{i}}$ Fiber Gratings, en terminología inglesa.

ii Optical frequency-division multiplexing, en terminología inglesa.
} 


\section{Fibra Óptica Monomodo}

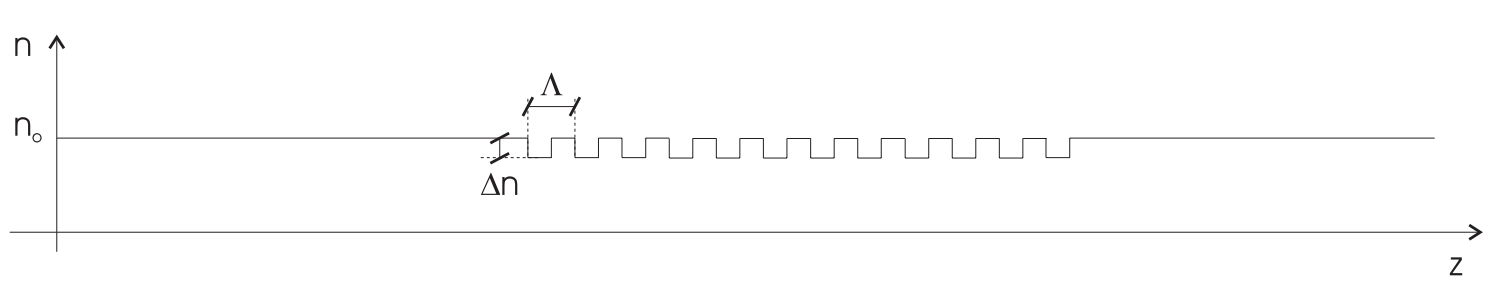

Figura 5.1: Ejemplo de red de difracción.

y con la tensión mecánica aplicada.

En cuanto a su aplicación a redes conformadoras de haces, se pueden distinguir dos casos: por un lado, la utilización de redes de difracción discretas grabadas sobre una misma fibra monomodo que permite disponer de un conjunto discreto de retardos [Bal94, Lem94, Ton96b, Mol95, Zmu97, Sor96, Cha96] y por otro lado, las redes de difracción con periodo variable que permiten generar retardos verdaderos continuamente variables. En este capítulo, se analiza profundamente el segundo caso, tanto sus prestaciones, como sus limitaciones, a la hora de aplicarse a redes conformadoras de haces.

Posteriormente, se presenta una arquitectura de red conformadora de haces que utiliza una red de difracción con variación lineal del periodo espacial del índice de refracción como dispositivo de retardo verdadero. Esta arquitectura ha sido probada, y sus parámetros más representativos a nivel de sistema, estimados a partir de los resultados del estudio previo de las redes de difracción.

\subsection{Redes de difracción en fibra}

Las redes de difracción sobre fibra óptica son dispositivos relativamente recientes y que, tras las mejoras realizadas durante la última década en los métodos de fabricación, están recibiendo un considerable interés por parte de diversos grupos de investigación, para su aplicación en diversos campos.

Físicamente, una red de difracción en fibra (Fig. 5.1) está constituida por un conjunto de discontinuidades en el índice de refracción de la fibra separadas periódicamente. La amplitud de las variaciones del índice de refracción es muy pequeña $\left(10^{-2}\right.$ a $\left.10^{-6}\right)$ respecto al índice medio de la fibra, $n_{o}$. Estas perturbaciones periódicas en el índice de refracción generan una serie sucesiva de ondas reflejadas y transmitidas, de forma que la magnitud $(\Delta n)$ y el periodo espacial $(\Lambda)$ de estas discontinuidades definirán la respuesta temporal y frecuencial (amplitud y fase) de la red de difracción, tanto en transmisión como en reflexión. Generalmente se utilizan las propiedades en reflexión de la red de difracción, mediante la adición de un 

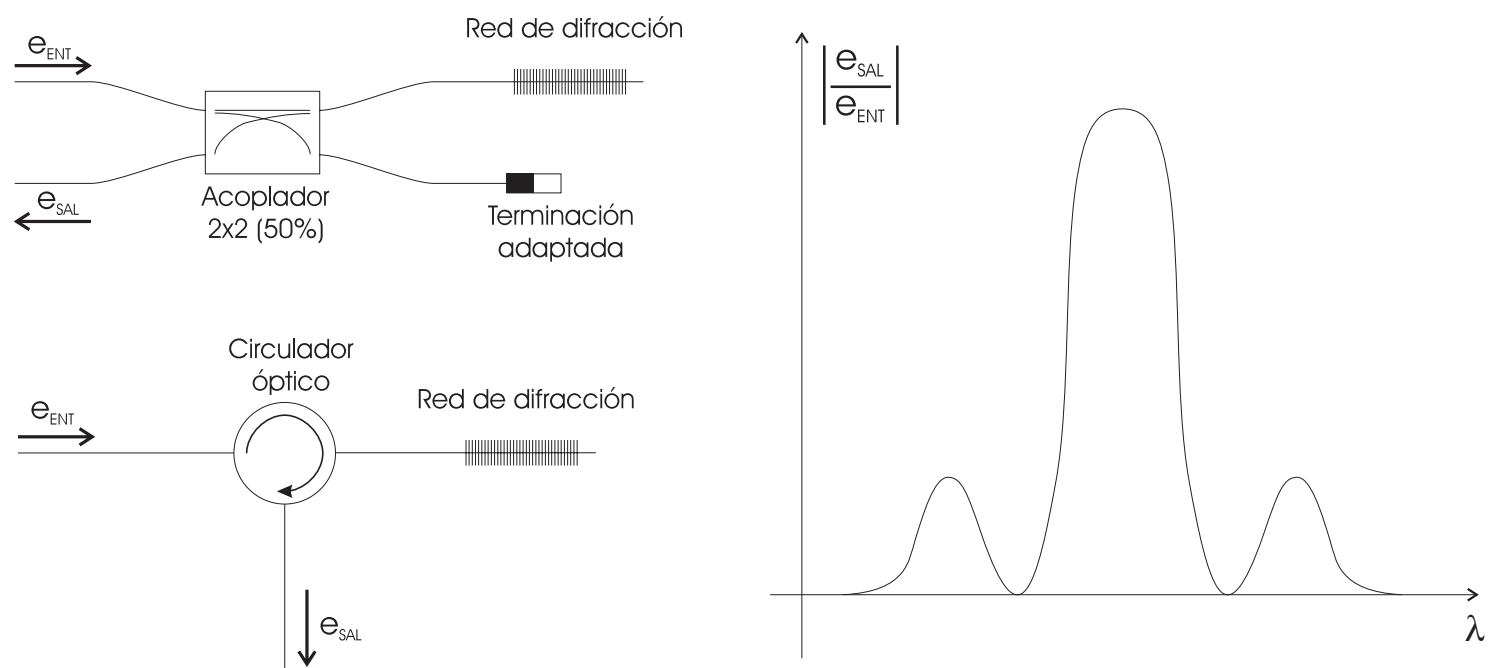

Figura 5.2: Configuraciones y respuesta típica de una red de difracción en reflexión.

circulador óptico o un acoplador óptico (Fig. 5.2). Con esta configuración, la red de difracción se comporta como un filtro selectivo en longitud de onda, mostrándose en la figura 5.2 una curva típica del módulo de la respuesta en frecuencia, donde se puede distinguir el lóbulo principal del filtro junto a los lóbulos secundarios más cercanos.

Los métodos de fabricación más modernos se basan en la fotosensibilidad de la fibra óptica a longitudes de onda entorno a $245 \mathrm{~nm}$ (ultravioleta); en concreto, se basan en los cambios permanentes en el índice de refracción de la fibra tras una exposición de la misma a una intensa radiación en el entorno de los citados $245 \mathrm{~nm}$. Las dos técnicas de fabricación de redes de difracción más utilizados son el método holográfico [Mel89] basado en el patrón de interferencia generado por dos haces incidentes sobre la fibra y el método de máscara de fase [Mar94, Hil93] que requiere un único haz de radiación ultravioleta incidente sobre una máscara interferente situada sobre la fibra a grabar.

Los parámetros de fabricación más importante son:

$\Delta n(z)$ Magnitud de la variación, sobre el valor medio, del índice de refracción del núcleo en función de la distancia sobre el eje de la fibra.

$\Lambda(z)$ Periodo espacial del índice de refracción del núcleo en función de la distancia sobre el eje de la fibra. En relación con el periodo espacial, se define también la frecuencia espacial como $K(z)=2 \pi / \Lambda(z)$.

$L_{\text {red }}$ Longitud total de la red de difracción.

En el caso de una red de difracción con una periodo espacial del índice de refracción constante $\left(\Lambda_{o}\right)$ a lo largo de toda la red, se define la longitud de onda de 
Bragg como aquella para la cual las sucesivas ondas reflejadas interfieren constructivamente y consecuentemente se obtiene la mayor reflectividad de la red, y viene definida por:

$$
\lambda_{B R A G G}=2 n_{o} \Lambda_{o}
$$

donde $n_{o}$ es el índice de refracción medio de la fibra. El valor de potencia reflejada máxima y el ancho de banda óptico dependerán tanto de la amplitud de la variación del índice de refracción como de la longitud total de la red de difracción.

El método de análisis de redes de difracción en fibra más extendido se basa en el estudio de las ecuaciones de acoplo contradireccional de modos en guías dieléctricas con perturbación periódica del índice de refracción. Cuando la amplitud y el periodo de la perturbación es constante, es posible obtener soluciones analíticas, siendo necesario acudir a métodos numéricos para analizar redes de difracción con amplitud y periodo de las perturbaciones variables con la distancia axial sobre la fibra [Kog76].

A la hora de analizar el comportamiento como dispositivo de una determinada red de difracción de longitud total $L_{\text {red }} \mathrm{y}$ con sus correspondientes funciones $\Delta n(z), \Lambda(z)$ no se suele trabajar con estos parámetros físicos, sino con la función de acoplamiento entre los modos progresivo y regresivo, $k(z)$, y la función de fase de la frecuencia espacial, $\phi(z)$, respectivamente. La función de acoplo, $k(z)$, también conocida como apodización de la red de difracción, depende de la magnitud de las perturbaciones del índice de refracción, pero también de las distribuciones transversales a la fibra de estas variaciones del índice de refracción y del campo eléctrico [PA96, Yar73]. Por otro lado, la función de fase de la frecuencia espacial, $\phi(z)$, consiste en la desviación de fase debida a la variación de la frecuencia espacial local a largo del eje de la fibra ${ }^{i}$ :

$$
\frac{d \phi(z)}{d z}=\Delta K(z)=K(z)-K_{o}=2 \pi\left(\frac{1}{\Lambda(z)}-\frac{1}{\Lambda_{o}}\right)
$$

En cuanto a las características más importantes de las redes de difracción, resaltando aquellas de mayor interés para la aplicación de redes de difracción como elementos retardadores (variables o discretos), se pueden citar [Col96]:

- Comportamiento insensible a la polarización.

- Dispositivo realizado directamente sobre fibra.

- Bajas pérdidas de inserción, pese a la necesidad de utilizar un circulador óptico $(1-2 \mathrm{~dB})$ o un acoplador $(\sim 7 \mathrm{~dB})$.

- Respecto a una longitud de fibra con dispersión similar, la red de difracción no sufre de no-linearidades ópticas significativas.

\footnotetext{
iSe asume una perturbación proporcional a $\cos \left(K_{o} z+\phi(z)\right)$ con $K_{o}$ constante e igual a la frecuencia espacial alrededor del centro de la red de difracción $(z=0)$.
} 
La elección adecuada de las funciones de acoplo y de fase así como de la longitud total de la red de difracción, de acuerdo con las prestaciones esperadas de la red es un punto crucial en el análisis y síntesis de redes de difracción para cualquier aplicación, y en concreto para aplicaciones de redes conformadoras de haces. Hace apenas tres años, se ha presentado un nuevo método de fabricación que permite realizar redes de difracción con variación lineal del periodo espacial utilizando cualquier función de apodización deseada [Loh95]. Además, existen nuevas técnicas de fabricación que aumentan día tras día la longitud máxima disponible para una red de difracción [Col96]. Ambos eventos no hacen sino aumentar los márgenes de libertad a la hora de sintetizar redes de difracción con las característica deseadas.

\subsubsection{Redes de difracción con periodo constante}

Para la aplicación de redes de difracción como filtros selectores en longitud de onda, y como líneas de retardo programables es interesante estudiar las redes de difracción con periodo espacial constante; las cuales, permiten obtener una solución analítica, en el caso de considerar acoplo constante; siendo necesario trabajar con soluciones numéricas, para el caso más general de redes de difracción con acoplo variable.

\section{Redes de difracción con periodo constante y acoplo constante}

El coeficiente de reflexión de campo de una red de difracción de longitud $L_{\text {red }}$, acoplo $k_{o}$ y periodo $\Lambda_{o}$, ambos constantes, se puede expresar como [Hau84]:

$$
\rho=\frac{-j k_{o} \operatorname{senh}\left(L_{r e d} \sqrt{k_{o}^{2}-\delta^{2}}\right)}{\sqrt{k_{o}^{2}-\delta^{2}} \cosh \left(L_{\text {red }} \sqrt{k_{o}^{2}-\delta^{2}}\right)+j \delta \operatorname{senh}\left(L_{\text {red }} \sqrt{k_{o}^{2}-\delta^{2}}\right)}
$$

donde

$$
\delta=\beta-\frac{\pi}{\Lambda_{o}}=\beta-\beta_{B R A G G} \approx \frac{d \beta}{d \omega}\left(\omega-\omega_{B R A G G}\right)=\frac{\Delta \omega}{v_{g}} \approx \frac{n_{o} \Delta \omega}{c}
$$

es la desviación de frecuencia normalizada, siendo $c$ la velocidad de la luz en el vacío y $\beta$ la constante de fase de una onda electromagnética de pulsación $\omega$ progagándose en una fibra con índice de refracción medio $n_{o}$ con una velocidad de grupo $v_{g}$.

Agrupando términos en la ecuación (5.3) se puede llegar a una expresión del coeficiente de reflexión que sólo dependerá del coeficiente de acoplo normalizado $k_{o} L=k_{o} \cdot L_{\text {red }} \mathrm{y}$ de la desviación de frecuencia normalizada $\delta L=\delta \cdot L_{\text {red }}$ :

$$
\rho=\frac{-j k_{o} L \operatorname{senh}\left(\sqrt{\left(k_{o} L\right)^{2}-(\delta L)^{2}}\right)}{\sqrt{\left(k_{o} L\right)^{2}-(\delta L)^{2}} \cosh \left(\sqrt{\left(k_{o} L\right)^{2}-(\delta L)^{2}}\right)+j \delta L \operatorname{senh}\left(\sqrt{\left(k_{o} L\right)^{2}-(\delta L)^{2}}\right)}
$$

de donde se puede concluir que el comportamiento de una red de difracción con acoplo y periodo constante queda totalmente especificado respecto a la frecuencia normalizada, $\delta L$, una vez se conoce el acoplo normalizado, $k_{o} L$. 
Se define la reflectividad de la red de difracción en términos de potencia óptica como $|\rho|^{2}$. Cuando se considera una desviación de frecuencia normalizada igual a cero, se cumplirá la condición de Bragg y la reflectividad será máxima, reflectividad que se puede calcular a partir de la ecuación (5.5) tomando $\delta=0$ :

$$
|\rho|_{M A X}^{2}=\tanh ^{2}\left(k_{o} L\right)
$$

ecuación que relaciona unívocamente la reflectividad máxima con el acoplo normalizado.

A partir de la ecuación (5.5) se han realizado una serie de simulaciones para redes de difracción con diferentes valores de acoplo normalizado $k_{o} L$, calculándose para cada caso los siguientes anchos de banda medidos en frecuencias normalizadas: ancho de banda entre ceros $\left(\Delta \delta L_{\text {ceros }}\right)$, ancho de banda a $-3 \mathrm{~dB}$ ópticos ${ }^{\mathrm{i}}\left(\Delta \delta L_{-3 d B o}\right)$ y ancho de banda a $-3 \mathrm{~dB}$ eléctricos $\left(\Delta \delta L_{-3 d B e}\right)$. En la tabla 5.1 se muestran los resultados obtenidos en función del módulo del coeficiente de reflexión a la frecuencia de Bragg de la red de difracción. Los valores de los anchos de banda, $\Delta \delta L$, pueden desnormalizarse partiendo de la ecuación (5.4), obteniéndose las siguiente expresiones:

$$
\begin{array}{r}
\Delta f=\frac{c \Delta \delta L}{2 \pi n_{o} L_{\text {red }}} \\
\Delta \lambda=\frac{\lambda_{\text {Bragg }}^{2} \Delta \delta L}{2 \pi n_{o} L_{\text {red }}}
\end{array}
$$

como ejemplo una red con una reflectividad de pico del $81 \%$ y una longitud total de $1 \mathrm{~cm}$, tendría una respuesta frecuencial centrada en $\lambda_{B R A G G}=1550 \mathrm{~nm}$ con un ancho de banda ${ }^{\text {ii }}$ a $-3 \mathrm{~dB}$ eléctricos de $12.3 \mathrm{GHz}$ o $0.1 \mathrm{~nm}$.

Los resultados mostrados en la tabla 5.1 permiten obtener unas expresiones aproximadas para el ancho de banda a -3 dB eléctricos de una red de difracción con acoplo y periodo constantes en función únicamente de su acoplo normalizado (directamente relacionado con la reflectividad máxima de la red a través de la ecuación (5.6)):

$$
\Delta \delta L_{-3 d B e} \approx \begin{cases}2+0.03 k_{o} L+0.83\left(k_{o} L\right)^{2} & \text { si } k_{o} L<1.5 \\ 1.25+1.95 k_{o} L & \text { si } k_{o} L>1.5\end{cases}
$$

Combinando las ecuaciones (5.7) y (5.9) se puede obtener para reflectividades altas, una expresión aproximada del ancho de banda a mitad de potencia eléctrica como:

$$
\Delta f_{-3 d B e} \approx \frac{4+6.4 k_{o} L}{L_{r e d}}
$$

\footnotetext{
${ }^{\mathrm{i} A n c h u r a ~ d e ~ b a n d a ~ m a ́ s ~ c o n o c i d a ~ p o r ~ s u ~ a c r o ́ n i m o ~ e n ~ i n g l e ́ s, ~ c o m o ~ F u l l ~ w i d t h ~ a t ~ h a l f ~ m a x i m u m, ~}$ $F W H M$.

${ }^{\text {ii }}$ Se ha asumido que $n_{o} \approx 1.46$.
} 


\begin{tabular}{|c|c||c|c|c|}
\hline$|\rho|_{\max }$ & $k_{o} L$ & $\Delta \delta L_{\text {ceros }}$ & $\Delta \delta L_{-3 d B o}$ & $\Delta \delta L_{-3 d B e}$ \\
\hline \hline 0,250 & 0,255 & 6,304 & 2,845 & 2,058 \\
\hline 0,500 & 0,549 & 6,379 & 3,080 & 2,266 \\
\hline 0,750 & 0,973 & 6,578 & 3,664 & 2,816 \\
\hline 0,800 & 1,099 & 6,656 & 3,879 & 3,029 \\
\hline 0,900 & 1,472 & 6,939 & 4,576 & 3,759 \\
\hline 0,950 & 1,832 & 7,273 & 5,284 & 4,539 \\
\hline 0,990 & 2,647 & 8,216 & 6,873 & 6,329 \\
\hline 0,999 & 3,800 & 9,861 & 9,035 & 8,690 \\
\hline
\end{tabular}

Tabla 5.1: Resultados de simulación de redes de difracción con acoplo y periodo constante en función de la reflectividad máxima a la longitud de onda de Bragg (Anchos de Banda).

donde $L_{r e d}$ se mide en centímetros y $\Delta f_{-3 d B e}$ en gigahercios. Por otro lado, la expresión exacta del ancho de banda entre ceros es la siguiente:

$$
\Delta \delta L_{\text {ceros }}=2 \sqrt{\left(k_{o} L\right)^{2}+\pi^{2}}
$$

Además del ancho de banda de la red de difracción, otro parámetro muy importante es el nivel y la posición de los lóbulos secundarios de la reflectividad de la red de difracción. En la tabla 5.2 se muestran las posiciones y niveles de los tres primeros lóbulos secundarios de las respuestas frecuenciales de las mismas redes de difracción con acoplo y periodo constante de la tabla 5.1. Como se puede comprobar en dicha tabla, si se desea que el filtro formado por la red de difracción presente unas pérdidas de inserción reducidas (reflectividad alta), los lóbulos secundarios de la respuesta en frecuencia son inaceptablemente elevados. Para conseguir altas reflectividades junto a lóbulos secundarios reducidos es necesario acudir a redes de difracción con acoplo variable; en concreto, con transiciones más suaves en los extremos de la red de difracción.

\section{Redes de difracción con periodo constante y acoplo variable}

Es conocido que para reflectividades bajas, el coeficiente de reflexión de una red de difracción en función de la desviación de frecuencia $(\rho(\delta))$ es, salvo ciertas constantes de fase, la transformada de Fourier de la función $k_{\phi}(z)=k(z) e^{j \phi(z)}$, en concreto:

$$
\rho(\delta L) \approx e^{-j\left(\frac{\pi}{2}+\delta L+\phi\left(\frac{-L}{2}\right)\right)} K_{\Phi}(2 \delta)
$$

donde $K_{\Phi}$ es la transformada de Fourier de $k_{\phi}$ [Kog76]. El resultado anterior, particularizado para el caso de redes de difracción con periodo constante $\phi(z)=$ cte, se reduce a que la respuesta en frecuencia es aproximadamente proporcional a la transformada de la función de apodización, $k(z)$. Por lo tanto, es razonable suponer 


\begin{tabular}{|c|c|c|c|c|c|c|c|}
\hline$|\rho|_{\max }$ & $K o L$ & $\delta L_{\text {sec } 1}$ & $N L S P_{1}$ & $\delta L_{\sec 2}$ & $N L S P_{2}$ & $\delta L_{\sec 3}$ & $N L P S_{3}$ \\
\hline 0,250 & 0,255 & 4,501 & $\begin{array}{c}0,06 \\
-13,1 \mathrm{dBo}\end{array}$ & 7,729 & $\begin{array}{c}0,03 \\
-17,7 \mathrm{dBo}\end{array}$ & 10,907 & $\begin{array}{c}0,02 \\
-20,6 \mathrm{dBo}\end{array}$ \\
\hline 0,500 & 0,549 & 4,527 & $\begin{array}{c}0,12 \\
-12,5 \mathrm{dBo}\end{array}$ & 7,745 & $\begin{array}{c}0,07 \\
-17,0 \mathrm{dBo}\end{array}$ & 10,918 & $\begin{array}{c}0,05 \\
-20,0 \mathrm{dBo}\end{array}$ \\
\hline 0,750 & 0,973 & 4,598 & $\begin{array}{c}0,21 \\
-11,2 \mathrm{dBo}\end{array}$ & 7,786 & $\begin{array}{c}0,12 \\
-15,6 \mathrm{dBo}\end{array}$ & 10,948 & $\begin{array}{c}0,09 \\
-18,6 \mathrm{dBo}\end{array}$ \\
\hline 0,800 & 1,099 & 4,626 & $\begin{array}{c}0,23 \\
-10,8 \mathrm{dBo}\end{array}$ & 7,803 & $\begin{array}{c}0,14 \\
-15,2 \mathrm{dBo}\end{array}$ & 10,959 & $\begin{array}{c}0,10 \\
-18,1 \mathrm{dBo}\end{array}$ \\
\hline 0,900 & 1,472 & 4,728 & $\begin{array}{c}0,30 \\
-9,4 \mathrm{dBo} \\
\end{array}$ & 7,864 & $\begin{array}{c}0,19 \\
-13,7 \mathrm{dBo} \\
\end{array}$ & 11,003 & $\begin{array}{c}0,13 \\
-16,6 \mathrm{dBo} \\
\end{array}$ \\
\hline 0,950 & 1,832 & 4,852 & $\begin{array}{c}0,37 \\
-8,2 \mathrm{dBo}\end{array}$ & 7,939 & $\begin{array}{c}0,23 \\
-12,4 \mathrm{dBo}\end{array}$ & 11,057 & $\begin{array}{c}0,16 \\
-15,2 \mathrm{dBo}\end{array}$ \\
\hline 0,990 & 2,647 & 5,215 & $\begin{array}{c}0,50 \\
-6,0 \mathrm{dBo}\end{array}$ & 8,166 & $\begin{array}{c}0,32 \\
-9,8 \mathrm{dBo}\end{array}$ & 11,221 & $\begin{array}{c}0,23 \\
-12,5 \mathrm{dBo}\end{array}$ \\
\hline 0,999 & 3,800 & 5,885 & $\begin{array}{c}0,64 \\
-3,9 \mathrm{dBo}\end{array}$ & 8,610 & $\begin{array}{c}0,44 \\
-7,2 \mathrm{dBo}\end{array}$ & 11,548 & $\begin{array}{c}0,33 \\
-9,7 \mathrm{dBo}\end{array}$ \\
\hline
\end{tabular}

Tabla 5.2: Resultados de simulación de la posición y nivel de los tres primeros lóbulos secundarios de la reflectividad para redes de difracción con acoplo y periodo constante en función de la reflectividad máxima a la longitud de onda de Bragg.

que incluso para redes con altas reflectividades, la utilización de funciones de apodización cuyas transformadas presenten lóbulos secundarios reducidos debería ofrecer redes de difracción con respuesta espectral con lóbulos secundarios de bajo nivel. Teniendo cuenta este resultado, se podrán aprovechar los resultados ya conocidos del enventanado de respuestas impulsionales de la teoría de filtros para conseguir reducir el nivel de los lóbulos secundarios hasta niveles aceptables. Además, se puede resaltar que cuando el periodo es constante, la reflectividad es simétrica respecto a $\delta=0[\operatorname{Kog} 76]$.

En la literatura, se ha analizado el efecto de estos enventanados, llegando a obtenerse unos lóbulos secundarios de -32 dBo para una función de apodización tipo coseno alzado, y de -74 dBo para apodización tipo Kaizer [Cro77]. En los casos de utilizar función de apodización, se puede estimar el ancho de banda a partir de obtener una longitud efectiva de la red de difracción, ponderada según la función de apodización. 


\subsubsection{Redes de difracción con periodo variable}

Las redes de difracción con periodo variable ${ }^{\mathrm{i}}$ se han aplicado satisfactoriamente a sistemas de compensación de la dispersión, limitando la variación del periodo al caso particular de variación lineal. Hasta 1997, no se conocía ninguna aplicación de redes de difracción con periodo variable a redes conformadoras de haces para agrupaciones de antenas; siendo entonces, cuando se presentaron simultáneamente las primeras referencias [Cru97, Cor97b, Rom97], entre ellas la correspondiente a resultados intermedios de esta Tesis Doctoral, la cual constituye el primer ejemplo de red óptica conformadora de haces basada en redes de difracción con periodo variable.

\section{Propiedades de las redes de difracción con periodo variable}

A continuación, se describe de una forma intuitiva el funcionamiento de una red de difracción con periodo variable, teniendo en cuenta que el comportamiento preciso de la red de difracción depende tanto de la variación del periodo, $\Lambda(z)$, como de la función de acoplo, $k(z)$, o de la longitud de la red, $L_{r e d}$ y se puede conocer a partir de la resolución numérica de las ecuaciones de acoplo de modos [Kog76]. $\mathrm{Su}$ comportamiento es similar al que se obtiene con un filtro de onda acústica de superficie $e^{\mathrm{ii}}$; se basa en que el periodo espacial del índice de refracción de la fibra no es constante, sino función de la distancia $(\Lambda=\Lambda(z))$, con lo que la longitud de onda de Bragg varía también con la distancia y por lo tanto el acoplamiento máximo entre las dos ondas contradireccionales se produce en diferentes puntos de la red, en función de la longitud de onda de la luz incidente. Consecuentemente, cada portadora óptica incidente en la red de difracción, se verá reflejada en una posición diferente, en función de la variación de la longitud de onda de Bragg a lo largo de la red, recorriendo diferentes caminos de ida y vuelta, y por lo tanto, serán diferentes los retardos de propagación asociados a cada longitud de onda (Fig. 5.3). Con un apropiado diseño del periodo espacial de la red de difración se puede conseguir la función retardo $\Leftrightarrow$ longitud de onda deseada. Por ejemplo, en redes de difracción con variación lineal de la frecuencia espacial, el retardo asociado con cada longitud de onda sería función lineal de la longitud de onda de la portadora.

De esta forma, con una red de difracción con periodo variable se puede conseguir un retardo continuamente variable, simplemente modificando la frecuencia óptica de la portadora.

A partir de la fase de la respuesta frecuencial de la red de difracción, $\theta_{H}(f)$, se

\footnotetext{
${ }^{\mathrm{i}}$ Las redes de difracción con variación de la frecuencia espacial de las perturbaciones en el índice de la fibra son más conocidas por su denominación inglesa, como redes con chirp o chirped fiber gratings.

ii Surface Acoustic Wave (SAW), en terminología inglesa.
} 


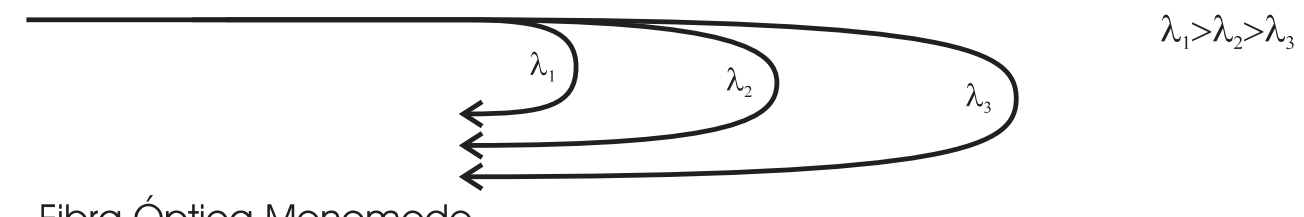

Fibra Óptica Monomodo

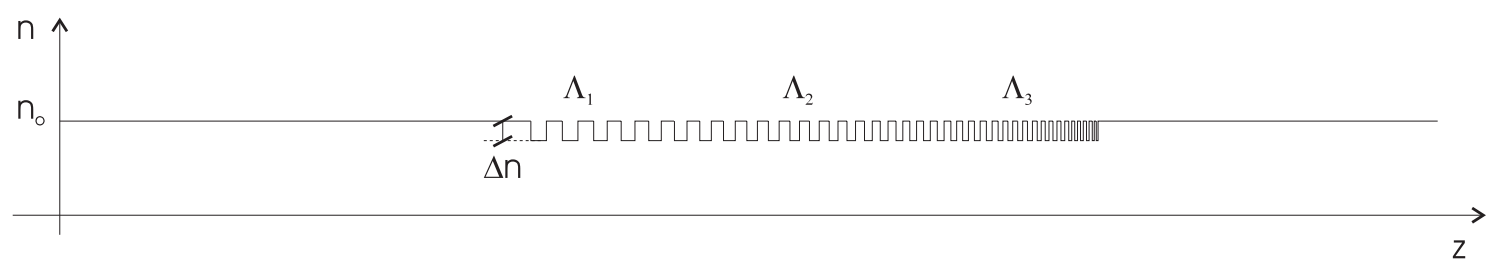

Figura 5.3: Ejemplo de red de difracción con periodo espacial variable mostrando como el retardo introducido es proporcional a la distancia.

puede obtener el retardo de grupo de la misma como:

$$
\tau(f)=-\frac{1}{2 \pi} \frac{d \theta_{H}(f)}{d f}
$$

En la figura 5.4 se muestra una respuesta típica de una red de difracción con acoplo constante y variación lineal de la frecuencia espacial. A partir de la gráfica del retardo de grupo en función de la longitud de onda se puede concluir que el comportamiento no constante del mismo va evidentemente asociado a una dispersión, la cual será positiva o negativa según el extremo por el que se acceda a la red de difracción.

\section{Redes de difracción con variación lineal de la frecuencia espacial}

En este apartado, se pasa a estudiar el caso particular de redes de difracción con variación lineal de la frecuencia espacial. La elección de este caso particular, se basa, por un lado, en que es el mejor analizado en la literatura, y por otro lado, a la mayor disponibilidad de dispositivos, sin necesidad de recurrir a la fabricación de redes de difracción a medida, gracias a su aplicación a redes compensadoras de dispersión para sistemas de comunicaciones ópticas.

Considerando una red de difracción de longitud total $L_{\text {red }}$ situada a lo largo del eje $\mathrm{z}$ entre las coordenadas $z=-L_{\text {red }} / 2$ y $z=L_{\text {red }} / 2$, se define la variación de la frecuencia espacial respecto a la frecuencia espacial en el centro de la red $\left(K_{o}, z=0\right)$ como:

$$
\Delta K(z)=\frac{2 F z}{L_{r e d}^{2}}
$$



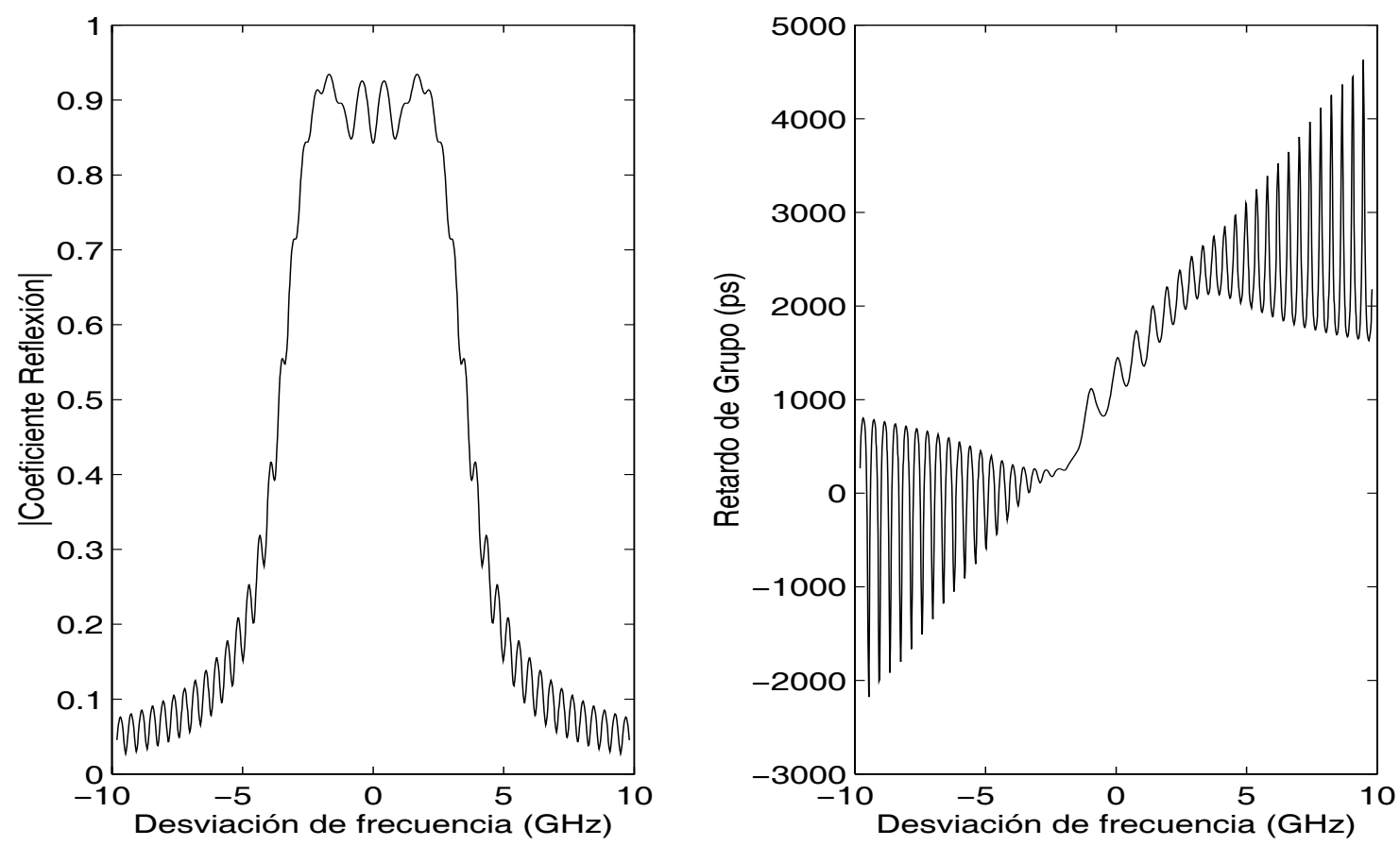

Figura 5.4: Ejemplo de respuesta en frecuencia de una red de difracción de longitud $L_{\text {red }}=25 \mathrm{~cm}$, con acoplo constante $K_{o} L=5 \mathrm{y}$ función de fase lineal con factor $F=50$. a) Magnitud de la función de transferencia en reflexión. b) Retardo de grupo de la función de transferencia en reflexión.

donde $F$ se define como el factor de la variación lineal de la frecuencia espacial. A partir de las ecuaciones (5.2) y (5.14) se puede obtener la expresión de la función de fase como:

$$
\phi(z)=F\left(\frac{z}{L_{r e d}}\right)^{2}
$$

De acuerdo con (5.14) el periodo de la red de difracción valdrá:

$$
\Lambda(z)=\frac{2 \pi}{K(z)}=\frac{2 \pi}{K_{o}+\frac{2 F z}{L_{r e d}^{2}}} \approx \frac{2 \pi}{K_{o}}\left(1-\frac{2 F z}{L_{r e d}^{2} K_{o}}\right)=\Lambda_{o}\left(1-\frac{F z \Lambda_{o}}{\pi L_{r e d}^{2}}\right)
$$

donde $\Lambda_{o}=2 \pi / K_{o}$ es el periodo de las perturbaciones en el centro de la red de difracción. A partir del periodo espacial, es posible calcular la longitud de onda de Bragg local, según:

$$
\lambda_{B R A G G}(z)=2 n_{o} \Lambda(z) \approx 2 n_{o} \Lambda_{o}\left(1-\frac{F z \Lambda_{o}}{\pi L_{r e d}^{2}}\right)=\lambda_{o}\left(1-\frac{F z \Lambda_{o}}{\pi L_{r e d}^{2}}\right)
$$

donde $n_{o}$ es el índice de refracción medio de la fibra y $\lambda_{o}=2 n_{o} \Lambda_{o}$ es la longitud de onda de Bragg correspondiente a una frecuencia espacial de $K_{o}$ y que se cumple 
para $z=0$. Por otro lado, el retardo de propagación de ida y vuelta respecto a $z=-L_{\text {red }} / 2$ en función de la posición de reflexión $(z)$ es:

$$
\tau(z)=2 n_{o} \frac{z+L_{r e d} / 2}{c}
$$

donde $c$ es la velocidad de la luz en el vacío. De acuerdo con (5.18) el margen de variación del retardo es:

$$
\Delta \tau_{M A X}=\frac{2 n_{o} L_{r e d}}{c}
$$

Asumiendo que una onda incidente de longitud de onda $\lambda$ se refleja básicamente en aquella posición del eje $\mathrm{z}$ para la que $\lambda \approx \lambda_{B R A G G}(z)$, se puede asignar un retardo de ida y vuelta para cada longitud de onda combinando (5.17) y (5.18):

$$
\tau=\tau_{o}-\left(\frac{4 n_{o}^{2} \pi L_{r e d}^{2}}{c F \lambda_{o}^{2}}\right) \Delta \lambda \quad \Delta \lambda \in\left[-\frac{F \lambda_{o}^{2}}{4 n_{o} \pi L_{r e d}}, \frac{F \lambda_{o}^{2}}{4 n_{o} \pi L_{r e d}}\right]
$$

donde $\tau_{o}=n_{o} L_{\text {red }} / c$ es el retardo correspondiente a $\lambda_{o}$ y $\Delta \lambda$ es la desviación de longitud de onda respecto a $\lambda_{o}$, siendo el ancho de banda de la red:

$$
\Delta \lambda_{\text {red }}=\frac{F \lambda_{o}^{2}}{2 n_{o} \pi L_{r e d}}
$$

Finalmente, (5.20) permite obtener la pendiente del retardo en función de la desviación de la longitud de onda como:

$$
\delta=\frac{\Delta \tau}{\Delta \lambda}=-\frac{4 n_{o}^{2} \pi L_{r e d}^{2}}{c F \lambda_{o}^{2}}
$$

Ecuaciones de diseño A partir de las fórmulas anteriores es posible establecer unas ecuaciones de diseño de redes de difracción con periodo lineal y sin apodización, que relacionen los parámetros de diseño de la red $\left(L_{r e d}, F\right)$ con las características de retardo esperado $\left(\tau_{M A X}, \Delta \lambda, \delta\right)$ :

- En función del retardo máximo deseado se elige la longitud de la red:

$$
L_{\text {red }}=\frac{c \tau_{M A X}}{2 n_{o}}
$$

- Para elegir el factor $\mathrm{F}$, dependiendo de cual sea la especificación $(\Delta \lambda$ o $\delta)$ se usará una de las ecuaciones siguientes:

$$
\begin{gathered}
F=\left(\frac{\Delta \lambda}{\lambda_{o}}\right) 2 \pi n_{o}\left(\frac{L_{r e d}}{\lambda_{o}}\right) \\
F=-\frac{4 n_{o}^{2} \pi}{c \delta}\left(\frac{L_{r e d}}{\lambda_{o}}\right)^{2}
\end{gathered}
$$

Por ahora, no se ha tenido en cuenta el acoplo, cuyo efecto debería ser similar al que presenta en las redes con frecuencia espacial constante, aunque aumentado. 
Efecto de la apodización en redes de difracción con variación lineal de la frecuencia espacial

Desgraciadamente, el diseño de una red de difracción con frecuencia espacial variable no consiste simplemente en la variación del periodo de las perturbaciones en la red, sino que al igual que en las redes con periodo constante, la elección de la función de apodización tiene una influencia decisiva en la aparición de lóbulos secundarios y de rizado en la respuesta frecuencial (amplitud y retardo de grupo) de la red de difracción.

En la figura 5.4 se muestra la respuesta de una red de difracción con acoplo constante, donde se puede comprobar el inaceptable nivel de rizado obtenido, tanto en la respuesta en magnitud, como en el retardo. El diseño de funciones de apodización óptimas, en cuanto a la linealidad de la respuesta de retardo, ha sido analizada profundamente en la literatura y los resultados obtenidos son extrapolables a la aplicación de agrupaciones de antenas [PA96].

\subsection{Líneas de retardo basadas en redes de difrac- ción}

\subsubsection{Líneas de retardo programable basadas en redes de difracción con periodo constante}

En 1994, se propuso la primera aplicación de redes de difracción para líneas de retardo sobre fibra óptica, basada en la utilización de redes de difracción de banda estrecha con periodo constante [Bal94, Lem94]. Ésta ha sido hasta ahora, la única aplicación de las redes de difracción a redes conformadoras de haces para antenas de agrupación, para la cual se han propuesto en los últimos años diversas arquitecturas.

En la figura 5.5 se muestra como es posible obtener sobre fibra una línea de retardo en radiofrecuencia, programable y dependiente de la longitud de onda, a partir de una agrupación de redes de difracción con periodo constante. El dispositivo mostrado en la figura 5.5 consiste en un circulador óptico conectado con una cierta longitud de fibra monomodo, sobre la cual se han grabado una serie de redes de difracción con periodo constante. Cada una de las redes de difracción está sintonizada a una longitud de onda diferente y por lo tanto, idealmente, refleja únicamente una banda estrecha de longitudes de onda, es decir, la red número 1 refleja un cierto ancho de banda alrededor de $\lambda_{1}$, la red número 2 alrededor de $\lambda_{2}$ y así sucesivamente. De esta forma, se pueden obtener diferentes retardos mediante la sintonización de la frecuencia óptica portadora, este retardo sufrido por la señal óptica se traslada a la señal moduladora obtenida tras la detección. La posición de la red de difracción seleccionada determinada el retardo sufrido por la señal moduladora de radiofrecuencia, 


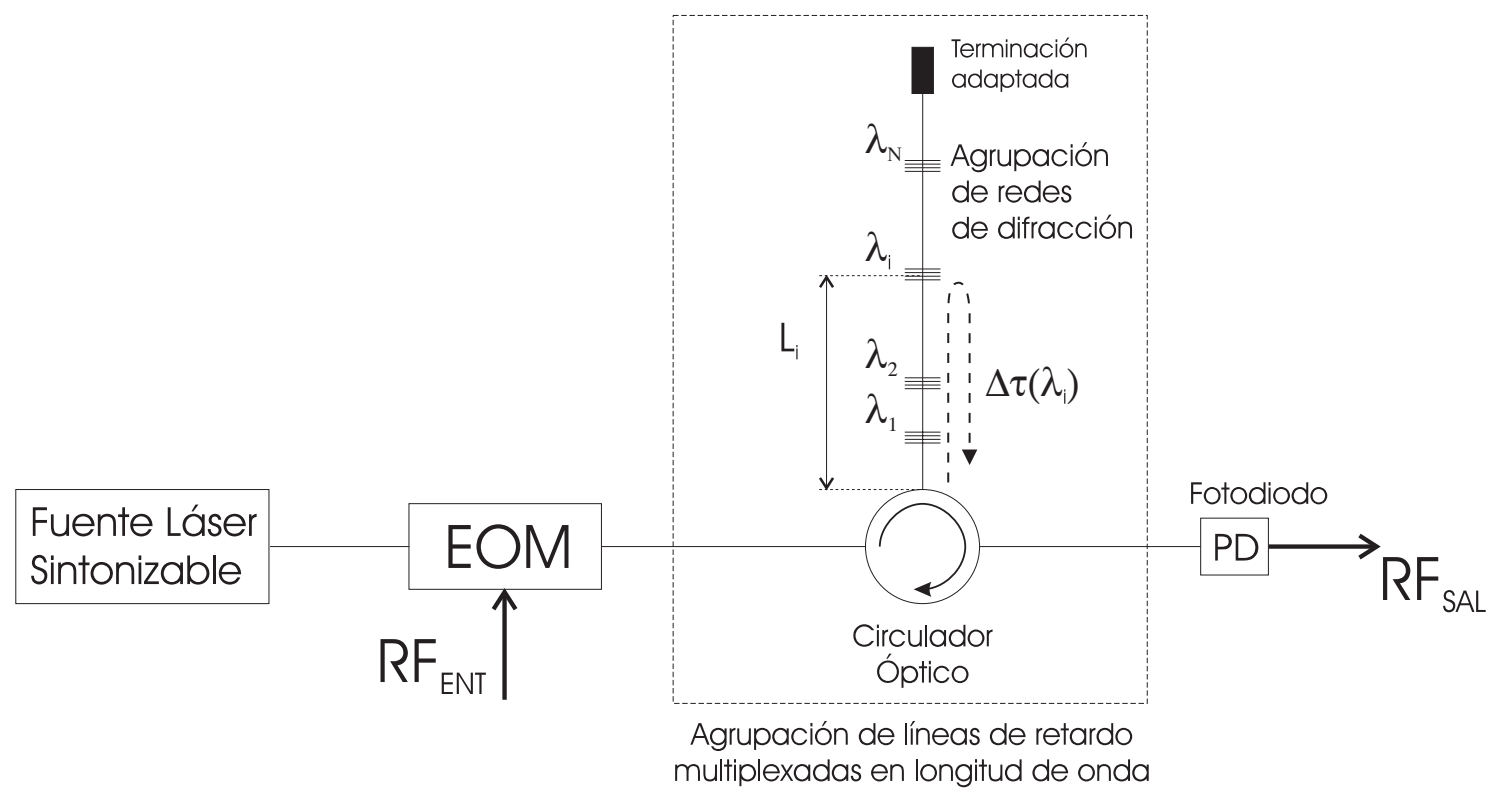

Figura 5.5: Ejemplo de línea de retardo basada en el uso de redes de difracción discretas.

siendo el retardo asociado a la red i-ésima:

$$
\Delta \tau\left(\lambda_{i}\right)=\frac{2 L_{i}}{n_{o} c}
$$

Gracias a que todas las redes de difracción se fabrican sobre la misma fibra, no son necesarios conectores ni conmutadores entre ellas. Es por esto, que a diferencia de otras estructuras para la generación de retardos verdaderos, en la basada en redes de difracción discretas, las pérdidas de inserción son básicamente independientes del número de retardos a implementar, gracias a la transmisión prácticamente total de las redes de difracción fuera de su banda de reflexión. Por consiguiente, las pérdidas asociadas a la agrupación de líneas de retardo de la figura 5.5 serán las propias del circulador óptico (alrededor de 2-3 dB) más las debidas a la reflectividad de la red de difracción seleccionada, tan alta como se desee con las limitaciones respecto a los lóbulos secundarios indicadas en el apartado 5.2.1.

Resumiendo, mediante una selección discreta de la longitud de onda, se consigue una selección discreta del retardo que sufrirá la señal moduladora. La precisión de los retardos conseguidos dependerá de la exactitud en el posicionamiento de las redes de difracción durante la fabricación, mientras que la resolución de los retardos dependerá de la longitud física de las redes y de la mínima separación entre dos redes contiguas. Esta separación es habitualmente del orden de dos veces la longitud de cada red de difracción [Mol95].

El número máximo de retardos obtenibles mediante esta técnica viene definido por el ancho de banda de la fuente sintonizable y por el ancho de banda de cada red de difracción, teniendo en cuenta un margen entre longitudes de onda contíguas, 


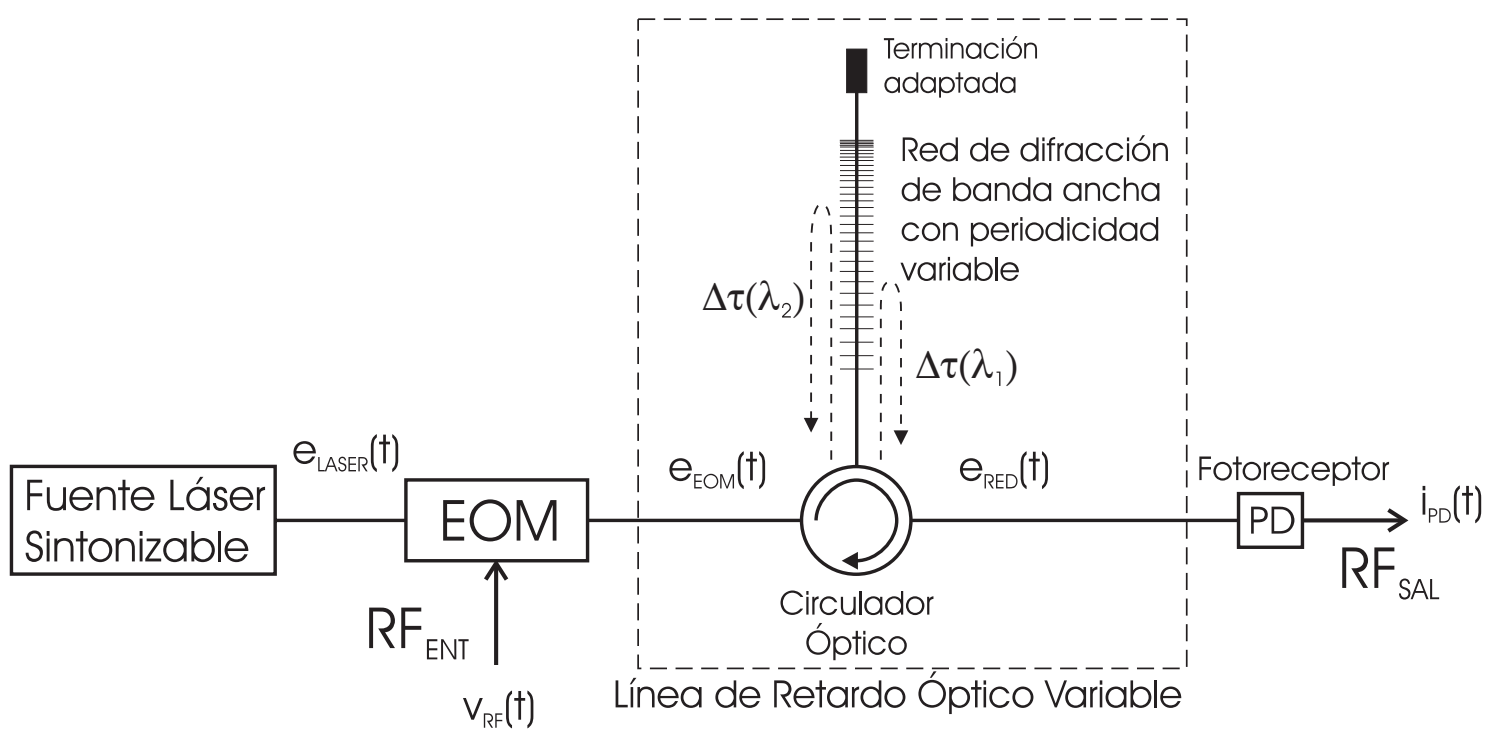

Figura 5.6: Ejemplo de línea de retardo basada en el uso de redes de difracción con periodo variable.

suficiente para reducir el efecto de posibles lóbulos secundarios de las respuestas frecuenciales de las redes de difracción.

\subsubsection{Líneas de retardo continuamente variables basadas en redes de difracción con periodo variable}

Como ya se comentó en la sección 5.2, una red de difracción con periodo variable permite obtener un retardo óptico variable, dependiente de la longitud de onda de la señal óptica. En el caso más habitual de variación lineal del periodo, se consigue una variación lineal del retardo sufrido por la señal con respecto a la longitud de onda. Una forma de conseguir una línea de retardo variable de radiofrecuencia basada en una red de difracción con estas características se muestra en la figura 5.6. En este esquema, una señal óptica contínua generada por un láser sintonizable es modulada en intensidad por una señal de radiofrecuencia. La señal óptica modulada sufrirá un retardo dependiente de la longitud de onda de la señal óptica portadora; retardo que se trasladará a la señal moduladora tras la detección.

La técnica más sencilla para la generación y distribución de señales ópticas moduladas por señales de radiofrecuencia es la modulación de intensidad (IM), ya sea de forma externa (tal y como se muestra en la figura 5.6) o interna a la fuente láser. Generalmente, se prefiere la modulación externa por el efecto, indeseado en la mayoría de casos, de modulación de la frecuencia del láser que aparece en los esquemas con modulación interna. Como se verá más adelante, esta segunda opción implica un peor rendimiento y una menor flexibilidad a la hora del tipo de modulación elegido, por lo que salvo en los casos en los que expresamente se indique lo contrario, en general, se trabajará con modulación externa. 


\subsubsection{Limitaciones en el uso de una red de difracción con periodo lineal como elemento de retardo verdadero en sistemas $\mathrm{IM} / \mathrm{DD}^{\mathrm{i}}$.}

En este apartado se tratarán únicamente las limitaciones inherentes a la propia estructura de la línea de retardo basada en redes de difracción con periodo lineal, considerando todos los elementos ideales. En posteriores apartados se tendrán en cuenta las limitaciones introducidas por la no idealidad de los componentes que forman la línea de retardo mostrada en la figura 5.6, tales como la inestabilidad en la frecuencia de oscilación de la fuente láser o el rizado en la respuesta en frecuencia de la red de difracción, tanto en módulo como en fase, es decir, tanto en reflectividad como en retardo de grupo.

\section{Análisis de los componentes de la línea de retardo contínuamente variable}

A continuación se analizarán los diferentes componentes que forman la figura 5.6, definiendo su comportamiento ideal.

Láser sintonizable Se trata de una fuente de señal óptica contínua cuya frecuencia de oscilación es variable dentro de un cierto ancho de banda, que por lo menos incluirá totalmente el ancho de banda de la red de difracción. En el caso más general, la potencia de la señal de salida también será sintonizable.

Modulador externo El modulador electro-óptico (EOM $\left.{ }^{\mathrm{ii}}\right)$ externo más conocido y utilizado es el modulador interferométrico Mach-Zehnder. Este modulador se basa en las propiedades electroópticas de materiales como el $\mathrm{LiNbO}_{3}$, cuyo índice de refracción puede modificarse al aplicar una tensión externa sobre el material. El funcionamiento de un modulador interferométrico Mach-Zehnder (Fig. 5.7) parte de la división de la señal óptica de entrada en dos caminos; caminos cuyas inserciones de fase son dependientes de unas tensiones eléctricas aplicadas externamente $\left(V_{1}\right.$, $V_{2}$ ). Variando las tensiones aplicadas a los electrodos conectados a ambos caminos, variarán sus índices de refracción y consecuentemente se variará el desfase sufrido por la señal óptica en su recorrido por ellos. A la salida del modulador interferométrico las señales provenientes de ambos caminos se combinan coherentemente de forma que en ausencia de tensiones externas, los campos ópticos en las dos ramas del interferómetro sufren un desfase idéntico e interfieren constructivamente. La interferencia de ambas señales sería constructiva si la diferencia de desfases entre ambos caminos fuera un múltiplo de $2 \pi$ y sería destructiva si el desfase diferencial fuera un múltiplo impar de $\pi$.

Si a la entrada del modulador mostrado en la figura 5.7 se tiene un campo

\footnotetext{
${ }^{\mathrm{i}}$ Intensity Modulation with Direct Detection, Se trata del enlace más sencillo de comunicaciones por fibra óptica, implica modulación de la intensidad óptica y detección directa de la potencia óptica.

${ }^{\text {ii }}$ Electro-Optical Modulator.
} 


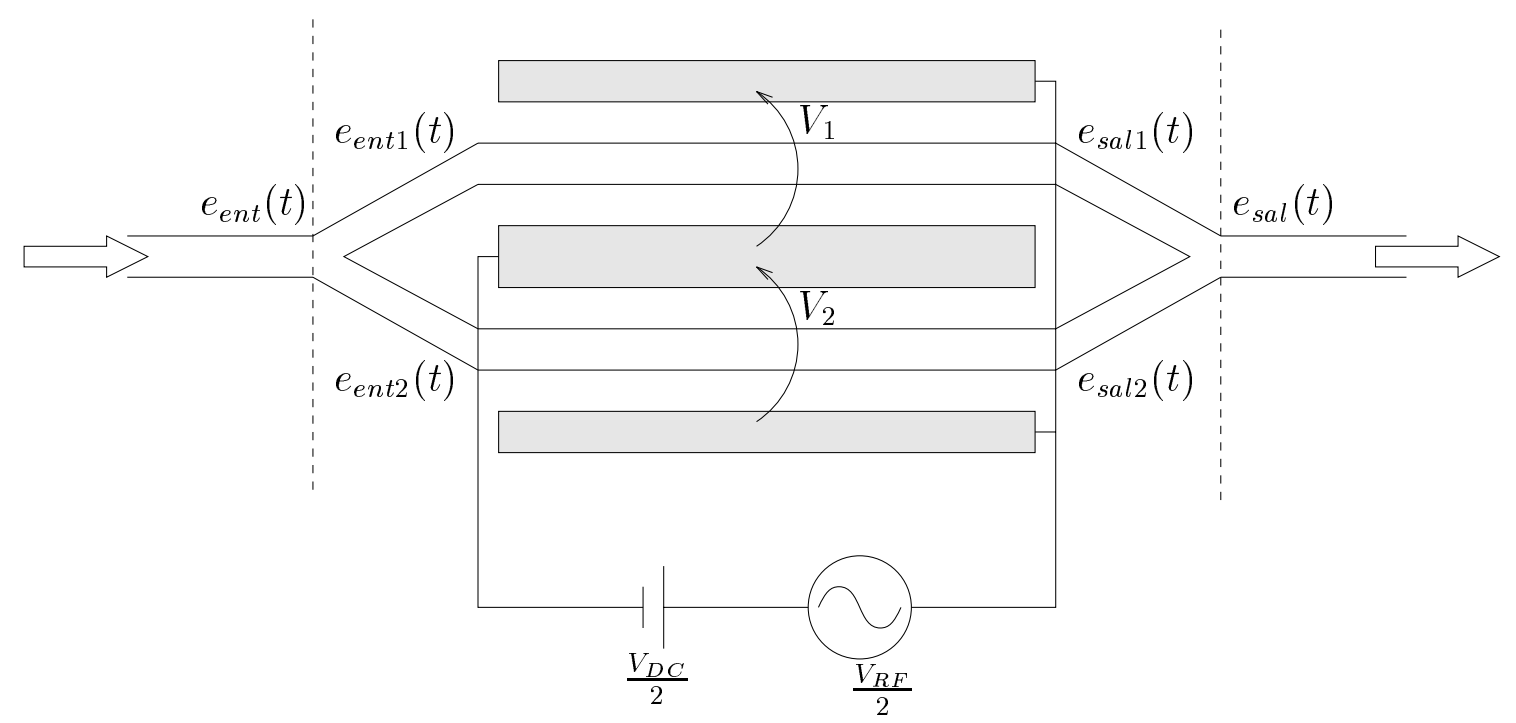

Figura 5.7: Esquema de modulador interferométrico Mach-Zehnder con alimentación simétrica.

óptico $e_{\text {ent }}(t)=A \cos \left(\omega_{c} t+\phi_{o}\right)$ y asumiendo divisiones y combinaciones de potencias ideales, se puede obtener la siguiente expresión para el campo óptico a la salida:

$$
e_{s a l}(t)=\frac{A}{2}\left[\cos \left(\omega_{c} t+\phi_{e}+\frac{\pi V_{1}}{V_{\pi}}\right)+\cos \left(\omega_{c} t+\phi_{e}+\frac{\pi V_{2}}{V_{\pi}}\right)\right]
$$

donde $\phi_{e}$ es un término que tiene en cuenta el retardo de propagación $\left(\tau_{\text {prop }}\right)$ en ausencia de tensión externai, mientras que $V_{\pi}$ es la tensión de conmutación o de salto de fase del modulador, es decir aquel valor de tensión que aplicado entre los electrodos del modulador provoca un desfase entre las dos ramas del modulador de $\pi$ radianes.

La potencia óptica media a la salida del modulador se puede calcular a partir de la envolvente de la expresión del campo, obtenida en (5.27), como:

$$
\overline{P_{s a l}}=\frac{\overline{P_{e n t}}}{2}\left[1+\cos \left(\frac{\pi\left(V_{1}-V_{2}\right)}{V_{\pi}}\right)\right]=\frac{\overline{P_{e n t}}}{2}[1+\cos (\Delta \phi)]
$$

o bien:

$$
\overline{P_{\text {sal }}}=\frac{\overline{P_{\text {ent }}}}{2}\left[\cos ^{2}\left(\frac{\Delta \phi}{2}\right)\right]
$$

donde:

$$
\Delta \phi=\frac{\pi\left(V_{1}-V_{2}\right)}{V_{\pi}}=\frac{\pi \Delta V}{V_{\pi}}
$$

En la figura 5.8 se representa la ecuación (5.28) en función de $\Delta V=V_{2}-V_{1}$. En la citada curva se pueden definir tres puntos de polarización como: punto de

$$
{ }^{\mathrm{i}} \phi_{e}=\phi_{o}-\omega_{c} \tau_{\text {prop }}
$$




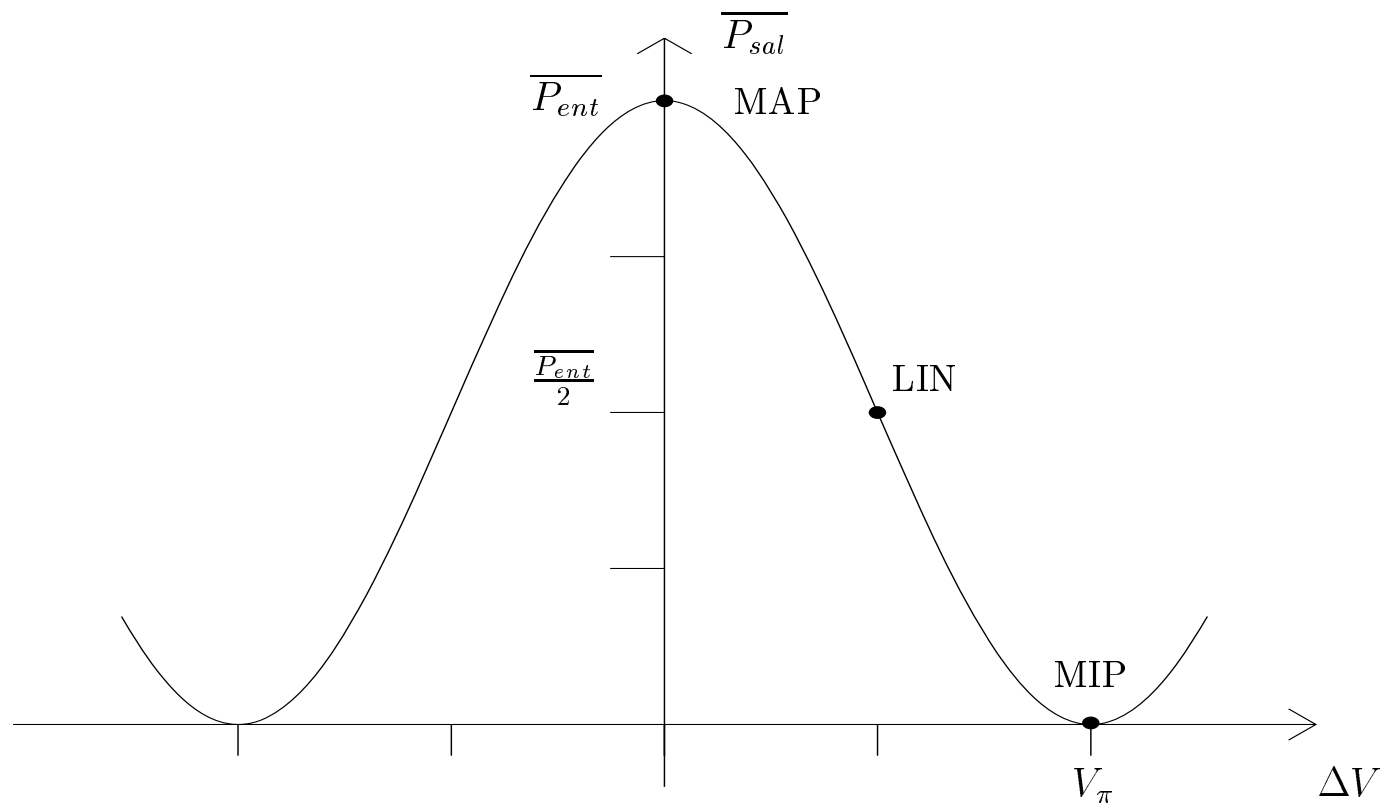

Figura 5.8: Potencia óptica de salida de un modulador Mach-Zehnder en función de la diferencia de tensión entre ambos electrodos.

máxima potencia óptica (MAP) correspondiente a $\Delta V_{D C}=0$; punto de mínima potencia óptica (MIP) correspondiente a $\Delta V_{D C}=V_{\pi}$ y punto de máxima linealidad (LIN) cuando $\Delta V_{D C}=V_{\pi} / 2$.

Circulador Asumiendo un comportamiento ideal, el circulador óptico presenta una transmisión total sin pérdidas entre accesos contiguos en el sentido de giro del mismo, y un aislamiento perfecto con el tercer acceso. Se ha comprobado que es necesario disponer de un aislamiento mayor de $40 \mathrm{~dB}$ para que el comportamiento del circulador no introduzca rizado sobre la respuesta, en magnitud y retardo, de la red de difracción [PA96, RM98].

Red de difracción con variación lineal del periodo Para caracterizar el comportamiento de la red de difracción, bastará con conocer la respuesta frecuencial de la misma $\left(H_{\text {red }}(\omega)\right)$ :

$$
H_{\text {red }}(\omega)=\left|H_{\text {red }}(\omega)\right| e^{j \theta_{\text {red }}(\omega)}
$$

Asumiendo un comportamiento ideal, se supondrá una respuesta frecuencial plana en amplitud y parabólica en fase dentro de la banda de paso de la red de difracción alrededor de $\omega=\omega_{c}$ :

$$
\left\{\begin{array}{l}
\left|H_{\text {red }}(\omega)\right|=1 \\
\theta_{\text {red }}(\omega)=\theta_{0}+\theta_{1}\left(\omega-\omega_{c}\right)+\frac{\theta_{2}}{2}\left(\omega-\omega_{c}\right)^{2}
\end{array}\right.
$$

donde se ha tomado un valor de reflectividad de la red de difracción del $100 \%$. Por otro lado, $\theta_{0}$ y $\theta_{1}$ son respectivamente el desfase y el retardo de grupo para $\omega=\omega_{c}$, mientras que $\theta_{2}$ es el término correspondiente a la dispersión de primer orden y 
equivale a un retardo de grupo lineal con la frecuencia como se puede comprobar en la siguiente expresión:

$$
\tau_{\text {red }}(\omega)=-\frac{d \theta_{r e d}(\omega)}{d \omega}=-\theta_{1}-\theta_{2}\left(\omega-\omega_{c}\right)
$$

La ecuación (5.33) permite comprobar como se puede variar linealmente el retardo introducido por la red de difracción mediante la sintonización de la frecuencia de la fuente óptica.

A partir de la ecuación anterior, resulta evidente que incluso el comportamiento ideal de la red de difracción va a dar lugar a fenómenos de dispersión.

El parámetro $\theta_{2}$ puede relacionarse con la pendiente, $\delta$, del retardo de grupo en función de la desviación en longitud de onda definida en la ecuación (5.22) como:

$$
\theta_{2}=-\frac{d \tau_{r e d}}{d \omega} \approx \frac{\Delta \tau_{r e d}}{\Delta \omega}=\frac{\lambda_{c}^{2}}{2 \pi c} \frac{\Delta \tau_{r e d}}{\Delta \lambda}=\frac{\lambda_{c}^{2} \delta}{2 \pi c}
$$

Fotodetector El comportamiento de un fotodetector ideal funcionando dentro de su ancho de banda de trabajo viene caracterizado por la responsividad del mismo, $\mathcal{R}$, de forma que la corriente eléctrica de salida es proporcional a la envolvente de la potencia óptica incidente $i_{P D}(t)=\mathcal{R} \overline{P_{o p t i c a}}(t)$.

\section{Limitaciones}

Una vez se han definido comportamientos ideales de los componentes de la línea de retardo de la figura 5.6, las limitaciones a su utilización vendrán impuestas por la dispersión de la red de difracción; así como por el efecto conjugado de esta dispersión con la distorsión introducida por los elementos no lineales de la línea de retardo, el modulador interferométrico y el fotodetector ${ }^{\mathrm{i}}$.

La dispersión generada por el elemento retardador crea dos problemas:

1. Atenuación dispersiva, debida al salto de fase entre las bandas laterales ópticas superior e inferior.

2. Distorsión inducida por la dispersión ${ }^{\mathrm{ii}}$.

A continuación pasan a estudiarse ambos efectos, a partir de la línea de retardo variable mostrada en la figura 5.6, asumiendo un comportamiento ideal de los componentes que la forman, tal y como se han definido en el apartado anterior.

\footnotetext{
${ }^{\mathrm{i}}$ Como se verá más adelante, en condiciones ideales, el efecto de la distorsión de los elementos no lineales puede considerarse prácticamente despreciable para sistemas IM/DD sin dispersión, con moduladores interferométricos polarizados en LIN y bajos índices de modulación.

${ }^{\text {ii }}$ Dispersion-induced distortion, en terminología inglesa.
} 


\section{Atenuación dispersiva en redes de difracción con periodo variable}

La principal limitación al uso de redes de difracción con periodo espacial variable como unidades de retardo verdadero variable de banda ancha es la degradación de la potencia de radiofrecuencia debida a la dispersión cromática introducida por la red de difracción.

En la modulación de intensidad convencional y debido a la no linealidad del proceso de modulación, la portadora óptica se modula para generar un campo óptico con un espectro formado por la portadora y dos bandas laterales formadas por múltiples armónicos de la señal moduladora. Para el caso de moduladores trabajando en su zona más lineal e índices de modulación bajos, las rayas espectrales significativas se reducen a tres: portadora, fundamental en banda lateral superior y fundamental en banda lateral inferior (Modulación en Amplitud convencional, AM). En el receptor óptico (Detección Directa), se generan dos señales de batido, correspondientes al batido de cada una de las bandas laterales con la portadora óptica; estas señales de batido interfieren constructivamente para formar una única componente a la frecuencia moduladora.

Por otro lado, si la señal óptica modulada se hace pasar a través de una red de difracción con periodo variable, la dispersión cromática de la misma provocará que cada componente espectral experimente diferentes retardos en función de la frecuencia de la señal moduladora y de las características de dispersión de la red de difracción (el parámetro $\theta_{2}$ o $\delta$ para el caso de variación lineal). A la salida del detector óptico, esta diferencia de retardos provoca un desfase entre las señales de batido, lo cual produce una degradación en la potencia de la señal compuesta, al dejar de ser totalmente constructiva la interferencia entre ambas señales. A medida que aumenta la frecuencia de la señal moduladora, aumenta la separación de ambas bandas laterales y el efecto de la dispersión es aún mayor, llegándose para un valor determinado a una anulación total de la potencia de la señal de radiofrecuencia, al encontrarse en contrafase las dos señales de batido correspondientes.

Asumiendo un comportamiento ideal de la fuente sintonizable, el campo óptico a la entrada del modulador interferométrico Mach-Zehnder tendrá la expresión:

$$
e_{\text {laser }}(t)=A_{o} \cos \left(\omega_{c} t+\phi_{o}\right)
$$

Si además, se considera que la señal de entrada al EOM es un tono de frecuencia $f_{R F}$ más un nivel de tensión fijo de polarizacion, $v_{R F}(t)=V_{D C}+V_{R F} \operatorname{sen}\left(\omega_{R F} t+\right.$ $\left.\phi_{R F}\right)$, y que esta señal se aplica simétricamente a ambos electrodos del modulador interferométrico ${ }^{\mathrm{i}}$ a partir de la ecuación (5.27) se obtiene la expresión del campo

\footnotetext{
${ }^{i} \mathrm{Si}$ las tensiones aplicada a ambos electrodos son $V_{1}$ y $V_{2}$, se cumplirá $V_{1}=\frac{v_{R F}(t)}{2}$ y $V_{2}=$ $-\frac{v_{R F}(t)}{2}$.
} 
óptico a la salida del modulador indicada en la ecuación (5.36):

$$
\begin{aligned}
e_{\text {eom }}(t)=\frac{A_{e}}{2}[\cos ( & \left.\omega_{c} t+\phi_{e}+\frac{\pi V_{D C}}{2 V_{\pi}}+\frac{\pi V_{R F}}{2 V_{\pi}} \operatorname{sen}\left(\omega_{R F} t+\phi_{R F}\right)\right) \\
& \left.+\cos \left(\omega_{c} t+\phi_{e}-\frac{\pi V_{D C}}{2 V_{\pi}}-\frac{\pi V_{R F}}{2 V_{\pi}} \operatorname{sen}\left(\omega_{R F} t+\phi_{R F}\right)\right)\right]
\end{aligned}
$$

donde $A_{e} \mathrm{y} \phi_{e}$ difieren de $A_{o} \mathrm{y} \phi_{o}$ de acuerdo, respectivamente, con las pérdidas y la fase de inserción del modulador. Simplificando:

$$
e_{\text {eom }}(t)=A_{e} \cos \left(\omega_{c} t+\phi_{e}\right) \cos \left(\frac{\pi V_{D C}}{2 V_{\pi}}+\frac{\pi V_{R F}}{2 V_{\pi}} \operatorname{sen}\left(\omega_{R F} t+\phi_{R F}\right)\right)
$$

Con el fin de identificar las diferentes componentes espectrales de la señal modulada, primeramente se ha calculado el desarrollo en serie de Fourier del segundo factor de la ecuación (5.37), obteniéndose el siguiente resultado:

$$
e_{\text {eom }}(t)=A_{e} \cos \left(\omega_{c} t+\phi_{e}\right) \sum_{n=-\infty}^{\infty} J_{n}\left(\frac{\pi V_{R F}}{2 V_{\pi}}\right) \cos \left(n \omega_{R F} t+\frac{\pi V_{D C}}{2 V_{\pi}}+n \phi_{R F}\right)
$$

donde $J_{n}(\beta)$ es la función de Bessel de primera especie de orden $n$ y con argumento $\beta$.

Desarrollando la ecuación (5.38):

$$
\begin{aligned}
e_{\text {eom }}(t)=\frac{A_{e}}{2} & \sum_{n=-\infty}^{\infty} J_{n}\left(\frac{m_{o}}{2}\right) \cos \left(\omega_{c} t+n \omega_{R F} t+\phi_{e}+n \phi_{R F}+\frac{m_{d c}}{2}\right) \\
& +\frac{A_{e}}{2} \sum_{n=-\infty}^{\infty} J_{n}\left(\frac{m_{o}}{2}\right) \cos \left(\omega_{c} t-n \omega_{R F} t+\phi_{e}-n \phi_{R F}-\frac{m_{d c}}{2}\right)
\end{aligned}
$$

donde $m_{o}=\frac{\pi V_{R F}}{V_{\pi}}$ y $m_{d c}=\frac{\pi V_{D C}}{V_{\pi}}$ son, respectivamente, el índice de modulación de la intensidad óptica y el índice de polarización.

Tras aplicar en el segundo sumatorio un cambio de variable $(m=-n)$ y la propiedad de simetría de las funciones de Bessel $J_{-n}(\beta)=(-1)^{n} J_{n}(\beta) \forall n \in \mathbb{Z}$, se obtiene:

$$
\begin{aligned}
& e_{\text {eom }}(t)=\frac{A_{e}}{2} \sum_{n=-\infty}^{\infty} J_{n}\left(\frac{m_{o}}{2}\right) {\left[\cos \left(\omega_{c} t+n \omega_{R F} t+\phi_{e}+n \phi_{R F}+\frac{m_{d c}}{2}\right)\right.} \\
&\left.+(-1)^{n} \cos \left(\omega_{c} t+n \omega_{R F} t+\phi_{e}+n \phi_{R F}-\frac{m_{d c}}{2}\right)\right]
\end{aligned}
$$

Aplicando propiedades trigonométricas ${ }^{\mathrm{i}}$ y reagrupando términos se obtiene la expresión final del campo óptico a la salida de un modulador electro-óptico simétrico:

$$
e_{e o m}(t)=A_{e} \sum_{n=-\infty}^{\infty} J_{n}\left(\frac{m_{o}}{2}\right) C_{d c}(n) \cos \left(\omega_{c} t+n \omega_{R F} t+\phi_{e}+n \phi_{1}\right)
$$

\footnotetext{
${ }^{\mathrm{i}}(-1)^{n} \cos (x)=\cos (x+n \pi)$.
} 
donde $\phi_{1}=\phi_{R F}+\frac{\pi}{2}$ y $C_{d c}(n)=\cos \left(\frac{m_{d c}}{2}-\frac{n \pi}{2}\right)$.

Para un circulador ideal y aplicando la respuesta en frecuencia definida en (5.31) al campo óptico a la salida del modulador (ecuación (5.41)) se obtiene la siguiente expresión para el campo a la salida de la línea de retardo formada por el circulador y la red de difracción:

$$
\begin{aligned}
& e_{\text {red }}(t)=A_{e} \\
& \sum_{n=-\infty}^{\infty} J_{n}\left(\frac{m_{o}}{2}\right) C_{d c}(n) \cos \left(\omega_{c} t+n \omega_{R F}\left(t+\theta_{1}\right)+\phi_{r e d}+n \phi_{1}+\frac{\theta_{2}}{2} n^{2} \omega_{R F}^{2}\right)
\end{aligned}
$$

donde $\phi_{\text {red }}=\phi_{e}+\theta_{0}$.

Finalmente, para obtener la expresión de la señal eléctrica detectada a la salida del fotoreceptor, $i_{p d}(t)$, es necesario calcular la envolvente de la potencia óptica incidente; para lo cual, a continuación se pasa a descomponer el campo incidente, $e_{\text {red }}(t)$, en las componentes en fase y cuadratura:

$$
\begin{aligned}
e_{r e d}(t) & =A_{e} \cos \left(\omega_{c} t+\phi_{r e d}\right) \\
& \cdot \sum_{n=-\infty}^{\infty} J_{n}\left(\frac{m_{o}}{2}\right) C_{d c}(n) \cos \left(n \omega_{R F}\left(t+\theta_{1}\right)+n \phi_{1}+\frac{\theta_{2}}{2} n^{2} \omega_{R F}^{2}\right) \\
& -A_{e} \operatorname{sen}\left(\omega_{c} t+\phi_{r e d}\right) \\
& \cdot \sum_{n=-\infty}^{\infty} J_{n}\left(\frac{m_{o}}{2}\right) C_{d c}(n) \operatorname{sen}\left(n \omega_{R F}\left(t+\theta_{1}\right)+n \phi_{1}+\frac{\theta_{2}}{2} n^{2} \omega_{R F}^{2}\right)
\end{aligned}
$$

La señal eléctrica que se obtendría a la salida de un fotoreceptor ideal de responsividad $\mathcal{R}$ sería:

$$
\begin{aligned}
& i_{p d}(t)= \mathcal{R} P_{\text {red }}(t)= \\
& A_{p d}\left[\sum_{n=-\infty}^{\infty} J_{n}\left(\frac{m_{o}}{2}\right) C_{d c}(n) \cos \left(n \omega_{R F}\left(t+\theta_{1}\right)+n \phi_{1}+\frac{\theta_{2}}{2} n^{2} \omega_{R F}^{2}\right)\right]^{2} \\
&+A_{p d}\left[\sum_{n=-\infty}^{\infty} J_{n}\left(\frac{m_{o}}{2}\right) C_{d c}(n) \operatorname{sen}\left(n \omega_{R F}\left(t+\theta_{1}\right)+n \phi_{1}+\frac{\theta_{2}}{2} n^{2} \omega_{R F}^{2}\right)\right]^{2}
\end{aligned}
$$

donde $A_{p d}=\mathcal{R} \cdot A_{e}^{2} / 2$. En la ecuación (5.44) aparecen separados los términos correspondientes a las frecuencias de batido de la banda lateral inferior y la superior, cuando, en banda base, ambas bandas laterales dan lugar a las mismas frecuencias, con el fin de obtener un resultado más intuitivo se puede reorganizar el resultado de la ecuación (5.44) separando los sumatorios en los índices positivos y negativos. Posteriormente, si se aplica sucesivamente el cambio de variable $m=-n$ en los sumatorios de índices negativos, la propiedad de las funciones de Bessel $\left(J_{-n}=\right.$ $\left.(-1)^{n} J_{n}\right)$, la propiedad trigonométrica $\left((-1)^{n} \cos (x)=\cos (x-n \pi)\right)$ y se reagrupan 
términos, se puede llegar a la siguiente expresión:

$$
\begin{aligned}
i_{p d}(t) & =A_{p d}\left[\left[J_{0}\left(\frac{m_{o}}{2}\right) C_{d c}(0)\right.\right. \\
& \left.+2 \sum_{n=1}^{\infty} J_{n}\left(\frac{m_{o}}{2}\right) C_{d c}(n) \cos \left(n \omega_{R F}\left(t+\theta_{1}\right)+n \phi_{1}\right) \cos \left(\frac{\theta_{2}}{2} n^{2} \omega_{R F}^{2}\right)\right]^{2} \\
+ & {\left.\left[2 \sum_{n=1}^{\infty} J_{n}\left(\frac{m_{o}}{2}\right) C_{d c}(n) \cos \left(n \omega_{R F}\left(t+\theta_{1}\right)+n \phi_{1}\right) \operatorname{sen}\left(\frac{\theta_{2}}{2} n^{2} \omega_{R F}^{2}\right)\right]^{2}\right] }
\end{aligned}
$$

El resultado de la ecuación (5.45) es válido para cualquier punto de polarización del modulador y para cualquier nivel de potencia eléctrica incidente, es decir para cualquier índice de modulación óptico. Para el caso habitual de modulación en amplitud convencional, el modulador interferométrico se polariza en la zona lineal (LIN, $V_{D C}=V_{\pi} / 2$ ), por lo que $m_{d c}=\pi / 2$. Generalmente el índice óptico de modulación, $m_{o}$, es bastante pequeño y se pueden realizar una serie de simplificaciones en el resultado genérico, a partir de las siguientes aproximaciones de las funciones de Bessel de primera especie ${ }^{\mathrm{i}}$ :

$$
\left\{\begin{array}{l}
J_{0}(\beta) \approx 1 \\
J_{1}(\beta) \approx \frac{\beta}{2} \\
J_{n}(\beta) \approx 0 \quad n>1
\end{array}\right\} \quad \beta<<1
$$

Aplicando estos resultados a la ecuación (5.44) y tras alguna operación se obtiene la siguiente expresión aproximada:

$$
\begin{aligned}
i_{p d}(t) \approx & \frac{A_{p d}}{2}\left[1+\frac{m_{o}}{2} \cos \left(\omega_{R F}\left(t+\theta_{1}\right)+\phi_{R F}+\frac{\pi}{2}\right) \cos \left(\frac{\theta_{2}}{2} \omega_{R F}^{2}\right)\right]^{2} \\
& +\frac{A_{p d}}{2}\left[\frac{m_{o}}{2} \cos \left(\omega_{R F}\left(t+\theta_{1}\right)+\phi_{R F}+\frac{\pi}{2}\right) \operatorname{sen}\left(\frac{\theta_{2}}{2} \omega_{R F}^{2}\right)\right]^{2} \\
= & \frac{A_{p d}}{2}\left[1+m_{o} \cos \left(\omega_{R F}\left(t+\theta_{1}\right)+\phi_{R F}+\frac{\pi}{2}\right) \cos \left(\frac{\theta_{2}}{2} \omega_{R F}^{2}\right)\right. \\
& \left.+\frac{m_{o}^{2}}{4} \cos ^{2}\left(\omega_{R F}\left(t+\theta_{1}\right)+\phi_{R F}+\frac{\pi}{2}\right)\right]
\end{aligned}
$$

despreciando el término que incluye el factor $m_{o}^{2}$ por trabajar con índices de modulación pequeños, la componente alterna de la señal eléctrica a la salida del fotodetector será:

$$
i_{p d-a c}(t) \approx-\frac{A_{p d} m_{o}}{2} \operatorname{sen}\left(\omega_{R F}\left(t+\theta_{1}\right)+\phi_{R F}\right) \cos \left(\frac{\theta_{2}}{2} \omega_{R F}^{2}\right)
$$

donde, por un lado, se comprueba la traslación al dominio eléctrico del retardo óptico introducido por la red de difracción, $\theta_{1}$; mientras que, por otro lado, se puede

iAplicando estas simplificaciones a la ecuación (5.41) es fácil comprobar que el campo óptico a la salida del modulador interferométrico se reduce a una modulación en amplitud convencional. 
observar claramente el efecto de atenuación dispersiva por el cual la potencia de la señal detectada viene afectada por un factor cosenoidal dependiente del producto de la dispersión de la red de difracción, $\theta_{2}$, y de la frecuencia de modulación de la señal de radiofrecuencia, $\omega_{R F}$. Este efecto ya conocido en los enlaces de comunicaciones IM/DD sobre fibra monomodo es el mismo que se obtendría con una fibra monomodo cuya dispersión total (producto de la longitud de la fibra, $L$, por el parámetro de dispersión de la misma, $D$ ) fuera igual a la pendiente del retardo de grupo de la red de difracción, $\delta(\mathrm{ps} / \mathrm{nm})=L(\mathrm{~km}) D(\mathrm{ps} / \mathrm{nm} \cdot \mathrm{km})$ [Mes84, Sch95].

Como conclusión del resultado de la ecuación (5.48) y aplicando la igualdad (5.34) se puede decir que la degradación de potencia de radiofrecuencia en líneas de retardo basadas en redes de difracción con periodo variable usando esquemas IM/DD con modulación de amplitud convencional y bajos índices de modulación, en función de la frecuencia de RF y de la pendiente del retardo de grupo de la red de difracción con variación lineal del periodo espacial es:

$$
\text { Aten }_{\text {disp }}\left(f_{R F}\right)=\frac{\left|H\left(f_{R F}\right)\right|^{2}}{|H(0)|^{2}}=\cos ^{2}\left(\frac{\lambda_{c}^{2} \pi \delta}{c} f_{R F}^{2}\right)=\cos ^{2}\left(\frac{\pi c \delta}{\omega_{c}^{2}} \omega_{R F}^{2}\right)
$$

de la expresión anterior se obtiene una secuencia alternada de máximos y nulos, correspondiendo la primera frecuencia para la cual la potencia baja a la mitad del máximo al ancho de banda disponible, cuya expresión es:

$$
f_{\max }=\sqrt{\frac{c}{4 \lambda_{c}^{2} \delta}}
$$

Es interesante comentar que a partir de la ecuación (5.49) es posible concluir que las sucesivas cancelaciones de la señal moduladora tienen lugar cuando la diferencia entre los retardos de las dos bandas laterales es igual a un número entero de periodos de la señal moduladora.

En la figura 5.9, se puede observar la atenuación que sufriría una señal de radiofrecuencia en función de la frecuencia en un sistema IM/DD en el cual el único elemento dispersivo fuera una red de difracción con una pendiente igual a $\delta=850 \mathrm{ps} / \mathrm{nm}$, cumpliéndose la limitación en frecuencia definida en (5.50) que para el caso de la figura es de $6.1 \mathrm{GHz}$.

Esta limitación en el ancho de banda de enlaces dispersivos, podría eliminarse mediante la compensación de la pendiente del retardo de grupo alrededor de la portadora óptica, únicamente en el caso de que ésta fuera fija. Para el caso de líneas de retardo continuamente variables basadas en redes de difracción con periodo variable será necesario acudir a otras técnicas de modulación si se desea eliminar la limitación impuesta por (5.50).

Como se verá más adelante, esta limitación en el ancho de banda disponible puede evitarse mediante el uso de modulación en banda lateral única con portadora $^{\mathrm{i}}$ [Smi96] o también mediante técnicas heterodinas (autoheterodinas o pseudoautoheterodinas (basadas en técnicas ópticas de elevación en frecuencia)) en lugar

\footnotetext{
${ }^{\mathrm{i}}$ Single Sideband plus Carrier $(S S B+C)$, en terminología inglesa.
} 


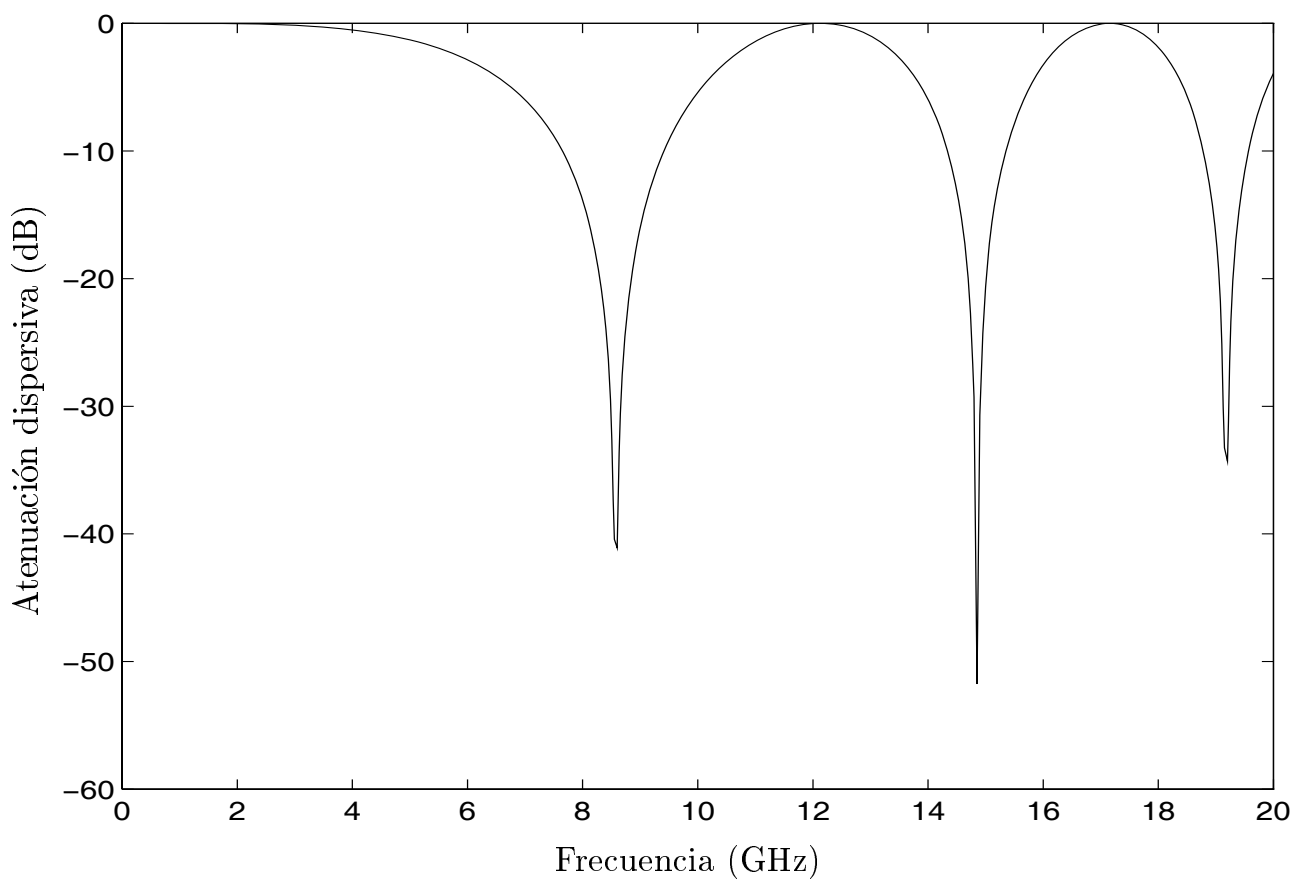

Figura 5.9: Atenuación dispersiva de una señal de radiofrecuencia en la transmisión a través de un enlace IM/DD que implementa una línea de retardo con una red de difracción con periodo variable lineal $\left(\delta=850 \mathrm{ps} / \mathrm{nm}, \lambda_{c}=1550 \mathrm{~nm}\right.$ y $\left.m_{o p t}=0.1\right)$.

de la modulación de amplitud convencional, aunque está última técnica requiere generadores más complejos y es de banda estrecha [Gol92]. Ambos esquemas de modulación permiten continuar trabajando en recepción con detección directa.

\section{Distorsión inducida por la dispersión en redes de difracción con variación lineal del periodo}

La no linealidad de los procesos de modulación (conversión tensión eléctrica de entrada a potencia óptica de salida junto a la respuesta no lineal de la misma (Fig. 5.8)) y de detección (conversión de potencia óptica a intensidad eléctrica) conllevan irremediablemente la aparición de fenómenos de distorsión, e intermodulación en sistemas con portadoras múltiples. La presencia de elementos dispersivos en la línea de retardo variable basada en redes de difracción acentuará el efecto de estos fenómenos.

Distorsión armónica Tal y como se muestra en la ecuación (5.41), la modulación externa de la señal óptica del láser con un tono de radiofrecuencia provoca la aparición de un conjunto infinito de rayas espectrales alrededor de la portadora óptica y separadas entre sí por la frecuencia de la señal moduladora. Aún en el caso de escoger como punto de polarización del modulador interferométrico el correspondiente a la zona más lineal (LIN, $V_{D C}=V_{\pi} / 2$ ) y realizando las aproximaciones para índices 
de modulación reducidos de (5.46) se puede llegar a una expresión como [CG97a]:

$$
e_{e o m}(t) \approx \frac{A_{e}}{\sqrt{2}} \sqrt{1-m_{o} \operatorname{sen}\left(\omega_{R F} t+\phi_{R F}\right)} \cos \left(\omega_{c} t+\phi_{e}\right)
$$

expresión que para el caso de modulación directa del láser se convierte en:

$$
e_{\text {laser }}(t)=A_{o} \sqrt{1+m_{o} \cos \left(\omega_{R F} t+\phi_{R F}\right)} \cos \left(\omega_{c} t+m_{f} \operatorname{sen}\left(\omega_{R F} t+\phi_{R F}\right)+\phi_{o}\right)
$$

donde $m_{o}$ y $m_{f}$ son respectivamente los índices de modulación de la potencia y de la frecuencia ópticas del láser, mientras que $\omega_{R F}$ y $\omega_{c}$ son las pulsaciones moduladora y portadora.

Tanto (5.51) como (5.52) son expresiones de señales ópticas moduladas en intensidad, modulación que tras el proceso de conversión óptico-eléctrico se convierten en modulación de amplitud.

El efecto sobre las ecuaciones (5.51) y (5.52) de la dispersión de enlaces de fibra monomodo ya ha sido analizado en la literatura [Ih90, Mes84, Phi91, CG97a], demostrándose para ambos casos que en sistemas con índices de modulación bajos y enlaces sin dispersión la señal detectada a la salida del fotodetector está prácticamente libre de distorsión. A medida que aumenta la dispersión del enlace óptico, aumenta el contenido en armónicos de la señal detectada, siendo el comportamiento para el caso de modulación directa, peor, en términos generales, que el obtenido para modulación externa [Ih90, CG97a]. Para el caso de la línea de retardo basada en redes de difracción con periodo variable, la situación es similar al del enlace dispersivo. A continuación se analizará la distorsión inducida por dispersión en la línea de retardo, únicamente para el caso de modulación externa, pues, por extrapolación de lo obtenido para enlaces de fibra, los resultados son mejores que con modulación directa.

Volviendo a la expresión exacta del campo óptico a la salida del modulador interferométrico mostrada en la ecuación (5.41), se pueden identificar las potencias asociadas a cada raya espectral tal y como se indica en la siguiente ecuación:

$$
\operatorname{Potencia}\left(\omega_{c}+n \omega_{R F}\right)=\frac{A_{e}^{2}}{2} J_{n}^{2}\left(\frac{m_{o}}{2}\right) C_{d c}^{2}(n)
$$

En la figura 5.10 se muestran las potencias de las diferentes componentes espectrales del campo óptico ${ }^{i}$, para tres puntos de polarización del modulador interferométrico simétrico (potencia óptica máxima (MAP, $V_{D C}=0$ ), potencia óptica mínima (MIP, $V_{D C}=V_{\pi}$ ) y zona más lineal (LIN, $\left.V_{D C}=V_{\pi} / 2\right)$ ) y para diversos valores de índice de modulación. Los resultados muestran como la polarización en MIP provoca que a la salida del EOM no exista portadora y sólo armónicos impares de la señal moduladora, alrededor de la frecuencia óptica portadora; mientras que, la polarización en MAP, implica portadora más armónicos pares de la moduladora.

${ }^{\text {i}}$ Por simetría, sólo se muestran las rayas espectrales correspondientes a la portadora y la banda lateral superior. 


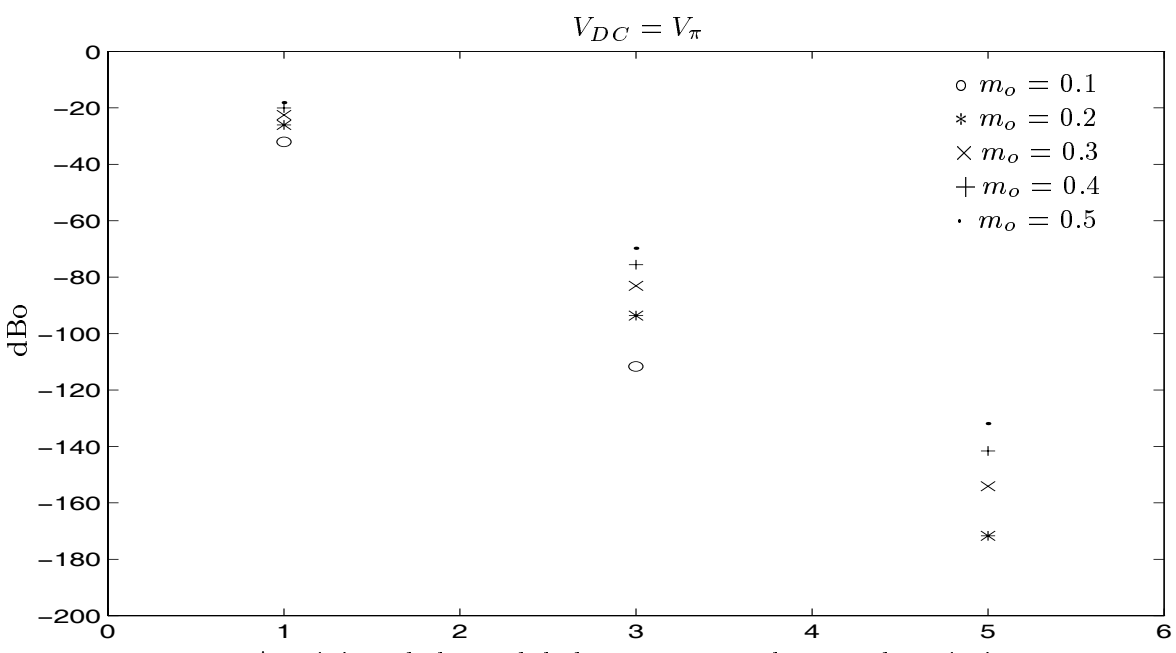

a)

Armónicos de la moduladora respecto a la portadora óptica

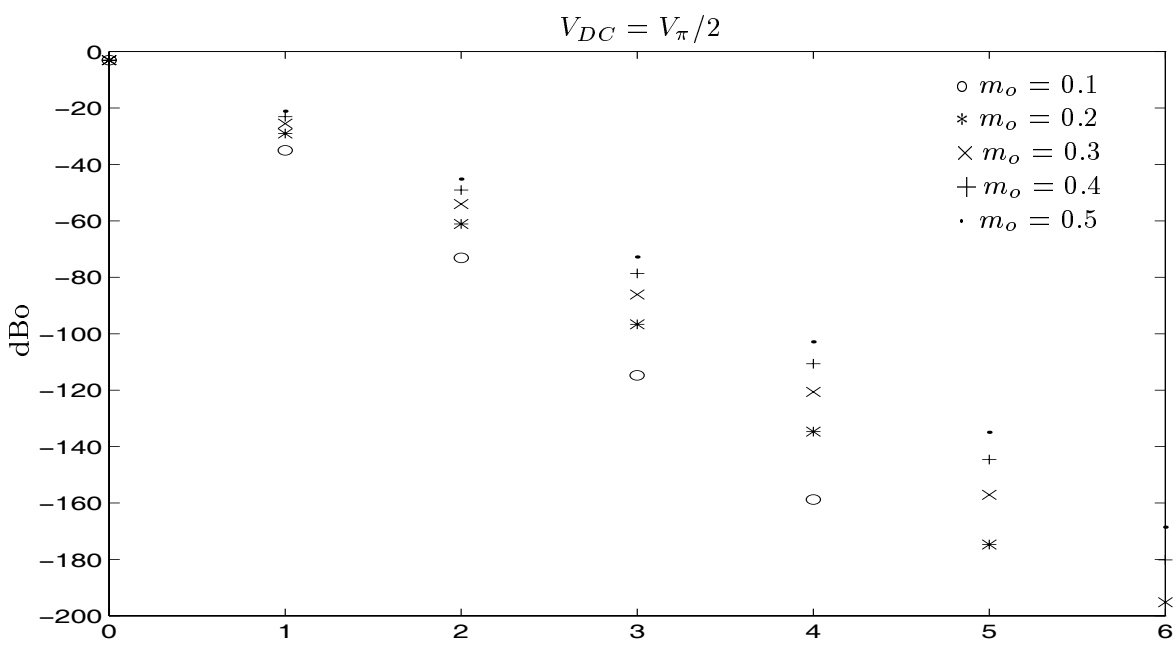

b)

Armónicos de la moduladora respecto a la portadora óptica

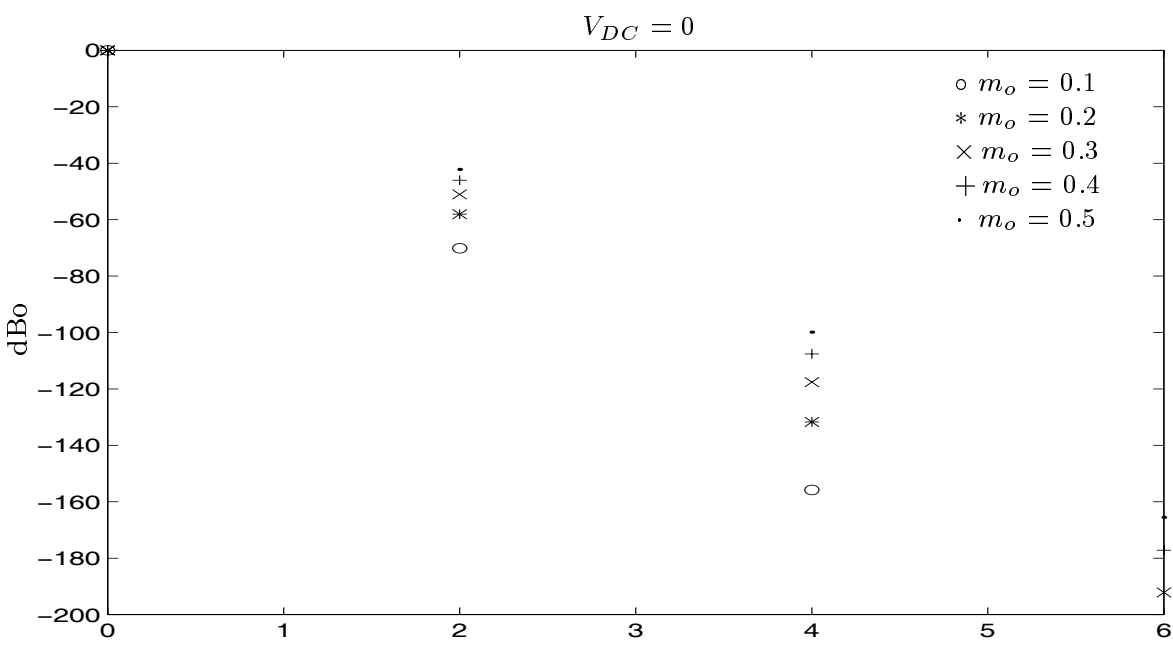

c)

Figura 5.10: Potencia relativa de los primeros armónicos de la señal moduladora alrededor de la portadora óptica a la salida de un EOM simétrico. a) Polarización en MIP. b) Polarización en LIN. c) Polarización en MAP. 
Por lo que respecta a la polarización del EOM en LIN, el campo óptico de salida tendrá tanto portadora como todos los armónicos de la moduladora, aunque con niveles decrecientes en función del orden de cada armónico. En todos los casos, el aumento del índice de modulación provoca un aumento del nivel de los armónicos, mayor cuanto mayor sea el orden del mismo.

Una vez la señal óptica modulada pasa por la red de difracción con periodo lineal y es detectada en el fotodetector, se demostró que la expresión exacta de la corriente detectada viene definida por las ecuaciones (5.45) o (5.44). Para identificar cada uno de las componentes espectrales presentes en estas expresiones será necesario realizar algunas simplificaciones. Partiendo de (5.44):

$$
\begin{aligned}
i_{p d}(t)= & A_{p d}\left[\sum_{n=-\infty}^{\infty} E_{n} \cos \left(n \omega_{R F}\left(t+\theta_{1}\right)+n \phi_{1}+\frac{\theta_{2}}{2} n^{2} \omega_{R F}^{2}\right)\right]^{2} \\
& +A_{p d}\left[\sum_{n=-\infty}^{\infty} E_{n} \operatorname{sen}\left(n \omega_{R F}\left(t+\theta_{1}\right)+n \phi_{1}+\frac{\theta_{2}}{2} n^{2} \omega_{R F}^{2}\right)\right]^{2}
\end{aligned}
$$

donde $E_{n}=J_{n}\left(\frac{m_{o}}{2}\right) \cdot C_{d c}(n)$. Operando dentro de los términos entre corchetes:

$$
\begin{aligned}
i_{p d}(t)= & A_{p d} \sum_{r=-\infty}^{\infty} \sum_{s=-\infty}^{\infty} E_{r} E_{s}\left[\cos \left(r \omega_{R F}\left(t+\theta_{1}\right)+r \phi_{1}+\frac{\theta_{2}}{2} r^{2} \omega_{R F}^{2}\right)\right. \\
& \left.\cos \left(s \omega_{R F}\left(t+\theta_{1}\right)+s \phi_{1}+\frac{\theta_{2}}{2} s^{2} \omega_{R F}^{2}\right)\right] \\
& +A_{p d} \sum_{r=-\infty}^{\infty} \sum_{s=-\infty}^{\infty} E_{r} E_{s}\left[\operatorname{sen}\left(r \omega_{R F}\left(t+\theta_{1}\right)+r \phi_{1}+\frac{\theta_{2}}{2} r^{2} \omega_{R F}^{2}\right)\right. \\
& \left.\operatorname{sen}\left(s \omega_{R F}\left(t+\theta_{1}\right)+s \phi_{1}+\frac{\theta_{2}}{2} s^{2} \omega_{R F}^{2}\right)\right] \\
= & A_{p d} \sum_{r=-\infty}^{\infty} \sum_{s=-\infty}^{\infty} E_{r} E_{s} \cos \left((r-s) \omega_{R F}\left(t+\theta_{1}\right)+(r-s) \phi_{1}+\frac{\theta_{2}}{2}\left(r^{2}-s^{2}\right) \omega_{R F}^{2}\right) \\
= & A_{p d} \sum_{p=-\infty}^{\infty} \sum_{s=-\infty}^{\infty} E_{p+s} E_{s} \cos \left(p \omega_{R F}\left(t+\theta_{1}\right)+p \phi_{1}+\frac{\theta_{2}}{2}\left((p+s)^{2}-s^{2}\right) \omega_{R F}^{2}\right) \\
= & A_{p d} \sum_{p=-\infty}^{\infty} \sum_{s=-\infty}^{\infty} E_{p+s} E_{s} \cos \left(\frac{\theta_{2}}{2}\left(p^{2}+2 s p\right) \omega_{R F}^{2}\right) \cos \left(p \omega_{R F}\left(t+\theta_{1}\right)+p \phi_{1}\right) \\
& -A_{p d} \sum_{p=-\infty}^{\infty} \sum_{s=-\infty}^{\infty} E_{p+s} E_{s} \operatorname{sen}\left(\frac{\theta_{2}}{2}\left(p^{2}+2 s p\right) \omega_{R F}^{2}\right) \operatorname{sen}\left(p \omega_{R F}\left(t+\theta_{1}\right)+p \phi_{1}\right) \\
= & \sum_{p=-\infty}^{\infty} I_{I}(p) \cos \left(p \omega_{R F}\left(t+\theta_{1}\right)+p \phi_{1}\right)-\sum_{p=-\infty}^{\infty} I_{Q}(p) \operatorname{sen}\left(p \omega_{R F}\left(t+\theta_{1}\right)+p \phi_{1}\right)(5.55)
\end{aligned}
$$


donde

$$
\begin{aligned}
& I_{I}(p)=\sum_{s=-\infty}^{\infty} E_{p+s} E_{s} \cos \left(\frac{\theta_{2}}{2}\left(p^{2}+2 s p\right) \omega_{R F}^{2}\right) \\
& I_{Q}(p)=\sum_{s=-\infty}^{\infty} E_{p+s} E_{s} \operatorname{sen}\left(\frac{\theta_{2}}{2}\left(p^{2}+2 s p\right) \omega_{R F}^{2}\right)
\end{aligned}
$$

Otra forma de expresar (5.55) es:

$$
\begin{aligned}
i_{p d}(t)=A_{p d}\left[I_{I}(0)+\sum_{p=1}^{\infty}\right. & \left(I_{I}(p)+I_{I}(-p)\right) \cos \left(p \omega_{R F}\left(t+\theta_{1}\right)+p \phi_{1}\right) \\
& \left.\quad-\sum_{p=1}^{\infty}\left(I_{Q}(p)-I_{Q}(-p)\right) \operatorname{sen}\left(p \omega_{R F}\left(t+\theta_{1}\right)+p \phi_{1}\right)\right]
\end{aligned}
$$

A partir de (5.56) se pueden demostrar las siguientes propiedades de simetría:

$$
\begin{aligned}
I_{I}(p) & =\sum_{s=-\infty}^{\infty} E_{p+s} E_{s} \cos \left(\frac{\theta_{2}}{2}\left((p+s)^{2}-s^{2}\right) \omega_{R F}^{2}\right) \\
& =\sum_{k=-\infty}^{\infty} E_{k} E_{k-p} \cos \left(\frac{\theta_{2}}{2}\left(k^{2}-(k-p)^{2}\right) \omega_{R F}^{2}\right) \\
& =\sum_{k=-\infty}^{\infty} E_{k-p} E_{k} \cos \left(\frac{\theta_{2}}{2}\left((k-p)^{2}-k^{2}\right) \omega_{R F}^{2}\right)=I_{I}(-p) \\
I_{Q}(p) & =\sum_{s=-\infty}^{\infty} E_{p+s} E_{s} \operatorname{sen}\left(\frac{\theta_{2}}{2}\left((p+s)^{2}-s^{2}\right) \omega_{R F}^{2}\right) \\
& =\sum_{k=-\infty}^{\infty} E_{k} E_{k-p} \operatorname{sen}\left(\frac{\theta_{2}}{2}\left(k^{2}-(k-p)^{2}\right) \omega_{R F}^{2}\right) \\
& =-\sum_{k=-\infty}^{\infty} E_{k-p} E_{k} \operatorname{sen}\left(\frac{\theta_{2}}{2}\left((k-p)^{2}-k^{2}\right) \omega_{R F}^{2}\right)=-I_{Q}(-p)
\end{aligned}
$$

Aplicando ambos resultados a la ecuación (5.57) se obtiene el resultado final:

$$
\begin{array}{r}
i_{p d}(t)=A_{p d}\left[I_{I}(0)+2 \sum_{p=1}^{\infty} I_{I}(p) \cos \left(p \omega_{R F}\left(t+\theta_{1}\right)+p \phi_{1}\right)\right. \\
\left.-2 \sum_{p=1}^{\infty} I_{Q}(p) \operatorname{sen}\left(p \omega_{R F}\left(t+\theta_{1}\right)+p \phi_{1}\right)\right]
\end{array}
$$


ecuación de la que es inmediato extraer la potencia asociada a cada armónico del tono moduladori:

$$
\operatorname{Potencia}\left(n \omega_{R F}\right)=2 A_{p d}^{2} \cdot\left(I_{I}^{2}(n)+I_{Q}^{2}(n)\right)
$$

excepto para $n=0$ donde:

$$
\text { Potencia }(0)=P_{d c}=A_{p d}^{2} \cdot I_{I}^{2}(0)
$$

Claramente, los dos factores que más van a influir en el nivel de los armónicos de la señal detectada son:

- El proceso de modulación: tanto la tensión de polarización, $V_{D C} / V_{\pi}$, como el índice de modulación óptico, $m_{o}$.

- La dispersión de la línea de retardo, de acuerdo con el producto $\phi_{\text {disp }}=\theta_{2} * \omega_{R F}^{2}$.

En las figuras 5.11 y 5.12 se puede comprobar el efecto de la distorsión inducida por la dispersión para diferentes casos; donde se han representado los niveles de potencia de las diferentes componentes espectrales de la señal detectada (ec. (5.60)) normalizados respecto a $A_{p d}^{2}$. En concreto, se muestran los niveles de los seis primeros armónicos de la señal moduladora junto al nivel de contínua de la señal detectada. En la primera figura, se representan los niveles de los armónicos para los puntos de polarización LIN, MIP y MAP, parametrizando los resultados respecto al índice de modulación óptico; mientras que en la figura 5.12 se representan los niveles de los armónicos para cinco valores de dispersión de la red de difracción.

Como conclusiones de los resultados de ambas gráficas, se puede comprobar que al polarizar el modulador en MAP o en MIP no se dispondrá a la salida del fotodetector de componente fundamental de la señal moduladora, siendo la componente de mayor nivel, el segundo armónico; resultado esperable si se observa en la figura 5.10, que para ambos casos todas las componentes de la señal óptica a la salida del EOM están separadas entre sí múltiplos del doble de la frecuencia moduladora.

Como diferencia principal entre la polarización en MIP y en MAP está el nivel de contínua de la señal detectada, que al depender del nivel medio de la potencia óptica incidente es mucho más reducido en el caso MIP; en concreto, es ligeramente superior al nivel del segundo armónico. Además, se puede comprobar en la figura 5.12, que la dispersión de la red de difracción, no afecta en absoluto al nivel del segundo armónico del caso MIP, resultado muy interesante y que se aplicará posteriormente.

De los resultados de la figura 5.12 se concluye que el punto de polarización más sensible a la dispersión de la red de difracción, en cuanto a la distorsión inducida por la misma, corresponde al caso de modulación lineal (LIN), por lo que será el caso que se estudiará más en detalle a continuación. Observando el nivel de la componente fundamental para la polarización en LIN, se puede apreciar el comienzo del efecto de la atenuación dispersiva.

\footnotetext{
iSalvo que se indique lo contrario, se asumirá que la potencia a la salida del fotodetector esta referenciada a una resistencia unidad $R_{C A R G A}=1 \Omega$.
} 


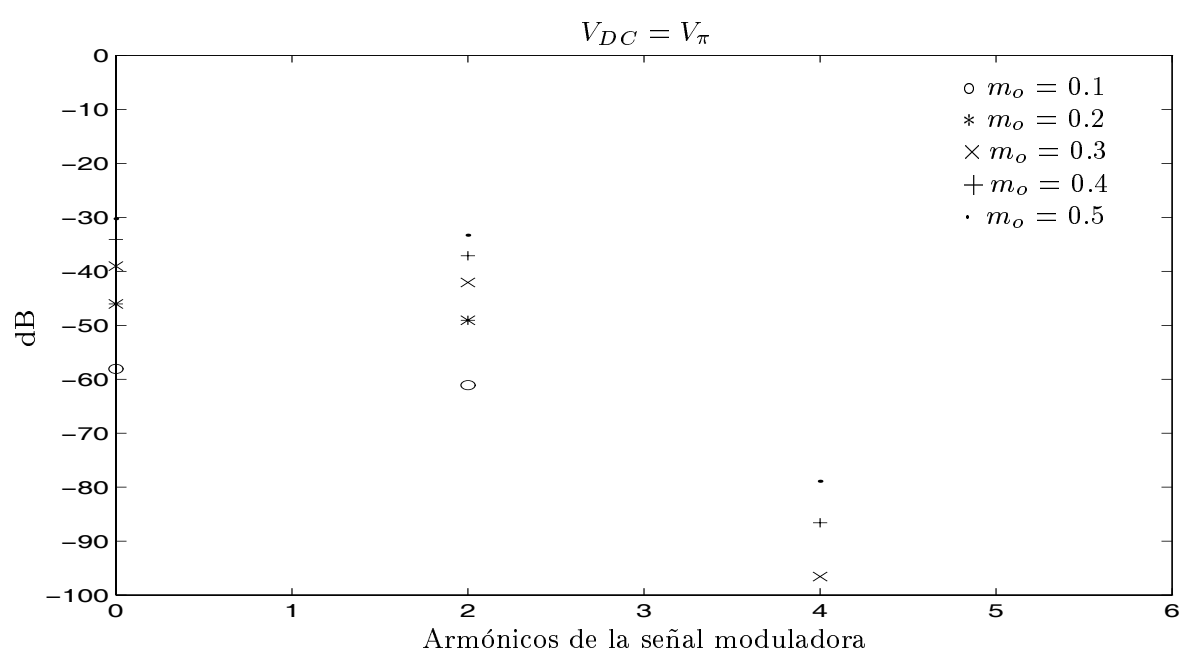

a)

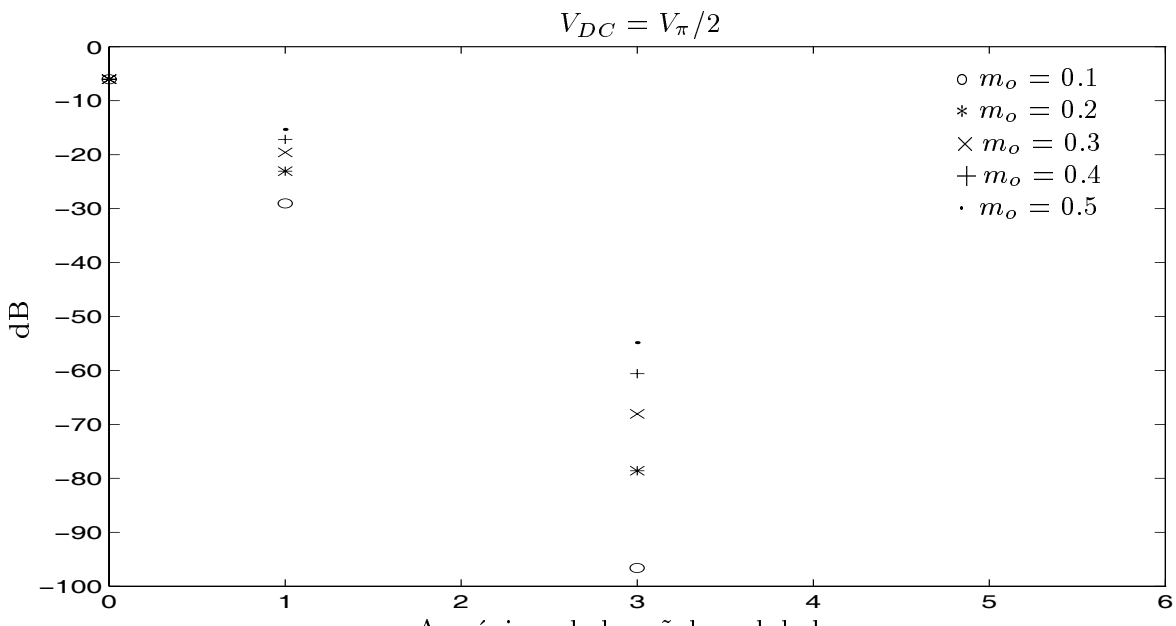

b)

Armónicos de la señal moduladora

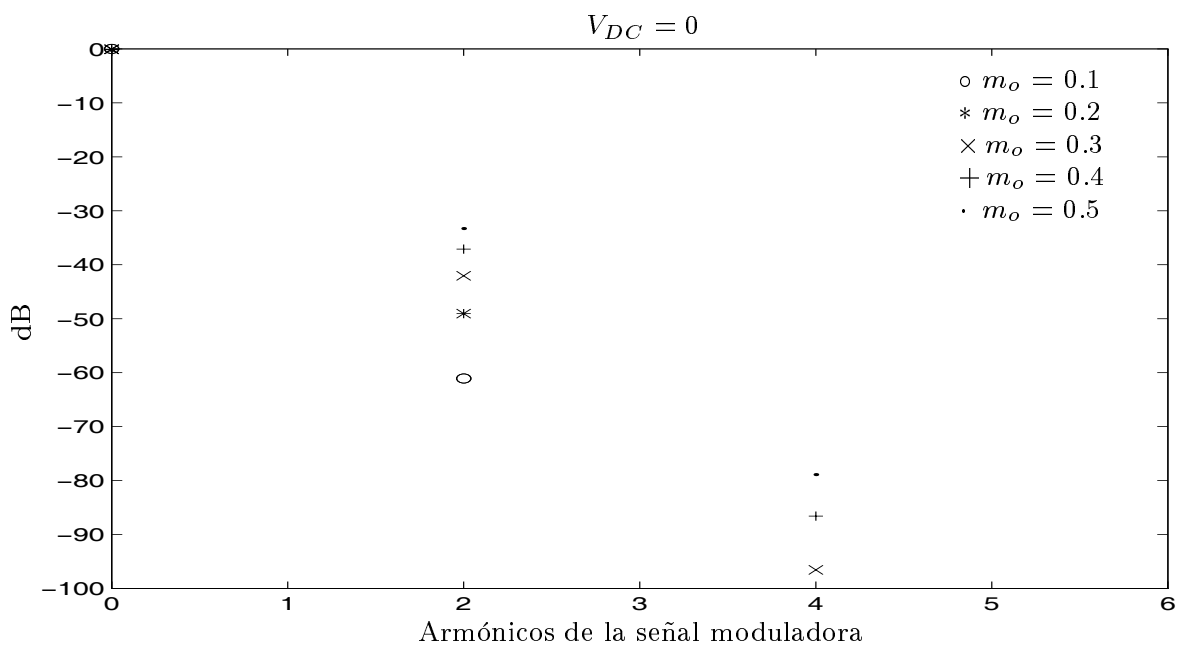

c)

Figura 5.11: Potencias relativas de los armónicos de la señal moduladora a la salida del fotodetector de una línea de retardo variable con una red de difracción con dispersión $\delta=100 \mathrm{ps} / \mathrm{nm}$, parametrizadas respecto al índice de modulación del EOM $\left(f_{R F}=1 \mathrm{GHz}\right)$. a) Polarización en MIP. b) Polarización en LIN. c) Polarización en MAP. 
a)
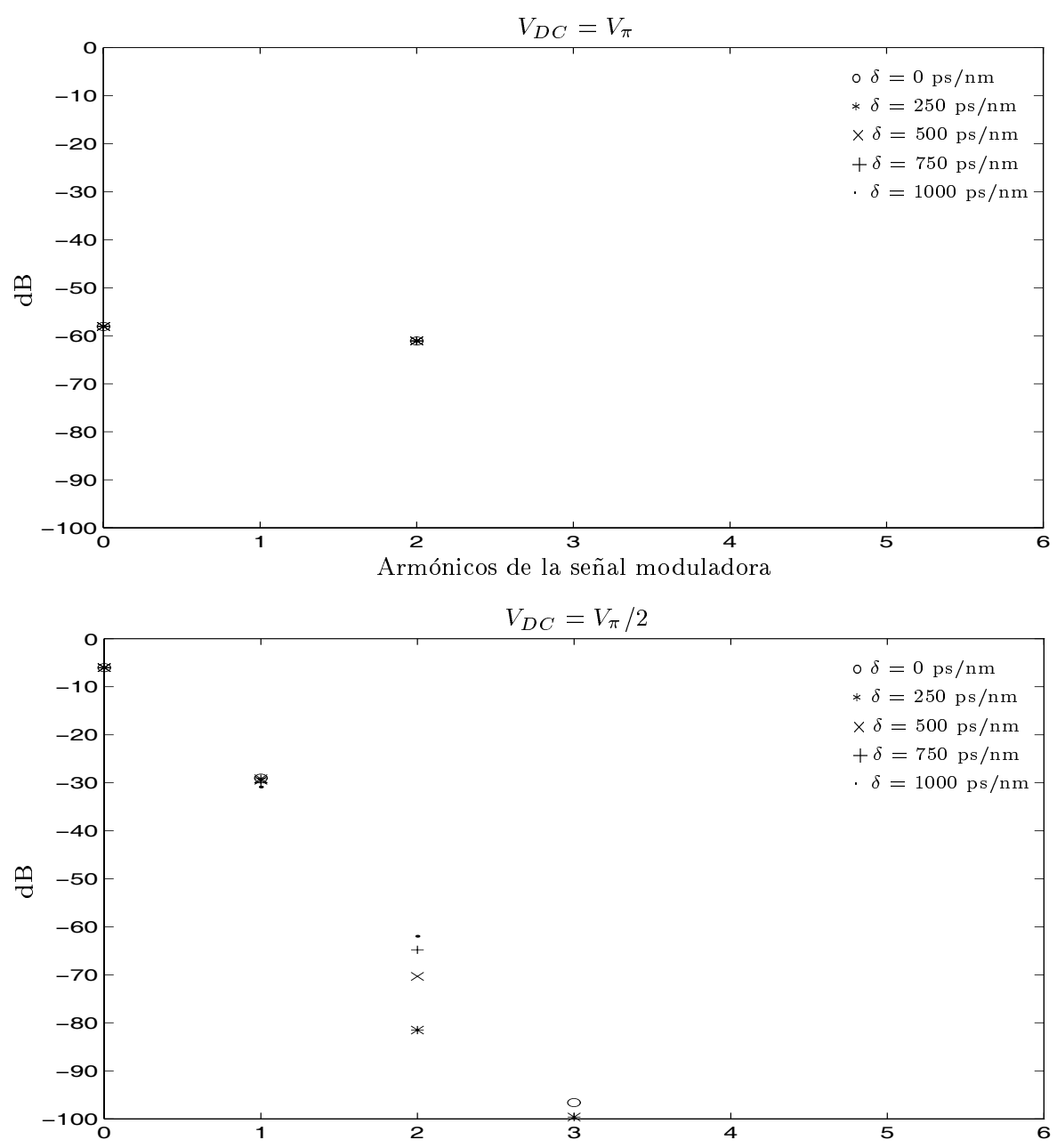

b)

Armónicos de la señal moduladora

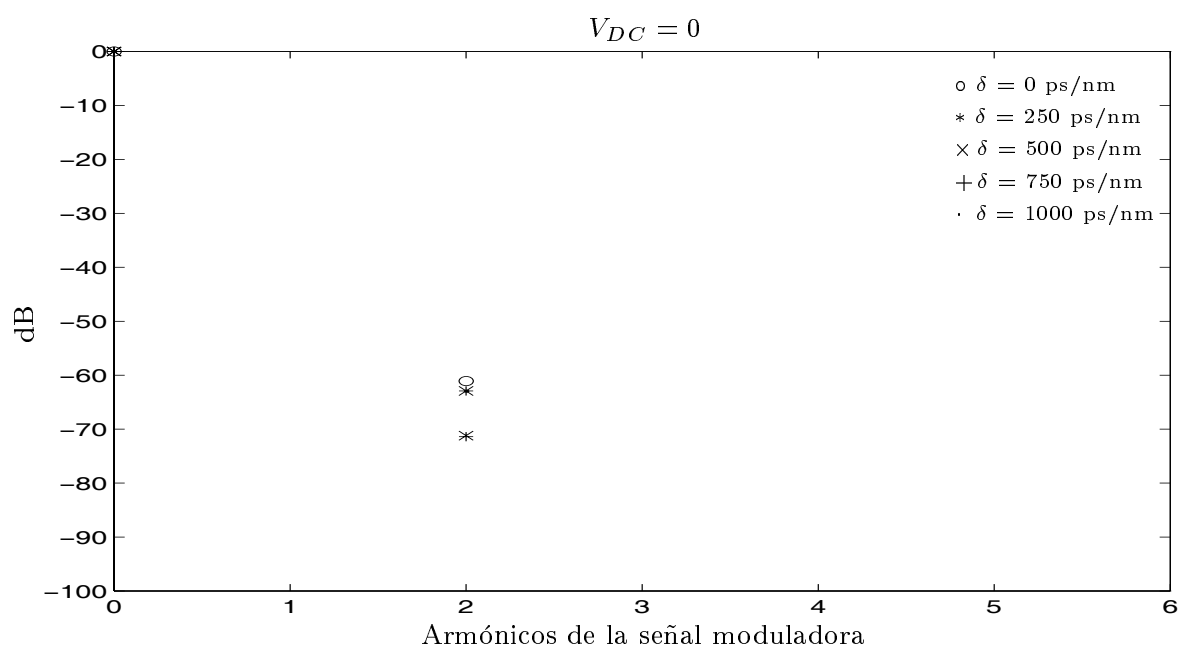

c)

Figura 5.12: Potencias relativas de los armónicos de la señal moduladora a la salida del fotodetector de una línea de retardo variable con modulación externa $\left(m_{o}=0.1\right)$, parametrizadas respecto a la dispersión de la red de difracción $\left(f_{R F}=5 \mathrm{GHz}\right)$. a) Polarización en MIP. b) Polarización en LIN. c) Polarización en MAP. 
Teniendo en cuenta el resultado de la ecuación (5.34), y tomando una longitud de onda central de $1550 \mathrm{~nm}$, se puede obtener la siguiente expresión para el producto de dispersión:

$$
\phi_{d i s p}(\mathrm{rad})=\theta_{2} \omega_{R F}^{2}=\frac{\lambda_{c}^{2} \delta}{2 \pi c} \omega_{R F}^{2} \approx 5 \cdot 10^{-5} f_{R F}^{2}(\mathrm{GHz}) \delta(\mathrm{ps} / \mathrm{nm})
$$

ecuación que permite referenciar de forma rápida el parámetro $\phi_{\text {disp }}$ a valores de frecuencia de señal moduladora y de parámetro de dispersión de la red de difracción; como ejemplo un valor de $\phi_{\text {disp }}=0.5$ rad equivale a una señal de $f_{R F}=10 \mathrm{GHz}$ con una red de $\delta=100 \mathrm{ps} / \mathrm{nm}$; o bien a una señal de $f_{R F}=3.16 \mathrm{GHz}$ con una red de $\delta=1000 \mathrm{ps} / \mathrm{nm}$.

Particularizando, para el caso de modulación en la zona lineal del EOM, se presentan a continuación unas gráficas que relacionan el nivel de los cinco primeros armónicos de la frecuencia moduladora en la señal detectada obtenidos a partir de la ecuación (5.60) normalizando los resultados respecto a $A_{p d}^{2}$. En la figura 5.13, se muestra cómo varía el nivel de estos armónicos, en función del producto de dispersión de la línea de retardo, $\phi_{d i s p}$, para dos valores de índice de modulación óptico; mientras que las figuras 5.14 y 5.15 presentan las variaciones del nivel de los cinco armónicos en función del índice de modulación óptico, que por otro lado, es proporcional a la tensión de radiofrecuencia de entrada al modulador.

Analizando los resultados de la figura 5.13, se puede destacar que la existencia de dispersión dentro del sistema no sólo introduce la atenuación dispersiva en el fundamental de la señal moduladora, tal y como se describió en el punto anterior; si no, que provoca que el nivel de los armónicos que antes no existían (armónicos pares en el caso del modulador trabajando en la zona lineal) aumentan de forma proporcional al producto de dispersión, $\phi_{d i s p}$, hasta un cierto nivel de saturación. Para el caso de los armónicos que existen incluso para sistemas sin dispersión (armónicos impares en el caso de modulador trabajando en la zona lineal), el efecto de la dispersión es el de la introducción de nulos de transmisión, pero en ningún caso se aumenta el nivel de potencia obtenido para el caso sin dispersión. También en la figura 5.13 se puede comprobar como el nivel de saturación para cada armónico se reduce al aumentar el nivel del mismo.

En las figuras 5.14 y 5.15 se representa la relación entre los niveles de potencia de los diferentes armónicos de la señal moduladora a la salida del fotodetector frente a la potencia de la señal de entrada (expresada a partir del índice de modulación óptico) de la línea de retardo con una red de difracción, particularizados para cuatro valores del producto de dispersión de la línea de retardo, $\phi_{d i s p}$. En los dos primeros casos $\left(\phi_{\text {disp }}=0\right.$ y $\left.0.1 \mathrm{rad}\right)$, no aparecen todavía los fenómenos de atenuación dispersiva y se puede comprobar cómo, la introducción de una pequeña dispersión implica la aparición de los armónicos pares sin afectar prácticamente al nivel de los armónicos impares. Para los valores más altos del producto de dispersión, comienza a notarse el efecto de la atenuación dispersiva, que aparece primero en los armónicos de niveles más altos, como se pudo comprobar en la figura 5.13. 


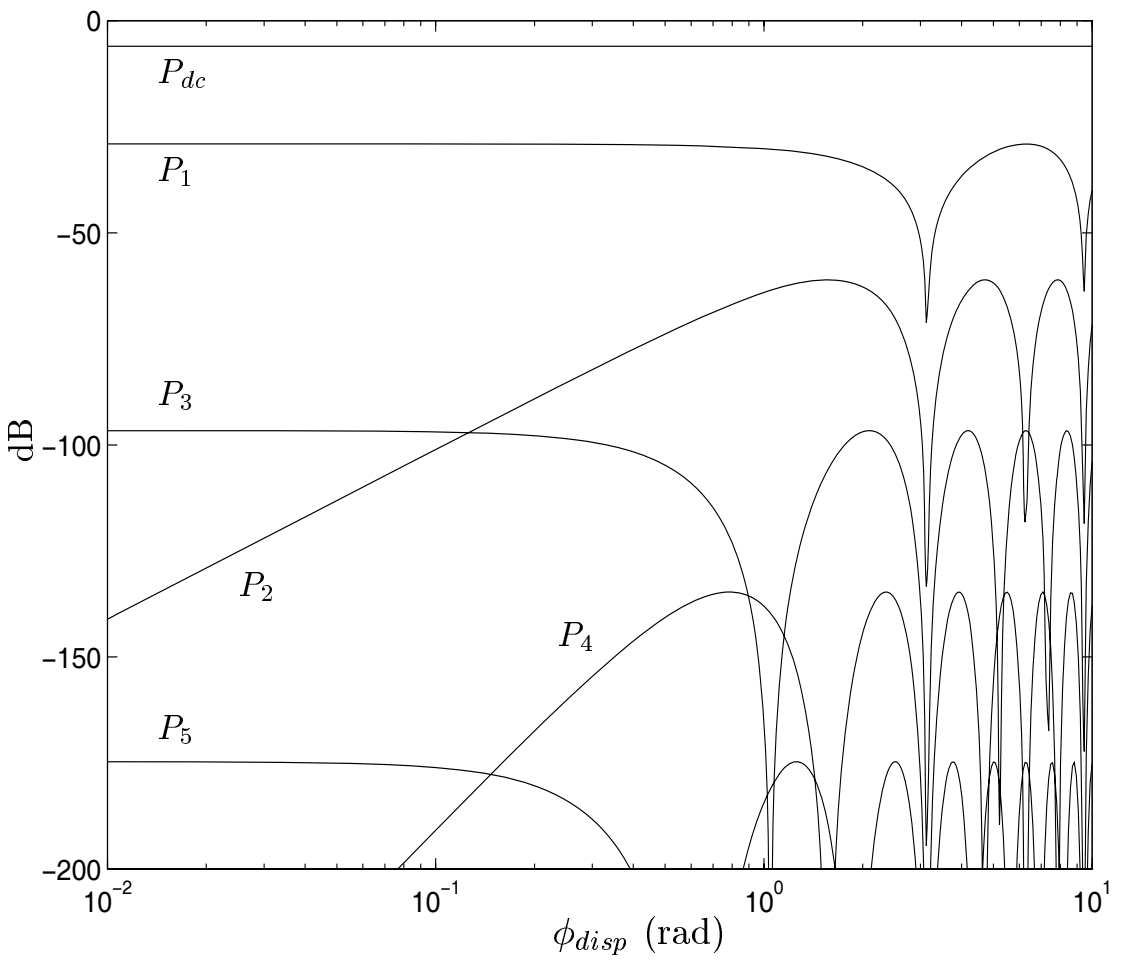

a)

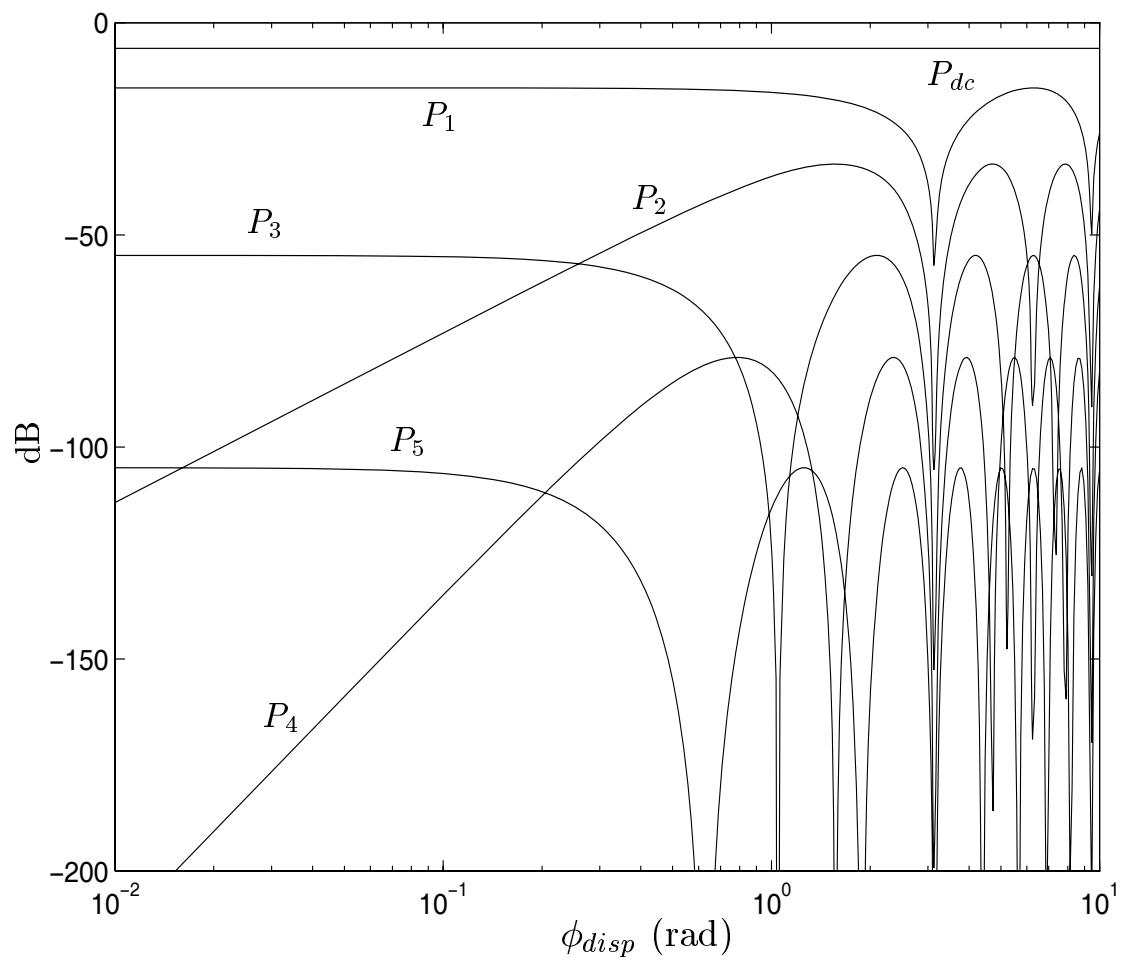

b)

Figura 5.13: Potencias relativas de contínua y de los cinco primeros armónicos de la moduladora a la salida del fotodetector, en función del producto de dispersión, $\phi_{\text {disp }}$, para un modulador trabajando en la zona lineal. a) $m_{o}=0.1$. b) $m_{o}=0.5$. 
a)
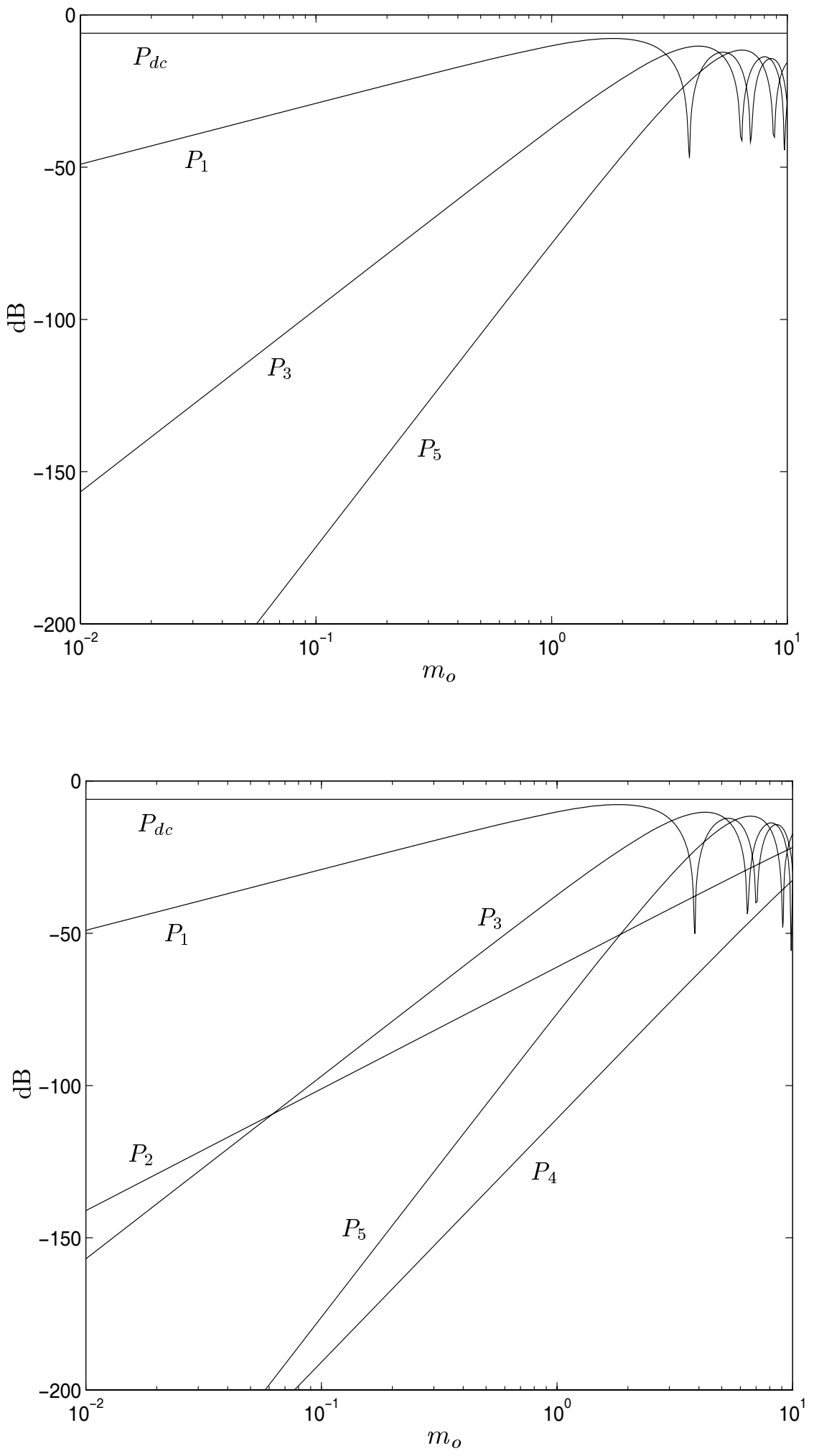

Figura 5.14: Potencias relativas de contínua y de los cinco primeros armónicos de la moduladora a la salida del fotodetector, en función del índice de modulación, $m_{o}$, para un modulador trabajando en la zona lineal. a) $\phi_{\text {disp }}=0 \mathrm{rad}$ y b) $\phi_{\text {disp }}=0.1 \mathrm{rad}$. 


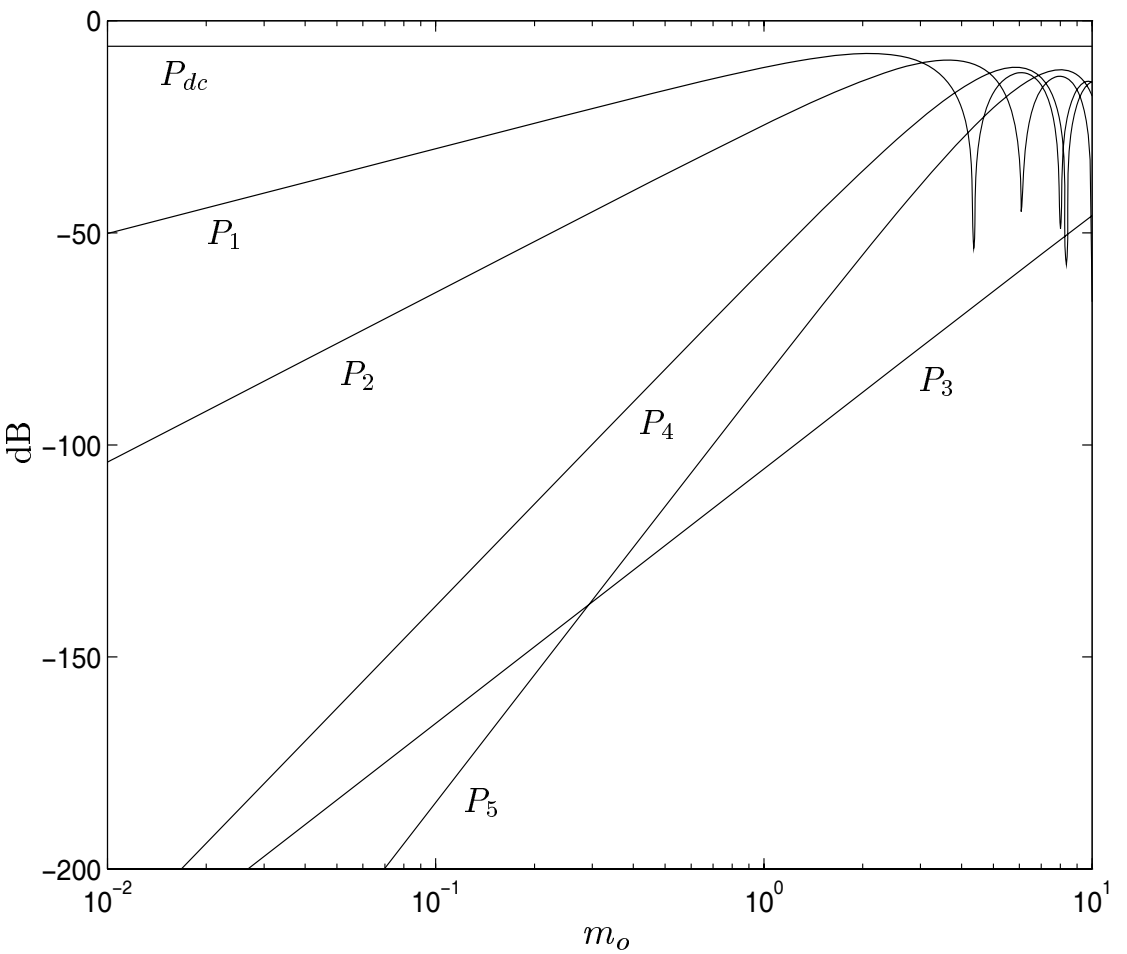

a)

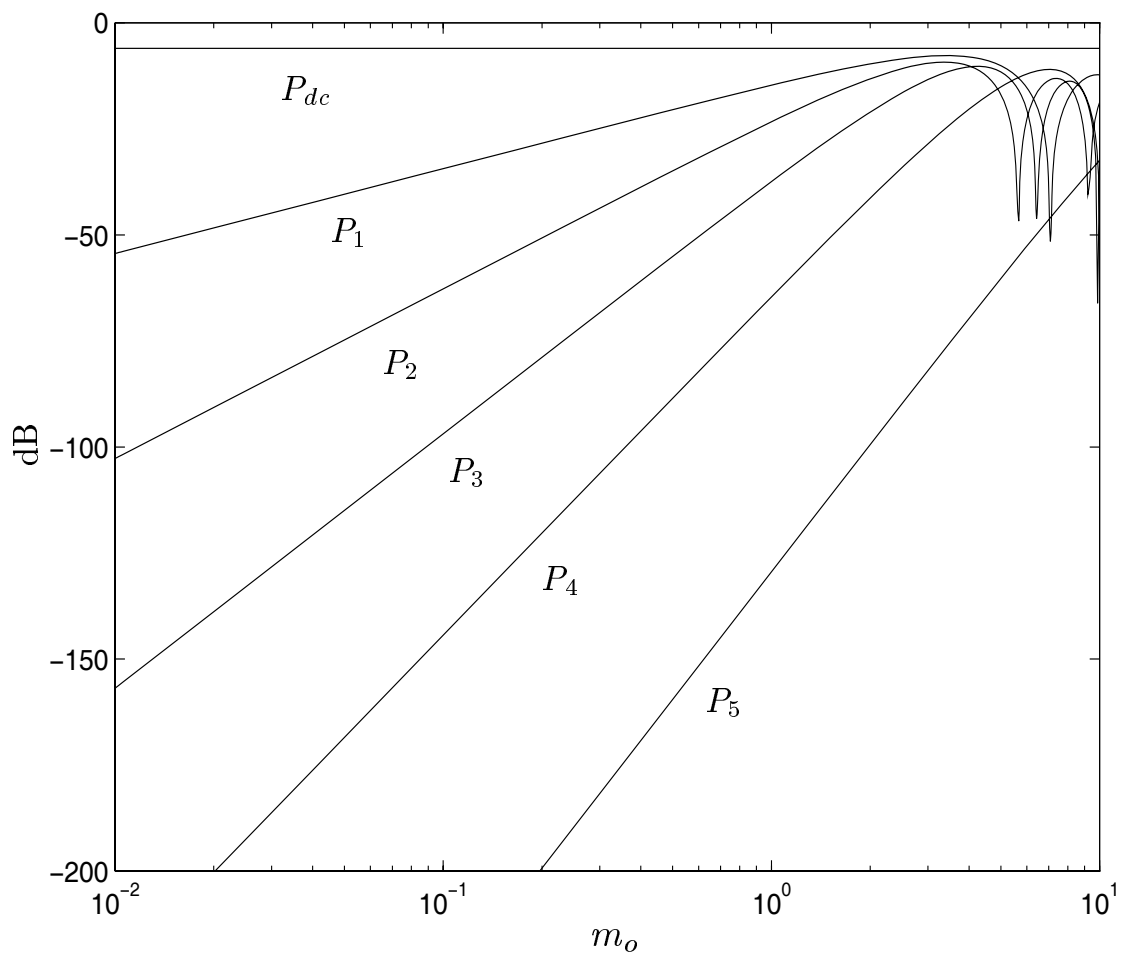

b)

Figura 5.15: Potencias relativas de contínua y de los cinco primeros armónicos de la moduladora a la salida del fotodetector, en función del índice de modulación, $m_{o}$, para un modulador trabajando en la zona lineal. a) $\phi_{d i s p}=1 \mathrm{rad}$. b) $\phi_{d i s p}=2 \mathrm{rad}$. 
Intermodulación A continuación se estudiarán los productos de intermodulación generados en los procesos de conversión electro-ópticos y el efecto que sobre la magnitud de los mismos tiene la dispersión de la red de difracción con periodo variable; para ello es necesario realizar un desarrollo similar al realizado previamente para una señal monotónica, pero incluyendo dos tonos de distintas frecuencias a la entrada del modulador interferométrico simétrico de la figura 5.6.

Asumiendo que el campo óptico a la entrada del modulador viene definido por la expresión (5.35) y que la señal eléctrica de entrada al EOM está formada por dos tonos de distinta amplitud $\left(V_{R F 1}\right.$ y $\left.V_{R F 2}\right)$ y frecuencia $\left(f_{R F 1}\right.$ y $\left.f_{R F 2}\right)$ más un cierto nivel de polarización, $v_{R F}(t)=V_{D C}+V_{R F 1} \operatorname{sen}\left(\omega_{R F 1} t+\phi_{R F 1}\right)+V_{R F 2} \operatorname{sen}\left(\omega_{R F 2} t+\right.$ $\left.\phi_{R F 2}\right)$, y que ésta se aplica de forma simétrica a los electrodos del modulador, es posible obtener la siguiente expresión para el campo óptico a la salida del modulador:

$$
\begin{aligned}
& e_{\text {eom }}(t)=A_{e} \cos \left(\omega_{c} t+\phi_{e}\right) \cdot \\
& \quad \cos \left(\frac{\pi V_{D C}}{2 V_{\pi}}+\frac{\pi V_{R F 1}}{2 V_{\pi}} \operatorname{sen}\left(\omega_{R F 1} t+\phi_{R F 1}\right)+\frac{\pi V_{R F 2}}{2 V_{\pi}} \operatorname{sen}\left(\omega_{R F 2} t+\phi_{R F 2}\right)\right)
\end{aligned}
$$

donde $A_{e} \mathrm{y} \phi_{e}$ difieren de $A_{o} \mathrm{y} \phi_{o}$ de acuerdo, respectivamente, con las pérdidas y la fase de inserción del modulador.

Con el fin de simplificar los resultados y sin pérdida de generalidad, se puede definir una pulsación común $\omega_{u}$ como aquella que cumpla:

$$
\begin{aligned}
& \omega_{R F 1}=n \omega_{u} \\
& \omega_{R F 2}=m \omega_{u}
\end{aligned}
$$

Aplicando propiedades conocidas de las funciones de Bessel de primera especie, es posible desarrollar la ecuación (5.62) de la siguiente forma:

$$
\begin{aligned}
& e_{\text {eom }}(t)=A_{e} \cos \left(\omega_{c} t+\phi_{e}\right) \cdot \\
& \sum_{k=-\infty}^{\infty} \sum_{l=-\infty}^{\infty} J_{k}\left(\frac{m_{o 1}}{2}\right) J_{l}\left(\frac{m_{o 2}}{2}\right) \cos \left(\frac{m_{d c}}{2}+k\left(n \omega_{u} t+\phi_{R F 1}\right)+l\left(m \omega_{u} t+\phi_{R F 2}\right)\right)
\end{aligned}
$$

donde $m_{o 1}=\frac{\pi V_{R F 1}}{V_{\pi}}, m_{o 2}=\frac{\pi V_{R F 2}}{V_{\pi}}$ y $m_{d c}=\frac{\pi V_{D C}}{V_{\pi}}$. Tras un desarrollo similar al realizado en las ecuaciones (5.39) a (5.41) se llega a la siguiente expresión para el campo óptico a la salida del modulador interferométrico modulado por dos tonos [CG97b]:

$$
\begin{aligned}
e_{\text {eom }}(t)= & A_{e} \sum_{k=-\infty}^{\infty} \sum_{l=-\infty}^{\infty}\left[J_{k}\left(\frac{m_{o 1}}{2}\right) J_{l}\left(\frac{m_{o 2}}{2}\right) \cos \left(\frac{m_{d c}}{2}-\frac{k \pi}{2}-\frac{l \pi}{2}\right)\right. \\
& \left.\cos \left(\omega_{c} t+k\left(n \omega_{u} t+\phi_{R F 1}\right)+l\left(m \omega_{u} t+\phi_{R F 2}\right)+\phi_{e}+\frac{k \pi}{2}+\frac{l \pi}{2}\right)\right]
\end{aligned}
$$

Con el fin de identificar separadamente todas y cada una de las componentes espectrales del campo expresado en (5.65), se puede hacer el cambio de variable 
$q=k n+l m$ con la precaución de que $l=(q-k n) / m$ sea siempre entero, para lo cual se define $J_{\frac{q-k n}{m}}^{*}=J_{\frac{q-k n}{m}}$ para $\frac{q-k n}{m} \in \mathbb{Z}$ y cero para el resto:

$$
\begin{array}{r}
e_{\text {eom }}(t)=A_{e} \sum_{q=-\infty}^{\infty} \sum_{k=-\infty}^{\infty} J_{k}\left(\frac{m_{o 1}}{2}\right) J_{\frac{q-k n}{m}}^{*}\left(\frac{m_{o 2}}{2}\right) \cos \left(\frac{m_{d c}}{2}-\frac{k \pi}{2}-\frac{\frac{q-k n}{m} \pi}{2}\right) \\
\cos \left(\omega_{c} t+\phi_{e}+q \omega_{u} t+k \phi_{1}+\frac{q-k n}{m} \phi_{2}\right)
\end{array}
$$

con $\phi_{1}=\phi_{R F 1}+\frac{\pi}{2}, \phi_{2}=\phi_{R F 2}+\frac{\pi}{2}$. Separando en parte real y parte imaginaria:

$$
e_{\text {eom }}(t)=A_{e} \sum_{q=-\infty}^{\infty} I_{q} \cos \left(\left(\omega_{c}+q \omega_{u}\right) t+\phi_{e}\right)-A_{e} \sum_{q=-\infty}^{\infty} Q_{q} \operatorname{sen}\left(\left(\omega_{c}+q \omega_{u}\right) t+\phi_{e}\right)
$$

donde

$$
\begin{aligned}
I_{q} & =\sum_{k=-\infty}^{\infty} J_{k}\left(\frac{m_{o 1}}{2}\right) J_{\frac{q-k n}{m}}^{*}\left(\frac{m_{o 2}}{2}\right) C_{D C}^{k, q} \cos \left(k \phi_{1}+\frac{q-k n}{m} \phi_{2}\right) \\
Q_{q} & =\sum_{k=-\infty}^{\infty} J_{k}\left(\frac{m_{o 1}}{2}\right) J_{\frac{q-k n}{m}}^{*}\left(\frac{m_{o 2}}{2}\right) C_{D C}^{k, q} \operatorname{sen}\left(k \phi_{1}+\frac{q-k n}{m} \phi_{2}\right)
\end{aligned}
$$

$\operatorname{con} C_{D C}^{k, q}=\cos \left(\frac{m_{d c}}{2}-\frac{k \pi}{2}-\frac{\frac{q-k n}{m} \pi}{2}\right)$. Finalmente:

$$
e_{\text {eom }}(t)=A_{e} \sum_{q=-\infty}^{\infty} A_{q} \cos \left(\left(\omega_{c}+q \omega_{u}\right) t+\phi_{e}+\phi_{q}\right)
$$

con $A_{q}=\sqrt{I_{q}^{2}+Q_{q}^{2}}$ y $\phi_{q}=\arctan \left(\frac{Q_{q}}{I_{q}}\right)$. Aprovechando los resultados de las ecuaciones (5.42) a (5.44), es posible obtener las siguientes expresiones para las señales $e_{\text {red }}(t)$ e $i_{p d}(t)$ de la figura 5.6:

$$
e_{\text {red }}(t)=A_{e} \sum_{q=-\infty}^{\infty} A_{q} \cos \left(\left(\omega_{c}+q \omega_{u}\right) t+\phi_{\text {red }}+\phi_{q}+\theta_{1} q \omega_{u}+\frac{\theta_{2}}{2} q^{2} \omega_{u}^{2}\right)
$$

donde $\phi_{\text {red }}=\phi_{e}+\theta_{0}$.

$$
\begin{aligned}
i_{p d}(t)=A_{p d}\left[\sum_{q=-\infty}^{\infty} A_{q}\right. & \left.\cos \left(q \omega_{u}\left(t+\theta_{1}\right)+\phi_{q}+\frac{\theta_{2}}{2} q^{2} \omega_{u}^{2}\right)\right]^{2} \\
+ & A_{p d}\left[\sum_{q=-\infty}^{\infty} A_{q} \operatorname{sen}\left(q \omega_{u}\left(t+\theta_{1}\right)+\phi_{q}+\frac{\theta_{2}}{2} q^{2} \omega_{u}^{2}\right)\right]^{2}
\end{aligned}
$$

donde $A_{p d}=\mathcal{R} \cdot A_{e}^{2} / 2$. 
Con el fin de identificar claramente cada componente espectral de la señal $i_{p d}(t)$ se procederá de forma similar a como se hizo en el caso de modulación por un tono:

$$
\begin{aligned}
i_{p d}(t) & =A_{p d} \sum_{r=-\infty}^{\infty} \sum_{s=-\infty}^{\infty} A_{r} A_{s}\left[\cos \left(r \omega_{u}\left(t+\theta_{1}\right)+\phi_{r}+\frac{\theta_{2}}{2} r^{2} \omega_{u}^{2}\right)\right. \\
& \left.\cos \left(s \omega_{u}\left(t+\theta_{1}\right)+\phi_{s}+\frac{\theta_{2}}{2} s^{2} \omega_{u}^{2}\right)\right] \\
& +A_{p d} \sum_{r=-\infty}^{\infty} \sum_{s=-\infty}^{\infty} A_{r} A_{s}\left[\operatorname{sen}\left(r \omega_{u}\left(t+\theta_{1}\right)+\phi_{r}+\frac{\theta_{2}}{2} r^{2} \omega_{u}^{2}\right)\right. \\
& \left.\operatorname{sen}\left(s \omega_{u}\left(t+\theta_{1}\right)+\phi_{s}+\frac{\theta_{2}}{2} s^{2} \omega_{u}^{2}\right)\right] \\
= & A_{p d} \sum_{r=-\infty}^{\infty} \sum_{s=-\infty}^{\infty} A_{r} A_{s} \cos \left((r-s) \omega_{u}\left(t+\theta_{1}\right)+\phi_{r}-\phi_{s}+\frac{\theta_{2}}{2}\left(r^{2}-s^{2}\right) \omega_{u}^{2}\right) \\
= & A_{p d} \sum_{p=-\infty}^{\infty} I_{I}(p) \cos \left(p \omega_{u}\left(t+\theta_{1}\right)\right)-A_{p d} \sum_{p=-\infty}^{\infty} I_{Q}(p) \operatorname{sen}\left(p \omega_{u}\left(t+\theta_{1}\right)\right)
\end{aligned}
$$

donde

$$
\begin{aligned}
& I_{I}(p)=\sum_{s=-\infty}^{\infty} A_{p+s} A_{s} \cos \left(\phi_{p+s}-\phi_{s}+\frac{\theta_{2}}{2}\left(p^{2}+2 s p\right) \omega_{u}^{2}\right) \\
& I_{Q}(p)=\sum_{s=-\infty}^{\infty} A_{p+s} A_{s} \operatorname{sen}\left(\phi_{p+s}-\phi_{s}+\frac{\theta_{2}}{2}\left(p^{2}+2 s p\right) \omega_{u}^{2}\right)
\end{aligned}
$$

Arreglando el resultado de la ecuación (5.72) y simplificando:

$$
i_{p d}(t)=A_{p d}\left[I_{I}(0)+\sum_{p=1}^{\infty} I_{p d}(p) \cos \left(p \omega_{u}\left(t+\theta_{1}\right)+\phi_{p d}(p)\right)\right]
$$

donde

$$
\begin{aligned}
I_{p d}(p) & =\sqrt{\left(I_{I}(p)+I_{I}(-p)\right)^{2}+\left(I_{Q}(p)-I_{Q}(-p)\right)^{2}} \\
\phi_{p d}(p) & =\arctan \left(\frac{I_{Q}(p)-I_{Q}(-p)}{I_{I}(p)+I_{I}(-p)}\right)
\end{aligned}
$$

ecuación de la que es inmediato extraer la potencia asociada a cada armónico de la frecuencia común:

$$
\operatorname{Potencia}\left(n \omega_{R F}\right)=\frac{A_{p d}^{2} \cdot I_{p d}^{2}(n)}{2}
$$

excepto para $n=0$ donde Potencia $(0)=P_{D C}=A_{p d}^{2} \cdot I_{I}^{2}(0)$.

El nivel de los productos de intermodulación presentes a la salida de la línea de retardo de la figura 5.6, cuando la entrada eléctrica consiste en una señal multiportadora depende de los siguientes factores: 
- Número de portadoras, $N$.

- Índice de modulación de cada portadora, $m_{o i}$.

- Separación en frecuencia entre las portadoras.

- Fase de cada portadora, $\phi_{R F i}$.

- Punto de polarización del modulador intererométrico, $V_{D C} / V_{\pi}$.

- Dispersión de la red de difracción, $\delta$.

El análisis realizado previamente está limitado a dos portadoras en la señal de radiofrecuencia; pero, aún en ese caso, los grados de libertad son muchos; por lo que, los resultados presentados a continuación están limitados a los casos de dos portadoras con idéntico índice de modulación $\left(m_{o 1}=m_{o 2}\right)$ e igual fase de cada portadorai.

En cuanto al modulador Mach-Zehnder, los resultados presentados a continuación incluyen únicamente el punto de polarización lineal, pues debido a la alta no linearidad del modulador polarizado en los puntos de potencia óptica máxima y mínima su uso queda limitado a señales de banda muy estrecha en conjunción con sistemas de elevación en frecuencia.

En las figuras 5.16 se muestra el nivel (relativo a $A_{p d}^{2}$ ) de los armónicos y productos de intermodulación a la salida de una línea de retardo con una red de difracción que introduce una dispersión $\delta(\mathrm{ps} / \mathrm{nm})$, cuando la entrada la forman dos señales de 5 y $6 \mathrm{GHz}$ que modulan un EOM con un mismo índice de modulación $m_{o}$. En la figura 5.16a se puede comprobar, cómo, para un valor pequeño de dispersión, el comportamiento de los productos de intermodulación al aumentar el índice de modulación es el esperado, con aumento proporcionales al orden de cada producto; mostrándose en la figura los productos de intermodulación de segundo orden (suma y diferencia), los productos de tercer orden diferencia e incluso los productos de quinto orden diferencia.

Por otro lado, al ir aumentando la dispersión de la red de difracción (Fig. 5.16b), el efecto es pequeño sobre las frecuencias fundamentales y los productos de intermodulación de tercer orden, traduciéndose en una atenuación de los niveles detectados; mientras que, para los productos de orden par, el aumento de la dispersión de la línea de retardo, se convierte en un incremento importante del nivel de los mismos. Este efecto es similar al observado en los armónicos de distorsión de la sección anterior (Fig. 5.13).

En las aplicaciones de redes conformadoras de haces, es habitual trabajar con señales de banda estrecha, por lo que tendría poco sentido analizar los productos de intermodulación. Ahora bien, el gran ancho de banda que ofrecen las redes

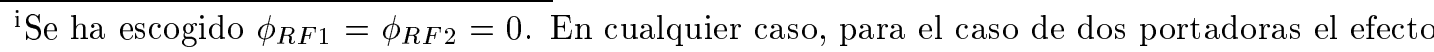
de la diferencia de fase entre las mismas no es muy importante. 


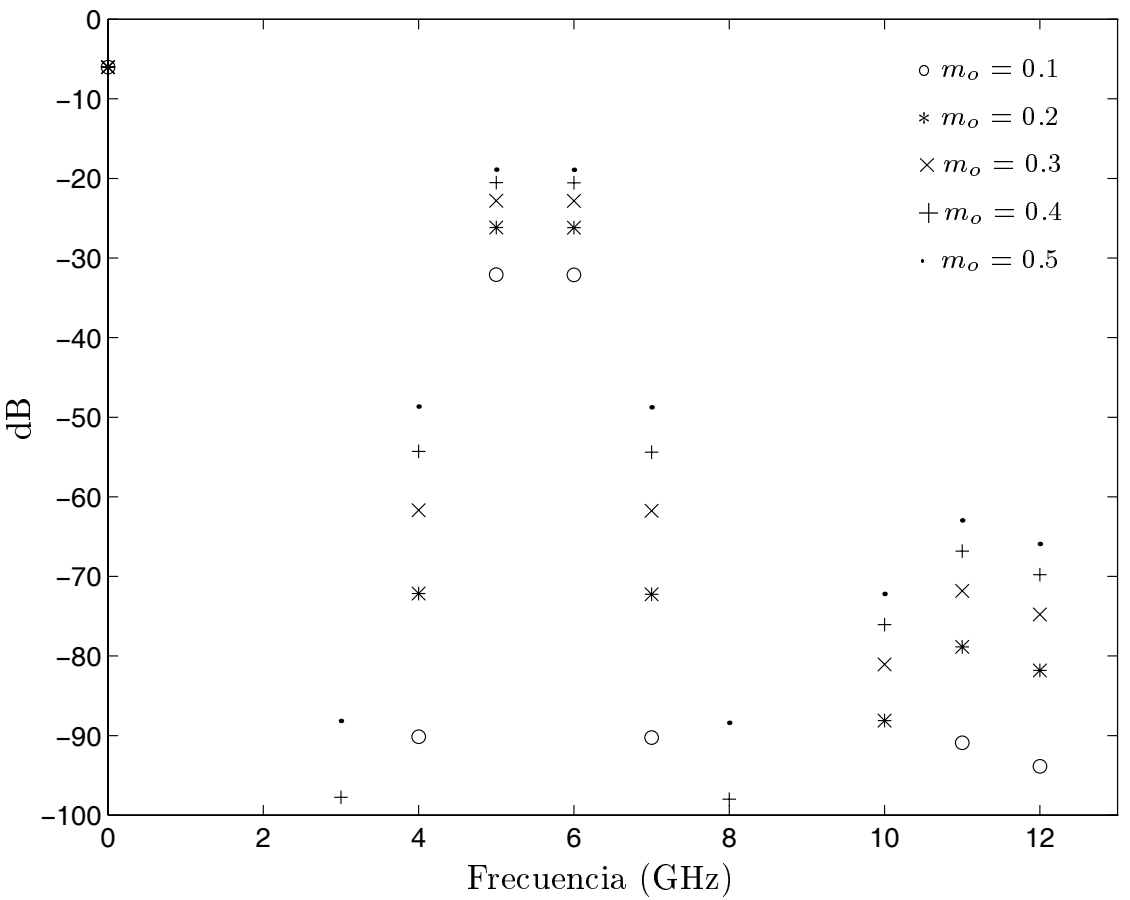

a)

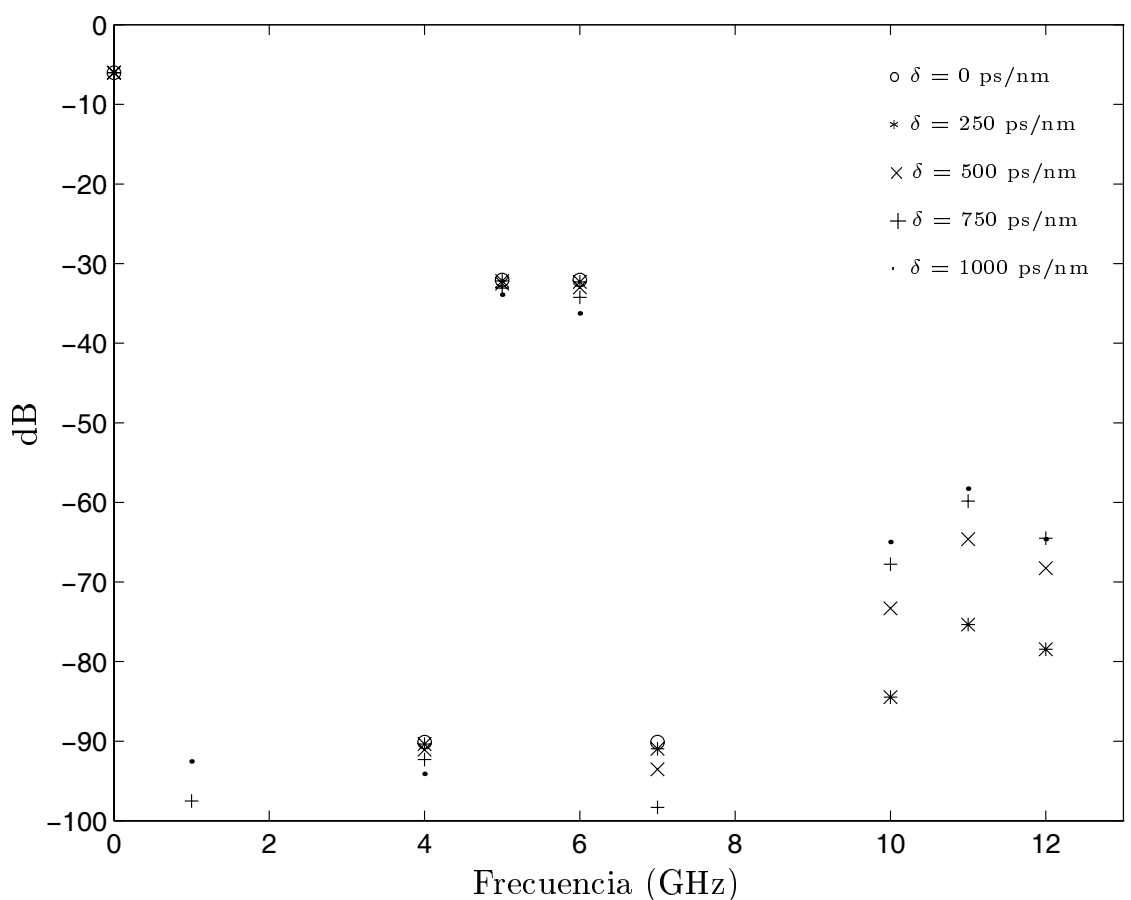

b)

Figura 5.16: Potencia relativa de los armónicos y de los productos de intermodulación a la salida del fotodetector para una línea de retardo con pendiente $\delta(\mathrm{ps} / \mathrm{nm})$ y un modulador interferométrico polarizado en la zona lineal y modulado por dos tonos de $5 \mathrm{GHz}$ y $6 \mathrm{GHz}$ con índices de modulación $\left(m_{o}=m_{o 1}=m_{o 2}\right)$. a) Resultados para $m_{o}=0.1,0.2,0.3,0.4$ y 0.5, y $\delta=100 \mathrm{ps} / \mathrm{nm}$. b) Resultados para $\delta=0$, 250, 500, 750 y $1000 \mathrm{ps} / \mathrm{nm}$, y $m_{o}=0.1$. 
conformadoras de haces ópticas, permiten su utilización en sistemas multiportadora y de banda ancha; siendo, por tanto, interesante el estudio de estos términos. En concreto, el término más interesante corresponde a los productos de intermodulación de tercer orden diferencia $\left(P_{3 I M_{-}}\right)$, ya que son los de mayor nivel dentro de aquellos cuyas frecuencias pueden estar dentro del ancho de banda del sistema. Por todo esto, se pasa a analizar con más detalle los productos de intermodulación de tercer orden diferencia.

En la figura 5.17, se muestra el efecto de la dispersión de la línea de retardo, expresada para la frecuencia más baja del par de tonos de entrada $\left(\phi_{\text {disp }}^{R F 1}=\theta_{2} * \omega_{R F 1}^{2}\right)$, sobre los niveles relativos de las frecuencias fundamentales y de los productos de intermodulación de tercer orden diferencia para tres combinaciones de frecuencias relativas de los dos tonos. Los resultados de esta figura muestran como el nivel de los citados productos de intermodulación se mantiene constante, hasta que entra en juego la dispersión de la línea de retardo; como uno de los productos $P_{3 I M}$ - está por debajo de las dos frecuencias fundamentales, mientras que el otro producto está por encima, cada uno de ellos comienza a sufrir los efectos de la atenuación dispersiva para valores inferiores y superiores de $\phi_{\text {disp }}^{R F 1}$, respectivamente, de los correspondientes a las frecuencias fundamentales $\omega_{R F 1} \mathrm{y} \omega_{R F 2}$.

Por otro lado, en las figuras 5.18 y 5.19 se puede observar el efecto del aumento del nivel de las señales de radiofrecuencia de entrada al EOM sobre los niveles detectados de los fundamentales y los productos $P_{3 I M_{-}}$, en presencia de dispersión en la línea de retardo según $\phi_{d i s p}^{R F 1}=0.1 \mathrm{rad}$ y $\phi_{\text {disp }}^{R F 1}=1 \mathrm{rad}$, respectivamente. 
a)

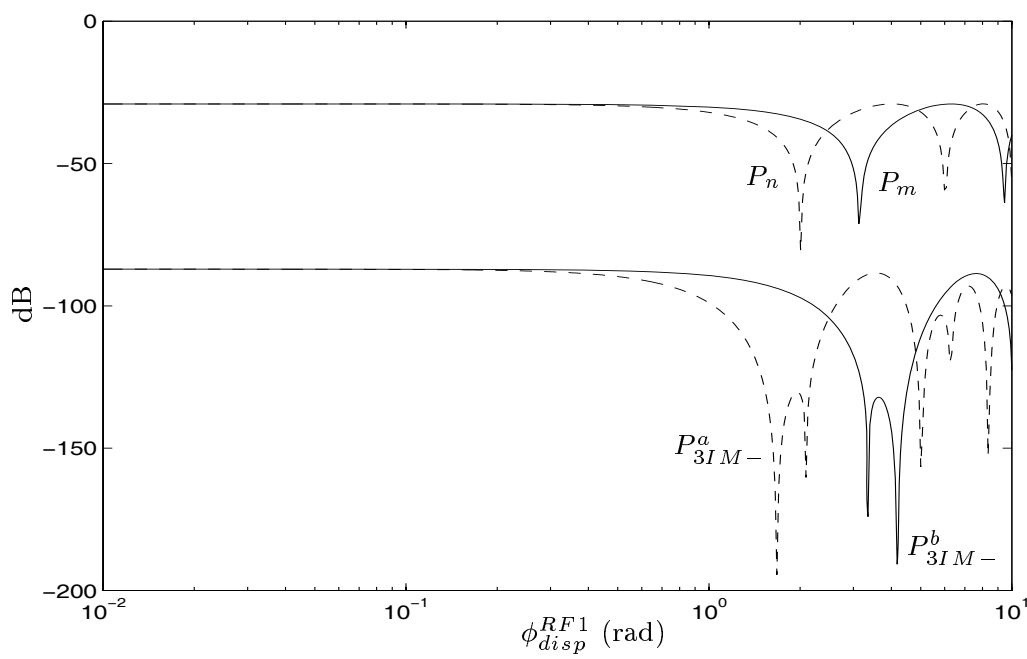

b)

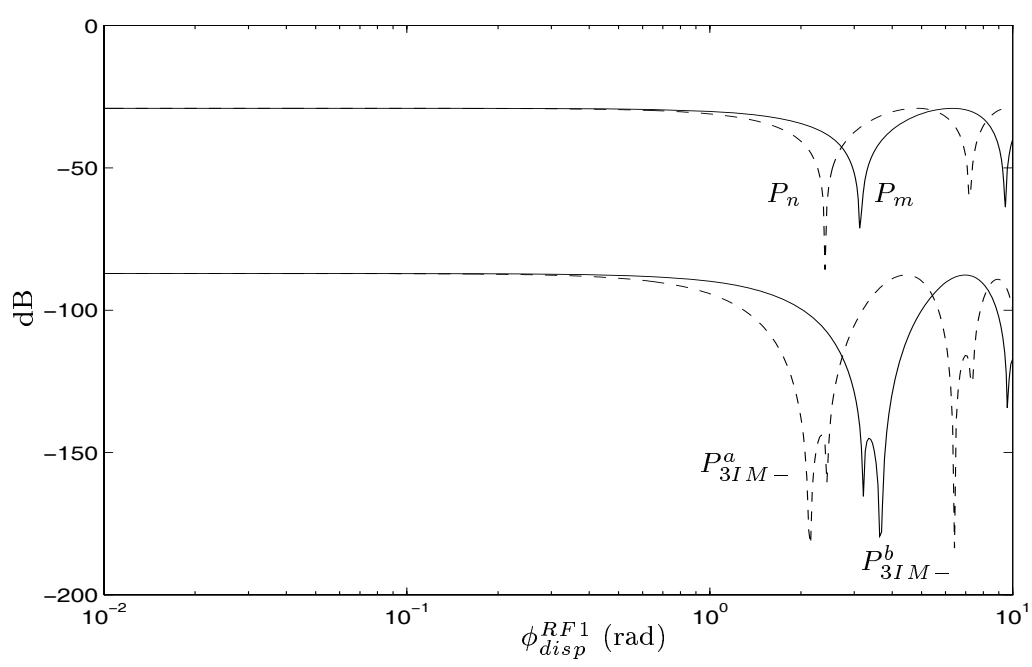

c)

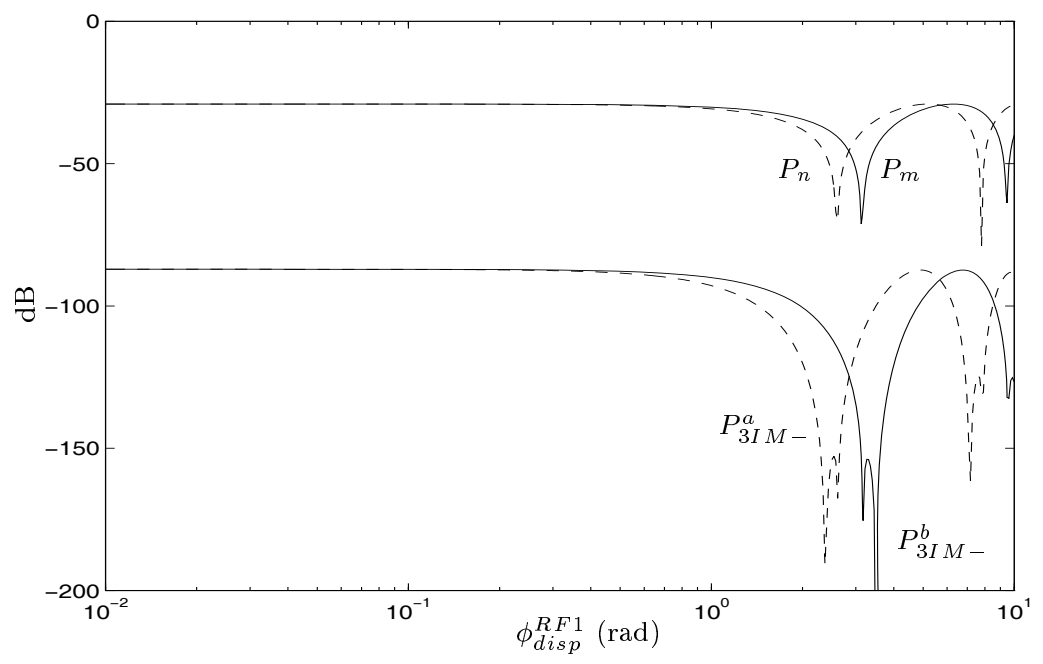

Figura 5.17: Potencia relativa de las frecuencias fundamentales y de los productos de intermodulación de tercer orden diferencia a la salida del fotodetector en función del producto de dispersión de la línea de retardo para $\omega_{R F 1}=n \omega_{u}, \phi_{\text {disp }}^{R F 1}$; tomando $m_{o 1}=m_{o 2}=0.1$. a) $n=4, m=5$. b) $n=7, m=8$. c) $n=10, m=11$. 
a)

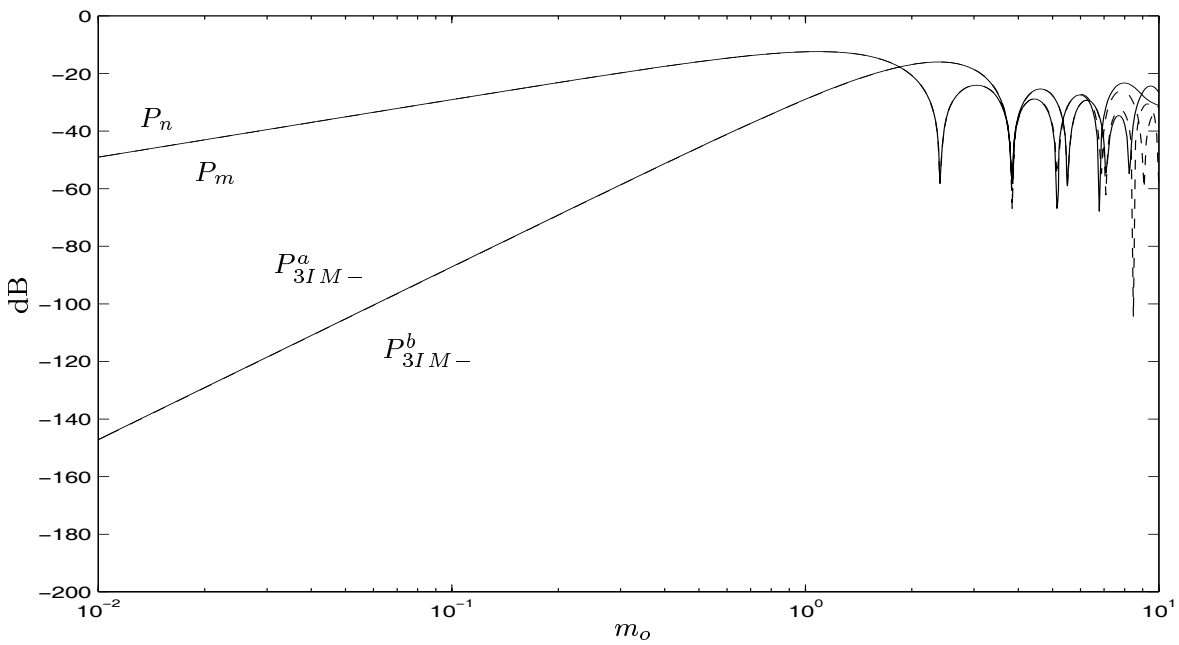

b)

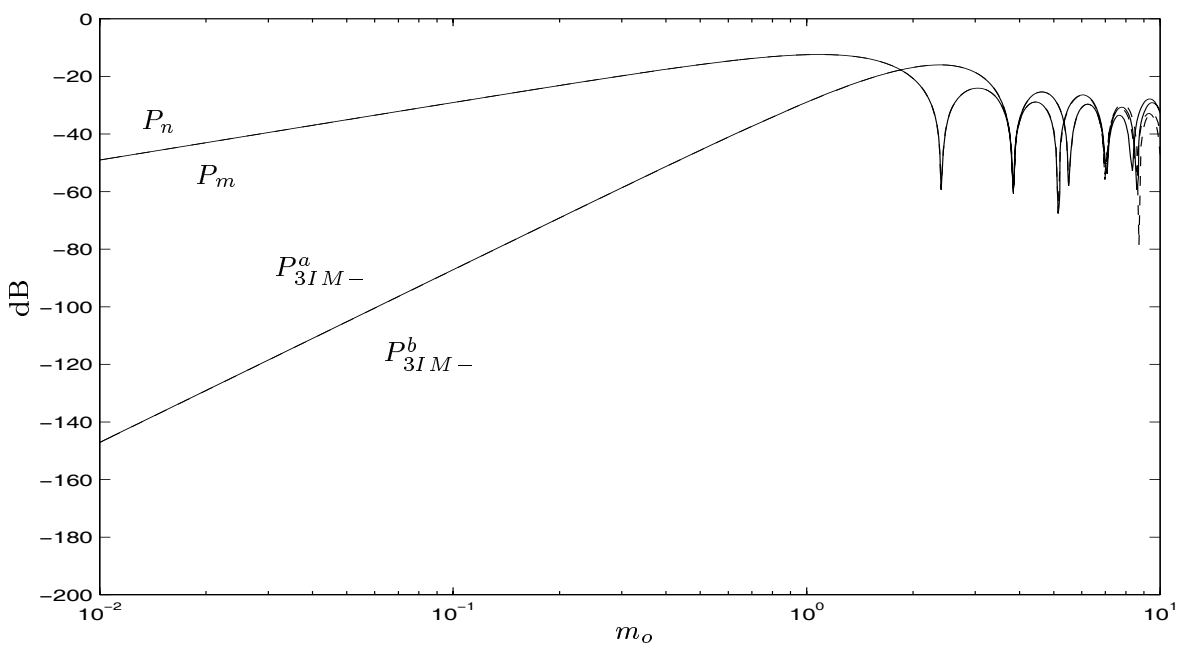

c)

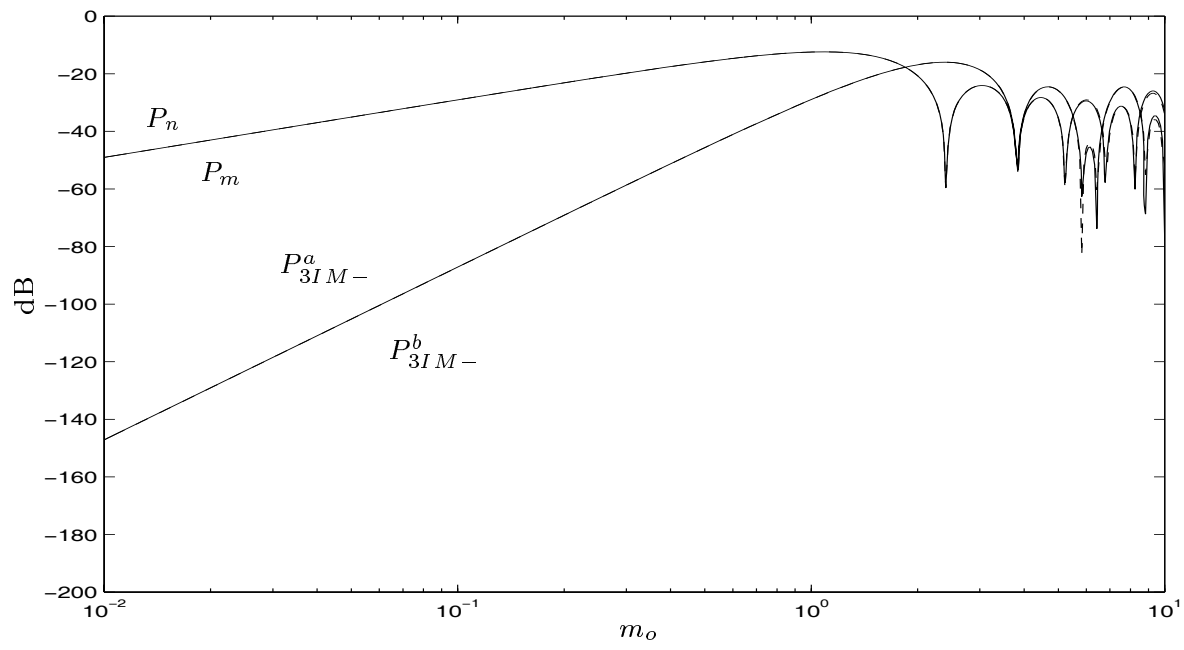

Figura 5.18: Potencia relativa de las frecuencias fundamentales y de los productos de intermodulación de tercer orden diferencia a la salida del fotodetector en función del índice de modulación óptico $\left(m_{o}=m_{o 1}=m_{o 2}\right)$ para una dispersión de $\phi_{\text {disp }}^{R F 1}=0.1$ rad. a) $n=4, m=5$. b) $n=7, m=8$. c) $n=10, m=11$. 
a)

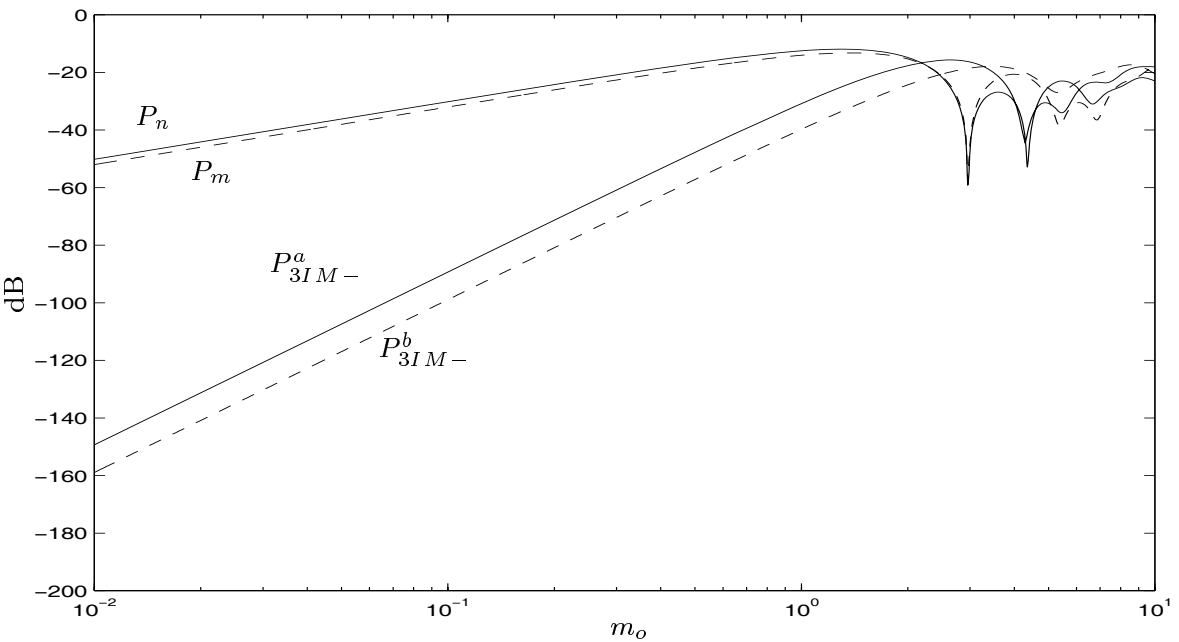

b)

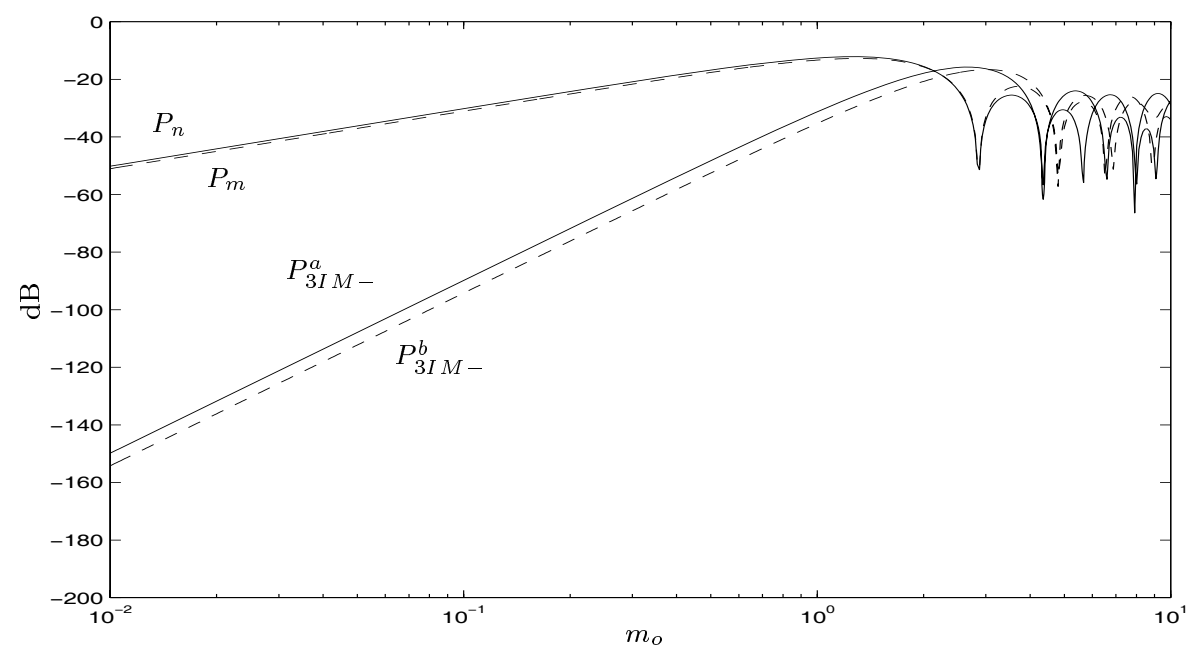

c)

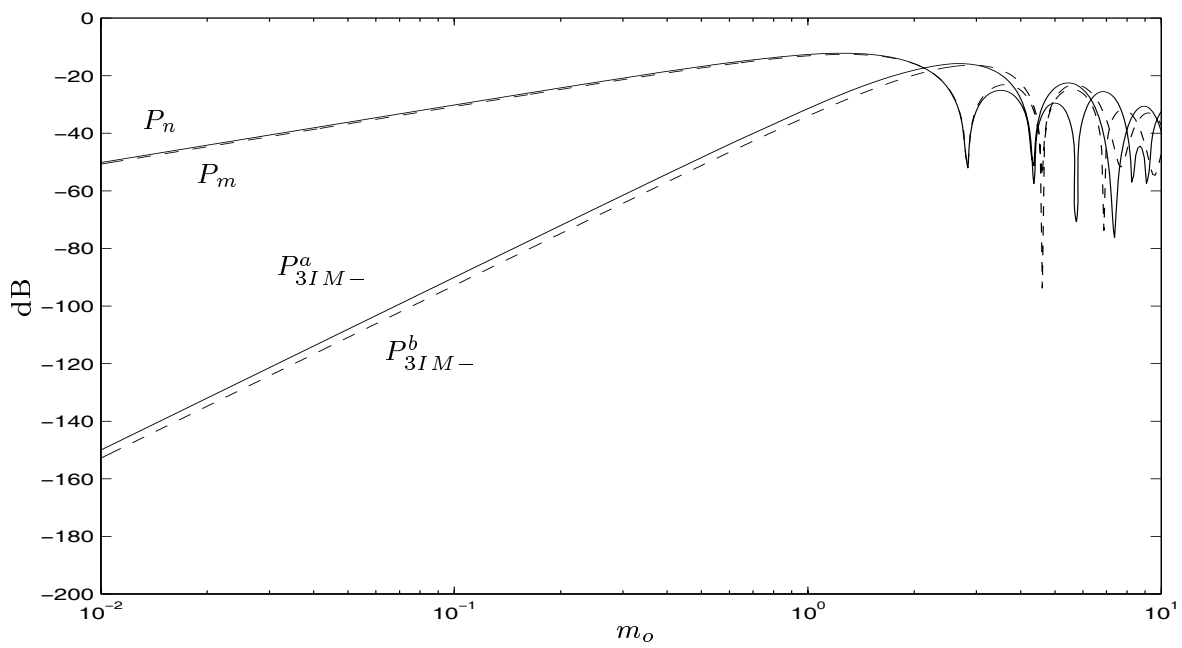

Figura 5.19: Potencia relativa de las frecuencias fundamentales y de los productos de intermodulación de tercer orden diferencia a la salida del fotodetector en función del índice de modulación óptico $\left(m_{o}=m_{o 1}=m_{o 2}\right)$ para una dispersión de $\phi_{\text {disp }}^{R F 1}=1 \mathrm{rad}$. a) $n=4, m=5$. b) $n=7, m=8$. c) $n=10, m=11$. 


\section{Aplicación de modulaciones distintas de la modulación de intensidad con- vencional}

Después de mostrar las limitaciones que la dispersión de la red de difracción introduce en las prestaciones de la línea de retardo, se ha estudiado la posibilidad de utilizar esquemas de modulación diferentes de la modulación de intensidad convencional. En primer lugar, se ha analizado la modulación en banda lateral única con portadora $\left(\mathrm{SSB}+\mathrm{C}^{\mathrm{i}}\right)$ con el objetivo de reducir o eliminar la atenuación dispersiva que aparecía debida al diferente retardo entra ambas bandas laterales.

Otros esquemas de modulación analizados han sido las modulaciones heterodinas, con la intención de aplicar el resultado visto previamente de que polarizando en MIP, no afecta la dispersión introducida por la red de difracción al nivel del segundo armónico; pero, como se verá posteriormente, su aplicación sólo es válida para señales de banda estrecha y de alta frecuencia.

Modulación en banda lateral única Habitualmente, en un modulador interferométrico Mach-Zehnder (Fig. 5.7), la señal de entrada (polarización y moduladora) se aplica simétricamente entre ambos electrodos $(+V / 2$ y $-V / 2)$. Por otro lado, en el caso de querer obtener un modulador en banda lateral única es conocido que se necesita disponer de la señal moduladora y de su transformada de Hilbert ${ }^{\mathrm{ii}}$ y modular con ambas señales la portadora en cuadratura.

Una forma de conseguir este objetivo es trabajar con un modulador MachZehnder con control independiente de ambos electrodos y escoger la tensión a aplicar a los electrodos de tal forma que se cumplan los criterios presentados a continuación.

Sean los valores de tensión indicados en las ecuaciones (5.78) las expresiones generales de las tensiones aplicadas a ambos electrodos, incluyendo un término fijo más un tono de igual amplitud, pero, distinta fase en cada electrodo:

$$
\begin{aligned}
& V_{1}=V_{D C 1}+V_{R F} \operatorname{sen}\left(\omega_{R F} t+\phi_{1}\right) \\
& V_{2}=V_{D C 2}+V_{R F} \operatorname{sen}\left(\omega_{R F} t+\phi_{2}\right)
\end{aligned}
$$

en ese caso, los criterios que deberán cumplirse para obtener una modulación en banda lateral única son:

- Aplicar a cada uno de los dos electrodos la señal moduladora y la transformada de Hilbert respectivamente, es decir la señal original más la misma señal con

\footnotetext{
${ }^{\mathrm{i}}$ Single SideBand plus Carrier.

${ }^{\mathrm{ii}}$ La transformada de Hilbert de una señal es el resultado de pasar la señal por un filtro cuya función de transferencia sea:

$$
\begin{array}{ll}
H(\omega)=-j & \forall \omega>0 \\
H(\omega)=+j & \forall \omega<0
\end{array}
$$

es decir, un filtro que desfase $90^{\circ}$ todas las frecuencias; respuesta que se puede conseguir a frecuencias de microondas con un híbrido de banda ancha cuyas salidas estén desfasadas $90^{\circ}$.
} 


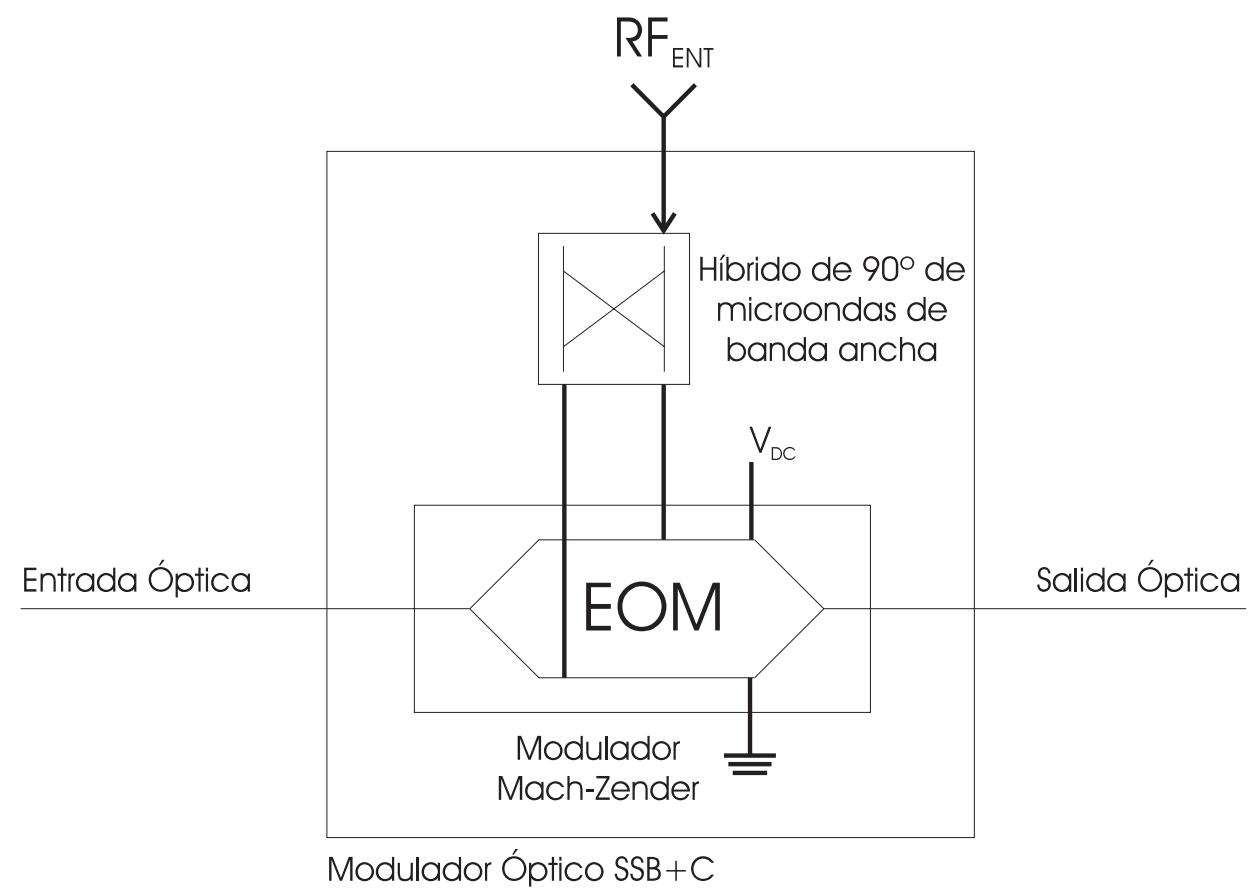

Figura 5.20: Modulador electro-óptico dual aplicado como modulador en banda lateral única con portadora $(\mathrm{SSB}+\mathrm{C})$.

todas sus componentes desfasadas $90^{\circ}$.

$$
\left|\phi_{1}-\phi_{2}\right|=\frac{\pi}{2}
$$

- Aplicar una diferencia de tensión contínua entre ambos electrodos tal que la diferencia de fase inducida sobre la portadora óptica sea $\pi / 2$, es decir:

$$
\left|V_{D C 1}-V_{D C 2}\right|=\frac{V_{\pi}}{2}
$$

Considerando el esquema de la figura 5.6, donde en este caso el EOM es un modulador dual como el mostrado en la figura 5.20, el campo óptico a la salida del modulador alimentado de acuerdo a las ecuaciones (5.78) sería:

$$
\begin{aligned}
e_{e o m / s s b}(t)=\frac{A_{e}}{2}[\cos & \left(\omega_{c} t+\phi_{e}+\frac{\pi V_{D C 1}}{V_{\pi}}+\frac{\pi V_{R F}}{V_{\pi}} \operatorname{sen}\left(\omega_{R F} t+\phi_{1}\right)\right) \\
& \left.+\cos \left(\omega_{c} t+\phi_{e}+\frac{\pi V_{D C 2}}{V_{\pi}}+\frac{\pi V_{R F}}{V_{\pi}} \operatorname{sen}\left(\omega_{R F} t+\phi_{2}\right)\right)\right]
\end{aligned}
$$

donde $A_{e}$ y $\phi_{e}$ diferirán de la amplitud y fase de entrada al modulador $\left(A_{o}\right.$ y $\left.\phi_{o}\right)$ de acuerdo con las pérdidas y la fase de inserción del modulador, respectivamente.

De la misma forma que se hizo en el caso de la modulación de amplitud convencional y con el fin de identificar las diferentes componentes espectrales de la señal 
modulada, se ha calculado por separado el desarrollo en serie de Fourier de ambos sumandos en la ecuación (5.81), obteniéndose el siguiente resultado:

$$
\begin{aligned}
e_{e o m / s s b}(t) & =\frac{A_{e}}{2}\left[\sum_{n=-\infty}^{\infty} J_{n}\left(\frac{\pi V_{R F}}{V_{\pi}}\right) \cos \left(\omega_{c} t+\phi_{e}+\frac{\pi V_{D C 1}}{V_{\pi}}+n \omega_{R F} t+n \phi_{1}\right)\right. \\
& \left.+\sum_{n=-\infty}^{\infty} J_{n}\left(\frac{\pi V_{R F}}{V_{\pi}}\right) \cos \left(\omega_{c} t+\phi_{e}+\frac{\pi V_{D C 2}}{V_{\pi}}+n \omega_{R F} t+n \phi_{2}\right)\right]
\end{aligned}
$$

donde $J_{n}(\beta)$ es la función de Bessel de primera especie de orden $\mathrm{n}$ de argumento $\beta$. Simplificando la expresión anterior se llega al siguiente resultado:

$$
e_{e o m / s s b}(t)=A_{e} \sum_{n=-\infty}^{\infty} J_{n}\left(m_{o}\right) \cos \left(\left(\omega_{c}+n \omega_{R F}\right) t+\phi_{o}+n \phi_{m}\right) \cos \left(\phi_{v}+n \phi_{d}\right)
$$

donde

$$
\begin{aligned}
m_{o} & =\frac{\pi V_{R F}}{V_{\pi}} \\
\phi_{c} & =\phi_{e}+\frac{\pi\left(V_{D C 1}+V_{D C 2}\right)}{2 V_{\pi}} \\
\phi_{m} & =\frac{\phi_{1}+\phi_{2}}{2} \\
\phi_{v} & =\frac{\pi\left(V_{D C 1}-V_{D C 2}\right)}{2 V_{\pi}} \\
\phi_{d} & =\frac{\phi_{1}-\phi_{2}}{2}
\end{aligned}
$$

Teniendo en cuenta las ecuaciones (5.79) y (5.80), se obtiene que el factor $\cos \left(\phi_{v}+\right.$ $n \phi_{d}$ ) de la expresión (5.83) toma el valor:

$$
\cos \left(\phi_{v}+n \phi_{d}\right)=\cos \left(\frac{\pi}{4} \pm \frac{n \pi}{4}\right)
$$

factor que se anulará cuando se cumpla la condición:

$$
n=4 i \pm 1 \quad \forall i \in \mathbb{Z}
$$

donde la ambigüedad del signo depende del signo de las diferencias $V_{D C 1}-V_{D C 2}$ y $\phi_{1}-\phi_{2}$, cuyo módulo viene definido por (5.79) y (5.80). En concreto, si ambas diferencias son positivas y teniendo en cuenta las ecuaciones (5.87), (5.88) y (5.89) se desprende que los términos del sumatorio que cumplan $n=1+4 i \forall i \in \mathbb{Z}$ se anularán y por lo tanto no existe potencia para las pulsaciones $\omega=\omega_{c}+(1+4 i) \omega_{R F}$ $\forall i \in \mathbb{Z}$, es decir, para $\omega=\omega_{c}-3 \omega_{R F}, \omega=\omega_{c}+\omega_{R F}$ o $\omega=\omega_{c}+5 \omega_{R F}$ por ejemplo. En el caso particular, habitual por otro lado, de señales con bajos índices ópticos de modulación $\left(m_{o}\right)$ y aplicando las aproximaciones de las funciones de Bessel mostradas en la ecuación (5.46) y la propiedad de simetría de las funciones de Bessel 
$J_{-n}(\beta)=(-1)^{n} J_{n}(\beta) \forall n \in \mathbb{Z}$, se obtiene que los dos únicos términos significativos del desarollo de la ecuación (5.83) serían los correspondientes a la portadora óptica $(n=0)$ y a la banda lateral inferior $(n=-1)$ :

$$
e_{e o m / s s b}(t) \approx \frac{A_{e}}{2}\left[\sqrt{2} \cos \left(\omega_{c} t+\phi_{c}\right)-m_{o} \cos \left(\left(\omega_{c}-\omega_{R F}\right) t+\phi_{c}-\phi_{m}\right)\right]
$$

quedando demostrado que aplicando a los electrodos de un modulador interferométrico las tensiones mostradas en la ecuaciones (5.78), con $V_{D C 1}=V_{D C 2}-V_{\pi} / 2 \mathrm{y}$ $\phi_{1}=\phi_{2}-\pi / 2$ se obtiene una señal óptica modulada en banda lateral única inferior ${ }^{\mathrm{i}}$ con portadora, siempre y cuando se trabaje con índices de modulación bajos.

Volviendo a la expresión general de (5.83) y a la figura (5.6), es posible obtener la expresión del campo óptico a la salida de la línea de retardo formada por el circulador y la red de difracción ideales, definida ésta última de acuerdo con las ecuaciones (5.31) y (5.32); obteniéndose:

$$
\begin{aligned}
e_{\text {red } / s s b}(t) & =A_{e} \\
& \sum_{n=-\infty}^{\infty} J_{n}\left(m_{o}\right) C_{n} \cos \left(\omega_{c} t+\phi_{\text {red }}+n \omega_{R F}\left(t+\theta_{1}\right)+n \phi_{m}+\frac{\theta_{2}}{2} n^{2} \omega_{R F}^{2}\right)
\end{aligned}
$$

donde $\phi_{\text {red }}=\phi_{c}+\theta_{0}$ y $C_{n}=\cos \left(\phi_{v}+n \phi_{d}\right)$.

Operando de forma análoga al caso de modulación de amplitud convencional se puede obtener la intensidad de corriente detectada a la salida de un fotoreceptor ideal de responsividad $\mathcal{R}$ a partir de la envolvente del campo óptico incidente definido en la ecuación (5.92):

$$
\begin{aligned}
i_{p d / s s b}(t) & =A_{p d}\left[\sum_{n=-\infty}^{\infty} J_{n}\left(m_{o}\right) C_{n} \cos \left(n \omega_{R F}\left(t+\theta_{1}\right)+n \phi_{m}+\frac{\theta_{2}}{2} n^{2} \omega_{R F}^{2}\right)\right]^{2} \\
& +A_{p d}\left[\sum_{n=-\infty}^{\infty} J_{n}\left(m_{o}\right) C_{n} \operatorname{sen}\left(n \omega_{R F}\left(t+\theta_{1}\right)+n \phi_{m}+\frac{\theta_{2}}{2} n^{2} \omega_{R F}^{2}\right)\right]^{2}
\end{aligned}
$$

donde $A_{p d}=\mathcal{R} \cdot A_{e}^{2} / 2$.

Con el fin de identificar claramente cada uno de los armónicos de la señal moduladora, presentes en la señal detectada y siguiendo una reorganización similar a

${ }^{\mathrm{i}}$ Otras combinaciones de tensiones de polarización y desfase entre las señales moduladoras pueden dar lugar a modulaciones en banda lateral única superior o inferior. A partir de las expresiones generales para las tensiones de los electrodos, (5.78), se obtiene:

\begin{tabular}{|c|c|c|}
\hline$V_{D C 1}-V_{D C 2}$ & $\phi_{1}-\phi_{2}$ & BANDA LATERAL \\
\hline \hline$V \pi / 2$ & $\pi / 2$ & INFERIOR \\
\hline$-V \pi / 2$ & $\pi / 2$ & SUPERIOR \\
\hline$V \pi / 2$ & $-\pi / 2$ & SUPERIOR \\
\hline$-V \pi / 2$ & $-\pi / 2$ & INFERIOR \\
\hline
\end{tabular}


la realizada en el caso anterior se llega tras una serie de operaciones al siguiente resultado:

$$
\begin{aligned}
& i_{p d / s s b}(t)=A_{p d}\left[\sum_{n=1}^{\infty}(-1)^{n} J_{n}\left(m_{o}\right) C_{-n} \cos \left(n \omega_{R F}\left(t+\theta_{1}\right)+n \phi_{m}-\frac{\theta_{2}}{2} n^{2} \omega_{R F}^{2}\right)\right. \\
& \left.+J_{0}\left(m_{o}\right) C_{0}+\sum_{n=1}^{\infty} J_{n}\left(m_{o}\right) C_{n} \cos \left(n \omega_{R F}\left(t+\theta_{1}\right)+n \phi_{m}+\frac{\theta_{2}}{2} n^{2} \omega_{R F}^{2}\right)\right]^{2} \\
& +A_{p d}\left[-\sum_{n=1}^{\infty}(-1)^{n} J_{n}\left(m_{o}\right) C_{-n} \operatorname{sen}\left(n \omega_{R F}\left(t+\theta_{1}\right)+n \phi_{m}-\frac{\theta_{2}}{2} n^{2} \omega_{R F}^{2}\right)\right. \\
& \left.+\sum_{n=1}^{\infty} J_{n}\left(m_{o}\right) C_{n} \operatorname{sen}\left(n \omega_{R F}\left(t+\theta_{1}\right)+n \phi_{m}+\frac{\theta_{2}}{2} n^{2} \omega_{R F}^{2}\right)\right]^{2}
\end{aligned}
$$

Finalmente:

$$
\begin{aligned}
& i_{p d / s s b}(t)=\frac{A_{p d}}{2}\left[J_{0}\left(m_{o}\right) C_{0}\right. \\
& +\sum_{n=1}^{\infty} J_{n}\left(m_{o}\right)\left[W_{n} \cos \left(n \omega_{R F}\left(t+\theta_{1}\right)+n \phi_{m}\right) \cos \left(\frac{\theta_{2}}{2} n^{2} \omega_{R F}^{2}\right)\right. \\
& \left.\left.+X_{n} \operatorname{sen}\left(n \omega_{R F}\left(t+\theta_{1}\right)+n \phi_{m}\right) \operatorname{sen}\left(\frac{\theta_{2}}{2} n^{2} \omega_{R F}^{2}\right)\right]\right]^{2} \\
& +\frac{A_{p d}}{2}\left[\sum _ { n = 1 } ^ { \infty } J _ { n } ( m _ { o } ) \left[Y_{n} \operatorname{sen}\left(n \omega_{R F}\left(t+\theta_{1}\right)+n \phi_{m}\right) \cos \left(\frac{\theta_{2}}{2} n^{2} \omega_{R F}^{2}\right)\right.\right. \\
& \left.\left.+Z_{n} \cos \left(n \omega_{R F}\left(t+\theta_{1}\right)+n \phi_{m}\right) \operatorname{sen}\left(\frac{\theta_{2}}{2} n^{2} \omega_{R F}^{2}\right)\right]\right]^{2}
\end{aligned}
$$

donde

$$
\begin{aligned}
W_{n} & =(-1)^{n} C_{-n}+C_{n} \\
X_{n} & =(-1)^{n} C_{-n}-C_{n} \\
Y_{n} & =-(-1)^{n} C_{-n}+C_{n}=-X_{n} \\
Z_{n} & =(-1)^{n} C_{-n}+C_{n}=W_{n}
\end{aligned}
$$

El resultado de la ecuación (5.95) corresponde al caso general, para cualquier valor de índice de modulación. Generalmente el valor del índice óptico de modulación, $m_{o}$, se escoge pequeño con el fin de reducir el gran número de armónicos presentes en la ecuación (5.95); en ese caso, y aplicando las aproximaciones indicadas en la ecuación (5.46) se puede simplicar la ecuación (5.95) obteniéndose el siguiente 
resultado:

$$
\begin{aligned}
i_{p d / s s b}(t) \approx \frac{A_{p d}}{8}[ & \left.\sqrt{2} \pm m_{o} \cos \left(\omega_{R F}\left(t+\theta_{1}\right)+\phi_{m} \pm \frac{\theta_{2}}{2} \omega_{R F}^{2}\right)\right]^{2} \\
& +\frac{A_{p d}}{8}\left[m_{o} \operatorname{sen}\left(\omega_{R F}\left(t+\theta_{1}\right)+\phi_{m} \pm \frac{\theta_{2}}{2} \omega_{R F}^{2}\right)\right]^{2} \\
= & \frac{A_{p d}}{8}\left[2+m_{o}^{2} \pm 2 \sqrt{2} m_{o} \cos \left(\omega_{R F}\left(t+\theta_{1}\right)+\phi_{m} \pm \frac{\theta_{2}}{2} \omega_{R F}^{2}\right)\right]
\end{aligned}
$$

despreciando el término $m_{o}^{2}$ por trabajar con índices de modulación pequeños, la componente alterna de la señal eléctrica a la salida del fotodetector será:

$$
i_{P D s s b-a c}(t) \approx \pm \frac{A_{p d} \sqrt{2} m_{o}}{4} \cos \left(\omega_{R F}\left(t+\theta_{1}\right)+\phi_{m} \pm \frac{\theta_{2}}{2} \omega_{R F}^{2}\right)
$$

donde el operando \pm con signo positivo corresponde a la modulación en banda lateral única inferior y el signo negativo a la superior.

Una cualidad muy importante de la modulación en banda lateral única con portadora es que permite la detección directa, por lo que su utilización en esquemas IM/DD es casi inmediata. Como comprobación de esta propiedad, simplemente habría que sustituir en la ecuación (5.97) el caso particular de transmisión por una línea de retardo sin dispersión $\left(\theta_{2}=0\right)$.

Como se puede comprobar a partir de la comparación de las ecuaciones (5.48) y (5.98), la utilización de modulación en banda lateral única con portadora elimina el efecto de atenuación dispersiva en el retardador con red de difracción con periodo variable. La dispersión introducida por la red de difracción en el campo óptico se traslada al dominio eléctrico, pero sin afectar al nivel de la potencia detectada. El efecto de la dispersión presente en la señal detectada, puede eliminarse en el dominio eléctrico, mediante líneas dispersivas eléctricas.

Modulaciones autoheterodinas En términos generales, los sistemas con detección heterodina, se basan en mezclar la señal modulada con un tono ${ }^{\mathrm{i}}$ cuya frecuencia, $\omega_{O L}$, está separada de la frecuencia portadora de la señal modulada, $\omega_{c}$, por la frecuencia intermedia, $\omega_{I F}$, deseada a la salida del detector, cumpliéndose la relación $\omega_{O L}=\omega_{c} \pm \omega_{I F}$. Al trabajar con sistemas de comunicaciones ópticas es más adecuado hablar de señal óptica fija $\left(\omega_{f i j a}\right)$, portadora óptica modulada $\left(\omega_{\text {port }}\right)$, y señal de radiofrecuencia detectada $\left(\omega_{R F}\right)$; de forma que se cumpla $\left.\omega_{\text {fija }}=\omega_{\text {port }} \pm \omega_{R F}\right)$.

En sistemas de comunicaciones ópticas, la detección heterodina está incluida dentro de los sistemas ópticos coherentes, debido a que, a diferencia de la detección directa, requiere coherencia en la fase, tanto de la portadora modulada como de la señal de frecuencia fija. Esta necesidad de coherencia en la fase, no implica directamente que el sistema sea síncrono, pero impide la utilización de modulación

\footnotetext{
iTambién conocida como señal de oscilador local, definición no muy adecuada para los sistemas autoheterodinos, donde no existe ningún oscilador o fuente en el receptor.
} 


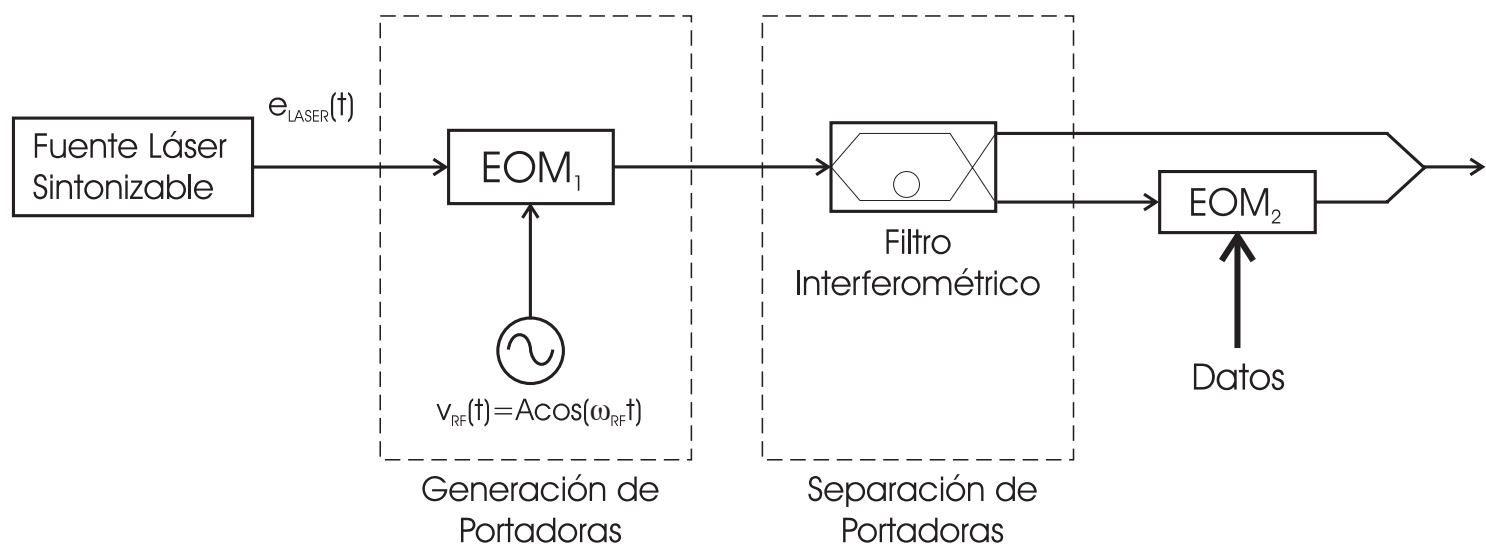

Figura 5.21: Generación de señal óptica autoheterodina con una única fuente óptica [Hof95].

directa, debido a la modulación de frecuencia indeseada que aparece asociada a la modulación directa.

El propio proceso de detección en un fotodetector, provoca un mezclado de las señales presentes en la señal óptica incidente, por que lo que para conseguir una detección heterodina en sistemas de comunicaciones ópticas, se debe disponer en la señal óptica incidente de la suma de la señal de frecuencia fija y de la señal modulada; el proceso de batido de ambas señales provoca la aparición de un gran número de productos de batido que deberán ser filtrados adecuadamente para obtener los términos deseadosi. En el caso de que la señal de frecuencia fija se genere en el transmisor y no en el receptor, de forma que se transmita junto a la señal modulada, se estará hablando de sistemas autoheterodinos.

La aplicación de técnicas autoheterodinas implica una mayor complejidad y un mayor coste del transmisor, pues es necesario generar dos portadoras ópticas separadas la frecuencia de radiofrecuencia deseada y posteriormente se ha de modular una de ellas. Un esquema adecuado para ello, es el mostrado en la figura 5.21, el cual requiere una única fuente óptica, lo que puede redundar en menores requerimientos en cuanto a anchura de línea y ruido de fase de la fuente óptica, siempre y cuando los diferentes retardos de cada portadora sean inferiores al tiempo de coherencia del láser.

El funcionamiento del generador de la figura 5.21 se basa en un utilizar un primer modulador interferométrico Mach-Zehnder $\left(\mathrm{EOM}_{1}\right)$ polarizado en MIP y modulado

${ }^{\mathrm{i} E n}$ concreto, si el campo óptico a la entrada del fotodetector es $e(t)=A x(t) \cos \left(\omega_{f i j a} t\right)+$ $B \cos \left(\omega_{\text {port }} t\right)$, donde $\omega_{\text {fija }}=\omega_{\text {port }}+\omega_{R F}$, a la salida del fotodetector aparecerían los siguientes grupos de componentes:

- $x(t)$ modulada alrededor de $\omega_{f i j a}-\omega_{\text {port }}=\omega_{R F}$.

- $x(t)$ en banda base.

- Armónicos de distorsión y productos de intermodulación de $x^{2}(t)$ en banda base. 


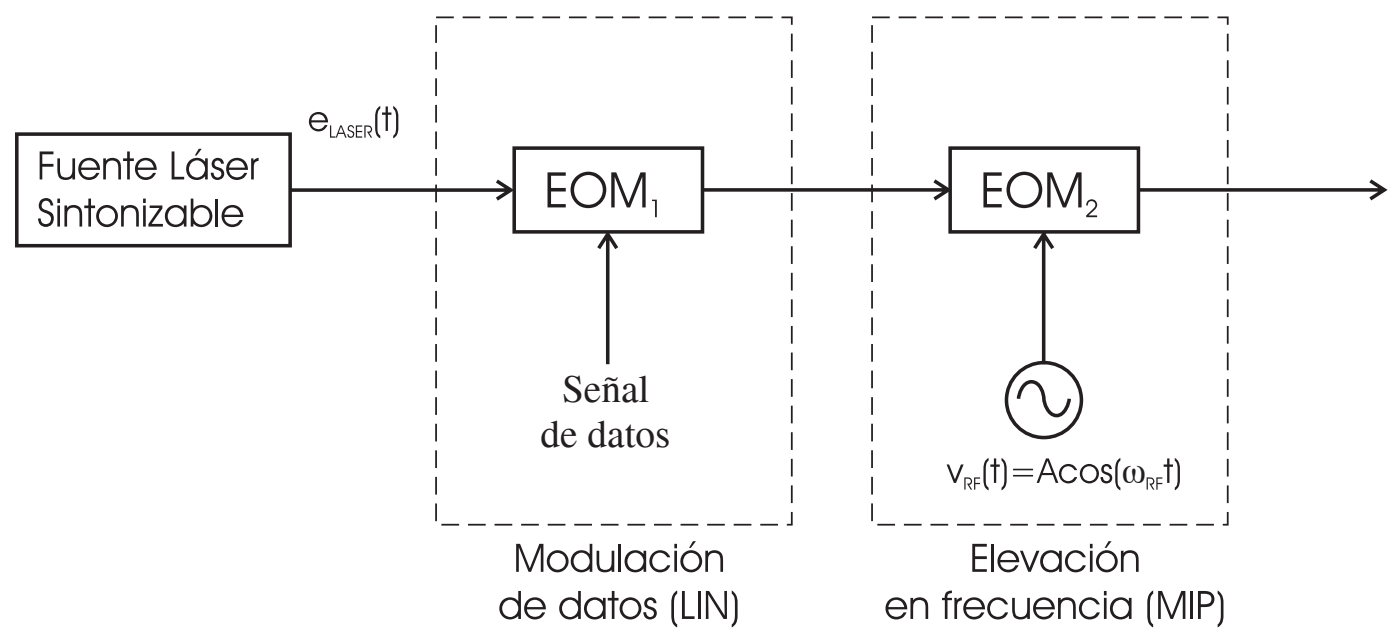

Figura 5.22: Modulador óptico pseudo-autoheterodino.

con una señal de radiofrecuencia de frecuencia mitad de la deseada, $\omega_{I N}=\omega_{O L} / 2$; de esta forma, a la salida del modulador $\mathrm{EOM}_{1}$, aparecerá una señal cuyo espectro está formado por armónicos impares de la frecuencia eléctrica de entrada, $\omega_{I N}$, alrededor de la frecuencia óptica de entrada al modulador, $\omega_{c}$. Al polarizar el modulador en MIP la componente a la frecuencia de la portadora óptica es nula. Si además se escoge un índice de modulación adecuado para que el nivel del primer armónico de cada banda lateral de la señal óptica sea máximoi, a la salida de un modulador ideal se dispondrá básicamente de dos portadoras ópticas separadas $\omega_{O L}$, cada una de ellas con una potencia $4.7 \mathrm{~dB}$ por debajo de la potencia de la señal óptica incidente. El último paso consiste en separar ambas portadoras, modular una de ellas con la señal de banda estrecha y volver a combinar ambas portadoras.

El generador autoheterodino de la figura 5.21 no es adecuado para la línea de retardo variable, pues el bloque de separación de las portadoras debería ser capaz de seguir las variaciones de la frecuencia central óptica de la fuente láser sintonizable.

Modulación pseudo-autoheterodina Debido a las limitaciones del esquema de modulación de la figura 5.21, se ha analizado el modulador pseudo-autoheterodino mostrado en la figura 5.22, formado por dos moduladores externos en cascada, el primero de los cuales $\left(\mathrm{EOM}_{1}\right)$ es polarizado en LIN y modulará la señal óptica del láser con la señal de datos, de forma que la señal óptica modulada se introduce en un segundo modulador $\left(\mathrm{EOM}_{2}\right)$ que polarizado en MIP y con un nivel de señal moduladora adecuado, duplicará el espectro óptico de entrada alrededor de la portadora óptica, generando por tanto dos señales separadas dos veces la frecuencia de la señal moduladora de $\mathrm{EOM}_{2}$ [Fus97].

Para analizar el comportamiento del modulador pseudo-heterodino, se partirá de una señal de datos modelada como un tono a una frecuencia intermedia dada.

${ }^{\mathrm{i}}$ De acuerdo con (5.41) el nivel de las componentes a las frecuencias $\omega_{c} \pm \omega_{O L}$ depende de $J_{1}\left(\frac{m_{o}}{2}\right)$, función cuyo valor máximo tiene lugar para $m_{o}=\frac{\pi V_{O L}}{V_{\pi}}=3.68$. 
El campo óptico a la salida del primer modulador Mach-Zehnder polarizado en LIN y modulado por una señal de frecuencia intermedia con un índice de modulación reducido puede aproximarse a partir de 5.51 como [CG97a]:

$$
\begin{aligned}
& e_{e o m 1}(t) \approx \frac{A_{e}}{\sqrt{2}}\left(1-\frac{m_{o 1}}{2} \operatorname{sen}\left(\omega_{I F} t+\phi_{I F}\right)\right) \cos \left(\omega_{c} t+\phi_{e}\right) \\
&=\frac{A_{e}}{\sqrt{2}} \cos \left(\omega_{c} t+\phi_{e}\right)-\frac{A_{e}}{4 \sqrt{2}} m_{o 1} \operatorname{sen}\left(\left(\omega_{c}+\omega_{I F}\right) t+\phi_{e}+\phi_{I F}\right) \\
&+\frac{A_{e}}{4 \sqrt{2}} m_{o 1} \operatorname{sen}\left(\left(\omega_{c}-\omega_{I F}\right) t+\phi_{e}-\phi_{I F}\right)
\end{aligned}
$$

Asumiendo un comportamiento lineal del modulador interferométrico, respecto a la señal óptica, se puede aplicar el resultado obtenido para una única portadora óptica a la entrada, cuando el modulador se modula simétricamente, expresado en la ecuación (5.41):

$$
\begin{aligned}
e_{\text {eom } 2} & =e_{\text {eom } 2 c}+e_{\text {eom } 2 \text { sup }}+e_{\text {eom } 2 i n f} \\
= & \frac{A_{e e}}{\sqrt{2}} \sum_{n=-\infty}^{\infty} J_{n}\left(\frac{m_{o 2}}{2}\right) C_{d c}(n) \cos \left(\omega_{c} t+n \omega_{O L} t+\phi_{e e}+n \phi_{1}\right) \\
& -\frac{A_{e e} m_{o 1}}{4 \sqrt{2}} \sum_{n=-\infty}^{\infty} J_{n}\left(\frac{m_{o 2}}{2}\right) C_{d c}(n) \operatorname{sen}\left(\left(\omega_{c}+\omega_{I F}\right) t+n \omega_{O L} t+\phi_{e e}+\phi_{I F e}+n \phi_{1}\right) \\
& +\frac{A_{e e} m_{o l}}{4 \sqrt{2}} \sum_{n=-\infty}^{\infty} J_{n}\left(\frac{m_{o 2}}{2}\right) C_{d c}(n) \operatorname{sen}\left(\left(\omega_{c}-\omega_{I F}\right) t+n \omega_{O L} t+\phi_{e e}-\phi_{I F e}+n \phi_{1}\right)
\end{aligned}
$$

donde $C_{d c}(n)=\cos \left(\frac{m_{b 2}}{2}-\frac{n \pi}{2}\right), \phi_{1}=\phi_{O L}+\frac{\pi}{2}$, mientras que $A_{e e}, \phi_{e e}, \mathrm{y} \phi_{I F e}$ difieren de $A_{e}, \phi_{e} \mathrm{y} \phi_{I F}$ de acuerdo, respectivamente, con las pérdidas y la fase de inserción del modulador $\mathrm{EOM}_{2}$.

Convirtiendo los tres sumatorios en un único sumatorio y simplificando se obtiene:

$$
\begin{aligned}
e_{\text {eom } 2}=\frac{A_{e e}}{\sqrt{2}} \sum_{n=-\infty}^{\infty} J_{n}\left(\frac{m_{o 2}}{2}\right) C_{d c}(n)\left[\cos \left(\omega_{c} t+n \omega_{O L} t+\phi_{e e}+n \phi_{1}\right)\right. \\
-\frac{m_{o 1}}{4} \operatorname{sen}\left(\left(\omega_{c}+\omega_{I F}\right) t+n \omega_{O L} t+\phi_{e e}+\phi_{I F e}+n \phi_{1}\right) \\
\left.\quad+\frac{m_{o 1}}{4} \operatorname{sen}\left(\left(\omega_{c}-\omega_{I F}\right) t+n \omega_{O L} t+\phi_{e e}-\phi_{I F e}+n \phi_{1}\right)\right]
\end{aligned}
$$

Si la señal óptica a la salida del modulador $\mathrm{EOM}_{2}$ (5.101) se pasa a través de una red de difracción cuya respuesta de fase venga definida por (5.32) se obtendrá 
a la salida:

$$
\begin{gathered}
e_{\text {red }}(t)=e_{\text {redc }}+e_{\text {redsup }}+e_{\text {redinf }}=A_{\text {red }} \sum_{n=-\infty}^{\infty} J_{n}\left(\frac{m_{o 2}}{2}\right) C_{d c}(n) \\
{\left[\cos \left(\omega_{c} t+n \omega_{O L}\left(t+\theta_{1}\right)+\phi_{r e d}+n \phi_{1}+\frac{\theta_{2}}{2} n^{2} \omega_{O L}^{2}\right)\right.} \\
-\frac{m_{o 1}}{4} \operatorname{sen}\left(\left(\omega_{c}+\omega_{I F}\right) t+n \omega_{O L}\left(t+\theta_{1}\right)+\phi_{r e d}+\phi_{I F e}+n \phi_{1}+\theta_{1} \omega_{I F}+\frac{\theta_{2}}{2}\left(n \omega_{O L}+\omega_{I F}\right)^{2}\right) \\
\left.+\frac{m_{o 1}}{4} \operatorname{sen}\left(\left(\omega_{c}-\omega_{I F}\right) t+n \omega_{O L}\left(t+\theta_{1}\right)+\phi_{r e d}-\phi_{I F e}+n \phi_{1}-\theta_{1} \omega_{I F}+\frac{\theta_{2}}{2}\left(n \omega_{O L}-\omega_{I F}\right)^{2}\right)\right]
\end{gathered}
$$

donde $A_{\text {red }}=\frac{A_{e e}}{\sqrt{2}}, \phi_{\text {red }}=\phi_{e e}+\theta_{0}$.

Separando en términos en fase y en cuadratura y simplificando, es posible calcular la intensidad eléctrica detectada por un fotodetector ideal de responsividad $\mathcal{R}$, a partir de la potencia de envolvente del campo óptico, obteniéndose:

$$
\begin{aligned}
i_{p d}(t)= & \mathcal{R} P_{\text {red }}(t) \\
= & A_{p d}\left[\sum _ { n = - \infty } ^ { \infty } J _ { n } ( \frac { m _ { o 2 } } { 2 } ) C _ { d c } ( n ) \left[\cos \left(n \omega_{O L}\left(t+\theta_{1}\right)+n \phi_{1}+\frac{\theta_{2}}{2} n^{2} \omega_{O L}^{2}\right)\right.\right. \\
& -\frac{m_{o 1}}{2} \cos \left(n \omega_{O L}\left(t+\theta_{1}\right)+n \phi_{1}+\frac{\theta_{2}}{2} \omega_{I F}^{2}+\frac{\theta_{2}}{2} n^{2} \omega_{O L}^{2}\right) \\
& \left.\left.\operatorname{sen}\left(\omega_{I F}\left(t+\theta_{1}\right)+\phi_{I F e}+\theta_{2} n \omega_{O L} \omega_{I F}\right)\right]\right]^{2} \\
& +A_{p d}\left[\sum _ { n = - \infty } ^ { \infty } J _ { n } ( \frac { m _ { o 2 } } { 2 } ) C _ { d c } ( n ) \left[\operatorname{sen}\left(n \omega_{O L}\left(t+\theta_{1}\right)+n \phi_{1}+\frac{\theta_{2}}{2} n^{2} \omega_{O L}^{2}\right)\right.\right. \\
& -\frac{m_{o 1}}{2} \operatorname{sen}\left(n \omega_{O L}\left(t+\theta_{1}\right)+n \phi_{1}+\frac{\theta_{2}}{2} \omega_{I F}^{2}+\frac{\theta_{2}}{2} n^{2} \omega_{O L}^{2}\right) \\
& \left.\left.\operatorname{sen}\left(\omega_{I F}\left(t+\theta_{1}\right)+\phi_{I F e}+\theta_{2} n \omega_{O L} \omega_{I F}\right)\right]\right]^{2}
\end{aligned}
$$

donde $A_{p d}=\mathcal{R} \cdot A_{\text {red }}^{2} / 2$. Arreglando el resultado anterior para que los sumatorios sólo incluyan términos positivos, y tras una serie de simplificaciones, se puede llegar 
al siguiente resultado:

$$
\begin{aligned}
i_{p d}( & t)=A_{p d}\left[J_{0}\left(\frac{m_{o 2}}{2}\right) C_{d c}(0)\left(1-\frac{m_{o 1}}{2} \cos \left(\frac{\theta_{2}}{2} \omega_{I F}^{2}\right) \operatorname{sen}\left(\omega_{I F}\left(t+\theta_{1}\right)+\phi_{I F e}\right)\right)\right. \\
& +2 \sum_{n=1}^{\infty} J_{n}\left(\frac{m_{o 2}}{2}\right) C_{d c}(n)\left[\operatorname { c o s } ( n \omega _ { O L } ( t + \theta _ { 1 } ) + n \phi _ { 1 } ) \left[\cos \left(\frac{\theta_{2}}{2} n^{2} \omega_{O L}^{2}\right)\right.\right. \\
& \left.-\frac{m_{o 1}}{2} \cos \left(\frac{\theta_{2}}{2} \omega_{I F}^{2}+\frac{\theta_{2}}{2} n^{2} \omega_{O L}^{2}\right) \operatorname{sen}\left(\omega_{I F}\left(t+\theta_{1}\right)+\phi_{I F e}\right) \cos \left(\theta_{2} n \omega_{O L} \omega_{I F}\right)\right] \\
& +\frac{m_{o 1}}{2} \operatorname{sen}\left(n \omega_{O L}\left(t+\theta_{1}\right)+n \phi_{1}\right) \operatorname{sen}\left(\frac{\theta_{2}}{2} \omega_{I F}^{2}+\frac{\theta_{2}}{2} n^{2} \omega_{O L}^{2}\right) \\
& \left.\left.\cos \left(\omega_{I F}\left(t+\theta_{1}\right)+\phi_{I F e}\right) \operatorname{sen}\left(\theta_{2} n \omega_{O L} \omega_{I F}\right)\right]\right]^{2} \\
& +A_{p d}\left[-J_{0}\left(\frac{m_{o 2}}{2}\right) C_{d c}(0) \frac{m_{o 1}}{2} \operatorname{sen}\left(\frac{\theta_{2}}{2} \omega_{I F}^{2}\right) \operatorname{sen}\left(\omega_{I F}\left(t+\theta_{1}\right)+\phi_{I F e}\right)\right. \\
& +2 \sum_{n=1}^{\infty} J_{n}\left(\frac{m_{o 2}}{2}\right) C_{d c}(n)\left[\operatorname { c o s } ( n \omega _ { O L } ( t + \theta _ { 1 } ) + n \phi _ { 1 } ) \left[\operatorname{sen}\left(\frac{\theta_{2}}{2} n^{2} \omega_{O L}^{2}\right)\right.\right. \\
& \left.-\frac{m_{o 1}}{2} \operatorname{sen}\left(\frac{\theta_{2}}{2} \omega_{I F}^{2}+\frac{\theta_{2}}{2} n^{2} \omega_{O L}^{2}\right) \operatorname{sen}\left(\omega_{I F}\left(t+\theta_{1}\right)+\phi_{I F e}\right) \cos \left(\theta_{2} n \omega_{O L} \omega_{I F}\right)\right] \\
& -\frac{m_{o 1}}{2} \operatorname{sen}\left(n \omega_{O L}\left(t+\theta_{1}\right)+n \phi_{1}\right) \cos \left(\frac{\theta_{2}}{2} \omega_{I F}^{2}+\frac{\theta_{2}}{2} n^{2} \omega_{O L}^{2}\right) \\
& \left.\left.\cos \left(\omega_{I F}\left(t+\theta_{1}\right)+\phi_{I F e}\right) \operatorname{sen}\left(\theta_{2} n \omega_{O L} \omega_{I F}\right)\right]\right]^{2}
\end{aligned}
$$

El resultado anterior corresponde a la solución exacta de la corriente detectada por el fotoreceptor, para una señal óptica modulada en amplitud a $\omega_{I F}$ y posteriormente modulada por un segundo EOM con una moduladora de frecuencia $\omega_{O L}$ y que atraviesa una red de difracción con una respuesta de fase definida por (5.32). Dependiendo del punto de polarización del segundo EOM, el resultado particular obtenido difiere considerablemente. Para el caso particular de polarización en mínima (MIP), se muestra a continuación la expresión aproximada obtenida, asumiendo un índice de modulación óptico en el $\mathrm{EOM}_{2}\left(m_{o 2}\right)$ reducido; para ello, se ha tomado únicamente el primer término significativo (distinto de $J_{0}$ ) de la expresion exacta y 
tras una serie de operaciones se llega al siguiente resultado:

$$
\begin{aligned}
i_{p d-m i n}(t) \approx \frac{A_{p d} m_{o 2}^{2}}{8}\left[1+\frac{m_{o 1}^{2}}{8}\right. & -m_{o 1} \cos \left(\theta_{2} \omega_{O L} \omega_{I F}\right) \cos \left(\frac{\theta_{2}}{2} \omega_{I F}^{2}\right) \operatorname{sen}\left(\omega_{I F}\left(t+\theta_{1}\right)+\phi_{I F e}\right) \\
& -\frac{m_{o 1}^{2}}{8} \cos \left(2 \theta_{2} \omega_{O L} \omega_{I F}\right) \cos \left(2 \omega_{I F}\left(t+\theta_{1}\right)+2 \phi_{I F e}\right) \\
& +\left(1+\frac{m_{o 1}^{2}}{8} \cos \left(2 \theta_{2} \omega_{O L} \omega_{I F}\right)\right) \cos \left(2 \omega_{O L}\left(t+\theta_{1}\right)+2 \phi_{1}\right) \\
& -\frac{m_{o 1}}{2} \cos \left(\frac{\theta_{2}}{2}\left(2 \omega_{O L}+\omega_{I F}\right) \omega_{I F}\right) \operatorname{sen}\left(\left(2 \omega_{O L}+\omega_{I F}\right)\left(t+\theta_{1}\right)+\phi_{I F e}+2 \phi_{1}\right) \\
+ & \frac{m_{o 1}}{2} \cos \left(\frac{\theta_{2}}{2}\left(2 \omega_{O L}-\omega_{I F}\right) \omega_{I F}\right) \operatorname{sen}\left(\left(2 \omega_{O L}-\omega_{I F}\right)\left(t+\theta_{1}\right)-\phi_{I F e}+2 \phi_{1}\right) \\
& -\frac{m_{o 1}^{2}}{16} \cos \left(\left(2 \omega_{O L}+2 \omega_{I F}\right)\left(t+\theta_{1}\right)+2 \phi_{I F e}+2 \phi_{1}\right) \\
& \left.-\frac{m_{o 1}^{2}}{16} \cos \left(\left(2 \omega_{O L}-2 \omega_{I F}\right)\left(t+\theta_{1}\right)-2 \phi_{I F e}+2 \phi_{1}\right)\right]
\end{aligned}
$$

donde se puede comprobar que el término deseado $\omega_{R F}=2 \omega_{O L}+\omega_{I F}$ aparece con el término de retardo debido a la red de difracción y que la atenuación dispersiva se debe a un factor que varía de forma más lenta con la frecuencia que en el caso de modulación de intensidad convencional.

Como conclusión del resultado de la ecuación (5.105) y aplicando la igualdad (5.34) se puede decir que la degradación de potencia de radiofrecuencia en esquemas IM/DD con modulación pseudo-autoheterodinas y bajos índices de modulación, en función de la frecuencia de RF, de la frecuencia de IF y de la pendiente del retardo de grupo de la red de difracción con variación lineal del periodo espacial es:

$$
\operatorname{Pow}_{\text {deg }}\left(f_{R F}\right)=\frac{\left|H\left(f_{R F}\right)\right|^{2}}{|H(0)|^{2}}=\cos ^{2}\left(\frac{\lambda_{c}^{2} \pi \delta}{c} f_{R F} f_{I F}\right)=\cos ^{2}\left(\frac{\pi c \delta}{\omega_{c}^{2}} \omega_{R F} \omega_{I F}\right)
$$

En la figura 5.23, se compara la atenuación dispersiva de una línea de retardo basada en una red de difracción con una pendiente en la respuesta del retardo de $\delta=850 \mathrm{ps} / \mathrm{nm}$, si se utiliza modulación AM convencional o si se usa modulación pseudo-autoheterodina con una frecuencia intermedia de $1 \mathrm{GHz}$. Los resultados muestran claramente como la modulación pseudo-autoheterodina consigue aumentar considerablemente el ancho de banda disponible en la línea de retardo, siempre que la señal a retardar sea de un ancho de banda suficientemente estrecho $(1 \mathrm{GHz}$ en este caso). Estos resultados confirman los resultados obtenidos previamente para esta modulación en aplicaciones de antenas remotas [Fus97]. Como principal inconveniente de este esquema de modulación, se debe citar la necesidad de disponer de dos moduladores en cada línea de retardo, además del bajo nivel de señal recibido debido a los dos procesos de modulación y al proceso de mezclado en el fotoreceptor. 


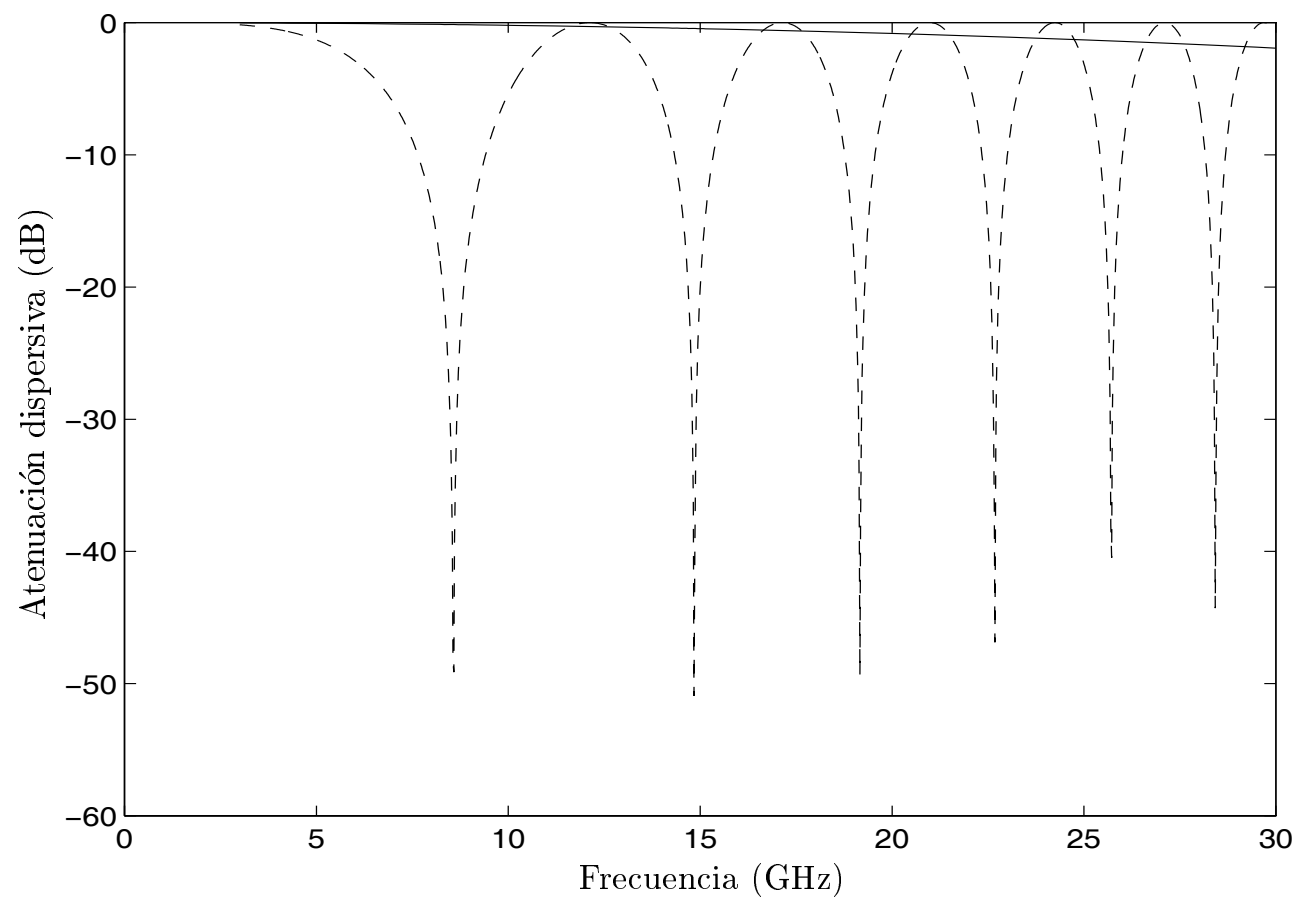

Figura 5.23: Atenuación dispersiva del término de la señal detectada $\omega_{R F}=$ $2 \omega_{O L}+\omega_{I F}$ en la transmisión a través de un enlace IM/DD con modulación pseudoheterodina que implementa una línea de retardo con una red de difracción con periodo variable lineal $\left(\delta=850 \mathrm{ps} / \mathrm{nm}, \lambda_{c}=1550 \mathrm{~nm}, m_{\text {opt }}=0.1, \omega_{I F}=1 \mathrm{GHz}\right)$. En traza discontínua, la atenuación si se utilizara modulación AM convencional para la misma frecuencia detectada.

\subsubsection{Comparación entre el uso de varias redes de difracción discretas y el uso de una única red de difracción con periodo variable}

Como ventajas para los retardadores basados en redes de difracción discretas se debe resaltar que dentro del ancho de banda del filtro, todas las señales sufren el mismo retardo, pues éste va asociado al retardo del camino de ida y vuelta, que es el mismo para todas las frecuencias dentro del ancho de banda de reflexion de la red difracción; aún cuando pudiera haber mínimas diferencias entre las dos bandas laterales o entre distintas componentes frecuenciales de la señal. Luego, en principio, las líneas de retardo basadas en redes de difracción discretas no sufren atenuación dispersiva, pero las redes de difracción deben tener el ancho de banda adecuado, teniendo en cuenta posibles desviaciones de la longitud de Bragg de la red o de la frecuencia óptica de la fuente sintonizable en función de la temperaturai. Esto implicará, que la red de difracción tenga una longitud suficientemente grande, de acuerdo con (5.10) y la tabla 5.1, lo que para una longitud de onda de $\lambda_{c}=1550 \mathrm{~nm}$ y una frecuencia

\footnotetext{
${ }^{i}$ Para la longitud de Bragg de la red de difracción, se puede esperar una variación de $0.01 \mathrm{~nm} /{ }^{\circ} \mathrm{C}$ [Bal94, Lem94]
} 
de $f_{R F}=12 \mathrm{GHz}$, se traduce en una longitud de red de $L_{r e d} \approx 1 \mathrm{~cm}$. Una separación física habitual entre redes de difracción discretas en líneas de retard es de 2 veces la longitud de la misma [Mol95], lo que equivale a un retardo incremental de 200 ps, que es, aproximadamente, dos veces y media el periodo de la señal de radiofrecuencia, siendo por tanto, una resolución excesivamente grande e inviable.

Como ventajas de los retardadores basados en redes de difracción con periodo variable comentar que no hay limitaciones de retardos debida a dimensiones físicas de las redes de difracción, pero sí las hay debidas a la no linealidad (rizado) de la respuesta de amplitud y de fase de la red de difracción.

\subsubsection{Otros retardadores variables en fibra basados en dis- positivos altamente dispersivos}

En la literatura se pueden encontrar otros dispositivos ópticos sobre fibra utilizados como retardadores variables y que se basan en elementos dispersivos por lo que presentarán las mismas limitaciones que la línea de retardo aquí presentada [Oue94]:

- Acoplador intermodal con variación lineal de la posición de acoplo en función de la longitud de onda. Como ventaja citar que trabaja en transmisión con lo que se evita el coste y las pérdidas del circulador óptico (2 dB en circuladores ópticos experimentales y $3 \mathrm{~dB}$ en dispositivos comerciales [Fuj93]).

- Fibras con núcleos diferentes y variación lineal del grosor. Al igual que el dispositivo anterior trabaja en transmisión por lo que se evita la utilización de circuladores.

- Ecualizador óptico [Oze92] y ecualizador óptico en tecnología planar [Tak94].

- Interferómetro Gires-Tournois (GTI) [Cim90].

\subsection{Arquitectura de red conformadora de haces controlada ópticamente}

A partir de la línea de retardo basada en una red de difracción con periodo variable, se diseña una nueva arquitectura de red conformadora de haces para señales de banda ancha con control óptico de la alimentación de la agrupación, es decir, con control óptico de la conformación del haz [Cor97b]. Se trata de un conformador monohaz, cuya configuración en transmisión se muestra en la figura 5.24.

En el conformador de la figura 5.24, se utiliza una correspondencia biunívoca entre una longitud de onda y cada uno de los elementos de antena (o subagrupaciones). Las salidas ópticas de $N$ fuentes láser sintonizables $\left(\lambda_{1}\right.$ a $\left.\lambda_{N}\right)$ se combinan y 


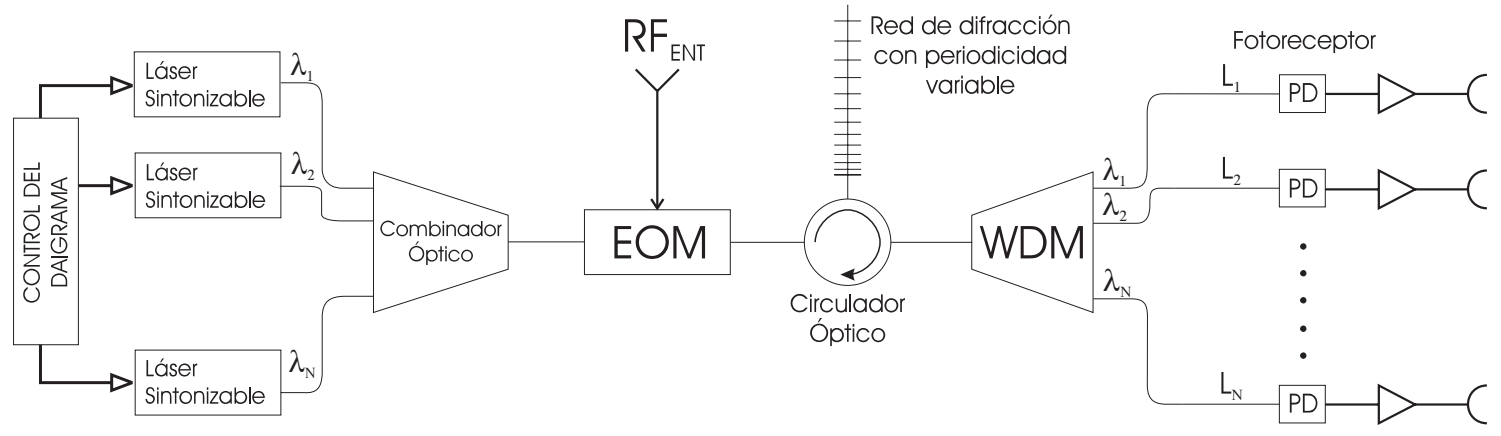

Figura 5.24: Diagrama de bloques en transmisión de la red conformadora de haces controlada ópticamente basada en una red de difracción como elemento de retardo verdadero variable.

son moduladas por la misma señal de microondas, utilizando un único modulador electro-óptico externo (EOM). Las portadoras ópticas moduladas pasarán a través de un circulador, hacia una red de difracción de banda ancha y con variación lineal del periodo. Tal y como se ha visto previamente, el retardo sufrido por cada portadora óptica variará linealmente con el valor de la longitud de onda de cada portadora. Las $N$ portadoras retardadas $\left(\lambda_{1}\right.$ to $\left.\lambda_{N}\right)$, serán finalmente separadas por medio de un demultiplexor de longitud de onda (WDM) y a continuación se pasarán a cada elemento tras la correspondiente fotodetección (PD) y amplificación para adecuar su nivel a los elementos de la agrupación. Dependiendo de los valores las $N$ portadoras y de los márgenes de excursión alrededor de las mismas, el demultiplexor WDM podría implementarse como un divisor óptico $1 \times N$ seguido de $N$ redes de difracción de banda estrecha con periodo constante, actuando como filtros selectores.

La conformación del haz se consigue a través de la sintonización de las longitudes de onda de cada láser ${ }^{\mathrm{i}}, \lambda_{i}$, alrededor de su posición central, tal y como se muestra en la figura 5.25. El máximo retardo necesario en cada elemento, $\pm \tau_{\max }$, dependerá de la separación entre los elementos de antena, $d$, y del máximo ángulo de desviación del haz principal de la agrupación, $\theta_{\max }$, siendo para el caso de una agrupación lineal de $N$ elementos:

$$
\tau_{\max }(\mathrm{ps})=16.7(N-1) d(\mathrm{~cm}) \operatorname{sen}\left(\theta_{\max }\right)
$$

como ejemplo, para una agrupación con 10 elementos $(N=10)$, separados entre sí por $d=3 \mathrm{~cm}$ (que corresponde a $\lambda / 2$ para $f=5 \mathrm{GHz}$ ) y escogiendo $\theta_{\max }= \pm 45^{\circ}$, el máximo retardo requerido sería 320 ps. En la figura 5.26, se muestra como varía el retardo máximo en función del ángulo de barrido máximo tomando el producto $N \cdot d$ (tamaño total de la agrupación) como parámetro; como conclusión de la misma, indicar que con un conformador que ofrezca un retardo máximo de \pm 250 ps en cada elemento, se puede alimentar una agrupación de seis elementos a una frecuencia de $f=2 \mathrm{GHz}$ barriendo un sector angular de $\pm 25^{\circ}$; pasando el sector a ser de $\pm 35^{\circ}$ si $N=10$ y $f=5 \mathrm{GHz}$ ó $\pm 45^{\circ}$ si $N=15$ y $f=10 \mathrm{GHz}$.

\footnotetext{
${ }^{\text {i}}$ De hecho, sólo se necesita sintonizar $N-1$ láseres.
} 


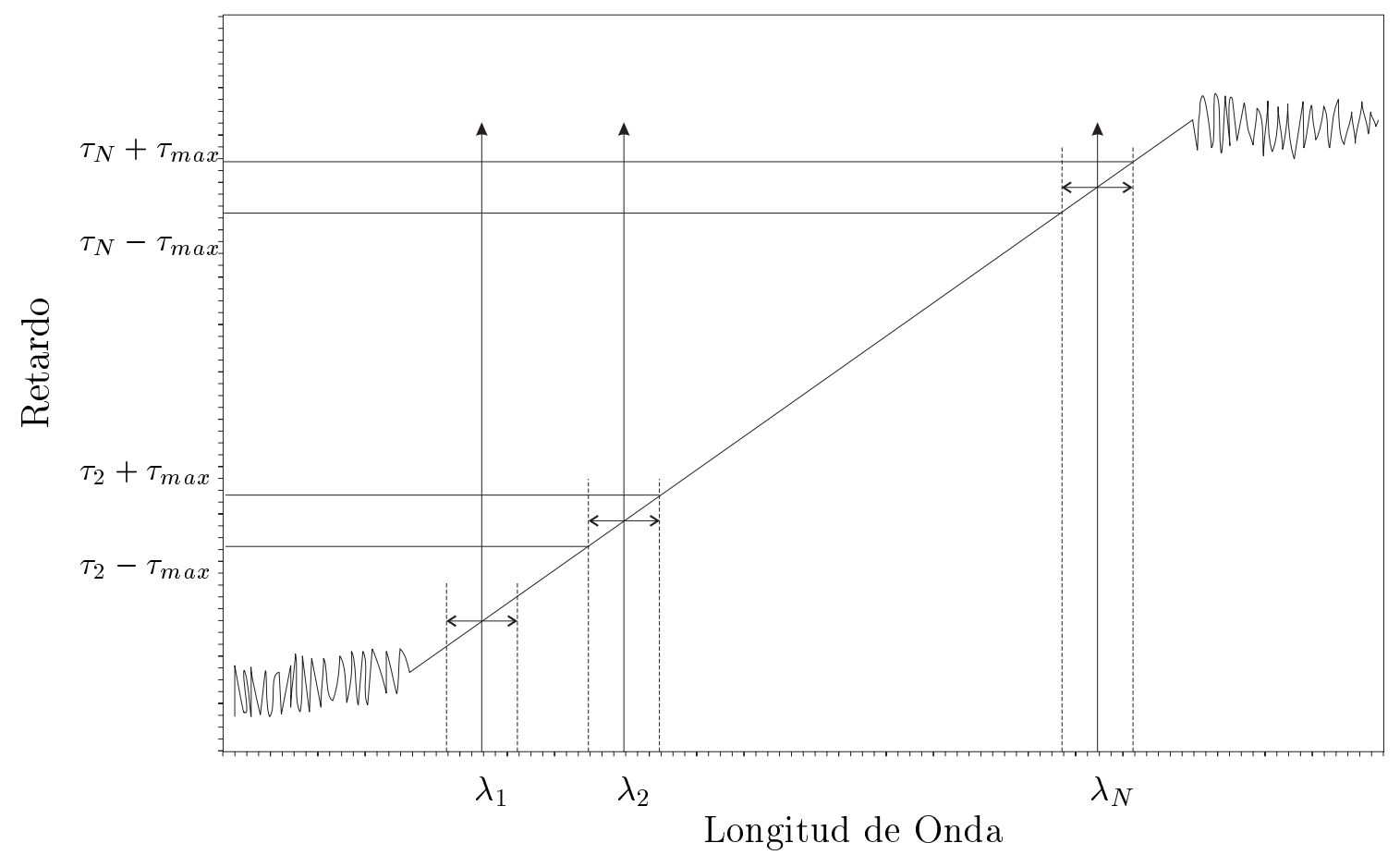

Figura 5.25: Respuesta ideal de una red de difracción con variación lineal del periodo y posición nominal de las $N$ longitudes de onda de la red conformadora, mostrando el margen de sintonización disponible.

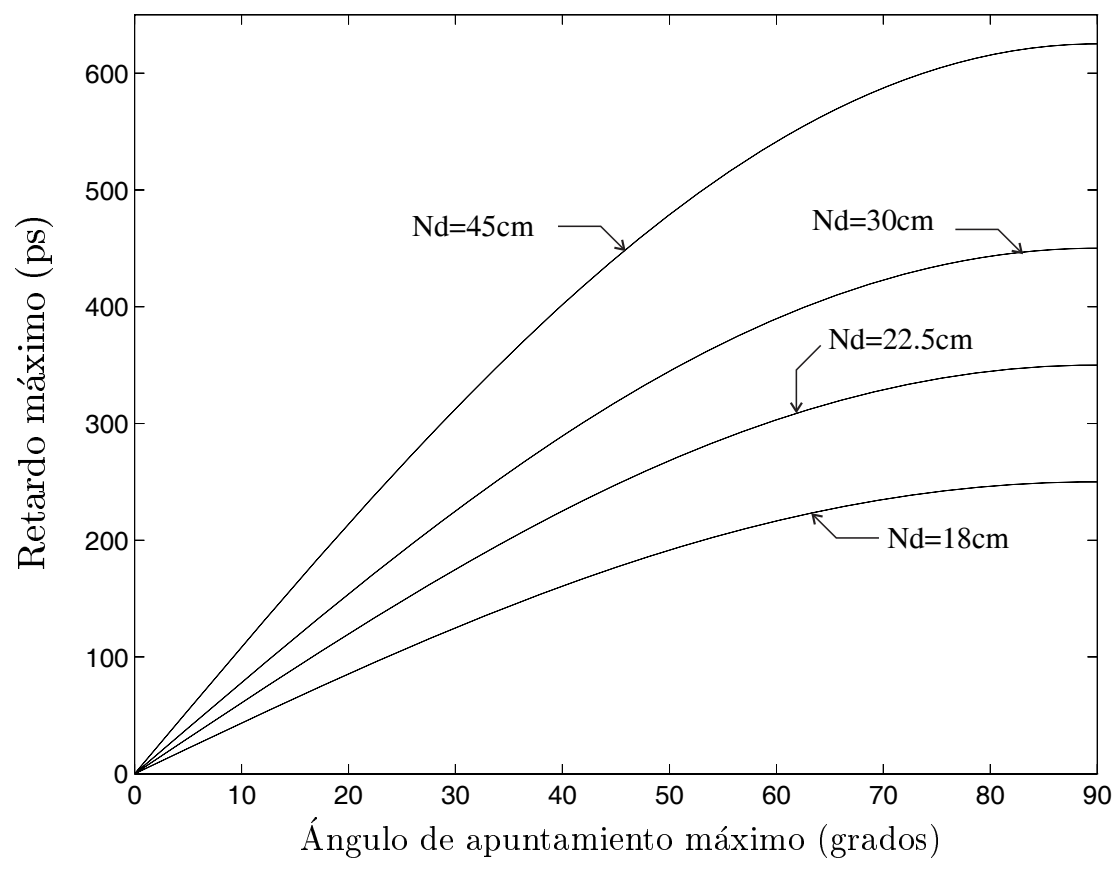

Figura 5.26: Retardo máximo $\left( \pm \tau_{\max }\right)$ en función del ángulo de barrido $\left( \pm \theta_{\max }\right)$ respecto al ángulo de reposo para diferentes valores del producto $N \cdot d$. 
Cuando todos los láseres estén sintonizados a su longitud de onda central, el haz debe apuntar en la dirección central dentro del sector a barrer; generalmente, esa dirección de reposo será la normal a la agrupación, para lo cual se requiere que todos los elementos de antena se alimenten en fase y será necesario añadir una cierta longitud extra de fibra estándar monomodo, $L_{i}$, en la rama de cada elemento para compensar el retardo, $\tau_{i}$, correspondiente a cada longitud de onda central, $\lambda_{i}$, como se muestra en la figura 5.24. El valor de $L_{i}$ se puede expresar en función de la pendiente media, $\delta$, de la respuesta de retardo de grupo de la red difracción, de la separación entre longitudes de onda centrales, $\lambda_{i}-\lambda_{1}$, y del índice de refracción de la fibra, $n_{o}$, como:

$$
L_{i}(\mathrm{~cm})=\delta(\mathrm{ps} / \mathrm{nm})\left[\lambda_{i}(\mathrm{~nm})-\lambda_{1}(\mathrm{~nm})\right] \frac{0.03}{n_{o}}
$$

tras añadir las citadas longitudes de fibra compensadoras, es como si el retardo de sistema dejara de ser una respuesta lineal y pasara a ser una respuesta tipo diente de sierra.

El rango de variación de longitud de onda necesario en cada fuente láser sintonizable, $\Delta \lambda$, depende del retardo máximo necesario y del coeficiente de dispersión, $\delta(\mathrm{ps} / \mathrm{nm})$, de la red de difracción con variación lineal del periodo en la forma:

$$
\Delta \lambda=33.3 \cdot \frac{(N-1) \cdot d(\mathrm{~cm}) \cdot \operatorname{sen}\left(\theta_{\max }\right)}{\delta(\mathrm{ps} / \mathrm{nm})}
$$

Asumiendo una separación entre longitudes de onda adyacentes de una vez y media la excursión de longitud de onda de cada láser y una pendiente del retardo de grupo constante dentro del ancho de banda total de la red de difracción, $\Delta \lambda_{\text {red }}$, el máximo número de diferentes elementos de antena (o subagrupaciones) sería:

$$
N_{\text {max }}=\sqrt{\frac{\Delta \lambda_{\text {red }} \cdot \delta(\mathrm{ps} / \mathrm{nm})}{50 \cdot d(\mathrm{~cm}) \cdot \operatorname{sen}\left(\theta_{\max }\right)}}
$$

En la figura 5.27 se muestra el esquema de red conformadora controlada ópticamente para trabajar en modo recepción, equivalente al presentado en la figura 5.24.

\subsubsection{Prestaciones de la red óptica conformadora de haces}

En este apartado se muestra un resumen de las prestaciones de la arquitectura propuesta de red óptica conformadora de haces, haciendo especial hincapié en aquellos puntos novedosos que difieren de resultados ya conocidos de los enlaces ópticos analógicos con modulación externa [Ste87, Ala95, Ack97, Cox97], como son el efecto de la dispersión de la red de difracción y el efecto del comportamiento no ideal de los componentes del conformador; en concreto, los láseres, la red de difracción o el demultiplexor en longitud de onda. 


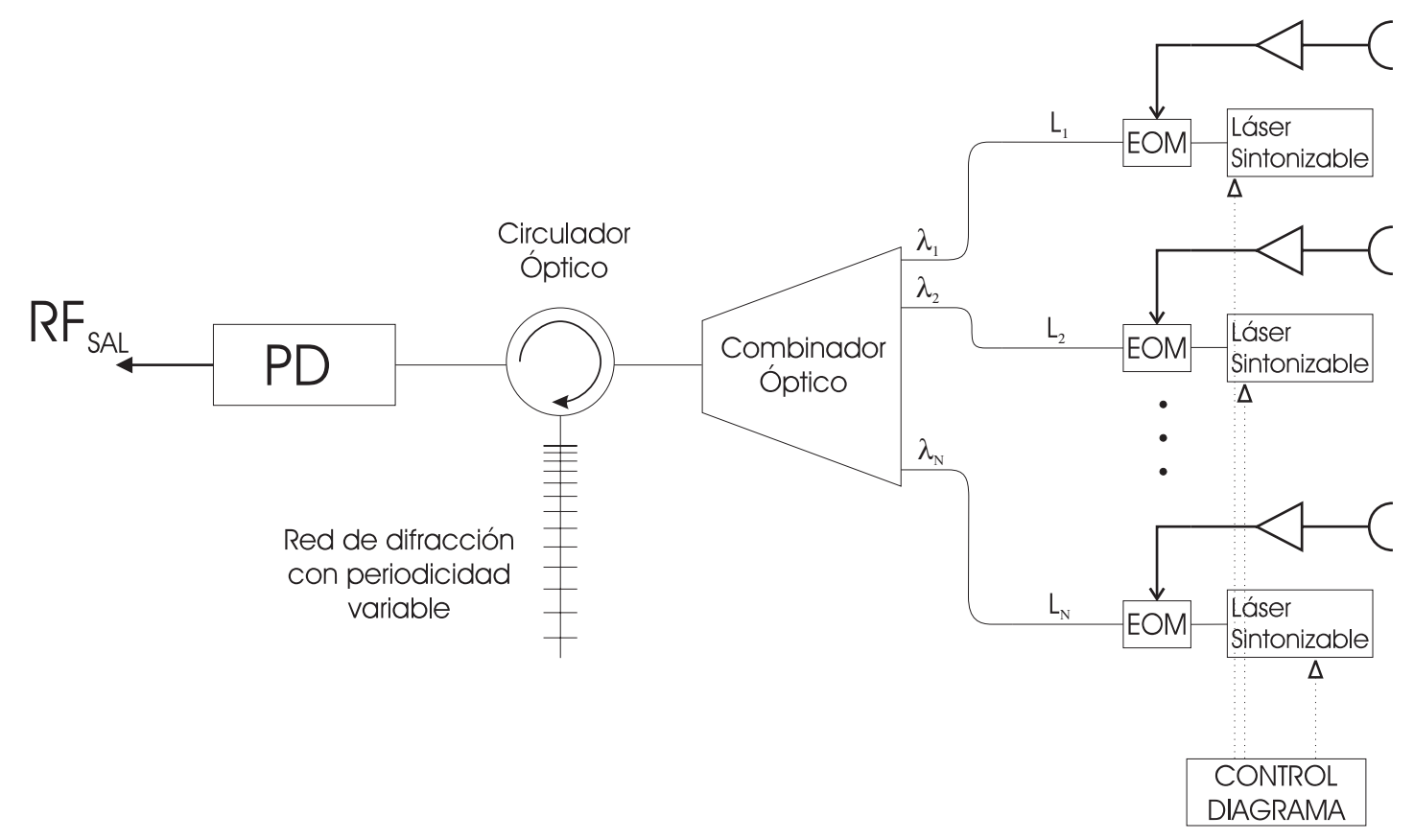

Figura 5.27: Diagrama de bloques en recepción de la red conformadora de haces controlada ópticamente basada en una red de difracción como elemento de retardo verdadero variable.

\section{Ancho de banda}

El ancho de banda de la red conformadora de haces vendrá limitado por el ancho de banda mínimo de cada uno de sus componentes. Existen dispositivos comerciales con anchos de banda de varias decenas de gigahercios tanto para el modulador electroóptico como para el fotoreceptor [Cox97], por lo que la limitación puede venir por la dispersión introducida por la red de difracción de acuerdo con la ecuación (5.50), si se utiliza modulación de intensidad convencional. Si se dispone de un modulador dual y de un híbrido de banda ancha, es posible utilizar modulación SSB $+\mathrm{C}$ y obtener un conformador libre de limitaciones en ancho de banda debidas a la dispersión de la red de difracción; de forma que serán otros componentes, como el híbrido, el modulador o el fotodetector los que establezcan el ancho de banda de utilización. Otra solución, aunque de aplicación más limitada, consistiría en la utilización de un esquema de modulación pseudo-autoheterodino, cuya limitación en ancho de banda se ha demostrado en secciones previas que es claramente inferior a la modulación de intensidad convencional. La modulación pseudo-autoheterodina presenta como ventaja que la propia modulación permite la elevación en frecuencia de la señal a transmitir, por lo que la señal de radiofrecuencia de entrada al conformador será de baja frecuencia.

A medida que aumenta la frecuencia de la señal de radiofrecuencia a aplicar al conformador, deja de ser despreciable el ancho de banda de la señal óptica modulada y deberá tenerse en cuenta a la hora de especificar los anchos de banda de los filtros 
selectores del demultiplexor, así como su respuesta en frecuencia. Por ejemplo para una señal de $f_{R F}=25 \mathrm{GHz}$ el ancho de banda de una modulación de amplitud ideal alrededor de $\lambda_{c}=1550 \mathrm{~nm}$ será de $\Delta \lambda=0.4 \mathrm{~nm}$.

\section{Balance de potencias}

La ganancia de una rama independiente del conformador (Fig. 5.6) cuando se utiliza modulación de intensidad convencional en LIN será:

$$
G_{R A M A}=\frac{P_{R F-s a l}}{P_{R F-e n t}}=\frac{K_{O}}{2}\left[\mathcal{R} t_{o d} L t_{f f} \frac{P_{\text {láser }}}{2}\right]^{2} R_{C A R G A}
$$

donde $R_{C A R G A}$ es la impedancia de carga del fotodetector, $t_{o d}$ son las pérdidas por acoplamiento del mismo, $t_{f f}$ son las pérdidas del modulador de fibra a fibra, $P_{\text {laser }}$ es la potencia óptica de salida del láser y $K_{O}$ se define como la respuesta del modulador externo y se puede expresar en función del índice de modulación óptico, $m_{o}$, y de la potencia de entrada al modulador, $P_{R F-e n t}$, como:

$$
K_{O}=\frac{m_{o}^{2}}{P_{R F-e n t}}=\frac{\pi^{2} 2 R_{E O M}}{V_{\pi}^{2}}
$$

siendo $R_{E O M}$ la resistencia de entrada del modulador Mach-Zehnder.

Las pérdidas, $L$, mostradas en (5.111) incluye las pérdidas debidas a los conectores, $L_{\text {conec }}$, al circulador, $L_{\text {circ }}$, a los diferentes trozos de fibra monomodo, $L_{f o}$, a la red de difracción, $L_{r e d}$, y a los acoplos láser/fibra $L_{\text {acoplo }}$.

Se puede comprobar en (5.111) la típica dependencia de la ganancia de los sistemas modulados externamente, con el cuadrado de la potencia óptica. Sustituyendo valores típicos de dispositivos comerciales, se puede obtener la siguiente estimación para la ganancia de cada rama [Cox97]:

$$
\begin{aligned}
G_{R A M A} & =\frac{2 \cdot 50 \pi^{2}}{2 \cdot 8^{2}}\left[0.9 \cdot 10^{-0.3} \cdot 10^{-0.2-0.1-0.2} \cdot 10^{-0.3} \frac{0.01}{2}\right]^{2} 1000 \\
& =0.98 \cdot 10^{-3}=-30 \mathrm{~dB}
\end{aligned}
$$

En el caso de utilizar un divisor de potencia y filtros selectores para cada una de las ramas en lugar de un demultiplexor, la división de potencia implicará pérdidas por división en un factor $L_{d i v}=10 \log (N)$ que se añadirán a las pérdidas totales, $L$.

Las pérdidas de cada rama del conformador son muy bajas y deberá incluirse amplificación en el sistema; siendo posible situarla a tres niveles: incluir un preamplificador a la entrada del EOM que a su vez deberá adaptar el nivel de la señal al margen dinamico del sistema, un amplificador óptico de erbio dopado (EDFA ${ }^{\mathrm{i}}$ ) o un amplificador de radiofrecuencia en postdetección que adecuara el nivel de señal al

\footnotetext{
${ }^{\mathrm{i}}$ Erbium-doped fiber amplifier.
} 
necesario en los elementos de la agrupación. Una vez especificadas las características precisas del conformador, se deberá prestar especial atención a la elección combinada de estas tres opciones, pues ésta tiene un efecto inmediato sobre el ruido y el margen dinámico del sistema [Ala95].

\section{Ruido}

Asumiendo que son despreciables las componentes de ruido debidas a la partición de modos y a la reflexión entre el láser y la fibra monomodo, se puede afirmar que en un enlace IM/DD, como el correspondiente a una rama del conformador, hay cinco fuentes de ruido principales [See93]:

- Ruido térmico en la entrada del modulador electro-óptico y en el propio modulador:

$$
N_{\text {térmico-ent }}=4 k \cdot T \cdot B W \cdot R_{E O M}
$$

donde $k$ es la constante de Boltzmann y $T$ la temperatura absoluta.

- Ruido de intensidad relativa $\left(\mathrm{RIN}^{\mathrm{i}}\right)$ del láser, siendo la potencia de ruido a la salida del fotodetector:

$$
N_{R I N}=I_{d c}^{2} \cdot 10^{(R I N / 10)} \cdot B W \cdot R_{C A R G A}
$$

donde el parámetro $R I N(\mathrm{~dB} / \mathrm{Hz})$ define el comportamiento de la fuente, $B W$ es el ancho de banda de trabajo y $I_{d c}$ es el valor medio de la corriente a la salida del fotodetector que es proporcional a la potencia óptica media incidente sobre el detector.

- Ruido del amplificador optico, en el caso de que se utilizaran.

- Ruido shot debido al flujo de corriente en el fotodetector, cuyo valor es:

$$
N_{s h o t}=2 q \cdot\left(\overline{I_{d c}}+I_{o s c}\right) \cdot B W \cdot R_{C A R G A}
$$

siendo $q=1.6 \cdot 10^{-19} \mathrm{C}$ y $I_{\text {osc }}$ la corriente de oscuridad del fotodetector.

- Ruido térmico en el fotodetector y su resistencia de carga, siendo su expresión:

$$
N_{\text {térmico-pd }}=4 k \cdot T \cdot B W \cdot R_{C A R G A}
$$

Es interesante resaltar la dependencia de las tres contribuciones de ruido con la potencia óptica incidente sobre el fotodetector; en concreto, el ruido RIN es proporcional al cuadrado de la potencia óptica media incidente, mientras que el ruido shot es proporcional a la potencia óptica y el ruido térmico es independiente

\footnotetext{
${ }^{\mathrm{i}}$ Relative Intensity Noise.
} 
de la señal detectada. Dependiendo de los parámetros específicos de cada sistema, esta dependencia permitiría aumentar el rango dinámico del conformador y reducir el factor de ruido, mediante la inserción de un amplificador óptico que aumentara la potencia óptica sobre el detector haciendo que la componente principal del ruido fuera el ruido de intensidad, el cual a su vez puede ser reducido considerablemente con la utilización de láseres de estado sólido con frecuencias de relajación bajas [Cox97].

En enlaces IM/DD donde las pérdidas del enlace son altas, el ruido dominante pasa a ser el ruido termico, pero en el caso del conformador óptico, al presentar unas pérdidas medias, es de esperar que el ruido dominante pase a ser el ruido RIN o el ruido shot; sobre todo, si se decide utilizar un amplificador óptico para compensar las pérdidas del enlace.

\section{Distorsión. Puntos de intercepción}

A partir de los resultados mostrados en las figuras 5.14 y 5.15 y de la expresión (5.60) se puede obtener el punto de compresión a $1 \mathrm{~dB}$ de una rama del conformador, en función del producto de dispersión de la línea de retardo definido en (5.61); resultado que se muestra en la figura 5.28a.

Se puede comprobar que mientras no aparece el fenómeno de atenuación dispersiva, el punto de compresión a $1 \mathrm{~dB}$ es el mismo que se tendría para una línea de retardo sin dispersión $\left(m_{o}^{1 d B c}=1.138\right)$. Por otro lado, al atenuarse la componente fundamental, por la dispersión de la red de difracción, aumenta el índice de modulación de entrada correspondiente al punto de compresión a 1 dB. Este fenómeno, no implica que aumente la potencia de salida, más bien al contrario, al aparecer la atenuación dispersiva, es posible aumentar el índice de modulación a la entrada sin llegar al punto de compresión a $1 \mathrm{~dB}$, pero realmente, si se compara el punto de compresión a la salida (Fig. 5.28b) se puede comprobar como la potencia de compresión a $1 \mathrm{~dB}$ (normalizada respecto a $A_{p d}^{2}$ ) a la salida de la rama del conformador disminuye cuando la señal sufre atenuación dispersiva.

Aplicando los resultados presentados en la sección 5.3.3 sobre los productos de intermodulación de una de las ramas del conformador, es posible obtener el punto de intercepción de tercer orden en función de la dispersión de la red de difracción, expresada ésta, para la menor de las frecuencias de los dos tonos de radiofrecuencia de entrada, $\omega_{R F 1}$. En las figuras 5.29 se muestran los resultados obtenidos, para diferentes posiciones relativas de los dos tonos de entrada. En concreto, se presentan los niveles de entrada y de salida del punto de intercepción de tercer orden, expresados, respectivamente, como el índice de modulación de ambos tonos en el EOM y como nivel de potencia relativa a $A_{p d}^{2}$.

A la hora de extraer el punto de intercepción, se debe considerar que la dispersión de la red de difracción afecta de forma diferente a las frecuencias de interés, es decir, a las dos fundamentales y a los dos productos de intermodulación de tercer orden 


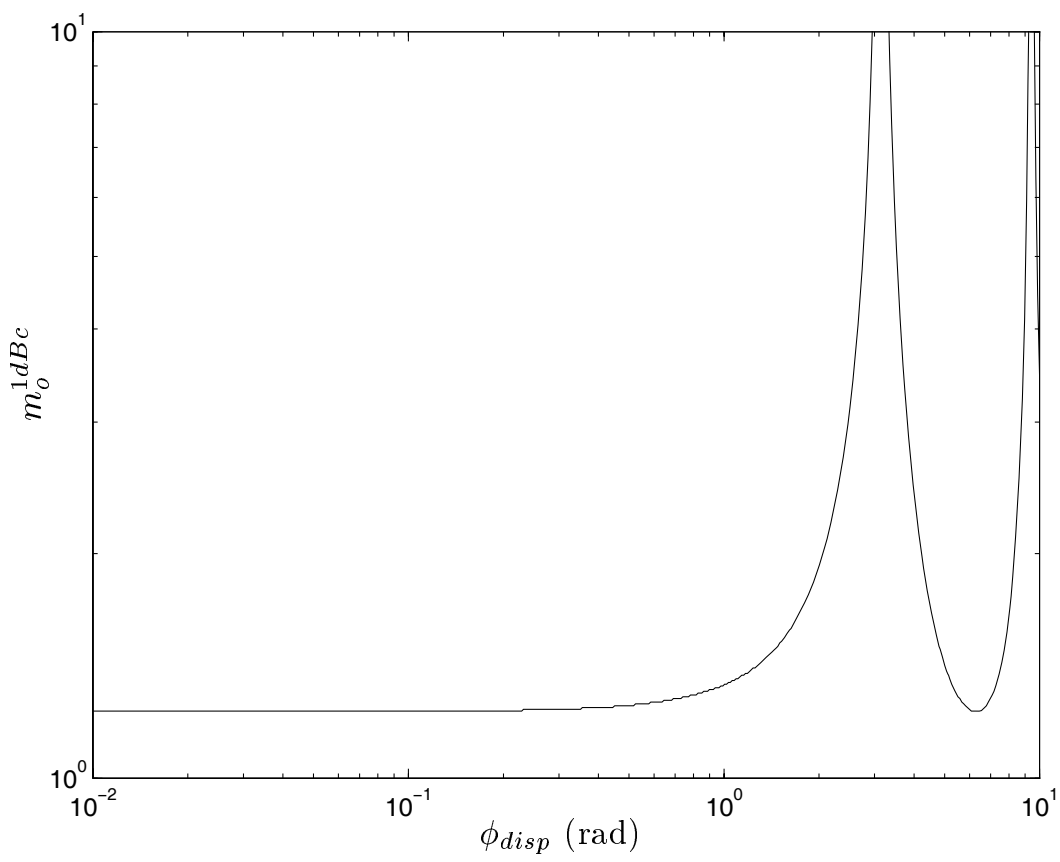

a)

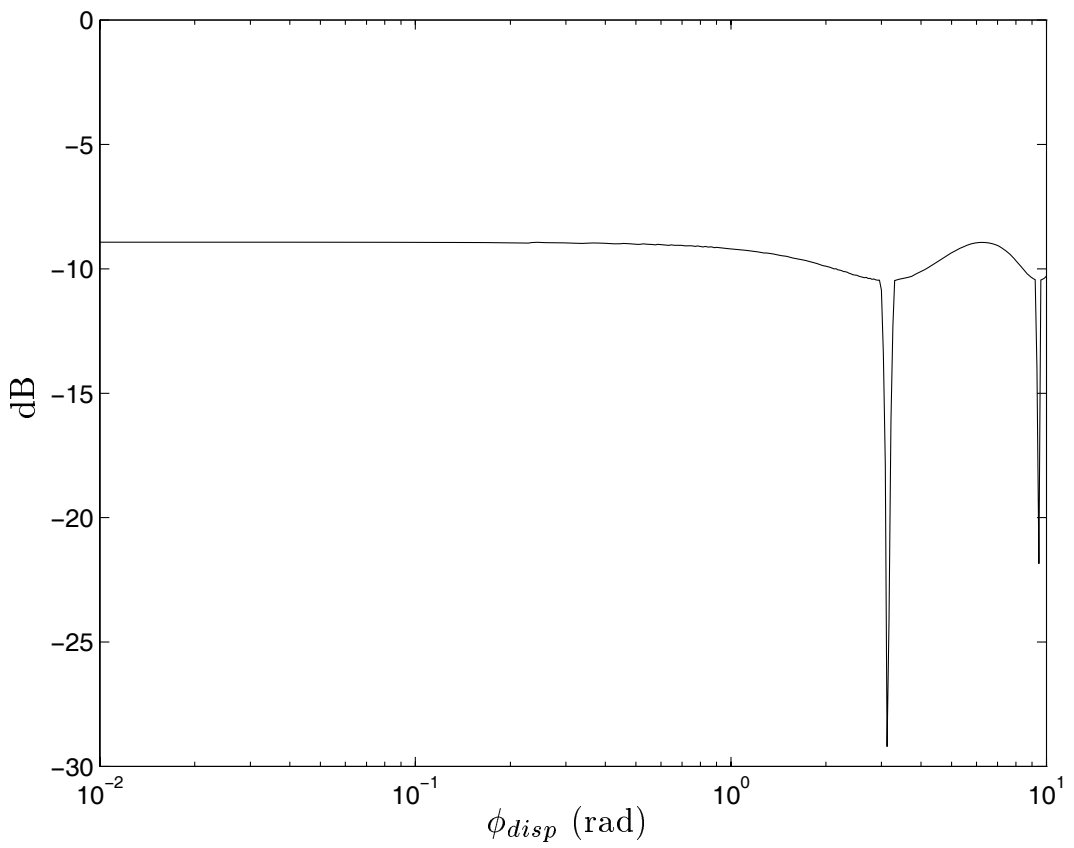

Figura 5.28: Efecto de la dispersión de una rama del conformador sobre el punto de compresión a $1 \mathrm{~dB}$, en función del producto de dispersión, $\phi_{\text {disp }}$. a) Índice de modulación a la entrada del EOM, correspondiente al punto de compresión a $1 \mathrm{~dB}$, $m_{o}^{1 d B c}$. b) Nivel de potencia relativa de la señal detectada a la salida del fotodetector en el punto de compresión a $1 \mathrm{~dB}$. 
a)
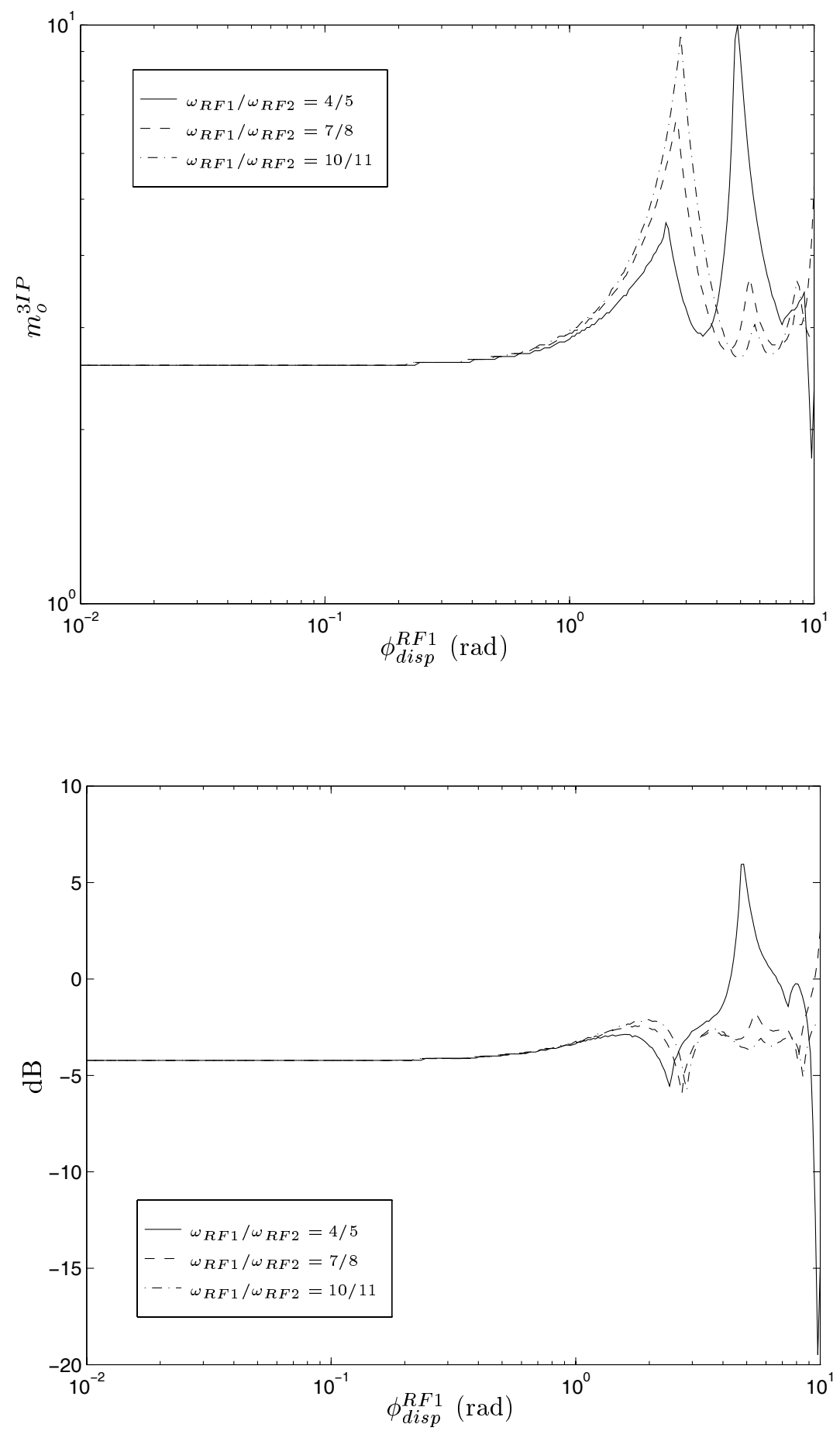

b)

Figura 5.29: Efecto de la dispersión de una rama del conformador sobre el punto de intercepción de tercer orden, en función del producto de dispersión, $\phi_{\text {disp }}$. a) Índice de modulación a la entrada del EOM, correspondiente al punto de intercepción de tercer orden, $m_{o}^{3 I P}$. b) Nivel de potencia relativa de la señal detectada a la salida del fotodetector en el punto de intercepción de tercer orden. 
diferencia. Por esta razón, se ha tomado como criterio coger siempre el nivel más alto de potencia para ambas parejas de frecuencias. Al igual que ocurría con el punto de compresión a $1 \mathrm{~dB}$, mientras no aparece la atenuación dispersiva, el punto de intercepción de tercer orden del conformador óptico es el constante.

A medida que aumenta la dispersión de la rama del conformador, ésta comienza afectando en primer lugar al producto de intermodulación de tercer orden diferencia inferior, a continuación a la fundamental inferior, a la fundamental superior y al producto de tercer orden diferencia superior. En todos los casos, el efecto consiste en una disminución de la potencia detectada, como se vió en la figura 5.17; esta atenuación irá afectando de forma diferente a cada frecuencia, lo que provoca las oscilaciones del punto de intercepción de tercer orden mostradas en la figura 5.29b. Evidentemente, estas oscilaciones son mayores, cuanto más separadas estén las frecuencias de los dos tonos de entrada.

\section{Margen dinámico}

El margen dinámico disponible es un parámetro de vital importancia a la hora de contrastar las prestaciones de una red conformadora de haces para agrupaciones de antenas. Es habitual trabajar con dos parámetros para medirlo; por un lado, el margen dinámico limitado por compresión, y por otro lado, el margen dinámico libre de espúreos (SFDR ${ }^{\mathrm{i}}$. El SFDR sólo tendrá sentido en el caso de trabajar con señales multifrecuencia, caso por otro lado cada día más habitual en los sistemas modernos de comunicaciones.

Considerando que las componentes más importantes de ruido son las correspondientes al ruido RIN, al ruido shot y al ruido térmico en el receptor, se obtiene como expresión del ruido total a la salida del fotodetector ${ }^{\text {ii }}$ :

$$
\begin{aligned}
N_{\text {total }}=N_{R I N}+N_{\text {shot }}+N_{\text {térmico-pd }} & \\
= & {\left[I_{d c}^{2} \cdot 10^{(R I N / 10)}+2 q \cdot \overline{I_{d c}}+4 k \cdot T\right] \cdot B W \cdot R_{C A R G A} }
\end{aligned}
$$

obteniéndose, para los valores típicos utilizados en (5.113), junto a un valor del parámetro RIN del láser de $R I N=-150 \mathrm{~dB} / \mathrm{Hz}$, y una temperatura de $290 \mathrm{~K}$, una potencia de ruido total a la salida del fotodetector de $-126 \mathrm{dBW} / \mathrm{MHz}$.

Durante el desarrollo de esta Tesis, se ha trabajado con potencias detectadas normalizadas respecto al factor $A_{p d}^{2}$ y calculadas sobre una resistencia de carga unidad, para desnormalizar los resultados, teniendo en cuenta parámetros reales de una rama del conformador, se deberá aplicar la siguiente igualdad:

$$
A_{p d}^{2}=\mathcal{R}^{2} t_{o d}^{2} t_{f f}^{2} P_{\text {láser }}^{2} L^{2}
$$

\footnotetext{
${ }^{\mathrm{i}}$ Spurious-free dynamic range.

${ }^{i}$ Se ha considerado nula la corriente de oscuridad del fotodetector.
} 
a)
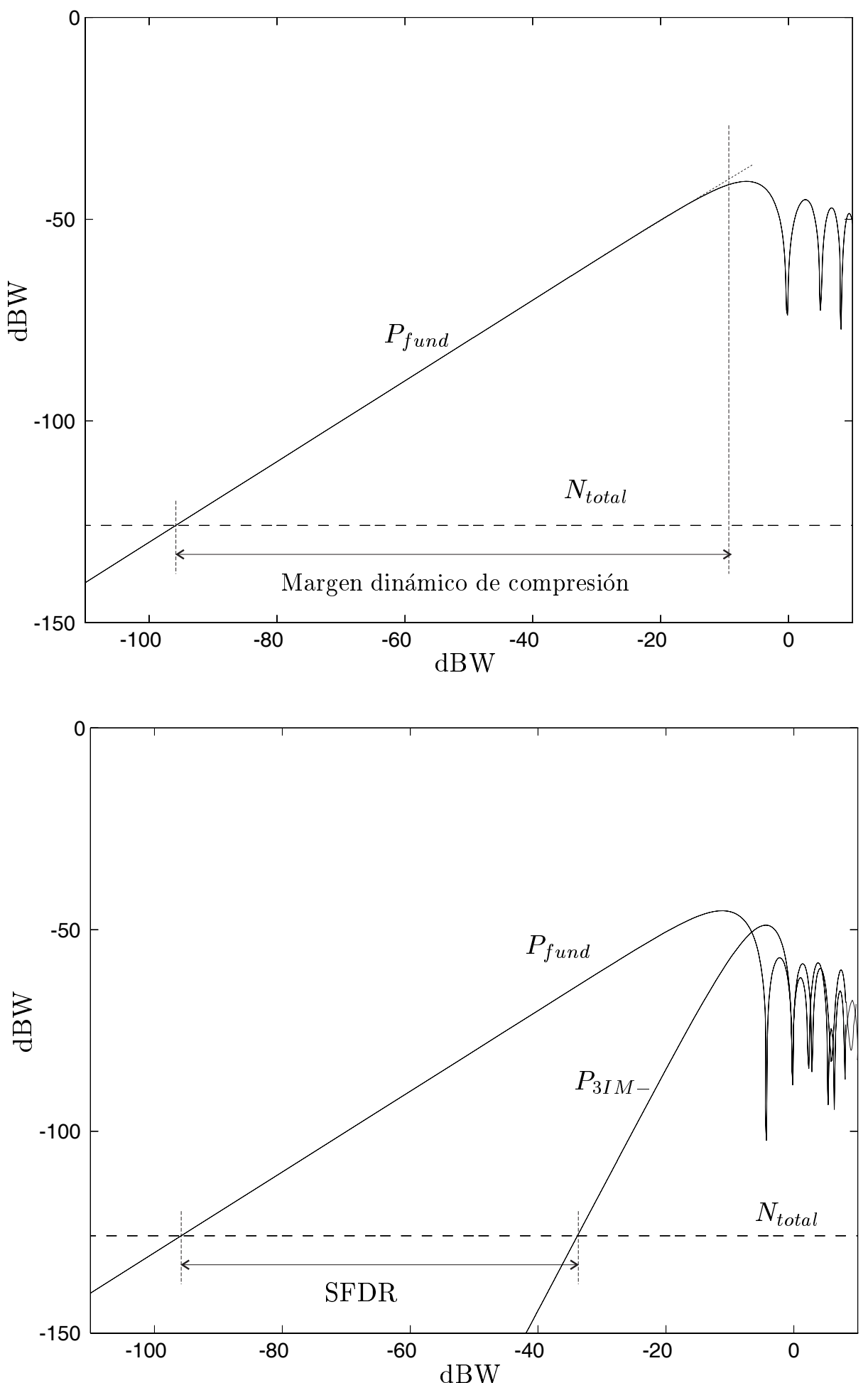

b)

Figura 5.30: Obtención de los márgenes dinámicos del conformador óptico basado en redes de difracción con variación lineal del periodo. a) Margen dinámico de compresión. b) Margen dinámico libre de espúreos, SFDR. 
que se utilizará para desnormalizar las expresiones de las potencias de los diferentes armónicos y productos de intermodulación expresados en (5.60) y (5.77), además de multiplicarlas por $R_{C A R G A}$.

Las potencias de entrada al modulador se habían respresentado hasta ahora en función del índice de modulación óptico, $m_{o}$, el cual está relacionado con la potencia de radiofrecuencia de entrada al modulador electro-óptico según la igualdad (5.112).

Una vez se ha calculado la potencia de ruido detectada total y tras desnormalizar los resultados obtenidos en el apartado 5.3.3 para los parámetros de distorsión y los productos de intermodulación, es posible obtener el margen dinámico de compresión y el margen dinámico libre de espúreos. En la figura 5.30 se representa el cálculo de los mismos, obteniéndose unos valores de 84 dBMHz y 60 dBMHZ ${ }^{2 / 3}$ para el margen dinámico de compresión y el SFDR, respectivamente.

\section{Acoplamiento entre ramas del conformador}

Una posible fuente de errores en el conformador consiste en las imperfecciones en el proceso de separación de las portadoras correspondientes a cada elemento de la agrupación (Fig. 5.24). Ya sea a través de la utilización de un divisor 1 a $N$ junto con $N$ filtros individuales, o bien de un demultiplexor en longitud de onda, la capacidad de selección de los mismos será finita, existiendo una cierta atenuación, no finita de las portadoras adyacentes a la portadora de cada elemento. Esta eliminación imperfecta del resto de portadoras, implicará que en cada elemento se dispondrá de la señal ideal, junto a una combinación de las señales atenuadas del resto de elementos; generalmente, estas señales estarán más atenuadas, cuanto mayor sea la distancia respecto a la portadora del elemento considerado.

Se puede comprobar fácilmente, que a la salida del fotodetector del elemento n-ésimo se dispondrá de una señal de amplitud y fase [RM98]:

$$
\begin{aligned}
A_{n} & =\sqrt{\left(\sum_{i=1}^{N} a_{i} \cos \left(\phi_{i}\right)\right)^{2}+\left(\sum_{i=1}^{N} a_{i} \operatorname{sen}\left(\phi_{i}\right)\right)^{2}} \\
\Phi_{n} & =\arctan \left(\frac{\sum_{i=1}^{N} a_{i} \operatorname{sen}\left(\phi_{i}\right)}{\sum_{i=1}^{N} a_{i} \cos \left(\phi_{i}\right)}\right)
\end{aligned}
$$

donde $a_{i}$ y $\phi_{i}$ son las amplitudes y fases de cada una de las $N$ señales eléctricas, tras el filtrado de la portadora n-ésima y la posterior detección. Idealmente, estas amplitudes vendrán afectadas por la respuesta del filtro centrado en la portadora n-ésima y los desfases vendrán definidos por la red de difracción y la sintonía de los láseres. 
A partir de (5.120) se puede ver que el efecto del filtrado no ideal de las $N$ portadoras se traduce en errores de amplitud y fase en cada elemento; siendo estos errores, dependientes del retardo asignado a cada elemento (que para una frecuencia dada, se traduce en las fases $\left.\phi_{i}\right)$.

En la figura 5.31 se muestra el efecto del filtrado no ideal sobre una agrupación de diez elementos espaciados media longitud de onda. En concreto, se muestran los valores cuadráticos medios de los errores de fase y amplitud de la alimentación de los elementos de la agrupación, además del error en el apuntamiento del haz. Estos resultados se representan en función del ángulo de barrido y parametrizados respecto a la pendiente del filtro selector de cada portadora expresada como el número de decibelios de potencia óptica que se atenúa la señal a una distancia igual a la separación entre portadoras, $\Delta \lambda$. Se observa como el efecto de la no idealidad del filtro se traduce en un error en la alimentación de la agrupación, donde el error RMS de fase máximo ocurre para un apuntamiento de $30^{\circ}$, aproximadamente, respecto a la dirección normal a la agrupación, mientras que el error de amplitud máximo ocurre justamente para la dirección normal a la agrupación.

Para valorar la importancia de los errores mostrados en la figura 5.31, se puede ir a la figura 2.17 o a las ecuaciones A.12 y A.18 del apéndice A. Para el caso en el que la atenuación de los filtros selectores cae solamente $5 \mathrm{dBo}$ cada $\Delta \lambda$ respecto al pico del filtro, los errores que se provocan en la alimentación de la agrupación implican, en el peor caso, un nivel de lóbulos secundarios de fondo respecto al principal de $-23.9 \mathrm{~dB}$ y una variación en la ganancia del haz de $-0.2 \mathrm{~dB}$. Para los otros dos casos mostrados en la figura 5.31, los resultados obtenidos están por encima de los $45 \mathrm{~dB} / 60 \mathrm{~dB}$, por lo que se puede concluir que una caida de $15 \mathrm{dBo} / \Delta \lambda$ en los filtros selectores de longitud de onda ofrece unos resultados excelentes para esta agrupación; de hecho, los errores para una pendiente de $20 \mathrm{dBo} / \Delta \lambda$ son casi inapreciables en la figura 5.31. Para agrupaciones de otros tamaños, habría que realizar un análisis similar, teniendo en cuenta las expresiones del apéndice A.

Por lo que respecta al error de apuntamiento (Fig. 5.31c), a pesar de los pequeños valores de los mismos; conviene resaltar que un error de $0.45^{\circ}$ en el apuntamiento del haz, se convierte para una agrupación transportada sobre un satélite geoestacionario, en aproximadamente $300 \mathrm{~km}$ de error sobre la superficie terrestre.

\section{Conformación de haces}

La estructura presentada permite obtener cualquier distribución en la alimentación de la agrupación y a diferencia de otras arquitecturas de redes ópticas conformadoras no está limitada a distribuciones con fase lineal [Esm93, Ton96a, Ton96b]. Además, si las fuentes sintonizables permiten la variación de la potencia de salida, la libertad a la hora de escoger la distribución en amplitud y fase es total, siendo además controlable remotamente, desde el punto donde se encuentren las fuentes y no desde el entorno de la agrupación. 
a)

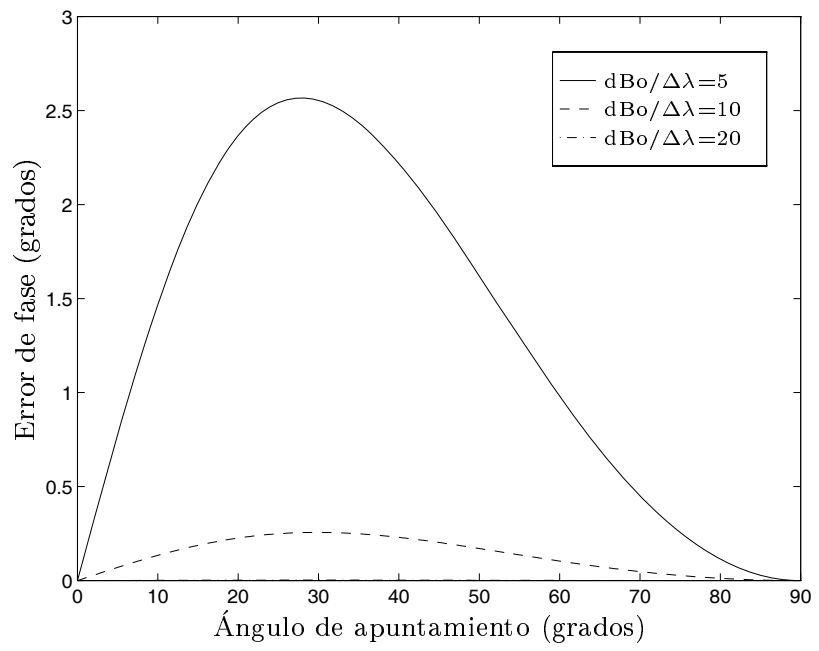

b)
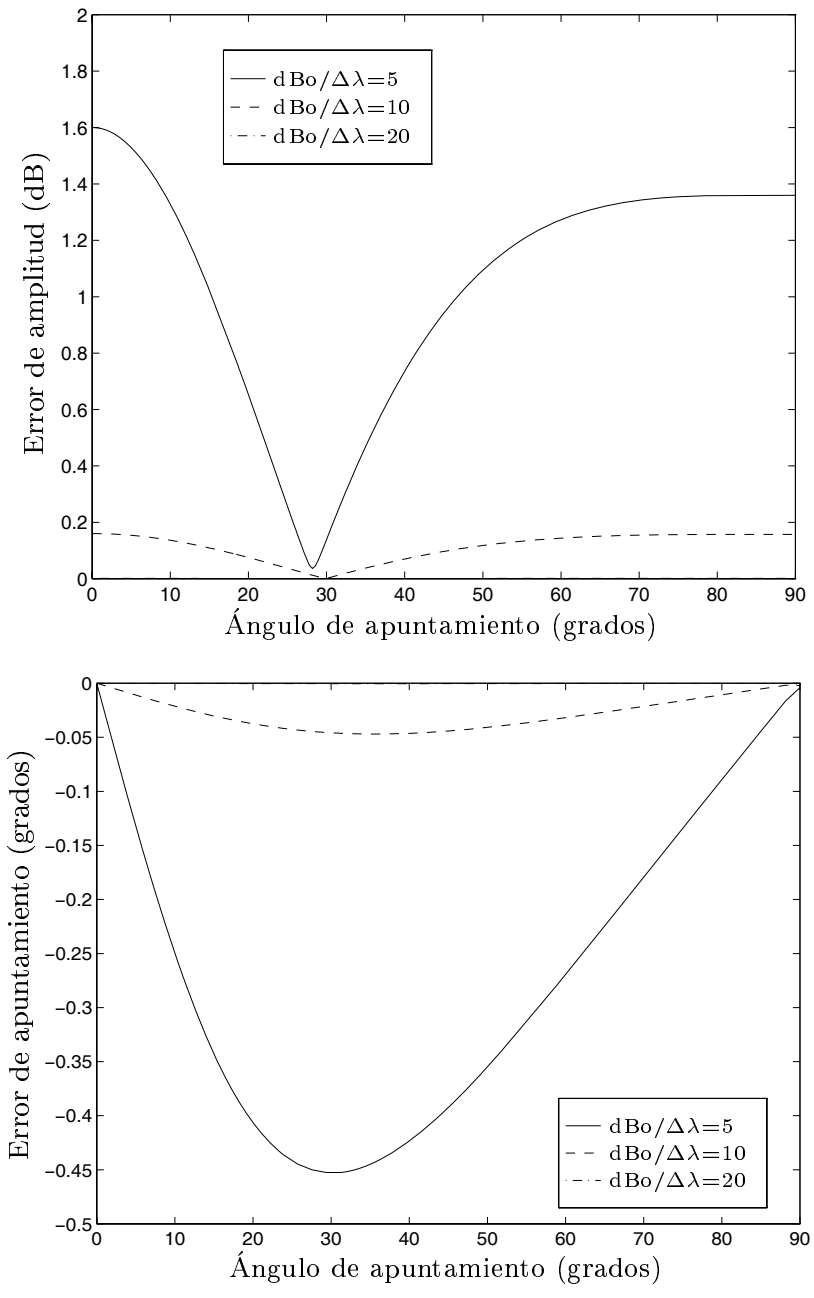

Figura 5.31: Efecto del filtrado no ideal en la separación de las portadoras ópticas, sobre una agrupación de diez elementos con separación entre elementos $d=\lambda / 2$, en función del ángulo de apuntamiento y de la caída del filtro entre portadoras $(\mathrm{dBo} / \Delta \lambda)$. a) Valores RMS del error de fase en la alimentación. b) Valores RMS del error de amplitud en la alimentación. c) Error de apuntamiento del haz principal. 


\section{Errores. Efecto sobre el diagrama de radiación}

Para una rama cualquiera del conformador, el error total en el retardo introducido por la misma puede estimarse a partir de dos fuentes de error: por un lado, la estabilidad en longitud de onda de la fuente láser, $\Delta \lambda_{\text {laser }}$, y por otro lado, el rizado que presente el retardo de grupo de la red de difracción respecto a una respuesta lineal ideal, $\sigma_{\text {red }}$. La estabilidad en longitud de onda del láser, puede traducirse a precisión de retardo para cada rama del conformador, teniendo en cuenta la respuesta de la red de difracción alrededor de cada longitud de onda central, $\lambda_{i}$, de acuerdo a:

$$
\sigma_{\text {laser }}\left(\lambda_{i}\right)=\Delta \lambda_{\text {laser }} \cdot \delta_{i}
$$

En cuanto al rizado del retardo de grupo de la red de difracción, $\sigma_{\text {red }}$, éste deberá estimarse en el ancho de banda de sintonización, $\Delta \lambda_{i}$, alrededor de cada longitud de onda central, $\lambda_{i}$, y de la pendiente media alrededor de la misma, $\delta_{i}$.

$$
\sigma_{\text {red }}^{2}\left(\lambda_{i}, f_{R F}\right)=\frac{1}{\Delta \lambda_{i}} \int_{\lambda_{i}-\frac{\Delta \lambda_{i}}{2}}^{\lambda_{i}+\frac{\Delta \lambda_{i}}{2}}\left[\tau_{\text {red }}(\lambda)-\left(\tau_{\text {red }}\left(\lambda_{i}\right)+\delta_{i} \cdot\left(\lambda-\lambda_{i}\right)\right)\right]^{2} d \lambda
$$

Si se combinan ambos fuentes de error independientes se puede obtener la precisión total como:

$$
\sigma_{\text {TOTAL }}^{2}\left(\lambda_{i}, f_{R F}\right)=\sigma_{L A S E R}^{2}\left(\lambda_{i}\right)+\sigma_{r e d}^{2}\left(\lambda_{i}, f_{R F}\right)
$$

\subsection{Medidas del conformador óptico}

En la figura 5.32 se muestra la configuración utilizada en las medidas de la respuesta de una rama de la red óptica conformadora que incluye la red de difracción con periodo variable. La red de difracción utilizada es una red apodizada de $40 \mathrm{~cm}$ de longitud con una variación lineal del periodo dentro de un ancho de banda de $\Delta \lambda_{\text {red }}=4 \mathrm{~nm}$ entre $1547 \mathrm{~nm}$ y $1551 \mathrm{~nm}$ como se puede comprobar en las curvas de caracterización (Fig. 5.33) de la misma suministradas por el fabricante. La pendiente media de la respuesta del retardo de grupo en función de la longitud de onda es de aproximadamente $835 \mathrm{ps} / \mathrm{nm}$.

\subsubsection{Atenuación dispersiva}

De acuerdo con (5.50), la dispersión cromática de la red de difracción, limitará el ancho de banda en sistemas IM/DD con modulación de intensidad convencional. La figura 5.34 muestra la medida de la atenuación dispersiva sufrida por la señal detectada de microondas debido a este efecto, para $\lambda_{1}=1547.5 \mathrm{~nm}$, cuando se utiliza modulación AM [Cor97a]. El ancho de banda medido, libre de atenuación dispersiva, 


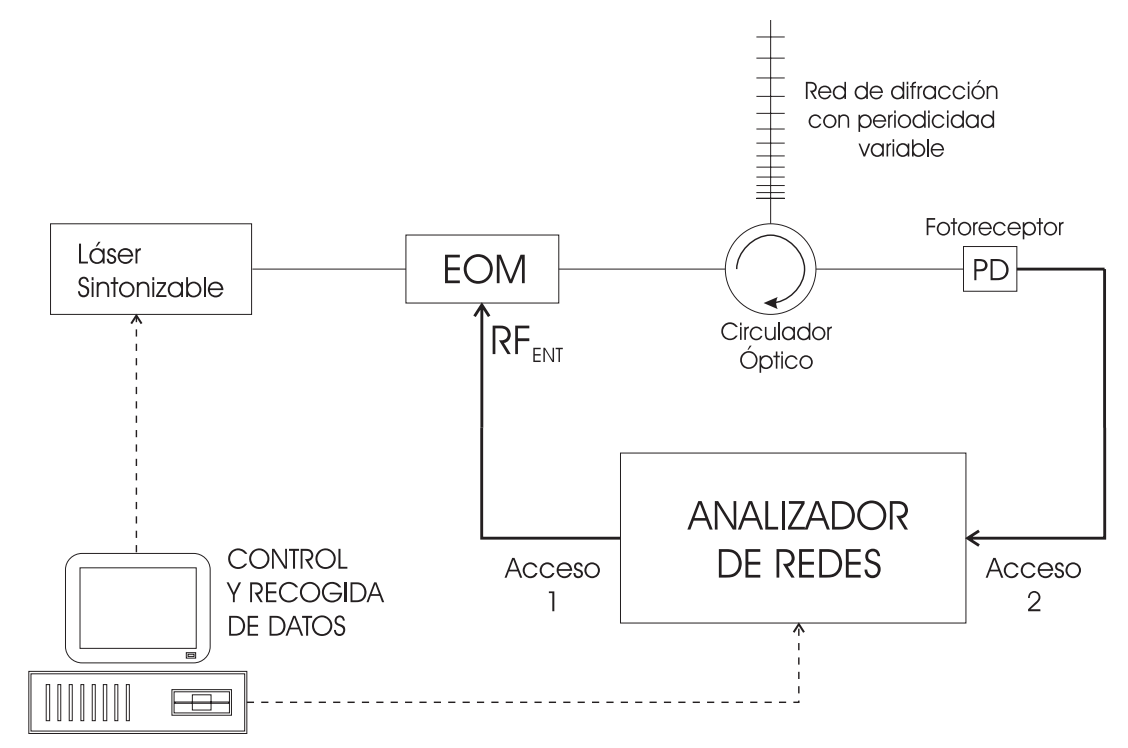

Figura 5.32: Configuración del experimento para la caracterización de los retardos relativos de una única rama de la red óptica conformadora.

es de $6.3 \mathrm{GHz}$, como se podía esperar de (5.50) teniendo en cuenta que alrededor de esa longitud de onda, la pendiente del retardo de grupo es de $\delta_{1} \approx 800 \mathrm{ps} / \mathrm{nm}$. Las zonas del espectro alrededor de los siguientes máximos (12 y $17.5 \mathrm{GHz}$ según la figura 5.34) permitiría trabajar al conformador en banda estrecha siempre y cuando la pendiente de la red de difracción fuera lineal dentro del ancho de banda de la red de difracción, $\Delta \lambda_{\text {red }}$.

Posteriormente, se sustituyó el modulador electro-óptico convencional por un generador $\mathrm{SSB}+\mathrm{C}$ basado en un modulador dual como el mostrado en la figura 5.20, obteniéndose los resultados mostrados en la figura 5.34; que demuestran la eliminación de la atenuación dispersiva dentro del ancho de banda del híbrido de microondas utilizado [Cor98].

Como última medida de los diferentes esquemas de modulación estudiados, se utilizó la modulación pseudo-autoheterodina, cuyos resultados se muestran en 5.35 confirmando que se aumenta considerablemente el margen de frecuencias libre de atenuación dispersiva.

\subsubsection{Caracterización de la red conformadora. Retardos}

Con el fin de mostrar y comprobar las posibilidades del conformador presentado en esta Tesis, se ha considerado una agrupación lineal de cuatro elementos $(N=4)$, partiendo de medidas del experimento de la figura 5.32 que corresponde a una rama del esquema general de la figura 5.24.

Asumiendo una separación entre elementos de $d=4 \mathrm{~cm}$ y un margen de barrido completo $\left( \pm 90^{\circ}\right)$, el retardo máximo en cada elemento será de $\tau_{\max }=200 \mathrm{ps}$, 


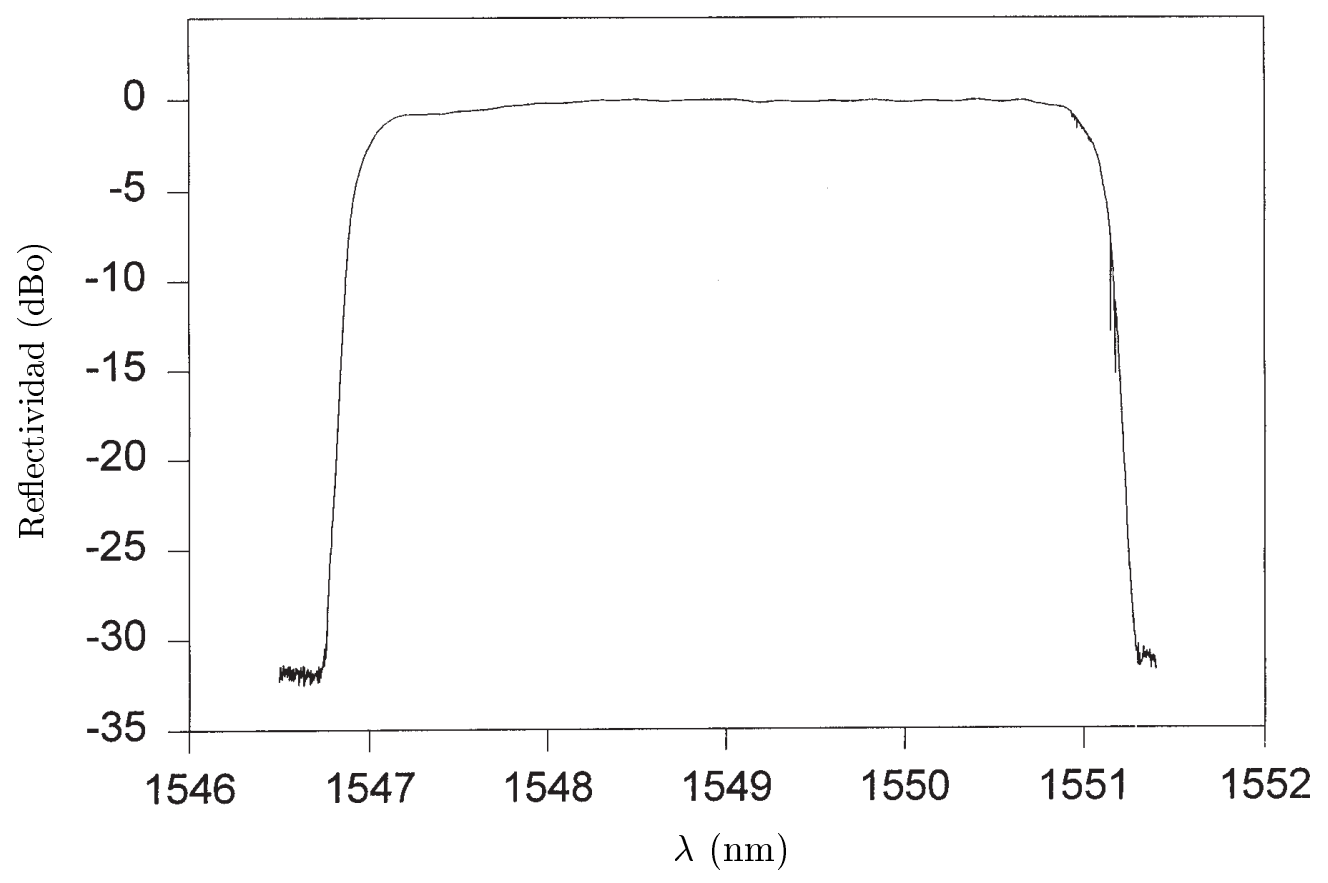

a)

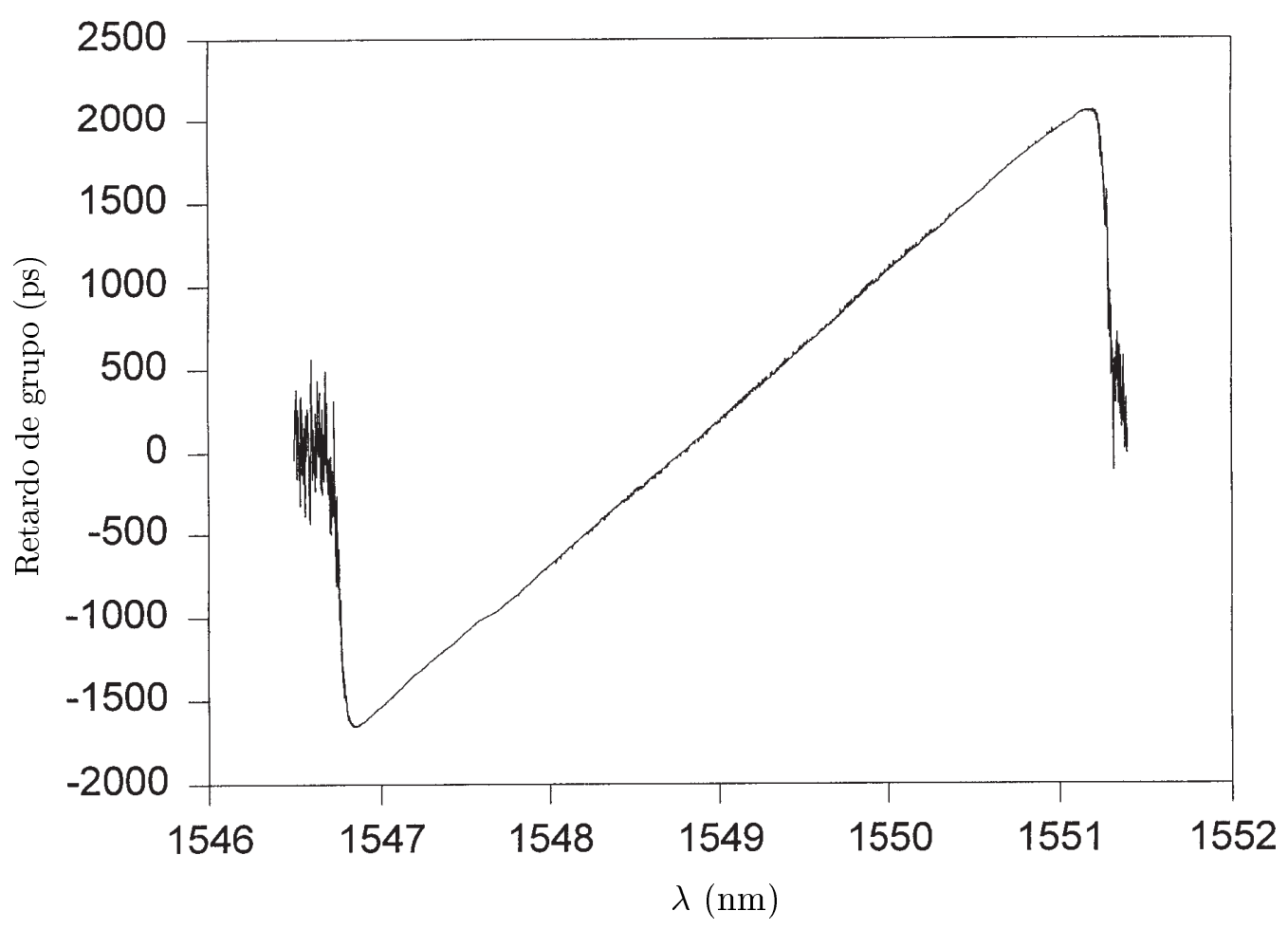

Figura 5.33: Respuesta en reflexión de la red de difracción utilizada en los experimentos. a) Respuesta en amplitud. b) Retardo de grupo. 


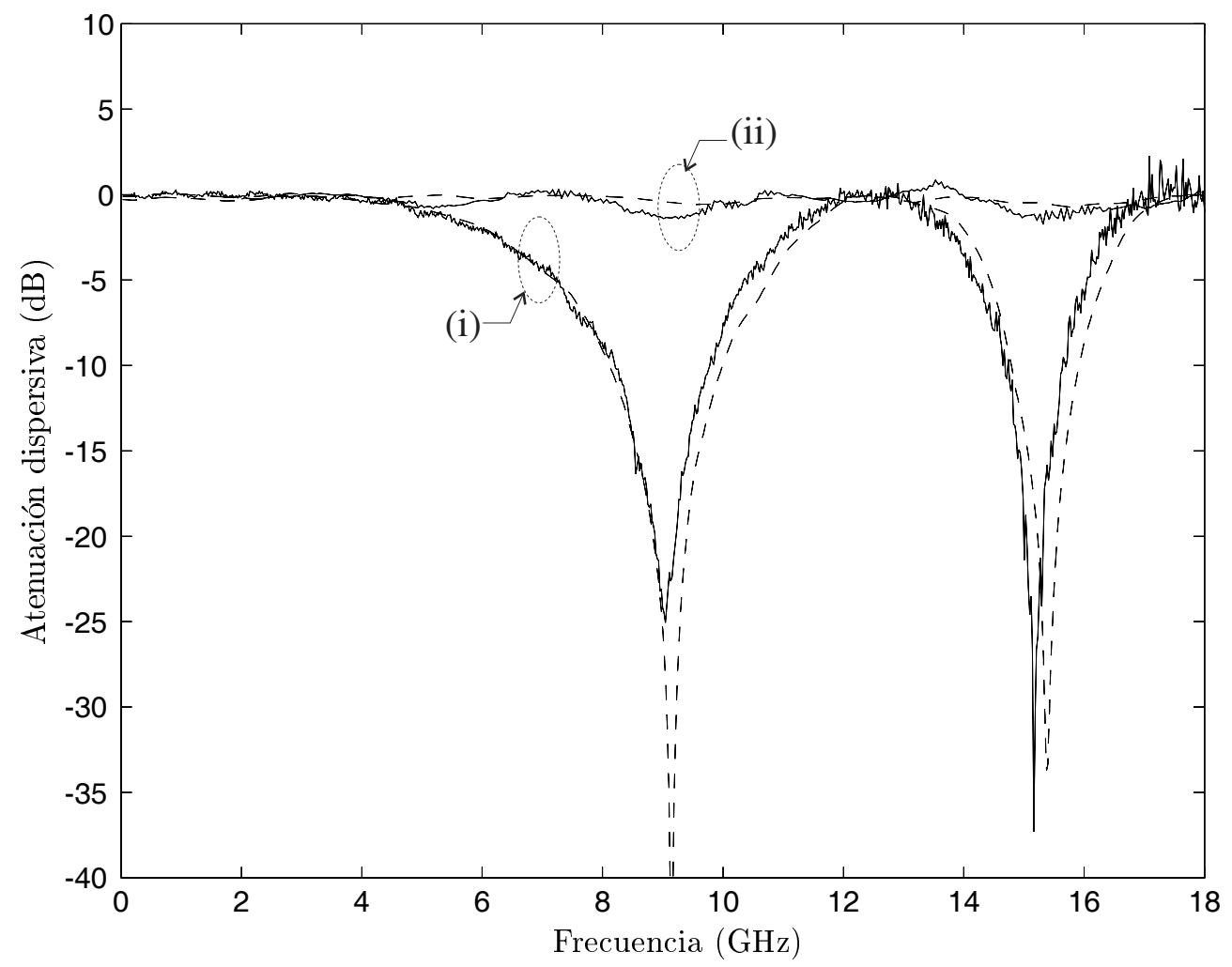

Figura 5.34: Medidas y simulaciones de la atenuación dispersiva de una señal de radiofrecuencia en la transmisión en un sistema IM/DD (modulaciones AM y SSB+C) que incluye una red de difracción $(\delta=850 \mathrm{ps} / \mathrm{nm})$. (i) Traza contínua: medidas AM IM/DD. Traza discontínua: simulación AM IM/DD. (ii) Traza contínua: medidas $\mathrm{SSB}+\mathrm{C}$ IM/DD. Traza discontínua: simulación SSB+C IM/DD. $\left(\lambda_{c}=1547.5 \mathrm{~nm}\right.$ y $\left.m_{o}=0.1\right)$.

necesitándose una excursión de longitud de onda en cada láser de $\Delta \lambda \approx 0.5 \mathrm{~nm}$. Se han escogido como valores para las cuatro longitudes de ondas centrales de cada pareja láser/elemento de $\lambda_{1}=1547.5 \mathrm{~nm}, \lambda_{2}=1548.5 \mathrm{~nm}, \lambda_{3}=1549.5 \mathrm{~nm}$ y $\lambda_{4}=1550.5 \mathrm{~nm}$.

Se midió el retardo de grupo dentro del ancho de banda completo de la red de difracción (1547 nm a $1551 \mathrm{~nm}$ ) para dos frecuencias distintas, 2 y $5 \mathrm{GHz}$. A partir de la medida del retardo de grupo, se puede extraer, para cada rama, la pendiente media, $\delta_{i}$, dentro de un ancho de banda de $\Delta \lambda=0.5 \mathrm{~nm}$ alrededor de cada longitud de onda central, $\lambda_{i}$, y las longitudes extra de fibra monomodo, $L_{i}$, (proporcionales a los retardos relativos al retardo introducido para $\lambda_{1}$ ); obteniéndose los resultados resumidos en la tabla 5.3.

Una vez compensados los retardos relativos a las longitudes de onda centrales, se puede obtener la respuesta del retardo de grupo incremental alrededor de cada una de las cuatro longitudes de onda y para ambas frecuencias de microondas. Los resultados se muestran en la figura 5.36 donde se aprecia la linealidad de los mismos 


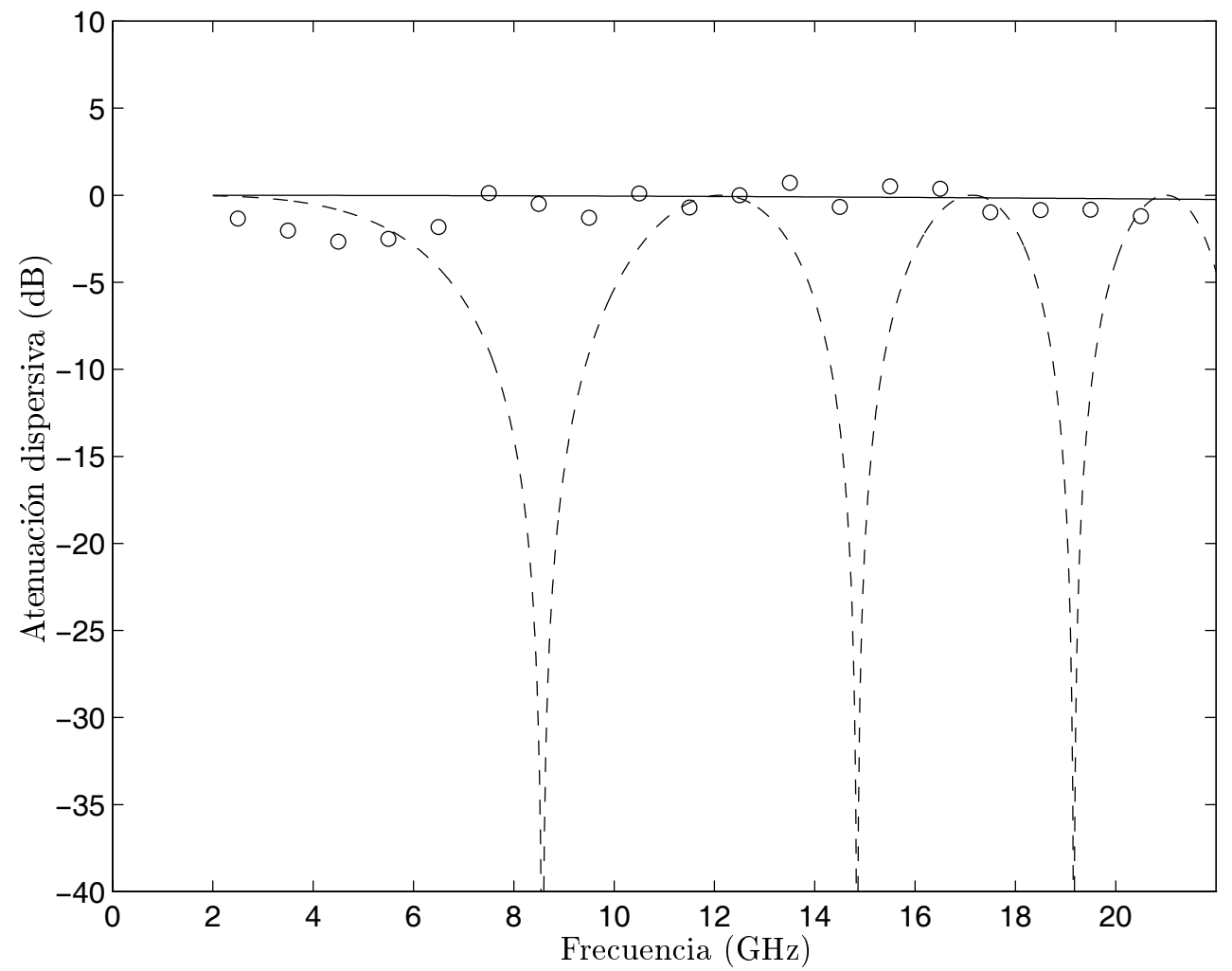

Figura 5.35: Medidas (traza contínua) y simulación (circunferencias) de la atenuación dispersiva del término de la señal detectada $\omega_{R F}=2 \omega_{O L}+\omega_{I F}$ en la transmisión a través de un enlace IM/DD con modulación pseudoheterodina que implementa una línea de retardo con una red de difracción con periodo variable lineal $(\delta=850 \mathrm{ps} / \mathrm{nm}$, $\left.\lambda_{c}=1547.5 \mathrm{~nm}, m_{\text {opt }}=0.1, \omega_{I F}=0.5 \mathrm{GHz}\right)$. En traza discontínua, simulación de la atenuación si se utilizara modulación AM convencional para la misma frecuencia detectada.

y su independencia respecto a la frecuencia de la señal de microondas.

Como primer paso para estimar la precisión de los retardos obtenidos con el conformador, es necesario calcular el parámetro $\sigma_{\text {red }}$ presentado previamente en la ecuación (5.122). En la tabla 5.4 se muestran los resultados obtenidos asumiendo un ancho de banda de $0.5 \mathrm{~nm}$ alrededor de cada longitud de onda central. En la citada tabla se ha añadido también la precisión media para cada frecuencia de microondas.

Para estimar la precisión total, falta por considerar la precisión temporal debida a la inestabilidad de la longitud de onda del láser; para ello, se debe partir de la estabilidad en longitud de onda del láser y aplicando (5.121) calcular la precisión temporal para cada rama; finalmente, se puede aplicar (5.123) para obtener la precisión total. En la tabla 5.5, se ofrecen los resultados de la precisión total para cada rama y para ambas frecuencias consideradas, asumiendo una precisión en cada fuente láser de $0.01 \mathrm{~nm}$.

De acuerdo con los resultados de la tabla 5.5 la precisión en retardo total del 


\begin{tabular}{|c|c|c|c|}
\hline $\begin{array}{c}\lambda_{i} \\
(\mathrm{~nm})\end{array}$ & $\begin{array}{c}\text { Retardo relativo medio } \\
\mathrm{a} \lambda_{i}(\mathrm{ps})\end{array}$ & $\begin{array}{c}\text { Longitud compensadora } \\
L_{i}(\mathrm{~cm})\end{array}$ & $\begin{array}{c}\text { Pendiente media } \\
\delta_{i}(\mathrm{ps} / \mathrm{nm})\end{array}$ \\
\hline \hline 1547.5 & 0 & 0 & 787 \\
\hline 1548.5 & 824.1 & 16.93 & 819 \\
\hline 1549.5 & 1653.7 & 33.98 & 850 \\
\hline 1550.5 & 2505.8 & 51.49 & 865 \\
\hline
\end{tabular}

Tabla 5.3: Retardo medio y pendiente para cada una de las cuatro longitudes de onda centrales para 2 y $5 \mathrm{GHz}$, considerando $\Delta \lambda=0.5 \mathrm{~nm}$.

\begin{tabular}{|c||c|c|c|c|c|}
\hline$\sigma_{\text {red }}$ & $\begin{array}{c}\lambda_{1} \\
1547.5 \mathrm{~nm}\end{array}$ & $\begin{array}{c}\lambda_{2} \\
1548.5 \mathrm{~nm}\end{array}$ & $\begin{array}{c}\lambda_{3} \\
1549.5 \mathrm{~nm}\end{array}$ & $\begin{array}{c}\lambda_{4} \\
1550.5 \mathrm{~nm}\end{array}$ & $\bar{\sigma}$ \\
\hline \hline $2 \mathrm{GHz}$ & $3.87 \mathrm{ps}$ & $3.83 \mathrm{ps}$ & $2.90 \mathrm{ps}$ & $4.04 \mathrm{ps}$ & $3.69 \mathrm{ps}$ \\
\hline $5 \mathrm{GHz}$ & $2.81 \mathrm{ps}$ & $3.37 \mathrm{ps}$ & $2.49 \mathrm{ps}$ & $2.53 \mathrm{ps}$ & $2.82 \mathrm{ps}$ \\
\hline
\end{tabular}

Tabla 5.4: Desviación estándar del retardo de grupo respecto a la pendiente $\delta_{i}$.

sistema es de $8.94 \mathrm{ps}$; precisión en tiempo equivalente a una precisión en fase de $16.1^{\circ}$ para $f_{R F}=5 \mathrm{GHz}$ o de $6.44^{\circ}$ para $f_{R F}=2 \mathrm{GHz}$. Estos resultados equivalen a la precisión que ofrece un desfasador digital binario de microondas de tres dígitos y medio a $5 \mathrm{GHz}$ o bien un desfasador de cinco dígitos para una frecuencia de trabajo de $2 \mathrm{GHz}$.

En el experimento aquí considerado, la mayor contribución al error total en el retardo, viene de la inestabilidad del láser, para la cual se ha tomado un valor ligeramente pesimista. Por consiguiente, con una ligera mejoría en la estabilidad del láser, los resultados en cuanto a precisión temporal de la red conformadora serían sinceramente excelentes. Por ejemplo, una reducción de la estabilidad del láser a la mitad de su valor considerado, $\Delta \lambda=0.005 \mathrm{~nm}$, implicaría una reducción de la precisión temporal hasta un valor RMS de $5.31 \mathrm{ps}$, lo que es equivalente a un error RMS de fase de $3.8^{\circ}$ para una frecuencia de $f_{R F}=2 \mathrm{GHz}$, es decir, casi la resolución de un desfasador digital de seis dígitos. 

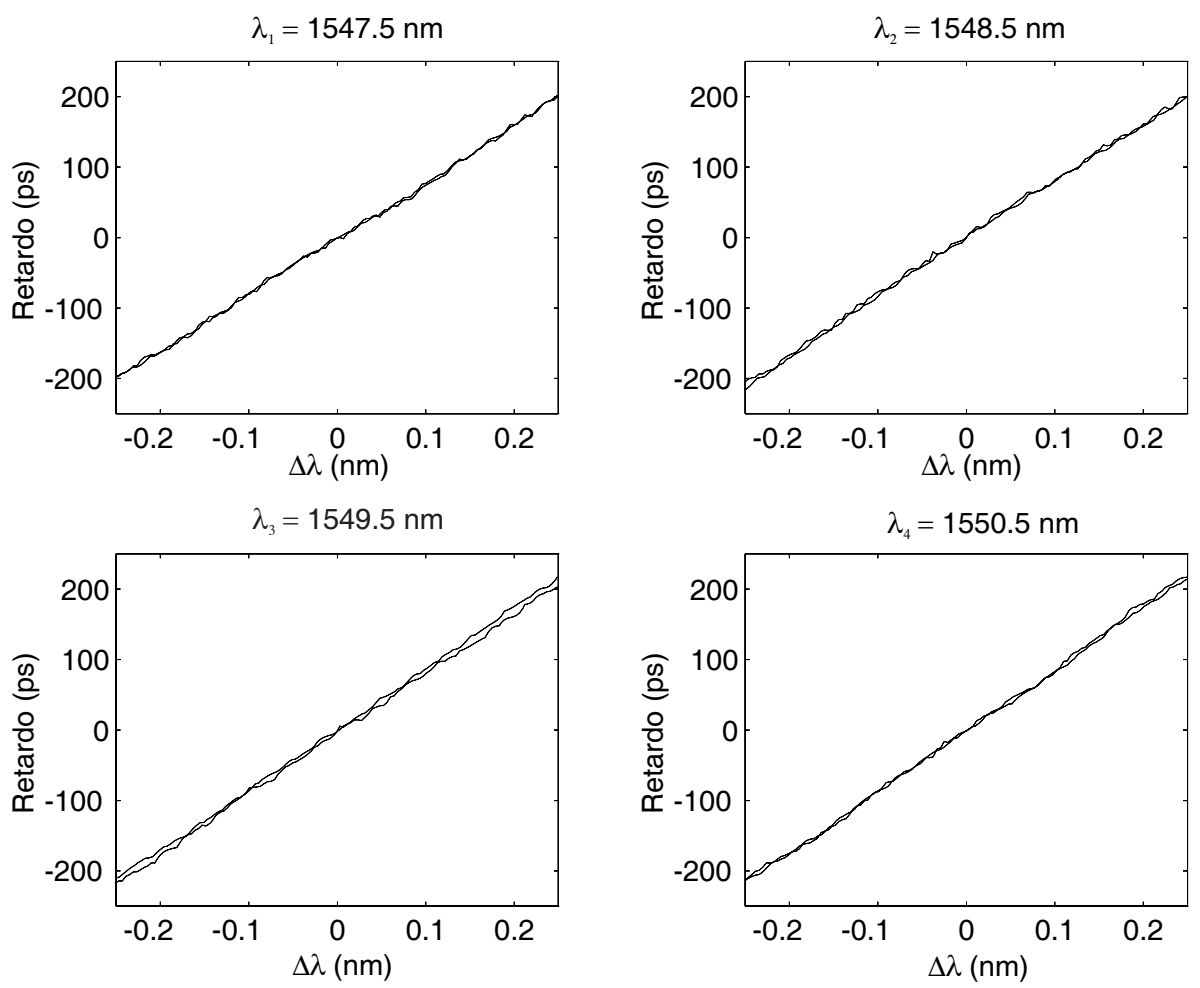

Figura 5.36: Medida de los retardos correspondiente a cada uno de los cuatro elementos de la agupación para dos frecuencias de microondas diferentes (2 y $5 \mathrm{GHz}$ ) en función de la desviación respecto a la longitud de onda central $\left(\Delta \lambda_{i}\right)$. Cada figura corresponde a una longitud de onda diferente.

\begin{tabular}{|c||c|c|c|c|c|}
\hline$\sigma_{\text {TOTAL }}$ & $\begin{array}{c}\lambda_{1} \\
1547.5 \mathrm{~nm}\end{array}$ & $\begin{array}{c}\lambda_{2} \\
1548.5 \mathrm{~nm}\end{array}$ & $\begin{array}{c}\lambda_{3} \\
1549.5 \mathrm{~nm}\end{array}$ & $\begin{array}{c}\lambda_{4} \\
1550.5 \mathrm{~nm}\end{array}$ & $\bar{\sigma}$ \\
\hline \hline $2 \mathrm{GHz}$ & $8.77 \mathrm{ps}$ & $9.05 \mathrm{ps}$ & $8.98 \mathrm{ps}$ & $9.55 \mathrm{ps}$ & $9.09 \mathrm{ps}$ \\
\hline $5 \mathrm{GHz}$ & $8.35 \mathrm{ps}$ & $8.86 \mathrm{ps}$ & $8.86 \mathrm{ps}$ & $9.01 \mathrm{ps}$ & $8.77 \mathrm{ps}$ \\
\hline
\end{tabular}

Tabla 5.5: Valor RMS del error total en el retardo de una rama del conformador óptico. 


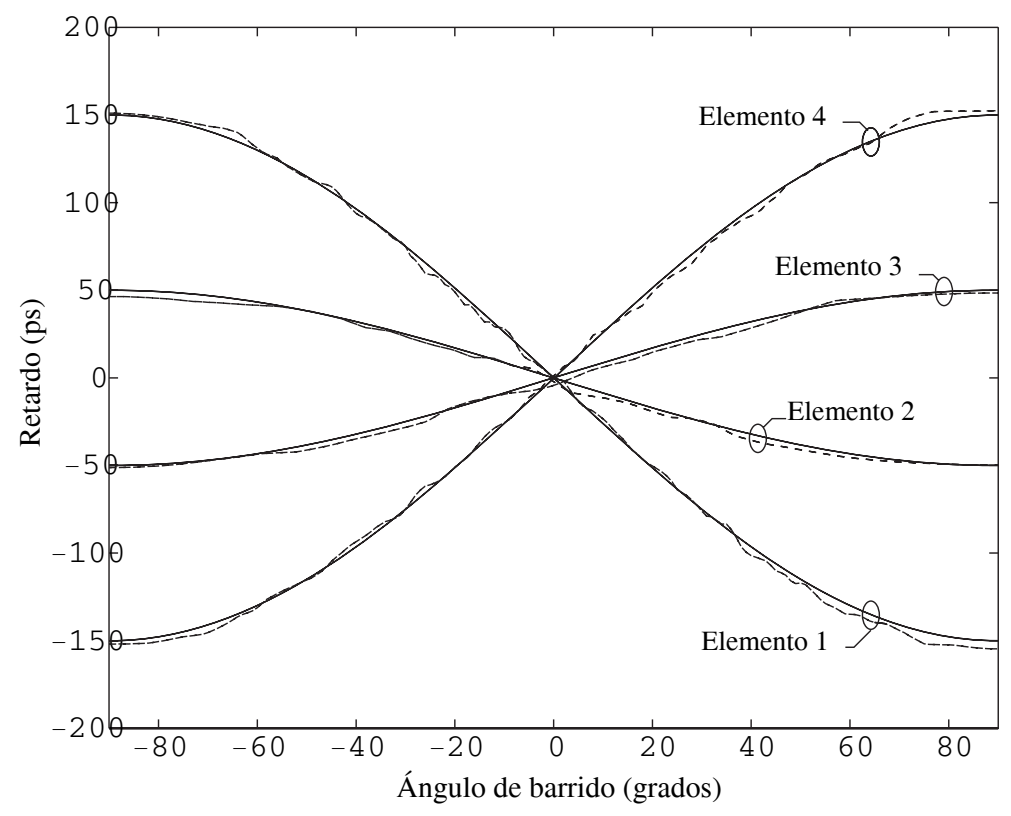

Figura 5.37: Medidas (traza discontínua) de retardo en cada elemento en función del ángulo de apuntamiento cuando $f_{R F}=5 \mathrm{GHz}$. Se muestran también los resultados teóricos con traza contínua.

\subsubsection{Propiedades de conformación}

Tras insertar las longitudes de fibra monomodo compensadoras, el controlador de diagrama de la figura 5.24 se encargará de seleccionar la longitud de onda adecuada teniendo en cuenta la pendiente $\delta_{i}$ extraída de las medidas. Por ejemplo, para la agrupación lineal bajo estudio y realizando un barrido del haz principal mediante la inserción de un retardo progresivo en la alimentación de la agrupación, el conformador ofrecería los retardos para cada elemento mostrados en la figura 5.37, en función del ángulo de apuntamiento del haz para una frecuencia de $f_{R F}=5 \mathrm{GHz}$. En la misma figura se muestran los retardos teóricos necesarios para el apuntamiento, observándose una excelente concordancia con las que se obtendrían con el conformador óptico.

En cuanto a resultados de radiación, en la figura 5.38, se muestra el diagrama de radiación que se obtendría con una agrupación de cuatro elementos isotrópicos separados entre sí $d=4.8 \mathrm{~cm}$ y trabajando a $f_{R F}=5 \mathrm{GHz}$; escogiendo como parámetros para el conformador los anteriormente indicados. Los resultados obtenidos se comparan en la misma figura con el resultado ideal, presentando el conformador un comportamiento muy próximo al esperado. 


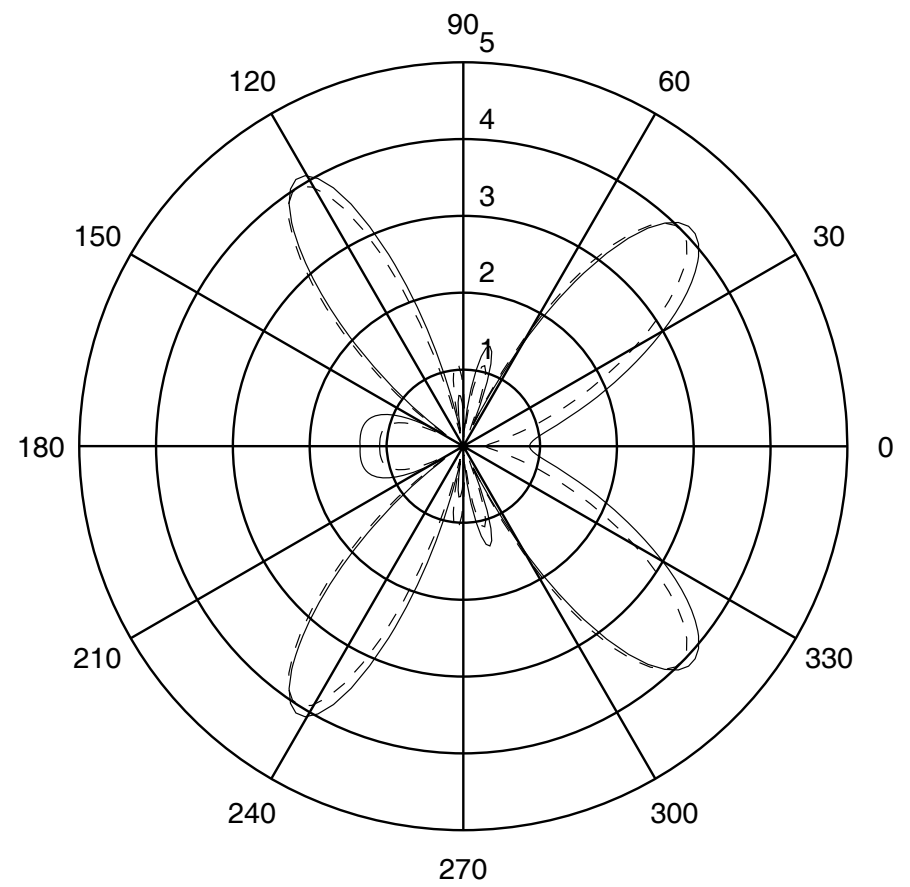

Figura 5.38: Diagrama de radiación ideal (traza discontínua) y simulación del diagrama de radiación a partir de las medidas obtenidas de una rama del conformador (traza contínua) para una agrupación lineal de cuatro elementos isotrópicos con un apuntamiento de $45^{\circ}$.

\subsection{Conclusiones}

En este capítulo se ha analizado la viabilidad de utilizar redes de difracción con periodo variable como elementos de retardo de radiofrecuencia en un enlace IM/DD con modulación de amplitud convencional. Los resultados demuestran la validez de este nuevo dispositivo, limitada únicamente por los fenómenos asociados a la dispersión inherente en la red de difracción. Estas limitaciones se han analizado y cuantificado, permitiendo estimar las prestaciones de un sistema concreto.

Se han analizado diferentes modulaciones además de la modulación de intensidad convencional con el fin de comprobar si las limitaciones seguían siendo válidas, demostrándose analíticamente que la modulación en banda lateral única con portadora no tiene limitación en cuanto al ancho de banda de trabajo, manteniendo la posibilidad de utilizarse en enlaces ópticos IM/DD. Además se ha comprobado que las modulaciones autoheterodinas pueden ser también válidas para señales de banda estrecha, ofreciendo como ventaja añadida, la elevación en frecuencia de la señal de información.

En cuanto a la aplicación de líneas de retardo basadas en redes de difracción con periodo lineal a agrupaciones de antenas se ha presentado una arquitectura de red conformadora de haces totalmente nueva cuyas prestaciones se han evaluado, 
mostrando unos resultados altamente interesantes y confirmando todas las cualidades esperadas de una red óptica conformadora de haces, junto a una libertad total a la hora de seleccionar la distribución de amplitudes y retardos asignados a cada elemento de la agrupación. A partir de las medidas realizadas sobre una rama del conformador, se han estimado las propiedades de radiación de la arquitectura para un caso particular de agrupación con cuatro elementos, consiguiéndose unos resultados excelentes. 



\section{Capítulo 6}

\section{Conclusiones y líneas abiertas}

\subsection{Conclusiones}

Los resultados obtenidos en esta Tesis cumplen los objetivos planteados al inicio de la misma.

La aplicación de tecnología monolítica a redes conformadoras de haces ha buscado introducir en el diseño y caracterización de los circuitos monolíticos, las tolerancias intrínsecas al proceso de fabricación.

En el caso de agrupaciones desfasables, se ha realizado un modelado exhaustivo y riguroso del efecto conjunto de los errores aleatorios y de cuantificación de cada desfasador de una agrupación, con el fin de aplicar, posteriormente, una ponderación no convencional a los pesos de los dígitos de cada desfasador y poder estimar sus prestaciones. El modelado del efecto de los errores, para los casos de ponderación binaria y no binaria, ha demostrado la capacidad de ésta última, para tolerar errores aleatorios en los dígitos del desfasador, ofreciendo unas mejores prestaciones que las soluciones conocidas. Además, esta tolerancia a los errores, es independiente del origen de los mismos, ya sean debidos al proceso de fabricación, a errores de diseño o a variaciones de la frecuencia de trabajo. En definitiva, se obtiene un desfasador más robusto. Como demostración práctica de este enfoque, se ha diseñado un desfasador de seis dígitos no binarios para una frecuencia de trabajo de $24 \mathrm{GHz}$. A pesar de los errores mostrados por algunos de los dígitos del desfasador, la utilización de ponderación no binaria ha permitido obtener unas prestaciones similares a las especificaciones realizadas. Se ha demostrado que una adecuada estimación estadística de los errores, junto con el conocimiento de la calibración que se va a realizar a posteriori, permite escoger la ponderación de cada dígito del desfasador de forma óptima, consiguiendo la resolución especificada con un margen suficiente frente a la presencia de errores.

La utilización de circuitos monolíticos como celdas básicas para la construcción de una red conformadora de haces para antenas multihaz, ha presentado dos retos. 
En primer lugar, la caracterización de un método sencillo, para el diseño del conformador a partir de las especificaciones de las distribuciones periódicas de los elementos de la agrupación y de las posiciones de los máximos de los haces a conformar. En segundo lugar, se debía comprobar la viabilidad de obtener los circuitos requeridos con un comportamiento adecuado, desde el punto de vista de los parámetros de radiación de la agrupación.

El primer objetivo, ha dado lugar a una recopilación completa de resultados teóricos relacionados con el diseño eficiente de redes conformadoras multihaz para agrupaciones lineales y planas, como extensión y generalización del concepto de matriz de Butler, estableciéndose criterios para identificar y diseñar aquellas redes conformadoras para antenas multihaz que son más adecuadas para su implementación mediante tecnología monolítica, gracias a una utilización exhaustiva de un mismo circuito básico sencillo. Tras la identificación de una celda básica $3 \times 3$, adecuada para su utilización como elemento constructor de conformadores de diferentes tamaños, se ha procedido a su diseño y fabricación. Se ha buscado una configuración, que, cumpliendo las especificaciones realizadas, presente una mayor simplicidad y un menor tamaño, con el fin de aumentar el rendimiento del circuito y reducir la variabilidad del mismo. A partir del análisis de esta estructura, se ha detectado la alta sensibilidad de su comportamiento a las tolerancias del proceso en cuanto a las capacidades de los condensadores. Con el fin de paliar este efecto, se modificó el diseño, introduciendo la posibilidad de sintonizar el circuito. Las medidas efectuadas sobre ambas celdas, confirman todas las consideraciones realizadas, demostrándose la utilidad del diseño sintonizable. Las celdas diseñadas tienen como objetivo su utilización en redes conformadoras de mayor tamaño, por lo que se han analizado también las prestaciones de estos conformadores basados en las celdas básicas, verificándose la aplicabilidad del nuevo concepto de red conformadora de haces de peso y volumen reducidos.

En esta tesis se ha presentado una novedosa estructura de línea de retardo de banda ultra-ancha, para la cual se han analizado diferentes esquemas de modulación ópticos. Además, se ha caracterizado la línea de retardo en cuanto a distorsión, intermodulación, ruido y margen dinámico; parámetros críticos a la hora de utilizar, en sistemas de comunicaciones o sistemas radar, este elemento como parte de una agrupación de antenas.

Esta línea de retardo se ha presentado como elemento básico de una red conformadora de haces altamente flexible, mediante la multiplexación y demultiplexación de tantas longitudes de onda como elementos o subagrupaciones presente la agrupación. Se han mostrado ecuaciones que permiten dimensionar la agrupación a partir de las características de los componentes o, en sentido inverso, establecer las especificaciones de los componentes a partir de las dimensiones de la agupación. 


\subsection{Contribuciones de la Tesis}

Dentro del trabajo realizado en la elaboración de esta Tesis, y de los resultados resumidos en la sección anterior, se pretende destacar a continuación, aquellos aportaciones realizadas por el autor. Brevemente:

- Modelado de los errores aleatorios, combinados con los de cuantificación, para desfasadores digitales, incluyendo el efecto de diferentes niveles de calibración.

- Aplicación del modelado anterior al análisis de la utilización de ponderación no binaria en los pesos de los dígitos de un desfasador digital variable. Estimación de los parámetros estadísticos del error total, así como del efecto de los mismos sobre el diagrama de radiación. Establecimiento del rango de viabilidad de la ponderación no binaria, en comparación con ponderación binaria convencional.

- Recopilación de algoritmos aplicables a la optimización de redes conformadoras que realicen la implementación analógica de la transformada discreta de Fourier para agrupaciones unidimensionales y bidimensionales. Obtención de criterios de eficiencia a la hora de diseñar el conformador.

- Demostración de la viabilidad de la utilización de circuitos monolíticos simples como celdas básicas de redes conformadoras de haces para antenas multihaz de banda estrecha, estableciendo las prestaciones y limitaciones de la misma.

- Caracterización completa de un nuevo tipo de línea de retardo continuamente variable utilizando redes de difracción con variación lineal del periodo.

- Introducción de una nueva arquitectura de red óptica conformadora de haces, basada en la utilización de una red de difracción con variación lineal del periodo, como elemento común de obtención de los retardos de los elementos.

\subsection{Líneas abiertas y trabajos de continuación}

El trabajo presentado en esta Tesis permite su continuación en diferentes direcciones, no sólo, en varias líneas de investigación, algunas de las cuales está actualmente en marcha, si no incluso, se puede hablar de aplicaciones inmediatas en diseños reales. En concreto:

\section{Tecnología monolítica}

- Extensión del modelado de los errores de fase, al caso de circuitos atenuadores o amplificadores, digitales, para el control de la amplitud de elementos de agrupaciones de antenas, y al caso conjunto, con errores de amplitud y fase. 
- Obtención de topología eficientes para las celdas básicas de tamaños pequeños, con especial incidencia sobre, la sensibilidad de los diseños a variaciones de los procesos, y la reducción de las pérdidas.

- Extender la matriz de Butler a agrupaciones conformadas sobre superficies simples, buscando criterios de eficiencia en cuanto a la modularidad y simplicidad de las interconexiones de los módulos.

\section{Tecnología fotónica}

- Medida y caracterización del diagrama de radiación de la nueva arquitectura de red conformadora de haces óptica.

- Estudio sobre la utilización de amplificación óptica en la red conformadora de haces. Compromiso entre amplificación óptica, preamplificación y postamplificación.

- Análisis de la sensibilidad de la arquitectura del conformador óptico a los errores introducidos por los elementos reales que la forman.

- Aplicación de la línea de retardo basada en redes de difracción con variación lineal del periodo a otras arquitecturas de conformadores ópticos. 


\section{Apéndice A}

\section{Análisis estadístico del efecto de los errores de iluminación de una agrupación de antenas}

\section{A.1 Caso general}

Sea el factor de la agrupación de una agrupación de antenas con N elementos:

$$
F_{o}(\theta, \phi)=F_{o}(\hat{r})=\sum_{n=1}^{N} A_{n} e^{j \alpha_{n}} e^{j k \vec{r}_{n} \hat{r}}
$$

donde $A_{n} e^{j \alpha_{n}}$ corresponde a la función de iluminación de los elementos de la agrupación.

Los errores en la iluminación de la agrupación pueden venir por tres motivos:

- Elementos fallidos. Probabilidad de que un elemento siga funcionando: $P$.

- Error en la amplitud de cada elemento: $A=A_{n}\left(1+\Delta_{n}\right)$.

- Error en la fase de cada elemento: $\alpha=\alpha_{n}+\delta_{n}$.

De esta forma, el factor de la agrupación, teniendo en cuenta estas tres fuentes de error pasaría a ser:

$$
F(\theta, \phi)=\sum_{n=1}^{N} p(n) A_{n}\left(1+\Delta_{n}\right) e^{j \delta_{n}} e^{j \alpha_{n}} e^{j k \hat{r} \vec{r}_{n}}
$$

donde $p(n)$ es una función aleatoria que toma el valor 1 con una probabilidad $P, \mathrm{y}$ 0 con una probabilidad $(1-P)$. 
Entendiendo una realización del proceso "factor de la agrupación", como el resultado de la ecuación (A.2) para una agrupación concreta; se pueden calcular ciertos estimadores estadísticos del citado proceso, teniendo en cuenta que los resultados obtenidos hacen referencia a un conjunto de muestras de diagramas de radiación de diferentes agrupaciones con las mismas distribuciones estadísticas en amplitud y fase y no a un conjunto de muestras de direcciones del espacio dentro de un único diagrama de radiación.

El valor cuadrático medio del factor de la agrupación, es decir el diagrama de radiación de potencia medio, será:

$$
\begin{aligned}
\overline{|F(\theta, \phi)|^{2}}= & \frac{F(\theta, \phi) \cdot F^{*}(\theta, \phi)=\overline{\left(\sum_{n=1}^{N} p(n) A_{n}\left(1+\Delta_{n}\right) e^{j \delta_{n}} e^{j \alpha_{n}} e^{j k \hat{r} \vec{r}_{n}}\right)} .}{\left(\sum_{m=1}^{N} p(m) A_{m}\left(1+\Delta_{m}\right) e^{-j \delta_{m}} e^{-j \alpha_{m}} e^{-j k \hat{r}_{m}}\right)}
\end{aligned}
$$

Tras agrupar los dos sumatorios, aplicar el teorema del límite central y separar los términos deterministas, se llega a:

$$
\begin{aligned}
& \overline{|F(\theta, \phi)|^{2}}= \\
& \quad \sum_{n=1}^{N} \sum_{m=1}^{N} \overline{p(n) p(m)\left(1+\Delta_{n}\right)\left(1+\Delta_{m}\right) e^{j\left(\delta_{n}-\delta_{m}\right)}} A_{n} A_{m} e^{j\left(\alpha_{n}-\alpha_{m}\right)} e^{j k \hat{r}\left(\vec{r}_{n}-\vec{r}_{m}\right)}
\end{aligned}
$$

El término $(T)=\overline{p(n) p(m)\left(1+\Delta_{n}\right)\left(1+\Delta_{m}\right) e^{j\left(\delta_{n}-\delta_{m}\right)}}$ de (A.4) toma distintos valores dependiendo de si los índices $n$ y $m$ son iguales o distintos:

$$
\begin{array}{ll}
(T)=\overline{p^{2}(n)} \cdot \overline{\left(1+\Delta_{n}\right)^{2}}=\overline{p^{2}(n)} \cdot\left(1+\overline{\Delta_{n}^{2}}+2 \overline{\Delta_{n}}\right) & \text { si } n=m \\
(T)=\overline{p(n)}^{2} \cdot\left(1+{\overline{\Delta_{n}}}^{2}\right) \cdot \overline{e^{j \delta_{n}}} \cdot \overline{e^{-j \delta_{n}}} & \text { si } n \neq m
\end{array}
$$

donde se ha considerado que las tres fuentes de error $p(n), \Delta_{n}$ y $\delta_{n}$ son procesos independientes entre sí y además también lo son tanto los errores de amplitud como los de fase de elementos de la agrupación diferentes. Además, se ha asumido que los estadísticos de los errores $\left(\overline{p(n)}, \overline{p(n)^{2}}, \overline{\Delta_{n}}, \overline{\Delta_{n}^{2}}, \overline{\Delta_{n}}, \overline{\delta_{n}^{2}}\right)$ son iguales para todos los elementos.

Dividiendo el sumatorio doble de (A.4) en dos términos y sustituyendo (A.5) y (A.6) se obtiene el siguiente resultado:

$$
\begin{aligned}
\overline{|F(\theta, \phi)|^{2}} & =P \cdot\left(1+\overline{\Delta^{2}}+2 \bar{\Delta}\right)\left(\sum_{n=1}^{N} A_{n}^{2}\right) \\
& +P^{2} \cdot\left(1+\bar{\Delta}^{2}\right) \cdot \overline{e^{j \delta}} \cdot \overline{e^{-j \delta}}\left(\sum_{n=1}^{N} \sum_{m=1, m \neq n}^{N} A_{n} A_{m} e^{j\left(\alpha_{n}-\alpha_{m}\right)} e^{j k \hat{r}\left(\vec{r}_{n}-\vec{r}_{m}\right)}\right)
\end{aligned}
$$


donde se ha tenido en cuenta que $\overline{p^{2}(n)}=P$ y $\overline{p(n)}=P$. Se puede observar que el término entre paréntesis del segundo sumando de (A.7) corresponde a la expresión del diagrama de radiación de potencia de (A.4) sin errores, es decir es el diagrama de radiación de potencia ideal $\left(\left|F_{o}(\theta, \phi)\right|^{2}\right)$, excepto por el hecho de que no incluye el término $n=m$. Tras sumar y restar simultáneamente ese término, el resultado final para el diagrama de radiación de potencia medio será:

$$
\begin{aligned}
& \overline{|F(\theta, \phi)|^{2}}=\left[P^{2} \cdot\left(1+\bar{\Delta}^{2}\right) \cdot \overline{e^{j \delta}} \cdot \overline{e^{-j \delta}}\right]\left|F_{o}(\theta, \phi)\right|^{2} \\
& \quad+\left[P \cdot\left(1+\overline{\Delta^{2}}+2 \bar{\Delta}\right)-P^{2} \cdot\left(1+\bar{\Delta}^{2}\right) \cdot \overline{e^{j \delta}} \cdot \overline{e^{-j \delta}}\right]\left(\sum_{n=1}^{N} A_{n}^{2}\right)
\end{aligned}
$$

Comentar que la ecuación (A.8) muestra que, al considerar los errores de iluminación, el diagrama de radiación medio es igual al diagrama de radiación ideal multiplicado por un cierto factor más un valor constante para cualquier dirección del espacio $(\theta, \phi)$. Este valor constante para todas las direcciones del espacio, corresponde a un valor residual de fondo que impide la obtención de niveles de lóbulos secundarios reducidos. Con el fin de obtener cual es el valor de nivel de lóbulo principal a secundario correspondiente a ese nivel residual de fondo, será necesario normalizar el diagrama de radiación de potencia dividiendo por su valor máximo. De (A.8) y utilizando la ecuación (2.9) se puede obtener el valor del diagrama en la dirección de máxima radiación como:

$$
\begin{aligned}
& \overline{\left|F\left(\theta_{o}, \phi_{o}\right)\right|^{2}}=P^{2} \cdot\left(1+\bar{\Delta}^{2}\right) \cdot \overline{e^{j \delta}} \cdot \overline{e^{-j \delta}}\left(\sum_{n=1}^{N} A_{n}\right)^{2} \\
& \quad+\left[P \cdot\left(1+\overline{\Delta^{2}}+2 \bar{\Delta}\right)-P^{2} \cdot\left(1+\bar{\Delta}^{2}\right) \cdot \overline{e^{j \delta}} \cdot \overline{e^{-j \delta}}\right]\left(\sum_{n=1}^{N} A_{n}^{2}\right)
\end{aligned}
$$

de los dos sumandos de la ecuación anterior, el primero es generalmente mucho mayor que el segundo por serlo el factor $\left(\sum A_{n}\right)^{2}$ respecto a $\left(\sum A_{n}^{2}\right)$, por lo tanto:

$$
\overline{\left|F\left(\theta_{o}, \phi_{o}\right)\right|^{2}} \approx P^{2} \cdot\left(1+\bar{\Delta}^{2}\right) \cdot \overline{e^{j \delta}} \cdot \overline{e^{-j \delta}}\left(\sum_{n=1}^{N} A_{n}\right)^{2}
$$

Utilizando (A.10) para normalizar (A.8) se obtiene:

$$
\begin{aligned}
\overline{\left|F_{N}(\theta, \phi)\right|^{2}} \approx\left|F_{o N}(\theta, \phi)\right|^{2} \\
+\frac{\left(1+\overline{\Delta^{2}}+2 \bar{\Delta}\right)-P \cdot\left(1+\bar{\Delta}^{2}\right) \cdot \overline{e^{j \delta}} \cdot \overline{e^{-j \delta}}}{P \cdot\left(1+\bar{\Delta}^{2}\right) \cdot \overline{e^{j \delta}} \cdot \overline{e^{-j \delta}}} \frac{\left(\sum_{n=1}^{N} A_{n}^{2}\right)}{\left(\sum_{n=1}^{N} A_{n}\right)^{2}}
\end{aligned}
$$

donde $\left|F_{o N}(\theta, \phi)\right|^{2}$ es el diagrama de radiación normalizado sin errores teniendo en cuenta el resultado de (2.9). 
De acuerdo con (A.11) el nivel de los lóbulos secundarios respecto al nivel del lóbulo principal (NLSP) será:

$$
\overline{\sigma^{2}} \approx \frac{\left(1+\overline{\Delta^{2}}+2 \bar{\Delta}\right)-P \cdot\left(1+\bar{\Delta}^{2}\right) \cdot \overline{e^{j \delta}} \cdot \overline{e^{-j \delta}}}{\eta N P \cdot\left(1+\bar{\Delta}^{2}\right) \cdot \overline{e^{j \delta}} \cdot \overline{e^{-j \delta}}}
$$

donde se ha introducido la eficiencia de iluminación de la agrupación $(\eta)$, definida como:

$$
\eta=\frac{\left(\sum_{n=1}^{N} A_{n}\right)^{2}}{N \sum_{n=1}^{N} A_{n}^{2}}
$$

Para una agrupación lineal de elementos sin pérdidas isotrópicos, separados entre sí media longitud de onda, se puede comprobar que la directividad de la agrupación vale [All63]:

$$
D_{o}=D_{o}\left(\theta_{o}, \phi_{o}\right)=\frac{\left|F_{o}\left(\theta_{o}, \phi_{o}\right)\right|^{2}}{P_{o}^{e n t}}=\frac{\left|F_{o}\left(\theta_{o}, \phi_{o}\right)\right|^{2}}{\sum_{n=1}^{N} A_{n}^{2}}
$$

donde $P_{o}^{e n t}$ es la potencia a la entrada de la agrupación.

$\mathrm{Al}$ existir elementos fallidos y errores en la amplitud de la alimentación, la potencia media a la entrada de la agrupación pasa a ser:

$$
\overline{P^{e n t}}=\overline{\sum_{n=1}^{N} p(n) A_{n}^{2}\left(1+\Delta_{n}\right)^{2}}=\sum_{n=1}^{N} \overline{p(n)} A_{n}^{2} \overline{\left(1+\Delta_{n}\right)^{2}}=\overline{P(1+\Delta)^{2}} \sum_{n=1}^{N} A_{n}^{2}
$$

por lo tanto aplicando (A.9):

$$
\begin{aligned}
\frac{D}{D_{o}}=\frac{P_{o}^{e n t}}{P^{e n t}} \frac{\left|F\left(\theta_{o}, \phi_{o}\right)\right|^{2}}{\left|F_{o}\left(\theta_{o}, \phi_{o}\right)\right|^{2}}=\frac{1}{P \overline{(1+\Delta)^{2}}}\left[P^{2} \cdot\left(1+\bar{\Delta}^{2}\right) \cdot \overline{e^{j \delta}} \cdot \overline{e^{-j \delta}}\right. \\
\left.+\left[P \cdot\left(1+\overline{\Delta^{2}}+2 \bar{\Delta}\right)-P^{2} \cdot\left(1+\bar{\Delta}^{2}\right) \cdot \overline{e^{j \delta}} \cdot \overline{e^{-j \delta}}\right] \frac{\left(\sum_{n=1}^{N} A_{n}^{2}\right)}{\left(\sum_{n=1}^{N} A_{n}\right)^{2}}\right]
\end{aligned}
$$

simplificando:

$$
\frac{D}{D_{o}}=\frac{P \cdot\left(1+\bar{\Delta}^{2}\right) \cdot \overline{e^{j \delta}} \cdot \overline{e^{-j \delta}}}{1+\overline{\Delta^{2}}+2 \bar{\Delta}}+\left(1-\frac{P \cdot\left(1+\bar{\Delta}^{2}\right) \cdot \overline{e^{j \delta}} \cdot \overline{e^{-j \delta}}}{1+\overline{\Delta^{2}}+2 \bar{\Delta}}\right) \frac{1}{N \eta}
$$


Para agrupaciones lineales con separación entre elementos iguales distinta de media longitud de onda, existen en la literatura expresiones exactas para el cálculo de la directividad distintas de la mostrada en (A.14) [Mai93a]. Aún así, el resultado (A.17) que permite calcular la reducción en directividad debida a los errores en la iluminación es una buena aproximación para cualquier agrupación, plana o lineal, sobre todo para agrupaciones con un gran número de elementos.

Para agrupaciones con un número de elementos suficientemente grande el segundo término de la ecuación anterior es despreciable, quedando:

$$
\frac{D}{D_{o}} \approx \frac{P \cdot\left(1+\bar{\Delta}^{2}\right) \cdot \overline{e^{j \delta}} \cdot \overline{e^{-j \delta}}}{1+\overline{\Delta^{2}}+2 \bar{\Delta}}
$$

\section{A.2 Expresiones particulares}

Las expresiones generales (A.12) y (A.17) se pueden simplificar una vez se conoce la estadística de los errores de fase y amplitud de la iluminación. Por ejemplo, en agrupaciones de antenas es habitual calibrar la red de distribución para que los errores de amplitud y fase tenga media nula $(\bar{\Delta}=0, \bar{\delta}=0)$, obteniéndose en ese caso:

$$
\begin{gathered}
\overline{\sigma^{2}} \approx \frac{\left(1+\overline{\Delta^{2}}\right)-P \cdot \overline{e^{j \delta}} \cdot \overline{e^{-j \delta}}}{\eta N P \cdot \overline{e^{j \delta}} \cdot \overline{e^{-j \delta}}} \\
\frac{D}{D_{o}} \approx \frac{P \cdot \overline{e^{j \delta}} \cdot \overline{e^{-j \delta}}}{1+\overline{\Delta^{2}}}
\end{gathered}
$$

Si además, los errores de fase siguen una distribución gausiana de varianza $\sigma^{2}=$ $\overline{\delta^{2}}$ se puede demostrar fácilmente que:

$$
\overline{e^{-j \delta}}=\overline{e^{j \delta}}=\frac{1}{\sqrt{2 \pi \overline{\delta^{2}}}} \int_{-\infty}^{\infty} e^{j \delta} e^{-\frac{\delta^{2}}{2 \bar{\delta}^{2}}} d x=\frac{2}{\sqrt{2 \pi \overline{\delta^{2}}}} \int_{0}^{\infty} \cos (\delta) e^{-\frac{\delta^{2}}{2 \bar{\delta}^{2}}} d x=e^{-\frac{\bar{\delta}^{2}}{2}}
$$

Por lo tanto, el nivel de los lóbulos secundarios residuales respecto al lóbulo principal $(N L S P)$ para una agrupación cuyos errores de amplitud y fase presenten una media nula, siendo la distribución que siguen los errores de fase una distribución gausiana, será:

$$
\overline{\sigma^{2}} \approx \frac{\left(1+\overline{\Delta^{2}}\right)-P \cdot e^{-\overline{\delta^{2}}}}{\eta N P \cdot e^{-\bar{\delta}^{2}}}=\frac{\left(1+\overline{\Delta^{2}}\right) e^{\overline{\delta^{2}}}-P}{\eta N P}
$$

y la variación en la directividad en la dirección de máxima radiación:

$$
\frac{D}{D_{o}}=\frac{P \cdot e^{-\overline{\delta^{2}}}}{1+\overline{\Delta^{2}}}
$$


Asumiendo que la varianza del error de fase es un número pequeño es posible aproximar el término exponencial $\left(e^{\overline{\delta^{2}}}\right)$ por su desarrollo en serie de Taylor alrededor de cero, de forma que se obtiene la expresión habitual en la literatura para el nivel de lóbulos secundarios residuales y para la reducción de la directividad o ganancia:

$$
\begin{gathered}
\overline{\sigma^{2}} \approx \frac{\left(1+\overline{\Delta^{2}}\right) e^{\overline{\delta^{2}}}-P}{\eta N P} \approx \frac{\left(1+\overline{\Delta^{2}}\right)\left(1+\overline{\delta^{2}}\right)-P}{\eta N P} \approx \frac{1-P+\overline{\Delta^{2}}+\overline{\delta^{2}}}{\eta N P} \\
\frac{D}{D_{o}} \approx \frac{P}{\left(1+\overline{\Delta^{2}}\right) \cdot e^{\overline{\delta^{2}}}} \approx \frac{P}{\left(1+\overline{\Delta^{2}}\right)\left(1+\overline{\delta^{2}}\right)} \approx \frac{P}{1+\overline{\Delta^{2}}+\overline{\delta^{2}}}
\end{gathered}
$$

\section{A.3 Errores de fase}

Para el caso particular en el que sólo existen errores de fase, la expresión del nivel de los lóbulos secundarios respecto al lóbulo principal $(N L S P)$ queda simplificado como:

$$
\overline{\sigma^{2}} \approx \frac{1-\overline{e^{j \delta}} \cdot \overline{e^{-j \delta}}}{\overline{e^{j \delta}} \cdot \overline{e^{-j \delta}}} \cdot \frac{1}{N \eta}
$$

y la directividad:

$$
\frac{D}{D_{o}}=\overline{e^{j \delta}} \cdot \overline{e^{-j \delta}}+\frac{1-\overline{e^{j \delta}} \cdot \overline{e^{-j \delta}}}{N \eta} \approx \overline{e^{j \delta}} \cdot \overline{e^{-j \delta}}
$$

Generalmente la distribución de los errores de fase sigue una estadística simétrica por lo que $\overline{e^{j \delta}}=\overline{e^{-j \delta}}$. Para el caso particular de distribución gausiana y teniendo en cuenta (A.21):

$$
\begin{gathered}
\overline{\sigma_{\text {GAUSIANA }}^{2}} \approx \frac{1-e^{-\overline{\delta^{2}}}}{e^{-\overline{\delta^{2}}}} \cdot \frac{1}{N \eta} \\
\frac{D_{G A U S I A N A}}{D_{o}}=e^{-\overline{\delta^{2}}}+\frac{1-e^{-\overline{\delta^{2}}}}{N \eta} \approx e^{-\overline{\delta^{2}}}
\end{gathered}
$$

Si los errores de fase siguieran una distribución uniforme entre $\left[-\delta_{Q},+\delta_{Q}\right]$, se cumplirá:

$$
\overline{e^{-j \delta}}=\overline{e^{j \delta}}=\int_{-\delta_{Q}}^{\delta_{Q}} e^{j \delta} \frac{1}{2 \delta_{Q}} d x=\frac{1}{2 \delta_{Q}}\left[\frac{e^{\delta_{Q}}-e^{-\delta_{Q}}}{j}\right]=\frac{\operatorname{sen}\left(\delta_{Q}\right)}{\delta_{Q}}=\operatorname{sinc}\left(\delta_{Q}\right)
$$

y por lo tanto:

$$
\overline{\sigma_{U N I F O R M E}^{2}} \approx \frac{1-\operatorname{sinc}^{2}\left(\delta_{Q}\right)}{\operatorname{sinc}^{2}\left(\delta_{Q}\right)} \cdot \frac{1}{N \eta}
$$




$$
\frac{D_{U N I F O R M E}}{D_{o}}=\operatorname{sinc}^{2}\left(\delta_{Q}\right)+\frac{1-\operatorname{sinc}^{2}\left(\delta_{Q}\right)}{N \eta} \approx \operatorname{sinc}^{2}\left(\delta_{Q}\right)
$$

En el caso de un error de fase que sea la suma de dos errores con estadísticas distintas $\delta=\delta_{1}+\delta_{2}$, siendo $\delta_{1}$ y $\delta_{2}$ variables aleatorias estadísticamente independientes, es fácil comprobar que:

$$
\overline{e^{j \delta}} \cdot \overline{e^{-j \delta}}=\overline{e^{j \delta_{1}} e^{j \delta_{2}}} \cdot \overline{e^{-j \delta_{1}} e^{-j \delta_{2}}}=\left(\overline{e^{-j \delta_{1}}} \cdot \overline{e^{j \delta_{1}}}\right) \cdot\left(\overline{e^{-j \delta_{2}}} \cdot \overline{e^{j \delta_{2}}}\right)
$$





\section{Apéndice B}

\section{Características del proceso D02AH}

Dentro de las rondas de diseños que habitualmente se organizan en la sección XRM de ESTEC, en el año 1994, se escogió el nuevo proceso D02AH de la compañia Philips Microwave Limeil (PML), todavía en periodo de pruebas, con el fin de contrastar sus prestaciones. Este proceso es un proceso Pseudomorphic High Electron Mobility Transistor (P-HEMT) con una longitud de puerta de $0.2 \mu$ m especialmente adecuado para aplicaciones en la banda de microondas y ondas milimétricas hasta $70 \mathrm{GHz}$.

Dentro de las tareas desarrolladas en el grupo de trabajo que se formó al efecto, algunas de ellas como el ajuste de los modelos de dispositivos activos (pequeña y gran señal) como la inclusión de los mismos en los programas habituales de diseño o la generación de macros para automatizar la generación de la distribución espacial de cada componente a partir de sus dimensiones, son cubiertas en otras publicaciones internas de la sección XRM [CG94, BB94]. Hay que tener resaltar que al tratarse de un proceso en pruebas en aquel momento, la realización de estas tareas conlleva una relación muy próxima con el personal de PML, de forma que el intercambio de modelos y macros dió lugar a que resultados obtenidos por autor fueran distribuidos a grupos de diseñadores de todo el mundo.

Las características principales (para una descripción detallada dirigirse al manual de diseño [PML94]) son:

- Hetero-epitaxia con una capa activa (GaInAs) pseudomórfica.

- PHEMT de deplexión con puerta hundida de anchura $0.2 \mu \mathrm{m}$ :

$$
\begin{aligned}
& -\mathrm{Vt}=-0.9 \mathrm{~V} \\
& -\mathrm{Ft}=62 \mathrm{GHz} \\
& -\mathrm{NF}=0.9 \mathrm{~dB} @ 12 \mathrm{GHz} \\
& - \text { Gass }=11.5 \mathrm{~dB}
\end{aligned}
$$

- Posibilidad de realizar huecos pasantes metalizados ${ }^{\mathrm{i}}$ a través del sustrato de

\footnotetext{
${ }^{\mathrm{i}}$ Via holes.
} 
$100 \mu \mathrm{m}$.

- Resistores de GaAs (195 $\Omega / \square)$.

- Protección completa $S i_{3} N_{4}$ asegurando una alta fiabilidad.

- $\mathrm{SiO}_{2} / \mathrm{Si}_{3} \mathrm{~N}_{4}$ aislamiento entre capas.

- Metalización $(0.03 \Omega / \square)$ gruesa $(1.25 \mu \mathrm{m})$ de oro para interconexiones e inductores en espiral.

- Amplio rango de relaciones C/S para los condensadores MIM (400 o 45 pF/mm²). 


\section{Apéndice $\mathrm{C}$}

\section{Elementos de conmutación a $24 \mathrm{GHz}$ en el proceso D02AH}

\section{C.1 Introducción}

A la hora de escoger como dispositivo de conmutación entre PHEMTs y diodos, se ha escogido los primeros debido principalmente a su bajo consumo y a la facilidad para aislar la señal de radiofrecuencia de las tensiones de alimentación en contínua.

\section{C.2 Conmutador serie}

Se han estudiado varios PHEMTs, con diferentes tamaños de puerta, números de dedos y carga de terminación de la puertai. En la tabla C.1 se muestra un resumen de los resultados.

Como comentario a la tabla C.1 se puede resaltar el mal comportamiento en cuanto a la relación (pérdidas de inserción/aislamiento) debido al bajo valor de la reactancia del conmutador en su estado $\mathrm{OFF}$, valor que viene definido principalmente por $C_{d s}$ (a $24 \mathrm{GHz}$ ), lo cual reduce considerablemente $Z_{O F F}$. La solución más habitual para este problema es la inserción de una inductancia $\left(L_{r e s}\right)$ entre drenador y fuente que resuene con $C_{d s}$, evitando de esa forma este efecto indeseado.

Tras aplicar esta técnica, se obtuvieron los resultados mostrados en las tres últimas columnas de C.1. La resonancia en el conmutador serie implica algunos beneficios para el estado OFF como un mejor aislamiento, lo cual implica a su vez una mejor desadaptación desde el punto de vista de la ' $T$ ' del SPDT. De todas formas, en la implementación real de estas inductancias se utilizará una línea de transmisión de alta impedancia, lo que probablemente modificará los resultados de la simulación

\footnotetext{
${ }^{\mathrm{i}} \mathrm{CC}$ : puerta cortocircuitada; CA: puerta en circuito abierto.
} 


\begin{tabular}{|c||r|r|r|r|r|}
\hline PHEMT & $\begin{array}{r}S_{11}(\mathrm{~dB}) \\
\text { ON } / \text { OFF }\end{array}$ & $\begin{array}{r}S_{21}(\mathrm{~dB}) \\
\text { ON } / \text { OFF }\end{array}$ & $\begin{array}{l}S_{11}^{\text {res }}(\mathrm{dB}) \\
\text { ON/OFF }\end{array}$ & $\begin{array}{r}S_{21}^{\text {res }}(\mathrm{dB}) \\
\text { ON/OFF }\end{array}$ & $\begin{array}{r}L_{\text {res }} \\
(\mathrm{nH})\end{array}$ \\
\hline \hline 4x15 CA & $13 / 1,5$ & $2,3 / 8,5$ & $13 / 0,9$ & $2,3 / 20,3$ & 1,53 \\
\hline 4x15 CC & $10 / 1,1$ & $3,2 / 14$ & $11 / 1$ & $3,1 / 22$ & 2,91 \\
\hline 2x60 CA & $17,5 / 2,7$ & $1,2 / 6$ & $17,5 / 1,6$ & $1,2 / 15,3$ & 1,02 \\
\hline 2x60 CC & $12 / 2,5$ & $3,8 / 11$ & $12,5 / 2,4$ & $3,8 / 21$ & 1,765 \\
\hline 2x120 CA & $23 / 5,5$ & $0,6 / 3,2$ & $23 / 3$ & $0,6 / 10,6$ & 0,54 \\
\hline 2x120 CC & $14 / 6$ & $3,4 / 6,9$ & $14 / 5,5$ & $3,4 / 20$ & 0,65 \\
\hline 4x90 CA & $26 / 8$ & $0,4 / 2$ & $26 / 4$ & $0,4 / 8,3$ & 0,35 \\
\hline 4x90 CC & $7,1 / 5,4$ & $5,7 / 8,2$ & $7,2 / 6,9$ & $5,7 / 20$ & 0,49 \\
\hline 6x75 CA & $28 / 9,5$ & $0,35 / 1,5$ & $28 / 5$ & $0,35 / 7,2$ & 0,28 \\
\hline 6x75 CC & $4,8 / 4,2$ & $7,4 / 9,2$ & $4,8 / 5,8$ & $7,3 / 9,1$ & 0,43 \\
\hline
\end{tabular}

Tabla C.1: Resultados para diferentes tamaños de conmutador en serie.

debido a las pérdidas y al comportamiento no totalmente inductivo de este elemento. Relacionado con esto, el valor de la inductancia debe ser lo suficientemente bajo como para ser implementado fácilmente con una línea de transmisión equivalente; en concreto, se cumple que una línea de transmisión de impedancia $Z_{o}$, constante de fase $\beta$ y longitud $\ell$ presenta una inductancia equivalente:

$$
L(\mathrm{nH}) \approx \frac{Z_{o} \cdot \operatorname{sen}(\beta \ell)}{2 \pi \cdot f(\mathrm{GHz})} \leq \frac{Z_{o}}{2 \pi \cdot 24} \approx \frac{Z_{o}}{150}
$$

por lo tanto, para valores habituales de $Z_{o}$, la inductancia estará por debajo de $1 \mathrm{nH}$.

Los conmutadores con la puerta cortocircuitada muestran un peor comportamiento debido a las resistencia y reactancia extra añadida por los elementos del modelo del conmutador situados entre drenador y puerta y entre fuente y puerta.

La elección de un conmutador adecuado depende del compromiso entre las bajas pérdidas de inserción de los transistores anchos de puerta y el alto aislamiento de los que tienen un valor bajo de anchura de puerta.

Ambos, el 4x90 CA y el 2x120 CA, muestran un valor muy bueno de pérdidas de inserción, pero sus propiedades de aislamiento impiden su utilización como conmutador.

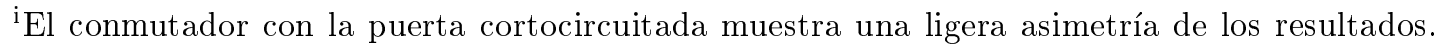
De todas formas, estas pequeñas diferencias no se muestran en este apéndice, ya que no son importantes a la hora de seleccionar el tamaño del conmutador. 


\begin{tabular}{|c||r|r|r|r|r|}
\hline PHEMT & $\begin{array}{r}S_{11}(\mathrm{~dB}) \\
\text { ON/OFF }\end{array}$ & $\begin{array}{r}S_{21}(\mathrm{~dB}) \\
\text { ON/OFF }\end{array}$ & $\begin{array}{l}S_{11}^{\text {res }}(\mathrm{dB}) \\
\text { ON/OFF }\end{array}$ & $\begin{array}{c}S_{21}^{\text {res }}(\mathrm{dB}) \\
\text { ON/OFF }\end{array}$ & $\begin{array}{r}L_{\text {res }} \\
(\mathrm{nH})\end{array}$ \\
\hline \hline 4x15 CA & $19,4 / 6,9$ & $0,3 / 5,2$ & $30 / 6,9$ & $0,23 / 5,2$ & 1,33 \\
\hline $4 \times 15 \mathrm{CC}$ & $16,6 / 6,9$ & $0,3 / 5,2$ & $30 / 6,9$ & $0,26 / 5,2$ & 1,1 \\
\hline 2x60 CA & $15,9 / 4,2$ & $0,5 / 8,4$ & $26 / 4,2$ & $0,4 / 8,4$ & 1 \\
\hline $2 \times 60 \mathrm{CC}$ & $12,9 / 4$ & $0,9 / 8,5$ & $20 / 4$ & $0,7 / 8,5$ & 0,5 \\
\hline $2 \times 120 \mathrm{CA}$ & $11 / 2,3$ & $1,2 / 12,6$ & $20,5 / 2,3$ & $0,8 / 12,6$ & 0,53 \\
\hline $2 \times 120 \mathrm{CC}$ & $9,6 / 2,3$ & $1,8 / 12,7$ & $16,2 / 2,3$ & $1,4 / 12,7$ & 0,45 \\
\hline $4 \times 90 \mathrm{CA}$ & $8 / 1,6$ & $1,9 / 15,4$ & $17,4 / 1,6$ & $1,3 / 15,4$ & 0,35 \\
\hline $4 \times 90 \mathrm{CC}$ & $6,4 / 1,6$ & $3 / 15,6$ & $13,3 / 1,6$ & $2,1 / 15,6$ & 0,265 \\
\hline $6 \times 75 \mathrm{CA}$ & $6,3 / 1,3$ & $2,5 / 17$ & $15,7 / 1,3$ & $1,5 / 17$ & 0,275 \\
\hline $6 \times 75 \mathrm{CC}$ & $4,8 / 1,3$ & $3,9 / 17$ & $12,2 / 1,3$ & $2,4 / 17$ & 0,198 \\
\hline
\end{tabular}

Tabla C.2: Resultados para diferentes tamaños de conmutador paralelo.

\section{C.3 Conmutador paralelo}

Por otra parte, se ha estudiado también la configuración paralelo para conmutadores con las mismas características que para el caso serie; mostrándose los resultados en la tabla C.2.

El conmutador paralelo no se suele utilizar en solitario, ya que para diseñar un SPDT, se necesitan dos líneas de cuarto de onda entre la ' $\mathrm{T}$ ' de bifurcación y los dos conmutadores.

La introducción de la inductancia resonante, implica en el caso del conmutador paralelo un mejor aislamiento y una mejor adaptación en el estado ON.

\section{C.4 Conmutador serie/paralelo}

Con el fin de mejorar la relación (pérdidas de inserción/aislamiento) del conmutador, se ha estudiado una configuración serie/paralelo:

Todos los PHEMTs utilizados, trabajan con la puerta en circuito abierto, debido a las mejores prestaciones mostradas en los datos anteriores.

Por ahora, el principal problema de este conmutador es el todavía alto valor de los parámetros de adaptación $S_{11}$ y $S_{22}$ en el estado OFF (columnas 2 y 3 de la tabla C.3), pues esto implica que al introducir estos conmutadores en un SPDT, el efecto de carga de la rama OFF sobre la ' $\mathrm{T}$ ' de bifurcación no es en absoluto el esperado circuito abierto (realmente, una carga de alta impedancia). Y este problema es el más importante en el diseño de un SPDT. 


\begin{tabular}{|c||c|c|c|c|c|}
\hline PHEMTs & $\begin{array}{c}S_{11}(\mathrm{~dB}) \\
\text { ON/OFF }\end{array}$ & $\begin{array}{c}S_{22}(\mathrm{~dB}) \\
\mathrm{ON} / \mathrm{OFF}\end{array}$ & $\begin{array}{c}S_{21}(\mathrm{~dB}) \\
\mathrm{ON} / \mathrm{OFF}\end{array}$ & $\begin{array}{c}L_{\text {res }}^{\text {ser }} \\
(\mathrm{nH})\end{array}$ & $\begin{array}{c}L_{\text {res }}^{\text {par }} \\
(\mathrm{nH})\end{array}$ \\
\hline \hline $4 \times 504 \times 15$ & $19,7 / 3,6$ & $17,9 / 9,2$ & $1,07 / 10,4$ & - & - \\
\hline $4 \times 504 \times 15$ & $20,4 / 2,9$ & $18,3 / 11$ & $1,06 / 19,5$ & 0,597 & - \\
\hline $4 \times 504 \times 15$ & $24 / 3,7$ & $25,4 / 9,7$ & $1,01 / 10,3$ & - & 1,5 \\
\hline $4 \times 504 \times 15$ & $24 / 2,9$ & $25,4 / 11$ & $1,01 / 19,5$ & 0,597 & 1,5 \\
\hline $2 \times 1204 \times 15$ & $20,1 / 4,1$ & $18,3 / 9$ & $0,94 / 10$ & - & - \\
\hline $2 \times 1204 \times 15$ & $20,7 / 3,4$ & $18,7 / 10,8$ & $0,94 / 18,2$ & 0,54 & - \\
\hline $2 \times 1204 \times 15$ & $26,2 / 4,2$ & $27,6 / 9,5$ & $0,88 / 9,9$ & - & 1,5 \\
\hline $2 \times 1204 \times 15$ & $26,2 / 3,4$ & $27,6 / 10,8$ & $0,88 / 18,2$ & 0,54 & 1,5 \\
\hline $4 \times 904 \times 15$ & $20,5 / 5,65$ & $18,9 / 8,27$ & $0,73 / 8,2$ & - & - \\
\hline $4 \times 904 \times 15$ & $20,9 / 4,97$ & $19,2 / 10,3$ & $0,73 / 15,6$ & 0,353 & - \\
\hline $4 \times 904 \times 15$ & $32 / 5,8$ & $34 / 8,7$ & $0,67 / 8,15$ & - & 1,5 \\
\hline $4 \times 904 \times 15$ & $32 / 4,97$ & $34 / 10,3$ & $0,67 / 15,6$ & 0,353 & 1,5 \\
\hline $6 \times 754 \times 15$ & $20,4 / 6,4$ & $19,1 / 7,95$ & $0,64 / 7,5$ & - & - \\
\hline $6 \times 754 \times 15$ & $20,8 / 6,1$ & $19,4 / 10$ & $0,64 / 14,3$ & 0,278 & - \\
\hline $6 \times 754 \times 15$ & $36,3 / 6,6$ & $39 / 8,3$ & $0,59 / 7,4$ & - & 1,5 \\
\hline $6 \times 754 \times 15$ & $36 / 6,1$ & $38 / 10$ & $0,59 / 14,3$ & 0,278 & 1,5 \\
\hline
\end{tabular}

Tabla C.3: Resultados para diferentes tamaños de conmutador serie/paralelo. 


\begin{tabular}{|r||c|c|c|c|c|c|c|c|}
\hline PHEMT & $\begin{array}{c}S_{11} \\
(\mathrm{~dB})\end{array}$ & $\begin{array}{c}S_{22} \\
(\mathrm{~dB})\end{array}$ & $\begin{array}{c}S_{33} \\
(\mathrm{~dB})\end{array}$ & $\begin{array}{c}S_{21}(\mathrm{~dB}) \\
\mathrm{ON} / \mathrm{OFF}\end{array}$ & $\begin{array}{c}S_{11}^{\text {res }} \\
(\mathrm{dB})\end{array}$ & $\begin{array}{c}S_{22}^{\text {res }} \\
(\mathrm{dB})\end{array}$ & $\begin{array}{c}S_{33}^{\text {res }} \\
(\mathrm{dB})\end{array}$ & $\begin{array}{c}S_{21}^{\text {res }} \\
\text { ON } / \mathrm{OFF}\end{array}$ \\
\hline \hline Se 4x15 & 13,7 & 13,3 & 1,3 & $2,8 / 12,4$ & 14,1 & 13,1 & 0,9 & $2,6 / 24,3$ \\
\hline Se 2x120 & 15 & 18 & 4,7 & $2,2 / 7,4$ & 34,3 & 45,8 & 3,3 & $1,4 / 15,5$ \\
\hline Se $6 \times 75$ & 12,2 & 13,8 & 7,7 & $2,7 / 5,3$ & 20,6 & 22,7 & 5,8 & $1,5 / 11,9$ \\
\hline Par 4x90 & 10 & 7,8 & 1,67 & $2,6 / 19,1$ & 23,5 & 20,2 & 1,7 & $1,9 / 20$ \\
\hline Par 2x120 & 13,6 & 10,7 & 2,5 & $2 / 16,9$ & 38,9 & 28 & 2,5 & $1,65 / 17$ \\
\hline $\begin{array}{r}\text { Se 4x90 } \\
\text { Par 4x15 }\end{array}$ & 8,6 & 9,6 & 7,7 & $3,1 / 12,2$ & 19,7 & 21,5 & 10,1 & $1,8 / 20,5$ \\
\hline $\begin{array}{r}\text { Se 4x50 } \\
\text { Par 4x15 }\end{array}$ & 10,8 & 12 & 9 & $2,5 / 14,8$ & 32,8 & 40,8 & 10,9 & $1,7 / 24$ \\
\hline $\begin{array}{r}\text { Se 2x120 } \\
\text { Par 4x15 }\end{array}$ & 10,4 & 11,5 & 8,7 & $2,5 / 14,3$ & 26,5 & 30,3 & 10,7 & $1,7 / 23$ \\
\hline
\end{tabular}

Tabla C.4: Resumen de resultados de simulación para diversas configuraciones de conmutadores SPDT.

\section{C.5 SPDT}

Para escoger la mejor configuración para un SPDT, se debe tener en cuenta el compromiso que existe entre conseguir unas pérdidas de inserción reducidas para el camino ON y un alto aislamiento para el camino OFF, pues un alto aislamiento implica una mala adaptación, de forma que el efecto de carga del camino OFF degrada las prestaciones del conmutador, tal y como se puede apreciar en la tabla C.4. 



\section{Bibliografía}

[Ack97] E.I. Ackerman and A.S. Daryoush. Broad-Band external modulation FiberOptic links for Antenna-Remoting applications. IEEE Transactions on Microwave Theory and Techniques, 45(8):1436-1442, August 1997.

[Ala95] K.E. Alameh, R.A. Minasian, and N. Fourikis. High capacity optical interconnects for phased array beamformers. IEEE Journal of Lightwave Technology, 13(6):1116-1120, 1995.

[All63] J.L. Allen. The theory of array antennas. M.I.T. Lincoln Lab Tech. Rep. 323, ESA/ESTEC, 1963.

[And88] L.P. Anderson, F. Boldissar, and D.C.D. Chang. Antenna beamforming using optical processing. In Proceedings SPIE Vol. 886 (Optoeletronic Signal Processing for Phased-Array Antennas), pages 228-232, 1988.

[Ass69] R.N. Assaly and L.J. Ricardi. A theoretical study of a multielement scanning feed system for a parabolic cylinder. IRE Transactions on Antennas and Propagation, 17:601-605, 1969.

[Aul60] W.H. Von Aulock. Properties of phased array. IRE Transactions on Antennas and Propagation, 9:1715-1727, 1960.

[Aya82] Y. Ayasli. Microwave switching with GaAs FETs. Microwave Journal, 25(11):61-74, November 1982.

[Bah88] Bahl. Microwave Solid-State Circuit Design. John Wiley \& Sons, Inc., 1988.

[Bal94] G.A. Ball, W.H. Glenn, and W.W. Morey. Programmable fiber optic delay line. IEEE Photonics Technology Letters, 6(6):741-743, June 1994.

[BB94] A. Betti-Berutto. D02AH passive components manual, macros \& electrical models. XRM internal report, ESA/ESTEC, 1994.

[Ber91] T. Berceli, W.D. Jemison, P.R. Herczfeld, A.S. Daryoush, and A. Paolella. A double-stage injection-locked oscillator for optically fed phased array antennas. IEEE Transactions on Microwave Theory and Techniques, 39(2):201-208, February 1991. 
[Bha86] K.B. Bhasin and D.J. Connolly. Advances in gallium arsenide monolithic microwave Integrated-Circuit technology for space communications systems. IEEE Transactions on Microwave Theory and Techniques, 34(10):994-1001, October 1986.

[Bla60] J. Blass. The multidirectional antenna: A new approach to stacked beams. IRE International Convention Record, 8(1):48-50, 1960.

[Bon95] Y. Bonnaire. Multilayer structure for BFN, June 1995. Dassault Electronique Fax Document N ${ }^{\circ} 592$.

[Bro91] E. Brookner. Practical Phased-Array Antenna Systems, chapter 3 Antenna Array Fundamentals, pages 3-25. The Artech House Antenna Library. Artech House, 1991.

[Bro97a] E. Brookner. Major advances in phased arrays: Part I. Microwave Journal, 40(5):288-294, May 1997.

[Bro97b] E. Brookner. Major advances in phased arrays: Part II. Microwave Journal, 40(6):84-92, June 1997.

[Buc72] G.J. Buck. Quantization and reflection lobe dispersion. In A.A. Oliner and G.H. Knittel, editors, Phased Array Antennas, pages 222-229. Artech House, 1972. Proceedings of the 1970 Phased Array Antenna Symposium.

[Buc94] B. J. Buck. Electrical apparatus with digitally set transfer function. Unites States Patent 5.327.107, July 1994.

[But61] J. Butler and R. Howe. Beamforming matrix simplifies design of electronically scanned antennas. Electronic Design, 9:170-173, April 121961.

[Car73] K.R. Carver, W.K. Cooper, and W.L. Stutzman. Beam pointing errors of planar phased arrays. IEEE Transactions on Antennas and Propagation, 21(3):199-202, March 1973.

[CG94] J.L. Corral González. D02AH large signal models. XRM Internal Report XRM/122.94/JLC, ESA/ESTEC, 1994.

[CG95a] J.L. Corral González. Design of $24 \mathrm{GHz}$ phase shifter. XRM Internal Report XRM/085.95/JLC, ESA/ESTEC, May 1995.

[CG95b] J.L. Corral González. Design of $24 \mathrm{GHz}$ phase shifter (RFOW measurements addendum). XRM Internal Report XRM/085.95/JLC, ESA/ESTEC, September 1995.

[CG95c] J.L. Corral González. Software for beamforming network analysis. XRM Internal Report XRM/159.95/JLC, ESA/ESTEC, September 1995. 
[CG97a] J.L. Corral González. Distorsión inducida por la dispersión. Modulación directa y externa. LCO internal report, Departament de Comunicacions. Universitat Politècnica de València, Septiembre 1997.

[CG97b] J.L. Corral González. Modulador Mach-Zehnder con electrodo dual. LCO internal report, Departament de Comunicacions. Universitat Politècnica de València, Junio 1997.

[Cha96] Y. Chang, H.R. Fetterman, D.A. Cohen, A.F.J. Levi, and I.L. Newberg. Optically-controlled serially-fed phased array transmitter. In Proceedings of the International Topical Meeting on Microwave Photonics, pages 201204, WE3-3, Kyoto, Japan, December 1996.

[Cim90] L.J. Cimini, Jr., L.J. Greenstein, and A.A.M. Saleh. Optical equalization to combat the effect of laser chirp and fiber dispersion. IEEE Journal of Lightwave Technology, 8(5):649-659, May 1990.

[Coh94] E.D. Cohen. Active electronically scanned arrays. In 1994 IEEE MTTSymposium Digest, pages 1323-1326, TH1A-1, 1994.

[Col96] M.J. Cole, H. Geiger, R.I. Laming, S.Y. Set, M.N. Zervas, W.H. Loh, and V. Gusmeroli. Continuously chirped, broadband dispersion-compensating fibre gratings in a 10 gbits/s $110 \mathrm{~km}$ standard fibre link". In 22nd European Conf. Optical Comm., pages 5.19-5-22,ThB.3.5, 1996.

[Cor96] F. Coromina, J. Ventura-Traveset, J.L. Corral, Y. Bonnaire, and A. Dravet. New multibeam beamforming networks for phased array antennas using advanced MMCM technology. In 1996 IEEE MTT-Symposium Digest, pages 79-82, TU1D-3, San Francisco, CA, USA, June 17-21 1996.

[Cor97a] J.L. Corral, J. Martí, J.M. Fuster, and R.I. Laming. True Time-Delay scheme for feeding optically controlled Phased-Array antennas using ChirpedFiber gratings. IEEE Photonics Technology Letters, 9(11):1529-1531, November 1997.

[Cor97b] J.L. Corral, J. Martí, S. Regidor, J.M. Fuster, R. Laming, and M. J. Cole. Continuously variable true Time-Delay optical feeder for Phased-Array antenna employing chirped fiber gratings. IEEE Transactions on Microwave Theory and Techniques/Journal of Lightwave Technology-Special Issue on Microwave Photonics, 45(8):1531-1536, August 1997.

[Cor98] J.L. Corral, J. Martí, J.M. Fuster, and R.I. Laming. Dispersion-Induced bandwidth limitation of variable time delay lines based on linearly chirped fibre gratings. Electronics Letters, 34(2):209-211, January 1998.

[Cox97] C.H. Cox, III, E. Ackerman, R. Helkey, and G.E. Betts. Techniques and performance of Intensity-Modulation Direct-Detection analog optical links. IEEE Transactions on Microwave Theory and Techniques, 45(8):13751383, August 1997. 
[Cro77] P.S. Cross and H. Kogelnik. Sidelobe suppression in Corrugated-Waveguide filters. Optics Letters, 1(1):43-45, July 1977.

[Cru97] J.L. Cruz, B. Ortega, M.V. Andrés, B. Gimeno, D. Pastor, J. Capmany, and L. Dong. Chirped fibre Bragg gratings for phased-array antennas. Electronics Letters, 33(7):545-546, March 1997.

[CSE] CSELT. Design of wideband Butler matrixes. CSELT Internal Report.

[Dar86] A. Daryoush, P. Herczfeld, V. Contarino, A. Rosen, Z. Turski, and P. Wahi. Optical beam control of millimeter wave phased array antennas for communication. In 16th European Microwave Conference Proceedings, pages 864-869, Dublin, Ireland, 1986.

[Dar87a] A.S. Daryoush, R. Glatz, P.R. Herczfeld, and M.P. Baccarini. Ku-band $(14 \mathrm{GHz})$ fiber optic communication link for distributed antennas in the Space Station. In Proceedings SPIE Vol. 756 (Optical Technologies for Space Communication Systems, Los Angeles, CA), pages 173-182, January 1987.

[Dar87b] A.S. Daryoush, P.R. Herczfeld, R. Glatz, and A.P.S. Khanna. Phase and frequency coherency of multiple optically synchronized $20 \mathrm{GHz}$ FET oscillators for satellite communications. In 1987 IEEE MTT-Symposium Digest, pages 823-826, W-4, Las Vegas, NV, 1987.

[Dev91] L.M. Devlin, B.J. Buck, and C.G. Eddison. An MMIC based beam forming module for active phased arrays. In Proceedings of an ESA Workshop on Advanced Beamforming Networks for Space Applications, pages 5.3.1-5.3.7, November 1991.

[Dev93] L.M Devlin, B.J. Buck, D. Leistner, W. Huber, and G. Braun. Technical evaluation of beam forming network for adaptive phased array. (Final Report) ESTEC-Contract 8762/90/NL/US, ESA/ESTEC, 1993.

[Dub92] F. Dubos and C. Mavrocordatos. Rain radar follow on feasibility study. Study Report ESTEC order 114054, ESA/ESTEC, April 1992.

[DuF85] E.C. DuFort. Optimum low sidelobe high crossover multiple beam antennas. IEEE Transactions on Antennas and Propagation, 33(9):946-954, September 1985.

[DuF92] E.C. DuFort. Optimum networks for simultaneous multiple beam antennas. IEEE Transactions on Antennas and Propagation, 40(1):1-7, January 1992.

[ESA94] ESA/REF/335. Low mass RF FFT Beam-Forming network. European Space Agency Patent, 1994. 
[ESA95] ESA. ESA at TELECOM'95, Geneva, 3-11 october 1995. http://www.esrin.esa.it/htdocs/tidc/Press/Press95/press37.html, 22 September 1995. ESA Press Release [N 37-95] - Paris.

[Esm93] R.D. Esman, M.Y. Frankel, J.L. Dexter, L. Goldberg, M.G. Parent, D. Stilwell, and D.G. Cooper. Fibre-optic prism true time-delay antenna feed. IEEE Photonics Technology Letters, 5(11):1347-1349, November 1993.

[Esp94] Alcatel Espace. Archimedes phase a study. Task 4 Final Report-wp40000 ATES 043517 STB 0340, ESA/ESTEC, November 1994.

[Far66] G.F. Farrell and D.H. Kuhn. Mutual coupling effects of triangular grid arryas by modal analysis. IEEE Transactions on Antennas and Propagation, 14:652-654, 1966.

[Fra72] J. Frank. Bandwidth criteria for phased array antennas. In A.A. Oliner and G.H. Knittel, editors, Phased Array Antennas, pages 243-253. Artech House, 1972. Proceedings of the 1970 Phased Array Antenna Symposium.

[Fra95] M.Y. Frankel and R.D. Esman. True time-delay fiber-optic control of an ultrawideband array transmitter/receiver with multibeam capability. IEEE Transactions on Microwave Theory and Techniques, 43(9):2387-2394, September 1995 .

[Fra96] M.Y. Frankel, P.J. Matthews, and R.D. Esman. Two-dimensional fiberoptic control of a true time-steered array transmitter. In 1996 IEEE MTTSymposium Digest, pages 1577-1580, TH3E-2, San Francisco, USA, 1996.

[Fuj93] Y. Fujii. Compact High-Isolation Polarization-Independent optical circulator. Optics Letters, 18(3):250-252, February 11993.

[Fus97] J.M. Fuster, J. Marti, and J.L. Corral. Chromatic dispersion effects in Electro-Optical upconverted Millimetre-Wave fibre optic links. Electronics Letters, 33(23):1969-1970, 6th November 1997.

[Gab76] W.F. Gabriel. Adaptive arrays - an introduction. Proceedings of the IEEE, 64(2):239-272, February 1976.

[Gal95] K. P. Galligan, R. Viola, and J.I. González-Núñez. ARCHIMEDES: Satellite DAB using Multi-Regional highly inclined orbits. ESA internal report, ESA/ESTEC, 1995.

[Gal97] J.T. Gallo and R. DeSalvo. Experimental demonstration of optical GuidedWave butler matrices. IEEE Transactions on Microwave Theory and Techniques, 45(8):1501-1507, August 1997.

[Gar89] G.J. Gardiner, M.W. Geen, and D.C. Smith. Design techniques for GaAs MESFET switches. In 1989 IEEE MTT-Symposium Digest, pages 405-408, L-17, 1989. 
[Gas78] R.A. Gaspari and H.H. Yee. Microwave GaAs FET switching. In 1978 IEEE MTT-Symposium Digest, pages 58-60, 1978.

[Gat95] G. Gatti. Design guidelines for microwave monolithic integrated circuits. XRM Internal Report XRM/017.95/GG, ESA/ESTEC, 1995. Issue 3, rev 0 .

[Gol92] L. Goldberg, R.D. Esman, and K.J. Williams. Generation and control of microwave signals by optical techniques. IEE Proceedings-J, 139(4):288295, August 1992.

[Gop85] A. Gopinath and J.B. Rankin. GaAs FET RF switches. IEEE Transactions on Electron Devices, ED-32(7):1272-1278, July 1985.

[Gou92] A.P. Goutzoulis, D.K. Davies, and J.M. Zomp. Hybrid electronic fiber optic wavelength-multiplexed system for true time-delay steering of phased array antennas. Optical Engineering, 31(11):2312-2322, November 1992.

[Gri83] J.W.R. Griffiths. Adaptive array processing: A tutorial. Proceedings IEE $H$ and $F, 130(1)$, February 1983.

[Gue86] A. Guessoum and R.M. Mersereau. Fast algorithms for the multidimensional discrete fourier transform. IEEE Transactions on Acoustics, Speech, and Signal Processing, 34(4):937-942, August 1986.

[Gup90] R. Gupta, L. Holdeman, H. Carlson, J. Potukuchi, K. Hogan, and K. Pande. Manufacturing technology and yield studies for MMIC 5-bit digital attenuators and Phase-Shifters. In 1990 IEEE GaAs IC Symposium, pages 305-308, New Orleans, USA, 1990.

[Gup91] R. Gupta, T. Hampsch, H. Gerson, A. Zaghloul, R. Sorbello, and F. Assal. Beam-Forming matrix design using MMICs for a Multi-Beam PhasedArray antenna. In 1991 IEEE GaAs IC Symposium, pages 41-44, 1991.

[Hal90] P.S. Hall and S.J. Vetterlein. Review of radio frequency beamforming techniques for scanned and multiple beam antennas. IEE Proceedings Pt. H, 137(5):293-303, October 1990.

[Har93] R.F. Harrington. Field Computation by Moment Methods. IEEE Press Series on Electromagnetic Waves. IEEE Press, 1993.

[Hau84] Hermann A. Haus. Waves and Fields in Optoelectronics. Prentice-Hall Series in Solid State Physical Electronics. Prentice-Hall, 1984.

[Hay87] S.G. Hay. Subreflector shaping to improve Multiple-Beam performance of a cassegrain antenna. Electronics Letters, 23(15):789-791, 1987. 
[Her86] P.R. Herczfeld and A.S. Daryoush. Integrated optic components for optically controlled phased array antenna-system considerations. In Proceedings SPIE Vol. (Integrated Optical Circuit Engineering IV, Cambridge, MA, USA), pages 174-181, September 1986.

[Her87] P.R. Herczfeld and M. Kam. System architecture of MMIC-based large aperture arrays for space applications. In Proceedings SPIE Vol. 756 (Optical Technologies for Space Communication Systems, Los Angeles, CA), pages 164-172, January 1987.

[Her88a] P.R. Herczfeld and A.S. Daryoush. Recent developments related to an optically controlled microwave phased array antenna. In Proceedings SPIE Vol. 996 (High Data Rate Atmospheric and Space Communications, Boston, MA), pages 108-115, 1988.

[Her88b] P.R. Herczfeld and A. Paolella. Optical gain control of a gaas mmic transmit-receive module compatible with optical spatial filters. In IEEE AP-S International Symposium, June 1988.

[Hil93] K.O. Hill, B. Malo, F. Bilodeau, D.C. Johnson, and J. Albert. Bragg gratings fabricated in monomode photosensitive optical fiber by UV exposure through a phase mask. Applied Physics Letters, 62(10):1035-1037, 1993.

[Hof95] R. Hofstetter, H. Schmuck, and R. Heidemann. Dispersion effects in optical Millimeter-Wave systems using Self-Heterodyne method for transport and generation. IEEE Transactions on Microwave Theory and Techniques, 43(9):2263-2269, September 1995.

[Hsi85] J.K. Hsiao. Design of error tolerance of a phased array. Electronics Letters, 21(19):834-836, September 12th 1985.

[Ih90] C.S. Ih and W. Gu. Fiber induced distortions in a subcarrier multiplexed lightwave system. IEEE Journal on Selected Areas in Communications, 8(7):1296-1303, September 1990.

[Ima92] Y. Imai, E. Sano, and K. Asai. Design and performance of wideband gaas mmics for high-speed optical communication systems. IEEE Transactions on Microwave Theory and Techniques, 40(2):185-190, February 1992.

[Jaz63] A.S. Jazi. Wide angle microwave lens for line source applications. IEEE Transactions on Antennas and Propagation, 11:623-632, 1963.

[Jes87] N.V. Jespersen, A.C. Heath, and E.S. Roller. Optical control considerations for phased array antennas. In Proceedings SPIE Vol. 756 (Optical Technologies for Space Communication Systems, Los Angeles, CA), pages 156-163, January 1987. 
[Joh93] S.T. Johns, D.A. Norton, C.W. Keefer, R. Erdmann, and R.A. Soref. Variable time delay of microwave signals using high dispersion fibre. Electronics Letters, 29(6):555-556, March 1993.

[Kah62] W.H. Kahn and H. Kurss. The uniqueness of the lossless feed network for a multibeam array. IRE Transactions on Antennas and Propagation, 10:100-101, January 1962.

[Kap86] P.D. Kaplan. Predicting antenna sidelobe performance. Microwave Journal, 29(9):201-206, September 1986.

[Koe84] G.A. Koepf. Optical processor for phased array antenna beam formation. In Proceedings SPIE Vol. $4^{\text {ry }}$ (Optical Technologies for Microwave Applications, Washington, DC), pages 75-81, May 1984.

[Kog76] H. Kogelnik. Filter response of nonuniform almost-periodic structures. The Bell System Technical Journal, 55(1):109-127, January 1976.

[Kom91] J.J. Komiak and A.K. Agrawal. Design and performance of octave S/CBand MMIC T/R modules for Multi-Function phased arrays. IEEE Transactions on Microwave Theory and Techniques, 39(12):1955-1963, December 1991.

[Law88] M. Lawrence. Optics illuminate next-generation radar systems. Microwaves \& $R F$, pages 165-191, June 1988.

[Lem94] L.J. Lembo, T. Holcomb, M. Wickham, P. Wisseman, and J.C. Brock. Lowloss fiber optic time-delay element for phased array. In Proceedings SPIE Vol. 2155 (Optoelectronic Signal Processing for Phased-Array Antennas, Los Angeles, CA), volume 2155, pages 13-23, 1994.

[Lev78] A.M. Levine. Fiber optics for radar and data systems. In Proceedings SPIE Vol. 150 (Laser 8 Fiber Optics Communications, Bellingham, MA), pages 185-192, 1978.

[Li97] R.L.Q. Li and R.T. Chen. 3-bit substrate-guided-mode optical true-timedelay lines operating at $25 \mathrm{GHz}$. IEEE Photonics Technology Letters, 9(1):100-102, January 1997.

[Lis91] Marco Lisi. Active beam-forming network activities at alenia spazio. In Proceedings of an ESA Workshop on Advanced Beamforming Networks for Space Applications, pages 1.9.1-1.9.15, 1991.

[Lo88] Y.T. Lo and S.W. Lee. Antenna Handbook "Theory, Applications and Design", chapter 18 Practical Aspects of Phased Array Design, pages 1826:18-30. Van Nostrand Reinhold, 1988.

[Loh95] W.H. Loh, M.J. Cole, M.N. Zervas, S. Barcelos, and R.I. Laming. Complex grating structures with uniform phase mask based on moving fiber scanning beam technique. Optics Letters, 20:2051-2053, 1995. 
[Mac87] T. Macnamara. Simplified design procedures for butler matrices incorporating $90^{\circ}$ hybrids or $180^{\circ}$ hybrids. IEE Proceedings Pt. H, 134(1):50-54, February 1987.

[Mag94] J. Magarshack. A new digital phase shifter architecture suitable for MMIC's. IEEE Transactions on Microwave Theory and Techniques, 42(1):154-156, January 1994.

[Mai82] R.J. Mailloux. Phased array theory and technology. Proceedings of the IEEE, 70(3):246-291, March 1982.

[Mai93a] R.J. Mailloux. Phased Array Antennas Handbook. Artech House Antenna Library. Artech House, 1993.

[Mai93b] R.J. Mailloux. Phased array error correction scheme. Electronics Letters, 29(7):573-574, April 1993.

[Mar94] J. Martin and F. Ouellette. Novel writing technique of long and highly reflective in-Fibre gratings. Electronics Letters, 30(10):811-812, May 12th 1994.

[Mat97] P.J. Matthews, M.Y. Frankel, and R.D. Esman. A wideband, fiber-optic, true time-steered array receiver. In 1997 IEEE MTT-Symposium Digest, pages TU4E-1, 1997.

[McF79] J. McFarland. The $\mathrm{RN}^{2}$ multiple beam array family and beam forming matrix. In 1979 IEEE AP/S International Symposium Digest, Seattle, Washington, June 1979.

[McL80] W.V. McLevige and V. Sokolov. Microwave switching with ParallelResonated GaAs FETs. IEEE Electron Devices Letters, EDL-1(8):156-158, August 1980.

[McL81] W.V. McLevige and V. Sokolov. Resonated GaAs FETs devices for microwave switching. IEEE Transactions on Electron Devices, ED-28(2):198204, February 1981.

[Mel89] G. Meltz, W.W. Morey, and W.H. Glenn. Formation of bragg gratings in optical fibers by a transverse holographic method. Optics Letters, 14:823825, 1989.

[Mer79] R.M. Mersereau. The processing of hexagonally sampled Two-Dimensional signals. Proceedings of the IEEE, 67(6):930-949, June 1979.

[Mer83] R.M. Mersereau and T.C. Speake. The processing of periodically sampled multidimensional signals. IEEE Transactions on Acoustics, Speech, and Signal Processing, 31(1):188-194, February 1983. 
[Mes84] G.J. Meslener. Chromatic dispersion induced distortion of modulated monochromatic light employing direct detection. IEEE Journal of Quantum Electronics, QE-20(10):1208-1216, October 1984.

[Mil64] C.J. Miller. Minimizing the effects of phase quantization errors in an electronically scanned arrays. In Proceedings 1964 Symposium Electronically Scanned Array Techniques and Applications, volume 1, pages 17-38. RADC, Griffiss AFB, NY, 1964. RADC-TDR-64-225.

[Mol95] A. Molony, C. Edge, and I. Bennion. Fibre grating time delay element for phased array antennas. Electronics Letters, 31(17):1485-1486, 1995.

[Moo64] H.J. Moody. The systematic design of the butler matrix. IEEE Transactions on Antennas and Propagation, AP-11:786-788, 1964.

[Mot88] R.C. Mott et al. Monolithic transmit modules for a multibeam ku-band phased array antenna. In 18th European Microwave Conference Proceedings, pages 759-763, September 1988.

[Opp89] Alan V. Oppenheim and Ronald W. Schafer. Discrete-Time Signal Processing, chapter Computation of the Discrete Fourier Transform, pages 587-622. Prentice Hall Signal Processing Series. Prentice Hall, 1989.

[Oue94] F. Ouellette, J.F. Cliché, and S. Gagnon. All-fiber devices for chromatic dispersion compensation based on chirped distributed resonant coupling. IEEE Journal of Lightwave Technology, 12(10):1728-1738, October 1994.

[Oze92] Takeshi Ozeki. Optical equalizers. Optics Letters, 17(5):375-377, 1992.

[PA96] Daniel Pastor Abellán. Redes de Difracción Sobre Fibra Óptica Para Procesamiento de Señales Fotónicas. Tesis doctoral, Escola Tècnica Superior d'Enginyers de Telecomunicació de València, Octubre 1996.

[Pao88] A. Paolella and P.R. Herczfeld. Optical control of a GaAs MMIC Transmit/Receive module. In 1988 IEEE MTT-Symposium Digest, pages 959962, JJ-3, New York, NY, USA, May 1988.

[Phi91] M.R. Phillips, T.E. Darcie, D. Marcuse, G.E. Bodeep, and N.J. Frigo. Nonlinear distortion generated by dispersive transmission of chirped IntensityModulated signals. IEEE Photonics Technology Letters, 3(5):481-483, May 1991.

[PML94] France Philips Microwave Limeil. D02AH Design Manual. PML-G-SC0008-E/APD, 1.1 edition, March 1994.

[Ram87] P. Ramanujam, P.I.B. Clarricoats, and R.C. Brown. Offset spherical reflector with low sidelobe radiation. IEE Proceedings Pt. H, 134(2):199-204, 1987. 
[Riz97] N.A. Riza. Analog vector Modulation-Based widely tunable frequency photonic beamformer for Phased-Array antennas. IEEE Transactions on $\mathrm{Mi}$ crowave Theory and Techniques, 45(8):1508-1512, August 1997.

[RM98] Santos Regidor Marta. Aplicación de las redes de difracción a las arquitecturas conformadoras de haces. estudio de diferentes técnicas electroópticas de modulación. Proyecto final de carrera, Escola Tècnica Superior d'Enginyers de Telecomunicació de València, Mayo 1998.

[Roe92] A.G. Roederer. Semi-Active multimatrix satellite antennas. In Proceedings of the COST-223 - ESA Workshop on Active Antennas, pages 4.2.1-4.2.14, June 1992.

[Rom97] J.E. Román, M.Y. Frankel, P.J. Matthews, and R.D. Esman. Time-steered array with a chirped grating bemaformer. Electronics Letters, 33(8):652653, 1997.

[Ros86] A. Rosen et al. Optically controlled lateral PIN diodes and microwave control circuits. RCA Review, 47(4):443-456, December 1986.

[Ruz50] J. Ruze. Wide-Angle Metal-Plate optics. Proceedings of the IRE, 38:53-69, 1950.

[Sae93] R. Saedi, J. Wen, N. Samant, A. Daryoush, D. Sturzbecher, and P. Herczfeld. Comparison of CPU level data mixing to $\mathrm{T} / \mathrm{R}$ level data mixing architectures in optically controlled phased arrays. In 1993 IEEE MTTSymposium Digest, pages 501-504, N-3, Atlanta, GA, 1993.

[Sch95] H. Schmuck. Comparison of optical millimetre-wave system concepts with regard to chromatic dispersion. Electronics Letters, 31(21):1848-1849, October 1995.

[See93] A. Seeds. Optical technologies for phased array antennas. IEICE Transactions on Electron., E76-C(2):198-206, February 1993.

[Sha61] E.D. Sharp. A triangular arrangement of Planar-Array elements that reduces the number needed. IRE Transactions on Antennas and Propagation, 10:126-129, March 1961.

[She68] J.P. Shelton. Multibeam planar arrays. Proceedings of the IEEE, 56(11):1818-1821, November 1968.

[Sko90a] M. Skolnik. Radar Handbook. McGraw-Hill, 2nd edition, 1990.

[Sko90b] M. Skolnik. Radar Handbook, chapter 7.6 Quantization Effects, pages 7.437.49. McGraw-Hill, 2nd edition, 1990.

[Sko90c] M. Skolnik. Radar Handbook, chapter 7.9 Phase shifters, pages 7.63-7.68. McGraw-Hill, 2nd edition, 1990. 
[Smi96] G.H. Smith, D. Novak, and Z. Ahmed. Novel technique for generation of optical SSB with carrier using a single MZM to overcome fiber chromatic dispersion. In International Topical Meeting on Microwave Photonics, pages 5-8, PDP-2, Kyoto (Japan), December 1996.

[Soa83] R. Soares et al. Applications of GaAs MESFETs, chapter 10 Systems Applications of GaAs MESFETs. Artech House, 1983.

[Sor92] R.A. Soref. Optical dispersion technique for Time-Delay beamsteering. Applied Physics Letters, 31(35):7395:7397, December 1992.

[Sor96] R.A. Soref. Fiber grating prism for true time delay beemsteering. Fiber and Integrated Optics, 15:325-333, 1996.

[Ste62] S. Stein. On cross coupling in Multiple-Beam antennas. IRE Transactions on Antennas and Propagation, 10:548-557, September 1962.

[Ste87] W.E. Stephens and T.R. Joseph. System characteristics of direct modulated and externally modulated rf fiber-optic links. IEEE Journal of Lightwave Technology, 5(3):380-387, March 1987.

[Str97] D. Strack, G. Ferrell, A. Piloto, J. Gipprich, J. Fisher, J. Kennedy, D. Heffernan, and S. Caswell. Low cost T/R modules for planar arrays. In 1997 IEEE MTT-Symposium Digest, pages WE2D-1, 1997.

[Tak94] K. Takiguchi, K. Okamoto, S. Suzuki, and Y. Ohmori. Planar lightwave circuit optical dispersion equalizer. IEEE Photonics Technology Letters, 6(1):86-88, January 1994.

[Tan72] R. Tang. Survey of Time-Delay beam steering techniques. In A.A. Oliner and G.H. Knittel, editors, Phased Array Antennas, pages 254-260. Artech House, 1972. Proceedings of the 1970 Phased Array Antenna Symposium.

[Tem72] D.H. Temme. Diode and ferrite phaser technology. In A.A. Oliner and G.H. Knittel, editors, Phased Array Antennas, pages 212-218. Artech House, 1972. Proceedings of the 1970 Phased Array Antenna Symposium.

[Ton96a] D.T.K. Tong and M.C. Wu. A novel multiwavelength optically controlled phased array antenna with a programmable dispersion matrix. IEEE Photonics Technology Letters, 8(6):812-814, June 1996.

[Ton96b] D.T.K. Tong and M.C. Wu. Programmable dispersion matrix using Bragg fibre grating for optically controlled phased array antennas. Electronics Letters, 32(17):1532-1533, August 1996.

[Tun86] S. Tun and N. Adatia. Dual shaped offset gregorian reflector antenna for contoured beam coverage of europe. In IEEE AP-S International Symposium, pages 169-172, 1986. 
[Vos89] D.I. Voskresenskii et al. Electrooptical Arrays. Springer Verlag, 1989.

[Whi62] W.B. White. Pattern limitations in Multi-Beam antennas. IRE Transactions on Antennas and Propagation, 10:430-436, July 1962.

[Whi74] J.F. White. Diode phase shifters for array antennas. IEEE Transactions on Microwave Theory and Techniques, 22(6):658-674, June 1974.

[Yar73] A. Yariv. Coupled-Mode theory for Guided-Wave optics. IEEE Journal of Quantum Electronics, QE-9(9):919-933, September 1973.

[Yng88] K.S. Yngvesson, J.F. Johansson, Y. Rahmat-Samii, and Y.S. Kim. Realisable feed element patterns and optimum aperture efficiency in multiple beam antenna systems. IEEE Transactions on Antennas and Propagation, AP-36:1637-1641, 1988.

[Zmu97] H. Zmuda, R.A. Soref, P. Payson, S. Johns, and E.N. Toughlian. Photonic beamformer for phased array antennas using a fiber grating prism. IEEE Photonics Technology Letters, 9(2):241-243, February 1997. 



\section{Publicaciones relacionadas con la Tesis}

La realización de esta Tesis Doctoral ha dado lugar a las siguientes publicaciones y actas de congresos:

\section{PUBLICACIONES EN REVISTAS INTERNACIONALES}

- J.L. Corral, J. Martí, S. Regidor, J.M. Fuster, R. Laming, J. Cole "Continuously variable true time delay optical feeder for phased array antenna employing chirped fiber gratings", IEEE Transactions MTT/Journal of Lightwave Technology- Special Issue on Microwave Photonics, 45(8):1531-1536, August 1997.

- J.L. Corral, J. Martí, J.M.Fuster, R.I. Laming, M.J. Cole. "True Time Delay Scheme for Feeding Optically Controlled Phased Array Antennas Using Chirped Fibre Gratings". IEEE Photonics Technology Letters, 9(11):1529-1531. November 1997.

- J.L. Corral, J. Martí, J.M. Fuster, R.I. Laming. "Dispersion-induced bandwidth limitation of variable true time delay lines based on linearly chirped fibre gratings". Electronics Letters, 34(2):209-211. January 1998.

PUBLICACIONES INTERNAS DE LA AGENCIA ESPACIAL EUROPEA

- Juan L. Corral. "D02AH large signals models". ESA/ESTEC Internal Report. Ref: XRM/122.94/JLC. July 1994.

- Juan L. Corral. "Design of a $24 \mathrm{GHz}$ Phase Shifter". ESA/ESTEC Internal Report. Ref: XRM/085.95/JLC. May 1995.

- Juan L. Corral. "Design of a low mass RF FFT Beamforming Network". ESA/ESTEC Internal Report. Ref: XRM/101.95/JLC. June 1995.

- Juan L. Corral. "Software for Beamforming Analysis". ESA/ESTEC Internal Report. Ref: XRM/159.95/JLC. September 1995.

- Juan L. Corral. "Design of a $24 \mathrm{GHz}$ Phase Shifter (RFOW measurements addendum)". ESA/ESTEC Internal Report. Ref: XRM/085.95/JLC. September 1995.

\section{ACTAS DE CONGRESOS INTERNACIONALES}

- F. Coromina, J. Ventura, J.L. Corral, Y. Bonnaire y A. Dravet. "New multibeam beamforming networks for phased array antennas using advanced MMCM technology", 1996 IEEE MTT-Symposium Digest, pp 79-82, San Francisco, June 1996. 
- F. Coromina, J. Ventura y J.L. Corral. "New highly miniaturised multibeam RF beamforming networks for phased array antennas using MMIC and multilayer technologies", Second European workshop on Mobile/Personal Satcoms EMPS'96. Rome, October 1996.

- J.L. Corral, J. Martí, J. Capmany, R.I. Laming y M.J. Cole. "True Time Delay Scheme for Continuous Optical Control of Phased Array Antennas Employing Chirped Fiber Gratings". 1997 European Microwave Conference. Paper B-9.3. Tel-Aviv, September 1997.

- J.L. Corral, J. Martí, J.M. Fuster, D. Sánchez. "Continuously Variable True Time-Delay Optical Feeder For Phased-Array Antenna based on Chirped Bragg Optical Fibre Gratings". COST 260 Workshop on Smart Antennas. Dubrovnik (Croatia). 11 $11^{t h}-12^{\text {th }}$ December 1997.

- J.L. Corral, J. Martí y J.M. Fuster. "Optical beamforming network based on chirped fiber gratings continuously variable true-time-delay lines", 1998 IEEE MTT-Symposium Digest, Paper TH2C-5. Baltimore, $8^{\text {th }}-12^{\text {th }}$ June 1998.

\section{Otras publicaciones científicas del autor}

- J.M. Fuster, J. Martí, V. Polo y J.L. Corral. "Fiber-optic microwave link employing optically amplified electrooptical upconverting receivers". IEEE Photonics Technology Letters. Vol. 9, no. 8, pp. 1161-1163. August 1997.

- J.M. Fuster, J. Martí y J.L. Corral. "Chromatic dispersion effects in electrooptical upconverted millimetre-wave fibre optic links". IEE Electronics Letters. Vol. 33, no. 23, pp. 1969. 13 November 1997.

- V.E. Boria y J.L. Corral. "Modelización de discontinuidades en guía rectangular y su aplicación al diseño de filtros paso-banda", VIII Symposium Nacional URSI 93 València, pp 541-545.

- V.E. Boria y J.L. Corral. "Expansion of Libra Senior Library with new elements for rectangular waveguides" EESOF Users Group Symposium 1993.

- J.M. Fuster, J. Martí y J.L. Corral. "Experimental reduction of chromatic dispersion effects in electro-optical up-converted millimetre-wave fiber-optic links". OFC'98. February 1998. 